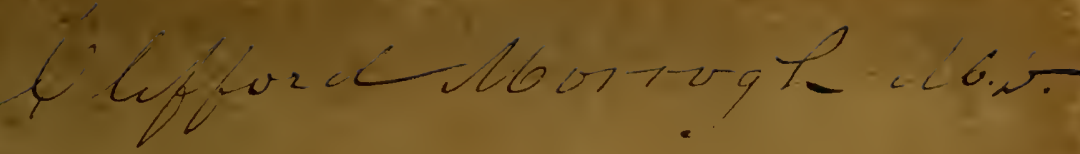 A TREATISE \\ LOCALIZED ELECTRIZATION,
}

AND ITS APPLICATIONS TO PATHOLOGY AND THERAPEUTICS.

\section{BY DR. G. B. DTCHEN NE.}

TRANSLATED FROM THE THIRD EDITION OF THE ORIGINAL,

By HERBERT TIBBITS, M.D.,

LICENTIATE OF THE ROYAL COLLEGE OF PHYSICIANS OF LONDQN;

MEDICAL SUPERINTENDENT OF THE NATIONAL MOSPITAL FOR THE PARALYSED AND EPILEPTIC.

WITH NUMEROUS ILLUSTRATIONS,

AND NOTES AND ADDITIONS BY THE TRANSLATOR.

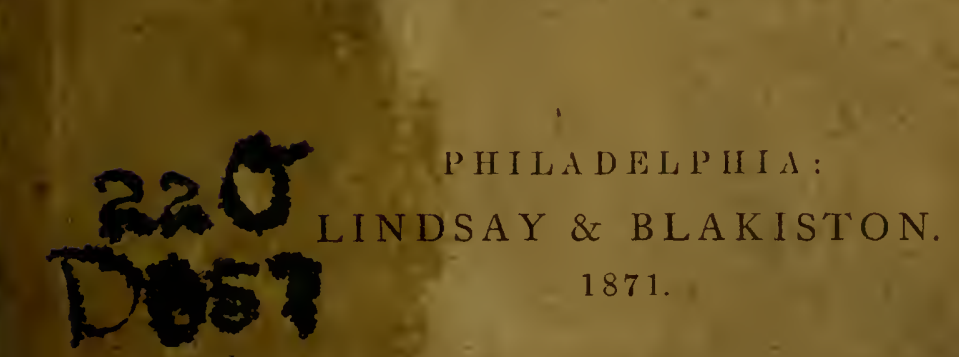



S Muonoge 



\title{
WORKS ON OBSTETRICS,
}

\author{
DISEASES OF. WOMEN, \\ AND CHILDREN.
}

PUBLISHED BY

\section{LindSAY \& BLAKISTON,}

\section{Medical Publishers and Booksellers,}

No. 25 South Sixth Street, Philadelphia.

JUST READY.

\section{A Manual of Midwifery.}

Including the Signs and Symptoms of Pregnancy, Obstetric Operations, Diseases of the Puerperal State, \&c., \&c. By Alfred Meadows, M.D., Member of the Royal College of Physicians, \&c., \&c. First American from the Second London Edition, with numerous Illustrations. Price,. . . $\$ 3.00$

"Those who read the first edition of this work will bear us out in thinking that Ir. Meadows's Manual forms one of the most convenient, practical, and concise books yet published on the subject. It was especially good as a sturlent's manual, and the author has, in his second edition, songht to make it of equal value to the practitioner. The part which treats of obstetric operations has been well revised, and has received numerous additions, and the several chapters on Unnatural and Complex Labors likewise comprise much new matter. Upwards of ninety new engravings have been inserted in this edition, and, with a view to facilitate reference, the author has furnished it with a very full and complete table of contents and index. We can cordially recommend this manual as aecurate and practical, and as containing in a small compass a large amount of the kind of information suitable alike to the student and practitioner."- 


\section{Cazeaux's Great Work on Obstetrics.}

A Theoretical and Practical Treatise on Midwifery, including the Diseases of Pregnancy and Parturition. Fifth American, translated from the Seventh French Edition. Revised and Annotated by S. Tarnier, Adjunct Professor in the Faculty of Medicine of Paris. Greatly enlarged and im. proved. Over 1100 royal octavo pages, with numerous Lithographic and other [l]ustrations on Wood.

Price, bound in cloth, bevelled boards, . . \$6.50 " " leather, . . . . . 7.50

\section{The Change of Life in Health and Disease.}

A Practical Treatise on the Nervous and other Affections incidental to Women at the Decline of Life. By Edward Јон TiLt, M.D. From the Third London Edition. In one volume, Octavo. Price, . . . . . . . . $\$ 3.00$

"The great abilities of Dr. Tilt, his extensive knowledge and his literary power, are well shown in this book, and we are pleased to say that it has substantial and peculiar merits. It contains many sage, practical recommendations, and will amply repay perusal."-Edinburgh Medical Journal.

\section{Dillenberger's Handy Book}

Of the Treatment of Women and Children's Diseases, according to the Vienna Medical School.

Part I. The Diseases of Women.

Part II. The Diseases of Children.

Translated from the Second German Edition, by P. Niсноц, M.D. One volume $12 \mathrm{mo}$. Price, . . . . .\$1.75

\section{The Practice of Medicine and Surgery}

Applied to the Diseases and Accidents incident to Women. By W. H. BYFord, A.M., M.D., Professor of Obstetrics and Diseases of Women and Children in the Chicago Medical College, \&c. Second Edition, Revised and Enlarged, with additional Illustrations. Price, . . . . . \$5.00

The rapill sale of the first elition of this book, which was exhausterl in a little more than a year, has enabled the author to carefully revise the whole work, add many improvements, anı to make a large addition of new matter, without, however, materially increasing the size of the volume. 


\section{Byford on the Chronic Inflammation and}

Displacements of the Unimpregnated Uterus. A New, Enlarged, and thoroughly Revised Edition, with numerous Illustrations. Price, . . . . . . . . . . . . \$3.00

"This work will be found particularly useful to general practitioners. The different subjects are treated plainly and concisely, anı just such information is given as will be of the most practical value. While prolixity is avoided, nothing of real importance is omitted. In prepariug the second edition it has been the object of the author to add to the usefulness of the work by thoroughly revisiug, correcting, enlarging, and illustrating it."-Cincinnati Medical Repertory.

\section{The Diagnosis and Treatment of Diseases of} Women, including the Diagnosis of Pregnancy. By Graily Hewitt, M.D., Lecturer on Midwifery and Diseases of Women and Children at St. Mary's Hospital Medical School, \&c. New Edition, with a new series of Illustrations. \$5.00

\section{A Practical Treatise on the Diseases of Chil-} dren. By J. Forsyth Meigs, M.D., Fellow of the College of Physicians of Philadelphia, \&c., \&c., and William Pepper, M. D., Physician to the Philadelphia Hospital, \&c., \&c. Fourth Edition, thoroughly Revised and greatly Enlarged. Over 900 royal octavo pages. Price, . . . . . \$6.00

"The most thorongh and practical work on the subject now before the profession."

A Practical Treatise on the Diseases of Infancy and Childhood. By 'Thomas Hawkes Tanner, M.D. The Second Edition, Revised and Enlarged, by Alfred Meadows, M.D., London, M.R.C.P., Physician to the Hospital for Women, and to the General Lying-in Hospital. One volume, Octavo. Price, . . . . . . . \$3.50

\section{A Clinical Treatise on the Diseases of Chil-}

dren. By Thomas Hillier, M.D., Physician to the Hospital for Sick Children, and to University College Hospital, \&c., \&c. Octavo. Price, . . . . . . . . \$3.00 


\section{The Surgical Diseases of Infancy and Child-}

hood. By J. Holmes, M.A., Surgeon to the Hospital for Sick Children, \&c. Second Edition, Revised and Enlarged. Octavo. Price, . . . . . . . . . . . . \$9.00

The Functions and Disorders of the Reproductive Organs in Childhood, Youth, and Advanced Life. By William Acton, M.D. A New American from the last London Edition. Octavo. Price, . . . . . . $\$ 3.00$

\section{Feeticide, or Criminal Abortion.}

By Hugh L. Hodge, M.D., Emeritus Professor in the University of Pennsylvania. Price in paper, $30 \mathrm{cts}$; in cloth, 50

\section{The Philosophy of Marriage}

In its Social, Moral, and Physical Relations. With an Account of the Diseases of the Genito-Urinary Organs, the Physiology of Generation in the Animal and Vegerable Kingdoms, \&c., \&c. By Michael Ryan, M.D., Member of the Royal College of Physicians and Surgeons in London, \&c. Price, . \$1.00

\section{On Intermarriage.}

Or, the Mode in which, and the Causes why, Beauty, Health, and Intellect result from certain Unions, and Deformity, Disease, and Insanity from others. With Illustrations. By Alexander WAlKer, author of "Woman," "Beauty," Sc., \&c. 1 2 mo. Price, . . . . . . . . . . . \$1.50

LINDSAY \& BLAKISTON'S complete Descriptive Catalogue of their publications, together with a classified and priced list of all recent Medical Books, American and English, furnished or mailed free upon application. 
A TREATISE

on

LOCALIZED ELECTRIZATION. 



\title{
A TREATISE
}

oN

\section{LOCALIZED ELECTRIZATION,}

\author{
AND ITS APPLICATIONS TO PATHOLOGY \\ AND THERAPEUTICS.
}

BY D R. G. B. D LCHEN N E.

TRANSLATED FROM THE THIRD EDITION OF THE ORIGINAL,

By HERBERT TIBBITS, M.D.,

LICENTIATE OF THE ROYAL COLLEGE OF PHYSICIANS OF LONDON;

MEDICAL SUPERINTENDENT OF THE NATIONAL HOSPITAL FOR THE PARALYSED AND EPILEPTIC.

WITH NUMEROUS ILLUSTRATIONS, AM I THE

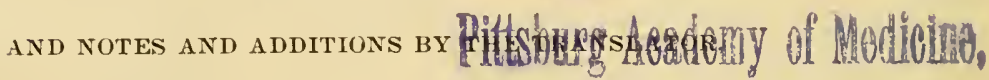
AOT TO BE RETROTIA

P H I L A D E L P H I A :

$$
120.2700
$$

LINDSAY \& BLAKISTON. 


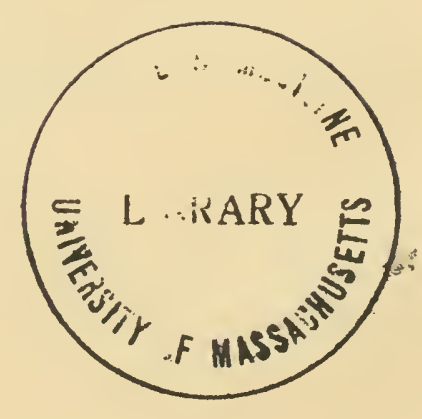




\section{NOTE BY THE TRANSLATOR.}

Tuis translation is made from the third edition of the original Work, now in course of passing through the press. The present Part includes all that had been printed at the time of the investment of Paris by the German army, and, in consequence of that investment, it is published before the original is giren to the world.

Duchenne's great work on 'Localized Electrization' is not only a well-nigh exhaustive treatise on the medical uses of electricity, but it is also an elaborate exposition of the different diseases in which electricity has proved to be of value as a therapeutical and diagnostic agent. No similar treatise exists in the English language: indeed the work is unique.

It has not been without hesitation that I have ventured to add certain notes to the original. These additions have been made with strict reference to the requirements of English medical practitioners, and they have particularly in view to facilitate the practice of medical electricity. In making them, I have been guided partly by the large experience of the National Hospital for the Paralysed and Epileptic, and partly by the observations derived from private practice.

H. T.

70, Guilford Street, Russell Square, W.C.,

Decumber, 1870.

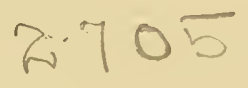





\title{
CON'TENTS OF PART I.
}

\author{
ME'THODOLOGY.
}

\section{CHAPTER I.}

Medical Electricity .. $\quad$.. 3

$\begin{array}{lllllllllll}\text { Part I.-Static Electricity } & \text {. } & \text {.. } & \text {. } & \text {. } & \text {. } & & \text {. } & \text {.. } & \text {.. } & 4\end{array}$

$\begin{array}{lllllllll}\S & \text { I. Physiological properties } & \text {. P } & \text {. } & \text {.. } & \text {. } & \text {.. } & \text {.. } & 4\end{array}$

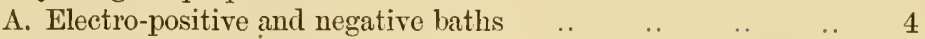

B. Sparks and the Leyden jar $\quad \begin{array}{llllllll} & \text {. } & \text {. } & \text {. } & \text {. } & & \text {.. } & 5\end{array}$

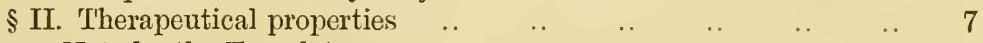

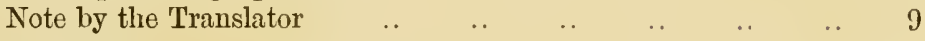

$\begin{array}{llllllll}\text { PART II.-Contact Electricity }(\text { Galvanism) } & . & & \ldots & \ldots & \ldots & \ldots & 10\end{array}$

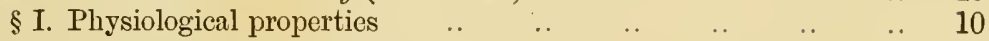

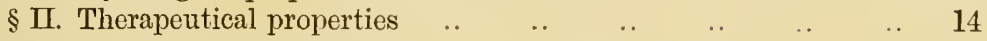

$\begin{array}{lllllllll}\text { PART III.-Induced Electricity } & . & & . & \ldots & \ldots & \ldots & \ldots & 17\end{array}$

$\S$ I. Physiological action of the current of the first helix, and of the

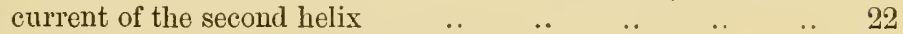

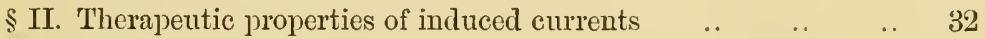

Different denominations of the various kinds of electricity $\quad . . \quad 36$

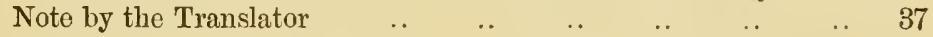

\section{CHAPTER II.}

\section{Localized Electrization.}

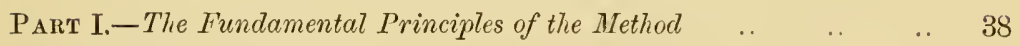

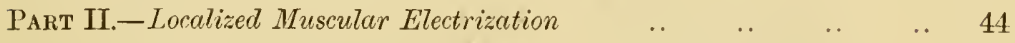

§ I. Localized muscular electrization by static electricity $\quad \ldots \quad$.. 44

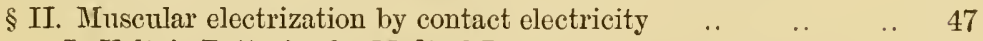

I. Voltaic Batteries for Medical Purposes

A. Batteries of large surface with inconstant currents $\quad . .48$

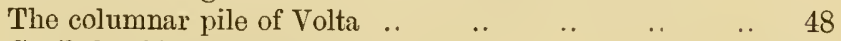

$\begin{array}{lllllll}\text { Cruikshank's trough battery .. } & \text {.. } & \text {.. } & \text {. } & \text {.. } & 48\end{array}$

$\begin{array}{llllllll}\text { The cylindrical glass battery .. } & \text {.. } & \text {.. } & \text {.. } & \text {.. } & 49\end{array}$

$\begin{array}{llllllll}\text { Porcelain trough battery } & \text {.. } & \text {.. } & \text {. } & \text {. } & \text {.. } & 49\end{array}$

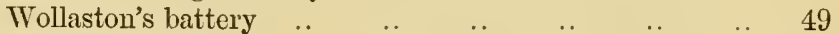

$\begin{array}{lllllllll}\text { Berzelius's battery } & . & & \text {. } & \text {.. } & \text {.. } & \text {. } & \text {. } & 49\end{array}$

B. Batteries of large surface with constant currents .. $\quad$.. 50

$\begin{array}{llllllll}\text { Daniell's battery } & \text {. } & \text {.. } & \text {. } & \text {.. } & \text {.. } & \text {.. } & 50\end{array}$

$\begin{array}{lllllllll}\text { Grove's battery } & \text {. } & \text {.. } & \ldots & \text {.. } & \text {.. } & \text {.. } & 50\end{array}$ 
Parelles' balloon battery

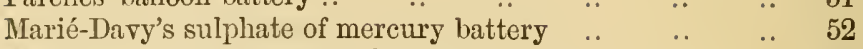

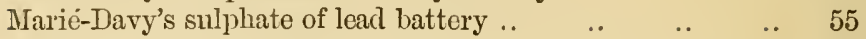

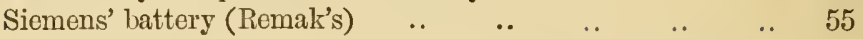

Note by the Translator:-(a) Stöhrer's battery; (b) the Becker-

$\begin{array}{llllllll}\text { Muirhead battery } & . & & . & & & & \\ \end{array}$

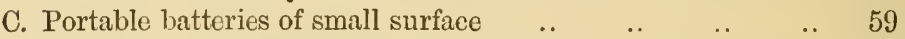

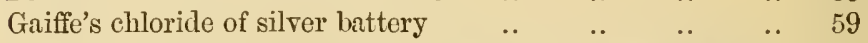

Small columnar pile of the Conservatoire des Arts et Métiers 61

Note by the Translator:-Hammond's columnar battery $\quad$.. 61

Galvanic chains of Golberger and Pulvermacher $\quad$.. $\quad$.. 63

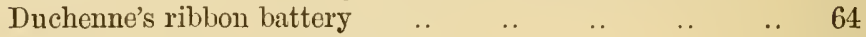

$\begin{array}{lllllll}\text { Mathieu's small tension-battery } & \ldots & \ldots & \ldots & & . . & 65\end{array}$

Note by the Translator:-Foveaux's portable battery $\quad$.. 66

Rulumkorff's portable constant-current battery $\quad$.. $\quad$.. 217

II. Operative Procedures for Localized Galvanization $\quad$.. $\quad$.. 69

§ III. Localized electrization by induced electricity, or localized

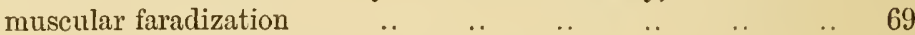

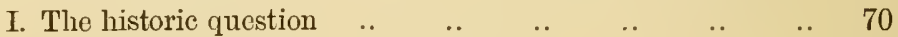

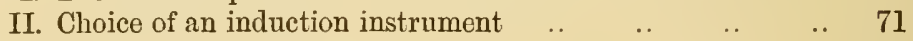

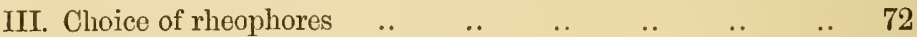

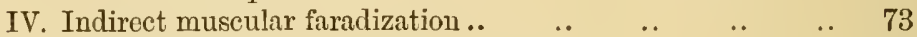

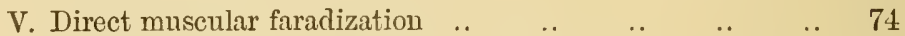

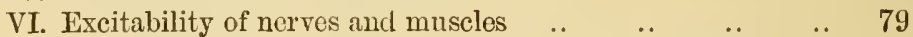

A. Excitability of the motricity of nerves, and the con-

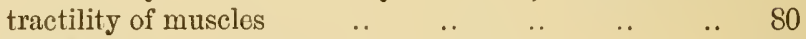

B. Excitability of the sensibility of muscles $\quad \ldots \quad$.. $\quad 82$

C. The points of selection in the practice of localized

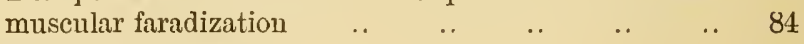

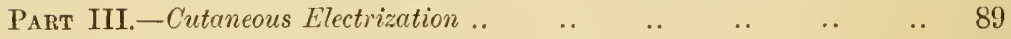

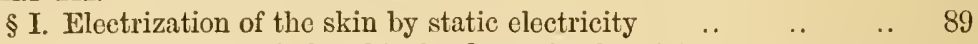

$\S$ II. Electrization of the skin by dynamic electricity, or cutaneous

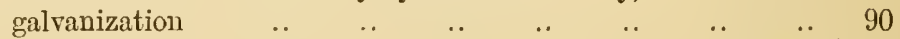

$\S$ III. Electrization of the skin by the induced current, or cutancous

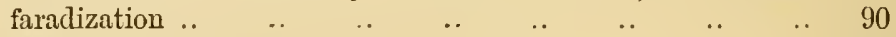

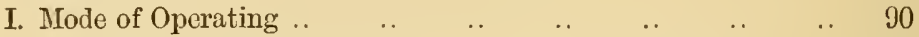

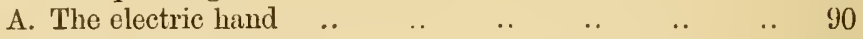

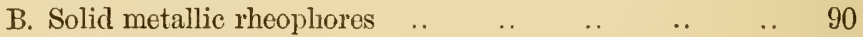

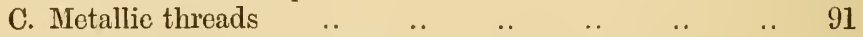

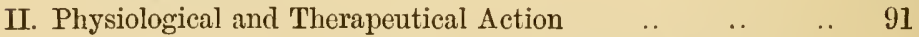

ParT 1V.-Faradization of Internal Organs, the Genital Organs, and of

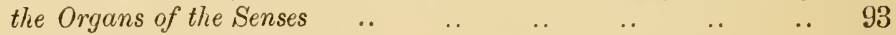

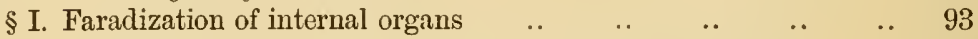

1. Furadization of the rectum and muscles of the anus .. $\quad$.. 93

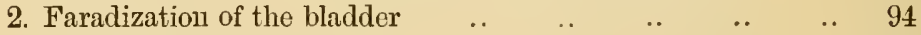

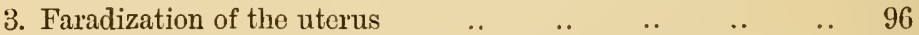

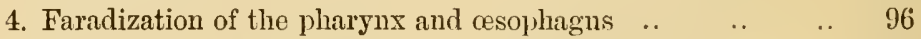

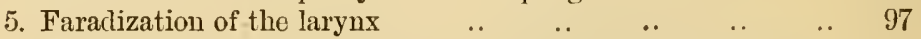

Note by the Translator :-Ziemssen on laryngeal electrization . 98

6. Faradization of the stomach, the liver, the lungs, and the

$\begin{array}{lllllllll}\text { heart } & . . & . . & . & . & & & & \end{array}$ 
7. Faradization of the diaphragm PAst

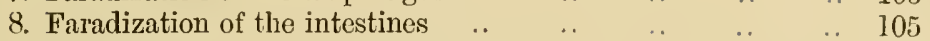

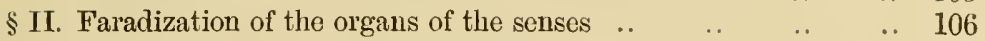

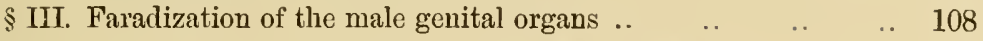

PART V.-The General Effects of Localized Faradization _. . . 109

\section{CHAPTER III.}

Historical and Critical Odservations upon the Principal Methods of Electrization .. $\quad$.. $\quad$.. $\quad$.. 114

Pant I.-On the Value of Electro-puncture, as applied to the Treutment $\begin{array}{lllllllll}\text { of Paralysis } & \text {. } & \text {.. } & \text {.. } & \text {.. } & \text {.. } & \text {.. } & \text {.. } & \text {.. }\end{array}$

Part II.-On the Value of Electrization by Reflex Action, as applied to

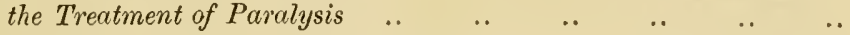

$\S$ I. Passage of induced currents from the nerve-extremities to the

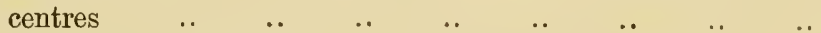

\$II. Excitation by reflex action in passing induced currents from

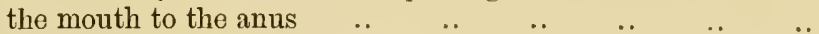

\$III. Excitation of the innervation of the heart and respiratory organs by means of cutaneous faradization of the precordial region

I. In the treatment of asphyxia

II. In the treatment of grave functional disorders of the cardiae circulation and the respiration, symptomatic of a dynamic

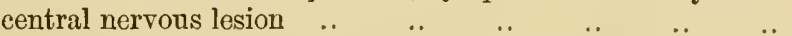

PART III.-On the Therapeutic value of Localized Faradization ..

134

$\S$ I. Brief exposition of elinical facts, which establish that localized faradization is of great value in the treatment of atrophic

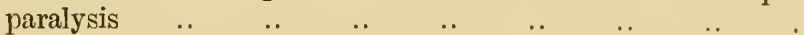

$\$$ II. Critical examination of the electro-physiological experiments which have served as the foundation of the attacks recently directed against the therapentic valne of localized faradization

$\S$ III. Upon what class of nerves does localized faradization exert its influence in order to increase the activity of the local cireulation?

PART IV.-The Therapeutical value of Galvanization by Interrupted Currents, and by Continuous Currents, direct or indirect..

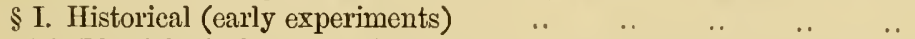

\$ II. Physiological action of continnous currents, direct or inverse, applied to the nerves of man, upon sensibility, or muscular contractility :-I. Duchenne's first experimental researches ..

II. The experimental researehes of Remak $\quad . \quad$.. $\quad .$.

\$III. Duchenne's latest researches on the therapentic action of 173 galvanization by interrupted or by continuous currents in the treatment of paralytic or nervous affections, as compared with localized faradization

Results of Dnchenne's investigations into the therapentic application of direct localized musenlar galvanization by intermittent currents 
Results of Duchenne's investigations into the therapeutic application of reflex galvanization by continuous currents, mingled with feeble intermissions (labile currents), as compared with

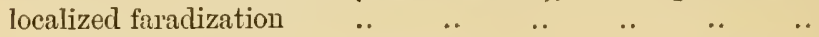

A. Atrophic paralysis consecutive to traumatic lesions of

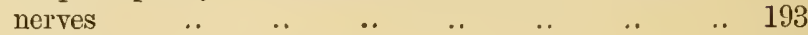

$\begin{array}{lllllllll}\text { B. Lead-palsy } & \text {. } & \text {.. } & \text {.. } & \text {.. } & \text {.. } & \text {.. } & \text {.. } & 194\end{array}$

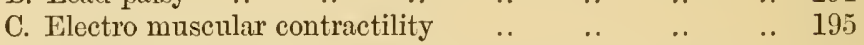

$\begin{array}{llllllll}\text { D. Paralytic contractions } & \text {. } & \text {. } & \text {.. } & \text {.. } & \text {.. } & 197\end{array}$

E. Functional spasms, scrivener's palsy, neuralgic contractions called rheumatic, reflex ascending contractions

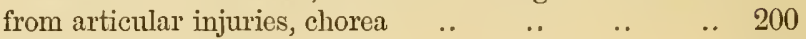

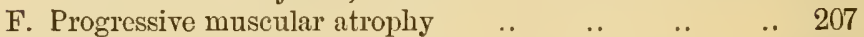

Galvanization by permanent continuous constant currents .. 208

The therapeutic action of galvanization of the skin, as compared

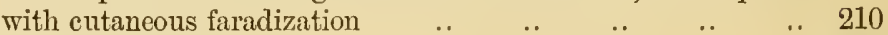

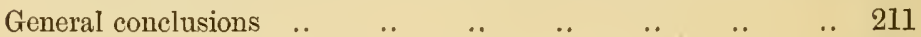

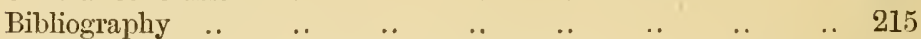

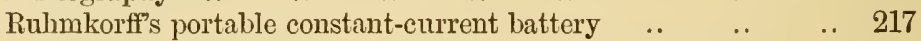

Note by the Translator:-Ziemssen on certain questions con-

cerning the value of galvanic currents in medicine .. $\quad . . \quad 219$

\section{CHAP'TER IV.}

Electro-Medioal Instruments with Regard to their applidation in Pathology, in Physiology, and in Therapeutics .. 232

PART I.-The Properties which Instruments should possess I .. $\quad$. 232

PAnT II.-The Author's large Double-Current Tolta-Faradic Instiument 243

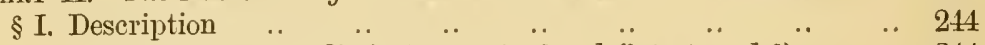

A. Large volta-faradic instrument, closed (latest model) $\quad$.. 244

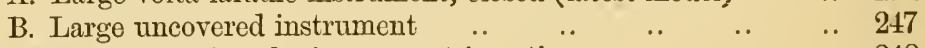

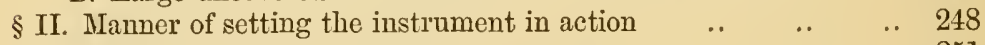

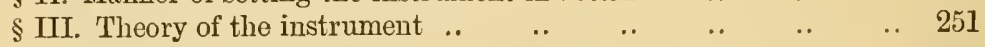

PART III.-Small Double-Current Volta-Faradic Instrument $\quad . . \quad$.. 266

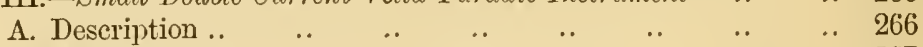

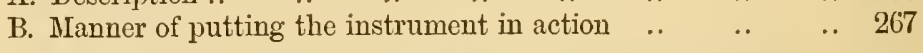

PART IV._The Author's Double-Current Magneto-Faradic Instrument .. 269

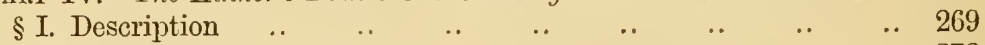

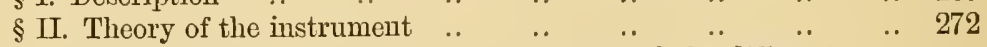

$\S$ III. Explanation of its principal properties, and the different com-

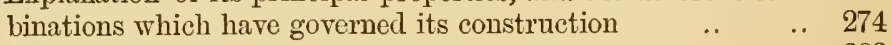

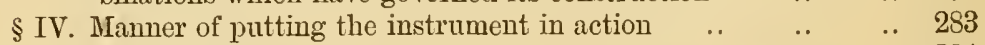

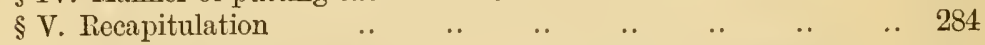

PART V.-Historical and Critical Account of Induction Instruments .. 287

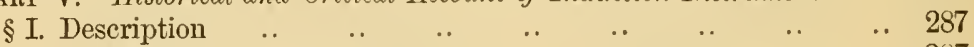

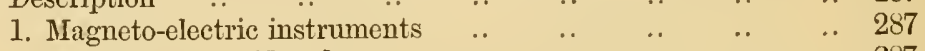

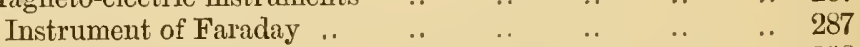

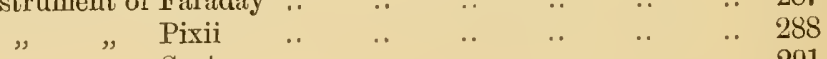

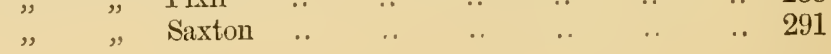


CONTENTS OF PART I.

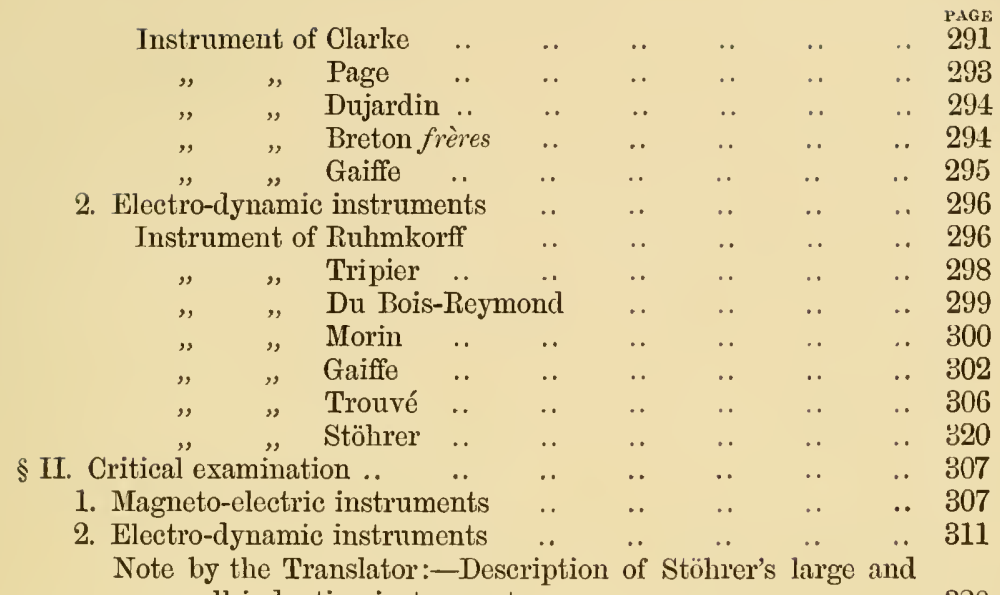

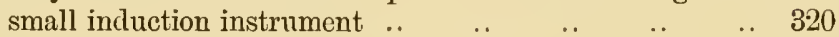





\section{ON \\ LOCALIZED ELECTRIZATION,}

AND ITS APPLICATIONS IN PATHOLOGY AND THERAPEUTICS.

PART I.-MEEHODOLOG Y. 



\title{
LOCALIZED ELECTRIZATION.
}

\section{CH A P T E R I.}

\author{
MEDICAL ELEC'TRICITY.
}

Three rarieties of electricity are employed in medicine-friction electricity, contact electricity, and induced electricity. ${ }^{1}$ The first is also called static electricity; the last two are included under the term dynamic electricity.

The first question, which suggests itself with regard to them, the importance of which is self-evident, is as follows:-In their physiological and therapeutical applications, when it is desired to limit their action within certain organs, is it a matter of indifference which of the three varieties is employed? In other words, are the physiological and therapeutical properties of the three varieties identical?

I have no intention to set forth the various and discordant opinions which lave prevailed upon this point. To do so would be to enter into historical questions, which I am desirous as far as possible to leave unnoticed. But I must observe that several physicians, who have made a special study of the therapentical applications of electricity, have remarked certain differences between the properties of the static and of the dynamic form. Fabré Palaprat, for example, taught that static electricity was chiefly applicable to paralysis of sensation, and to the excitation of the muscles of relation; whilst, on the contrary, contact electricity was only adapted for the excitation of the muscles of organic life, or of delicate organs, such as the eye, the ear, \&c.

These statements have been reproduced in many special treatises, although they did not rest upon any serious research; and although, as I shall presently demonstrate, they were not in harmony with the physiological properties of the different forms of electricity. It is therefore not surprising that they should have been neglected

[1 The two former varieties of electricity are now commonly termed by physicists franklinic electricity and voltaic electricity. It is greatly to be desired that this ter- minology should be adopted in works devoted to the medical application of electricity. $-H . T$.] 
in practice. It would be wrong, however, to suppose that the therapeutical action of electricity is always the same, whatever its source. I make no claim to have entirely solved this difficult question; but I lope to establish that friction electricity, contact electricity, and induced electricity possess special physiological and therapeutical properties, and that each of them will fulfil particular indications.

\section{Part I.}

STATIC ELECTRICITY.

\$ I.-Physiological Properties.

The principal methods employed for the application of static electricity are, as is well known, electrization by simple contact, called the electric bath; electrization by sparks; and, lastly, electrization by the Leyden jar. The other methods do not appear to me sufficiently important to require discussion.

\section{A.-Electro-positive and Negative Baths.}

The electric bath has long been ranked among the most valuable therapeutical agents. According to Giacomini, ${ }^{2}$ the positive form is applied and acts in the following manner:- "The patient, being insulated, is placed in contact with the conductor of the electrical machine. The whole surface of the body is thus electrified, while the air which surrounds the body is rendered electro-negative. The positive electricity by which the organism is charged is confined to the surface, and probably accumulates in the skin, by virtue of the law above-mentioned, for it has no effect upon any internal organs. Neither the pulse, nor the secretions, nor the intellectual functions, nor the respiration, undergo any particular change; and the accumulated electricity which constitutes the bath, escapes from all points of the epidermis, from the hair, beard, and nails," \&c.

Giacomini attributes a hyposthenic influence to the electronegative bath, which withdraws from the body a greater or smaller quantity of its natural electricity. This is done by insulating the patient, and by placing his body, and especially the diseased part, in connection with the cushion or rubber of the machine. The plate, being then turned, discharges vitreous electricity in proportion as it has accumulated. It is evident, according to Giacomini, that the electricity yielded by the cushion is derired from the nerres of the patient, instead of from the ground, as in the case of the positive bath.

2 Cited in La Bibliothèque du Médecin Practicien. Paris, 1850. Vol. xiv. 1. 90. 
This electro-physiological theory has certainly not been founded upon experiment; for a healthy person, subjected to an electric bath, either positive or negative, does not exhibit any symptoms of an influence either excitant or hyposthenic.

\section{B.-Spartis, and the Leyden Jar.}

Whatever may be the form of exciter that is brought into connection with the conductor of an electric machine, and whatever may be the distance that separates this exciter from the skin, the electricity furnished by the machine in action will reunite with that of the body at the epidermic surface, with a greater or less degree of tension. A pointed exciter allows the electricity to escape easily; with a knob the tension is greater and the sparks are less frequent; but each of these forms discharges itself by a single spark. An exciter having a plane surface discharges itself by several sparks at once (from two to five), if it be at a little clistance from the skin.

A metallic brush acts, in this respect, like a plane surface: that is, it gives off only two or three sparks at a time, whatever the number of threads of which it is composed. ${ }^{3}$

Static electricity applied by the exciter-of which the several actions have just been described-produces always the same sensations, differing only in their degree. These sensations may be compared to those produced by the impact of a small sulustance striking upon the slin. They are always disagreeable, however weak the electric tension. The feeling is never very acute, nor like that of a burn, or a puncture, whatever form of exciter may be employed. After a time the skin reddens and becomes more sensitive. To render the discharge more painful, it is necessary to employ a degree of tension that can only be obtained by a Iseyden jar of moderate size, sufficiently charged; but then the excitation will be no longer limited to the skin, and will produce the effects to be described hereafter.

Under weak tension, the action of static electricity can always

3 A brush of badger's hair has been used as an exciter; and this, being in connection with an electric machine, and being helil at a short distance from the skin, occasions a sensation of coolness and a very slight pricking. Such a brush, itself a bad conductor, is charger with positive electricity, the excess of which escapes from the extremities of the hairs, to unite with the electricity of the surrounding air. The effect is a series of rapill and successive re-runions which determine a current of air to the surface of the brush. It is this current, analogous to that which is formed at the surface of the plate of an electric machine in movement, which causes the sensation of coulness produced by the vicinity of the electrified brush of badger's hair. It is evident that this method of electrization is illusory; since the natural electricity of the body undergoes no appreciable infuence. 
be limited to the skin. Such is the tension obtained from an electric machine of medium power: which is hardly enough to produce contraction of the superficial muscles, especially if the cellular tissue be rather abuudant; and, if produced, the contractions will be incomplete.

Although it is possible to confine the action of static electricity to the skin, it is not possible to concentrate its power upon the muscular tissue or upon the sub-cutaneous nerres. In fact, the superficial action of this form of electricity is inseparable from its deep action. In the latter the electric recomposition is still effected at the epidermic surface, and produces a cutaneous sensation that obscures the muscular.

The tension obtainerl by means of a Leyden jar enables the current to penetrate through a considerable thickness of tissue. Thus the current from the jar is sufficient to produce energetic muscular contraction; but however feeble may be the tension, so long as it can be measured by Lane's electrometer, it always produces a shock, that is to say, a contraction and a sensation which extends beyond the point excited, and which is felt most acutely in the nervous centres.

If the exciter be placed over a nervous trunk, the sensation is like that produced by a severe contusion of the nerve, and is followed by a numbness that extends almost to the ultimate ramifications. With a sufficient degree of tension, acting either upon a nerve or upon muscular tissue, the shock is so strong that the whole limb, or even the whole borly, seems as if struck by lightning.

At the spot at which a Leyden jar is discharged, the slin gradually loses colour over an area of two or three centimetres in radius, and becomes of a dull white in a few seconds. The nervous papilla stand erect upon the blanched surface, which is also somewhat lowered in temperature. The person on whom the experiment is tried sometimes experiences a feeling of numbness. Analogous phenomena appear to take place in the muscular tissue suljacent to the portion of skin that is acted upon. I have had occasion to make an electric discharge upon the surface of a denuded muscle, and this muscle lost somewhat of its colour, over a small space, for some time. The cutaneous local phenomena last generally from twenty to thirty minutes; and once, in a delicate person, I saw them continue for three-quarters of an hour. After this time, the blanched spot passes, in a few minutes, from a dull white to an erythematous redness, and undergoes an elevation of temperature appreciable by the thermometer, and sometimes by the sensations of the patient. 
It is universally known that the discharges of static electricity are very small in quantity; and that for this reason their calorific and electrolytic effects are feeble.

Finally, the exciting action of the negative pole is greater than that of the positive, as is also observed in galvanic electricity. ${ }^{4}$

The organic phenomena just described appear to me to establish: (1) that the discharge of a Leyden jar produces at first, locally, a profound torpor, by suspending, for a time, the capillary circulation, and by diminishing the calorification in the tissues upon which it acts; (2) that the excitement produced by this method of electrization is only a lind of reaction, as shewn by the erythematous redness, and by the augmentation of temperature in the parts formerly bleached; (3) that the reaction is established with different degrees of facility.

\section{$\S$ II.-Therapeutical Properties.}

The electro-positive bath, formerly used as a general excitant of the surface of the body, is now commonly abandoned, its therapentical being as little discoverable as its physiological action.

It is not the same, it is said, with the electro-negative bath, which is ranked by the Italian school among their most valuable hyposthenisants. According to Giacomini, the patient subjected to this bath is de-electrized, is consequently depriver of a greater or less quantity of a stimulant analogous to heat, and undergoes a real hyposthenisant effect. Erysipelatous tissues may be seen to become blanched under its influence; and chronic inflammations undergo an unquestionable improvement. Headaches, and neuralgic pains, have been instantly relieved by this lind of electric flux, as by the application of ice, which abstracts heat, and perhaps, at the same time, electricity also. Such are the virtues attributed to the electro-negative bath.

This notion of an electric flux may impress the imagination; but it is difficult to believe that the hyposthenic effect is less doubtful than the excitant property once attributed to the positive bath. It would, however, be wrong to condemn a therapentic agent solely on the ground of the nullity of its physiological action, especially when it is supported by an authority so great as that of Giacomini. It is therefore necessary to determine by fresh trials what is the actual value of the electro-negative bath.

4 This important fact was discovered by M. Chanvean, the Director of the anatomical and physiological courses at the Imperial Veterinary School at Lyons. Sce his memoir: 'Théorie des effets phy- siologiques produits par l'électricite transmise dans l'organisme animal à l'état de courant instantané, et à l'état de courant continu. 
Electrization by sparks produces a sensation analogous to that of whipping, and appears principally adapted for cases in which it is necessary slightly to stimulate the skin; although it would be insufficient if very active stimulation were desired. In fact, to obtain such active stimulation, it would be necessary to have recourse to static electricity of high tension, such as would be furnished by the Leyden jar, and which would also produce such muscular contractions and general shock as to complicate the action upon the skin, and to contra-indicate the employment of the agent. Electrization by sparks is, therefore, useless in cases of profound and obstinate anzsthesia, or where it is wished to produce an instantaneous revulsion, such as would be effected by the actual cautery.

Electrization by sparks, eren when they are yielded by a powerful machine, and by splerical exciters, is only able to produce contraction of some superficial or very excitable muscles, such as the platysma, the upper half of the sterno-mastoid, the margin of the trapezius, and some muscles of the face. In order that static electricity may affect the muscles generally, especially when they are covered by an abundance of cellular tissue, it would be necessary to employ a high tension by means of a Leyden jar. But the resulting shock, which would possibly extend to the nervous centres, would render such an operation dangerous or impracticable.

The knowledge of the local organic changes produced by the discharge of a Leyden jar enables us better to appreciate the therapentic action. It has been already shewn that an organ so electrified only undergoes excitation after having suffered, for some time, from all the effects of torpor. It is easy to conceive that if any organ were exposed to discharges too frequent or too powerful, or if its vitality were already depressed, the stage of electric torpor might be indefinitely prolonged.

However favourable may be the conditions under which it is attempted to practise muscular electrization by the Leyden jar, it will always be imprudent to expose the patient to many discharges. Hence it would be impossible to electrify all the muscles in cases of paralysis of one or more of the limbs.

Moreover, the operation is always painful; since the cutaneous excitation inseparable from the use of static electricity increases in proportion to the increase of tension.

It appears, then, on the whole, that the excitation of muscles by static electricity ought generally to be excluded from practice, and the more so since it may be replaced by another electric agent, which will be described in the fhird section (induced electricity); 
and which excites muscular contractility and sensibility in a more energetic and effectnal manner than the former, without any of the inconveniences that I have described. I purpose to point out in the sequel the cases in which its employment will be useful or necessary.

It is, however, incontestable that static electricity, which for many years was used exclusively in medical practice, has wrought some cures seemingly marvellous. These results shew only that certain paralyses are always cured by electricity, in whatever form and manner it may be employed. Unfortunately, these cases liare been so rare that this mode of using electricity has not borne the test of time, and has been many times laid aside by physicians after having been taken up with some degree of enthusiasm.

[Friction electricity is used somewhat largely at the National Hospital for the Paralysed and Epileptic. It has been found in the praetice of this Hospital, and in private cases coming under my own observation, of considerable value in the treatment of the following affections:-

(a) Fuciul Neurulgia, which has resisted other modes of treatment, is occasionally relieved with rapidity, and permanently, by drawing sparks along the traeks of the affected branch or branches of the trifacial nerre. Oceasionally also, but less commonly, electrization by sparks has partially or altogether relieved obstinate sciatica.

(b) Facial spasm.-A remarkable instance of the effect of electrization by sparks in relieving facial spasm (tic convulsif) occurred in the case of a hospital patient under the eare of Dr. Radcliffe. The patient was a female forty-eight years of age, and she had suffered for thirteen years from spasm of the muscles of the left side of the face. The distortion produced by the spasm was very great, and was apt to be so much exaggerated by slight emotion, even such as would be caused by having to address a stranger, as to make speaking difficult, and to prevent proper attention to her occupatiou as a small shopkeeper. Dr. Radcliffe directed an experimental trial of electrization by sparks along the lines of the nerves distributed to the affected muscles. After the third applieation the spasm was manifestly relieved, the distortion being diminished, and the consecutive paroxysms oceurring less frequently. By persisting with this treatment thrice weekly over a period of two months so great an amount of relief was obtained that little distortion of the face remained, and the patient was able to pursue her business with comfort.

(c) Hysterical Aphonia.-Electrization by sparks over the larynx has proved in the practice of the hospital so effective in the relief of cases of emotional aphonia, even those of long standing, that it is now almost invariably used in the treatment of these eases before having recourse to induced electricity. In six or seven recent cases, electrization by sparks repeated twice or thrice effected a complete cure. One of these cases was of nine, another of six montlss' duration. The remainder had lasted from four weeks to three months. The seventh ease did not receive any benefit from the use of static electricity, and the other forms of the agent proved equally ineffective. The case recovered slowly under general treatment.

(d) Iysterical hiyperasthesia.-Electrization by sparks over the affected spot has often proved of great benefit in removing the localized exeessive sensitiveness not unfrequently found in liysterical cases, particularly in the spinal region.

(e) Trrmor, whether general or local, is sometimes largely relieved hy insulating the patient, and tharging lin with positive elcetricity for a 
period of from twenty minutes to half an hour. For an examination of the probable influence of friction electricity in the treatment of painful and spasmodic affections consult Dr. Radeliffe's 'Lectures on Epileptic, Spasmodic, Neuralgic, and Paralytic Disorders of the Nervous System.'-II. T.]

\section{Part II.}

\section{CONTACT ELECTRICITY (GALVANISM).}

The term dynamic electricity, that is to say, electricity in motion, has been used to denote the electricity produced by contact (galvanism), and the electricity eaused by induction. This form, obtained from voltaic piles, or from an electro-magnetic apparatus, possesses physiological and therapentical properties essentially different from those of static electricity.

The most important property of dynamic electricity, regarded from a physiological or therapeutical point of view, is that by which its action can be directed to, and limited within, almost any organ of the body. It is shown, indeed, by my experiments, that we may at pleasure confine this agent to the skin, or may cause it to penetrate the skin, withont puncture or incision, and may limit its action to the subjacent organs, the nerves, the muscles, or even the bones. Of this I shall give evilence in the sequel.

The cutaneous sensation prodnced by dynamic electricity may range from a simple titillation to the most acute pain. The excitation thus produced may always, by the use of special means, be confined entirely to the skin, whatever be the power of the current.

The same electro-dynamic force, directed upon a muscle, or upon a nerve-trunk, has power to produce the most energetic muscular contraction, without any of the general shock that characterises the action of static electricity, and that usually forbids its employment.

Such are the principal effects to be obtained from dynamic electricity, which includes contact electricity (galvanism) and induced electricity.

But each of these forms of dynamic electricity possesses special properties, physical, chemical, and physiological, such as to prevent them from being employed indifferently in practice. These special properties may even fulfil certain determinate indications, and they therefore require to be separately considered.

\section{§.-_Physiological Properties.}

Quantity and tension being equal, every form of clectro-motor 
apparatus of contact has the same physiological properties, whatever may be the nature of the elements composing it. ${ }^{5}$

The apparatus can be constructed so as to furnish electricity in greater quantity, or of higher tension, according to the effects that it is desired to obtain ; but whatever is done, the different effects are produced simultaneously, and cannot be completely isolated from one another.

The intermittent galvanic current exerts a triple physiological action at each intermission : one at the moment of completing the current, a second at the moment of breaking it, a third during the interval between these acts. The physiological action produced at the instant of interrupting the circuit is weaker than that at the instant of completing it. Thus, with a pile of thirty pairs of Bunsen's elements, muscular contraction is not produced, in the human subject, by interrupting the current; even when moist rheophores are applied over a portion of skin corresponding to the surface of a muscle: but by the same procedure, the electro-physiological effect is very manifest at the completion of the current. 'The power of the interruption increases, however, in proportion to the number of elements in the battery. It appears to me that a Bunsen's pile of 120 pairs produces at the moment of interruption a contraction about equal to that produced by a pile of twenty pairs at the moment of completion.

The physiological action produced in the period between the completion and the interruption of the current is more manifest the longer this period is prolonged. The current passing during the period is called continuous.

Galvanic electricity can be applied either by continuous or by interrupted currents.

The continnous current, limited to the skin, excites, besides the phenomena of sensibility above described, an organic effect more or less considerable, from a simple erythema to the production of an eschar. This organic effect, due to the calorific and electrolytic action of galvanism, is produced but gradually; at least if the battery employed be not very powerful.

A constant and very intense continuons current, traversing a limb longitudinally, appears only to produce fibrillar and oscillatory or irregular contractions; - provided that the current was feeble at the moment of its completion, and was then rapidly raised to its maximum. Such, at least, was the result of a trial made upon myself with a pile of 120 pairs of Bunsen's elements. 'The continuous

5 The quantity of eleetricity furnished | surface of the elements. The tension is hy a pile is in direct proportion to the $/$ in proportion to their number. 
current also produces phenomena of calorification in the deepest parts of the organism. The sensation that I experienced was such as might be produced by the circulation of a hot fluid in the limb galvanized. After a certain time, a deep continuous current produces a sensation of heat that becomes insupportable; but I have not found that the limb is actually raised in temperature. ${ }^{6}$ By

6 When I first recorded this experiment, I committed the error of not describing the manuer in which it was performed; and as it has appeared to some to be difficult, if not impossible, I now detail the cireumstances:- At the time when M. Despretz, Nember of the Institute of Franee, conducted at the Sorbonne his beautiful researches into the simplieity of the elementary bodies, I found in his laboratory a Bunsen's pile of 120 elements of the ordinary size, and with the carbon in the middle. It was with this pile that I worker. It is probable that it no longer possessed its full power; since it had been a long time in action, and had been used for numerous experiments. Still its power was such that, on grasping in each hand a metallic rheophore, euvered with leather thoroughly wetterl, I found it diffienlt to suppoit the shock oansed by the completion of a current of from forty to fifty elements. In order not to expose myself to the shock of the action of 120 elements, I opposed to the passige of the eurrent the resistance of a column of distilled water, ten centimetres in length, by placing in the eirenit a tube filled with the water; such as will be deseribed hereafter (Chap. IV.) under the name of a moderating tube. After having reeeived the shock of the completion of the current, which was thus greatly weakened, I gradually increased its power, by diminishing the distance that it had to pass through water, until the continuous current reached me in its full force. I was surprised to find that I could bear this current long enough to effect the experiment of which I have recorded the results. My skin remained unchanged, because my hands and the rheophores were freely wetted, beeause the epidermis of the palm of the hand is thick, and because the experiment was only of short duration. The skin of other parts of the body, if exposed to such a current, would donbtless have been burnt more or less deeply, and would have suffered intolerable pain. The method pursued hat the advantage, by avoiling cutaneous pain, of rendering it possible to analyse the deeper seusations caused by the passage of such a current in a longitudiual direetion throngh the nerves and muscles of the upper extremities. I was unable to continue the experiment with the pile of 120 conples longer than from thirty to forty seconds; although I could beir the current from 20 or 30 comples for from ten to twenty minutes. Subsequently I tried experiments with a pile of 40 elements of protosulphate of mereury, sueh as I shall describe shortly (one of these elements is nearly equivalent to two of Daniell's). I immersed each of my hunds in a basiu placed in eommunication with one of the electrodes; and in this way allowed the continnous current to pass for nearly ten minutes. I could have borne it for a much longer time. The first time that I proceeded in this way, I felt, after two minutes of the continuous eurrent, a kind of fibrillar oscillatory contraction in the flexors of the thumb anil fingers. A sensation of somewhat painful stiftness, in the sime regions, led me to infer the existence of a continued spasm, althongh I could not prove this by the movements of the tlumb and fingers. 'The operation wis followed by a feeling of fatigne in the arms. On the day folluwing, the same experiment, performed in the same manner and under similar conditions, yielded an almost identical result. The feeling of fatirue that I experienced in the anterior regions of the forearms and arms, if not exactly painful was at least very uneomfortable. I have repeaterl the experiment very often upon myself, and also upon other persons; and I liave always observed, during the passage of the continuous current, sometimes the fibrillar contractions, sometimes only the sensation of stiffiness in the museles of the forearm, or of the arm, but in an irregular manner. A similar trial made with the lower extremities by placing the fect in basins connected with the poles of the pile, produced only numbness and fatigue; I lave sometimes seen fibrillar contractions. Each experiment commonly lasted from ten to twenty minutes. (I shall return to this subject in the third chapter.)

I ought to add that, in all my experiments made mpon eitlier the superior or inferior extremities, by plunging the hands or feet in basins of water conneeted with the pile, sunsations of pricking were folt 
diminishing the number of pairs of elements, the phenomena above described diminish; and, when there are only fifteen or twenty couples, they become at last inappreciable.

Is the continuous current really an hyposthenisant of nervous force? If we may apply to mankind the results obtained by vivisection, the reply should be in the affirmative. We know, in fact, that a continuous current, passed for a certain time through the nerves or the limbs of an animal, not only diminishes the motor power, but even produces paralysis. I shall shew in the sequel, by the results of experiments, that this kind of hyposthenisant action is not appreciable in man. (See Chap. III.) With regard to the action of the continuous current upon sensation, although after a certain time it produces numbness of the limb through which it passes, I do not believe that it can be considered as an hyposthenisant of the nervous system, or, in other words, as an anæsthetic.

The sensibility of the skin appears to me to be more acutely excited by a galvanic current of rapid intermission than by a continuous current; although the opposite holds good for their organic action. Thus the continuous current produces a more rapid erythema, vesication, or destruction of the skin. The erection of the nervous papillæe, and the disorganization of the tissues, should be ascribed to the action of the continuous current; and are effects to which the periods of completion and of interruption contribute but very feebly. It is for this reason, doubtless, that the rapidly intermittent current disorganizes the skin much less than the continuous current. But, inasmuch as it is impossible to avoid the continuous action that takes place between the commencement and conclusion of each intermittence, so it is impossible to excite the skin galvanically without the production of more or less organic change. The calorific action of the negative (zinc) pole is greater than that of the positive (copper or carbon pole). ${ }^{7}$

Of all the varieties of electricity, it is the galvanic that acts most powerfully upon the retina, when applied to the face by means of moist rheophores. A galvanic current directed upon the nerves of the fifth pair, or even upon their ultimate ramifications, exerts a special action upon the retina; producing three luminous sensa-

in the skin of the back of the hands or feet respectively. These sensations progressively increased, and were soon replaced by one analogous to that produced by the action of a strong sinapism. There was, however, no vesication, even although the current was continued for half an hour. (I shall refer hereafter to the practical importance of this point.) Lastly the sensibility was always most strongly excited in the part or limb that was placed in connection with the negative pole.

7 It has long betn known that the other cliemical (electrolytic) and physiological phenomena are more pronounced at tlie zinc pole. 
tions (phosphenes) at each intermission. These sensations are felt, the first and most powerful, at the completion of the circuit; the second, much less powerful, at its interruption; and the third, so feeble as only to be perceptible in the dark, in the interval between the two former. To whatever point of the face, or hairy scalp, the moist galvanic rheophores are applied, they always produce a succession of luminous sensations of a very dazzling kind, even with an extremely weak current-provided that the region excited is supplied by the fifth pair. 'The luminous sensation only affects the retina on the same side as the galvanic excitation; and it becomes larger and more brilliant as the exciters are brought nearer to the median line. Lastly, the light is perceived on each side, when the galvanic exciters are placed on the median line, where the ramifications of the fifth pair of each side appear to be blended together. We may even produce the electric phosphene by galvanizing the chorda tympani; but I lave not been able to do this with induced electricity. (I shall return to this subject at a convenient opportunity.) The knowledge of this special property of galvanism, which is only found in a degree iufinitely less developed in other kinds of electricity, is of the highest importance in medicine, as the sequel will shew.

It is, however, on the contractility of muscle that the intermittent galvanic current displays a physiological power infinitely greater than the continuous current.

The galvanization of muscle by intermissions excites more acute sensations than the electrization by induced currents.

What is the action of continuous currents upon the vascular nerves? I shall return to this important question in the third chapter, which is devoted to a critical examination of the different methods of electrization, and in which, after having tested comparatively the therapeutical action of continuous currents in the treatment of atroplic paralysis and of atrophy, I shall have to inquire whether the beneficial results obtained by the different kinds of electricity can be explained on physiological grounds; or, in other words, whether we have any accurate knowledge of their action upon the circulation and upon the vascular or trophic nerves.

\section{II.-Therapentical Properties.}

The galvanic excitation, which, when confined to the skin, may produce there, besides very acute sensation, a considerable organic effect, such as erythema, or vesication, or even an eschar, fulfils certain special indications. It is adapted, for example, to the cases in which it is desired to obtain at once an acute immediate 
action and a durable effect, analogous to that of the moxa, or of cauterization by hot iron.

MC. Matteuci, ${ }^{8}$ drawing therapeutic deductions from his vivisections, has advised the employment of a continuous ascending current in tetanus, as an hyposthenisant of the nervous system. He says it is natural to suppose that the passage of a continuous current along a tetanized limb would destroy that state by producing paralysis. In support of his theory, this celebrated physicist cites a case in which tetanus was cured by the influence of the contimuous current. The fact is important; but it requires to be confirmed by further experience. He has also endeavoured to establish, on electro-physiological data, a method of galvanization by continuous currents, for the treatinent of paralyses of sensation and of motion. I purpose to examine the value of these electrotherapeutic deductions in the third chapter, which is devoted to a critical notice of the different methods of electrization.

The galvanization of muscles by intermittent currents may certainly be employed with advantage in the treatment of paralyses of movement, and of muscular atrophy ; but the inconveniences inherent in this method of electrization (its calorific action and the size and unwieldiness of the necessary apparatus) render the method by induction generally preferable.

The chemical properties of electricity by contact may be advantageously applied in therapentics. We know, for instance, how happily they have been utilized by surgeons for the coagulation of the blood within aneurismal saes. ${ }^{9}$

What is the therapentic value of continuous currents in atrophy and atrophic paralysis? I shall return to this question in the third chapter.

Galvanization of the skin is not applicable to the treatment of cutaneous anæsthesia, because it produces vesications and eschars. But the calorific action of galvanism may sometimes be employed with benefit in surgery, in the destruction of certain tumours, and in the cauterization of wounds, either used as an actual cautery in

8 Traité des Phenomènes Électro-Physiologiques des Animaux. Paris, 1814.

${ }^{9}$ Although this application of galvanic electricity to the treatment of aneurisms lias been for some time abandoned, it is not impossible that it may one day be revived with some success. I think it will first be necessary to examine what conditions of size of surface and number of elements should be combined, in order to obtain considerable electrolytic action, while the calorific action is masked as much as possible.

The positive pole of the pile exerts a coagulating action; while the negative pole, on the contrary, acts as a solvent. The idea has been entertained of applying this special property of the positive pole to the treatment of certain fibro-plastic deposits which form between the laminæ of the cornea. The first attempts of this kind appear to have been crowned witl success. 
the manner of Middeldorf, or to produce electrolysis, as by MLI. Nélaton and Ciniselli. ${ }^{1}$

By reason of its power to excite the retina, galvanism shonld be applied to the face with great cireumspection. The Iuminous sensation produced by it is so dazzling that it may injure the sight if the application is too prolonged, the intermissions too rapid, or the current too intense. The following case is an example:-

Case I.-For about a month I had electrified a patient, suffering from paralysis of the muscles of one side of the face, with my electro-dynamic apparatus (an induction apparatus with battery). Improvement was

1 It was in France that the calorific action of galvanism was first employed as a means of cauterization. Recamier and Pravaz, in 1841, attempted to destroy cancers of the neck of the uterus by a galvanic current. In 1850 M. Nélaton also made some very felicitous applications of the calorific properties of galvanism; and was ably seconded by Mr. Jules Regnauld, then ehief physician in his clinique, who contrived a galvanic battery of suall size, easy application, and great heating power (Gaz. des Hôpitaux, 1852). II. Nélaton thought that this method of canterization might be employed with advantage in cases where the diseased part was decply situated-as in the pharynx, for example. After him MI. Alph. Amussat, and in Germany, Dr. Middeldorf, of Breslau, have employed, in analogous cases, the canterizing power of galvanic currents, with no less success than M. Nélaton.

'The electrolytic action of galvanic currents has also been applied with good results to the destruction of certain tumours. Huniphry Davy, in 1807, was the first to show the power of electricity to produce decomposition of the body, by means of the following experiment. He subjected a morsel of muscular fibre to the action of a pile of 150 pairs for five days. The fibre hardened and dried rapidly; and, when incinerated, it left no trace of saline residue. The potash, soda, and ammonia had gone over to the negative pole; the three mineral acids and the phosphoric acid to the positive pole. Davy obtained similar results by subjecting to the action of the battery living borlies and the leaves of plants. Matteuci has also shewn the action of the pile upon living animal substances. In the writings of Crusel we find the fundamental principles of the electrolytic method. In 1841 he published his first work, - (Sur le galvanisme comme moyen curatif chimique contre les moladies locales), and since then, up to 1849 , he sent a succession of memoirs and papers to the Academy of Sciences, in which he first called the method the electrolytic treatment. The Academy, when solicited to make a report, declared that "these communications, in the opinion of the commission appointed to examine them, do not seem sufficiently developed to allow of a judgment being formed upon the method." The essays fell into oblivion; and the author limself abandoned the practice in order to return to the gilvanic cautery, for which he has since substituted a hydrogen flame for the destruction of tumours. In 1860, Ciniselli recalled attention to the researches of Crnsel upon the electrolytic or decomposing properties of galvanism as applied to the destruction of morbid growths, and made a communication upon the sulject to the Surgical Society of Paris, from which I have taken the following extract: "My first attempts at electro-puncture have led me to the axiom that, in applying electric currents to the tissues, it is not always by the calorific action that scars are produced. They are formed also, even frequently, when there is no appreciable elevation of the temperature of the conductors." In 1862, M. Ciniselli, in a new work, proved that the tissue changes produced by electric currents are analogons to those prodnced by potential catustics. In July 1861, MI. Nélaton had the merit to be the first to popularize electrolysis in France. - (Nélaton, Comptes rendus de l'Acad. des Scienccs, 1861). He published a most interesting account of the destruction of a naso-pharyngeal polypus by the electrolytic method. I need not farther continue this history; and will only add that M. Ciniselli employed a columnar Voltaic pile, composed of from 20 to 40 or more elements; and II. Nélaton a Bunsen's battery of nine elements, each sixteen centimetres in height by eight in width, and of high tension. 
gradually produced. The exciters were applied daily to the paralyzed muscles without inconvenience, although the apparatus was of considerable power. The sight was never in any way affected; and the patient never perceived any luminous appearance during the operation. One day, the inventor of a new galvanic apparatus applied to me to make trial of his machine. Not being then aware of any facts that shewed the necessity for cantion, I directed the current of this machine, at its weakest, upon the paralysed muscles, which contracted, but much less strongly than they ordinarily did under the use of the induction apparatus. At the same moment the patient saw a considerable light in the eye of the corresponding: side. He called out, "Your room seems all on fire!" $\mathrm{He}$ begged of me that I would desist from making the application. On recovering from the dazzling he complained of much obscuration of the sight; and could not see towards the side on which the galvanism had been applied. The other eyo did not suffer. I ordered him an immediate footbath, and a blood-letting on his return home. The sight did not improve; and, notwithstanding the use of various stimulants and of an appropriate treatment, only very slight amendment was obtained.

It is to this accident that $I$ owe the discovery of an action of galvanism on the retina, far more powerful than that of induced electricity: a discovery which led me, in its tmm, to researches into the different properties of the various kinds of electricity with regard to the production of phosphenes.

This unfortunate circumstance, not possible to have been foreseen, ought not to be lost to science. It furnishes therapeutic applications that are of great importance to the sick; shewing that induced electricity, furnished by an electro-dynamic apparatus even of great power, has no sensible action upon the retina, while galvanism, even in a very feeble dose, energetically influences that organ. It is therefore legitimate to hope that the judicious employment of the electricity of contact may produce therapentic effects that will be useful in the purely nerrous affections of the eye. Such expectations have been confirmed by experience.

To sum up-1. Galvanic electricity acts strongly upon the retina, when the excitation is directed upon parts in which the fifth nerve ramifies. 2. This kind of electricity may be an useful therapeutic agent in purely dynamic affections of the sight. 3. On the contrary, induced electricity, yielded by an electro-dynamic apparatus, acts very feebly upon the retina. 4. But, for the reason that induced electricity is insufficient when it is necessary to stimulate the retina, it is exclusively adapted for the cases in which it is wished to stimulate the facial muscles, without exposing the retina itself to the perils of over-stimulation.

\section{PAR'T III.}

\section{INDUCED ELECTRICITY.}

Although it forms no part of my present plan to explain the principles that govern the development cf induced currents (prin- 
ciples that may be found in any treatise upon physics), I think it necessary to state certain general considerations, which must be kept in mind by all who use this kind of electricity in electrophysiological experiments, or in the science or practice of medicine. Addressing myself to a medical public, generally but little conversant with the technicalities of treatises on physics, I shall avoid the use of these technicalities; and, in order to be intelligible to all readers, shall express myself as much as possible in ordinary terms.

The different forms of induction apparatus derive their force, as is well known, either from a galvanic pile, or from an artificial magnet. The former are called electro-dynamic, the latter electromagnetic instruments. Both are composed of a wire of copper, covered with silk, of variable length and thickness, rolled in tight spirals so as to form a coil, in the centre of which is placed either a bar of soft iron or a magnet. In most of the electro-dynamic instruments a second copper wire, finer and longer than the first, is rolled around it so as to form a second coil. The ordinary electro-magnetic apparatus has only a single wire, rolled around an electro-magnet, or around each arm of a magnet.

It is also well known that, to obtain the action of an electrodynamic instrument with two coils, we complete the communication between the extremities of a circuit formed by an electro-motor and by the wire rolled round the central bar of soft iron (the wire of the primary coil). At the instant when this circuit is completed, a change is wrought in the electric state of the wire, and also of the soft iron-which latter becomes for the time a magnet. If the circuit be opened, a new electric and magnetic change is produced; the natural electricity of the wire returns to its normal state, and the iron loses its magnetism. It is only at the moments when these changes are produced that the phenomena of induction are manifested in the primary coil, by the material influence of the spirals and of the temporary magnet upon the spirals themselves; so that no electro-physiological phenomena are observed in the intervals. (The current produced under these conditions is called the extra-current). If we place in the circuit a living contractile organ, the muscle of a frog for example, at the moment when the circuit is completed the muscle contracts, and then returns to its state of repose. If, then, the circuit be re-opened the muscle again contracts, but this contraction is much less energetic than the former. The same electro-physiological phenomena are produced by the current proceeding from the wire rolled around the first one, and indnced by it. And if, in place of a muscle, we put a galvanometer in the circuit of wire, we see the needle deviate at 
every completion and interruption of the current, but in opposite directions for the two.

The current that is instantaneously developed in the second coil, at the noment when the circuit formed by the first coil and the battery is completed, pursues an opposite direction to that of the current of the battery. It is, doubtless, for this reason that it has been termed the negative or inverse induced current. On the contrary, the current inducerl in the second coil, at the moment when the circuit of the first coil is interrupted, travels in the same direction as that of the generator current, and is called the positive or direct induced current. Lastly, it may be shown that the tension of the induced is infinitely greater than that of the extra current. It therefore follows that the identity of the two currents is not perfect; and I shall hereafter show, experimentally and by clinical ubservation, that the two currents differ essentially as regards their physiological action.

I have stated that the circuit developed in the first wire has been called by authors the extra or inductive current; and that of the second or superimposed wire the induced current of the first kiud. I myself formerly called them the current of the first and of the second lind. I now discard these appellations, in order not to introduce confusion into scientific nomenclature, and because the new terms that I propose are most in accordance with reason and physiology. For the future I shall not employ the expressions formerly used to denote the different currents, because these expressions are liable to be misinterpreted by the generality of practitioners, who are not familiar with the history of the ancient nomenclature. I shall speak of the extra current as the current of the first coil ; and of the current of the first kind, of authors, as the current of the second coil. I hope that these names, perfectly intelligible to everybody and not opposed to any hypothesis, will be generally accepted by the profession. ${ }^{2}$

2 The experimental researches that $\mathbf{I}$ published some years ago, and of which I may say that their exactness has never been disputed, although the facts have been variously interpreted, have shown in an incontestable mauner that the two induction currents possess each their special qualities, denending not only upon the length and thickness of the wire, but also upon the mode of induction. It was therefore necessary to denote the two currents by torms perlectly clear and precise; for which purpose it was necessaly to morlify the existing nomenclature: a proceeding that should never be attempted without absolute necessity. I felt, however, that I was justified in :m endeavour to express in the clearest manner the current with which we are now concerned. Perhaps I ought to explain on what the general nomenclature of induced curreuts is founded.

That has happened with regard to the curreuts of induecd electricity, which commonly lappeus with regiril to many other matters; the nomenclature of the subject has been built up gradually, in aceorlance with the growth of science. The first discovery of Faradiy was simply that a fine metallic thread, rolled around another thread that was traversed by a current, became itself the seat of an 
Let us now examine what occurs when an electro-magnetic apparatus is pot in action. A bar of soft iron is placed at a short distance from the poles of a magnet, in such a manner that it may alternately approach and recede from them by a rotatory movement. We place the soft iron at first crossways with the magnet, and complete the circuit of metallic thread, rolled either round the soft iron (as in the instruments of Pixii or of Clark) or round the magnet (as in the instruments of Dujardin or of Breton) by placing between its free extremities either a galvanometer or the living muscle of a frog. If now we communicate a movement of rotation to the soft iron, so as to bring it opposite the poles of

instantaneous current, at the moment when the circuit formel by the inner thread was either interrupted or completed. Hence the expression "the induced current," that of the fine thread; and "the inductive current," that proceeding from the battery. But it was discovered, after a time, that the spirals of the inductive wire acted one upon another, so as to produce also a special induced current at the moment of opening and of closing the circuit. This special current, distinct from that produced by the battery, but produced in the same wire, was called the cxtra current. It was next ascertained that separate helices superimposed upon the primary spiral traversed by the current from the battery, induced currents one on another; occasioning a succession of secondary induced currents. It became necessary to distinguish these by names. M.M. Henry (P'rinceton), Becquerel, Abria, De la Rive, ${ }^{*}$ Pouillet, and others, used the terms currents of the secoml order, of the third, fourth, and fifth orders and so on, for the currents induced in the second, third, fourth, and fifth helices. And, by a singular contradiction, they retained the term extra current, for the current induced in the first helix; and they called the indurtive current, or current of the battery, the current of the first order. They knew, however, that the extra current, prolneed, as has been said, by the action of the inductive current upon its own spiral, behared in all respects like the other induced currents, with which it ought to be classed. I have thought it most reasonable to call this extra current, the first current induced, the induced cur rent of the first order, after the principle of nomenclature established by the physicists above cited. In my electro-dynamic and electro-magnetic instruments, in which the two threals occupy the places of the first and second spirals, or superimposed helices, mentioned above, we obtain the induced currents of the first and of the second order.

This brief historical retrospect exhibits the insulficiency or confusion of the nomenclature hitherto employed. For those not familiar with a certain conrentional language (and few practitioners are thus familiar), the names first, second, and third order would indicate currents of the same kind, but classed according to their listinctive characters or their superposition, while the name extra current would appear to indicate one of another kind.

There is yet another source of confusion in the received nomenclature, in which somc call the current of the battery the current of the first order, while others call it the inductive currot, and reserve the name current of the first order for that of the second spiral. Finally, M. de IIoncel distinguishes the currents of the first two helices as the primary and the secondary currents. Which of all these is the classical nomenclature?

In electrodynamic instruments, instead of excluding, so to speak, the current of the first helix from the family of inducel currents, it will be found, I think, more reasonable and more philosophic to call it the current of the first orler; since the other currents superior to it have been distinguished by physicists, according to the superposition of the helices traversed, as the currents of the seconil, third, fourth, fifth, and sixth orders. But, in order to avoid any confusion with terms alrealy in use, I propose, in place of calling the extra current the current of the first orler, to call it the current of the first helix; and to call the current next superior to it the current of the second lielix. 
the magnet, the needle deviates from the magnetic meridian, or the muscle contracts. Presently the needle returns to its former position, or the inuscle to repose. If a fresh movement be given to the iron, so as to make it once more transverse to the poles, a fresh deviation of the galvanometer needle will take place in a direction opposite to the former; or the muscle will again contract as energetically as at first.

These phenomena of induction are produced in the electromagnetic precisely as in the electro-dynamic instruments, by virtue of a modification wrought in the state of the magnet and of its soft iron, and in the state of the copper wire rolled round the soft iron or the magnet.

The theory is as follows:- When the soft iron is brought opposite to the poles of the magnet, the natural electricity of the iron is decomposed by the magnet, is attracted to the opposite poles and recomposed. There results a neutralisation of the artificial wagnet, and an induced modification of the electricity of the copper wire, the spirals of which also act upon one another. When the soft iron is withdrawn from the magnet by rotation, the magnetic fluid of the magnet regains its liberty and accumulates at the poles. The soft iron, the polarisation of which ceases at the same moment, returns to its normal state, and the copper wire sustains a fresh induction.

If at the moment when these electrical modifications occur we interrupt the circuit formed by the wire, the power of induction is very considerably increased. Nearly all electro-nagnetic instruments are arranged so that these interruptions of the current only take place when the soft iron arrives opposite the poles of the magnet. It follows that they produce, by the rotation of the iron, one indnotive action that is very feeble, inappreciable by man, and one that is powerful: the first when the iron is transverse to the poles of the magnet, the second when it is opposite to them.

In the electro-magnetic instruments the interruptions of the eurrent are affected by a little bobbin (commutator) placed upon the axis of the soft iron (armature). The latter is put in morement by a mechanism of toothed wheels, so that the rotation of the iron may be rendered extremely rapid.

This rapidity of rotation is essential to the force and even to the action of electro-magnetic instruments, for the reasons following :-

When the current of an electro-dynamic instrument is interrupted, the induction and magnetization of the soft iron cease abruptly with the cessation of their producing cause;-and the change from a great magnetic intensity to zero oceurs suddenly, and withont transition in the central soft iron of the boblibin. 
With electro-magnetic instruments it is different. If the magnetic current be established by the juxtaposition of the soft iron with the poles of the magnet, the interruption takes place only at the moment when the iron is transverse to the poles. If the soft iron slowly describe the arc of a circle in order to assume this position, the magnetic current established between the magnet and the iron will diminish gradually until it ceases.

But if the iron revolve very rapidly the passage of the current from its maximum to zero takes place in a time so short as to be nearly equivalent to a sudden interruption, as in the volta-electric instruments, and to produce a powerful induction. We should hence imagine that the inductive force of an electro-magnetic instrument would be greater the more rapid the rotation of its soft iron. It is found, however, that in these instruments the power diminishes when a certain rapidity of intermission is exceeded ; as happens also in electro-dynamic instruments furnished with Masson's wheel. This phenomenon, which has long perplexed physicists, is produced by a mechanical cause, and depends on a vice of construction that I will explain hereafter.

Prior to my researches, the electro-magnetic instruments had only a single helix. I caused to be constructed an apparatus of double induction, in which the two helices, superimposed and fixed upon a magnet possess, as I will show hereafter, differential properties like the helices of electro-dynamic instruments with double current. The theory and description will be given in the fourth chapter.

The foregoing observations not only show that induced electricity cannot furnish a continuous current, being essentially intermittent or instantaneous, but also that each intermission is composed of two currents opposite in their direction. It has also been shown that the current at the end of each intermission is the only one that exerts a powerful physiological action. Lastly, the ordinary electro-maguetic instruments have no action except in rapid intermission; while the electro-dynamic instruments furnish intermissions either rapid or slow at pleasure.

\section{\$ I.-Different physiological action of the current of the first helix, and of the current of the second helix.}

The excitation of the skin by induced electricity, however long continued and however intense, produces no organic action beyond erection of the papillæ and a little erythema. There are persons, however, in whom the skin is liable to inflammation under the influence of the slightest cause; and in them the current of 
the second helix, which exerts, as we shall see, an elective action on the cutaneous sensibility, may produce an erythema that will last for many days.

In the present state of science, no difference has been established physiologically between electricity communicated to organs from the induced current of the first or of the second helix. We shall see facts, howerer, which prove incontestably that each of these currents acts physiologically in an especial manner. ${ }^{3}$ In the experiments by which they are demonstrated, it is necessary first to equalise the power of each helix by holding a moist rheophore in each hand. (It is known that, on thus placing oneself in the circuit of an induced current, each intermission communicates to the upper extremities a shock that extends to the wrists, the elbows, the shoulders, or even the chest, according to the strength of the current).

A.-The current of the second helix excites the retina more vividly than does that of the first helix, when applied to the face or to the eyeball by means of moist excitors.

This special property of the current of the second helix, the production of stronger luminous sensations than the current of the first helix, is infinitely more developed in my electro-magnetic apparatus with two spirals ${ }^{4}$ than in my electro-dynamic apparatus. Thus the current of the second helix of my electro-magnetic apparatus acts powerfully on the retina, applied to any point of the face, even when a very feeble current is employed; while the current of the second helix of the volta-electric instrument only produces luminous sensations when comparatively powerful; and even then only when the moist rheophores are placed on the

3 Abria alone has endeavoured to determine the comparative power of physiological action possessed by the second, third, fourth, fifth, sixth, and seventh helices; or, in ordinary language, of the current of the first, second, third, fourth, fifth, and sixth order; but he has not studied the differential action of the extra current (current of the first lelix), and of the current of the first order (current of the second helix), the only difierence that it is inportant to know in practice, since the differential actions described by Abria are quite insignificant therapeutically. I sliould say also that there is no resemblance between the researches of Abria and my own. Abria simply took the rheopliores in lis hands to compare the different action of induced currents of difierent orders; and when any current gave him more powerful shocks in the upper extremities, he inferred that its physiological power was greater. This was all. In my experiments, as we sliall see hereafter, I first equalised the strength of the currents that I wisled to compare; and then studied their differential action upon the sensibility of the skin, of the muscles, of the retina, \&c. Abria was unable to undertake such researches, because he did not know how to localize the electric force in certain organs; a proceeding that requires before all things, special anatomical and physiological knuwledge.

The apparatus with two spirals, or of double induction, which I was the first to construct, will be fully described in the fourth chapter. 
points of emergence of branches of the trifacial nerves, or on the eyeball itself. The flame then perceived is paler than that from the-current of the second helix of the electro-magnetic instrument, and impresses the retina less vividly. The current of the first helix of the electro-magnetic instrument produces no more impression of light than that of the electro-dynamical instrument. Lastly, the luminous sensation that is due to the action of the current of the second helix of the electro-magnetic apparatus is far from being so strong as that which is excited by electromotor instruments.

B.-WThe current of the second helix excites the cutaneous sensibility more vividly than the current of the first helix.

This phenomenon was established as follows:-Two electrodynamic or electro-magnetic instruments were set in action, so arranged that one furnished the current of the first, the other the current of the second helix; and they were so graduated as to act with equal force upon muscular contractility. If then the skin was excited by these alternately, it was always observed that the sensation produced was much less, under the influence of the current of the first helix than under that of the second.

such are the differential properties of the currents of the first and of the second helices, properties the discovery of which goes back to 1848 , which I described to the Academy of Sciences in 1849 , and which were set forth in the former edition of this work in 1855. Since then, my experiments upon the differential properties of induced currents having been facilitated by the use of a commutator of the helices that I have added to my instruments, by which I can change rapidly from one current to another, and more readily compare their physiological effects, I have observed that the induced currents possess other special properties of no less physiological and therapeutical importance. These I proceed to describe.

C.-The current of the first helix excites, more powerfully than that of the second, the sensibility of certain organs placed more or less deeply beneath the skin.

I was led to a knowledge of this fact while repeating, in the presence of M. Béclard, Associate Professor of the Faculty of Medicine, the experiments that display the differential properties of currents, and that I have described above. My learned companion observed to me that the current of the first helix produced in him a much more acute sensation than the current of the 
second helix, when he held both rheophores in the same hand. The observation led me to institute a series of experiments, which, repeated frequently, and on many persons, have always given the same results.

If, after having equalized the force of the currents of the first, and of the second helix, by taking in eacli hand a moist rheophore, and obtaining from each current an action that is felt, let us say, as far as to the elbows, their action is compared anew by taking both cylinders into one hand, freely moistened (it being well understood that the cylinders do not touch each other), it will be found that the sensation produced by the current of the first helix is more acute, and more penetrating than that produced by the current of the second. These phenomena of sensibility are the more pronounced, the more rapid the intermissions. The sensations and contractions, limited to the hand that holds the two moist rheophores, result from the direct action of the current upon the subcutaneous nerves of the palmar surface of the hand and fingers, and upon the muscles of that region.

The current of the first helix produces also a more acute sensation when it is directed upon the muscles of other regions of the body.

There are some muscles in which it is easy, by reason of their isolation, to display this special action of the current of the first helix upon the muscular sensibility. I may mention, as examples, the deltoid and the supinator longus, on the middle part of which the exciting sponges may be placed as near together as possible. If in such experiments we place the two currents in ilentical conditions by equalizing their tension (by means of a tube of water), we may see how the current of the first helix excites most the muscular sensibility. But when we experiment in regions where the muscular layers are more or less thick, and are superimposed on one another, the difference of action of the two currents on the muscular sensibility is less easy to display; because the current of the second helix is of a greater tension than that of the first, and, as I will soon explain, its reconposition takes place more deeply - so that a large number of muscular fibres are thrown into contraction, and sensation is proportionately augmenterl.

It would be reasonable to infer that the sensibility of other organs more or less deeply seated beneath the skin would also be more acutely excited by the current of the first helix than by that of the second. I have proved this for many of them; for the mixed nerves, the testes, the bladder, the rectum; and for these the difference of sensation excited by the two currents is considerable. 


\section{D. - The current of the second helix produces more energetic reflex contractions that that of the first helix.}

'This proposition follows from the foregoing experiments, which should be completed in the following manner:-

Having first equalised the action of the two currents on the sensibility of subcutaneous organs, by holding both moist rheophores in the same hand, a rheophore should then be taken in each hand. It will be found that, of currents which act with equal force in producing sensation, that of the second helix will produce contractions which ascend much higher up the arms.

It is necessary to explain the mode of physiological action in these phenomena. The contractions observed in the upper extremities when a moist rheophore is held in each hand, are manifested above the point excited, that is, in a direction contrary to that of the nervous force. They are due to the reaction of the spinal cord, excited by the electric current which passes along the limbs, from the extremities to the centre. I shall return, in Chapter III., \$3, to this mode of electrization, which I have denominated the mode by reflex action.

E.- When the moist theophores are applied to the surface of the skin, the current of the second helix penetrates the tissues to a greater depth than that of the first.

I arrived at the above conclusion in the following manner. I had frequent occasion to localize electric excitations in muscles that had lost their contractility and their electric sensibility, consequent upon some injury to their nerves; as, for instance, to the radial nerve. When I employed the current of the first helix, I was able to limit the excitation to the paralysed muscle, even when the strength of the current was considerable. There was neither sensation nor contraction. But, if I replaced the current of the first helix by that of the second, after having equalised the two by taking the two rheophores in each hand, the muscles of the anterior region of the fore-arm would be thrown into contraction, and the patient experienced sensation.

It is evident that, in the latter case, the current had penetrated more deeply than in the former, and had been recomposed in the muscles of the anterior region, after traversing the interosseus space. I have seen similar phenomena in the leg, when the internal or external popliteal nerve had been wounded; that is to say, that the current of the second helix excited at once the paralysed and the sonnd muscles, while that of the first, apparently 
of equal strength, was limited in its action to the paralysed muscles only.

It has, therefore, been shown by the foregoing:-

1. That the current of the first helix excites most acutely the sensibility of certain subcutaneons organs; the nerves, the muscles (proportionately increasing their contractility), the rectum, the bladder, the testes, the epididymis, and the spermatic cord.

2. That the current of the second helix acts most powerfully upon the cutaneous sensibility, upon the sensibility of the retina, and penetrates most deeply into the tissues.

\section{F.-Theories of the differential physiological properties.}

Whatever be the cause of the differences observed in the physiological properties of the currents of the two helices in every double-induction apparatus, differences observed in varying degrees in different instruments, according to the varieties in diameter and relative length of their coils, it is incontestable that these properties, so important physiologically and therapentically, and which cannot be combined in a single helix,-it is incontestable, I repeat, that these properties are not physiologically identical. It is this that $\mathrm{I}$ mean to express by saying, apart from any physical theory, that the currents of the first, and of the second helix possess different physiological properties.

With regard to the physical theory of these differences, esteeming myself fortunate to have pointed out facts of such great practical importance, that had previously escaped observation, I prefer leaving their explanation to others, to physicists, more able than myself to deal with them. In a communication addressed, ${ }^{5}$ in 1856 , to the Academy of Medicine, upon certain new physiological properties of induced currents, I confined myself to expressing an opinion that there was some connection between the degree of tension of the currents, and their power to penetrate deeply beneath the skin. If I did not attempt to explain also the physical causes of the other differences, it was not for want of experimental inquiry into the subject. ${ }^{6}$

5 Duchenne, Note sur quelques propriétes differentielles des courants d'induction de premier et de seconde ordre (Bull. de l'Acad. de Méd., 18 Mars 1856, t. xxi. p. 538). See also Rapport de M. Bouvier (ibid., p. 671).

6 The following experiment will show that the currents of the first and of the second helices possess different degrees of tension. If, after having equalised the two currents in the manuer deseribed in the text, we measure their physiological power anew, after causing them to traverse a portion of distilled water in a glass tube, it will be found that the current of the first helix is much more weakened than that of the second. When my most powerful induction instrument is in action at its highest grarle, and its two currents are equalised, the current of the first order ceases to be perceptible after having passed through distilled 
My first idea, when I had thoroughly convinced myself of the difference in the effect of the two currents upon the sensibility of the skin, the idea that would naturally have presented itself to any physicist, was that this difference might perhaps be explained by the difference of tension. I was induced by this belief to make certain experiments, the results of which were entirely negative. Being unable to solve my problem, or, in other words, being unable to find the explanation that I sought, I thought it best to abstain from publishing my experiments. In this decision I now think I was mistaken; since many physicists of the first reputation and of the highest talent have attributed the different properties of the eurrents solely to the difference in their tension.

"The extra current," they say, "and the current of the first order, do not enjoy elective properties over this or that function; but they have an action more or less energetic by reason of their tension,-tension which they owe to the size, the inductive power, and the insulation of the wire that they traverse. Thus an induced current, produced in a wire much longer, and of much less diameter than the first, has a much greater tension than the extra current produced in a thicker and shorter wire. Thus powers and properties, apparently different, result from the greater tension of the current that produces them."

From the foregoing theory critical deductions have been framed with regard to the arrangement and properties of my electrodynamic and electro-magnetic instruments, made, as is well-known, with reference to their application in medicine. Since these deductions diminish the value of my physiological researches, and of the practical considerations that flow from them, I think it my duty to make known my own experiments with regard to this important question, experiments which seemed to me to prove that the physiological differences between the induced currents of the first and second helices were not solely due to differences in their tension; that their properties are really special to each, and, at present, are not capable of explanation.

In order that my experiments should be simpler, and more decisive, I studied the phenomena of induction without the inter-

water for a distance of eight centimetres; while that of the second helix is still very appreciable after passing throngh thirty centimetres. These phenomena are the same, whether the eurrents be furnished by an eleetro-dynamic or an electro-magnetic apparatus. They show that the current of the first helix overcomes the resistance of the water with mueh more difticulty than that of the second; or, in other words, that the former has a much less degree of tension than the latter.

${ }^{7}$ Traité des applications de l'étectricité à la therapeutique, par A. Becquerel, 1857, p. 58. I may mention that the physical part of this book is due to the assistance of II. E. Becquerel, whose learning and abilities are world renowned. 
vention of magnetic force; that is, by producing induction by the initial voltaic current alone, and waiting to test afterwards the influence of temporary magnetism as an initial force also producing induction.

a.-In my electro-dynamic instruments with double current, the difference in diameter and length of the two wires which produced the extra current (current of the first helix) and the current of the second helix, did not alone explain the different action of those currents on the sensibility.

Experiment.-I constructed two helices, nine centimetres in length, of copper wire, covered with silk. In one the wire was one millimetre in diameter, and 200 metres in length; in the other a sixth of a millimetre in diameter, and 1000 metres in length. In the centre of the helices was a space, intended to receive a bar of soft iron or a bundle of iron wire, but left empty during the present experiment.

In this state, the current of a pair of Bunsen's elements, circulating with intermissions throngh each of the separate helices, gave origin to two instantaneous currents of unequal power. That which was developed in the thicker and stonter wire (the extra current of anthors) exerted a physiological action infinitely greater than that of the fine wire.

I then equalised the physiological force of the two currents, by placing the moist rheophores one in each hand, or both in the same hand, or on the plane of the muscles, and by then causing the current of the thick wire to pass through a tube filled with distilled water of such a length that the shocks produced were equal to those of the current of the fine wire.

If, after thus equalising the physiological power of the two currents, their action on the cutaneous sensibility was compared, it was found that the fine and long wire did not excitc this sensibility more acutely than the thick and short.

It follows from this experiment that an extra current developed in a helix of very long and slender wire (1000 metres in length, and one-sixth of a millimetre in diameter) does not excite the cutaneous sensibility more actively than an extra current developed in a wire much shorter and thicker (200 metres in length, and a little less than a millimetre in (liameter).

It is also eviclent that, if the action of a current upon the cutaneous sensibility bore any direct proportion to the degree of tension, the extra current developed in a fine wire should act upon the skin much more powerfully than that developed in a wire thicker and shorter. This does not require to be demonstrated.

b.-The fine and long wire of my electro-dynamic apparatus of double current only acquires its special action upon the cutaneous sensibility when its current is induced by a thicker wire.

If the same helix of fine wire, that was used in the foregoing experiment, was exposed to the inductive influence of the helix of thicker wire, the current circulating in its coils instantly acquired the special property by which the sensitiveness of the skin is 
acutely excited; the property that I lave made known as being peculiar to the current of the second helix. The experiment was tried as follows:-

Experiment.-I mounted the two helices used in the preceding experiment, and made a second fine helix, the same as the former, to cover the helix of larger wire. Into the latter I conducted an intermitting current of the same electro-motor. The force of the current of the second helix, when produced by the induction of the first, was very considerably augmented (as would be forescen). In order to compare the special properties of this second helix, yielding an induced current, with those of the similar helix, yielding only an extra current, I caused the current of the former to pass through a water regulator, until it was impossible, with the moist hand to distinguish one current from the other. They might therefore be thought to be perfectly equal, with regard to their physiological action upon the sensibility of the skin. But on limiting their action to the surface of the skin, and testing them alternately, the induced current of the fine wire was found to produce much more sensation than the fine-wire extra current.

We have therefore seen in this experiment, that a helix made of wire 1000 metres in length, and one sixth of a millimetre in diameter, and of which the power, as the source of an extra current, scarcely equalled that of a helix made from a wire 200 metres in length, and one millimetre in diameter, acquired an enormous increase of power, as regards the cutaneous sensibility, when excited by an inductive helix of the thicker and shorter wire.

It will doubtless have been observed that in the foregoing experiments I refrained from placing the soft iron in the centre of the inductive helix; that is to say, that magnetism had no influence upon the phenomena of induction that I have described. My experiments were couducted in this manner for the purpose of showing how little foundation there is for the opinions of certain writers, who have ascribed the special action of the current of the second helix to the influence of magnetism. "There is," says M. E. Beequerel, "another observation that should be made. M. Ducheme, by placing in his instruments a second helix over the first, has thonght he obtained from it only a current of a superior order to that of the first. But, from such an arrangement, the result is very complicated: because, in the second helix, as in the first, the predominant action is due to the influence of the central magnet."

This opinion is valueless, in the face of the experiments above recorded; because in them the differential action of the two currents was displayed quite independently of the presence of the temporary magnet. If I had placed a bar of soft iron in the centre of the inductive helix of large wire, the iron would have become strongly magnetic, and would have re-acted upon the induced current of the helix, increasing its power; as may easily be 
slown. This increase of the power of the current of the first helix would have increased in proportion, by its influence, the power of the current of the second helix. This is all. To say that the magnet acts directly upon the second helix, and confers special properties upon its current, is mere hypothesis, and the objection is set aside by my experiments.

We may therefore say that the special action of the current of the second helix upon the cutaneous sensibility, or the special action of a helix of fine and long wire induced by a helix of wire that is thicker and shorter, cannot be explained entirely by the great tension of the current. What, then, is the exact explanation of the phenomenon? This is a question that ought to employ the ingenuity of the learned physicists who have criticised my researches; and who, I repeat, by reason of their special knowledge are far more competent than myself to enter upon such inquiries.

Let us, however, admit for an instant that the greater action of the current of the second helix upon cutaneous sensibility is due solely to the greater tension of this current. How, then, shall we explain why the current of the first helix, the tension of which is infinitely less, excites much more acutely than the other the sensibilities of certain sub-cutaneous organs (muscles, nerves, bladder, rectum, testes)? How, also, shall we explain the elective action of the current of the second helix upon the sensibility of the retina ${ }^{8}$

8 MI. Chaureau, endeavouring in a recent book to generalize on the plıysiological effects of induced currents, has concluded from some experiments on animals:-" 1 . That the physiological effect of elcetricity is the result of a mechanical disturbance of the molecules in the track of the currents. 2. That this disturbance depends entirely upon the tension of the currents, and is not directly influenced by the quantity of electricity set in motion. 3. That the various portions of the trick of the electricity, in an animal conductor, do not undergo the same degree of mechanical excitation; because the tension, instead of being uniform throughout the conductor, is always stronger at the extreme points, and, especially, at the point of escape (negative pole)."

'The experiments marle upon the human subject, by the aid of localized electricity, and described in the text, show that the energy of all the physiological effects of an induced current, far from being always in direct proportion to its tension, is sometimes greater under the influence of a current of lower tension (the current of the first helix, or the extra current of authors).

I should observe also, that it is known in physics, and may be shown by experiment, contrary to the statement of MI. Chatuvean, that the electric current has less tension at the negative than at the positive pole; so that a more powerful physiological action at the negative pole cannot be attributed to the greater tension.

Lastly, M. le vicomte Dumoncel gave additional support to the position that I defend, in a work that he addressed in 1859, to the Academy of Sciences. He has shown by experiment that the physiolngical power of an induced current is not always due to its tension; and his experiment is described in the following communication, with which this ingenious and learned physicist has favoured me.

"We may," he says, "conclude from this experiment, that the tension is not the sole cause of the power of induced currents to produce physiological effects; and that the different effects observed by M. Duchenne, from the action of secondary currents and extra currents, 
We cannot, therefore, in the present state of knowledge, find a satisfactory physical theory of the differential physiological properties of the currents of the first and of the second helix; and we must be content, for the time, with a simple recognition of incontestable facts.

\section{\$ II.-Differential therapeutic properties of the induced currents.}

Induced electricity is the only therapentic agent which, limited to the skin, is capable of producing acute cutaneous sensation which ceases suddenly with the operation, which can be graduated from simple titillation to the most acute pain, either through all intermediate degrees, or by passing suddenly from one extreme to

camnot be entirely clue to the differences in their tension.

"I have demonstrated by the apparatus represented in the figure fig. 1); which is composed of two straight electro-magnets $\mathrm{B}$ and $\mathrm{D}$, furnisherl with induction bobbins at their polar extremities, that when the two armatures $\mathrm{E}$ F and $\mathrm{G}$ II

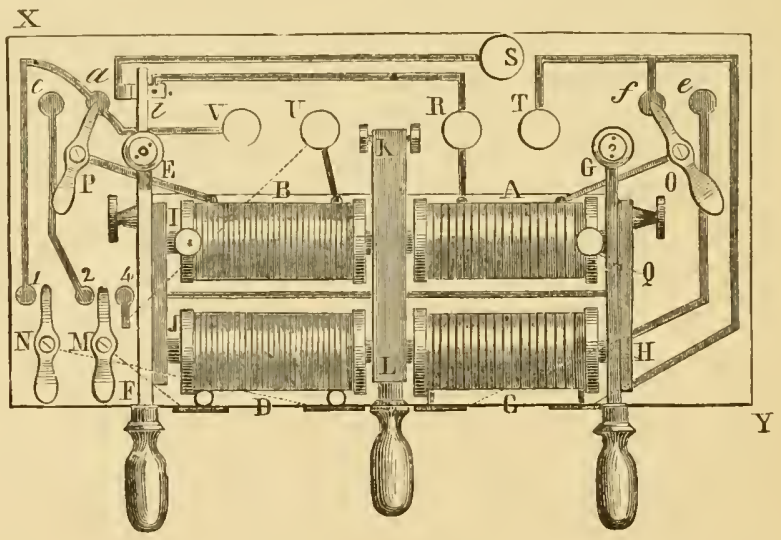

Fig. 1.

are let down over the poles of the be cited; and I have shown that, under magnets, the induced currents excited by certain circumstances, the inverse current, the closing and opening of the inductive which never gives appreciable physiocurrent across the bobbins $\mathrm{A}$ and $\mathrm{C}$, logical effects, yields some that are more although more intense in their action energetic than those of the direct current. upon the galvanometer than when the Furthermore, we know that the negative armature $\mathbf{E} F$ is raised, give much more pole of the induced current re-acts most powerful sliocks in the latter case; and yet the two currents, measured by the rheostat, have the same tension. It is impossible to ascribe this difference of action to the difference in the rapidity of the demagnetization of the magnetic centres in the two cases; because the same effects are produced by interrupting the two inductive and induced currents simnltaneously by means of a double tootled whecl. MIoreover, this effect is not the only me of its kind that conld powerfully upon the animal economy; although the experiments of M. Riess and myself have shown that the current of the positive pole possesses the greater tension. We must therefore conclude, as I have said, that the reciprocal relations between the nervous system and the action of electricity are not sufficiently understood, in the present state of science, to furnish us with à priori explanations of all the phenomena that have been ohscrvel." 
the other, without ever disorganizing the skin, or leaving any trace behind further than a slight erythema or a little elevation of the papillæ. It is evident that such an agent will fulfil a number of indications; whether we wish to excite the sensibility of the skin, as in cases of cutaneous anæsthesin; or whether we wish to produce some revulsive local action, as in neuralgic and rheumatic pains. This cutaneous excitation may be repeated frequently, and may be applied to all points of the surface, even of the face, since it leaves no mark behind; and it may be controlled in accordance with the excitability of every patient, or of any region of the body.

There is often need for an intense current in the treatment of certain muscular affections. In such cases, only induced electricity is applicable; since it does not exert the calorific action of contact electricity.

'The chemical or electrolytic action of induced electricity is so feeble, that it is useless as a means of coagulating the blood, in the treatment of aneurisms. Induction instruments will, however, even when of small size, exert a considerable influence upon contractility, and this greatly facilitates their employment.

\section{A.-The different physiological actions of the induced curvents of the first and of the second helia cannot be applied inclifferently in practice.}

The two induced currents (of the first and of the second helix) the physiological properties of which are so dissimilar, should exert a different therapeutic action, answering, for each of then, to some special indications. Although empirical trial has often played a principal part in therapentics, we should nevertheless be guided in such empiricism by the physiological action of medicinal agents. The path that I have followed, in the therapeutical use of the currents of the two helices, lias been that which was traced out for me by physiological experiment. If, however, the physiological differences between the currents had been scarcely sensible, I should in that case have drawn from them no therapeutical deductions; in order not to complicate unnecessarily the art of medical electricity, which, as we shall see, is sufficiently difficult already. There must be something more than slight varieties between these physiological properties. I may say, without exaggeration, as regards their effect on the cutaneous sensibility, that they differ as water that is warm differs from water that is boiling, or as iron slightly warm differs from iron that is white-hot.

We do not, indeed, find so great a difference between the two 
currents as regards their other properties; although even for these it is very notable; and if applied indifferently in certain cases, and under certain conditions, the currents will produce accidents more or less serious.

Nothing has enabled me to foresee the existence of these properties; and I have only become acquainted with them by long practical experience, and always at the expense of the patients operated upon ; or, in other words, by accidents. Among recent examples, in faradization of the testes, in a case of impotence, with the current of the second helix, (two moist rheophores, one in front the other behind, were applied to the scrotum at the level of the testes), the patient experienced some pain. Instead of diminishing the force of the current, I changed it for the current of the first helix (by turning the index of the commutator of helices of my electro-dynamic apparatus), thinking that, as the tension of the latter was far less, it might penetrate less deeply, and perhaps produce less sensation. 'The very contrary took place, the pain becoming much more acute. It seemed to the patient, so to speak, as if the testes were being torn from the cord. ${ }^{9}$ The same thing has occurred in every patient with whom I have repeated the same experiment. The epididymis and the cord are alike much more sensitive to the excitation of the first than of the second helix. Another time, having to treat paralysis of the bladder, I introduced one rheophore into that viscus, and another into the rectum (for the coustruction of the rheophores, see parge 95), and caused the passage of a sufficiently strong current from the second helix, with two intermissions in a second. The patient felt only a very bearable sensation. But, as the tension of the current was very great, it was difficult to avoid its penetration to the sacral plexus, in such a way as to prodnce powerful shocks in the lower limbs. I therefore changed suddenly, without any warning, to the current of the first helix, on account of its lower tension. The patient immediately complained of horrible pain in the hypogastrium. Three other patients, who were then under treatment, all complained of similar pains under the same circumstances. I have often repeated the experiment, when acting solely upon the bladder or the rectum with a double rheophore that will be described hereafter; and the local sensation has always been incomparably stronger from the current of the first

9 The patient was a distinguished pro- and the current of the first order have no fessor of physics; and certainly, after this elcetive properties with regard to this or argumentum ad hominem, he would no that function, but they have a more or longer hold the opinion of MIM. Bec- less energetic action, by reason of their querel, who wrote "The extra current tension." 
helix than from that of the second. These experiments are not harmless; because in many cases the acute sensations have been followed by neuralgic pains in the organs over-excited. The difference in the action of the two currents upon the sensibility of the retina is far from being as great as in the preceding cases; but I have seen many persons who have been inconvenienced by brilliant phosphenes, produced by the current of the second helix, when I have excited regions supplied by the branches of the fifth pair, such as the face or the tongue. Patients have been eren fearful about their sight, and have complained of muscæ volitantes, after such operations.

These facts show, therefore, that the currents of the two helices must not be employed indifferently or indiscriminately; and that the practitioner must be circumspect in the selection of one or the other of them.

\section{B.-Cases in which we should employ by preference the current of the first or of the second helix.}

The differential action of the currents of the two helices being known, it is easy to foresee in what cases the one or the other should be chosen.

The extreme energy with which the current of the second helix acts upon the sensibility of the skin, renders it a most valuable agent in the treatment of cutaneous anæsthesia, or in producing a cutaneous revulsion or shock greater, if desired, than that of the actual cantery, without any destruction of tissue, e.g., in rheumatoid and neuralgic affections. It is unnecessary to add that the current of the second helix will succeed in a number of cases in which that of the first would be wholly powerless. I have frequently seen cutaneous anæsthesia yield rapidly to the current of the second helix, when an apparatus possessing only the current of the first helix more powerful than the former in exciting muscular contraction, had failed to exert upon it any appreciable influence!

If it be desired to practise electric excitation upon deep masses of muscle, protected by thick aponeuroses or covered by an adipose subcutaneous cellular tissue, thick or cedematous, the current of the second helix perfectly fulfils the indication, by virtue of its greater tension; or, in other words, on account of its power of penetration.

Again, the current of the second helix, which acts less upon the muscular sensibility than that of the first, should be preferred when it is desired to provoke energetic contraction with as little 
pain as possible; as in children, and in cerebral paralysis, when it is necessary to avoid reaction of the nervous centres.

The current of the first helix, the tension of which is feeble, succeeds perfectly in cases where it is desired to excite acutely the sensibility and the contractility of small and superficial muscles, without going beyond their limits; as in paralysis with muscular insensibility, or local atrophy. In paralysis of the seventh pair, for instance, when the electric contractility is more or less weakened, it is indicated to excite acutely the muscles that are paralysed. These are of small thickness, and it is necessary to select for them the current of least tension (that of the first helix) when it is not wished to carry the excitation too deeply.

The singular property possessed by the current of the first helix, of exciting acutely the sensibility of the bladder and rectum, is valuable in cases where these organs are the subjects of simple anæsthesia, or when, at the same time, their contractility is lost or diminished. I shall describe hereafter a case of anæsthesia of the bladder, which by itself constitutes a serious affection, since the patient, feeling no desire to micturate, allows the organ to become distended, and is unconscious of its fulness until this is manifested by dribbling. By such distension, the bladder becomes paralysed secondarily. In such a case it is plain that the current of the first helix would be indicated; but in a case of muscular paralysis of the bladder, where one feared any over-excitation of the sensibility, the eurrent of the second helix, which acts porrerfully on the contractility and feebly on the sensibility of the organ, ought to be preferred. The same considerations are applicable to paralysis of the sensibility or of the contractility of the rectum. ${ }^{10}$

\section{Different denominations of the various linds of elestricity.}

It follows, from what has been said, that each of the sources of electricity, of which I have described the physiological and therapeutical properties, responds to special indications. It is, therefore, necessary to introduce a nomenclature to express clearly the employment of each.

'I'he word electrization should be used only in a general sense.

The application of frictional electricity may be called static

10 In the face of the facts set forth in the preceding pages, to maintain before an Acarlemy of Sciences, in the present day, that the extra crurent and the current of the first orler, independently of their tension, have no elective properties with regard to this or that funclion, is to omit from consideration evidence obtained by electro-physiological experiments and by a long course of clinical observation, or is a proof of such ignorance as to imply absolute incompetence to deal with any electro-physiological or therapentical question. 
electrization; and that of contact electricity, galvanization. But this last word has, in general, been employed indifferently in medical practice, to denote the use of either contact or induced electricity. The electro-physiological and electro-therapeutical considerations above laid down will render plain the disastrous effects of such confusion.

Since, then, it is necessary to coin a word to express exactly induced electricity and its application, may we not take the word from the name of the discorerer? The name of Galvani has been given to contact electricity; and I would give to induced electricity the name of Faraday. Thus induced electricity itself may be called faradism; and its employment may be called faradization. Such a nomenclature seems to me to be the more happy, since it not only establishes a well-marked distinction between induced and contact electricity, but also does honour to the name of a philosopher to whom medicine is indebted for a discovery far more valuable in therapentics than that of Galvani."1

[MIr. J. Netten Radcliffe has taken objection to the nomenclature adopted by Duchenne in respect of the application of "frictional" and "contact electricity" to medical purposes. The use of the word electrization, sometimes in a general, sometimes in a special and limited sense, is, he thinks, apt to confuse. The terms "frictional electricity" and "static electricity" are words, moreover, passing into disuse among physicists; while the terms "contuct electricity" and "galvanism" are almost entirely disused in physical science. Mr. Radcliffe thinks that it would be an advantage to have a scries of terms which, while securing the object which Duchenne had in view, would not clash with the nomenclature adopted by physicists. He writes, "The electricity of chemical action ('contact electricity,' 'galvanism,' so-called) is more correctly and generally designated after the name of the original discoverer, Volta, voltuic elcctricity. Faraday suggested that frictional electricity should be termed after the illustrious philosopher Franklin, whose name is especially connected with its early experimental sturly, franklinic electricity, and the name is now being widely adopted by pliysicists. By applying the method of terminology which Duchenne has so happily used in respect of the induced current, to other forms of electricity, a series of terms is obtained which would be accurate in form as the practice of nomenclature goes, true to science in fact, free from confusion, and particularly convenient in nsage. The series would be (1) faradaic electricity, or faradism; anrl, as respects the pathological and therapeutical application of the agency, furadization; (2) voltaic electricity, or voltaism; voltaization; (3) franklinic electricity, or franklinism; franklinization."-( 'The Practitioner," vol. i. p. 19.-H. T.]

11 At prescnt, the above nomenclature |although MIM. Bocquerel have main-" is universally used in practical medicine, tained that it was not acceptable. 


\section{CH A P'T E R I I.}

\section{LOCALIZED ELECTRIZATION.}

Part the First.

\section{The Fundamental Principles of the Method.}

AT the commencement of my researches, having adhered to the principles of electrization given in special treatises, and my first attempts having been, if not unfortunate, at least little encouraging, I was soon convinced that my ill success might be attributed to the imperfection of the operative proceedings until then in vogue, and to which I had had recourse. Reserving a critical examination of them for the next chapter, I may say here that their greatest fault is the difficulty of acting upon the diseased part without exposing healthy organs, or even the entire nervous system, to the inconveniences or dangers of the electric stimulation. Moreover, it is impossible, in using these methods, to attain to any exact study of the muscular electro-physiology, or electro-pathology.

It then suggested itself to me that it would be possible to obtain more important and more definite results, if I could either arrest electricity in the skin, without stimulating the subjacent organs, or cause it to penetrate the skin without influencing it, and to concentrate its power upon a nerve or a muscle; in a word, to make it penetrate to deeply-seated organs.

\section{§ I. How to direct electricity through organs? How to set limits to its action?}

This problem, so difficult in appearance, was very simple in reality. For its solution nothing was needed beyond a careful analysis of the phenomena daily seen in practice, on applying to the moist or dry skin, the rheophores ${ }^{1}$ of an induction-iustrument of medium power. The following are the principal facts that enabled me to accomplish the work I had undertaken. They furnish the basis of localized electrization.

1. If the skin, and the metallic rheophores are perfectly dry, and the cuticle of considerable thickness, as in persons who are much exposed to weather by their occupation, the current is recomposed on the surface of the epidermis, without reaching the dermis, and produces sparks and crackling, but no physiological phenomena.

${ }^{1}$ [Conductors or directors. - II. T.] 
2. If the two rheophores, one moist and the other dry, are placed on two points of the surface, the person experimented upon will feel, at the point where the dry rheophore has developed only physical efferts, a superficial and evidently cutaneous sensation. 'The opposite electricities, in such a case, are recomposed at the dry point; lut after having traversed the dermis by the aid of the moist rheophore.

3. If the skin be very slightly moistened, on a part where the epidermis is very thick, a sensation that is superficial, but stronger than in the preceding case, and without either sparks or crackling, is produced at the points where the dry metallic rheophores are placed. Here the electric recomposition takes place in the thickness of the skin.

4. Lastly, if the skin and the rheophores are both thoroughly moistened, the current produces neither sparks, nor crackling, nor burning sensation, but very variable phenomena of contractility or sensibility; accordingly as we act upon a muscle, or muscular fasciculus, a nerve, or an osseous surface. In the latter case an acute pain of a very peculiar character is experienced; insomuch that we should carefully avoid placing moist rheophores over such surfaces. ${ }^{2}$

It follows from these experiments that we may, in faradization, arrest at pleasure the power of electricity in the skin; and that, without puncture or incision, we may make the current traverse the skin, and may limit its action to subcutaneous organs, that is, to nerves, muscle, and even bone.

It is difficult to conceive, however, that electricity will act upon subcutaneous organs without, at the same time, physiologically exciting the skin itself. One is apt to think that the sensations produced by electrization of deep organs are due either to excitation of the skin only, or of the skin and the deep organs together.

2 I will attempt to generalize these phenomena. It is well known that tension elcetricity has a great tendency to escape by points. The same occurs with dynamic electricity, when recompositions occur between the epidermis and dry rheophores. In fact, the epidermis and the rheophores present each a number of asperities by which the fluids of oppositc denominations, proceeding from the battery or the inluction apparatus and the body, escape to be recomposed and neutralized; producing crejitation and sparks. If the skin has been trayersed, the excitation is then superficial, and can only produce cutaneous phenomena, that is, cutaneous sensations. But if the aspe- rities are abolished by a film of water, the electricity traverses the skin en masse and is recomposed deeply, either in the muscles or in bone, or in nerve; the more deeply the more intense the current. There will then be no more sparks or crackling, or cutaneous sensations; but only physiological phenomena in accordance with the functions of the organs that are excited. These latter phenomena display a difference between the physiological effects of tension electricity and dymamic electricity. 'The former always produces electric recomposition between the rheophore and the epidermis, in whatever manner the operation is performed. 
The following experiments may'serve to show that the sensation produced during faradization of the skin by dry rheophores, applied to an equally dry surface, is entirely the result of cutaneous excitation; and that the sensation produced by the application of very moist rheophores to the surface, on the level of a muscular plane, can only be attributed to direct excitation of the muscle.

First experiment.-Having found, in a wounded patient in the Hôtel Dien, a part of the external crural muscle laid bare, I applied over the muscle, and on the denuded portion, a dry metallic rheophore. The resulting contraction was accompanied by a dull sensation, special to electro-muscular contraction. I then placel the same rheophore on the plane of the same muscle, but upon nninjured skin, and I obtained only a burning sensation, withont muscular contraction. Having replaced the metallic rheophore by moist sponges contained in the exciting cylinders, and these being placed upon the skin, in a plane corresponding to the crural muscle, I obtained contraction, with the same peculiar dull sensation that was prodnced when the dry metallic rheophore was in contact with the denuded musele.

Second experiment.-A patient, in whom the radial nerve had been destroyed by a bullet-wound of the lower part of the arm, had lost the sensibility and the electric contractility of the muscles of the posterior region of the forearm; while the sensibility of the skin remained intact, from the integrity of the cutaneous nerves. I applied the dry metallic rheophores on the skin of the anterior and posterior antibrachial regions, and they prodnced an acnte burning sensation. I replaced the dry rheophores by the cylinders with wet sponges, which produced in the posterior region neither sensation nor contraction, althongl contractions attended by serisation were manifested in the anterior region; where also the burning sensation produced by the dry rheophores was replaced by the dull and peculiar sensation of muscular contraction.

I have many times repeated similar experiments in other pathological conditions, not only on muscles, but on mixed nervetrunks; and I have arrived at the conviction that the electric excitation may reach a muscle or a nerve, without exerting any action upon the skin in its course.

It should, however, be remembered, that in certain cases sensations really produced by the excitation of the skin, or of the cutaneous nerves, may be attributed to muscular sensibility alone. In order to explain how such an error may be avoided, it is necessary to enter into certain details.

(a). It has just been shown by experiment that, if the skin and the rheophores are sufficiently moist, and in perfect contact, the former is traversed by the current without being excited; and that the electric recomposition takes place more or less deeply in the subcutaneous tissues. But at the moment when the rheophores and the skin are brought into contact, and when contact as yet is not perfect, the surface presents certain asperities which occasion electric recompositions, attended by sensations of pricking and burning, and even by crepitation and sparks, especially if the current be that of the second helix, with its power of exciting 
cutaneous sensibility, and if the current be sufficiently strong. The same phenomena are repeated at the moment of separating the rheophores from the skin. In order to avoid this electrocutaneous excitation during the faradization of muscle, it is necessary to avoid establishing the current until the contact between the skin and the rheophores is complete. (I shall hereafter point out the precautions to be taken in order not to produce cutaneous sensations.)

(b). The moist rheophores act invariably upon the cutaneous nerves when placed over their course. In order to study the phenomena resulting from their excitation, the tension of the current should not be sufficient to penetrate to the muscles. The faradization of a cutaneous nerve produces a sensation which is continued along the course of the nerve, beyond the point excited, to the finest ramifications; where it occasions tingling and pricking, proportionate to the degree of intensity of the current, and the rapidity of the intermittences. The sensations are also more acute when the nerves are excited at a point near to their finest ramifications: thus they are produced more strongly by faradization of the collateral nerves of the fingers and toes, than of the cutaneous nerve higher up. The excitability of the cutaneous nerves differs greatly. It is carried to the highest degree in some of them; the frontal, for example, derived from the opthalmic of Willis, cannot be tonched withont provoking acute pain in the faradized point, spreading from thence to all the ramifications. In other nerves faradization is only appreciable by the extension of slight tingling over the regions of their distribution. The cutaneous nerves of the limbs are generally in this latter condition.

A knowledge of these phenomena renders it possible to distinguish the complex sensation, due to the simultaneous excitations of cutaneous nerve and muscle, from that which is the result of the excitation of muscle alone. Indeed, when the moist rheophores are placed on the plane of a muscle, and over an excitable cutaneous nerve, the participation of the latter is shown by the tingling or pricking which extends along its ramifications, and by a special pain, if the nerve be very sensitive, limited to the point excited. When the rheophores are so moved as to avoid the cutaneous nerve, the tinglings and the local pain cease instantly, and allow the purely muscular sensations to be perceived.

\section{$\S$ II. Is it possible to concentrate the electric force in a muscle?}

We are met here by an objection seemingly very serious, which wonld present itself naturally to all minds, and which nearly 
arrested me at the beginning of my researches. If it be true that we can concentrate the electric force in a muscle, is it also certain that the resulting excitation will not itself produce the phenomena called reflex, by reacting upon the nervous centres? In other words, is it not to be feared that the electrization of a muscle may provolie not merely its own contraction, but also that of other muscles? If it had been so, I should certainly have renounced my idea as a chimera, and all the researches that $I$ owe to its realization would have to be recommenced. The following experiments have convinced me that the reflex action of the cord does not disturb the muscular phenomena produced by localized electrization :-

First experiment.-Having removed the skin from the face of a living rabbit, I divided the facial nerve of one side only, in order that the muscles supplied by it might be cut off from all connection with the cord. I then applied electric excitation to each muscle of the face, alternately on the two sides. The muscles contracted separately, and equally on both sides.

Second experiment.-I then destroyed the brain of the same animal, in order to place the cord in a condition favourable to the production of reflex action, and again excited the muscles as before. The results were absolutely the same.

A similar experiment on the muscles of the lower limbs, after having excised a portion of one of the sciatic nerves, produced similar results.

Third experiment.-After having decapitated a number of frogs, I destroyed the spinal cord in some, leaving it intact in others. In all these frogs I threw individual muscles into contraction, even the little muscles of each claw, without any mingling, even in those with spinal cord unhurt, of the least contraction due to reflex excitement.

Fourth experiment.-When in the human subject we compare the contraction of the muscles of a perfectly anæsthetic limb with those of a limb in which sensation is perfect, we do not discover any difference between the two, as regards the manner in which the muscles respond to localized faradization. In both, each muscle contracts singly.

Fifth experiment.-In cases of cerebral hemiplegia (a condition favourable to the production of reflex phenomena) localized faradization produces isolated contractions as certainly on the diseased side as on the healthy one.

I may add that, in all my experiments upon men and animals, I have made single muscles and single fasciculi contract with as much facility and certainty as when acting on the muscles laid bare, and freshly removed from their positions. I have publicly repeated these comparative experiments at the Hôtel Dieu, at La Charité, and at the Lariboisière Hospital.

Sixth experiment.-In 1852, I made some interesting experiments upon a patient in La Charité (under M. Cruveilhier), in whom reflex phenomena were very easily developed. The lower limbs were entirely deprived of movement; and yet the slightest impression on any part of the skin covering them threw them into violent action which he was unable to control.

Cutaneous excitation of the upper limbs, however powerfnl, produced no movement of the lower. If the sensation experienced by the patient was slight, as when a finger was drawn orer his thigh, the morement would be trifling, and confined to the limb tonched; but if the sensation were greater, the movement would be more extensive, and would be executed by both limbs at once.

The movements consisted always of flexion of the thigh upon the pelvis, of the leg upon the thigh, and of the foot npon the leg. I never saw any contraction of the muscles antagonistic to the flexor's. The movements were 
evidently due to the reflex action of the cord, called forth by excitation of the skin of the paralysed limbs.

I wished to discover whether electro-muscular excitation would produce reflex action with the same facility, when individual muscles were made to contract. At the instant of applying the moist rheophores to the skin, on the surface over the tibialis anticus, the entire limb was thrown into the state of flexion described above. This was entirely due to the touch of the sponges wet with cold water; the apparatus being not then in action.

Immediately after the limb had fallen again into its habitual inertia, the rheophores remaining all the time in contact with the same points of skin, I discharged one intermission of an induced current of moderate strength. To my great surprise the tibialis anticus contracted singly, without producing any reflex action in the other muscles.

I repeated this experiment seven or eight times in succession (that is, with a single intermission of an indnced current each time), and obtained always the isolated contraction of the tibialis anticus, although the power of the instrument was gradually increased to its maximnm, and although the patient, each time, experienced a marked sensation. It was also easy to obtain, in the same manner, the contraction of every one of the muscles of the lower limbs.

I repeated the same esperiments, only employing a current of very rapid intermissions in place of that with the intermissions distant. (The effect upon the sensibility was heightened by the rapidity, a subject to which I shall recur in the following chapter). The phenomena changed; and the isolated muscular contraction was followed by energetic contractions due to reflex action. The very acnte sensation experienced by the patient had been transmitted to the nervous centres, and had prodnced reflex contraction.

It follows from the above that reflex contractions are produced during electro-muscular excitation only in certain pathological conditions; and that it is possible even in these conditions to make individual muscles contract singly, by directing upon them an induced current of slow intermissions, so as to produce only a moderate sensation.

It appears also to be shown that reflex action is more readily provoked by excitation of the skin, than by excitation of the muscular sensibility.

I used to accumulate here proofs in refutation of the objections urged against localized electrization in 1848; and, if I still recur to them, it is because a distinguished writer, M. J. Guérin, to whom science is indebted for valuable contributions on muscular pathology, has attempted, in a critical article published in the 'Gazette Médicale,' by objections founded upon the reflex action of the cord, to set aside localized electrization, and all the researches that flow from it.

From the whole of the facts, it appears, in the plainest manner, that electrization will cause a muscle to contract singly, without provoking other muscles to reflex contractions, even in the states most favourable to the latter phenomena.

It has hence been possible for me to create this method, which limits electric excitation to each of the organs, without its being necessiry to puncture or incise the skin. I shall now endeavour 
to explain the various procedures; and shall treat in succession :1, of muscular electrization ; 2 , of cutaneous electrization; and, 3 , of the electrization of internal organs, the organs of the senses, and of the genito-urinary apparatus.

\section{Part the Second.}

\section{LOCALIZED MUSCUIAAR ELECTRIZATION.}

In order to confine the electric action within one of the muscles, or the nerves which supply them, is it a matter of indifference which variety of electricity is used? This question can be answered only by studying muscular electrization separately; (1) by static electricity; (2) by contact electricity (muscular galvanization); and (3) by induced electricity (muscular faradization).

\$ I. Localized muscular electrization by static electricity.

I have already shown, in Chapter I., that static electricity cannot penetrate to muscular tissue without also exciting the skin, on the surface of which recomposition always occurs, and produces an electric spark. It, therefore, cannot be used for the comparative study of the sensibility of the skin and of the muscles, in electrophysiological and pathological researches.

The muscular contractions that it excites being inevitably abrupt, cannot be used for the study of muscular function.

Lastly, the shock that is inseparable from its employment, the rupture of capillary ressels that it occasions, the kind of torpor that it produces in organs, the bulk of the apparatus by which it is liberated, all these together, in a word, should restrain more and more the use of static electricity in medicine.

It would nevertheless be a great error to reject static electricity entirely, and always to prefer the dynamic, on the gronnd that the latter is not attended by the same inconveniences, and because it possesses special properties, marvellously appropriate, as we shall see, to localized electrization. In my hands static electricity has been of great ntility in certain cases in which the other varieties have been insufficient. ${ }^{3}$

In fact, the subcutaneous cellular tissue is sometimes so abundant, or so infiltrated by serosity, that the most intense currents from an induction-apparatus will not reach the muscles. We then may find, in the discharge of a Leyden jar, an electric tension strong enough to overcome the resistance of the tissue behind which the muscles or the nerves are sheltered. 
I use the following method of discharging the two electricities, accumulated in a Leyden jar, upon the muscle that I wish to excite, and of graduating the force in such a manner as not to produce a general shock.

The apparatus necessary consists of an electric machine, a Leyden jar, and a Lane's electrometer placed upon the table of the machine.

The interior coating of the Leyden jar, A (fig. 2), is placed in connection with the arms, $c$, of the conductor of the electric machine $\mathrm{B}$, by meaus of a conductor D. This interior coating also communicates with $\mathrm{E}$, the knob of one of the horizontal branches of the electrometer. This branch is insulated by the glass upright $\mathrm{F}$, on which it rests. The external coating of the jar is connected by the conductor $\mathrm{G}^{\prime}$ with the upright $\mathrm{H}$, which communicates with the second branch of the electrometer. 'Two excitors, terminating' in the knobs $J$ and $K$, mounted on long insulating handles of glass, are placed in connection, $J$ with the branch $\mathrm{H}^{\prime}$, $\mathrm{K}$ with the extremity $\mathrm{L}$, of the conductor of the electrical machine.

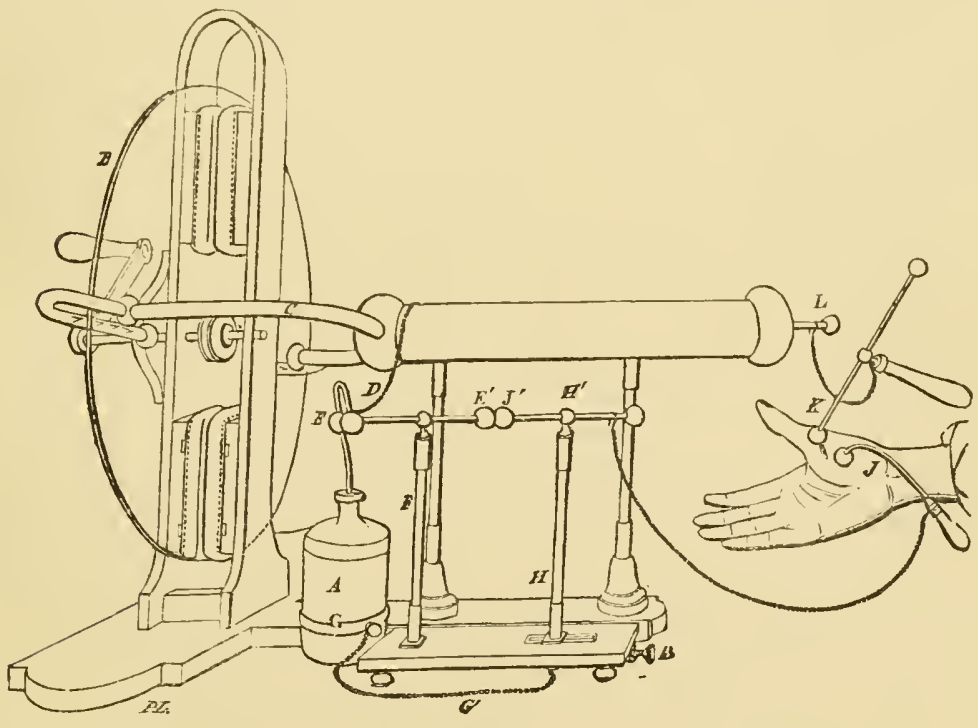

Fig. 2.

All things being thus arranged, the operator applies the excitor $J$, which receives the positive electricity of the exterior coating of the jar, to a point of skin over the muscle that he wishes to make contract; and when he desires to excite the contraction, he brings the other excitor, $K$, which receives the negative electricity of the interior coating, nearly but not quite in contact with the skin, over 
the surface of the same muscle. It is necessary that the extremities of the excitors should always be at least two centimetres distant from each other. The force of the discharge is regulated by the number of resolutions of the plate. If only a feeble contraction be desired, the excitor $K$ is brought near to the skin after one or two turus only. To obtain a stronger contraction, a greater or less number of additional turns must precede the discharge. It may happen, however, from error or inadvertence, that the jar is too powerfully charged; and that its discharge may produce either a very strong contraction, or an action that penetrates to one of the neighbouring muscles, or a very painful sensation, that is followed by general excitement contra-indicated in the particular case. It is to avoid such mischances, troublesome and sometimes dangerous, that I use Lane's electrometer. Of its two uprights, $H$ and F, the former is movable, and can be made to approach or recede from the latter by the screw II. A scale of millimetres, placed at the base of $\mathrm{H}$, indicates the distance between the balls $\mathrm{J}^{\prime}$ and $\mathrm{E}^{\prime}$. Before discharging the jar upon a muscle, I regulate the force by approximating or separating these balls, according to the degree of excitability of the muscle, the region in which it is situated, and the therapentic end that is desired. If I should be deceived about the number of turns of the plate, the jar cannot be overcharged, because the accumulated vitreous and resinous electricities will reumite and neutralize each other between the two knobs $\mathrm{E}^{\prime}$ and $\mathrm{J}^{\prime}$, as soon as they acquire sufficient tension to overcome the resistance of the intervening air ; a resistance which is well known to be in direct proportion to the distance between the knobs.

Is it desirable to have recourse to the advantages of the above procedure of muscular electrization, in the application of tension electricity? Is it not evident that it concentrates its action upon the inuscles that require treatment, without exposing healthy organs, and especially the nervous centres, to the dangers of an inopportune excitation? The procedure admits of clirecting very strong clischarges upon the muscles, when their pathological condition needs such, either from atrophy, or from diminution or loss of their irritability or sensibility.

When, on the contrary, the excitors are placed far apart, or one of them is held, according to common practice, in one hand of the patient, while the other is brought near to the diseased organ or part on which the therapentic action of electricity is to be directed, we see the local effect complicated by phenomena of shock more or less extended or general; so that we cannot, without danger, exceed very weak doses.

The phenomena of general shock are always the result of ex- 
citation of the nervous centres. They are analogous to an effect that I propose to discuss in Chapter III., under electrization by reflex action. This excitation of the nervons centres may, it is true, find its indication in electro-therapentics; but it should at least be avoided when the indication does not exist, or when its occurrence might be dangerous. It is this method of electrization that occasions, in certain conditions, serions accidents, as I shall show in the sequel; and yet mountebanks are permitted to practise it in public places!

It is a great mistake to suppose it possible to localize electric excitation in a muscle, by directing upon its surface the discharges of a powerful electric machine. To correct this mistake, it is sufficient to analyse the phenomena of interior recomposition which produce a lind of shock in return, and which must necessarily overrun the whole nervous system, when a portion of the natural electricity by which the body is pervaded escapes from a point on the cutaneous surface, to neutralize electricity of a contrary name proceeding from a machine in movement.

Another advantage in the employment of the Leyden jar is that it then becomes unnecessary to use an electric machine of large size and high price; which would with difficulty find a place in the consulting-room of the practitioner. A small machine with a single conductor, and with a plate fifteen or sixteen inches in diameter, is sufficient to charge a powerful Leyden jar; the action of which may always be diminished at pleasure by Lane's electrometer, used as described above.

\section{§ II. Muscular electrization by contact electricity, or localized muscular galvanization.}

I have said already, in the first chapter, which treats of the physiological and therapentical effects of the different kinds of electricity, that galvanic electricity, administered with an intermittent current, is distinguished from static, principally in that it can be made to penetrate the skin without exciting it, and that its action can be more easily localized in subcutaneous organs. We shall see that galvanic electricity is useful to localize electric action in the muscles or in the nerves that supply them.

Unfortunately, many inconveniences attend the application of this kind of electricity to muscular electrization.

The calorific and electrolytic action of galvanism, and its property of acutely exciting the retina when applied to the face, are enough, I think, to proscribe its employment in the study of muscular 
electro-physiology and pathology, especially that which concerns the individual muscular action, and the treatment, by intermittent currents, of paralyses of movement, particularly when the treatment requires frequent applications. What I bave before stated in the preceding (first) chapter, renders it unnecessary to dwell on this part of the subject.

\section{I.-Medical Batteries.}

\section{A.-Batteries of large surfuce and with inconstant currents.}

For a long period, the only batteries employed in medicine were those with inconstant currents, in which the electricity was producer by the action of sulphuric acid upon a plate of zinc; while a plate of copper was the collecting element. This was the pile of Volta; and it was tried in succession in many different forms.

All these forms, although they were for a long time used exclusirely, have now been abandoned for medical purposes; because

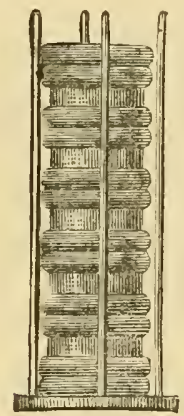

Fig. 3. they lose their power after a short period, and because they vary much during the course of even a single experiment, although it may last only from ten to fifteen minutes. I shall therefore content myself by briefly recalling them by the aid of figures and descriptions taken, for the most part, from the ardmirable treatise of $\mathrm{M}$. de la Rive. ${ }^{4}$

(a). The columnar pile of Tolta (fig. 3) is celebrated as being the first form in which the illustrious inventor realized his conception. It was speedily abandoned on account of the quick ressication of the circles of eloth or of paper which sepaColumnar Pile of Volta. rated the disks.

(b). The wooden trough battery with fixed metallic divisions, called after its inventor the Cruikshank battery (fig. 4), is the

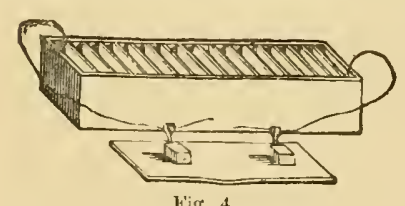

Fig. 4.

Cruikshank's 'Trough Battery. form in which the great battery was marle that was presented by Napoleon to the Ecole Polytechnique in 1806 . It was with this that Gay-Lussac and Thénard made their experiments in 1808. This form has many inconveniences, especially that it requires much time to prepare; and that, unless the partitions are very securely. fixed, they allow of communication between the fluid in the several cells.

${ }^{4}$ De la Rire, Traité d'Electricite théorique et pratique. Paris, 1854, t. i. 
(c). The Cruikshank battery was replaced by the cylindrical glass battery (fig. 5), already snggested by Volta, and known as the crown of crups (couronne de tasses) battery; or by the porcelain trough battery, with movible metallic elements (fig. 6).

The Royal Institution of London possesses a battery of this construction,

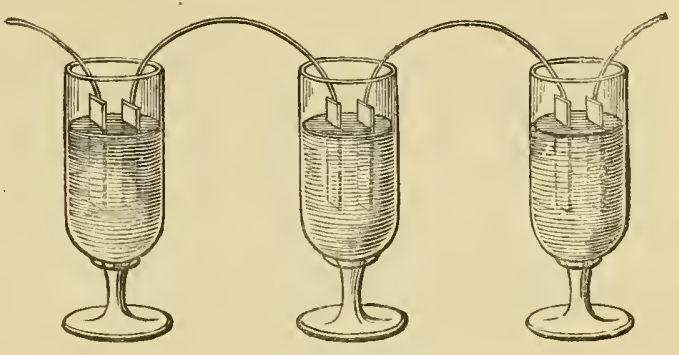

Fig. 5.-Cylindrical Glass Battery. containing two thonsand pairs of elements. It was with this that 1)avy and Faraday conducted their admirable experiments.

(d). Wollaston's battry (fig. 7) differs from the foregoing rhiefly in having the surface of the copper clouble that of the zinc, by which the power is increased.

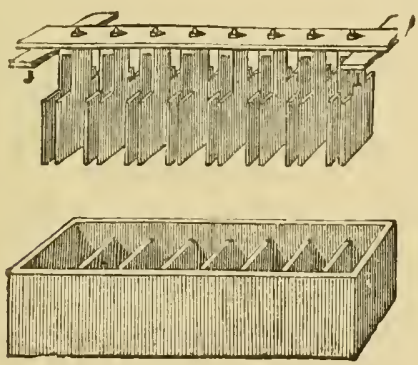

Fig. 6.-Porcelain Trough Battery. ${ }^{5}$

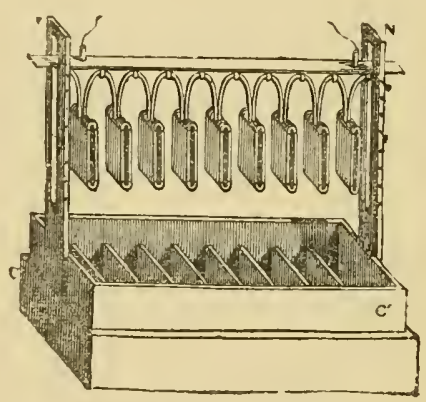

Fig. 7.-Wollaston's Battery.

(e). In the battery of Berzelius (fig. S), the copper surrounds the zine, without touching it, and serves to contain the liquid. In

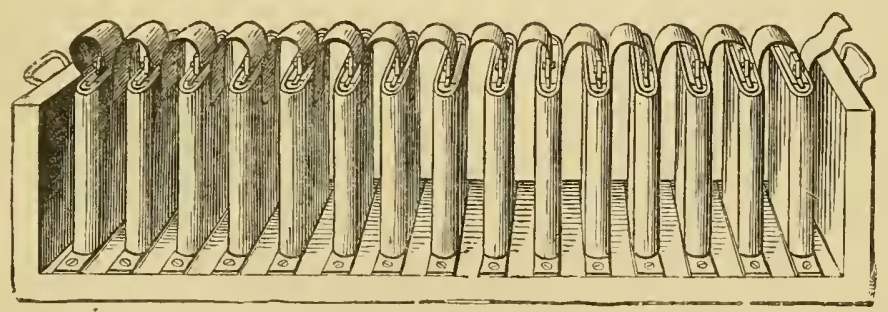

Fig. 8.- Berzelius's battery.

5 An arlangement intermediate between the trough battery strictly so called, and the battery of a circle of cups. 
practice its employment has been attended with inconveniences that have prevented its general adoption; although it rendered valuable service in the first researches into electro-magnetism.

\section{B.-Batteries of large surface and with constant currents.}

(a). Daniell's battery (fig. 9). In 1836, Daniell contrived a battery in which he succeeded in aroiding rapid weakening of the

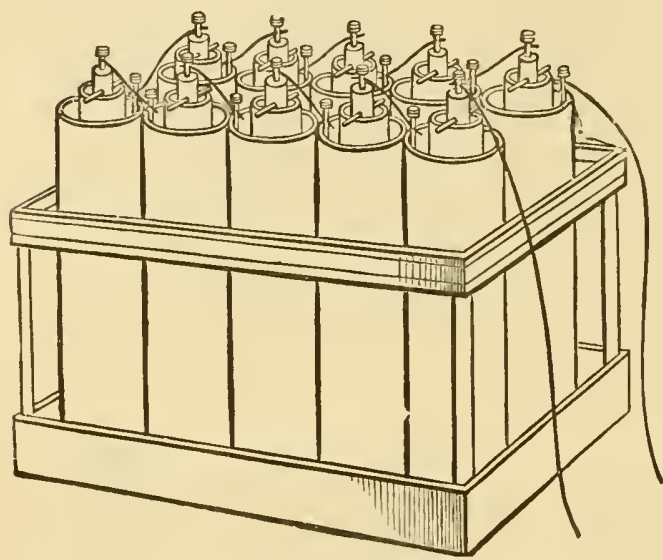

Fig. 9.-Daniell's battery. action, or variations of the power during the passage of the current. It is a battery with porous organic diaphragms, and with two liquids, a solution of sulphate of copper for the copper, and acidulated or saline water for the zinc. It is highly useful for protracted experiments, and especially for inquiries into the chemical effects of the current.

(b). Grove's constant battery (fig. 10) resembles that of Daniell in having two kinds of liquid and a diaphragm; but the copper is

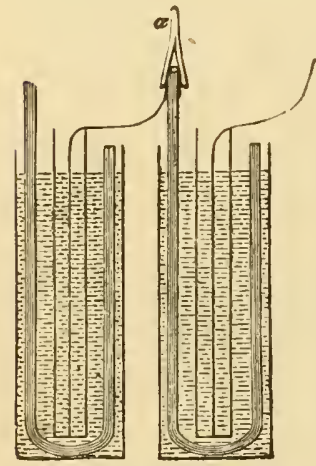

Fig. 10.-Grove's battery. replaced by platinum, the sulphate of copper by nitric acid, and the porous diaphragm is of smooth unglazed porcelain. This battery is the most useful for the production of the electric current; because it combines enormous power with a constancy only little less than that of Daniell.

(c). Bunsen's constant battery (fig. 11) is a modification of that of Grove by the substitution of carbon for the platinum. It is constant for a longer period, but is less energetic than that of Grove. It is much in use, especially in Germany.

The batteries of Bonijol and of Deleuil differ from that of Bunsen only in the form and place given to the carbon.

Among all the foregoing batteries, that of Daniell has been most generally employed for medical purposes. 
The large batteries with extensive surface, which are at present chiefly used in medicine, are the modified Daniell, the proto-

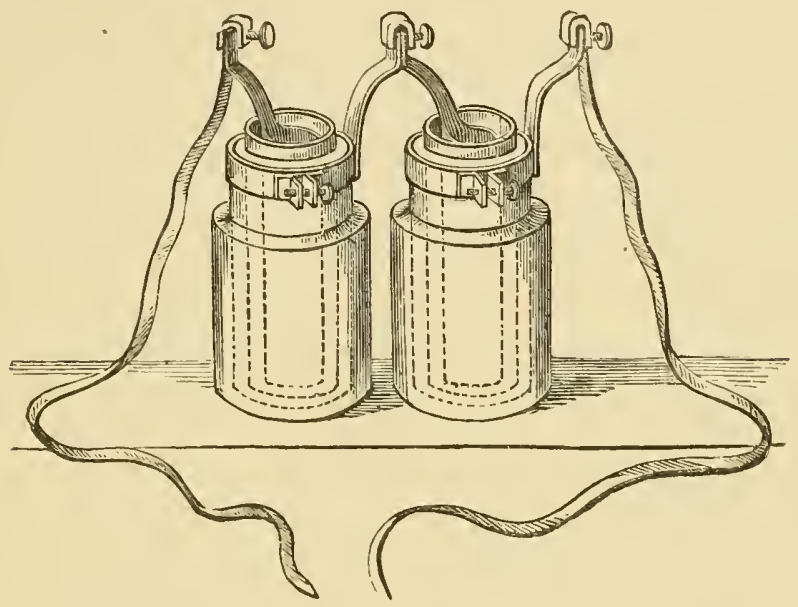

Fig. 11.-Buusen's battery.

sulphate of mercury battery of Marié-Davy, the sulphate of lead battery of Marié-Davy, and the battery of Siemens (Remak's). These forms will require more particular description.

(d). The balloon battery of Daniell, by Parelle (fig. 12). I used for a long period, for the application of continuous currents, one of the modified Daniell's batteries, which, of the forms then known, was that which furnished the most enduring and most steady, current. Instead of having the positive pole within, and the negative pole without, as in the original form, the poles of this apparatus had the contrary arrangement. The zine plates, 14 centimetres in height, were amalgamated, and immersed in a solution of sea-salt, placed between the porous cell and the external vessel. The porous cells containing the solution of snlphate of copper were surmonnted by glass balloons

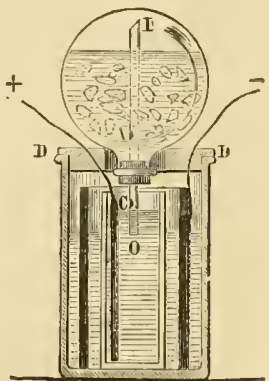

Fig. 12.-A Daniell's cel with Parelle's balloon. ${ }^{6}$ (inverted flasks) full of crystals of sulphate of copper, moistened with water. This arrangement gave way to the use of a very large porous cell, in the midlle of which was a grating supporting

${ }^{6} \mathrm{I}$, tube introduced into the balloon. immersed in the solution of sulplate of A. C, lateral openings in the tube. D, D, copper. O, porous cell containing the cover of wood, pierced by two openings solution. - conductor fixed to the zine for the conductors. + rod of copper that surrounds the porouscell. 
a considerable quantity of the crystals, and supplying the action of the battery for a long period.

For more than ten years, I have had constantly in action, in my room, twenty or twenty-five Daniell's elements of this arrangement, some to charge my apparatus, others to work a telecraph, bells, and an electric clock. To these, when united, I ard at pleasure a score of other elements of the same kind, which I have used since 1860 , for some new investigations of the action of continnous constant currents; investigations, the results of which will be stated in Chapter III.

Well, notwithstanding the greatest care, this pile is far from being constant; and the least change of temperature is sufficient to canse considerable changes in the force of its current between one day and the next. These variations of temperature increase or diminish the concentration of the saline solutions, whence result proportionate variations in the strength of the current. To avoid these considerable oscillations, it is necessary that the room in which the pile is placed should be constantly maintained at the same temperature, by an appropriate system of warming. This precaution is indispensable in winter, because a frost will diminish the power of the apparatus by one-fourth, one-half, or even more. Changes of temperature also occasion damage and accidents to the pile, which becomes encrusted, and the porous vases are broken by the crystallization that takes place in their pores. In summer, a Daniell's battery is always more powerful, because the saline solutions are more concentrated; but it is not the less exposed to considerable oscillations, only not so considerable as in the winter. It is said that a Daniell's balloon battery will act for six months; but, under the most farourable circumstances, I have al ways cleaned mine at least every three weeks. It requires experience to know how dirty and tedious is that manipulation; and it will be found necessary, on each occasion, to replace a greater or less number of the porous cells, and of the balloons, so that the maintenance of the apparatus becomes somewhat costly.

(e). Marié-Davy's battery of protosulphate of mercury. The desideratum that I have pointed out appear's to me to be fulfilled, in great measure, by the protosulphate of mereury battery of M. Marié-Dary (fig. 13). ${ }^{7}$ This battery has been used at the Ministry of the Interior, for the service of the electric telegraph, for a period of eight months, requiring no other care during that time, than an occasional addition of water to supply the loss by evaporation. The current appears to have been, in this application to telegraphy,

7 M. Marié-Davy presented his battery to the Academy of Sciences in 1854. 
as constant as that of a Daniell's pile. I have constructed in my rooms a battery of proto-sulphate of mercury, of forty elements, like that used at the Ministry of the Interior. I have kept it in action for comparison with the Daniell's battery, and for the performance of some new electro-physiological and therapeutical experiments upon the most constant currents. During more than eight months it has beu in action, preserving nearly all its original power. I have simply poured into it, from time to time, a little water to fill up the cells. This battery has not appeared to me to be so sensitive to variations of templerature as

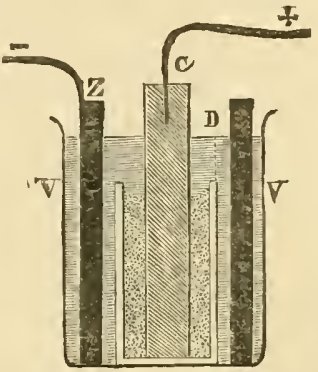

Fig. 13.-Cell from Marié-Davy's protosulphate of mercury buttery. that of Daniell; and, during the winter, it has not brokien a single porons cell. Its elements have only one-third the surface of those of Daniell; and, consequently, it occupies much less space, and gives a smaller quantity of electricity. It possesses, howerer, a greater electro-motive power than the battery of Daniell.

Unquestionably, in some respects, the battery of sulphate of mercury is inferior to Daniell's. Thus it polarises more; and wastes more rapidly under the influence of closure of the current by a metallic conductor, especially when the current is too long closed. Niy electric clock, for example, which closes the circuit for one-half of each second, exhansts in a few days (in three days) a sulphate of mercury battery, the porous cell of which has been filled by the salt; although it will go for three weeks with a Daniell's battery, preserving a sufficient force.

Fortunately, however, this polarisation and rapid exhaustion of the sulphate of mercury pile, under the influence of too prolonged a current, are not observed, or only in a slight degree, when the intra-polar conductor is organic, or, in other words, is a bad conduetor. 'Thus I have passed a continnous current from my pile, for twenty or thirty minutes, through the upper limbs, the hands being placed in basins of water, and each hand holding an electrode, without the current being much enfeebled after the operation. - For medical uses the importance of this fact is manifest.

One of the greatest inconveniences attending a Daniell's pile, especially for medical purposes, is that it exhausts and tarnishes itself in repose nearly as much as in action. Fifteen elements of my Daniell's pile had been at work for three weeks, for the ap-

s $Z$, zinc, C, carbon collector. D, porous cell containing the protosulphate of merexuy. V, external ressel. 
paratus in my rooms, for my bells, chamber telegraph, and electric clock, while other fifteen elements had been at rest for the same period. The latter were nearly as much soiled and enfeebled as the former.

I shall show hereafter how, by means of an apparatus that I have called the isolator and distributor of the battery currents, I have been able to moderate this rapid exhaustion. The sulphate of mercury battery, however, wastes but little during the intervals of its application, and may, without doubt, be kept in activity a considerable time, as much as six or eight months, for certain purposes. The power to preserve a sulphate of mercury battery for so long a time without having to devote to it incessant care, gives it an incontestable superiority over the Daniell's; and the aggregate of all these advantages induces me to give the preference to the sulphate of mercury, for the application of contimuous currents for physiological and therapeutical purposes.

The description of this battery is not yet to be found in standard works; and I will therefore describe here the one that I have had constructed upon the model of that at the Ministry of the Interior. Each element is composed (1) of an exterior glass cylinder ( $\mathrm{V}$, fig. $13)$; (2) of a cylinder of zinc, $\mathrm{Z}$; (3) of a porous cylindrical cell, $\mathrm{D}$; and of a piece of carbon, C, placed within it. The extemal cylinder, eight centimetres high and six in diameter, is one-third full of water, in which is immersed the zine. This is seren centimetres high, and is formed into a cylinder four centimetres and a half in diameter. The porous cell, as high as the zinc and threeand-a-half centimetres in diameter, is a third full of paste of protosulphate of mercury, ${ }^{9}$ in which is placed a piece of carbon twelve centimetres high, two-and-a-half wide, and twelve millimetres in thickness. A plate of copper, riveted and soldered at one extremity to the zinc of one element, is placed in communication with the carbon of the next. It is unnecessary to say that a battery formed of forty of such elements is put together like all other batteries for obtaining physiological effects. The details are given in elementary treatises on physics. Many inconveniences have led me to doubt the excellence of this battery. The plates of copper oxidize rapidly at their points of contact with the carbon; and break on account of their amalgamation with the mercury. I hare obviated these inconveniences by giving the pieces of carbon such a height that the acid mercurial solution

9 To make the paste, a sufficient quantity of water should be poured upon enough powdered proto-sulphate of mercury for all the clements, and mixed with

it into a paste. The preparation should be made at the time of setting up the pile. 
ascends with difficulty to the point of contact; and I have checked endosmosis by dipping the upper end of each carbon in melted wax, which, in cooling, fills the pores into which it has penetrated. For greater firmness, I have covered this upper end with a coat of varnish, except at the points of contact. Lastly, I have platinized the plates of copper at their points of contact.

(f). Marié-Davy's sulphate of lead battery.-The high price of protosulphate of mercury has probably stood in the way of its introduction into common use. This would doubtless be foreseen by the inventor; who has employed himself in endeavours to remove the obstacle. Moreover, the battery leaves us something to desire, as I have said, with regard to the constancy of its action.

It has occurred to this ingenious physicist: (1) to replace the mercurial paste by a paste made with porrdered sulphate of lead and a saturated solution of marine salt; (2) to substitute for the carbon a thin plate of timned copper, seren centimetres square, and rolled upon itself; (3) to fill the space between the porous cell and the external ressel with a concentrated solution of marine salt; (4) to coat with tin the plates of copper that form the connections between the two parts (zine and lead) of the battery.

Thus formed, the sulphate of lead battery affords a current of the greatest constancy. In a damp place and in an even temperature it has been in action for more than a year in my room, requiring no farther care than to replenish the saline solution from time to time, so as to keep it always at nearly the same level. It is much less costly to procure or to maintain than the battery with sulphate of mercury; and for all these reasons I prefer it to all others for the application of continuous or interrupted voltaic currents. But, as its electro-motor force is less by one-half than that of the sulphate of mercury, I have found it necessary to increase the number of the elements in order to meet the demands of practice. Moreorer, its bulk is such that it is only applicable in my own house.

(g). Siemens' battery (called also Remak's).-Quite recently, a battery, invented by a very distinguished Prussian engineer, M. Siemens, has been much extolled in Germany. It is often called also Remal's battery; from his having rendered its use general in medical practice.

This battery of Siemens' (fig. 14) is no more than a modification of Daniell's, in which the inventor has sought chiefly to increase resistance by placing between the porous cell that contains the sulphate of copper and the zinc a paste of paper and sawrlust 
moistened with water, and by increasing considerably the distance between the zinc and the porons cell. This particular arrangement affords the most constant current of any of the modifications of Daniell, and it is the one which will work

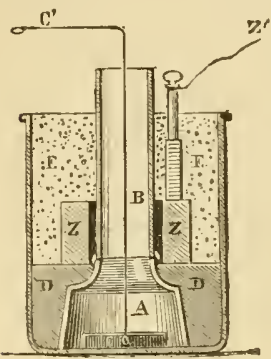

Fir. 14-Cell of Siemens' (or Remak's) battely.' for the longest time, they say, without requiring to be touched. Can it be said that it is not necessary to renew from time to time the crystals of sulphate of copper contained in the glass tube? After having become considerably weaker during the first three or four weeks, a Siemens' battery will, indeed, remain nearly stationary for a year or more. If it only becomes constant when it has lost threefourths of its power, this is paying a little dearly for the constancy.

This battery, which is quite sufticiently dear, is excellent for the application in medicine of continuous constant currents, but is defective for all other purposes.

For this reason I still prefer the employment of the sulphate of lead. It furnishes a current not less constant, it will work for a long time, not requiring to be cleaned, or to have the sulphate of lead renewed, before the lapse of about a year; it requires no other care than to pour, from time to time, a little salt water between the porous cell and the external vessel; its electrolytic action is not considerable; it is alapted for the application of continuous and constant currents to therapentics or to physiological experiments ; and it will at the same time work my domestic telegraph and my electric clock; and, finally, it is moderate in price, and not too bulliy.

[Stöhrer's Battery with Lifting Apparatus.-Stöhrer, of Dresden, has constructed a very excellent battery for medical purposes. This battery consists of carbon and zinc, without an earthenware cell. The carbon (fig. 15), hollow within, filled with sand, and closed by a glass stopper, serves for the reception of a concentrated solution of chromic acid in water. Of this solution, ten or twelve drops will suffice for a long time. In daily and continued nse, this portion must be renewed as often as the diluted sulphuric acid in the glass. The zine surrounds the carbon; but is kept from contact by glass insulators. These elements are placed in a circular glass cell, which serves for the reception of diluted sulphuric acid. This cell

1 A, porous diaphragm ecmented to the glass tube $\mathrm{B}$, filled witl a solution of sulphate of copper and with crystals. C. plate of copper fixed in the porous diaphragm, and communicating with the exterior by the wire $\mathbf{C}^{\prime}$. Z, a crlinder of zine surrounding the glass tube, and communicating with the exterior by the copper wire Z'. D, paste of paper placed between the diaphragm and the external vase, and below the zinc. E, saw-dust, filling all the space between the glass tube, the external rase, and the paper paste. The whole of the battery is moistened with water. 
is so arranged in the completed apparatus, that it can be moved vertically up and down; and ean be fixerl at any point. By this arrangement, the acid can be lorought into contact with the whole, or with part of the zinc and carbon; or, by shntting down the glass, can be excluded from them altogether. In

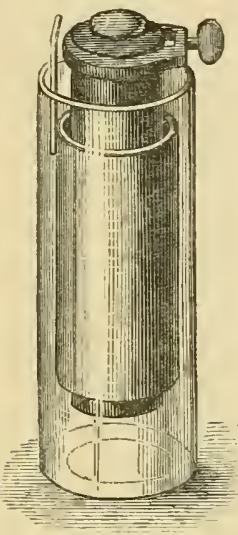

Fig. 15.-Stohrer's battery.

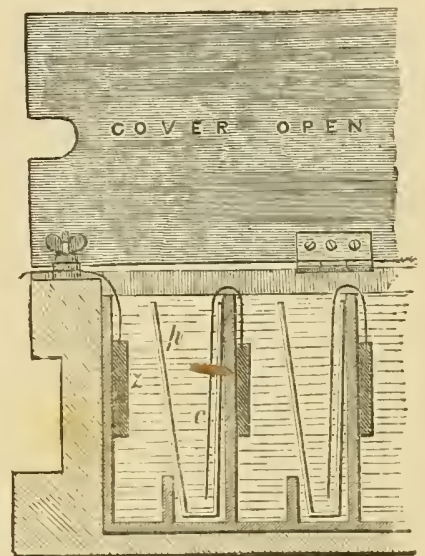

Fig. 16.-Muirhead's buttery.

the latter case, the acid will only fill the lower third of the glass. The advantages arising from being able to remove the elements at once and without difficulty from the exciting fluid, and from the equal facility with which they may be brought into aetion, are obvious. If the battery be in daily use, the zine wonld require to be re-amalgamated, and the acids renerred every six or eight weeks. Twenty-four or more pairs of the elements are arranged upon a stand to form a battery for medical use.

Muirhear's Battery.-This form of hattery, as adapted for mentical purposes by MIr. Becker, of the firm of Messrs. Elliott Brothers, is in use at the National Hospital for the Paralysed and Epileptic. It is a modification of Daniell's battery, and has been largely employed in this country for the electric telegraph. The tension of the battery is low, but the action is very uniform. The elements consist of a zinc plate (fig. 16, z), mamalgamated $\left(3 \frac{2}{2} \times 1 \frac{6}{8} \times \frac{1}{8}\right.$ inches), and of a thin copper plate, $3 \frac{6}{5} \times 3 \frac{2}{\mathrm{~s}}$ inches (fig. 16, c). The copper plate is immersed in a solution of sulphate of copper, placed in a porous cell (fig. 16, $p$ ); the zine plate immersed in simple water, contained, with the porous cell and its contents, in a quadrangular porcelain ressel. The porcelain ressels are constructed in couples, each holding two pairs of elements, and five of these comples are packed in a strong oak box. The arrangement of the battery adopted at the National Hospital for the Paralysed and Epileptic, as least complex, is that shown in fig. 17, in which ten boxes, containing 100 pairs of elements, are placed upon a simple open stand.

In using this battery for medical purposes, single pairs of elements are not bronght into action. The pairs, to the eightieth pair, are grouped in sets of five; and the remaining twenty are grouped in tons. The terminal wires of the colls thus arranged, are attached to buttons in rear of two revolving dises, numbered respectively from 5 to 45 , and, from 50 to 100 ; and by turning the dises, the operator, without detaching the conducting wires with which the rheophores are commected with the instrument, can bring into play the current from as many sets of cells as he desires.

When in daily use, fhe cells require to be recharged, and the zine plates cleaned every two months; and new zine plates are needed every cighteen 
months. Mr. Becker has added to the battery, as a means of ascertaining its state of action, and as a guide to the operator, an ingenionsly constructed tangent galvanometer. The hattery, when freshly charged, and all connections

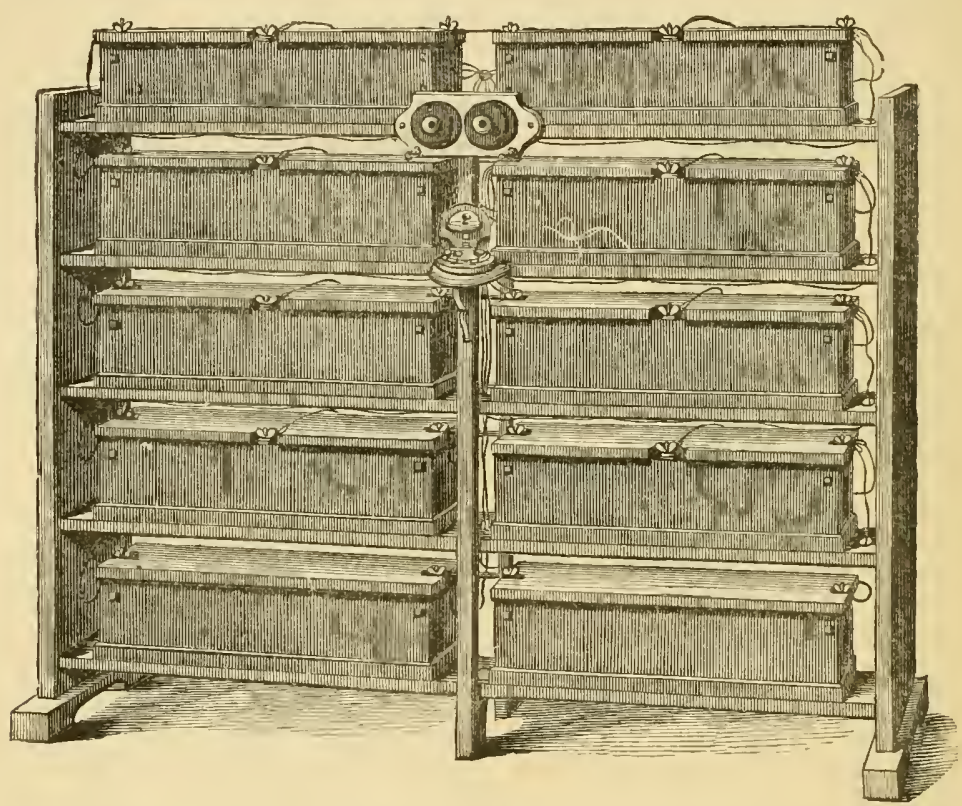

Fig. 17.-Muirhead's battery as arranged at the Nitional Hospital for the Paralysed and Epileptlc.

cleaned, is in its most effective state; but as the strength gradually diminishes, it is essential for the medical practitioner to know the degree of diminntion at any given time, in order that he may determine the number of cells to be used. The galvanometer coil has about one unit resistance, and in the foot of the instrument a boblin is inserted, which has abont 130 units' resistance, and which has no influence on the needle. This additional bobbin is inserted to make the resistance of the galvanometer somewhat equal to the resistance of the battery itself. The terminals are commected with a simple commutator, so that the current can be made to traverse the galvanometer or not.

When the battery is in perfect action, it has been found that-

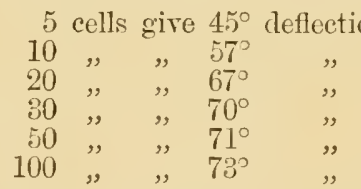

Should five cells give only $22^{\circ}$ deflection, the battery would be half its strength (if for the sake of illustration we take the angles for the expression of the strength instead of the tangents), and a higher number of cells should be placed in action, where before five were sufficient.

To show how additional resistance thrown into the circnit affects the reading of the galvanometer, the following observations were made by Mr. Becker. When 100 cells are used, then- 
10,000 B A units in circuit, gave $13^{\circ}$ deflection.

\begin{tabular}{|c|c|c|c|}
\hline 5000 & & & \\
\hline 3,000 & $"$ & " & $\begin{array}{l}25 \\
33^{\circ}\end{array}$ \\
\hline 2,000 & ", & " & $41^{\circ}$ \\
\hline 1,000 & 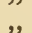 & ", & $55^{\circ}$ \\
\hline 500 & " & , & $63^{\circ}$ \\
\hline 300 & ", & 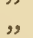 & $66^{\circ}$ \\
\hline 100 & , & " & $70^{\circ}$ \\
\hline
\end{tabular}

This table may give a hint to the operator, in what degree the resistance of more or less of the human body influences the effective strength of the battery, although it must be borne in mind that the human body has a very variable resistance.

The Becker-Muirhead Battery has been in use at the National Hospital for the Paralysed and Epileptic, and in my own private residence for several years. ${ }^{2}$ With ordinary watchfulness of its state of action, and care as to regularity in re-charging and cleansing, it has proved a very effective and trustworthy instrument. For a fixed, large surface, constant-current battery, it is the form best fitted, to the present, to meet the wants of the English practitioner. For, from the large extent to which Muirhead's battery is used for the electric telegraph in the kingdom, little difficulty is experienced in making good accidental damage, or necessary deteriorations, at a reasonable cost. Moreover, from the simplicity in detail of the instrument, it can be cleaned, re-charged, and repaired with facility by a person of ordinary intelligence.H. T.]

\section{C.-Portable batteries of small surface.}

In order to diminish certain inconveniences of the batteries of large surface, instruments have been designerl composed of a great number of small elements, which can be combined within a narrow compass; and which, in little bulk, possess sufficient electro-motor power, without our having to fear too great calorific or electrolytic action.

I proceed to give a description of several forms of these small electro-motor instruments, which constitute a real progress as regards their therapeutical application in certain cases; although they are, for the most part, far from combining all the conditions that are to be desired.

(a). Gaiffe's portable battery of chloride of silver.-II. A. Gaiffe, favourably known by the ingenious arrangements of his instruments for medical electricity, has sent me a description, or rather a detail, of a portable battery of constant continuous current that he has constructed. It is the chloride of silver battery of МII. Marié-Davy, E. Becquerel, and Warren de la Rue; who, at different times, have made communications with regard to it. The original idea belongs to M. Marié-Davy; ${ }^{3}$ and II. Gaiffe has

2 [In my own house the battery is placed in a closet on the area floor, and the conducting wires with dises brought into the consulting-room. I may add, incidentally, that this form of battery lends itself very readily to ingenious and orna- mental modes of packing, if such be required. Giving off no fumes or odours, it may be placed in any room without hesitation, never becoming offensive.II. $T$ :]

3 See, for the method of re-charging 
rendered it applicable to medical uses by the improvements that he has introduced. The following is the notice sent to me on the subject:-

"The apparatus (fig. 18) is fitted in a box, the size of which depends upon the number of elements that it contains. The hox

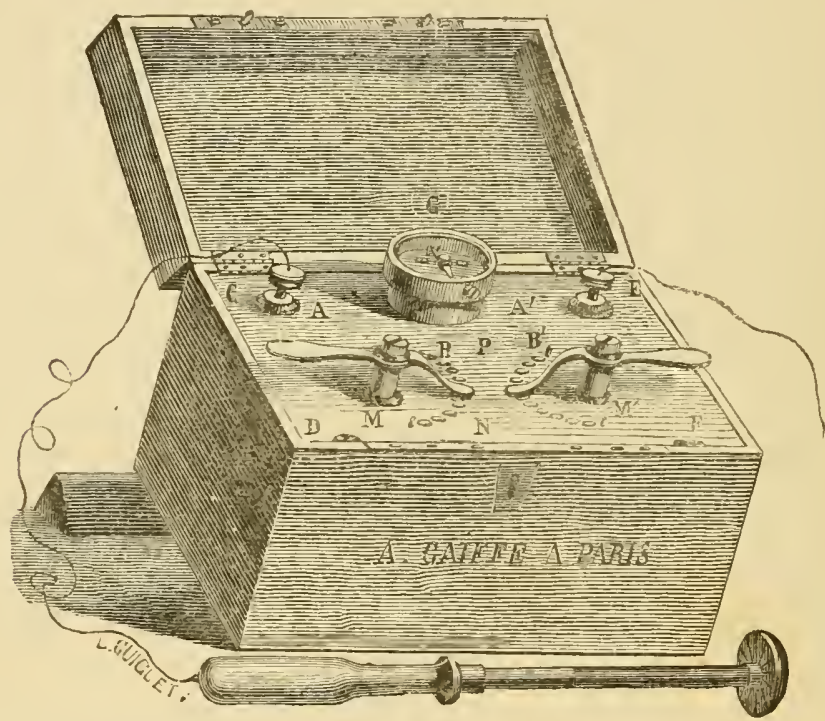

Fig. 19.- fiuiffe's portable buttery of chloride of silver.

for thirty-six pairs, represented in fig. 18 , is 20 centimetres high, 20 long, and 18 wide.

"A double top CDEF, which forms the upper part of the box and covers the battery, serves to support the various appendages necessary for the use of the instrument. These are, A, $\mathrm{A}^{\prime}$, electrodes which deliver the current, and from which the conducting wires proceed; $\mathrm{II}, \mathrm{II}^{\prime}$, hatudles by which to combine, in greater or less number, the elements of the battery, and to direct the current in the course desired; $G$, a galvanoscope, serving to show the passage of the current.

"All the prairs, being identical in size and arrangement, can be placed in the box one for another; and they are so constructed that they can only be placed in a position proper for their being combined. The various contacts are made automatically by putting the elements in place.

the cells, the fourth page of M. Gaife's $\mid$ instrument for the continuous and in printed instructions. The cells are that for the indueed current.

charged in the same manner in the 
"This general arrangement of the instrument greatly simplifies its manipulation, and will promote the extended employment of the continnous current.

"A pair of elements with chloride of silver, having eight square centimetres of working zinc surface, will correspond, as regards quantity, to a new and well charged pair of Remali's. The electromotor force is a little less, about in the ratio of 5 to 6 . In order to obtain the equivalent of a cumbrous Remak's battery of 24 pairs, it is sufficient to have 36 pairs of chloride of silver with eight centimetres of surface.

"I Lastly, the cost of the battery does not exceed thirty centimes the working hour, when the human body forms part of the circuit."

I have now experimented with this battery for about a month, and have been well satisfied with the results obtained. Its transport is as easy as that of an induction instrument; and its application requires none of the manipulation inevitable in the use of other batteries; but I have not had it under observation sufficiently long to be able to determine its actual value, or to say how far it fulfils the promises of the inventor with regard to its electro-motor force, and with regard to the constancy of its continnous current.

(b). Small columnar pile of the Conservatoire des Arts et Métiers. There has long been made in England a small columnar battery (a diminntive of Volta's pile), of which each disk, formed of two plates of copper and zine soldered together, lias a diameter of abont one centimetre and a laalf. This battery is formed of a greater or less number of conples. The disks, and the circles of cloth which separate the conples, all have a central perforation, by which they are strung on to a cotton wick, so as to form a little colımn. To excite the battery, the whole is dipped in vinegar. The circles of cloth and the cotton wick remain impregnated with the liquid for some time after they are withdrawn from it. The current of this pile is sufficiently steady; becanse the acid used (vinegar) attacks the metal plates slightly.

This little pile occupies only a small space, but it requires to be cleaned after every time of using, and thus occasions much loss of time. From the number and the small surfaces of its elements, it increases the physiological power of the battery while diminishing its bulk and calorific action. 'This was evidently an improvement, the utility of which, unfortunately, was little unclerstood, for the apparatus has remained almost unknown; and the idea which governs its construction has been too long neglected.

[Hammond's columnar battery.-Dr. William A. Hammond, of New York, has devised a very ingenions colmmnar battery, of the effects of which he 
speaks very highly, when he has liad occasion to make use of the intermupted galvanic current in the treatment of infantile paralysis, the variety he terms "organic," and to which Duchenne has given the desiguation "fatty atrophic paralysis of infancy" (faralysie atrophique graisseuse de lenfance). The following is Dr. Hammond's description of this instrument:-

"It consists of a series of elements formed of plates of perforated zine and copper, as shown in fig. 19. These plates are soldered together, the copper being bent over at the sides, the zine laid upon it, and the two securely fastened. A thin piece of wood is then placed between the plates, to prevent their heing pressed together. The elements rest upon a plate of hard rubher, and are kept in place by four hard rubber rods. Two other plates of hard rubber, having each a large hole in the centre, and four holes for the rods, rest on the top. These are kept in place by pins which pass through holes in the rods. The whole is hung to an iron or lorass support as shown in the figure, and a saucer underneath eatches the rinegar used to set the instrument in action.

"To arrange the apparatus, an element with a copper wire soldered to the copper plate, is placed upon the lower piece of hard rubber; npon the elcment, a piece of flannel, and so on. The elements are so placed that the copper is always below. The last element has a piece of copper wire soldered to the zine plate. Insulated wires are used to connect the poles with the electrodes.

"To set the apparatus in action, strong vinegar is poured upon the top. It passes throngh the elements, and moistens the flannel. If the plates

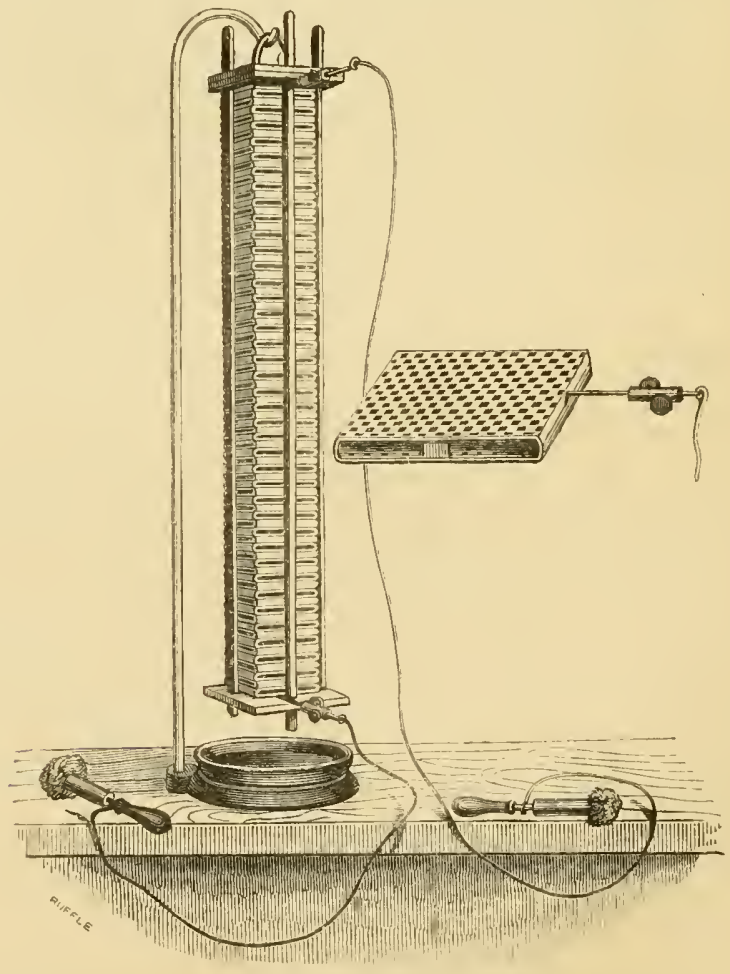

Fig. 19 -IIammond's columnar battery. 
were not perforated, the flannel wonld only be moistened at the edges, and thus a great loss of power would be the result.

"Copper" ganze may be nsed instead of perforated copper plates." _ - The Quarterly Jomrnal of Psychological Medicine and IIedical Jurisprudence,' vol. i. pp. 62,63.)-II.T.]

(c). Galvanic chains of Golberger and of Pulvermacher.-Of late years the little voltaic instruments known as galvanic chains have been introduced. They rest on the same principles as the foregoing; that is, they are formed of a great number of elements having but small surface.

The first chains of Golberger, which possessed no kind of property, because the elements that composed them were not disposed in the manner necessary to establish a galvanic current, soon gave origin to the galvanic chains of Pulvermacher (figs. 20 and 21).

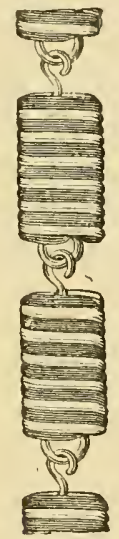

Fig. 20.-1'ulvermacher's galvanic chain.

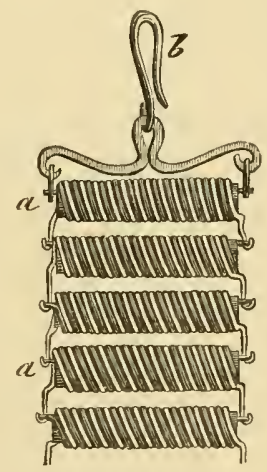

Fig. 21,-Another form of Pulvermacher's galvanic chain.

These are admirably combined to produce, in small bulk, galvanic currents of great physiological power, with but moderate calorific action. They are arranged in the following manner. Each couple is formed of a zinc wire, rolled in a close spiral, but with its coils not in contact, around a cylinder of wood one-and-a-half or two centimetres in length and five or six millimetres in diameter. These couples are united together by little rings of copper, the copper wire communicating with the zinc, and vice versa, forming chains varying in length according to the intensity that is desired. In this way may be formed batteries of 300 or 400 elements. The chains, having been steeped in vinegar, yield currents, and preserve this property for many hours; although they become much weakened in a very short time. Pulvermacher's chain, which

4 These batteries are nanufactured by Messrs. Chester, of New York. 
is thus a happy modification of the voltaic pile, has been principally intended to be employed as a topical application; and the electro-cutaneous excitation that it produces has been found useful in the treatment of obstinate rhemnatoid or neuralgic pains. But the continued interruptions due to its mobility and its rapid polarisation render it an apparatus ill suited for muscular electrization, and especially for the application of continuous currents.

[Pulvermacher has recently effected a considerahle improrement in the construction of his chain battery. He has discarded the cylinder of wood, and substituted for the wire thin zine and copper plate. The dimensions of the clements of the different sized chains remain the same, but each segment is formed of a cylinder of copper plate, perforated with longitudinal openings; and within the copper cylinder is a cylinder of zinc without perforations. The zinc is separated from immediate contact with the copper by a few stont threads. An mulroken link, which forms part of the copper cylinder, serves as the means for joining the sereral segments. This construction permits the withdrawal and renewal of the zine cylinder when necessary. - $11 . T$.

(d). Rilbon battery of Duchenne.-To avoid the inconvenience of mobility of the elements, I constructed a small voltaic apparatus, resting also on the principle of the multiplication of elements with diminution of their surfaces. It was formed of little cubes, a centimetre and a half in height, each composed of a band of zine and a band of copper, folded repeatedly upon themselves, and separated by a small diaphragm of linen, or, better still, of goldbeater's skin. These little cuber, connected by soldering, and so adapted that the bands of copper communicate with the bands of of zinc, were fixed in a wooden frame (fig. 2.2).

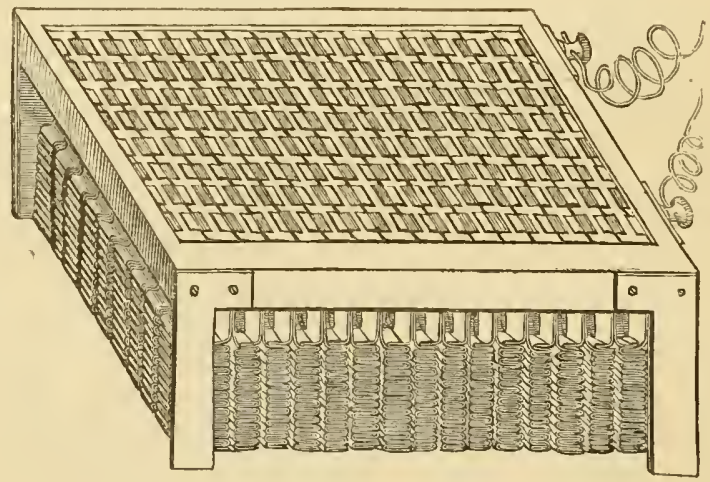

Fig. 22,-The Rilbon battery of Ruchenne.

One hundred and fifty of these little elements were arranged in a frame, having a surface of sixteen centimetres by twelve. I called the apparatus the ribbon battery. In order to put it in action, it was placed for a few minutes in a vessel of vinegar. A 
rheotome (instrument for causing intermissions) moving by clockwork, and connected with one of the poles, allowed me to obtain, at pleasure, intermissions of greater or less rapidity.

It is not necessary to describe the advantages of this ribbon battery, in which the elements are fixed and soldered to one another; advantages that I have not found in Pulvermacher's chains.

The latter form of battery is only fit to be used for topical applications; the former is more trustworthy, and more convenient, when it is wished to produce localized galvanization. Lastly, the ribbon battery is more powerful than a Pulvermacher's chain, and is not, like it, exposed to a rery rapid deterioration.

(e). Small tension battery of Alph. Mathieu. - I prefer to my ribbon battery the small electro-motor apparatus represented in fig. 23. Conceived in the same spirit, it differs from the ribbon

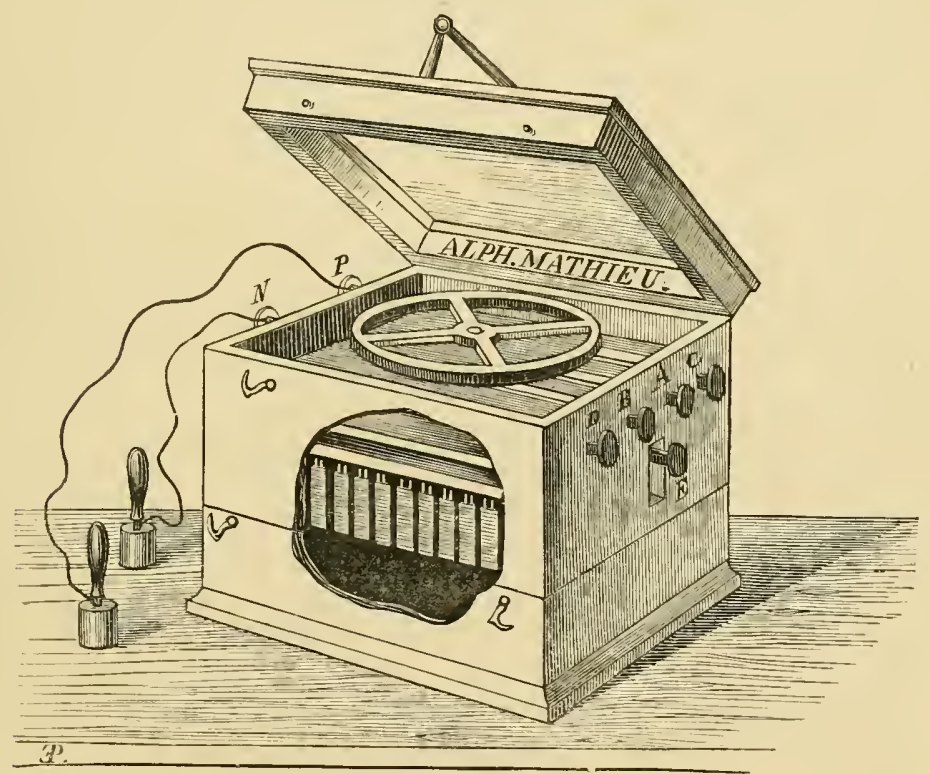

Fig. 23.-Electro-motor instrument of Alph. Mathieu.

battery in its more simple and more skilful construction, and in being easier of management, rather than in any difference of its elements. By means of an ingenious mechanism, forming part of the apparatus, a continuous current may be used, or one with intermissions either very rapid or more or less slow, through a flywheel which continues to revolve for from ten to fifteen minutes 
when once an impulse has been griven to it. ${ }^{5}$ The following is a description.

This battery, of small dimensions, is contained in a handsome box, divided into an upper and a lower part. The lower part receives the liquid required to charge the battery. In the ujper part, a tablet of open work receives the extremities of 400 cylindrical elements. These elements are contained one within another in pairs, one of brass in one of zinc, and are separated by a slight porous tube, rendering each pair equivalent to a galvanic couple. The liquid surrounds them on all sites, within and withont, and descends along the sides when the battery is in action.

Between the lid, which is closed by a glass, and the space oceupied by the battery, is placed the mechanism for intermissions. On the right are the knobs that govern this mechanism. The movement is furnished by a fly-wheel, started by a rack $\mathrm{C}$. The rapid intermissions are produced by the vibrations of a spring, struck by the fly-wheel. The slow intermissions are produced by means of two toothed wheels, also guided by the fly-wheel, and acting by means of pegs upon a lever arranged for the purpose. Each wheel produces a different intermission; and one or other of them is put in action by the play of a slide, worked by drawing ont the stop $A$.

On the left are the binding screws, N P, for fixing the conductors. For the continuous current, the knob D must be turned mutil it is stopped. The knob B, which may be turned more or less up to its point of arrest, serves to graduate, with perfect regularity, the electro-motor force of the current.

'The exciting liquid having been poured into the lower compartment, the elements are immersed by pressing upon the knob E. After a few minutes, they may be raised to their former position.

The pile is charged with common vinegar, or with pyroligneons acid, pure or diluted, or with glacial acetic acid, in the proportion of about ten grammes to the half litre of water, or even with salt and watcr.

If it be desired to carry the apparatus about, the liquid must be emptied out; and, this done, the battery will continue in action for a whole day, without a sensible diminution of force.

[Foveaux's portable battery. - M. Foveanx, of the firm of Messr's. Weiss and Co., has recently designed and constructed a portable battery, which surpasses all others with which I am acquainted for efficiency of action, compactness consistent with efficiency, and freedom from the annoyances which too often attach to batteries of which smallness of bulk is an important condition. This battery is formed of fifty pairs of Smee's elements, packed

3 This instrument is marle by the inventor, MI. A. Alph. Mathieu, mechanician. 
into a handsome mahogany box, which measures in length 14 inches, in breadth $7 \frac{1}{2}$ inches, and in height $10 \frac{1}{1}$ inches (fig. 24). The cells containing

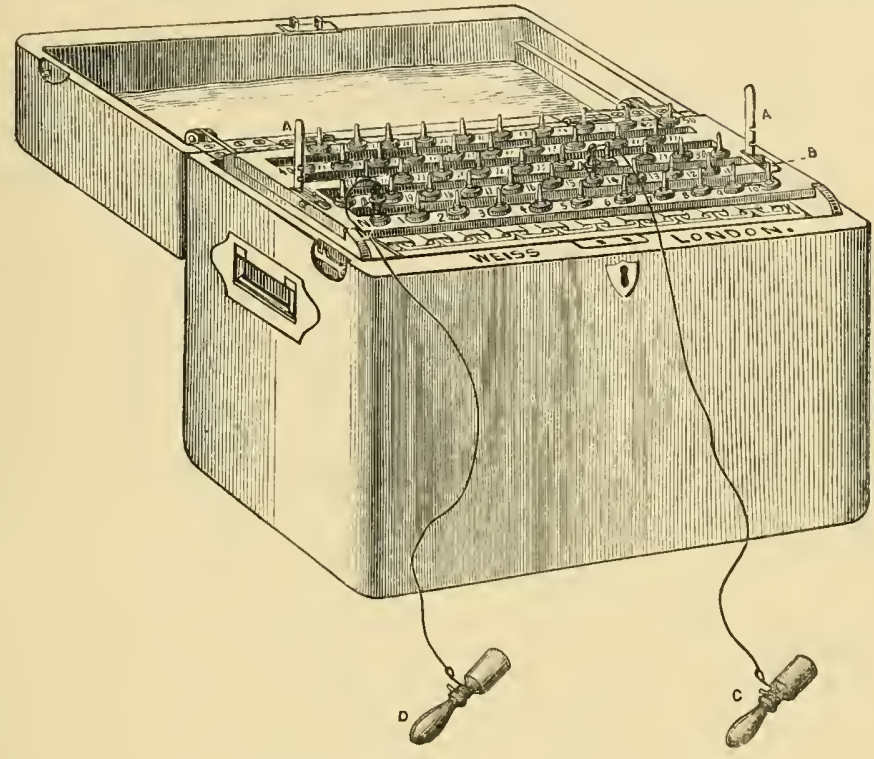

Fig. 24,-Foveaux's portable battery

the exciting fluid (dilnted sulphuric acid), and which are constructed of vulcanite, are attaehed to an ingeniously devised lifting arrangement. When the lid of the box is closed, and the battery is out of use, the cells and the contained exciting fluid are depressed beneath the elements, and the latter are no longer immersed. When the lid of the battery is raised, to place the instrument in use, the cells are elevated and the elements immersed in proportion to the extent to which the lid is thrown back. By this arrangement the zine is withdrawn from the destructive action of the acid when the apparatus is not in use, and the waste of the element may be obviated to the greatest extent. I have used this battery chiefly, as yet, in eases in which the interrupted galvanic current has been required for diagnostic or therapeutical purposes. A battery with the elements similarly arranged, but somewhat differently packed (an earlier arrangement of M. Foveaux's), was largely used for six werks at the National Hospital for the Paralysed and Epileptic, and at the end of that time was still effective. As a portable battery, and for diagnostic purposes, Foveanx's battery leaves little or nothing to be desired. It is exquisitely constructed, and fully supplies a long felt need.- H. T.]

It has been said already that the calorific and electrolytic action is diminished in the forms of apparatus just described (the columnar battery, Pulvermacher's chain, Duchenne's ribbon battery, Mathieu's battery), but it is not so much diminished that it cannot be used in case of need. In fact, all these instruments may be made entirely to disorganise the skin, causing it to pass through every stage of burning, when their action is limited to this organ. They may consequently replace, in a certain degree, 
the moxa and transcurrent cauterisation. ${ }^{6}$ They are even preferable to those means, since they may be made to produce an action more or less gradual, according to particular indications, while the pain ceases immediately after the formation of the eschar. 'Those who have prescribed the therapentic application of the batteries of small surface have too often forgotten the dangers or the inconveniences of their calorific or electrolytic action.

These instruments, having the power to produce energetic muscular contraction, appear, at first sight, likely to be very useful in the treatment of paralysis. Unfortunately, they rapidly become enfeebled; almost immediately, indeed, after being set in action. To illustrate this, it will be sufficient to relate a single experiment with a Pulvermacher's chain. I directed an intermittent current upon the biceps of the arm, the apparatus being at its maximum. The contraction of the muscle was such, at the first intermittence, that the fore-arm was strongly flexed upon the arm, and the patient experienced an acute sensation. But the second contraction was a little less strong than the first, and each successive contraction became weaker, until, after about twenty intermissions, the muscle scarcely contracted at all. I repeated this experiment frequently on different muscles, and found that the weakening was more rapid the more frequent the intermissions. The continuous current, again, exhausts the apparatus more quickly than the intermittent. These little instruments are powerful, therefore,-on the condition that they are not set in action! 'The weakening, however, is only temporary; and, if the current be out of use for a quarter of an hour, the apparatus will recover its original force.

The temporary weakening of these small voltaic piles, even under the influence of intermittent currents, joined to their continual and progressive exhaustion, renclers it very difficult to measure or calculate their action. On account of the sources of weakening to which their physiological action is exposed, these batteries of small surface are, it may be conceived, inapplicable to the localization of electric excitation in individual muscles or nerves, and especially for the electro-physiological and pathological researches which require extreme precision. How, indeed, can we measure a force that is subject to such variations? How can we graluate the dose to the excitability of the organs, so different in different states of health or of disease? ${ }^{7}$

6 If it be desired to produce deep and rapid cauterization, as by a moxa. it is necessary to use a battery of large surface, like that contrived by M. Regnault, and already described at page 16 ; or, still better, the powerful and ingenious pile of Mr. Grenet [or the battery specially constructed for the purpose by Stöhrer, of Dresden.-II. $T$ :]

7 IL. Remak, criticising my statements on the action of the galvanic emrent, lins written (Galvanotherapie, Paris, 1860, 
The considerable and rapid polarization produced in the small galvanic batteries by the continuous currents, prevents them from being used for the physiological or therapeutical study of such currents.

Among the galvanic batteries of large surface that I have described above, those only of which the current is most constant, and of which the electro-motor force is the greatest, are applicable to localized muscular galvanization.

\section{II.-Operative Procedures for Localized Galvanization.}

The precepts that it is necessary to observe, in order to practise localized muscular galvanization by intermittent currents, are absolutely the same with those about to be stated for the practice of localized muscular faradization.

\section{§II.-Localized electrization by induced electricity, or localized muscular faradization.}

Of the three kinds of electricity, induced electricity is the most fitted for muscular electrization, especially when that operation requires to be practised frequently, and for a long time. To be convinced of this, it is only necessary to recall what has been said in the first chapter, on the special therapentical and physiological effects of each kind. Thus faradization excites the most powerful muscular contractions, without at the same time acutely exciting the cutaneous sensibility, without producing shock, without throwing the organs into a kind of stupor, without rupturing capillary vessels; in a word, without occasioning, like static electricity, any accidents from which the patients might recover with difficulty. We know, again, that galvanism and faradism possess, in a nearly equal degree, the power to penetrate more or less deeply beneath the skin; or, in other words, to concentrate their action on muscles or nerves, without affecting the skin; but that the chemical and physiological properties of faradism are infinitely little developed as compared with those of galvanism, and are too weak to be appreciable during muscular faradization. We know, lastly, that faradization produces only a faint phosphene; and that its action upon the retina does not complicate in a distressing manner, like that of galvanism, the electrization of the muscles of

p. 46):- “M. Duchenne appears to be ignorant of the difference which exists between a constant and an inconstant chain; although he lives in Paris, near M. Becquerel, the celebrated inventor of constant chains." Is this good faith? or has he not understood the question at issue. which refers only to small voltaic batteries? I am not aware that M. Beequerel's constant ehains liave been used for medical purposes. 
the face. We have, therefore, good reason to say that faradic electricity, which in no way alters the tissues, is essentially medical electricity.

It is when we wish to limit exactly the power of electricity upon certain muscles or fasciculi, or upon individual nerves, that we understand the value of faradism, and the need of employing those induction instruments which unite all the properties that I have been describing.

\section{I.-The historic question.}

Before proceeding to explain the method of localized faradization, of which, whatever may be said by Mr. Becquerel, I deem myself to be unquestionably the inventor, it is necessary to glance at the historic question.

From the time when the pile of Volta was first applied to electrophysiological experiment, all the world has seen that rheophores, placed upon the moist skin, over masses of muscle, called those masses into contraction at the moment of completing or of breaking the circnit. No refinement of observation was needed in order to establish this fact, which, moreover, was mentioned in the accounts given of physiological experiments made with voltaic currents, prior to the discovery of induced electricity.

Still later, when induced currents were for the first time applied to electro-physiological research, it would have been necessary to be blind in order not to observe that the rheophores, when placed upon moist skin, produced contraction of the muscles beneath, as had long been known to be the ease with intermittent galvanic currents.

At the time when I commenced my experimental researches upon localized electrization, every one used moist rheophores in order to provoke, by their application to the surface of the skin, muscular contractions by means of any kind of battery or any apparatus of induction.

It was certainly not with such insufficient ideas, and by such coarse methods, that I have been able to establish the method that I have called localized electrization, and more especially localized faradization. This method was only really created an the day when I discovered the means of confining to the surface of the skin the action of even the most intense induced currents; and when I had shown, by experiments upon mankind in a normal state, as well as in certain pathological conditions, that it was possible to make induced currents penetrate the skin without exciting it, and to concentrate their action upon the organs beneath. It had not really deserved the name of localized elec- 
trization until it lad been rendered clear by special anatomical and electro-physiological knowledge. Then, with the help of induction instruments and of appropriate rheophores, it became possible for me to lay down the rules by which electric excitation may be limited to different depths, to each muscle, or group of muscles, to each nerve, and to a great number of internal organs.

I am indebted to the use of localized faradization, by the methods which I am about to explain, for the discovery of the individual actions of many of the muscles. ${ }^{6}$ This method, applied to certain pathological and therapentical investigations, has given very beautiful results that have been tested and confirmed by a large number of observations. ${ }^{8}$

\section{II.-Choice of an induction instrument.}

The choice of a good apparatus is of such importance, in the practice of localized faradization, that I shall devote a whole chapter (Chap. IV.) to the question. The considerations that I shall have to set forth cannot be well understood until I have given the anatomical and physiological data upon which localized faradization rests.

For the present I am content to say that, without the proper instruments, localized faradization could scarcely have been realized, and that, without their aid, I could not have opened the way for researches in electro-physiology, pathology, and therapentics, the importance of which is now well established.

8 See Duchenne, Recherches électrophysiologiques sur le méchanisme de la physionomie humaine, 1862; Physiologie des movements démontrée à l'aide de l'expérimentation électrique et de l'observation clinique, 1866.

9 Until now, I have been unwilling to enter upon the historical question that I have thus briefly set forth; because I wish to avoid the appeartnce of glorifying my own work. It appeared more suitable to leave the matter to those who were disinterested with regard to it. I find it necessary, however, to be more guarded, since the invention of my method of localized electrization has lately been claimed by a physicist of merit, Masson, Professor at the Ecole Polytechnique. This philosopher has acquired some fame in the history of induced electricity; but attention has chiefly been drawn to his name by the incessant claims of priority that he has addressed to the Academy of Sciences, with regard to the discovery of electricity by induction. Although his works are certainly in no way to be compared to those of the illustrious Faraday, although the Academy has proved that his pretensions are unfounded, his claims have this much of reason, that he believes himself to have been the first to observe the phenomena of induction. But how shall I characterise his new pretensions, since, notwithstanding his ignorance of anatomy and physiology, he claims priority of invention for a method of electrization that could only be created by the aid of special anatomical and physiological research, and which is not applicable without the exact knowledge, and the observation of the rules, which I have deduced from my experiments? Another physicist, M. Becquerel, has also supported the pretensions of Masson before the Academy of Sciences. He was equally incompetent with him, as regards questions of anatomy and electro-physiology. This must be his excuse. 


\section{III.-Choice of rheophores.}

Muscular faradization is practised, either by concentrating the electric excitation in a nervous plexus, trunk, or filament, which shall conduct it to the muscles dependent upon it, or by directing the excitation upon individual muscles or fasciculi. In these different operations, the rheophores should always be bronght as near as possible to each other.

The first mode of faradization produces general movements; that is, the contraction of many muscles or of all parts of a single muscle; and is called indirect muscular faradization. The second gives more partial morements, from the excitation of one muscle, part of a muscle, or a fasciculus; and is called direct muscular faradization.

Each of these modes of faradization requires a special proceeding that I will describe.

It is known that, on placing upon the skin the moist rheophores of an induction apparatus, the electricity concentrates its power in the organs placed immediately beneath the skin. Consequently, to excite muscular contraction, it is necessary to set the rheophores on points corresponding to the surface of the muscle or of its nerve; the rheophores being placed in communication with the intrapolar extremities of an induction apparatus by metallic conductors.

For the muscles of the trunk, which have a large surface, I apply moist sponges contained in cylinders (fig. 25), or metallic disks

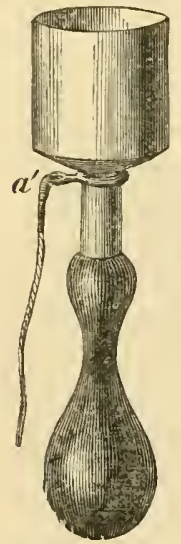

Fig. 25 .

Fig. 25 , insulating handle.

Fig. 26.-Netallic rheophore with (fig. 26) covered with wet leather. These are furnished with insulating handles, as in fig. 25.

To limit the electric action in muscles that present only a small surface, as those of the face, or the interossei, I employ conical metallic rheophores (figs. 27 and 28) which screw into separate handles. The conical rheophores are corered by leather soaked in water, ${ }^{1}$ and are pressed by their extremities to points over the muscles to be faradized. They also serve me to guide the electric action upon nerves, when I practise indirect muscular faradization.

The moist leather which covers the metallic rheophores opposes much less re-

${ }^{1}$ I habitually use the fingers of leather gloves, turned inside out. 
sistance than wet sponge to the faradic current. The difference is due to the different thickness of the two bad conductors that have to be traversed by a current directed upon the surface of the body 'Therefore, in certain cir' cumstances, I prefer the metallic rheophores of large surface (fig. 26), also covered with wet leather, to the moist sponges; because the former practically increase the power of the current. The large metallic rheophores have also the advantage that they may be more or less pressed upon the skin, without annoying the patient, as in the case of the sponges, by water being freely squeezed out from them.

\section{IV.-Indirect muscular faradization.}

Indirect muscular faradization requires, as may be supposed, an exact knowledge of the positions and the anatomical relations of the nerves. It is most practicable in the limbs, where most of the nervous trunks, subcutaneous at some point of their length, are accessible to rheophores.

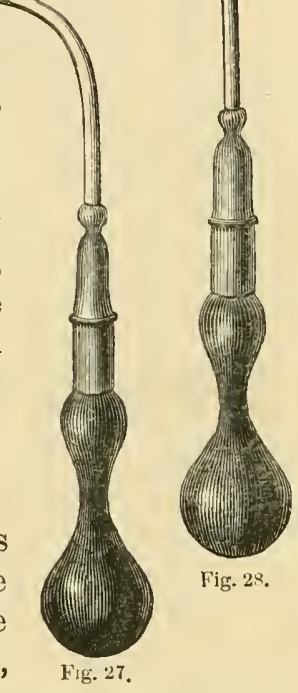

Fig. 27.-Olivary metallic rheophore.

Fig. 2*,-Conical metallic rheophore.

In the upper limbs, electrical action can be precisely limited to the median nerve, at the inner side of the lower third of the arm, and to the uluar, at its passage along the channel which separates the inner cordyle from the olecranon. Faradization of the radial may be practised at the outer side of the humerus, at the junction of its middle and lower thirds, where the nerve escapes from the humeral channel. It would be impossible not to stimulate directly, at the same time, some fibres of the triceps and brachialis muscles. The musculo-cutaneous nerve may be faradized in the axilla. It is also possible to localize the electric action in some terminal branches; for example, in those that supply the muscles of the thenar eminence, and in the collateril nerves.

In the lower limbs indirect muscular faradization is still more simple. The crural nerve is found in the fold of the groin, external to the femoral artery; and the popliteal nerves in the popliteal space. It should be known that electricity will not reach the internal popliteal nerve, which is protected by a great thickness of cellular tissue, unless the current be somewhat powerful. 
The sciatic nerve is only accessible at its origin in the pelvis, through the posterior wall of the rectum. The process for applying faradization to it will be explained hereafter.

In other regions, the practice of indirect muscular faradization becomes more difficult and more delicate. On the face, the trunk of the portio dura, concealed in the thickness of the parotid, is inaccessible to electro-excitation, whatever be the strength of the current. It can be reached at its issue from the stylo-mastoid foramen, by introducing a conical rheophore, covered with wet leather, into the external auditory meatus, and resting it on the lower cartilage; at this point the trunk of the nerve will be distant from the rheophore only three or four millimetres. Its branches may be faradized at their points of emergence from the parotid; and the contraction of the muscles supplied by them is a certain index that they are excited.

In the supra-clavicular region the rheophore, placed immediately above the clavicle, acts upon the brachial plexus; at the summit of the supra-clavicular triangle it is in relation with the external branch of the spinal, at a finger's breadth above the clavicle, and over the clavicular attachment of the trapezius the rheophore acts upon the nerve which supplies the serratus magnus and deltoid; at the same distance above the clavicle, but towards the inferior attachment of the sterno-cleido-mastoid, are the nerves which supply the pectoral, the flexors of the forearm upon the arm, and all the muscles of the anterior region of the forearm which receive the excitation. ${ }^{2}$ Lastly, over the scalenus anticus, the electric excitation is brought to bear upon the phrenic. I shall explain the method which should be employed for the faradization of this nerve, when I speak of the electro physiological and pathological study of the diaphragm. The great hypoglossal is almost subcutaneous at the level of the great cornu of the os hyoides, where it passes between the stylo-hyoid and the hyo-glossus. At this point the rheophores should be placed, when it is desired to faradize this nerve. I shall speak hereafter of the faradization of the glosso-pharyngeal, of the pneumo-gastric, and of the recurrent.

\section{V.-Direct muscular faradization.}

Direct muscular faradization consists, as I have already stated, in causing each muscle, or fasciculus of muscle, to contract indi-

2 I am certain that, in the different points above stated, we find, in the midst of the brachial plexus. the nervous fibres which go to form each of the nerves which arise from it; for I have repeated the experiments thousands of times, and always, on placing the rheophores on these points, I have seen contraction produced in muscles which receive spinal intluence from each of the nerves. 
vidually, or rather, in directly exciting the muscular tissue, by placing moist rheophores on points of skin corresponding to its surface.

It is right to administer to a muscle only the dose of electricity proportionate to its degree of excitability, which is variable with each. It is therefore necessary that the operator should always have one hand at liberty, ready to control, during faradization, the graduator of the apparatus. The same hand serves also to act on the intermissions of the current (in a manner to be explained hereafter). This part of the operation should never be entrusted to a stranger, because the physician should relax or press the movement of the rheotome, according to particular indications, which present themselves at any moment, even during the faradization of a single muscle. One hand only, the opposite to that which controls the graduation of the intermittences, should hold and manouvre the rheophores. The handle of one should be placed between the thumb and the index, that of the other between the middle and ring fingers, the fingers being placed so as to keep the handles in the palm of the hand. This method permits faradization to be practised with great rapidity. Figs. 29 and 30 are intended to show the manner of holding the rheophores.

In fig. 29, the cylindrical rheophores, fitted with moist sponges, or the disks covered with wet leather, are held in the left hand;

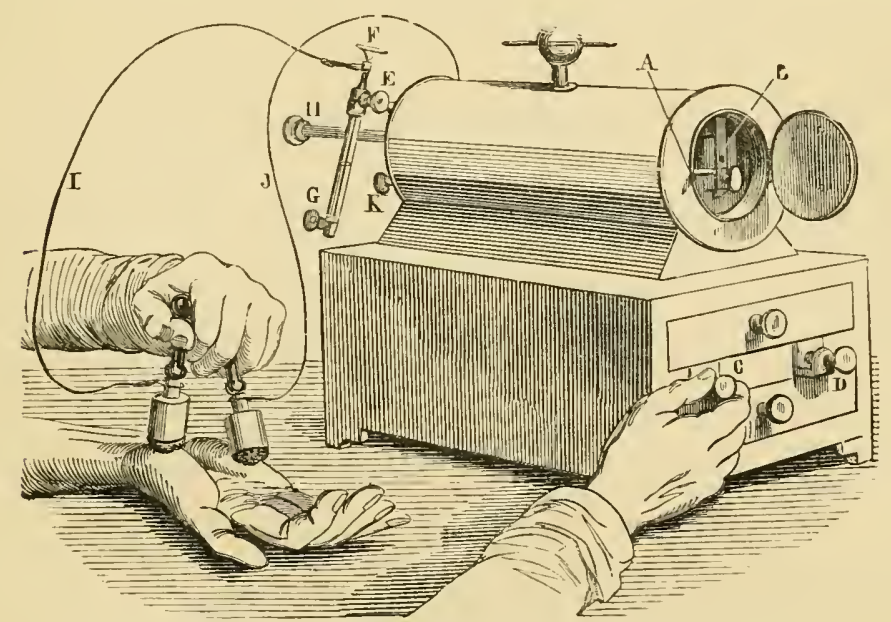

Fig. 29,-Method of holding the cylindrical or disk rheophores in a single hand.

while the right regulates the intermittence, by manipulation of the screw C. 
Fig. 30 represents the conical rheophores, covered with moist leather, held in the left hand, and placed upon the triangular muscle of the lips.

It is always necessary to place the rheophores orer the fleshy bodies of the muscles, and never over their tendons; because (as it is hardly requisite to state), the stimulation of the latter does not produce muscular contraction.

[Mr. J. Netten Radcliffe gives the accompanying illustration (fig. 31) of the method of holding the rheophores ('The Practitioner,' vol. i., p. 25).-H. T.]

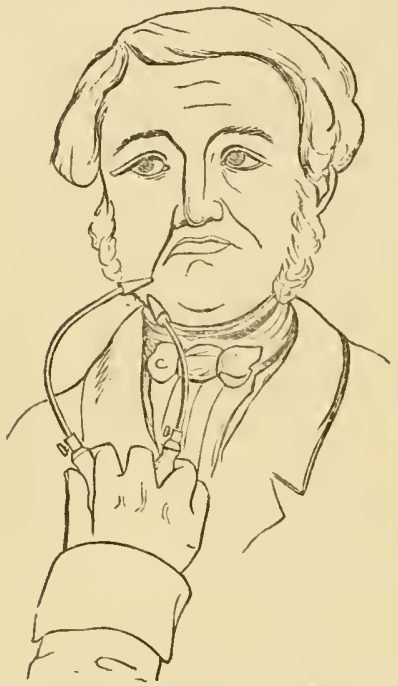

Fig. 30.-Method of holding the conical rheophores.

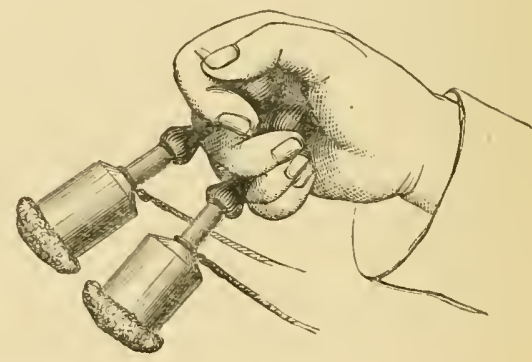

Fig. 31.-Mode of holding rheophores according to Mr, Radcliffe.

In order to faradize a muscle completely, it is necessary that the moist rheophores should cover the whole of its surface; and, when they are not large enough to do this, they should be applied in succession to all points of the surface. The thiclier the substance of the muscle, the more intense should be the current; because a weal current will only produce excitation of the superficial layers.

I proceed to develope this capital proposition, and to demonstrate its truth.

(a).-When the moist rheophores are placed on the upper part of a long muscle, we see, under the influence of an induced current of medium force, this part of the muscle swell, and we feel it harden; while, it the same rheophores are placed over the lower part of the muscle, the lower part swells and hardens in its turn. Rheophores applied to one point of a broad muscle cause contraction only of the fibres with which they are in relation; the neigh- 
bouring fibres remaining relaxed. It follows that the excitation of the muscle only oceurs at the points which are brought into connection with the rheophores.

(b). MIy researches have shown me that, under the influence of an apparatus of strong tension, the electricity penetrates deeply into the tissues. The following observations support this pro: position. In lead-palsy, as I shall have occasion to show, certain muscles of the posterior region of the forearm become atrophied, and do not contract under the influence of faradization. If the tension of the current be moderate, we do not see any morement of the limb when the rheophores are placed over the paralysed muscles; if the tension of the current be very great, we see that the muscles situated beneath those paralysed are thrown into contraction. In the former case, the electric excitation has been limited to the paralysed muscles; in the latter, it has penetrated these muscles to act on those covered by them. In very fat or oedematous persons electricity does not reach the muscles at all, unless by the aid of a current of strong tension. It follows, from these facts that, in the practice of direct muscular faradization, the tension of the current should be proportioned to the thickness both of the muscle to be excited and of the tissues to be traversed.

It is well understood that in all these experiments care must be taken not to excite the muscular nerves; either by placing the rheophores at a distance from their emergence or their immersion ; or, where the nerves cannot be avoided, by operating with a current of moderate tension. In fact, since the moist rheophores are only placed in relation with the surfaces of the muscles, and since the muscular nerves enter at the deep surfaces of all superficial muscles, it is certain that the contractions produced by a moderate current are not excited through the medium of nerve agency.

(c). When the moist rheophores are applied to points corresponding to the surface of a muscle, the excitation produced by the electric recompositions acts simultaneously upon all the anatomical elements (muscular fibres, motor, sensitive, and ganglionic nervous filaments, arteries and veins) which enter into the composition of the portion of muscle excited; while, if the contraction be produced indirectly, that is to say, by placing the rheophores over the motor-nerve trunk, at a distance from the muscle to be excited, only the muscular fibres are influenced. Consequently, the direct faradization of a muscle is not complete, even when its contraction en masse has been produced by the excitation of its nerve, if the rheophores have not been moved over all the points corresponding to the surface of the muscle.

Need I add that, in the living subject, when the muscles are 
excited by the process of direct faradization, it is impossible to act singly upon each of the aratomical elements, since all these elements ineritably receive, at the same time, the direct excitation of the current? Such a remark would be little less than a gratuitous insult to the intelligence of my readers. No one can be ignorant that, during life, these elements cannot be separated as in an anatomical preparation.

I have long adrocated direct muscular faradization in the treatment of affections in which the muscular properties are injured; and principally in atrophy and atrophic paralysis.

This doctrine rests upon a long experience, and proceeds from numerous facts related in the many memoirs that were reproduced in the previous edition. The doctrine, without donbt, has not been laid down explicitly enough in my writings; for certain anthors have attacked direct muscular faradization with violence. According to M. Remak, and according to other authors (his followers) I should only act upon the muscle, in this mode of electrization, through the medium of its proper nerve, and when placing my rheophores over its point of immersion. In order thus to travestie the method of direct muscular faradization, my opponents could not have read that I have advised, as stated above, the movement of the moist rheophores over all the points corresponding to the cutaneous surface of the muscles. 'That by this process the muscular nerve will sometimes be influenced, if the current is strong enough, is incontestable; but, in order for muscular faradization to be complete, it is nevertheless necessary to carry the excitation over all the muscular surface. It is true that M. Remak denies the existence of muscular irritability and sensibility. ${ }^{3}$ Consequently, according to him, what could be the utility of direct excitation of muscle?

Even admitting - what I dispute - that these properties could be as much excited through the intermediation of the nerves as when the rheophores are placed over the muscular tissne, would it be a matter of indifference, therapeutically, that the electric excitation should be applied directly to the tissue of the muscles, the capillaries, and the vaso-motor nerres which accompany them? MI. Remak and his pupils are too, good physiologists to maintain such a position. A little reflection should, therefore, make them

3 For M. Remak to make me say that in direct electrization, the muscnlar fibre contracts solely by resson of its irritability (Hall rian irritability) is to attribute to me an enomity that I have not committ d. (Leber methorlische elec- trisiring gelahmer Muslieln. Berlin, 1856). My reply to this strange criticism was publislied in a well-known and highly esteemed jomnal, Jahrbücher fiir gesammte Medizin, May 1856, and in Lat Revue medicale, 1856. 
understand the therapentical importance of direct muscular faradization.

Nothing can be more easy than this direct faradization, especially in the superficial regions of the trunk and limbs, to those who possess any knowledge of surface anatomy. For the deeper layers of the muscles of the limbs the process is more difficult; although most of these muscles offer some point of their tissue that is sub-cutaneous, and accessible to direct excitation.

I could show, by a synoptical table, the points on which the rheophores shonld be placed, in order to practise direct and partial faradization of the muscles. But such a work would be beyond the limits I have imposed upon myself; and would be of little value to those who have not forgotten their anatomy. 'The practitioner who desires to perfect himself in the art of localized faradization should, however, study myology from a special point of view; and should seek to learn exactly the points at which the superficial and the deeper nuscles come into relation with the surface. With regard to those inaccessible to direct faradization, the number of which is not great, we have always the power to excite them indirectly, that is to say, throngh the medium of their motor nerves.

\section{VI.-Excitability of nerves and muscles.}

The faradization of a mixed nerve or of a muscle always produces, in the normal state, contraction and sensation. It is very important for those who study the art of localized faradization, in its applications either to physiology, pathology, or therapentics, to be acquainted with the degree of excitability of the nerve or muscle upon which the electric stimulant is directed. If all organs possessed the same degree of excitability, the practice of this methor of faradization would be very easy. It would be sufficient to know the thickness of tissue to be traversed, and its resistance to the current, the proper state of dryness or humidity of the skin, and the best form of rheophores for obtaining deep or superficial action; to know superficial muscular anatomy, and the points of immersion or emergence of the motor nerves, so as to be acquainted with the parts on which the rheophores should be placed, either for the direct or indirect excitation of each muscle. Unfortunately the matter is not so easy; since each organ, each muscle, each nerve possesses its own degree of excitability, of contractility, or of electric sensibility.

It would be unsuitable to enter at great length into details on this point of the subject; but it is as necessary to mention certain 
phenomena, ignorance of which might lead to many errors, and sometimes eren to serious accidents.

\section{A.-Excitability of the motricity of nerves, and the contractility of muscles.}

The motricity ${ }^{4}$ of the external branch of the spinal nerve (respiratory nerve of Bell) is the most excitable. Hence the muscles or parts of muscles that it supplies will be thrown into action by a very feeble electric current. For example, the lower half of the sterno-cleido-mastoid, and the middle and inferior portions of the trapezins, are but little excitable. But, if we direct upon the superior half of the sterno-mastoid, or upon the external border of the upper half (the clavicular portion) of the trapezius, a current that is too weak to produce even the commencement of contraction in the other parts of the same muscles, we see the head bent, or the shoulder elevated, or the side excited, by a brusque and violent movement. If the rheophore is placed over the apex of the supraclavicular triangle, the same movements are energetically produced by the simultaneous contraction of the superior parts of the trapezius and of the sterno-mastoid. ${ }^{5}$ It seems to me to follow, from this experiment, that the extreme excitability, which exists only in limited portions of these muscles, is due to the presence of the external branch of the spinal nerve.

I proceed to show the dangers to which the patient is exposed during faradization, if the operator is not acquainted with the important fact just stated. At the beginning of my researches I could not find in writers any warnings to put me on my guard against the too numerous accidents that I have had to regret. The following, among others, occurred during faradization of the trapezius in a case of paralysis of the upper extremity. I had directed a tolerably strong current upon the upper half of the trapezius; and then, passing suddenly to the external border of that muscle, I placed a rheophore upon the apex of the supraclavicular triangle in such a manner as to tonch, at the same time, a portion of the upper half of the sterno-mastoid. The head executed a movement of rotation and inclination so forcible that the patient felt something crack, and had acute pain in the neck. He also experienced stunning, and tingling in the extremities; and required to be bled immediately. If the apparatus had been gradnated to its maximum, would there not have been an accident

4 'The power that a nerve possesses, when artificially stimulated, to excite muscular contraction.

$s$ The external branch of the spinal nerve is distributed to the upper half of the sterno-cleido-mastoid, and to the upper half of the trapezius, especially at its external m ırgin. 
of extreme gravity? 'This fact led me to the discovery of the great excitability of the respiratory nerve of Bell, which I believe to be the most excitable of all the nerves; but this discovery, as we have seen, cost me somewhat dear. ${ }^{6}$

${ }^{6}$ Remak has criticised the experiment which shows the great excitability of the respiratory nerve of Bell, by making, and commenting upon, the experiments following. He has placed a moist rheophore over the occipital extremity of the trapezins, and another over the spine of the scapula, according to the direction of the muscular fibres, and then passed a moderate induced current, from which he obtained only slight contractions. In another experiment, he left one of the rheophores in its former position (either that at the occiput or that at the scapula), and placed the other over the border of the trapezius, at the point of entry of the accessory nerve of Willis. The same current then produced elevation of the shoulder and inclination of the head. He has made analogons experiments upon the biceps of the arm, and has obtained similar results, that is to say, a contraction of the muscle en masse, from excitation of its motor nerve, and very weak contractions when the rheophores were placed over the extremities of the muscle. The only rational conclusion to be drawn from these expcriments is that excitation of a motor nerve produces contraction of all the fibres to which it is distributed (and hence a considerable movement); while faradization of the parts of a muscle remote from the points of immersion or emergence of a motor nerve, produces contraction only of those fibres that are in relation with the rheophores, and of these only the more superficial if the tension of the current be slight; whence a feeble and limited movement. By acting alternately upon bare muscle, and upon its nerve, it may be seen that this conclusion is perfectly correct. It is, therefore, not surprising that direct faradization should produce less movement than indirect; since a much smaller number of muscular fibrès are thrown into contraction by the former. Moreover, in these experiments of MI. Remak, the rheophores were placed at the extremities of the muscle (at the occipital extremity of the trapezins and on the spine of the scapula, and in the same way for the biceps), that is to say, over structures in great part aponeurotic $m$. tendinous, so that the contractions would necessarily be feeble, becanse only a few muscular fibres would be in relation with the rheophores. If M. Remak had placed his rheophore entirely upon muscular fibre,-for instance, a little above the midale portion of the border of the trapezius,-I am sure that he would have produced a marker contraction of the clavicular portion of the muscle, which he evidently could not obtain whilst the rheophores were placed over the aponeurotic extremities.

It is plain that it would be difficult to dednce any strict conclusion from experiments so badly performed. We shall sce, however, from his mamner of interpreting them, how ardent is the imngination of M. Remak. In the first place, he fails to see that the difference in the movements obtained in the several experiments depends upon the fact that, in the one case, the excitation of the muscular nerve causes the contraction of all the fibres to which it is distributed; while in the other, only a small number of fibres, namely those in the neighbourhood of the aponeurosis, are situated beneath the rheophores, and thus called into contraction. Every one else wonld conclude from this that the tendinous or aponeurotic fibres, in their normal state, are not irritable; but M. Remak, taking the aponeurotic for muscular fibres, draws from his experiments the following deduction against the Hallerian contractility, "'That which strikes us the most," he says, "is that these experiments, besides their practical significance" (this practical significance being, according to M. Remak, that the electric current should be exclusively directed upon the motor nerve at its point of inmersion or emergence-a retrograde precept as I have shown) "contribute essentially to the solution of the question, respectable by its antiquity, of the so-called irritability." The solntion of which M. Remak wishes to speak, is the reversal of the doctrine. A little above, in fact, introducing irrelevantly the question of the doctrine of irritability, he lias said, "It is scarcely necessary for me to point out how little these physiologival experiments justify the pretended docirine of Hallerian irritability."

If M. Remak has only such arguments as these in opposition to the Hallerian irritability. that doctrine has nothing to fear from his attacks. The micrographie. physiologist should know, better than any one, that such a question can only 


\section{B.--Excitability of the Sensibility of MIuscles.}

It is of much less importance to the operator, especially in the application of faradization to therapeutics, to know the degree of excitability of the motricity of the nerves, or of the electric contractility of the muscles, than to know what is the degree of sensibility dereloped by the faradization of these nerves or of these muscles. It is, indeed, the exaggerated sensibility of certain regions, or of certain subjects, that sometimes renders muscular faradization impracticable. When I set forth, in the sequel, the results of faradization applied to the treatment of certain kinds of paralysis, it will be seen how the exaltation of the electro-musetrlar sensibility can render localized faradization dangerons. It is, therefore, upon a knowledge of the degree of excitability of the electro-muscular sensibility that the art of localized muscular faradization, as applied to treatment, chiefly rests. Although the individual differences are greater in this respect than for the electromuscular contractility, $\mathrm{I}$ am convinced that it is possible to find an average that will serve as a general rule, just as there exists an average or ordinary dose of any medicine.

Without entering into the details of my researches on this interesting subject, I purpose to set forth, with regard to the excitability of the sensibility of each of the muscles, certain general facts which may be guides to the operator in direct museular faradization.

The electric sensibility is very acute and easily excited in the muscles of the face. This is due to the fifth pair of nerves, from which these museles receive filaments. In faradization of the facial muscles it is necessary to avoid placing the rheophores over points corresponding to the infra-orbital or mental nerves. Excitation of these nerves produces rery acute pain, which extends

be studied experimentally by placing under the microscope a innscular fibre, separated from all anatomical elements of nerve, and by then subjecting it to some stimulus-such, for example, as that of a galvanic or an induced current. But this is precisely what was done by M. Lebert, in 1842, with the musenlar fibre of insects, and afterwards of rabbits, and of logs: it is what has been since done hundreds of times by a great number of observers; and it is done every day by M. liobin, and is shown in his excellent lectures. If M. Remak, by chance, refuses to see what has been seen liy everyboly clse, I still ask if he be mi- acquainted with the researches of MII. C1. Beruard and Kölliker upon the action of woorara, which destroys immediately the excitability of the nerves and of the spinal cord, but leaves intact the irritability of the muscular fibre. 'This particular' action of the Indian poison would suftice, without the foregoing experiments, to establish the truth of the Hallerian doctrine. Let us ard to such faets those which are furnished by the researches of M. Longet, and by daily pathological observation, and we obtain an aggregate of proofs sufficient to carry conviction eren to the most obstinate minds. 
to a distance,- - to the incisor teeth, and sometimes to the base of the orbit.

Excitation of the frontal nerves, given off by the ophthalmic nerve of Willis, produces pains which radiate over the head; and it is for this reason that direct faradization of the frontal muscle, which is erossed by these nerres, is very painful. The orbicular miscles of the eyelicls, the depressor ala nasi, the levator labii superioris aleque nasi, the quadratus menti, the levator menti, the orbicularis oris, and the triangularis menti, are the most excitable. 'The order in which they have been enumerated indicates the degree of their sensibility. Next come the zygomaticus major and minor, the masseter, and the buccinator, which is comparatively little excitable. I rarely faradize the levator anguli oris, for fear of acting upon the infra-orbital nerve.

In the neck the platysma is as excitable as the superior half of the sternomastoid, and as the external border of the superior half of the trapezius.? 'The other muscles of the neck are much less excitable.

'The pectoralis major, and the muscles of the infra-spinous fossa, are moderately sensitive to electric excitation; the deltoid, and the muscles of the arm, a little less so. The muscles of the anterior region of the forearm are much more sensitive than those of the posterior region.

The longissimus dorsi, and the sacrolumbal muscles, have very little sensibility.

The muscles of the buttocks, and the tensor vagina femoris, are very sensitive to electric excitation; as compared with the muscles of the external and posterior regions of the thigh. Those of the internal crural region are more sensitive than those of the external crural region.

'The muscles of the posterior region of the leg have very little sensibility, when compared with those of the interior and external region.

It is possible to express in numbers the degree of excitability of each muscle and nerve; and such investigations will be the subject of a special work. I am so familiarised with the practice of localized faradization that $\mathrm{I}$ am able, with the help of my new apparatus, to administer to each muscle or nerve the dose of electricity required for the production of an energetic muscular contraction, and that without causing much pain. It requires

7 The extreme sensitiveness of the platysma leads me to suppose that it is supplied by the external branch of the spinal nerve.
${ }^{8}$ Of the muscles of the lower limbs, the tensor vagine femoris is the most sensitive to electric excitation. 
long study of faradization to attain to this degree of certainty; which fortunately does not seem to be absolutely necessary for the application of the method to therapentics.

It is perfectly certain, as I have shown, that a rheophore sufficiently moist, and in perfect contact with the skin, produces no sensations but such as are purely muscular; unless it chance to be placed orer the track of a cutaneous nerve. But it must not be forgotten that, at the moment of application to the skin, and before the perfect contact is established, a cutaneons blends with the muscular sensation. It follows that muscular faradization, especially with rapid intermissions, is much more painful at the moment when the rheophore is placed in contact with the skin. It is necessary to proceed as follows, in order to avoid exciting the mixed and very painful sensation. Before placing the rheophores upon the skin, they should be brought into contact with each other, so as to nentralize the current. When they are properly placed upon the skin, and lightly pressed down, they may be carefully separated a little, so that the electric recomposition may take place in the muscle that is to be excited. I adopt also another and equally simple method, which consists in not allowing the current to pass until the rheophores are placed in position over the muscle.

\section{C.- The points of selection in the practice of localized muscular faradization.}

The general precepts set forth in this paragraph are perfectly elear. The chief of them may be summed up in the following manner. When the electric excitation is localized in a nervous trunk, or in the muscular nerves proceeding from it, we hare indirect muscular faradization; but when the excitation is applied to the muscular tissue, we have direct muscular faradization.

(a). But, if the precept is clear, the execution is not always easy; especially when it is desired to produce contraction of individual muscules or fasciculi for some electro-physiological purpose.

The nervous trunks of the limbs are, for the most part, easily accessible to rheophores, since they nearly all present some point of their continuity immediately beneath the skin. It is not the same with the nerves of the muscles. Some of them, indeed, may be assailed at points remote from the muscles to which they are distributed (as, for example, the phrenic nerve, in front of the scalenus anticus; the nerve of the serratus magnus, above the clavicle), and near their points of emergence. With regard to other muscular nerves (those of the limbs), we can neither reach their points of emergence or of immersion withont acting upon a 
part of the muscular tissue behind the deep surface of which they are concealed. This, however, does not prevent the current from penetrating to them, when such an action is required.

An exact knowledge of the points of emergence and immersion of the muscular nerves is absolutely necessary in order to study the proper action or the state of the properties of muscles, or of those fasciculi which, physiologically considered, are often distinct muscles themselves. At the beginning of my researches, I found it a matter of the greatest difficulty to limit exactly the action of the current to each muscular bundle, because I did nut possess the knowledge. It was necessary to commence new anatomical studies, in order to determine, as exactly as possible (because in this respect there are certain individual differences), the points of exit and of entrance of the nerves; and by these I learnt to aroid the nerves, when I wished to excite single fasciculi; and to excite them, when I wished to obtain the contraction en masse of large muscles or fasciculi.

It is not always sufficient, however, to be able to avoid the points of exit or of entrance of the nerres, in order to obtain the isolated contraction of one of the bundles of a muscle. There are certain points of election on which the rheophores should be placed; and which must be sought in order to be perfectly known. I will take as an example the extensor communis digitorum. In a person in whom the forearm measures twenty-four centimetres from the olecranon to the styloid. process, the point of emergence of the nerve is, as a rule, six centimetres from the epicondyle. Before dividing, the nerre has a length of two centimetres; and its point of immersion is at twelve centimetres below the epicondyle; it being then diviled into two branches. With these anatomical data, it is evident that a rheophore of small surface placed over the nerve prior to its division,-_that is, orer the central part of the extensor communis at a point between six and eight centimetres below the epicondyle,-will produce simultaneous contraction of all the fisciculi of the muscle, if the current be sufficiently penetrating to reach the nerre. But at twelve centimetres below the epicondyle (the point of immersion) the rheophore must be brought into relation with two branches in order to produce the same effect. In other words, the rheophore must either cover a large surface, or only one of the branches will be excited, and only two fasciculi will be thrown into contraction.

If it be desired to obtain isolated contraction of each fasciculus of the extensor communis, it will evidently be necessary to avoid the nerve before its division, and to seek the points at which the muscular fibres constituting each fasciculus become sulcutaneous. 
'These points are: for the fasciculus of the index, on the inner side of the second radialis externus (extensor carpi radialis brevior) at from 10 to 14 centimetres below the epicondyle; for the fasciculns of the ring finger, a little below the epicondyle, on the plane of the posterior aspect of the ulna. It is requisite, in all these operations, to use a current that is only slightly penetrating (that of the first helix), and of moderate strength; in order that the excitation may not reach the deeper layers.

By following the anatomical data that I have set forth, I have been able to obtain with certainty, in the first patient presenting himself, isolated contraction of the fasciculi of the extensor communis, and also contraction of the other fasciculi that move the fingers and the thumb.

(b). It was with reference to the difficulties that the practice of localized muscular faradization presents even to an anatomist, if not possessed of these data, that I wrote, in the former editions, the following lines:- "It must not be supposed that it is sufficient to know the precepts that I have laid down, and to be an anatomist, in order to possess completely the art of making each muscle or fasciculus contract singly. To attain this degree of slitl, it is necessary to be initiated into a vast number of details, which I cannot enter upon without transgressing the limits that I have laid down."

Remak, misinterpreting initiated as if it referred to some mystery, played upon the word, and referred to it perpetually and malicionsly in lis brochure. Howerer, having knowledge of the general precepts already lairl down, he ought to understand, especially after having seen me make the application, that it would be possible to initiate limself into these anatomical details. He is silir since then to have done so, by seeking, in dissections, the points of immersion of the muscular nerres. ${ }^{9}$

9 In October, 1852, Remak dirl me the honour to be present, with MI.MI. the Professors liichter (of Dresden), Jackseh (of Prague), Hébra (of Vieuna), and other German savants, at a séun'e at which I demonstrated experimentally the functions of certain museles by electricity. M. Remak writes, in the brochure alreacly rited, that this seum e was of such interest that he gave an account of it, on his return from Paris, to the Nedical Society of Berlin. But he explains that my verbal explanations were confined to physiological demonstration; ami did not include my methou of localized electrization. Then, not having umlerstoorl how it was possible to make muscles contract singly, he arthls, "Whoever has seen the skill and confidence with which he (II. Dnchenne) throws the great muscles into contraction could almost complain of his intention to preserve his secret." (p. 11.)

When I experiment in the presence of pupils, I habitnally remember to explain the anatomical reasons that induce me to place the rheophores mon this or that point of election, in order to obtain either the contraction of a muscle en musse or the contraction of one of its fasciculi or portions. But, before the select auditory of which Remak formed part, I should have thought it out of place to deliver a lesson on anatomy. 
The directions given by Remak for obtaining the tetanic contraction of a muscle en masse teach nothing about the points of emergence and of immersion of the nerves; and they cannot even serve to supply a general rule. Thus, according to him, in order to make a muscle contract en masse, it is necessary to place one rheophore on a point of the border of the muscle towards which the entrance of its nervous trunk occurs. If this principle will hold good for some muscles, it will not for many others. In support of my assertion, I will take as an example one of the muscles which it is said may be made to contract by this procedure-the deltoid. On whatever point of the border of that muscle we apply the rheophores, we obtain only partial contractions; since, as the place of entry of the nerve is at the union of the upper third of the muscle with the lower two-thirds, and of the posterior third with the anterior two-thirds, the rheophores can only encounter its final ramifications. The principle of Remak is, on the contrary, applicable to the trapezins. 'T'he method that he employs to make

When, at that seunce, I was seen to produce contraction en mase of the serratus magnus, by placing the rheophores on the sile of the chest, by the anterior borker of the latissimus dorsi, was it necessary to explain that the rheophores were in relation with the nerve of the serratus maguus? When the same rheophores were placed at the juncture of the iniddle and lower thirds of the margin of the trapezins, was it necessary to say to those professors that I was then axciting the esterual branch of the spiual nerve from the cervical plesus? The contraction en masse was sufficient to show them what was done. This method of musenlar electrization by the intermediation of the nerves was the inclirect muscular faradization that I had cleseribed in a general manner, in my writings and my experimental seunces, withont entering into details about the points of emergence and immersion of the motor nerves. Again, when, during the same séance, I wished to obtain isolated contraction of the portions of the trapezius which are physiologically distinct muscles, they saw me avoil, as much as possible, the points of entrance of nerves, and place the rhenphores in succession on each of the parts that form what I have called the respiratory (clavicular), the elevator, or the adilnctor, portion of the trapezius.

During several hours that the seance lasted, Remak saw me produee, in succession, contraction of a large number of unscles en masse, and of separate fasci- enli-the former by exciting their nervous trumks, the latter by placing the rheophores upon them, beyoud the points of immersinu of the nerves. This great critic, howerer, who has witnessed the results that I obtained, and has made them the sulject of enlogy, obtained no glimpse of the particular methods that allowed me to obtain them! With whom is the blame? Did I trust too much to lis anatomical knowledge? Had microscopic anatomy caused him to forget that which can be seen with the naked eye? Such seems to be the only solution of the enigma.

Remak, who has thus represented me as a lind of prestigiator, whose dexterity he praises, but who preserves his secrets from him, says again, "It must equally be admitted that he (II. Dnchenne) has not yet perfectly fixed upon the auatomical conditions of the results that he obtains; and that he owes his knowledge of the aforesaid points (the points of election on which the rheophores should be placed) perhaps to cluance or ratler to the trials by which he has arrived at his method." Then Remak, for the honour of science, sets himself to work, and discovers that these famous points of election are the points of entry of the muscular nerves. Unfortumately for the priority of this discovery, the experincuts that he adduces to explain the anatomical reasons for these points of clection are precisely those that I performed before his eyes, at the seunce at which he was present in 1852. 
that muscle contract en masse is nearly that which he saw me practise in 1852 (without then understanding it). I say nearly, because Remak states that he places only one rheophore on the branch of the accessory nerre of Willis, while the other is placed on a point of the muscle. But it is impossible to produce contraction of all the parts constituting the trapezins except by placing the rheophores on the points of the cervical border of the muscle at which the two trapezian nerves penetrate: the one on the trapezian branch of the spinal (fire centimetres above the clavicle), the other below the trapezian branch of the cervical plexus (three centimetres above the claricle). Then, on the side excited, we see the head inclined, the shoulder raised, and the scapula drawn towards the median line: then we see the three portions of the muscle swell, and stand ont in relief bencath the skin.

In $18.55^{1}$ I wrote: "This work (in which would be shown the anatomical points on which the rheophores should be placed, in order to study the proper action of muscles or of their divisions) would certainly be very useful; but, with the desire to shorten it as much as possible, and to facilitate study, I intend to set forth the data, at some future time, in a synoptical table with figures. This project cannot be put in execution until I have entirely terminated my researches on muscular electro-physiology." 2

Taking up my idea, and desirous, without donbt, to put it more quickly in execution, a German writer, Dr. Ziemssen, ${ }^{3}$ has shown on anatomical plates the points on which the rheophores should be placed to produce isolated contraction of muscles; declaring, in the preface to his book, that he wishes to render the employment of my method of muscular electrization easy to erery practitioner.

We must not praise this work too much; for the writer unfortunately has fallen under the influence of the errors of Remak. He says that the method consists solely in always placing the rheophores over the points of immersion. Nothing, certainly, can be more simple and more easy than this; for which he has only nceled to use certain anatomical plates, as I had advised in my reply to MI. Remak. (The points of immersion of the nerves are shown with the greatest exactitude in the plates of MI. Ludovic Hirschfeld.* As these are drawn to half the natural size of an

1 De l'Electrisation localisée, Paris, 1855 , in Sro. p. 58.

2 It is well known that, since $1855, \mathrm{I}$ have never ceased to exert all my activity in the publication of new elertrophysiological researches. Why, then, should I be so bitterly reproached because I have not yet prepared the srnoptical table? I cannot double my existence.

3 Ziemssen, Die Electricitüt in der Medizin. Second edition. Berlin, 1866.

4 Neurologie; on Desription et Iconoaraphie du Système Nerveux. l'aris, $1853-4$. 
adult, it is possible, with the lelp of a compass, to ascertain exactly the points).

Since Dr. Ziemssen has made himself acquainted with my reply to MI. Remak, he should have understood, it seems to me, after the detailed explanations into which I entered, and which have been reproduced above:-

(1). That my manner of practising direct muscular excitation is not that which he has described as being mine. It is true, indeed, that I sometimes place my rheophores over the points of immersion or emergence of muscular nerves, in order to produce the contraction en masse of each muscle; but, again, I sometimes avoid these points of immersion, in order to obtain isolated action of the fisciculi, which often, indeed, are really independent muscles.

(2). That it is, consequently, not sufficient to mark, as he has done, the points of immersion of the muscular nerves; but that it is also necessary, as I have given an example in $\mathrm{p}$. 86, to point out the spots on which the rheophores should be placed when it is desired to throw only single portions or fasciculi into action. (These points are not always easy to find; and, as Remak says, I have sometimes had to seek them empirically.)

(3). That the figures, on which the points of emergence and of immersion of the nerre are marked by a sign, are certainly useful; but that they are not sufficient for practical purposes. It is necessary, in fact, and I cite, as an example, the faradization of the extensor communis digitorum or of its fasciculi, to choose a fixed marked point, and to make known the average distances between the marked point and the point of election.

\section{PART' the 'Third.}

\section{CUTANEOUS ELECTRIZATION.}

Cutaneous electrization may be practised by means of either static or dynamic electricity.

\section{\$ I.-Electrization of the skin by static electricity.}

In order to limit static electricity to the skin, it is necessary to use only a feeble degree of tension. Indeed, cutaneons excitation, by that form of electricity, does not exceed certain degrees of tension without penetrating more or less deeply to subcutaneous organs and producing shock. For this reason, the therapeutic action of cutaneous static electrization, which must be very weak in order to be confined to the skin, is seldom indicated and almost always insufficient, as I have shown already in Chapter 1. 


\section{\$ II.-Electrization of the slin by dynamic electricity; or cutaneous galvanization.}

I have already said that cutaneous excitation by dynamic electricity is practised by dry rheophores, applied to the dry skin, and that the resulting sensation ranges from simple tingling to acute pain. It is well known that galvanism does not act upon the skin withont causing an alteration more or less considerable (resication or cauterisation); while faradism produces no greater organic change than little papules or erythema. It follows that cutaneous galvanization should only be applied in certain cases; and that cutaneous faradization, which respects the tissues, is one of the most valuable and most frequently indicated therapeutic agrents.

\section{\$III.-Electrization of the stin by the induced current; or cutaneous faradization.}

These reasons have determined me to treat specially of cutaneous furadization; the methods of applying which are the same as for cutaneous galvanization.

\section{I.-MIOdE OF OPERATING.}

The different electro-cutaneons excitability of different regions of the body requires the nse of different methods of cutaneous faradization. The procedures are of three kinls: faradization by the electric hand, by solid metallic rheophores, and by electric threads.

Each of these methods possesses a special action, which may be rendered a vailable in thernpeutics; and I proceed to describe their application.

\section{A.-The electric hand.}

In this method a moist rheophore is used (a sponge contained in a cylinder) like that shown in fig. 25 . This is put into communication with one pole of the apparatus, and is then applied to some insensitive part of the patient's body. The other rheophore, in communication with the other pole, is held by the operator, who, laving first dried the skin to be faradized by some absorbent powder (lycopodium or rice starch), passes the back of his free hand, also dried, over the points which he wishes to excite.

\section{B.-Solid metallic rheophores.}

The skin must be dried as ahore described; althongh if the epidermis be very thick and hard, as it becomes from some occnpations, and chiefly on the hands and feet, which are often in more 
or less continuous contact with air and water, it may be necessary slightly to moisten the surface, in order that the electric excitation may penetrate to the dermis. Large dry metallic rheophores, cylindrical, olive-shaped, or conical, as shown in figs, $27,28,30$, and 31 , are then applied to the skin and moved over its surface. Rheophores of the first shape are used to excite, by their outer face, the slin of the chest and limbs; the second for faradization of the hairy scalp.

The rheophores should always be moved, more or less quickly, over the diseased parts. In certain special cases, when it is desired to produce a very strong revulsion over a very small surface, the point of the olive may be kept for some time stationary. When the conical rheophore is thus employed, it is called by patients the electric nail, from their comparing its action to that of a hot nail penetrating the skin. This method of proceclure is chiefly applicable to the neighbourhood of the vertebral column.

\section{C. - Metallic threads.}

The metallic threads (fig. 32) are employed in the form of a little brush, contained in a eylinder, which is screwed to an insulating handle. There are two methods of faradization by the metallic threads. They may be moved over the affected surface, or this surface may be lightly struck with the extremity of the brush; or they may be held in contact with the skin as long as the patient can bear them. The first proceeding las nuch analogy, as regards its action on the sensibility, to transcurrent cauterization. The second I have called electric fustigation. The third, seldom endured by patients, may be employed in certain deep-seated affections, - in the treatment of white swellings, for example, and to aid the absorption of tumours and enlarged glands. It may be described as the electric moxa.

\section{II.-Physiological and therapeutical action.}

The application of the electric hand to the excitation of the cutaneous sensibility produces, in the face, and under the influence of an intense current, a very acute sensation. On other parts of the body Fig. 32.-Metallic the sensation produced is hardly more than if the threads. hand rested long on the part. The smart crepitation cansed by the passage of the hand over the surface is the unly effect produced. 
The solid metallic rheophores act energetically upon the sensibility of the face, even with a slight current. They actively stimulate the skin of the trunk; but they are often powerless on the palms of the hands and the soles of the feet, whatever the intensity of the current.

The metallic threads triple the power of faradization upon the cutaneous sensibility; and are almost always the only agents capable of exciting it acutely on the palms of the hands and the soles of the feet, in profound anæsthesia of these regions.

The sensations produced by these several methods of faradization are different. On the face the electric hand produces the effect of a rough brush tearing the skin; and the metallic threads exert a deeper action. When they are kept stationary, the sensation is like that of hot needles piercing the tissues. The fustigation by threads produces a sensation that differs from this only in its duration. No other agent, not even fire, produces pain equal to that caused by metallic threads; if we may judge from the statements of patients who have also experienced the moxa and transcurrent cauterization. It is very difficult exactly to express these different sensations, and I have striven to do so by using the terms habitually employed for the purpose by patients themselves.

In the normal condition, the cutaneous excitability by electricity differs greatly in different parts of the body. It is necessary, in order to use faradization successfully in cutaneous anæsthesia or in various lesions of tactile sensibility, to know the differences, in this respect, between various regions in the healthy state.

The skin of the face is indebted to the fifth pair of nerves for exquisite sensibility, and its electric excitability is so great that the most feeble faradic current produces an acute sensation; even when its effect is scarcely appreciable on other parts of the body. The skin of the face is more sensitive to electric action near the middle line than elsewhere; thus its sensitiveness is greater on the eyelids, the nose, and the chin, than on the cheeks. 'The skin covering the upper eyelid, the alæ and point of the nose, the borders of the nostrils, the sub-nasal depression of the upper lip, and the line of junction of the labial skin and mucous membrane, are the points on which the electric excitation is felt the most acutely.

On the forehead the sensibility is less than on the face, and still diminishes as the hairy scalp is approached. In the latter it is comparatively slight; and is ouly excited by a tolerably powerful current.

The sensibility is notably greater on the neck and trunk than on the limbs; in the cervical and lumbar regions than in other parts 
of the trunk; on the internal and anterior aspects of the limbs than on their external and posterior aspects.

The skin of the palm of the hand possesses the least electric excitability, and that of the sole of the foot more, exeept at its middle and interual portion. In persons whose hands are much exposed to air and moisture, the sensibility of the skin covering them is so blunted that particular methods and a very strong current are necessary in order to excite it.

There is no other therapentic agent the action of which is comparable to that of cutaneons faradization. By this alone, the sensibility of the skin may be exeited to any extent, either in passing rapidly from a simple tingling to the most intense pain, or in passing gradually through all intermediate degrees. By this alone can an excitation be produced, which that of the actual cantery scarcely equals, without disorganising the tissues, without even raising the epidermis, however prolonged may be the operation. The sensation produced ceases suddenly, and nearly always completely, as soon as the rheophore is no longer in contact with the skin; and the instantaneousness of the action allows the electric stimulation to be carried rapidly to all points of the surface of the body.

This exposition of the chief properties of cutaneous faradization should afford an idea of the power of its therapentic action, and of the numerous services that it is calculated to afford in practice. These I shall have many occasions to establish in the course of the work.

\section{Partit the Fourth.}

Faradization of internal organs, the genital organs, and of the organs of the senses.

Most of the organs situated within the cavities of the body are accessible to faradic excitation, ${ }^{5}$ either directly, by the action of rheophores placed upon their tissues, or indirectly, by stimulation of the nerves that govern them. I proceed to describe the different methods that I lave adopted, in order to direct upon these organs the faradic excitation.

\section{$\S$ I. Faradization of Internal Organs.}

\section{Faradization of the rectum and muscles of the anus.}

Involuntary stools and descent of the rectum are often caused by paralysis of the sphineter and levator ani; and it may then be

${ }^{3}$ I repent that it is not sufficient that an organ slould be traversed by an electric current in order to be excited by it. It is necessary for that purpose, be directly stimulated. 
necessary to faradize these muscles. For this purpose a metallic olive (fig. 33), mounted on a stem of metal, insulated by gum

Fig. 33.-Rectal rheophore.

elastic, is introduced into the rectum, and then connected with one of the poles of an induction apparatus. A second (moist) rheophore is moved over the circumference of the anus. Then the apparatus is in action, the stem is so moved as to place the olive in contact with the muscles of the lower part of the rectum; that is, with the levator and sphincter ani. In order to excite the muscular coat of the rectum, the olive shonld be moved over all its internal surface. It is always necessary first to free the rectum from foecal matter, by means of enemata.

If it be desired to overcome constipation, due to insensibility of the mucous membrane of the rectum or to paralysis of the muscular coat, the rheophore must be introduced as before described, and moved over the whole surface of the intestine.

The margin of the anus is so sensitive that the slightest faradic

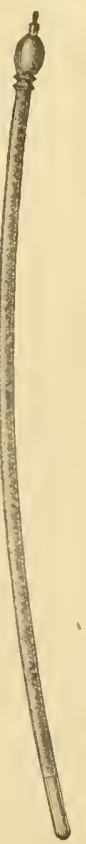
excitation produces insupportable tenesmus. Care must therefore be taken always to insulate the stem that bears the olive, unless it be wished to act upon the spliucter muscle.

\section{Furadizution of the bladder.}

In all faradic operations npon the bladder, the organ must first be emptied, as in faradization of the rectum. Withont this precantion, the excitation, instead of being linited to the parietes of the two organs, wonld extend to the sacral, or the hypogastric plexus.

In order to faradize the muscular fibres of the neck of the blatder, an olive-shaped rheophore is introduced into the rectum, as above described. A curved metallic sound (fig. 34), insulated, by being covered with an elastic catheter to within two or three centimetres of its vesical extremity, is then introduced into the bladder, and placed in connection with one of the poles of an induction apparatus. When this is in action, the sound is drawn back in 
such a manner that its extremity is brought in contact suceessively with all points of the neck of the blalder. The patient submitted to the operation feels contractions. that result from the excitation of the muscular fibres that go to form the sphincter of the neck of the bladder.

If it be desired to excite either the sensibility or the contractility of the bladder as a whole, the resical rheophore must be moved over all parts of the internal surface. It is not always necessary to have recourse to this operation in paralysis of the bladder, complieating paraplegia. It is sometimes possible, in such cases, to re-establish the function by energetic faradization of the muscular walls of the abdomen. The same operation will also often orereome the constipation that is habitual in paraplegia. Numerous facts in my possession appear to show that a certain number of cases of paralysis, both of the rectum and of the bladder, have no other cause than paralysis or debility of the abdominal walls.

The electric excitation of the rectum may be attended with so much inconvenience as to be a difficulty in the way of the procedure that I have deseribed, and to require to be aroided. For this purpose I introduce two rheophores into the bladder; and I have had made, by MI. Charrière, an instrument that I call the double vesical rheophore (figs. 35 and 36 ).

The double resical rheophore is formed of two flexible metallic stems, enclosed in an elastic catheter of double channel, which separates them from each other. The two stems have such terminations as are shown in fig. 36 ; so that when approximated, as in fig. 35 , they resemble an ordinary sound. The instrument is closed by pushing the elastic catheter forward, and is introduced into the bladder. The stems are then pushed forward for three or four centimetres, while the eatheter is held still, in such a

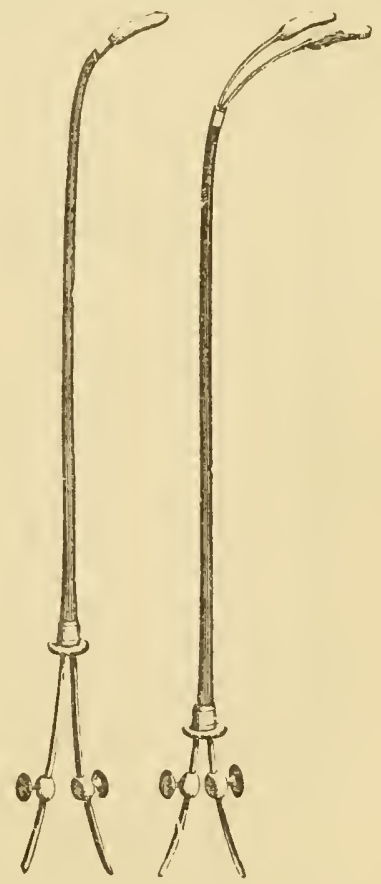

Fig. 35. Fig. 36.

Fig. 35.-1)ouble vesical rheojhore, clused.

Fig. 36.-The same, open. manner that the extremities may separate. Then, after each stem has been connected with the pole of an induction apparatus, the instrument is handled in the way already described. T'he donble gum 
cathether, which encloses the stems of the rheophores, must never be penetrated by moisture, because the currents would then pass from one rheophore to the other, and would be recomposed in the interior of the eatheter, instead of reaching the extremities. It is necessary to empty the bladder for this reason also.

\section{Faradization of the uterus.}

In certain cases of amenorrhœea, electric excitation of the neck of the uterus may be practised with advantage. For this pnrpose I use a rheophore like the double one for the bladder, but differing from the latter in the curvature of the stems, and in the larger size of the terminal plates (figs. 37 and 38 ). It is introduced closed, as in fig. 38 , into the vagina, and then the two plates are made to

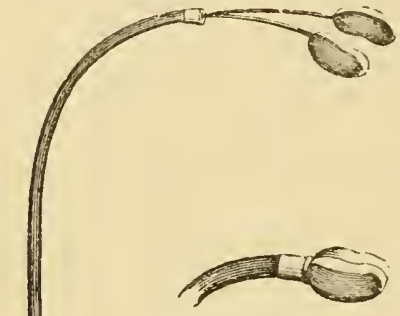
separate, as in fig. 37 , by pushing the stems onward through the double gum tube that covers them. The operator guides each of the plates by the index fingers of his free hand, and places them on the sides of the cervix. It then only remains to comnect the stems with the poles of an induction

Fig. 39. apparatus. 'The facts that I have observed show the good effects of this method of excitation in cases of obstinate amenorrhoen, that are not wholly dependent upon chloro-anrmia.

Fig. 37.

If it be desired to excite the organs

Fig. 37.-Uterine rheophore, open.

Fig. 38.-The same, closed. contained in the pelvis, by indirect faradization, the terminal olive of the rectal rheophore should be directed to the posterior wall of the rectnm. The current then traverses the coats of the intestine, and concentrates its action upon the sacral and hypogastric plexus behind it.

\section{Faradization of the pharynx and cesophagus.}

Faradization of the pharynx is practised by means of a rheophore called a pharyngeal. 'This consists of a metallic stem, very flexible, or jointed like an esophageal sound, terminating in an olive-shaped metallic extremity, three or four millimetres in diameter, and protected by a gum tube to insulate the stem. The curvature of the jointed rheophore may be increased or diminished 
at pleasure, by means of a mechanism for the purpose, so that, when introduced into the pharynx its olive-shaped extremity may reach to the inferior constrictor.

In order to faradize the constrictor muscles, the olive is moved over the surface of the cesophagus, while a moist rheophore is placed on the back part of the neck. The operator should beware of directing the olive against the lateral walls of the pharynx, which are in relation, from top to bottom, with the pneumogastric. If the rheophore were placed over the nerve, the electric action, instead of being limited to the pharynx, wonld be carried further, to organs the stimulation of which would be dangerous, or at least contra-indicated.

'To faradize the œsophagus, an osophageal sound is required, conveying and insulating a metallic stem, terminating in a small olive-shaped metallic bulb. This rheophore, thus insulated, acts ouly on those parts of the cesophagus that are in contact with the olive-shaped bulb; and hence it is necessary to move this bulb over every part of the organ that it is wished to excite. We know that the osophagus is in relation, in its cervical portion, with the left recurrent nerve, lodged in the groove that separates the œsophagus from the trachea, and that it is accompanied, in its thoracic portion, by both pneumogastric nerves, which are situated lower down, the left in front of it, and the right behind it. It is sufficient to mention these anatomical data, in order to show the difficulty, or even the impossibility, of avoiding, during faradization of the œsophagus, the conveyance of the excitation to nerves that supply the most important organs. The operation therefore requires much prudence.

\section{Faradization of the larynx.}

The muscles of the larynx that are concerned in phonation, except the thyro-arytænoid and the crico-arytænoid, are accessible to the direct action of faradization. I have practised the following method many times.

I introduce into the pharynx the pharyngeal rheophore, and carry it down below the posterior part of the larynx. 'The second (moist) rheophore being placed on the exterior, at the level of the crico-thyroid muscle; and the apparatus being in action, I move the pharyngeal rheophore, after having increased its curvature, in such a manuer as to bring its olive-shaped extremity into contact with the posterior face of the larynx, and I then move it up and down. In this operation the stimulus is carried directly and successively to the posterior crico-arytænoid and to the arytænoid 
by the pharyngeal rheophore; and to the crico-thyroid ${ }^{6}$ by the moist external rheophore.

Indirect faradization of the larynx is still more simple. By directing the extremity of the pharyngeal rheophore to the lateral portions of the inferior constrictor, we act upon the inferior laryngeal nerve, which supplies all the internal muscles of the larynx. The left inferior laryngeal may also be reached through the wesophagus.

My experience has shown that the above procedure would frequently be very advantageous in aphonia due to paralysis of the muscles of the larynx. I shall record, in the sequel, many cases in which I have practised the operation with success.

[Duchenme makes no reference to the use of the laryngoscope to facilitate direct faradization, or galvanization, of the muscles of the larynx. It is probable that his experience has shown that the intervention of the laryngoscope is practically rarely necessary. Precision of manipulation, however, is at all times to be cultivated, and this is only possible of the larynx by the aid of the laryngoscope. Ziemssen has treated the subject of laryngeal electrization with great perspicnity in his work Die ETertricität in der Medizin, and I append a translation of his observations. which I have no doubt will prove acceptable to the English practitioner,-H. T.

\section{ZIEMISSEN ON LARYNGEAL ELECTRIZATION.}

"The electric excitation of the nerres and muscles of the larynx has latterly become of great practical importance; on account of the increasing certainty of laryngoscopic diagnosis, and especially on account of the evidence thus obtained of the frequency of paralytic conditions of the laryngeal muscles, affecting them either singly or in groups.

"MItch labour has been bestored in recent years upon endeavours to find a satisfactory basis for electrical treatment of the larynx. The first attempts were to find the laryngeal branches of the ragus nerve in the neck, and to excite them through the soft parts superficial to them. These attempts, made upon patients suffering from some disorder of the nerves or muscles of the larynx, were sometimes followed by beneficial therapentical results; and it was thence inferred that the excitation of the nerves in question had been accomplished. This conclusion, I believe, was not justified; and it has still to be proved, notwithstanding the admitted recoveries, that it is generally possible to direct an electric current upon the superior and inferior laryngeal nerves through the skin. Gerhardt has been at the pains to test the effect of the excitation in the only practicable way, by means of the laryngeal mirror, and he obtained only negative or doubtful results. He found, in healthy persons, on applying the induced current to the regions of the neck that are superficial to the course of the recurrent nerves,-namely, for the superior laryngeal nerves to the vicinity of the superior cornua, for the recurrent nerves to the inferior cornua of the thyroid cartilage,-that he prodnced neither closure of the glottis, nor tension nor vibration of the vocal cords, nor audible change of tone. He observed, on the contrary, after excitation on both sides, a tremulous falling apart of the vocal cords after the utterance of vocal sounds: the glottis in one case attaining one-half, in another threefourths of its normal inspiration width. After excitation on one side, he observed a similar effect on the rocal cord of that side only.

- MT. Lorget has shown, by direct and | crico-thyroid performs an important part well-conceived experiments, that the in the act of phonation. 
"I have repeatedly witnessed the same phenomenon myself, both in patients and in healthy persons, and I have not been able to regard it as arising from excitation of the recurrent, but only as the result of an impression upon the larynx generally. I have seen this tremulous and uncertain condition of the vocal cords chiefly in sensitive and unpractised persons ; and in practised persons also upon whom some unusual impression had been made upon the larynx by canterization, faradism, or galvanism, although the special nerves and muscles of the organ had not been included in the circnit.

"While, therefore, I cannot admit that the result above described is a satisfactory test of successful excitation of the recurient, I think that we possess, in energetic detraction of the epiglottis, a certain sign of excitation of the superior laryngeal. This phenomenon may sometimes be produced from within, and chiefly from the pyriform sinus, which is crossed obliquely by the internal hranch of the superior laryngeal-sometimes running under an elevation of the mucons membrane (pliea nervi laryngii, Hyrtl.).

"The reason why it is so seldom possible to excite the inferior laryngeal nerves alone from the cutaneous surface may be found, I beliere, in their deep position, and in the layers of muscle by which they are covered, and which prevent, by their contraction, the pressing in of the electrode and the penetration of the current. These layers are formed by the platysma, the sterno-thyroid and sterno-hyoid, the omo-hyoid, the thyro pharyngens, and the crico-pharyngeus. 'The inaccessibility of the recurrent would also often be due to the enlarged lateral portions of the thyroid body; and the adipose tisstre of the neck may also hinder the pushing in of the electrode torrards the deeper parts.

"Although, therefore, it cannot be denied that, in particularly favourable conditions, with a thin neck, a small thyroid body, and a platysma not extending far forwards, it may be possible to isolate the laryngeal nerves from the surface; yet they generally, on account of their deep position, are withdrawn from the reach of direct excitation, and any attempt to act rpon them, especially upon the recurrent, from the skin, must be regarded as a highly doubtful procedure, not to be controlled by any satisfactory test of its success.

"We must, of course, fully admit that, by the percutaneous application of electric currents, therapentic results have been obtained in cases of paralysis of the vocal cords, and in disorders of innervation of all kinds, especially in hysterical subjects. The observations of Bamberger, Gerhardt, von Bruns, Tobold. Meyer, and Benedict, place this beyond doubt. Such knowledge, however, affords only an uncertain and deceptive basis, and does not assist in the special treatment of a diseased part, such as a single paralysed muscle, or group of muscles, with currents of a known intensity. On the one hand, we are quite uncertain whether the current will generally penetrate to the parts requiring excitation; and, on the other, the complicated functions of the recurrent nerve deprive us of the power of influencing, through its means, determinate groups of muscles; such as those that open or close the glottis, or that render tense the vocal cords.

"This deficiency of the percutaneous method has been perceived, of late year's, by other's beside myself. Duchemme, Mackenzie, Gerhardt, and von Bruns have all laid stress upon the possibility and necessity of direct electrization of special portions from the pharynx. Duchenne's observations lack the laryngoscopic basis; but the other authors named have employed the mirror as a guide to the operating hand. Gerhardt and von Bruns alone appear not to have gone beyond isolated experiments upon patients; while Mackenzie limits the direct action of the current to the vocal cords and the arytænoid cartilages, without having considered the complicated anatomical relations, or the possibility of a detailed diagnosis, and of a localization of the current corresponding thereto.

"The greater number of specialists in the domains of laryngeal disease and electro-therapeutics, as Tobold, Meyer, Benedict, and others, content themselves with the percutaneous method, on account of the difficulty of the 
internal application, especially because of the irritability of the mucous membrane of the month and larynx. The last-named author plainly declares the clirect excitation of the laryngeal muscles to be 'mnnecessary.'

"During several years that I have studied this question, I have applied myself, first, to perfect the method of application npon a number of persons habituated to the process, and, next, to demonstrate its ntility and therapeutical value in suitable cases. On the second question I have spoken elsewhere: ${ }^{7}$ and here I confine myself to the first.

"The direct electric excitation of the laryngeal muscles from the pharynx, which in all other cases I prefer to the percutaneous method, is inapplicable to one pair of muscles only; and these only are easily accessible from the surface. These are the crico-thyroid muscles (recte and obliqui, Henle). They extend, in the front of the neek, both vertically and obliquely (from above, downwards, and inwards), on both sides of the conoid ligament, from the thyroid to the cricoid cartilage; and they are thrown into strong contraction by two electrodes, placed one on each side of the conoid ligament. Not only can the approximation of the two cartilages be felt by a finger in their interspace; but a vocal note sounded during the contraction (by which the tension of the vocal cords is increased) will be raised about a semitone.

"The isolated excitation of the internal laryngeal muscles from the pharynx, is at first attended by great difficulties, as well for the operator as for the subject. For the first, rapidity in the introduction of instruments, without unnecessarily touching the root of the tongue or the fauces, complete knowledge of the anatomy of the parts, with special reference to the oljects of the procedure, steady and certain holding of the electrode in the right place, and, above all, paticnce, are required. For the patient, the at first constantlyoccurring ammoyances, snch as nansea, or even romiting, acute hyperæmia of the mucous membrane, cough, temporary aphonia, \&c. \&c., can only be overcome by weeks of steady persererance. By the necessary perseverance on both sides, we generally at last obtain such a tolerance of these sensitive regions to the contact of the sponge-covered electrode, and to the action of the electric stimulus, that the duration of each single excitation may be extended to half a minute. Many of the persons that I cxperimented upon, and of my laryngeal patients, became able, while the electrode rested, say in one simus pyriformis, not only to breathe quietly, but to execute swallowing movements, and to sound notes, during the passage of the current. It will be evident that movements of the internal soft parts during the action of the current cannot wholly be prevented, even after longer practice, since muscular contractions are produced, not only of a reflex character, but also by the direct action of the current itself. The entire disapjearance of the laryngeal image from the mirror, at first very perplexing; is soon prevented by practice.

"The strength of the induced or galvanic current that is applied is best determined by testing it, immediately before, upon the face. An induced current that produces distinct contraction of the frontal muscle, or of the corrugator supercilii, a galvanic current from eight to twelve of Siemens' elements, and producing faint twitching of the facial muscles, will be sufficient, on account of the small size of the laryngeal muscles, their superficial situation, and the moisture of the epithelial surface that covers them.

"As a laryngeal electrode, I use a slender sound, curved like a catheter, screwed into a wooden handle, and insulated up to its point. This point terminates in a little knob, which is covered by a thin layer of the finest sponge. The double excitor of Duchenne, in a somewhat modified shape, is ;ery useful for bilateral internal excitation. To begin with, an excitor may be made from an English gum catheter, by cutting off its extremity down to the eye, and by drilling a hole through the point of the stilet, so as to he able to fasten the sponge securely. Mackenzie's laryngeal galvanizer, and von

"See "Laryngosenpisches und Laryngotherapentischts," Deutschen Archiv für Klin. Medicin. Bi. II., Heft 3. 
Brun's laryngo-electrode are convenient instruments, since after their introduction the circuit can be made and broken at pleasure, by pressure of the finger upon a contrivance for the purpose. Such a contrivance is especially useful for the simultaneous introduction of both poles into the pharynx. An operator who does not possess these instruments, may allow the circuit to be completed, by an assistant, with a second electrode, furnished with a larger sponge covering, and applied either to any distant part of the body, or, in therapeutical procedures, to some selected spot of the surface over the larynx, as soon as the laryngo-electrode is in its place.

"The introduction of the laryngo-electrode should be accomplished quickly, with the right hand, and without any avoidable contact with the teeth, tongue, or fances; while its course is watched in the mirror held in the left hand.

"Among the immediate effects of the excitation, hyperæmia of the selected spot and its vicinity, increased mucous secretion, transient hoarseness, inclination to congh, and pain in the neck, are unavoidable. These effects mostly disappear in a few hours, or even sooner. The therapentical effect, where any such is to be expected, shews itself after the disappearance of the first effects; but, after the early sittings, has usually itself disappeared by the next morning. That the irritation of the mucous membrane of the posterior surfaces of the arytænoid cartilages, of the pyriform sinuses, or of the vocal cords, in long continued treatment, may occasion acnte inflammation, ulceration, or soreness, need not be feared, if pauses of a few days are made, from time to time, between the otberwise daily applications. Even in coutinuing the treatment for many months, I have never seen any injurious local action.

"The localization of the electric current upon the several museles is to be accomplished as follows :-

"The arytænoid muscle (trausversus) is the easiest to reach with the electrode. As soon as the point of the electrode is placed upon the posterior surface of the arytænoid cartilage, the circuit should be closed by an assistant, or in any other manner. By the shortening and contraction of the arytænoid muscle, the posterior surfaces of the arytænoid cartilages are arched, and the cartilages are approximated. On this account especial care is required, lest the occurrence of the contraction should cause the point of the electrode to slip from its place. The juxtaposition of the cartilages that is produced by the arytrenoid muscle is very energetic. Absence or incompleteness of the effect, in consequence of paralysis or paresis of the muscle, is a very important and conmon canse of aphonia and hoarseness, especially in the hysterical. The diagnosis of this condition presents no difficulty even to the inexperienced.

The crico-arytænoideus lateralis is to be reached at the bottom of the pyriform sinus, in a direction backwards, in the immediate vicinity of the onter margin of the plate of the cricoid cartilage; but it requires great dexterity and practice in laryngoscopic localization. 'The point of the electrode must take a course directly downwards, and must be firmly pressed, so as to reach the muscle by stretching the rather loose mucous membrane that covers it. The tendency of beginners is to bring the point of the electrode too far forwards in the pyriform sinus; and, in order to counteract this, I advise a larger and longer curve to be given to the electrode, and the handle to be depressed during the action.

"The effect of isolated excitation of the crico-arytænoideus lateralis is to produce slight rotation of the arytænoid cartilages, with movement forwards and inwards, so that the processus vocalis and the free edge of the vocal cord approach the middle line. The glottis is little influenced by this, only so far as the cartilage, and especially the vocal process, change their relative position to their fellows. If the patient inspire quickly and deeply during continuous contraction of the muscle, the vibiation of the prominent, and yet lax, vocal cords produces a loud jarring sound, and their vibrations may be clearly seel1. 
"The thyro-arytænoideus externus (Henle), or ary-syndesmicus (Luschka Merkel), which rests close against the anterior and upper margin of the cricothyroideus lateralis, and also the thyro-arytænoideus internus, lying somewhat further inwards, can both be reached from the pyriform fossa. The position of the electrode must be changed, so that its joint presses downwards, inwards, and forwards; while the handle is carried upwards and outwards, towards the upper teeth and angle of the mouth.

"The effect of the direct excitation of the thyro-arytænoideus, thus produced, consists only in a drawing of the arytænoid cartilages forwards and downwards. It is manifest that no tension of the rocal cords can be produced, so long as the arytrenoid cartilages are not fixed inwards and backwards by the arytænoidei transversales and the crico-arytænoidei postici; nor the thyroid and cricoid cartilages approximated by the cricothyroidei.

"In these excitations from the pyriform sinus, in which a certain degree of pressure is required, care must be taken not to confound mechanical movements impressed upon the parts of the laryux by the point of the electrode with movements resulting from muscular contraction. To prevent such an error, it is necessary to study the position of the arytrenoid cartilages, and of the soft parts, after the introduction of the electrode, with the circuit at first open, and then closed.

"The two thyro-arytænoidei interni can bo reached from the glottis, by placing the electrode, during inspiration, quickly and steadily upon, or between the vocal cords. By closure of the glottis by reflex action, the sponge-covered end of the electrode will be caught between either the true or the false rocal corcls. In the first case it touches the former by their margins, in the sceond, from above. This method of excitation is highly irritating to the mucous membrane of the larynx; and should not be often repeated during one sitting.

"The crico-arytrenoideus posticus, the actual dilatator glottidis, lies upon the posterior surface of the cricoid cartilage, on cither side of a central prominence. Accolding as it is wished to stimulate the right or the left muscle, the point of the electrode must be made to glide from the arytrenoid cartilage either to the right or left behind the plate of the cricoid cartilage. At first the constrictor muscles offer strong resistance; and it is sometimes necessary for the patient to swallow, in order that the electrode may be guided to its right place. Sometimes these muscles may he excited from the pyriform sinus, by carrying the electrode backwards and downwards. As soon as the muscle contracts and bulges, the electrode is liable to slip from its place; so that its position should be attended to.

"The effect is slight rotation of the arytrenoid cartilages on their axis, in a direction ontwards, and also morement of them backwards and ontwards, so that the glottis is completely opened. If the patient attempt to produce a note, while one muscle only is excited, a false and deeper note is produced, since only the vocal cord of the other side is brought into play.

"The muscles of the epiglottis, which reccive their nerves from the superior laryngeal, namely, the thyro- and ary-epiglottidei, can be excited either directly-from the lateral portions of the base of the epiglottis--or indirectly, where the interual branch of the superior laryngeal nerve passes through the laryngo-pharyngeal sinus.

"The glosso-epiglotticus, which consists, according to Luschka, only of a few terminal bundles of the longitudinalis lingux superior, in the neighbourhood of the glosso-epiglottic folds, can seldom require the attention of the surgeon. It can be reached, from the glosso-epiglottic ligament, more easily than any of the muscles that have been mentioned.

"The vagus nerve can be reached, according to Duchenne, from the œesophagus; and, according to Semuola and Gerhardt, from the surface of the neck. The first method presents many difficulties, since the operator works in the dark. The second is easier, but of still more doubtful value; since, on account of the deep position of the nerve, even when the electrode is in 
the right position (on the inner margin of the sterno-mastoid, immediately below the omo-hyoid), the thickness of the superincumbent soft parts protects the nerve in most cases, if not always. My experiments with both methods have, as yet, led to no positive results." $-H$. T.]

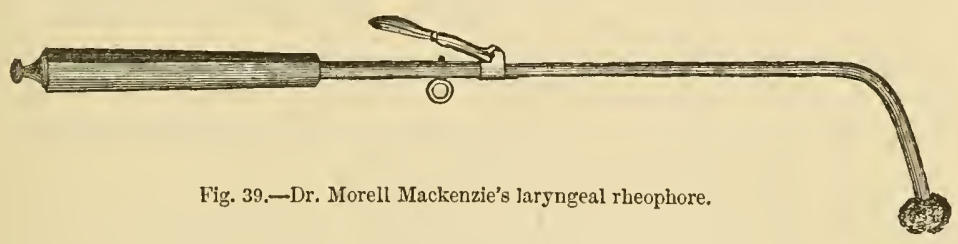

\section{6.-Faradization of the stomach, the liver, the lungs, and the heart}

Even when moist rheophores are employed, and whatever may be the strength of the current, the thickness of the thoracic and abdominal parietes will not permit the electric excitation to reach the regions situated within their cavities. Most of these organs may, however, be faradized indirectly, thanks to the pnenmogastric, which is accessible to rheophores in the pharynx and in the oesophagus. It is plain that the effects of faradization of the pneumogastric must vary according to the height at which that nerve is excited. From the lower part of the cesophagus, the excitation of the nerve is communicated solely to the stomach and the liver; while from the upper part of the pharynx, it is communicated to all the organs that the nerve supplies. To faradize the pneumogastric at its upper portion, the olive of the rheophore should be carried over the inferior and lateral part of the pharynx, and the circuit completed by placing the second rheophore on the nucha. ${ }^{8}$

When it is wished to limit the action to the stomach and liver an olive-shaped rheophore should be carried by an insulated stem, to the cardiac orifice, and the circuit completed by placing the second rheophore upon the epigastric region.

Is faradization of the pneumogastric sometimes indicated? At present, experience has taught us nothing worth notice as to the therapeutic value of the proceeding. I hope, however, that it may be applied with success to the treatment of certain obstinate

8 It is necessary to remind the reader that in its cervical portion the pneumogastric rests during its whole course upon the prevertebral muscles; that in the angular space it is covered posteriorly, on one side by the internal jugular vein, on the other by the common and the internal carotid artery; that the superior laryn- geal nerve, which springs from the inferior and internal portion of the gangliform plexus, lies below and to its imner side, and that, arrived at the side of the pharynx, at the level of the midlle constrictor muscle, it gives off the external laryngeal, and the nerve of arrest of Sion. 
nervous affections of the viscera of the chest or abdomen,--for instance, to gastralgia. It is needless to say that, in these cases, the faradization of the pneumogastric should be practised at different heights.

The procedure is not always free from danger. I need only recall the important organs that are subject to the pneumogastric, in order to impress upon my brethren the need of great caution in similar researches. 'The following accident occurred in my practice, and may serve as a warning to others. Whilst moving a rheophore over the lateral and superior parts of the pharynx, with a current of rapid intermission, but moderate intensity, the patient fell suddenly into syncope. When restored, he said that he had experienced a kind of suffocation, and an indefinable sensation. Since then, having faradized the pneumogastric many times at the same height, with one intermission per second, and with a very moderate current, the same accident has not occurred, but the præcordial sensation has been felt every time. I once saw the necessary caution neglected, in faradizing the pharynx of a young man, in whom that organ, and the velum palati, were paralyzed consecutively to an angina. A profound syncope was immediately produced by the operation; and, in this case I have no doubt that the pneumogastric had been irritated by the current.

Faradization of the pneumogastric at the lower part of the cesophagus may be practised without inconvenience.

Case II.-I made this application, in fact, a score of times, upon a woman named Célestine Bonhamy, twenty-three years of age. The metallic olive, with its insulated stem, was carried to the lower part of the osophagus, and was bulky enough to be in contact with the internal surface of the canal, so that the pneumogastries, which, at the cardiac surface, are placed close to the wall of the osophagus, one in front and the other behind, could not escape the action of the electric excitation. The rheophore (a moist sponge in a cylinder) was placed upon the epigastrium. The application was made with a view to modify the state of the digestive tube, which had seemed to be paralysed for a month. The stomach was enormously distended with gas, and the constipation obstinate. When I allowed the current to pass with one intermission a second, the patient felt moderately painful shocks at the level of the olive; the stem was shaken by an evident movement, and the patient liberated a considerable quantity of flatus. But no appreciable physiological phenomenon was produced during the operation; even when I had increased the force of the current to the maximum of the apparatus. Once I employed a very rapid current of slight intensity. The pain was very acute, the patient complained of a deep constriction; but this was all. There was no appreciable modification of temperature or circulation.

I cannot leave this subject without mentioning an. experiment that I made upon a man, after faradization of the pneumogastric. I examined his urine after this excitation (once only, it is true), and discovered the presence of glucose by the usual tests (liquor potassæe and Barreswell's solution). There was no glucose in his 
urine ordinarily. I believe this to be the first repetition, on man, of the experiments of M. Cl. Bernard upon animals : in whom he produced a temporary diabetes by faradization of the pneumogastrics. ${ }^{9}$

\section{7.-Faradization of the diaphragm.}

I shall explain the method of applying faradization to the diaphragm, in the electro-pathological study of that muscle.

[Duchemne's method of exciting the diaphragm is to place a conical metallic rheophore, covered with wet leather, on each phrenic nerve, to allow a very rapid current to pass, and to interrupt it at regular intervals to allow of expiration. In order to find the phrenic nerve, he presses the skin of the neck in opposite directions (outwards and inwards) by two fingers placed over the external border of the clavicular fascicnlus of the sterno-mastoid. The rheophore is then placed between the fingers, across the conrse of the nerve and pressed down.-II. T']

8.-The portion of intestine comprised between the stomach and the rectum, although inaccessible to direct faradization, may be excited by reflex action.

It is possible to excite the intestine indirectly, by a sort of reflex action, by placing the rheophores on two very distant points of the canal. Thus M. Leroy (d'Etiolles) has produced evacuations by passing galvanic currents from the month to the rectum. ${ }^{1}$ In 1854 (the time at which I made my experimental researches upon the functions of the diaphragm), I repeated this experiment upon horses, with MI. U. Leblanc, by passing an induced current of rapid intermissions. I confess that I was not able to obtain an alvine evacuation, whatever might be the intensity of the induced current. But we remarked that during its passage, if it were intense and very rapid, the sacro-spinal muscles, the respiratory muscles, and the motor muscles of the limbs, became tetanic. ${ }^{2}$

9 Leçons de Physiologie experimentale. Paris, 1856. Tome i.

1 Leroy (d'Etiolles), Memoire sur le traitement de lileus et des étranglements intestinaux internes, lu à l'Academie de Médécine, 1826, suivi d'un rapport de II. P. Dubois (Archives générales de Médécine, tome iii.). Rapport fait a la Société générale des naufrages (Asphyxie), Joumal de la Société générale des naufrages, tome iv. 1840.

"To stimulate the contractility of the digestive tube, there is," he says, "another and a more powerful means, more speedy and less hurtful than tobacco fumigations, namely, the establishment of a galvanic eurrent from the mouth to the anus. This contractile action was long ago displayerl by experiments upon animals; and one of the members of the council has proved, by application to the living human subject, that it is possible in this way, without risk, to produce energetic intestinal contractions. Lastly, M. Lestrohan has shown, by experiments upon aninals, the utility of the same curreut in asphyxia."

2 'The experiments were conducted in various ways. 1. In a horse lying upon its side, its feet secured by hobbles, the rheophores of my induction instrument were placed, the one in the mouth, and the other in the anus, the instrument working with rapid intermissions and a medium degree of intensity. They immediately produced general tetaniform convulsions in the limbs and trunk, but no alvine evacuation. During the 
This method of electrization of the intestine acts upon the spinal cord and upon the medulla oblongata witlı much more energy than the former. It is the excitation of the nervous centres which produces the tetanic contraction of so large a number of muscles; and it is therefore necessary to have recourse to the procedure very cautiously, and to administer only a gentle dose, lest we should produce asphyxia by artificial and prolonged contraction of the respiratory muscles, or by arrest of the heart's action. I shall return to this procedure, which has also been used in the treatment of asphyxia.

This proceeding, besides being dangerous in certain cases, could only be used in very slight intensity in the human subject; because the rheophore, applied to the mouth, would excite the extreme sensitiveness of the fifth pair of nerves, and would produce phosphenes. I attempted this operation on the patient mentioned above; but she could not bear it, eren when very slight. I then modified it as follows: one rheophore was placed in the cesophagus, near to the cardiac orifice, the other in the rectum. It was then possible to use a full current. The whole intestine was evidently excited, and the patient had daily colic and abdominal pains, but I did not succeed in orercoming the constipation.

I see no necessity to employ these proceedings, which are painful, and not free from inconvenience. By placing one rheo. phore in the rectum, and a second (moist) on the abdominal wall, I have often succeeded in obtaining an alvine evacnation. This metlod produced a stool in an hysterical woman who had suffered for three weeks from a constipation that MI. Briquet had been unable to overcome, and whose abdomen was enormously tympanitic. $^{3}$ By the same method I reliered internal strangulation of the intestine in a woman who would have undergone an operation if I had not succeeded. I shall recur to this case by-andby.

\section{§II.-Faradization of the organs of the senses.}

I proceed to set forth briefly the different methods of faradization that are most successful in paralysis of the senses.

first second, the respiration was suspended; but on its return, it was accelerated during the fire or six seconds that the experiment lasted.

The heart coutinued to beat throughout the whole experiment, and with incrcased rapidity. 2. In another horse that was about to be killed at Monf fauçon, and that I laid open, I did not observe any appreciable coutraction under the influence of the induced current passed, as in the former experiment, from the mouth to the anus, whatever might be its intensity. 'The tetaniform contractious of the limbs and of the trunk were carried to their utmost limits, so as to coustitnte opisthotonos.

3 Briquet, Traité clinique et therapeutique de lhystérie, p. 661. Paris, 1859. 


\section{A.-Action upon paralysis of touch.}

Apply moist rheophores on the track of the collateral nerves, and to the pulp of the fingers.

\section{B.-Action upon certain forms of paralysis of the retina (cmaurosis).}

I have shown in the preceding chapter, that galvanism possesses the special property of exciting the retina very acutely ; producing luminous sensations when moist rheophores are applied to parts of the face on which the branches of the fifth pair ramify; and that these sensations increase in acuteness as the rheophores approach the median line. It suffices to recall these facts, in order to know how to operate, when it is an indication to excite the retina, as in amaurosis. In defanlt of a galvanic current, it would be possible to use the induced current of the second helix of a magneto-faradic apparatus. The action of this current upon the retina, althongh less than that of galvanism, is still sufficiently powerful.

To direct excitation of the retina may be added electro-cutaneous excitation of the circumference of the orbit.

I shall hereafter describe the methods of faradization of the ocular muscles, in treating of paralysis of these muscles.

\section{C.-Action upon paralysis of hearing.}

(1). Fill the external auditory meatus with tepid water, and place a metallic rheophore-a sound, for example-in the water. Complete the circuit by a second rheophore on the nucha. (This method, to which I shall return, has allowed me to institute electro-physiological and pathological researches into the uses of the chorda tympani.)

The sensibility of the membrana tympani is very great; and hence it is necessary to avoid rapid intermissions and intense currents. I purpose to treat specially of certain forms of nervous deafness; and we shall then see that some of these forms, and even some of congenital deaf-mutism, have been successfully treated by these procedures.

\section{D.-Action on paralysis of smell.}

A moist rheophore being placed over the nucha, the second (a metal sound of small diameter, insulated by caontchouc except at its extremities) is moved over all points of the nasal mucous membrane.

It is often sufficient to excite very feebly the general sensibility 
of the mucous membrane, in order to recall the lost or weakened sense of smell.

\section{E.-Action upon paralysis of taste.}

The metallic rheophores must be moved orer the base and over the borders of the tongue.

Electric excitation of the senses of sight, hearing, smell, and taste, should be performed with extreme circumspection, because liable to react acutely upon the brain. It is, therefore, contraindicated in all cases in which it is necessary to avoid central excitement. In operations of this kind, we shonld always commence with a minimum dose, increase it gradually, and never produce much pain. It is also prudent to use a current of slow intermission.

\section{Faradization of the male genital organs.}

'The secretion of semen may be diminished or perverted; and it follows that the sexual appetite is not awakened by the generative instinct, and that erection is absent or incomplete. It may be indicated to act upon the secreting organ of the semen, the testis; and also upon the reservoirs in which this secretion is elaborated, the vesiculæ seminales.

Faradization of the testicle is extremely simple. The moist rheophores are placed upon the scrotum, over the testicle or the epididymis. The current traversing the skin, concentrates its action upon the glands. The sensation produced is very painful, and extends to the loins, if the current be intense, and especially if the intermissions are rapid. It is analogous to that produced by compression of the testicle or of the epididymis. The latter is more sensitive to electric excitation than the former. Faradization of either should be performed with a moderate current; since their orer-excitation may be followed by a very painful neuralgia, as happened in the case of a patient treated by myself. I may here repeat that the current of the first helix, even if of feeble tension, excites these organs more acutely than that of the second helix.

Faradization of the vesiculæ seminales is accomplished by the aid of the rheophore for the rectum already described. The bowel being first emptied, the rheophore is introduced, and so directed that its olive-shaped termination may be brought into relation with the resiculæ. For this purpose, it is sufficient to move the rheophore from right to left, and vice versâ. A strong current will penetrate the intestine, and reach the vesiculæ, exciting them cnergetically. I need not say that the circuit should le com- 
pleted, by placing a second rheophore on some insensitive part of the body. When there is no contra-indication, I introduce the second rheophore into the bladder, and excite the bas fond, so as to place the vesiculæ between the two rheophores.

The seminal fluid often dribbles away, not only in consequence of a kind of paralysis of the resiculæ, but also from paralysis of the levator and sphincter ani, and of the muscles of the urethra. It is then proper not only to faradize the vesiculæ, but also to direct the excitation upon all the muscles that concur in the act of ejaculation. Faradization of the levator and sphineter ani has been described. The bulbo and ischio-cavernous muscles are faradized like those of other regions of the borly ; by placing moist rheophores on points of the skin corresponding to their surface.

There may exist a kind of weakness or paralysis of the ejaculatory ducts, which occasions seminal losses, or a too rapid emission during sexual congress. This affection may be cured by faradization of the orifices of the ejaculatory ducts, by means of an urethral rheophore terminating in a little olive-shaperl metallic knob, the stem being insulated by a caoutchouc sound. The knob is introduced into the urethra as far as the orifice of the ducts, and the second moist rheophore is placed upon the perineum. I shall return to this important question when I treat specially of paralysis of the genito-minary organs.

The testes, the skin of the penis, the scrotum, the perinemm, the glans penis, and the urethra, may be in a state of complete anæsthesia. I have seen impotence that could be referred to no other canse than this general insensibility of the genital organs. In such a case I used the following methods of faradization. I excited the sensibility of the testicles by the method described above. Then a vesical rheophore was mored slowly along the urethra, acting principally upon the most irritable points, the fossæ navicularis. Lastly, electric fustigation by metallic threads was employed to restore the sensibility of the skin of the penis and scrotum. I shall return to this case hereafter.

\section{Part the Fifth.}

\section{The General Effects of Localized Faradization.}

Beyond the local and immediate action produced by electric recompositions, occurring within organs, faradization exerts also general effects, of which it is necessary to take account in practice. The influence of general excitation may increase the secretions, the perspiration for example; and it may make itself felt by this or that organ, according to individual predisposition. It is thus that, 
in amenorrhcea, the menstruation is recalled or modified by faradization, in whatever manner it is applied.

I have proved that electro-physiological excitation is limited, in general, to the points which are in relation with the rheophores; but it is a belief widely diffused among practitioners, that faradism always over-excites the nervous centres. If this belief be well founded, we may see how much danger would attend the use of such a therapentic agent, in the treatment of certain forms of paralysis, and especially of cerebral paralysis.

By taking care to keep the rheophores near tomether, it is certain that the action of the currents is felt but fecbly by the nervous centres. In the following chapter I shall describe the means of directing the excitation upon these centres, when it is necessary to excite them.

It would be easy for me to show that faradization reacts upon the nerrous centres, only by the intermediation of the sensibility that it over-excites. When we produce contractions, by the influence of a very intense and rapid eurrent, in muscles that are paralysed in consequence of cerebral hemorthage, and that are at the same time deprived of their sensibility, the electric excitation will be localized in the parts to which it is applied, the muscles will contract energetically, and the patient will not even have any conscionsuess of the process. Howerer frequent or long continued the operations, he will be in no way incommoded. If, on the contrary, the same current he applied, in the same patient, to muscles that retain their normal sensibility, it will not only produce immediate and very acute pains in the muscles themselves, but will produce also a general over-excitation that may be a source of danger to the brain.

It appears from these considerations that localized faradization reacts very faintly upon the nervous centres, when so practised as not to excite the sensibility too acutely; and when the rheophores are kept near together.

But there is a point which it is important to know in every application of electricity to man: I refer to the dynamic influence that may be exerted upon patients by the change in their natural electricity that is prodnced by electrization. In order to give any account of these phenomena, it is essential to explain the physical change that is wrought in the body by the action of the faradic current. At the moment when the rheophores of an induction apparatus are applied to the surface, the natural electricity of the dermis is decomposed, and opposite electricities collect towards each of the rheophores, and escape in sufficient quantity to neutralise the current that comes to trouble their repose. This 
nentralisation being effected, the two opposite electricities, by which the body is still penetrated, reunite to regain their normal state, and do not again separate until a new current comes to disturb them. Such is the series of physical phenomena produced, more or less frequently, in man; according to the rapidity and the frequency of the induced current that is directed upon his organs.

The modifications that are thus wrought in the natural state of the electricity of the human body do not produce, in general, any appreciable dynamic effect. But it should be known that, in certain nervous conditions difficult to analyse or to explain, man does not bear with impunity these changes in his electric state. There are then developed certain general phenomena, which, if they do not contra-indicate the use of faradization, at least show the necessity for cantion in its employment. It is well known that some persons are very sensitive to electric changes in the atmosphere. I know a lady who, during a thunderstorm, is stricken for some hours with general paralysis. I have also seen persons who, under the influence of faradization, suffer from singular nervous troubles. These general effects are not produced by the excitation of organs; but appear to depend upon the morlification of the electric state of the body. Thus faradization may produce dazzling, a feeling of faintness, or general numbness, althongh the operation, very slightly performed, has occasioned no local sensation. I have seen, in La Charité, a young paralysed girl so sensitive to the influence exerted upon her general state by indured currents, that faradization was contra-indicated in her case, although it produced no local sensation. I append an account of the case, which was one of much interest:-

C'ase III.-Salle St. Vincent, No. 26 (service de M. Andral)-Eugénie Thonvenir, aged sixteen, born in Paris, of good constitution and lymphatic temperament. Continual contraction of the flexors of the fingers and thuml, of the left hand; so that the fingers cannot be extended by any effort. The patient can herself flex and extend the left fore-arm. She walks without dragging the left leg; but is easily fatigued, and feels a weakness of that side. On the left, a needle thrust deeply into the tissnes, or a hard blow on an osseous surface, produces no sensation ; the hands and feet have lost tactile sensibility. In standing or walking, she feels the ground only with the plantar surface of the right foot. On the left side there is insensibility of the skin of the face, of the conjunctiva, and weakening of sight, loss of smell, taste, and of the general sensibility of the nasal and buccal mucous membrane. Although thus insensitive to impressions from withont, the patient suffer's from frequent and deep-seated pains in the left arm and leg. She cannot bear pressure on a fixed and limited spot below her breast, which is the seat of continued pain. She vomits five or six times daily, either food or drink. She has no attacks of hysteria, no strangulation, no palpitations, no abnormal heart murmurs; but a murmur in the carotid.

At the first séance for faradization, excitation of the skin, muscles, bones, or periosteum, and even of the nervous trunks, occasioned no kind of sensation 
on the diseased side. The muscles contracted in response to the excitation, but without the consciousness of the patient. At the second séance, as at the former, she felt some of the local sensations commonly produced by electric recomposition; but she complained of a kind of stitch in the left side, with suffocation, palpitations, dazzling, nausea, and cephalalgia. These phenomena were more severe, the stronger the current, and the nearer the rheophores were placed to the precordial region. If the rheophores were applied to the healthy side, the ordinary phenomena produced by electric recomposition were displayed without any general effects. These strange phenomena induced me to repeat the following experiment several times, in the presence of MHI. Andral, Therial, Lacaze, and the students in attendance upon M. Andral's clinique. We arranged so that the patient could neither see nor hear what was done; and then the rheophores were placed at the lower extremity of the left leg, so as to prodnce electric recompositions in the skin. As soonas the apparatus was set in action, the symptoms above described appeared. Leaving the rheophores in place, the course of the current was interrupted in a manner that the patieut could not know, and instantly the symptoms disappeared. The rheophores, placed upon the healthy side, produced either muscular contractions and sensations or a burning sensation, according to the tissue in which the electric recompositions occurred; but they never occasioned any of the general phenomena described above. This experiment repeated rery frequently, and on many days in succession, gavo always the same result. The patient was so sensible of the electric influence that she recognised the very faintest currents when applied to the diseased sicle; although the phenomena occasioned by the same currents, when applied to the sound side, were scarcely appreciable. Every time that she was exposed to the current, however feeble, and for however short a time, she suffered for a long period afterwards from pains in the head, disturbance of vision, general malaise, a stitch in the side, and a redoubled occurrence of romiting to which she had long been subject.

It is very difficult to find the key to these singular phenomena. The th ory of local electric recompositions is certainly not applicable to them; because, on the left side, the patient was deprived of sensation in the parts on which I acted, and did not experience any of the ordinary physiological effects of electric recompositions, occurring in the skin, in the muscles, \&c., \&c. They can only be explained by the reflex action of some important point in the nervous centres, under the influence of the peripheral excitation of certain zones; althongh the organs excited were themselves struck with insensibility.

An extreme reflex excitability of the nervous centres, of the heart, or of the respiratory organs may also be developed under the influence of certain pathological states. Faradization is then a two-edged weapon. However, by proper circumspection, we may always avoid danger. We may even, under certain circumstances, take a fortunate part in sustaining or in regulating functions essential to life, such as those of the heart, or of respiration. I shall have occasion to record a remarkable case, in which a slight electro-cutaneous excitation with the hand, on the precordial region, prolonged for many days the life of a patient who, during convalescence from membranous angina, and under the influence of 
diphtheritic poison, being in a state of extreme excitability, had attacks of grave syncope, and was constantly in danger of arrest of the heart's action.

The general nerrous disorders in question may sometimes be produced even when the subject is in his natural state. Thus, I could mention one of my confieres and friends, who cannot submit to electrization without experiencing vertigo, dazzling, \&c., however feeble may be the electro-physiological effects produced in him. I am unable to say what are the causes which predispose to this extreme susceptibility to electric influences.

From the facts stated above, I deduce the following propositions :-

1. The changes wrought in the state of the natural electricity, by which the human body is pervaded, may produce, independently of the local electro-physiological phenomena due to localized faradization, general effects of a particular kind.

2. Most commonly, these general effects are not appreciable. They are only manifested in certain rare idiosyncracies, or pathological conditions, the causes of which are usually unknown.

3. These general effects, the chief of which are giddiness, dazzling, nausea, and even vomiting, render the application even of localized electrization difficult. Sometimes they contra-indicate its employment.

4. We may, however, under certain circumstances, have recourse to it, in order to encounter serious disorders of the circulation or respiration. 


\section{CHAPTER III.}

HISTORICAL AND CRITICAL OBSERVATIONS UPON THE PRINCIPAL METHODS OF ELECTRIZATION.

Whatever preference I may declare for the method of electrization described in the foregoing chapter, has not been arrived at without long comparative study of each of the methods that have preceded it. In the present chapter I purpose to set forth the results of these researches.

Static electrization (by the electrical machine) is the most ancient of all modes. I hare already, in the first chapter, said enough about its physiological and therapeutical properties; and its application for the purposes of treatment is now generally abandoned. ${ }^{1}$

I propose, therefore, to examine as briefly as possible, and to compare, by the light of my own experience, the therapentic value: 1. of electro-puncture; 2 . of electrization by reflex action; 3 . of localized electrization by induction currents and by interrupted galvanic currents; 4 . of continuous constant currents; and I shall preface the therapeutical results of my own practice by certain physiological considerations.

\section{Part the First.}

\section{ON THE VALUE OF ELECTRO-PUNCTURE AS APPLIED TO THE TREATIENT OF PARALYSIS.}

\section{$\S$ I. Historical retrospect.}

The application of electro-puncture to the treatment of paralysis was a distinct step in advance, when Sarlandière introduced it into practice. In order to estimate correctly the services rendered to therapeutics by this method of electrization, it is necessary to recall what was then the state of medical electricity.

It is well known what enthusiasm this agent excited in its infancy, when the electrical machine was first invented. We may, indeed, find records of incontestable cures, clue to the use of electricity of tension, in the authors who were employed about the subject at the time in question, that is from 1743 to 1754. Kruger, professor at Helmstadt, was the first to use electricity as a curative

[' See Observations, p. 9.-H. T. $]$ 
agent, in the begimning of 1744: Two years later, in 1746, when the effects of the Leyden jar (the strong discharges of which had at first inspired great terror), had become familiar, Hermann Klyn cured, by this instrument, a woman who had been two years paralysed. After these observers came the Abbé Nollet, Privata of Venice, Sauvage, and Zindult, a Swedish physician, who used the jar in the treatment of chorea in $1753 .^{2}$

ILedical electricity, unjustly abandoned some years after its birth, had been taken up again with fresh ardour. Manduit was nominated reporter to a commission by the Royal Society of Medicine, in order to determine the actual worth of electricity in paralysis. Notwithstanding the brilliant report of this learned physicist, made in 1773 , and in which the cure of paralysis by electricity was represented to be the rule; notwithstanding the works of the Abbé Bertholon, in 1779, of Cavallo, in 1780, of James Larry, in 1800; and notwithstanding a great number of treatises which were published upon the subject in France, in England, in Germany, and elsewhere, electricity was unable to bear the test of time. The results obtained from it were not in accord with the hopes that had been entertained by some ardent minds, and there followed a period during which the agent suffered under disrepute.

The discovery made by Galvani in 1789, and, soon afterwards, the pile of Volta, afforded to medicine a new and precious source of electricity; of which numerous applications were made in practice, especially in Germany, from 1797 to 1804 . I shall return to this as a matter of history, when treating of the application of continuous currents. But, this time also, either that the possibly exaggerated predictions of experimenters were not generally realized in practice, or that the proper method of application was not known, or that the instruments then in use were insufficient or irregular in their action, or difficult and inconvenient of use, galvanism was unable to preserve medical electricity from general neglect.

Such was the state of things existing in France, at the time when Sarlandière conceived the ingenious idea of using acupuncture in order to direct and limit the power of electricity in the deeper parts of the organism. ${ }^{3}$ This method, which supplemented the weakness of the instruments by increasing the physiological

2 We may even carry back medical electricity to times still more remote; for that of the torpedo was employed by the ancients, even before Galeu, as a remedy against a certain number of diseases. Some physicians employ it even at the present day in the countries where the creature is found; and I have known an inhabitant of Martinique who was cured of facial hemiplegia by the discharges of the torpedo.

${ }^{3}$ Sarlandière, Mémoires sur l'élects:opuncture. Paris, 1825. 
action of the electricity, without exposing the patients to the startling effects of the Leyden jar, soon superseded the older modes of application, and gave new life to medical electricity. Magendie, by lis talent and by the authority of his name, powerfully contributed to its popularity.

\section{$\S$ II. Critical considerations.}

I shall next endeavour to show that electro-puncture is far from the attainment of the end that was proposed by Sarlandière and Magendie.

1. Electro-puncture does not exactly localize electricity in the organs.

I hope to afford proof of the foregoing proposition, by analyzing successively the phenomena of electro-cutaneous sensibility, and the phenomena of electro-muscular contractility and sensibility, that are witnessed under the influence of electro-puncture.

In thin patients, or in those whose subcutaneous cellular tissue is scanty, needles thrust into the skin, in such a manner as not to pass through the dermis, produce, even uncler the influence of a very feeble current, simultaneons excitation of the skin and of the muscles and nerves, if the needles are placed over the course of muscles or nerves. In order that the excitation may be confined within the skin, it is necessary that the cellular tissue should be either plentiful or infiltrated with serosity, and that the current should be extremely weak.

Electro-puncture cammot prodnce muscular contraction without, at the same time, exciting the sensibility of the skin. In order to prevent this effect, M. Pétrequin has covered the needles with a layer of caoutchonc, leaving only their extremities free, for an extent of one or two millimetres, commencing a millimetre from the point. But the covering of caoutchouc, which is not very thick, speedily becomes moistened and softened when the needle is plunged into the tissues. It then permits the passage of the current; and the skin is excited almost as acutely as if the needle were bare. Patients on whom I have made comparative trials with the insulated and with the common needles, have been unable to discover any difference between them as far as regards their action upon the skin.

The cutaneous sensation produced by electro-puncture masks the muscular sensation produced by the direct excitation of the muscle into which the needle is plunged. It might be supposed that the two sensations are the same, and that they blend together; but this is not the case, as may be shown by the following 
experiments. In wounded persons I have inserted needles in denuded portions of muscle, and also at points where the muscles were still covered by unbroken skin; and I have applied electricity in both situations. In the former, I produced the dull sensation proper to muscular excitation; such as is commonly produced by placing moist rheophores upon the healthy skin. In the latter the sensation was very acute; and resembled that which follows electro-cutaneous excitation, as in faradization by metallic threads upon a dry skin.

We cannot produce at pleasure, by means of electro-puncture, the contraction of a muscle or of a single fasciculus, or of a few fibres only. Indeed, although I have been careful to place the needles at a distance from nerve-trunks, the muscular contractions observed in my experiments have nearly always been irregular and unforeseen ; sometimes merely fibrillar, and limited to a certain radius from the needle, at other times involving an entire muscle, or even several muscles at once. It is evident that, in these cases, the effects have varied accordingly as the needle has come into contact with nervous filaments proceeding to one or to many muscles, or with muscular fibres alone. It follows that, in muscular electrization by acupuncture, chance alone will preside over the phenomena of contraction, and will dominate over the will of the operator.

Sarlaudière recommended that the needles should be placed at a distance from nerve-trunks; and believel that the therapeutic influence of electro-puncture was greatest when exerted upon the nervous terminations. Magendie had the hardihood to practise electro-puncture with needles that traversed the nerve-trunks themselves. The latter proceerling may appear easy to those who possess some anatomical knowledge; but it is by no means so simple as may be imagined by those who have not tried. If it be true, for instance, that we may sometimes sncceed in piercing the median, ulnar, and crural nerves, we cannot in like manner succeed with the radial, the sciatic, or the popliteal. In the face, we may place needles in the sub-orbital and mental nerves. But who could pretend to hit with any certainty either the trunk or the branches of the portio dura? The method appears to me to be nearly always impracticable. Moreover, it does not help us to confine the electric action to the nerve. We camnot reach the latter without piercing the skin, which is then inevitably excited.

\section{Electro-puncture is insufficient or inapplicable in ancesthesia or lesions of tactile sensibility.}

I shall hereafter show that, as a rule, in anæsthesia, cutaneous 
faradization will recall sensibility only in the points which are brought into contact with the rheophores. It thence follows that, in order to obtain a cure by electro-puncture, it would be necessary to cover, with a great number of needles, the whole of the portion of surface that had lost its sensibility. It is manifest that such an operation would be impracticable, especially if it were to be frequently repeated. In certain slight cases the excitation produced by a very small number of needles may be sufficient to restore the sensibility; but such cases are only exceptional.

Electro-puncture is not available for the restoration of tactile sensibility to the hand or to the sole of the foot; because we cannot insert needles into either the fingers or the plantar integument without danger of producing inflammation or whitlow.

\section{Electro-puncture, applied to the treatment of paralysis of move-} ment, does not appear to produce the results that are obtained by localized electrization.

Numerous and incontestable instances of success bear witness to the therapentic power of electro-puncture in the treatment of muscular paralysis; and it has done much more than the methods which preceded it. It must, howerer, yield the palm to localized faradization, by the aid of moist rheophores placed upon the sound skin; a method the importance of which I shall shortly have to establish.

There are no rules with regard to the manner of applying electro-pumcture in paralysis. In what points of the paralysed limbs should the needles be inserted? In what number should they be employed? What degree of power should be employed? All this is left to the determination of each operator.

Some practitioners, after the example of Magendie, have endeavoured to pass the needles into the nerve-trunks which supply the paralysed muscles. But it follows, from my investigations, that the electric excitation of nerre-trunks, in the treatment of paralysis, produces results that are but unsatisfactory. Moreover, if the mode of excitation were excellent, the electrization of nerve-trunks by puncture would almost always be impracticable, for the reasons already stated.

The method generally adopted is to place, at the superior and inferior parts of the limb, needles that are connected with the poles of a galvanic or faradic instrument. By this method the excitation is produced in a most unequal manner, and affects only a small number of muscles. Those muscles that do not receive the stimulus directly, commonly remain paralysed; some 
of them are excited by reflex action. The method borders upon that of electrization by reflex action, which forms the subject of the second part of this chapter.

In order that excitation should be complete, in the treatment of paralysis by electro-puncture, it would be necessary that needles should be inserted into each of the paralysed muscles. Moreover, as it seems to me to be demonstrated, by numerous facts, that the therapeutical action is limited, in general, to the points that are in contact with the rheophores, or at least that it extends over but a small radius, it would be necessary that the number of needles used to excite each muscle should be proportionate to its bulk and length. The operation would become a torture to which patients would never submit, especially in paralysis of some extent, by reason of the time that would be required and of the inevitable suffering that would be produced.

On the other hand, how simple and rapid is the localized faradization of each one of the paralysed muscles, by means of moist rheophores moved over the unbroken skin. In electro-puncture, the needle traverses the thickness of the muscles, whilst, in localized faradization, the moist rheophores act only upon their surface. We might, therefore, suppose that the electro-puncture would possess more therapeutic power than localized faradization. Such an opinion would be unfounded; for I have already shown that, on applying a moist rheophore to the surface of a muscle, the electric excitation penetrates the tissues the more deeply, the more intense the current. Faradization, therefore, by the aid of moist rheophores, will penetrate a muscle in the direction of its thickness as readily as by the aid of needles. Moreover, by moving the rheophores over the whole surface of the muscle, the electricity is distributed to every part of it, which can never be clone by electropuncture.

I have seen many cases of paralysis undergo improvement under the influence of localized faradization by moist rheophores, in which electro-puncture had previously completely failed.

If electro-puncture were as efficacious and as practicable as muscular faradization, the pain inseparable from the former method should lead us always to prefer the latter. I shall, moreover, show hereafter that painful electrization is dangerous in some forms of paralysis, in those of cerebral origin for example. Electro-puncture is therefore contra-indicated in such cases, and also in all those that require numerous applications. (We shall see that the cure of some forms of paralysis requires from sixty to eighty applications.)

It is especially in children that it is desirable to practise 
electrization without pain. How many parents, and even how many practitioners, would shrink from inflicting upon young paralytics the torture of electro-puncture, which is al ways painful, however feeble may be the current.

There are yet other inconveniences which attach themselres to electro-puncture, but which are not to be feared in connection with the method of localized electrization that I advise. I refer to the disorganization of the tissues through which the needles pass, and of the inflammations and abscesses excited by them. I need not dwell upon these accidents, which have been recorded by all the observers who have practised electro-puncture.

Lastly, electro-puncture frequently occasions severe and obstinate neuralgia. The following is an example. One of the pupils of Magendie, A. Bérard, consented to undergo an electro-physio- . logical experiment, and allowed the professor to perform electropuncture upon his face. His complaisance, or rather his curiosity, cost him dear; for the operation was followed by a facial neuralgia. from which he suffered cruelly for five or six months. He became one of the most brilliant professors of the Ecole de Paris, but, unfortunately, he was too soon lost to science. My information was derived from his brother, the professor of physiology.

The foregoing considerations, however, must not lead us to conclude that electro-puncture should be altogether abandoned. I believe, on the contrary, that in certain cases it may be a potent auxiliary to localized faradization. ${ }^{4}$

\section{Part the Second.}

ON THE VAlue OF ELECTRIZATION BY REFLEX ACTION, AS APPLIED TO THE TREATMENT OF PARALYSIS.

\section{$\S$ I. Passage of induced currents from the nerve-extremities to the centres.}

Before commencing the study of the therapeutic influence of this method of electrization it is important thoroughly to understand its physiological action.

\section{Physiological action.}

A.-Following the known laws of the propagation of the nervous fluid in the motor nerves, the motor action is exerted only in the

- Electro-puncture has been called upon | by destroying certain hepatic and ovarian to render great services in surgery in the treatment of hydrocele, by promoting the resolution of certain ganglionic tumours, cysts, and by coagulating the blood in aneurisms. 
direction of the nerve-branches which proceed to muscles, and never in the contrary direction. It follows that electrization of the nerve-terminations of limbs will never call into contraction muscles which receive their nerve-filaments from branches given off above the point excited.

This is, in fact, what occurs when we localize electric excitation in the terminations of nerves, by holding both the rheophores of a galvanic battery, or an induction apparatus, in one hand (with the well understood precaution that the circuit shall not be completed by allowing them to come into contact). We then experience some contraction of the muscles of the thenar and hypothenar eminences, produced by direct excitation of the muscles, with pricking and tingling in the fingers, under the influence of the equally direct excitation of the collateral nerves, or of the digital tissues; the muscles of the forearm do not enter into contraction.

But if, in place of holding both rheophores at the same level in a single hand, one is separated from the other, either by placing one in the hand and the other higher up on the limb, or by holding one in each hand, we then see contraction of the muscles of the fore-arm or arm, according to the degree of intensity of the current. The muscular contractions produced by this method of electrization are irregular, and do not correspond with the degree of pain that accompanies them. The pain, indeed, is not confined to the muscles in contraction, but extends to the wrists or the elbows, or even to the shoulders when the current is intense. The sensation in that case is very painful, and leaves behind it an aching of the joints.

In these various experiments the excitation is always greatest in the arm the hand of which grasps the positive pole, so that it receives an ascending current. From these two experiments I draw the following conclusions:-1. Electrization localized in the nerve-terminations of a limb produces only local phenomena in accordance with the functions of the organs excited; that is, sensations limited to the part. 2. In order to produce contractions of muscles situated above the nerve-terminations, it is necessary to separate the rheophores in such a manner that the currents may traverse the limbs, or rather their nerves, in a longitudinal direction; this is electrization by reflex action. 3. Lastly, the electrization by reflex action operates specially upon the seusibility, ascends to the posterior roots of the nerves, affects the anterior roots, and thus produces, in the muscles dependent upon the nerves the terminations of which have been excited, irregular contractions that are but little in harmony with the intensity of the sensations. 
Whenever the moist rheophores of an induction apparatus, applied to the skin, are a little apart from one another, in such positions that the current traverses the limb longitudinally, then the extremities of the limb, although they are not in communication with the rheophores, will exhibit muscular contractions from reflex action. If, for instance, we place one rheophore upon the anterior surface of the fore-arm, and the other near the spine in the cervical region, we shall not only see contraction of the muscle situated in the plane of the rheophore, but also of other muscles of the arm and fore-arm.

B.-Electrization of the nerve-extremities by reflex action is often practised by placing the extremities of the patient in two basins of water, each of which is connected with one of the rheophores of an induction instrument.

It is possible to apply faradization at once to the upper and lower limbs, and in a more general manner, by placing both hands in a basin of water communicating with one of the poles, and both feet in another basin communicating with the other. This method excites the nervous centres, and especially the spinal cord, in a very general and energetic matter, even when the current is of small intensity. If the intermissions are rapid, all the muscles of the limbs are thrown into tetanic contraction, and the accompanying sensation, which is felt chiefly in the joints, is very painful. At this degree of intensity this method of electrization is followed by severe lumbago.

We may classify under this kind of electrization the electric bath, which may be also called the electro-dynamic, in order to distinguish it from the bath of static electricity given by the friction electric machine. The former consists of a bath, the water of which is in contact with one of the rheophores of an induction instrument, or of a battery of intermittent current, while the other moist rheophore is applied to some part of the body that is not immersed; commonly to the upper and back part of the chest.

By this proceeding also, the spinal cord is excited in a general manner, nearly as much as by reflex faradization of the peripheral extremities of the nerves, and with the same energy.

C.-Electrization by centripetal or centrifugal currents with currents of induction.-In electrization by reflex action, physiologists have attached great importance to the direction of the currents, resting upon experiments which show that in certain animals the direct (centrifugal) currents act upon the motility, and the inverse (centripetal) currents upon the sensibility.

Induced currents, under ordinary conditions, cannot be said to 
be either direct or inverse. In fact, each intermission of the induced current (of the second coil) is produced by two successive currents travelling in contrary directions. The intermissions produced by all the ordinary rheotomes described in the sequel (the toothed wheel and the tremblers) afford these alternations. But it is sometimes desirable to avoid the altemations, especially in certain pliysiological experiments, or in therapentical applications, where it is wished to pass only a direct or an inverse current. In order to fulfil this indication, I had made, some twelve years ago, an instrument which is a modification of the wheel of Abria, joined to a commutator of the poles. This instrument is represented in fig. 40.

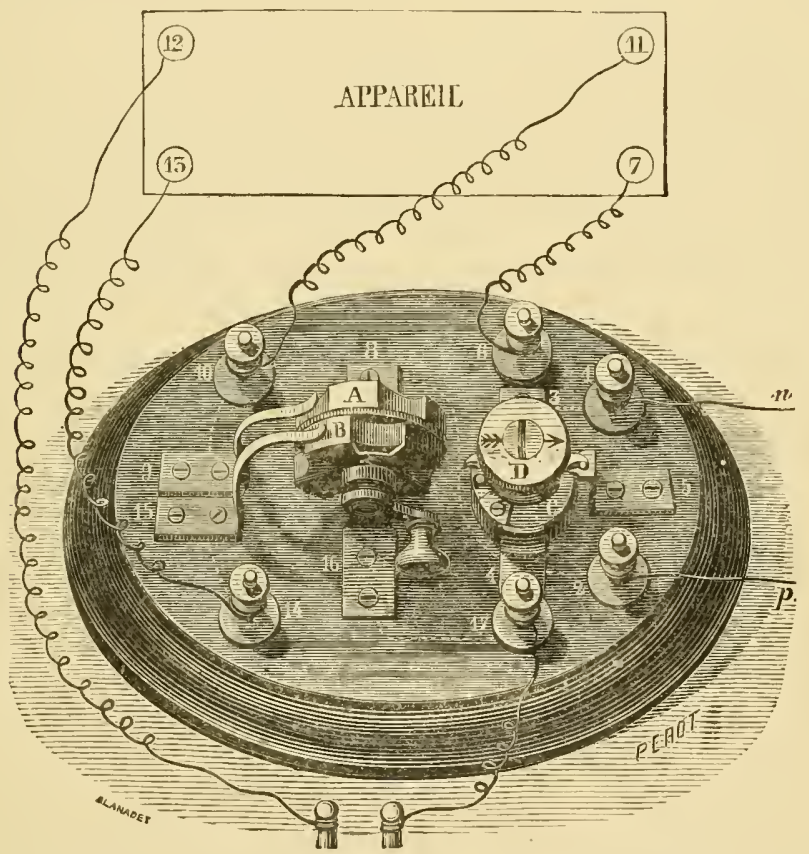

Fig. 40.-Distributor of the entrance and issue of induction currents, and commutator of the poles.

Like the wheel of Abria, it is composed of two toothed wheels, A, B, the teeth being alternately of wood and of brass. In the figure the shaded teeth represent those of wood, and the unshaded those of metal, mounted upon the same axis, but insulated; the brass teeth of each wheel communicate with the brass mounts 8 and 16 , in which turns the wooden axle common to both the wheels, which move together (thus differing from the instrument of Abria, in which the wheels turn independently). The two 
wheels are so arranged that their respective teeth only correspond by half their surfaces, so that the two springs 9 and 15 only rest at once upon the metallic parts of the teeth for one half of their extent.

Things being thus arranged, let us first follow the course of a battery current through the commutator $\mathrm{C}$ (fig. 40) and to the extremities of the wire of the primary coil of a double-induction volta-electric apparatus, represented by the knobs 7 and 11 . The two poles, $n, p$, of the pile are brought to 1 and 2, knobs which communicate with each of the isolated halves, 3 and 4 , of the commutator $\mathrm{C}$, so that they can be marle to change sides by turning the button $\mathrm{D}$ of the commutator from left to right, or vice versâ. One of these poles-suppose the negative-passes from 3 and 5 to 6 , a knob to which is attached a conductor in communication with 7 , one of the extremities of the wire of the primary coil; the other, or positive pole, $p$, goes from 2 and 4 to 8 , a metallic support communicating with the metallic teeth of the wheel A. At 9 is a spring in contact with these metallic teeth, and connected with 10, a knob to which is attached a conductor in communication with 11, the other extremity of the wire of the primary coil.

In these conditions the wheel $\mathrm{A}$ is only an ordinary rheotome, producing by each of its teeth, or at each intermission, while a rotatory motion is communicated to it, two currents in opposite directions, one at the completion and the other at the interruption of the circuit, in the wire of the second coil, the extremities of which, represented by 12 and 13, are connected by some metallic or organic conductor.

But if one of the extremities of this wire-13, for example,is conducted by a wire to 14 , and so to 15 , a spring resting on the metallic teeth of the wheel $B$, which themselves communicate with 16 , the support of this wheel, and with the knob 17, it is manifest that if the circuit of the second coil is completed by a conductor placed in communication with the knob 17, and with the knob 12, which represent the two extremities of the wire of the second coil, the organ placed in the circuit will receive only the current of interruption, when the wheel is turned from left to right, and only the current of completion when the wheel is turned from right to left. The comnection between the metallic teeth and the wood of the wheels $A$ and $B$ will explain how an organ placed in the circuit will receive at pleasure only the one current or the other.

In fact, the circuit of the second coil being completed, by the contact of the spring 15 with the metal teeth of the wheel $\mathrm{B}$, only 
at the moment when, during the revolution of the wheel from left to right, it breaks the inducing circuit of the primary coil, it is only the current of interruption that can be transmitted to the induced circuit. It is unnecessary to say that the contrary occurs when the wheels A and B are turned in the opposite direction.

In order to show the utility of the rheotome above described, it is sufficient to say that it has enabled me to investigate, physiologically and therapeutically-

1. The influence of centrifugal or centripetal induced currents.

2. The comparative values and the comparative effects of the induced currents of inteription and of completion.

3. The inflnence of centrifugal or centripetal indnced currents, with reversal of the poles at will.

\section{Therapeutic action in the treatment of paralysis.}

It is sometimes desirable to stimulate the nervous centres. In such cases we commonly have recourse to the various medicines which possess a special property of producing muscular contractions by their influence upon the nervous system. Electrization of the nerve-extremities by reflex action operates in an analogous manner. I have therefore applied it to the treatment of certain cases of paralysis in which I was able, without danger, to extend the stimulation to the nervous centres.

I have not always been able to congratulate myself upon the results obtained by this method. 'The paralytics that I have faradized by its means have, it is true, in some cases received benefit; but, consecutively to the treatment, some of them have experienced deep-seated pains in the course of the nerves the extremities of which had been excited, pains that have frequently been persistent, and have assumed the character of neuralgia. The energetic excitation of numerous nerve-branches, specially subservient to sensibility, explains the frequency of these accidents.

Under certain circumstances, faradization by reflex action has exposed the patients to consequences of the most serious character. The following case is an example:-

Case IV.-A medical student, M. X., æt. 22, had heen struck by apoplexy in 1851. The hemiplegia, which affected the left side, and which was due to cerebral hæmorrhage, had gradually disappeared in the course of a year. But the voluntary movements were impeded by the continued contraction of certain muscles, especially the flexors of the fore-arm and of the hand, a contraction which extended to a greater number when he wished to perform certain movements, or when he experienced any impression whatever. Such phenomena are not symptomatic only of a selerosis of the spinal cord, as seems to be established by modern research; but they may indicate a condition of hyperæmia which it is necessary not to over-excite, on pain of producing the most serions accidents. Ignorant of this danger, the student, 
who had imperfectly understood one of my publications, thought it possible to apply withont risk, in all cases, faradization to the treatment of cerebral hemiplegia, in six months or a year after the absorption of the hæmorrhage. The young man had not understood the difference between an electric excitation limited to the muscles, and a method that calls them into contraction by a powerful electrization by reflex action. Te shall see the result of his hope to hasten his recovery.

Having put in action an induction coil, with intermittences (by a trembler) of great rapidity, he took one of the metallic cylinders in each hand, so that the current passed throngh his brachial nerves from end to end. At the moment when it began to pass, his hands closed with great force, and he was unable to relinquish the cylinders. Feeling then that his paralysed limb was contracting very painfully, he had presence of mind to overturn the Bunsen's pile with his foot. The current was stopped instantly, but it was too late. Its action, althongh continned only for a few seconds, had already occasioned serions injury. The head was extromely painful, the contraction extended to the whole side, and the poor fellow, who was alone in his room, was found an hour later in convulsions upon the floor. He was remored to La Charité, mnder M. Piorry; and there I heard from himself the history I have related. His life was for many days in danger; and, notwithstanding repeated depletion, purgatives, and a blister to the nucha, painful and tetanoid contractions persisted for many weeks. At last, after a long sojourn in the liospital, he was able to leave; still suffering from contractions of the right side, much more general and much more severe than before he conceived the unfortunate notion of curing himself by reflex electrization.

If, in this case, the electric excitation had been limited to the paralysed muscles, notwithstanding the peculiar cerebral condition, and the hyperæmia which may exist at the point where a secondary sclerosis of the cord has been developed,-Conditions which, in my judgment, would contra-indicate even localized faradization,--these grave accidents, evidently due to over-stimulation of the centre, would not have taken place. I have learnt thus much by long experience; for I have many times faradized locally the muscles of patients who were in the same state as this young man, and without producing any inconvenience. I have come to the conclusion that localized applications are in such cases simply useless, but not dangerous when made with circumspection. At the commencement of my researches $I$ often faradized by reflex action the antagonists to the contracted muscles of hemiplegic patients, and I sometimes had occasion to repent doing it, so that it is necessary to be cautious in the employment of a method that has proved to be so dangerous.

Electro-dynamic baths, given in a dose so feeble as not to provoke muscular contraction, produce an excitation that increases muscular force in certain forms of general paralysis. I have often employed such baths with success.

The mode of application already referred to I think preferable, on account of its simplicity, to other and more complicated methods. 


\section{§ II.-Excitation by REFLex ACtion IN PASSING INDUCED} CURRENTS FROH THE MOUTH TO THE ANUS.

\section{Physiological action.}

The already describer methods of faradization by reflex action do not strongly excite the spinal cord, excepting at the points of origin of the brachial and lumbo-sacral plexuses. There is yet another procedure which produces a more general reflex excitation of the cord. I refer to the method by placing one rheophore of an induction apparatus in the mouth, and the other in the anus; the already mentioned mode of application of Leroy (d'Etiolles). 'The following is a brief account of the experiments by which I have studied the physiological action that it thus produces:-

Experiment 1.-I have already stated that in 1853, I repeated, upon a horse, the experiment of Leroy with an induced current. The eurrent was feeble; but strong enough for its reflex action to produce general tetanoid contractions in the limbs and trunk, during which the respiratory movements and the action of the heart were not suspended. 2. I repeated analogous experiments upon rabbits; and many were made, in my presence, in the laboratory of my friend MI. Liégois. With an excessively feeble current there was no appreciable result beyond a slight contraction of the tongue and of the muscles of the face. The force of the extra current of my instrument, which had been originally at its minimum, and not only so, but had been passed through a stratum of water in the moderator to be hereafter described, was then progressively increased until it produced tetanoid contractions of the muscles of the trunk and limbs. Neither the respiration nor the heart's pulsation were arrested, but they were suspended during one or two seconds. The experiment, repeated twice upon the same rabbit, and for from twenty to thirty seconds each time, gave always the same results. But, when the strength of the current was so much increased as to tetanize and render immovable the walls of the thorax, a needle thrust into the heart also ceased to oscillate.

We see, therefore, that when the rheophores are placed as described, a feeble induced current of rapid intermission can produce tetanic convulsions of the limbs and trunk, evidently by reflex action of the whole cord, without arresting either the circulation or the breathing.

Another fact not less important follows from these experiments, namely, that this mode of faradization produces a comparatively feeble reflex excitation of the medulla oblongata. Notwithstanding the general tetanic convulsions that are produced, the respiration and the heart's action, although suspended for one or two seconds, have continued during the twenty or thirty seconds during which the experiment lasted ; and it was only to be noticed that these movements were increased in rapidity.

Every experimenter knows how feeble a current, applied to the exposed pneumogastric, will arrest the heart and the respiration; 
and knows also that the most feeble faradization of the superior laryngeal suspends respiration instantly. Should he, therefore, donbt that the medulla has been really excited in the experiments during which a current of the same degree of force has been passed from the mouth to the anus, without producing great disturbance of the respiration?

It is necessary, as we have seen, that the current should attain a very high degree of tension, in order that the respiration shonld be completely stopped, or that the heart should cease to beat.

It is not even shown that, under these circumstances, the arrest of the respiration and of the heart is due to excitation of the medulla oblongata. Indeed, the tetanic contraction was not at its maximum, so that the inspiratory muscles, principally the diaphragm and the intercostals, which were only in a certain degree of rigidity, were able, under the influence of a great inspiratory effort, to contract yet more, and to produce morement. In the second experiment it is manifest that the maximum tetanic state of the same inspiratory muscles would be necessarily opposed to any respiratory movement of the thorax; by which may be explained the rapid death which befel the animals thus treated. The arrest, observed in the last experiments, of the oscillations of a needle thrust into the heart, does not prove that that organ had then ceased to beat; because the oscillations may have been stopped by the extreme rigidity of the intercostal muscles traversed by the needle, while in the former experiments these museles were not sufficiently rigid to stop them. The correctness of this explanation is shown by the fact that, when the muscles relaxed on the suspension of the faradization, although the rabbit had ceised to live, small oscillations of the needle again gave evidence of contraction of the heart.

'The facts and considerations that I have advanced show, therefore, that the mode of general faradization under discussion does not powerfully excite the medulla oblongata, and this is all that I endeavour to establish.

I do not maintain that the procedure exerts no influence upon the medulla oblongata. I do not think, indeed, that in these experiments, when one of the rheophores is placed in a region (the mouth) deriving its nerves from the medulla oblongata, and from a part of the pons Varolii, that these points of the nervous centres can possibly altogether escape excitation. Certain facts show, indeed, that there is a real reflex action of the medulla.

Thus, when the faradization has been moderate, we have seen that the cardiac and respiratory movements have been increased in frequency. Lastly, we may see that, in asphyxia, this mode of 
faradization of the cord by reflex action has power to re-excite respiration that has been suspended for some minutes; and that it will also re-establish and develop the contractions of the heart, when they are only appreciable by the feeble and very slow oscillations of a long needle thrust into the organ, although the current employed has been very feeble, and insufficient to produce reflex contractions in the limbs.

\section{Therapeutic action.}

Faradization by reflex action of the spinal cord, and of the merlulla oblongata is indicated in asphyxia; and experimentalists have thought that the best method of using it for this condition is by placing one of the rheophores in the mouth, and the other in the anus.

A.-History. This method of general electrization by reflex action was, as I have already stated, advised in 1825 by Leroy (d'Etiolles), who used the galvanic current in cases of asphyxia. It was again brought into notice, for the same purpose, by MII. Legros and Onimus, with the difference that they employed a battery of constant current, while Leroy had only the inconstant battery of Wollaston.

B.-My own first experiments. In 1854 , I made my first experiments upon the horse, with regard to the therapeutic action of Leroy's method, modified by the substitution of the induced for the galvanic eurrent.

An analysis of the physiological effects of this kind of faradization soon assured me, that it was the most certain method to excite the general reflex action of the cord; that the reflex contractions produced were very energetic; that with rapid intermissions they became tetaniform; and that with a very intense current, they might rapidly produce asphyxia by contraction of the muscles of the trunk, so that the method could not be used in any strong dose without danger.

But, as I found that in weak doses it reither arrested the respiration no: the circulation, although it still produced reflex contractions of the limbs, I dis not hesitate to apply it immediately to the human subject. It will be remembered that I experimented upon the hysterical patient whose case has been already mentioned. I repeated, under analogons circumstances, the same experiment upun others; and I proceed to describe what were the principal phenomena that $I$ observed.

Having commenced with a current of slight intensity, or too weak to produce reflex contractions in the limbs, the patients complained of no sensatious, but such as were occasioned by a 
slight contraction of the tongue, of the sphincter and levator ani, and by phosphenes; while the pulse and the respiration were a little increased in frequency. The procedure was perfectly well borne, as the current was not sufficiently intense to produce reflex contractions in the limbs. But, having in this way obtained no result, as against the maladies that were to be overcome, I gradually increased the intensity until reflex contractions were obtained; taking care, in order to avoid all danger, to diminish the speed of the intermissions to one or two in the second. The patients then experienced painful shocks, chiefly in the legs,pains which were followed, after the passage of the current, by a feeling of general lameness. In one patient, who suffered from complete anæsthesia and analgesia, I was able to produce, in consequence, during fifteen or twenty seconds, and with a weak current of very rapid intermission, general reflex tetanic contractions. The heart did not cease to beat, nor the respirations to be made; but the patient remained for some time dazzled by the phosphenes that were produced during the passage of the current.

From these experiments I concluded--1, that the method of faradization by reflex spinal action might be applied in practice to the human subject without any danger, and that it is perfectly borue so long as the current is not sufficiently intense to produce contraction of the limbs; 2, that it does not, indeed, produce arrest of the respiration, even when it produces tetaniform reflex contractions, unless these are too strong; but that it is difficult to say at what point the limits of safety would be exceeded; and that, 3 , in certain dynamic or morbid conditions, the slightest tetaniform coutractions might expose the patient to danger. For such reasons, founded upon experiments dating from 1854, I have been very circumspect in the use of general faradization, and have discountenanced it when practised in a degree of intensity to produce reflex contractions, or with very rapid intermissions.

C.-More recent experiments on the application of this method to the treatment of asphyxia, as compared with induced, and with constant continuous currents. General faradization by this method may succeed in the cure of asphyxia, without there being oceasion to increase the intensity of the current so as to produce reflex contraction.

This important fact was brought to ligh by M. Liégeois, in a very able report read by him before the Imperial Surgical Society on the 17th 1Larch, 1869, in the name of a commission composed of MI. Paul Broca and himself, upon a paper addressed to the Society by MLI. Legros and Onimus, on the 10th of December, 
1868 , on the use of continuous electric currents in cases of syncope, and in accidents caused by chloroform.

The authors (of the original paper) had said, "We will not pass in review the other means (other than the continuous current), that have been employed in the treatment of chloroform accidents. All, perhaps, with the exception of artificial respiration, have been useless; and we assert that the intervupted or induction currents, which have been extolled, cannot be condemned too strongly. The best means of completely arresting the action of the heart, when reakened by an anrsthetic, is to pass over it an induced current. We ought, therefore, to dread the use of an induction apparatns, which will determine the very result that it is desired to avoir."

After having describer the experiments conducted by MIM. Legros and Onimus in the presence of the commission, the reporter (II. Liégeois) has arrived at the conchn:ion that, notwithstanding the interest attaching to these experiments, the commission is bound to observe, that they do not exactly correspond to the title of the paper; and that they are only strictly applicable, in man, to asphyxia from chloroform, - an accident which, in his view, is less frequently fatal, and is more amenable to treatment, than chloroform syncope. Limited in its application to chloroform asphyxia, galvanization, as practised by MII. Legros and Onimus, is certainly of incontestable efficacy. But MI. Liégeois has also reported another experiment tried before the commission, which demonstrates, that the induced current, applied in an equivalent dose and by the same method, that of Leroy (d'Etiolles), restores from chloroform asphyxia as certainly as the continuous current. "Upon our request," he writes, "to MMI. Legros and Onimus, that they would test the influence of an induced current in our presence, a rat was chloroformed under the same bell-glass and in the same manner as the former ones. It was withdrawn when the respiration, the sensibility, and the general movements had completely ceased, although the action of the heart still continued. They then passed the current of a Dubois.Raymond apparatus from the mouth to the anus; but in order to approach as nearly as possible, with our apparatus, to the slight intensity of the continuous current, we requested the operator to employ the most feeble that the instrument would yield. The current was allowed to pass for a few seconds only, and, almost immediately on its interuption, the respiratory movements returned, the beats of the heart increased, then the sensibility and the voluntary movements were gradually restored : the animal was saved."

II. Liégeois has repeated this experiment upon a large number of rabbits. Mr. Duchemne, jun., and myself have repeated it both 
in the laboratory of Mr. Liégeois and in my own, with somewhat more precision, by making the induced current pass through the water moderator, to be described hereafter. We have also experimented comparatively, with the ascending and descending continuous current, in asphyxia by strangulation and in asphyxia from chloroform.

The therapeutical results, in all these experiments, have been precisely the same; and they fully justify the following conclusions in the report of ML. Liégeois:-

"After the experiments that have been related," he says, "it will be understood that it is impossible for us to assign to continuous currents, for asphyxia produced either by chloroform or by strangulation, a superiority of action over intermittent currents. In placing them on the same level, we believe that we shall state the truth."

In their practical aspect, the conclusions of MI. Liégeois are not less important. "If," he says, "we leave the laboratory for the region of practice, we cannot refrain from assigning to infuced currents the pre-eminence. In fact, in the first place, it is difficult to have the command of an apparatus like that of Remak (specially recommended by MII. Legros and Onimus for the application of continuous currents in asphyxia), composed of twenty, thirty, or forty of Daniell's elements, while it is very easy to obtain an induction instrument that is simple of management, of small bulk, and that may be carried by the surgeon wherever he goes."

"Moreover," continues MI. Liégeois, "by the confession even of MIM. Legros and Onimus, the continuons currents only restore the heart's action when applied to the cerebro-spinal axis. It is in vain, they said at our meeting, to apply them to the heart directly, to the diaphragm, or to the phrenic nerves; when thus employed, they never restore animals to life. But, in man, the efficacy of induced currents applied to these varions organs has already received confirmation from experience."

Upon the last point again, I am entirely in accorl with $\mathrm{M}$. Liégeois ; and, whenever I have been called to the aid of a person asphyxiated, or menaced by arrest of the heart's action or of the respiration by some grave disorder of innervation, I have always chosen to arm myself with an induction apparatus, not only because it is more portable, but also because, under such circumstances, it is capable of fulfilling many important indications which cannot be fulfilled by galvanization with continuous currents; principally in the maintenance of artificial respiration by faradization of the phrenic nerre (very preferable, in certain 
cases, as I shall shew hereafter, to either faradization, or galvanization by the method of Leroy (d'Etiolles); and in the application of cutaneous faradization as a stimulant of the respiration and of the cardiac functions by reflex action, in the treatment of asphyxia or of syncope. I shall have to treat hereafter of this last method of reflex stimulation of the cord.

It is not well, however, to be exclusive; and in cirenmstances where danger is urgent and has been unforeseen, the practitioner who is hastily called to a case of asphyxia or of syncope should know how to avail himself of any means that comes to his hand, of any battery or induction apparatus whatever. Let us see, then, how the surgeon should in such cases act; and how I have many times been called upon to act myself.

I. If we have command of an induction apparatus, whether graduated or not, and of whatever power, its current may be diminished and controlled in the following manner:-The water moderator, which I habitually place in the circuit when I wish to reduce the tension to a degree infinitely small, may be replaced by a wetted band of any kind of linen or cotton cloth, made from pocket-handkerchiefs, sheets, \&e., and from one to several yards in length, according to the power of the apparatus. This band should be laid out on a table, and one of its extremities shonld be connecterl with one of the electrodes of the instrument, the other with the conductor to the rheophore that is put into the month of the patient; the second rheophore, which is introduced into the anus, communicating by its conductor with the other electrode. Under such conditions, the tension of the current will be reduced to extreme weakness. Then, in order to obtain the necessary power, the length of the moist band must be gradually diminished by placing the conductor of the rheophore nearer and nearer to the electrode, until, using intermissions about one second apart, the commencement of reflex muscular contraction of the limbs is observed. It is necessary to operate with a degree of tension just below this, too weak to produce muscular contraction, and by passing from the anus to the mouth a current of rapid intermissions, until the respiration and the heart's action are re-established.

In case the surgeon has at his disposal a constant battery, combining all the desirable conditions (such, for example, as the large battery of Siemens, or the new small portable battery of Gaiffe, already described, but which are only to be found in the hands of a limited number of persons), a continuous current should be passed between the mouth and the rectum, without heed to its direction, which, according to my own experiments, has no influence upon the therapeutic result. 
If, in the place to which one may be called to an asphyxiated person, the only battery to be found is composed of too small a number of elements for the useful application of a constant current, such as the battery of a telegraph station, or if there should be only a battery of inconstant current, such as that of Wollaston, recommended by Leroy (d'Etiolles), it is necessary to pass a current of rapid intermissions between the month and the anus, without going so far as to produce reflex contraction. This may be done with as much prospect of success as will attend the use of the continuous current of a constant battery, composed of a large number of elements. ${ }^{4}$

§ III.-Excitation OF the inNervation of the HEART AND RESPIRATORY ORGANS BY MEANS OF CUTANEOUS FARADIZATION OF THE PRECORDIAL REGION.

A long clinical experience has taught me that excitation of the sensibility of the skin of the thorax, and especially of the precordial region, is one of the best means of treating asphyxia, or certain grave disorders of the innervation of the heart or respiratory orgaus, by reflex action. The correctness of this assertion will be shown by the relation of the most important clinical facts that I have collected.

\section{I.-Treatment of Asphyxia.}

Artificial respiration by means of faradization of the phrenic nerves is the mode of electric excitation that I generally prefer in the treatment of asphyxia; but in hands that are unskilful, or little accustomed to the operation, it is not free from danger to the patient. Under such circumstances I advise, as the more simple method, cutaneous faradization of the precordial region,

4 I cannot leare this part of the subject without recalling another method of galvanization suggested, in 1824 , by Leroy (d'Etiolles), lauded by him in the treatment of asphysia, and long employed in practice:-

"When the battery," he says, "is ready, the operator takes two fine pins prepared for the purpose, only six or seren millimetres in length, and terminated by discs which are surmounted by hooks or rings. These pins are intended to convey the galranic fluid beneath the skin, across the fibres of the chief respiratory muscle. 'The pins are thrust into the sicles of the patient up to their dises, between the seventh and eighth rib. One of the conductors is then attached to nne of the pins; and the operator, holding the other conductor, tonches with it, every two seconds, the head of the second pin on the opposite side of the chest. If no proper pins are at hand, a puncture may be made, which should not exceed the thickness of the skin, with a lancet, a penknife, or the points of scissors, and through this puncture the galvanic fluid will make its way. The punctures should be made in the situation already mentioned, between the seventh and eighth rib, on opposite sides of the chest, in the course of a line proceeding from the pit of the stomach to make the circuit of the body. 'The ends of the conducting wires shonld be made to touch the rounds, one of them in an intermittent mamner, as described for the pins." - Instructions sur laspluyxie (in Journal de la Société des Naufrages, t. iv. 1810). 
conducted according to the principles that I have laid down in treating of this method of faradization.

There are also circumstances in which electro-cutaneous excitation ought to be employed concurrently with faradization of the diaphragm. The latter is especially indicated in cases where it is urgent to compel the mechanical entrance of air into the lungs; the former is useful, or even necessary, when, after we have reestablished the respiration and the cardiac circulation, and have dissipated the cyanosis, the patient threatens to fall into a state of coma, symptomatic of cerebral ischæmia, dne to the poisoning of the blood either by carbonic oxide, or by the accumulated carbonic acid if the state of partial asphyxia has been of long duration. Then electro-cutaneous excitation, especially if applied to a certain zone of the precordial region, reacts upon those points of the nervous centres which govern the innervation of the breathing and of the heart's action.

The therapeutic value of this method, in the treatment of this kind of asphyxia, is placed beyond doubt by the following history :-

CASE V.-Asphyxia by carbonic oxide; absence of respiration; heart's action very feeble, and only discoveruble by auscultution; cure by cutineous furuli:ation of the precordial region.

A pastrycook's boy, fifteen years old, of ecentric character, had endeavoured to drown some imaginary troubles in drink; and hal the notion, in his intoxieation, to try to asphyxiate himself by creeping, during the night, into a sort of hole situated over his master's oven, where he remained to sleep himself sober. The next morming, the men who went to light the fire saw one of his feet, and drew him, seemingly lifeless, from the place where he had passed the night. Living in the same house, I was able to try, a few minutes later, to recall him to life by faradization. His face was blue, there was no respiration, a mirror held before his lijss remained untarnished, no julse could be felt, the action of the heart was not discoverable by the hand, but on auseultation, feeble valvular sounds were heard. While I made this examination my induction apparatus had been got ready by my son, who assisted me. I was abont to excite artificial respiration through the phrenic nerves, when I saw that the rheophores had been forgotten. The danger being very urgent, I instantly applied the metallic extremity of one of the conductors of the induced eurrent of my instrument (at its maximum, and with rapid intermissions) over the left nipple, and moved the other conductor about over tho apex of the heart. After a few seconds, slow and weak respiratory movements appeared, and increased progressively in frequeney and depth; and, at the end of a minute, the pulse conld be felt. Soon afterwards, the face assumed a slight expression of pain. In a few minutes the patient uttered a faint groan, and moved first the arms and then the trumk, as if to remove the conductor's from his ehest. Then he gave a lond ery, kieking with his feet at the people surrounding him, and began to reply to the questions that were asked. In five or six minutes the respiration and circulation were re-established, the colour of the face was natural, and the intelligence had returned.

He appeared to be saved. However, I ordered simapisms to be applied to the limbs and the precordial region, and warned his master that he might fall back into a state of coma, from the tuxic influence of the earbonic oxide. In fact, a few minutes after the suspension of the entaneous faradization, he 
no longer replied to questions; his respiration slackened, at times he seemed as if he forgot to breathe; his face beeame discoloured and his lips blanched; then the breathing beeame stertorous; he could not feel when pinched; in a word, he was asphyxiated afresh. He was quickly restored by eutaneous faradization of the precordial region, applied this time less severely, as it was quickly and aeutely felt. It was continued by my son for about twelve minutes, until the respiration, circulation, and intellectual faculties, were all completely restored, and he conld stand erect and walk.

Believing this cure to be only apparent, I ordered his removal to the Hotel Dieu, to the wards of Professor Trousseau, where I shortly followed him. Foreseeing that the carbonic oxide would continue its poisonous action, and produce a return of coma, I had plaeed sinapisms mpon his legs, and had directed the person who conducted him not to let him sleep. An hour later I found lim in the hospital, in a state of coma, into which he had fallen on the way, in spite of the efforts of his guide, who had in vain slapped him to try to keep him awake. It was necessary to return to the eleetro-cutaneous excitation of the precordial region, and to repeat it several times during the day, as well as to apply sinapisms orer the body, before we were entirely freed, towards the evening, from the tendency to consecutive coma, due to the poisonons influence of the carbonic oxide.

In this case we have seen a lad who was asphyxiated by carbonic oxide, in whom the respiration had ceased (it was not known for how long a time), and in whom the feeble action of the heart was only discoverable by the ear. 1. He recovered entirely, in a few minutes, under the influence of precordial electro-cutaneous excitation, his respiratory and cardiac functions. 2. He fell back many times into the same state of coma and asphyxia, shortly after the suspension of faradization, which, perseveringly re-applied on several occasions, during nearly a whole day, finally triumphed over the asphyxia.

It was incontestably and solely to the faradization that the rapid disappearance of the grave symptoms was in this case to be attributed. The proof of this assertion rests so plainly in the relation of the facts, that it seems useless to dwell farther upon the point.

I proceed to describe briefly another case of aspyhxia by carbonic oxide, which brings into prominence the therapeutic value of reflex excitation of the nervous centres, by cutaneons faradization; and at the same time sliews the necessity of continuing the application for a long time, until respiration is perfectly reestablished, under penalty of seeing the asphyxia return and lead to a fatal issue.

C'use VI.-I was called to a woman asphyxiated by carbonic oxide, in La Charité under the care of M. Andral, where she had been conveyed on the previous evening. In a short time I restored the respiration and the heart's aetion to their normal state, and had dispelled the coma. The patient had so far recovered her intelligence that she was able to give me information about what had happened to her, and about all the symptoms of her asphyxia. I believed her to be safe. But some hours later she relapsed into somnolence, and, although treated by sinapisms and even by blisters, she died during the course of tlie night. 
If, in this case, the faradization had been continued, as in the subject of Case V., the patient would probably have been saved. From this example, confirmed by what I have seen in others, I have come to the conclusion that patients asphyxiated by carbonic oxide, and in whom electrization has removed all traces of asphyxia, are still threatened, for a certain time, with a relapse into coma and asphyxia; and that it is consequently necessary to continue the peripheral excitation for a considerable time after the patients have been recalled to life, and to apply cutaneous furadization to the precordial region, as soon as any tendency to sleep or to coma becomes apparent. It was to the observation of these precepts that the young subject of Case V. owed his life.

It is important to add that the danger which threatens these patients is a result of the blood poisoning by carbonic acid produced by the asphyxia itself; and that it is greater in proportion to the length of time that the asphyxia has continued. In support of this proposition, I could cite many examples from my practice; but shall only relate one, and that concisely :-

Cuse VII.-I was called, some years ago, by MIM. Troussean and Chailly, to a case of asphyxia in a new-born infant. At birth it gave no sign of lifo (the labour had been long and diffieult), and it had been restored immediately l,y artificial respiration, made from mouth to month by Chailly; but, however prolonged and renewed this process, and in spite of stimulating frictions, it remained in a state of semi-asphyxia from two o'clock till eight in the morning. At the time of my arrival it had not breathed for about a minnte. I moved the metallic rleophores of my induction apparatus over the precordial region, and in five or six seconds witnessed respiratory movements, feeble at first, but becoming more and more developed, and leading, for the first time, to the ery of the new-born, until, under the influence of deep inspiration, the lividity of asphvxia had disappeared. We thonght all was safe. Nothing could express the astonishment and delight of the family at the result. The infant was the first grandson of one of the kings of finance. But ten minutes later it relapsed into coma, the breathing became more and more slow, the lips were once more livid, and, in a word, the asphyxia re-appeared. I suceeded again by the former process of faradization, to which I added artificial respiration by faradization of the phrenie nerves, practised for five or six seconds. Ten minutes later there was another relapse, followed by a renewed success from the eutaneous faradization, which this time aeted nore slowly. In another quarter of an hour the asphyxia gained the upper hand, and the infant died.

\section{II.-Treatment of gave functional disorders of the cardiac circula-} tion and the respiration, symptomatic of a dynamic central nervous les̈ion.

Excitation of the medulla by reflex action, by means of cutaneous faradization of certain zones of the thorax, will modify or cure:-1. grave disorders of the cardiac circulation, symptomatic of a paralytic condition of the vagus, producing syncope that is sometimes fatal, by stoppage of the lieart; 2 . disorders of the 
respiration, such as paresis or paralysis of the expiratory or bronchial muscles of Reissessen (paralysis of the lung); 3 . simple or comatose apnoea, producing consecutively an accumulation of bronchial mucus and asplıyxia.

A.-The following clinical observation is the proof of the two first assertions :-

CASE VIII.-Grave disorder of the cardiac innervation and of expiration, in consequence of diphtheritic poisoning.

Summarx.-Dhembranous angina attacking a large surface, in a lady twentyone yeur's old; diphtheritic poisoning producing, on the sixteenth duy, paralysis of the pharynx and the soft palate, and touarls the twenty-seventle day, after a miscurniage at three months and a hulf, other paralytic phenomena symptomatic of a lesion of the medulia; namely,-1. (irave disorder of the cardiac circulation, due to paralysis of the vagus, and removed ly slight cutuneous furatization of the precordial region. 2. Two days later, temporary diplopiu, lasting an hour, and immediately followed by hemiplegia of the left side; uhich was cured in an hour by cutaneous faradization of the paralysed parts. 3. Two or three hour's later, a return of grare functional clisorder of the circulution; cured again, after turo days of cutaneous furadization of the precordial reyion, practised night and duy at short intervals. 4. Grave functional disturbance of respiration, symptomatic of paralysis of the intrinsic expriratory muscles (bronchinl muscles of lieissessen) or, in other words, parulysis of the luny; removel immediately (for the space of half an hour or an hour), and on different occasions, by cutancous furatization of the dorsal aspect of the chest. 5. Lastly, formation of bronchial mucus from reuliness or paralysis of expiration, and death on the thinty-eighth duy, at tuo o'clock in the morning.

Nadame X., 21 vears of age, and three months advanced in pregnancy, was attacked, on the 22 nd February, 1869, with a membramous angina, following a cold. It seems superfluous to describe the symptoms of the angina, which, under the care of MIM. Barth, Ricord, H. Roger, and Descroizilles, charged specially to remain day and night near the patient, followed a regular course up to the time of convalescence; when, on the 10th of March, there appeared paralysis of the pharynx and of the soft palate. It followed that deglutition was rendered difficult, and that the patient conld take only liquids, somps, and slops. Nevertheless, Madame X. began to regain strength, and it was hoped that her convalescence was establisled, when, in the moruing of the 21st of March, severe uterine pains announced the approach of a miscarriage. Although this took place regularly, and produced no hrmorrhage, it was followed by derangements of the respiration and circulation, of extreme severity. IIM. Barth, Ricord, H. Roger, Campbell, and Descroizilles, who had passed the night in attendance, and who had successfully encomntered, by the aid of diffusible stimulants, a state of syncope that had several times placed her life in danger, were still unable to overcome very grave disorder's of the circulation and the breathing.

On the following morning, the 22 nd of March, my confrères wished to call in the aid of electricity. They asked me-1. Whether in such a case an electrical treatment afforded any hope of success; 2. By what mode of proceeding it would be possible to direct the therapentic action upon the fumctions or the parts of the nervous centres that were acted upon by the diphtheritic poison; and, 3. How, by faradization or galvanization, we might resist the syncope or the asphyxia that was imminent.

Before replying to these questions, I examined the patient; and the following were the chief conclitions that I observed:- Face very pale; lips discoloured; the nose and extremities cool; precordial weight and semse of suffocation; respiration a little panting, altliongh the rythm of the movements was normal, and there was no paralysis of the diaphragm, no empliysema, and no ráles; the pulse small and very frequent (136 to 140), irregular, 
and so intermittent that six or eight successive pulsations were often wanting; by anscultation of the heart it was impossible to recognize the rythm of the movements, and I heard only some slight dull sounds, unequal and much disordered. There was no elevation of temperature, but a paralysis of the pharymx and the soft palate, dating from a time much anterior to the appearance of the symptoms for which I had been summoned.

I recognized, in the whole of these cardiac and respiratory disorders, the characteristic phenomena of paralysis of the pnemmogastric nerves; and I conceived that, in this patient, the diphtheritic poison exercised a morbid influence over that part of the nervous centre-the fourth ventricle-that governs the immervation of the heart.

According to this hypothesis, what should be the therapeutic action of faradization? Excitation of the phrenic nerve, in a case where the diaphragm was not paralysed, and where there was no sign of asphyxia, did not seem to me likely to exert a beneficial influence upon the disturbance of the cardiac circulation, and upon the feeling of suffocation. Faradization of the pnenmogastric and superior laryngeal, at the point where these nerves are in relation with the sides of the pharynx, might, indeed, modify favomably the innervation of the heart and lungs; but the proceeding, although very easy of performance, is extremely dangerons; and, even in small doses, may arrest the heart's action and the breathing. It was not even to be thonght of.

As, however, it was needful to act, I determined to endeavour to modify the morbicl state of the centre, which gives origin to the nerves of the heart and lungs, by the electro-cutaneous excitation of a region in relation with it.

Was it not possible, however, that the diphtheritic poison, in modifying the dynamic state of the patient, had rendered her unusually sensitive to electric excitation? Should I not expose myself, by acting a little too powerfully, to a risk of stopping the heart?

After having well weighed the value of these and other considerations, I performed cutaneous faradization, by means of the electric hand (see page 90 ), over the precordial region, principally at the region of the apex of the heart, being careful to commence with an extremely feeble dose. I then gradually increased the intensity of the indnced current, the intermissions of which were rapid, until it prodnced slight tingling of the skin. We then had the satisfaction of secing my anticipations realized; for, after a few minntes, and during the passage of the current, the pulse diminished in rapidity, and became more full and regular; and after a while the precordial weight and sense of suffocation disappeared. It was necessary, lowever, to return to the cutaneous faradization several times during the day, in order to dispel definitively the diplitheritic perturbation of the innervation of the heart, which liad shown a tendency to recur.

In order to omit nothing, I should mention that, together with the precordial faradization, we employed and continued, during the whole course of the treatment, the diffusible stimulants internally, and the peripheral stimulants externally, which alone, had failed to improve the immervation of the heart; that oxygen was frequently inspired; and that tonies and such alimentation were given as the paralysis of the soft palate and the dysphagia wonld allow.

On the following days, no disorder of the circulation or respiration reappeared; and our patient suffered only from the palatal and laryngeal paralysis, against which I conld do nothing under such serions conditions. TVe thought life no longer in danger, when, on the 29th of March, abont four o'clock, the ctiphtheritic poisoning announced its returu by a diplopia that lasted an hour. Then, about five o'clock, Madame X. was suddenly struck by complete hemiplegia of the left sicle, with loss both of sensation and of motion, but with no impairment of the intelligence, of the leart's action, or of the respiration.

Considering this paralysis to be an evidence of a new invasion, by the diphtheritic poison, of a portion of the right half of the medulla oblongata 
(probably of the anterior pyramids or their prolongations), I did not hesitate to encounter it by the same means that had succeeded against the paralysis of the nerves of the heart and lungs; by the electro-cutaneous excitation practised by the hand over all the surface attacked by paralysis of motion and of sensation. On this occasion, also, we were not deceived in our hope; for, after eight or ten minutes of this slight peripheral excitation, the sensibility had in great part returned, and voluntary movement coon followed, although muscular furadization had not been practised. In four hours the hemiplegia had completely disappeared.

The hemiplegia, alas! was soon replaced by new indications of pneumogastric paralysis, more serious than those which had preceded them; for smspension of the heart's action returned more often and continued longer. I soon succeeded once more in removing this symptom, by slight faradization of the cutaneous precordial zone which, according to the theripentical results previonsly obtained in the case, seemed to be in relation with the origin of the pnermogastric. Unfortmately, this renewed snccess lasted only for about a quarter of an hour From this time we were compelled to maintain, day and night, an incessant strife agamst the tendency to stoppage of the heart. Every hour, or half hour indeed, the poor lacly was attacked by the same cardiac tronbles, which became more and more painful, and drew groans from her. On these occasions the pulse, which became small and irrognlar, improved again and again under the influence of electrocutaneons excitation of the precordial region; and the paticnt was able to compose herself to the sleep of which she had such need.

After two days of this struggle against the diphtheritic poison, the electrocutaneous excitation scemed to have tritumphed anew. The symptoms of pneumogastric paralysis had disappeared, and the rapid pulse had returned to its normal state and so remained.

But, unfortunately, this improvement was but a brief respite; for nervons disorders of a new kind shortly appeared, and this time affected only the respiration, which became sighing and more rapid. Ten or twelve normal respiratory acts were followed by about the same number of abnormal movements, which were more rapid in their character, and were each composcd of a full and long inspiration, followed by a short and fecble expiration. Nadame $X$. was soon awakened by a most painful orthopncea; she was suffocated, she said to me, as if between two mattresses. There was no blueness of the lips, becanse the air that freely entered the bronchi was sufficient for hæmatosis; althongh the air that there was no power to remove from the smaller ramifications, had become irrespirable, and this occasioned the dysinoea.

The electric hand moved over the precordial region afforded no relief. It was only by exciting the skin of the posterior region of the chest, that I was able, after the lapse of eight or ten ninntes, to render the breathing regular.

The paralysis of expiratory power returned after the lapse of an homr; and shewed itself, as on its first appearance, by sighing respiration, soon followed by most painful orthopncen, producing groans. The horizontal position, which she had long maintained for fear of syncope, became insupportable. She found no comfort but by sitting up in her bed, with the body bent forward; an attitude that she maintained until I had restored the breathing to its natural state. This paralysis of the bronchial muscles of Reissessen (the intrinsic expiratory) filled me from the first with great disquietude, althongh the hæmatosis had not appeared to suffer, judging from the colour of the lips and the face. But I could not fail to see the presence of a new danger, fully as great as those from which the patient had escaped. Indeed, from the weakness of her inspiratory movements, she had great difficnlty in expelling the abundant mucosity that had already formed in the larynx. She conld accomplish the expulsion only by very violent efforts, which left her completely exhausted. There was only too much reason to fear that, if this condition shonld be prolonged, the accumulation of mucosity in the bronchi would become a cause of asphyxia. 
Such unfortunately was the case, although I had succeeded for the moment in relieving the orthopnœa by slight electro-cutaneons excitation of the dorsal surface of the trunk, and had thus obtained some hours of repose. In the struggle in which I had been engaged against this paralysis of the lung, I had retained, up to the last hour, the hope that by prolonging the life of the patient we should at last exhanst the deadly influence of the diphtheritic poison, which had already been driven from other points of the spinal cord. Bnt we were at last compelled to recognize the near approach of deatl, when bronchial râles and a slight lividity indicated the commencement of asphyxia. From this time we had only to watch the progress of a long and painful struggle. Death took place on the 2nd of April, about two o'clock in the morning.

Remarks. - The case is interesting from many points of view; ${ }^{5}$ but, not to wander from my subject, it is necessary that I should bring into relief the influence that was exerted by cutaneous faradization over the grave disorders of the cardiac circulation, and over the paralysis of the intrinsic expiratory muscles of Reissessen;-in other words, over the paralysis of the lung, which, at the last, produced asphyxia and death.

The results obtained, under these circumstances, by cutaneons precordial faradization, so far surpassed my anticipations that I should doubt them even now, had they not been witnessed also by the learned physicians who called me to the case.

a. Let us examine these results in their physiological aspect.

The grave functional disorders of the cardiac circulation which, in the case of Madame X., I had at first to combat; that is, the extreme rapidity, smallness, and irregularity of the pulse, with syncope or the danger of syncope from stoppage of the heart, the

5 I lare suppressed, in the abore history, many interesting details that were not necessary to the demonstration of the electro-therapeutic questions that are under discussion. 'There is, howerer, one circumstance that deserves mention, on account of its importance, and that is, the absence of eleration of temperature during the production of norvous disorders of extreme malignity. I ascertained this by the thermometer, in conjunction with M. Descroizilles, who, during all the weeks of the illness, was scarcely ever absent from the patient. If, then, the severity of the diphtheritic intoxication could not be explained by excessive elevation of temperature, it is probable that the poisonous action may be explained by some change in the blood, of the nature of which we are ignorant. Such a hypothesis is supported by the circumstance that there was an intestinal hremor'hage with severe colicky pains, and severit successive evacuations, on the day following the first appearance of nervous dis- order. This was not mentioned in the history of the case.

False membranes having been found mingled in large quantity with the bloody stools, we all thought that the hæmorrhage probably proceeded from those parts of the intestinal surface upon which tho false membinnes harl been developel. These false membranes having been expelled two days after the abortion, we were for an instant led to suppose that they might be nterine débris, that had passed from the ragina at the moment of the alvine evacuation; but a microscopic examination shewed them to possess all the anatomical characters of false membrane. Their development over so large a surface led us to fear that the legree of poisoning might be in proportion to the extent of this surface, and male us better understand the full grarity of the case. Moreover, the presence of albumen, for some days, in the urine (another fact omitted from report), arleded still more to the peril in which Madame $\mathrm{X}$. was placed. 
precordial weight, and the suffocation, were in my judgment symptomatic of the paralysing influence of diphtheritic poisoning upon the origin (ganglion) of the vagus nerve. Such, indeed, are the symptoms which display themselves in an animal in which the pneumogastrics are divided; excepting, of course, the sensations of precordial weight and of suffocation, of which the animal could give no account. I have also observer the same symptoms in the last stage of disease (glosso-labio-laryngeal paralysis); that is, at the period when the anatomical lesion, which appeared to be an ascending sclerosis of the cord, with wasting of certain cells, had reached the ganglion of the vagus.

I observed that, whenever these functional disorders of the cardiac circulation appeared in Madame $\mathrm{X}$., they were removed, as if by enchantment, by cutaneons faradization of the precordial region; and if I departer from that region, moving my manual rheophore to other parts of the elnest, the patient experienced no benefit, and quickly pointed with her own hand to the precordial region, to indicate the point to which the electricity should be applied.

Since, in this kind of therapentic experimentation, the excitation of the sensibility of the skin, practised in the precordial region, was alone able to modify, and even to remove, by its reflex action, grave disorders of the cardiac circulation prodnced by a morbid state of the innervation of the pnenmogastric, it is reasonable to conclucle that there exists an intimate relation between the cutaneons sensibility of the precordial zone and the origin of the pneumogastric; or, in other words, that the former is in reflex communication with the latter.

Another therapentic result, observed during the progress of the case, confirmed the belief that a special reflex action is exerted upon the pnenmogastric ganglia through the cutaneous nerves of the precordial region; and this was the fact, that certain disorders of the respiration could only be overcome by cutaneous faradization of another thoracic region. It was found that faradization of the precordial region was powerless against the weakened expiration (paralysis of the lung, or of the bronchial muscles) which followed the pneumogastric paralysis; while faradization of the back of the chest relieved this weakened expiration on several occasions, so as to diminish or dispel, for a certain time, the sufferings of the patient, to give her some repose, and to delay, for several days, the asphyxia which at last terminated the scenc. We may therefore conclude also that the sensibility of the cutaneous zone of that thoracic region which corresponds to the posterior aspect of the lungs, is in special relation with the 
point of origin of the nervous fibres which supply the intrinsic expiratory muscles, or bronchial muscles of Reissessen.

$b$. Erery one must perceive the practical bearings of the foregoing physiological considerations; which lead directly to the following conclusions. 1. Electro-cutaneous excitation of the precordial region around the apex of the heart is one of the best means by which to encounter syncope from arrest of the heart's action, or other functional disorder of the cardiac circulation, symptomatic of a paralytic condition of the vagus, without organic lesion. 2. Electro-cutaneous excitation of the posterior region of the chest exerts a special therapentic action, in the treatment of paralysis of the intrinsic expiratory muscles.

'T'he following, in a few words, is the physiological explanation of the facts above stated. In the case of Madame X., the slight electro-cutaneous excitation of the precordial region, acting in a reflex manner upon the ganglion of the pneumogastric, restored to the nerve the power, of which its paralytic state had deprived it, to control the action of the heart. It had, in other phrase, put the heart once more under a curb, and hence followed regulation of the cardiac circulation, and disappearance of the grave nervous disorders that were symptomatic of pneumogastric paralysis. A similar theory is applicable to the therapeutic influence exerted by electro-cutaneons excitation of the back of the chest over paralysis of the power of expiration.

c. The case affords also valuable instruction, with regard to the choice of a method of electrization, and with regard to the manner of its application under similar circumstances.

When it was decided, in consultation with my colleagues, that reflex faradization of the medulla oblongata was indicated in the case of Madame X., I at first proposed to them to pass an induced current from the mouth to the anus. (I showed them that with my water molerator, or with a band of wetted linen in the circuit, the current could be so reduced as to be scarcely appreciable by the tongue, and could be gradually increased.) Notwithstanding the assurance that I possessed (from experiments that I had made upon animals, and even upon man), that I conld regulate at will the degree of this reflex excitation, I proposed to commence by slight action upon the skin of the precordial region, in order to discover what was the degree of excitability or of tolerance of the patient. It will be remembered that, after having used at first in this way a very feeble current, the intensity of which was progressively increased, the disorder of the cardiac circulation was relieved in a short time.

This result so far exceeded my expectations that I was at first 
unwilling to attribute it to the slight electro-cutaneous excitation that I had practised. In the cases of asphyxia already recorded, in which precordial faradization had given me a result not less important, I had found it necessary to increase the current to the highest degree of intensity. But when I beheld cutaneous faradization of the precordial region, although applied only of feeble intensity, and with the electric hand, overcome such grave disorders on successive occasions, I felt it necessary to place the fact on record.

If, instead, of acting with this prudence, and feeling my way, so to speak, to the degree of excitability of the patient, I had in the first instance passed an intense current, the result would have been wholly different. This, indeed, was shown in the case of Madame $\mathrm{X}$., by an accident, on one occasion of the recurrence of the disorder of innervation of the heart. I had all in readiness to relieve this disorder in the way that had always succeeded; but, the apparatus having been moved, its graduating cylinder had been entirely drawn out without my observing it. The acute excitation that the instrument then produced was followed by slight syncope, and momentary stoppage of the heart. However, the cutaneous faradization of the precordial region, that I practiser immediately afterwards, soon subdued this nervous disorder.

Physiological experiments perfectly explain such accidents. They show that a powerful excitation of the pnemogastric produces stoppage of the heart's action, and of the respiration.

The general conclusion to be drawn from the foregoing is, that, in certain morbid conditions, the general excitability is greatly increased; a proposition that I have already laid down. Consequently, if called upon to encounter, by the aid of cutaneous fararlization, grave nervous disorder, it is necessary to proceed gradually, and to proportion the intensity of the current to the degree of tolerance or of excitalility of the patient, under penalty of producing more.or less dangerous mischances.

Under the influence of ideas that are sufficiently old, and that have been promulgated anew by pliysiologists whose only practical experience upon the point is based upon their experiments on rats and rabbits, and who on this basis have scized upon the Academy of Sciences and the Surgical Society, I was disposed, as I have said, to pass a current, in the case of Madame X., from the month to the ants. But, when I observed her extreme excitability, I accepted it as a warning not to expose her to such a method of reflex excitation of the cord and the medulla oblongata,-a method infinitely more powerful than the very feeble cutaneous electrization that I put in practice. Even supposing that the former 
method had been free from danger, it is manifest that a proceeding in which one rheophore was placed in the mouth and the other in the anus would become unbearable, when it was necessary to repeat faradization very frequently for several days. It would then always be necessary to have recourse to cutaneous precordial faradization.

d. The clinical history of the case of Madame X., and the considerations which flow from it, show that it is important, in diphtheritic poisoning, to diagnose exactly the kind of paralysis that is symptomatic of a morbid condition of the medulla; for unon this diagnosis will depend special therapeutic indications. As we have seen, the disorders of the cardiac circulation require to be encountered by faradization of the cutaneous precordial zone, which is in reflex relation with the origin of the pneumogastrics; and paralysis of the intrinsic expiratory muscles, by faradization of a cutaneous zone on the posterior surface of the chest, which is in reflex relation with the nerves by which these muscles are controlled.

In September, 1S69, in conjunction with MII. Millard and A. Ollivier, I saw another case of diphtheritic poisoning; one which both confirms the foregoing observation and displays its importtance.

Case IX. - Without entering fully into the history of this patient, I may say that he appeared to have contracted membranous angina by kissing the mouth of one of his children who died from the disease. After having passed through the period of the formation of false membranes, which put his life in danger by obstructing the bronchi, and when making progress towards convalescence, the patient was attacked, about the twenty-fifth day, by paralytic symptoms which indicated the invasion of diphtheritic poisoning. There was paralysis of the soft palate and pharynx, diplopia, and slight hemiplegia of the fifth, seventh, hypoglossal, and inferior laryngeal nerves. Suddenly, on the twenty-eighth day, the intrinsic expiratory muscles were struck with paralysis; and, at the same time there was formed a considerable quantity of mucus which, obstructing the bronchi and not being expelled, threatened to produce asphyxia. The paralysis had appeared withont ferer; and we apprehended a speedy dcath. Electro-cutaneous excitation of the posterior region of the thorax rapidly re-established the expiratory power, and procured the immediate expulsion of the bronchial mucus. It required to be continued for several days in order completely to overcome the paralysis of the lung. The patient is now in safety, although still under treatment for other consequences of his illness (impairment of sensibility of the hands and feet, with paresis of the limbs). The whole history of the case will probably be published by M. Millard.

If, in the foregoing instances, I had had to deal with paralysis of the diaphragm, I should have employed cutaneous faradization of the base of the thorax; although it does not seem to me to be proved that this cutaneons region is in reflex relation with the origin of the phrenic nerve. My doubt upon the question is 
due to the failure of excitation of this region, in a case of diaphragmatic paralysis from diphtheritic poisoning, which was only cured by faradization of the phrenic nerve itself.

Case X.-A little girl four months old, who had suffered from diarrhcea for several days, became the subject of a rather large ulceration, covered with false membrane, in the neighbourhood of the umbilicus. Although the child had had neither croup nor angina, the diphtheritic nature of this false membrane was not donl,ted by MIM. Barthez and Trousseau. Some days later, the child was attacked by general paralysis, which continued for forty-eight hours, and was followed by complete aphonia, with extreme difficulty of respiration and deglutition. Every attempt at sucking brought on cough and choking; and nuder these perilous conditions, I was called to the case. I found, besides the conditions mentioned above, paralysis of the diaphragm, the signs of which will be described hereafter. After having used electrocutaneous excitation of the base of the thorax, without any appreciable result, I applied faradization to the phrenic nerves, and the diaphragmatic respiration soon became normal. The paralysis returned several times, but was always overcome in the same manner. After faradization of the soft palate, the pharynx, and the anterior surface of the neck at the laryngeal level, the infant sucked better and its roice improved. It was completely cured by a few applications.

Before leaving the subject, I should remark that, in the history of diphtheria, the varieties of paralysis incidental to the period of poisoning have not been analysed, or rather, have escaped observation. I hope it will suffice to have mentioned them, in order, for the future, to call attention to their occurrence. I believe they are not extremely rare; for, during the short time that my own inquiries have been directed to the subject, I have met with several examples.

B.-I come now to the therapeutic effect of excitation of the medulla oblongata by reflex action, by the aid of cutaneous faradization, in a kind of apnoea, a neurosis characterized by the absence of the besoin de respiver, and which, for a certain time, renders the respiratory movements infrequent, or even suspends them. I will cite, as an example, an instance in which I observed this singular functional disorder, and cured it by cutaneous precordial faradization, and by faradization of the diaphragm.

Case XI.-Neurosis characterized by a lind of amoa. Cure by cutaneous faradization of the precordial region, and by furadization of the phrenic nerve. The patient was a young man of 17 , of nervous temperament, in whom there was developed, after a disorder of the chest (pneumonia ?) a singular derangement of the respiratory functions. At intervals, he ceased to breathe for from thirty to sixty seconds, without feeling the least inconvenience, although a slight degree of cyanosis became apparent in his lips and face. 'Then, after this temporary arrest, came a deep, suspirions, noisy inspiration, such as it would be difficult to imitate by intentionally making an exaggerated sigh; and this singular, and as if spasmodic inspiration, would then be repeated many times in succession. At the commencement, this sequence of events only took place five or six times a day; but the recurrences become more and more frequent until they were almost contimial, and were most remarkable 
when the patient's mind was occupied ahout himself, and his attention was directed to his breathing. The sighs then succeeded each other almost withont cessation. This condition had continued for six reeks, withont affecting the general health, when the patient was sent to me by my friend, M. Barthez. I considered that I had to deal with a neurosis that consisted of a temporary abolition of the instinctive craving to breathe. According to this hypothesis, the sighs that followed the period of apncea would be only the satisfying of that craving, which remained unfelt until the deficiency of hæmatosine amounted almost to cymosis. Together with this, there was a spasm of the diaphragm, produced by the sighs and by the elevation of the epigastrium and hypochondria which followed them, or occurred when the patient thought about them. It seemed that each of these singular attacks presented two well-marked periods; one characterized by apnoea and cyanosis, the other by convulsive contraction of the inspiratory muscles and diaphragm.

Excitation of the diaphragm by faradization, practised every day for five or ten minutes, and with slow intermissions, had for its immediate effect to increase the functional disorder; but some time afterwards the sighs became less frequent. The application producing only a small degree of improvement, I employed also cutaneous faradization of the precordial region. From this time the disorder rapidly diminished; and, at the end of a month, the patient was completely cured.

The kind of weakening of the inspiratory nervous excitability, by which this case was characterised, alarmed II. Barthez and myself: It seemed to us to be symptomatic of a morbid condition of an important central point of the medulla. We felt that, if this condition increased, asphyxia must be the result. Influenced by this belief, and not thinking that the functional derangement was produced by any organic lesion, I had recourse to faradization of the precordial cutaneous zone that is in reflex relation with the pneumogastric, and, as has been said, was rewarded by complete success.

I have also employed the same mode of electro-cutaneous excitation whenever I have been called to patients in whom the respiratory nervous excitability had been weakened or paralysed, by whatever influences, to the approach of apnca; that is to say, so as to slacken considerably, or temporarily to abolish, the respiratory movements, and in this way to lead to asphyxia.

\section{Part the Thilid.}

ON THE THERAPLUTIC VALUE OF LOCALIZED FARADIZATION.

§. Brief exposition of clinical facts which estallish that localized faradization is of great value in the treatment of atrophic paralysis.

Wishing to bring about the prevalence of the therapeutic employment of continuous constant currents, Remak has charged 
the induced current with exerting a paralysing influence upon nerves. ${ }^{6}$

In presence of the knowledge of electro therapeutics gained by long clinical experience, and tested by many observers in Europe and even in America, to venture to say that induced currents exert a paralysing influence upon the nerves seems to me to be so far to disregard the truth that I have not thought it necessary to combat Remak's strange assertion. Clinical observation will render it prompt justice.

But I am unable any longer to keep silence upon the point, since recently some young philosophers, supported by experiments upon rats and rabbits, have again maintained, like Remak, that induced currents paralyse the nerves, and canse the disappearance, at the end of a very short time, of all their vital endowments. ${ }^{7}$

Clinical observation, I repeat, protests against such an assertion. The following are the words in which I have briefly summarized the facts which these observers fancy they can overturn: ${ }^{8}$

"In 1862 I reported ${ }^{9}$ some cases of atrophic paralysis, consecutive to traumatic lesions of mixed nerves, which were of many years' standing, and in which the paralysed limbs were so wasted that the muscles, which no longer responded to electrio excitation, appeared to be entirely destroyed. 'The temperature of these limbs was diminished, the cutaneous veins were very small, the skin discoloured, earthy, and often of a violet hue. Well, under the influence of direct muscular faradization, applied according to certain rules which I have laid down elsewhere, I have seen, in all these cases,- -1 . the temperature of the limbs quickly increase; 2. the cutaneous reins enlarge, and the skin return to its normal colour; 3 . the muscles gradually increase in size, and regain their tonic force, their sensibility, their voluntary contractility, and, much later, their electric contractility also."

Since 1852, a host of European observers have experimentally tested and confirmed the reality of these electro-therapentic results, and have borne testimony to them in numerous publications. I will select, as one of the most important among them, that of M. R. Philippeaux, of Lyons. In America, direct muscular faradiza-

${ }^{6}$ Remak, Galvanotherapie, etc., translated from German into French by Dr. A. Morpain, Paris, 1860, "Valeur" antiparalytic des courants induits," p. 447.

7 Onimus et Legros, "Del'influence des courants électriques sur la nutrition" (Gaz. des Hôp, 19 Janvier, 1860.) The same anthors had previously addressed a note upon the question to the Academie des Scienecs.
${ }^{8}$ Duchenne (de Boulogne), "Dela paralysie musculaire pseuto-hypertrophique" (Arch. génér. de Mlédécine, Janvier, 1868.

${ }_{9}$ Duchenne (de Boulogne) "De l'application de la faradisation loralisće au diagnostic, aù prognostir, et au traitement des paralysies consécutives aux lésions des nerfs mixtes" (mémoire couronné par la Société de MIédécine de Gaud, au concours de 1852. Annules de la Soc. de Méd. de Gaud). 
tion has been applied very recently, in the hospitals of Philadelphia, to the treatment of traumatic paralysis, by surgeons specially selected by Mr. Hammond, the chief surgeon of the army of the North, and in the cases of men wounded in the civil war. The following are the terms in which the therapentic results of this method of localized electrization have been described by these distinguished men. 'The only important means,' they say, 'in the treatment of paralysis arising from default of innervation, is faradization by the method of MI. Duchemne. . . . Most of our cases were from wounds that were of old standing when they came under our care. We can assure you that localized faradization has, in our hands, been of the utmost value. In some cases it has, at a single application, restored the power of movement to parts that had been long deprived of it; in others, a few applications have more or less completely restored the power of movement to an entire limb; and we have seen very few instances in which there las not been improved nutrition, and greater sensibility and strength, when we were able to continue the faradization sufficiently long. In a word, we cannot too strongly repeat that, until faradization has been used, no limb, however completely paralysed, should be abandoned by the surgeon as inenrable." 1

I might also addnce other kinds of paralysis, in which the lesion of nutrition is equally profound, and in which localized faradization is often successful. Moreover, clinical facts will be hereafter detailed, which prove incontestably the favonrable-I would almost say the marvellous-influence of localized faradization upon the nutrition and the motility of muscles. These facts will be found in the chapters specially devoted to the application of this method of electrization to traumatic paralysis of nerves, to paralysis consecutive to myelitis in adults and adolescents, to saturnine and rheumatic paralysis. We shall even see that, when applied to the fatty atrophic-spinal-paralysis of infincy, localized faradization acts not only upon the nutrition of the muscles, but also upon that of the entire limb, so that it prevents arrest of development of the bone.

\$II. Critical examination of the electro-physiological experiments which have served as the foundation of the attacks recently divected against the therapentic value of localized faradization. Their. inexactitude.

Althongh, during more than twenty years, I have obtained from localized faradization such results as I have described, and after I

I S. Weir Mitchell, George R. More- | Wounds and other injuries of Nerves, house, and William W'. Keen, 'Gunshot Philadelphia, 1868. 
hare seen these results confirmed, both in Europe and in America, by a host of the most competent observers, an endeavour is made to persuade me, on my objecting to deductions drawn from a few experiments upon rabbits, that I am myself under illusion!

As much as any one, I admire experimental medicine; and, without believing that the whole future of therapenties rests upon it exclusively, I have always held that it will exert a guiding and enlightening influence. But when, in defiance of all logic, it would lead us to cast doubt upon facts perfectly established by clinical observation, it is necessary that we should renomee it.

A.-Fortunately, in the ease with which we are now concernerl, we have not to find fault with experimentation itself, but with those who have experimented badly, or who have badly interpreterl the results of their experiments. I proceed to prove this, at least for those which are now under discussion.

In the rabbits, the sympathetic nerve was laid bare in the neck, slightly lifter by means of a thread placed under it, and then electrified, for two or three minutes, by powerful induced currents. The artery of the corresponding ear became contracted until it was no longer perceptible. On ceasing the electrization, the artery regained its size little by little; and finally became larger than it uas before the operation. After an instant, the temperature of that side was elevated above that of the other.'

There can be no question that, in these experiments, the dilatation of the vessels of the ear, consecutively to faradization of the eervieal sympathetic of the same side, was produced by paralysis of their vaso-motors. Is there not some cause for surprise that the sympathetic, alrearly exposed to the air, and perhaps more or less injured in the dissection, was not, so to speak, killed by the powerful induced current by which it was directly traversed for several mimutes; and that the ressels had still power slowly to regain their normal calibre?

'I'o proceed thus with experiments the object of which is to study the action of induced eurrents upon the nervous system, is but to contribute to toxicology. We know that there are certain poisons which, in small (loses, increase the action of the heart, but which would arrest that action if given in large doses. To expeet a therapeutic action from such a manner of applying induced currents is like maintaining that paralysis might be as readily cured by a thunderbolt as by electrieity wisely and methodically applied.

'There is certainly no need of experimental medicine to inform us that, in its therapentic aspect, localized faradization is a two- 
edged weapon. Empirical clinical observations very soon convinced me, at the beginning of my electro-therapeutical researches, that localized faradization, if applied to a muscle or nerve in too full a dose or for too long a time, may increase or eren produce paralysis or atrophy, instead of curing either. It was such clinical observation that led me to lay down the precept that muscular faradization should be moderate in degree, and that neither the whole application, nor the application to any single muscle, should be unduly prolonged.

With a powerful induced current, contracting the vessels during its passage through the sympathetic, and producing, after its cessation, a temporary diminution of their contractility (and hence their dilatation), the same authors have compared the relatively infinitely feeble action of continnous currents upon the same sympathetic, "under the influence of which," they say, "the activity of the cireulation was promoted during their passage, and for a long time after their cessation." 3

It appeared to follow, on the whole, from their experiments :-1. that induced currents, during their passage through the sympathetic, arrested or impeded the circulation by closing the ressels by a powerful excitation of the vaso-motor nerves; 2 . that they produced, after their passage, a passive dilatation of these vessels, and a consequent blood stasis within them, by the paralysis or exhaustion of their raso-motors; and, 3. that continuous currents, on the contrary, increased the artivity of the local circulation, both during and after their passage through the sympathetic.

Partisan and defender, as has been seen already, of the existence of different physiological properties in the different kinds of electricity, although the reason of such difference has not at present received complete explanation from actual physical data, I was disposed to hail the discovery of a special difference of action between the contiunous and the induced currents as a triumph of experimental medicine, applicable to therapeutics. But I must add that there remained in my mind some doubt with regard to the reality of the assigned difference; becanse, in the comparative experiments, the two forms of current had not been applied in equivalent force.

It was, therefore, important to verify the results said to have been obtained, and the following is a summary of experiments made upon rabbits by myself, in conjunction with my learned friend M. Liégeois, Professor of Physiology to the Paris Faculty of Medicine. Our results are far from agreeing with those of MIM. 
Onimus and Legros; and show that, in equivalent doses and under similar conditions, the continuous constant and the induced currents, when passed through the great sympathetic, exert an identical action upon the vaso-motors.

Experiments. $-\mathrm{A}^{7}$ continuous constant current (from a pile of from fifteen to thirty sulphate of lead elements) passing through the sympathetic in a portion three or four millimetres below the superior cervical ganglion, produced a slight but manifest contraction of the vessels, and a decoloration of the ear on the same side, during the whole time of the experiment.

When the sympathetic nerve had previonsly been strongly excited, or, in other words, when its excitability had already been more or less exhausted, as by the passage of a powerful induced current, or by section, the same continuous current of thirty elements, or even of fifteen, produced, instead of contraction of the vessels, their dilatation during the whole time of the experiment.

An extremely weak induced enrrent (the minimum of the extra current of my volta electric apparatus, passed through a column of distilled water twenty-two centimetres in height), and localized in the sympathetic in the same manner for three minntes, produced a slight contraction of the vessels of the ear during the first half minute, followed by dilatation during the rest of the experiment.

The same proceeding, repeated upon the sympathetic of a rabbit that had been subjected to a strong induced current for three minutes, an hour previously, but in which the ear had regained its normal vascularity, immediately produced dilatation of the vessels which rendered their finest ramifieations visible for three minutes.

A strong induced current passing in the same manner through the sympathetic of a rabbit, whether it had been the subject of previous experiment or not, produced, during the whole time of its passage (three minutes), a considerable shrinking of the ressels; followed, when the current was withdrawn, by an enormous and long persistent dilatation.

Interripted galvanic currents passed through the great sympathetic acted upon the ganglionic vaso-motors in the samo manner as induced currents.

\section{It follows from these experiments:-}

That a continuous constant current, directed upon the exposed great cervical sympathetic of a rabbit, the excitability of which has not been exhausted (neither by electrization in prior experiments, nor by manipulations, nor by long exposure to the air), produces, like an induced current of equivalent (i.e. very feeble) strength, a slight contraction of the vessels that are controlled by the nerve.

That the same current, acting with equivalent force upon a nerve already exhausted, can no longer produce contraction of vessels, but only moderate dilatation.

'That a powerful induced current, localized in the sympathetic, produces a considerable contraction of the vessels, followed, after the experiment, by enormous and protracted dilation; and that a powerful intermittent galvanic current acts in the same manner.

Finally, as a corollary to the foregoing, that, contrary to certain statements, the continuums constant currents and the induced 
currents, in equivalent doses, exert an identical action upon the sympathetic and the vaso-motor nerves.

$\S$ III. Upon what class of nerves does locatized faradization exert its influence in order to increase the activity of the local circulation?

\section{Upon the Vascular Constrictor Nerves?}

A.-Ramifications of the sympathetic nerve accompanying the arterioles, excited by localized faradization.-Localized electrization (either faradization or galvanism, localized by interrupted currents, ) is the only means by which we can directly excite the ramifications of the great sympathetic, that are distributed to the muscles of the limbs and of the surface of the trunk.

It has now been shown, by microscopic anatomy, that the ramifications of this nerve accompany the arterioles almost to their ultimate divisions, and that they are found embracing capillaries of the second order. This anatomical fact was discovered, in 1856, by MI. Ordoñez, ${ }^{4}$ and confirmed, in 1865, by my friend M. Gimbert (of Cannes), ${ }^{5}$ one of the most distinguished pupils of Professor Ch. Robin, in an excellent memoir, from which $I$ have taken fig. 41.

In this figure it will be seen that, on a level of the anastomoses of the arterial capillaries, the nerves present ganglia, G, from which proceed new filaments which spread over the capillaries, and terminate in pointed extremities. These are like the isolated fibres of Remak, R.

We can conceive, then, that in localized muscular electrization (which consists in moving moist rheophores, as nearly as possible approximated to each other, over all points of the surface covering a muscle, so as to produce electric recomposition in the thickness of the muscle, at varying deptis according to the intensity of the current), we may carry the excitation directly to the vaso-motor nerves which accompany the arteries to their divisions into capillaries of the second kind.

4 M. Ordoñez has mounted beautiful microscopic preparations, shewing the vaso-motor nerves accompanying the arterial vessels to their final ramification. He has nearly followed them to their terminations on the eapillaries of the second variety. In man, he has established this important anatomical relation in the small arteries of the retina and of the brain; and he informs me that he should have formd this same arrangement of other organs, if it had been possible to isolate them with the same facility. The dis- covery of M. Ordonez was annonnced in 1865, by M. G. Pouchet (Rapports du grand sympathique avec le système capillaire: leçon faite an Musémm d'histoire naturelle-Revue des cours scientifiques, etc., p. 709). To be just, I shonld add that M. Schweigger, of Berlin, had discovered the same anatomical fact at the same time, in the arterioles of the choroid and of the retina in man.

${ }_{5}$ Gimbert, Structure et texture des artères : Thèse de Paris, 1865. 


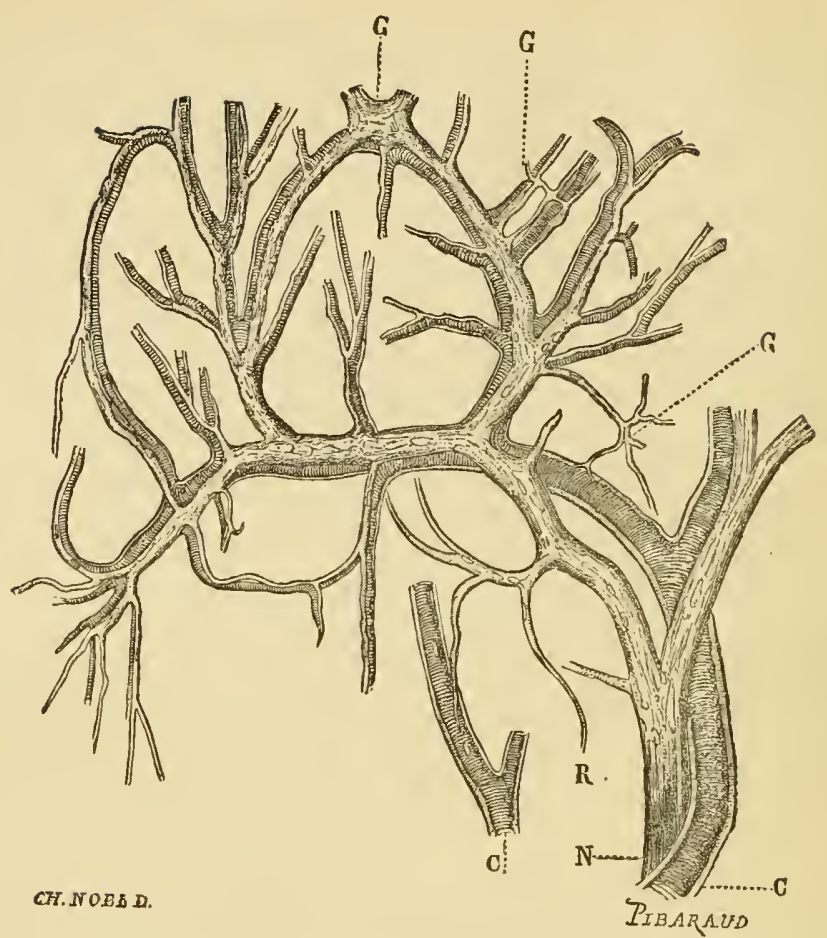

Fig. 41.-Vaso-motor nerves accompanying capillaries in the palatine wucous membrane of a frog (aiter Gimbert). ${ }^{6}$

B.-Tonicity of the vascular constrictors augmented by localized electrization.-Experiments show that the great sympathetic is highly excitable, at least in the cervical region, and that its excitability is quickly exhausted. Thus it has been mentioned previously that, under the influence of even a feeble induced or continuous current, a contraction of vessels is produced, presently followed by a dilatation of some persistence. This dilatation of vessels is with good reason held to depend upon a paralysed state of the vaso-motor nerves, producing an afflux of blood to the parts controlled by them, and an elevation of temperature, which has hence been called neuro-paralytic hyper:mia.

But the kind of weakening of vaseulat tonicity, which is the first effect of localized faradization, is only momentary, and is soon followed by a reaction. Indeed, both clinical observation, and the therapeutical results that have been obtained, tend to prove that,

$6 \mathrm{C}, \mathrm{C}$, Capillary vessels. N, raso-motor nerve. $G, G, G$, ganglions forming nerwous branches at the level of the anasto- point. 
as regards the ultimate result, the tonic force of the vessels is increased.

Such, at least, is the object that I propose to myself, and that an endeavour should be made to attain, when, in the treatment of atrophic paralysis, electric excitation is directed upon the ganglionic vaso-motors. It is indeed shown, both by clinical observation and by experiment, that neuro-paralytic hyperæmia is a morbid state that is seen in atrophic paralysis, consecutive to lesions of the cerebro-spinal centres or of the nerve-trunks. It is rational to conclude that any agency which increased the neuroparalytic hyperæmia would aggravate the pathological state. But this is precisely the opposite of what I lave witnessed, when, by the aid of faradization, I have cured the cases of atrophic paralysis that have been brought to me in such a condition. Under the influence of the treatment, I have seen the colour of the skin and the nutrition of the paralysed limb return in a short time to their normal state; while, at the same time, the paralysis underwent improvement: and I have considered, under these circumstances, that the vascular tonicity had been increased by the localizer faradization. On the whole, the clinical facts teach that, as far as regards therapentics, we should take into consideration the secondary and ultimate, rather than the immediate effects of electrization.

C.-Local circulation promoted by localized faradization.-Such has not been the reasoning of those who, on the contrary, attribute the therapeutic action of electrization on the nutrition to the slight degree of neuro-paralytic hyperæmia that is produced by the influence of a feeble current. To reluce their opinion into terms, we must say that, neuro-paralytic hyperremia being one of the morbid phenomena of atrophic paralysis due to lesions of the nervous centres or nerve-trunks, we should have to increase the neuroparalytic hyperemia in order to cure the paralysis. To diminish what woull otherwise appear paradoxical in their opinion, those who advance it have altered the physiological signification of the fact on which it rests, by saying that the increased activity of the circulation is produced by the influence of the current upon the sympathetic.

In order to accept this theory, it would be necessary to forget the experiments which show that this kind of neuro-paralytic hyperemia is passive; and that, in itself, it is powerless to affect the nutrition. I would recall the experiment of Snellen, ${ }^{7}$ many times since repeated, which proves this in an incontestable manner. I have seen the fact confirmed by clinical observation; and I shall

7 Snellen, Arch. für die hollandische Beiträge zur Natur und Heillinnde, 1857, rol. i. p. 206. 
hereafter relate a case in which neuro-paralytic hyperæmia of the conjunctiva and vessels of one eye, consecutive to a lesion of the cervical sympathetic, had continued, at a very high degree, for a period of two years, without in any way modifying the anatomical state of the hyperæmic parts; and without producing the slightest exudation; while a conjunctivitis of the other eye, due to exposure to a current of air, had rapidly displayed all the symptoms of acute inflammation, with a slight formation of pus.

It is, however, impossible to overlook, in a case of the cure of atrophic paralysis due to traumatic or spontaneons lesion of nerve or cord, such as I have already related, the influence of the faradization upon the activity of the local circulation, upon the nutrition of the muscles, and upon the general nutrition of the limb to which it is applied.

But how is this therapeutic action to be explained? I am unable to attribute it to a kind of gymnastic effect produced upon the muscles by faradization, because the muscles had lost their electric irritability. Neither do I believe that excitation only of the nervous fibres, which govern motricity and sensibility, wonld have power to produce the results. From all the evidence, I think that the direct muscular faradization promotes the capillary (local) circulation and the nutrition. I had not neglected to point out the last electro-therapentic fact, and to bring it into prominence in the earlier editions of this treatise; but I was then limited to setting forth the results of clinical observation in empirical fashion, since science was not sufficiently advanced to justify the framing of any theory whatever.

What has been the mechanism? In other words, by what class of nerves have the nutrition and the capillary circulation been promoted?

It is with fear that I approach this question. In all my earlier researches into the physiology of movement, the problems to be solved have always been in harmony with anatomy, with electromuscular experiments, and with clinical observation. On the contrary, in the new jath on which I am now to enter, I cannot make a step without proceeding from hypothesis to hypothesis, relying only upon the great discoveries of experiment.

\section{On the Nerves which pronote Local Circulation?}

A.-Historical retrospect.-The section of the great sympathetic, made by Pourfour-du-Petit in $1727,{ }^{8}$ is the fundamental fact

${ }^{8}$ Pourfour-du-Petit, "Mémoire dans|tent des esprits dans les yeux" (Mem. de lequel il est démontré que les nerf́s inter- l'Acad. des Sciences, Paris, 1727).

costaux fournissent des rumeaux qui por- 
which has served as a point of departure for numerous researches. Besides contraction of the pupil, Pourfour-du-Petit had also observed that the vessels of the conjunctiva lost their elasticity, and became distended by blood consecutively to the section. In 1840, II. Stilling, who had also recognised the action of the ganglionic system upon the contractility of vessels, called the special nerves conveying it the vaso-motors. M. Cl. Bernard announced in different publications, in 1851 and in 1855, the great physiological fact that section of the great sympathetic is constantly followed by a considerable afflux of blood to the parts of the head to which the nerve is distributed. ${ }^{1}$ M. Brown-Séqnard discovered, in 1852, that galvanization of the great sympathetic produced contraction of the ressels and diminution of temperature; and, consequently, that the principal phenomenon described by MI. Cl. Bernard, was due to paralysis of the blood-vessels. ${ }^{2}$ Three or four months later, MI. CI. Bernart, not knowing of MI. BrownSéquard's publication, announced the same fact to the Biological Society. ${ }^{3}$ H. Ludwig was the first to suggest the idea of irritating the glandular nerves, and especially the sub-maxillary gland, the principal vessels of which he laid bare, with their nerve-branches, after having isolated Wharton's duct, in which a canula was inserted, so as to exhibit the flow of saliva. The excitation of the nerve produced, in a short time; an extraordinary secretion of this liquid. (M. Lndwig enabled me to witness this beautiful experiment, when, in 1856, I attended the congress of naturalists at Vienna.) MI. Maurice Schiff instituted, in 1851, a series of experiments upon the chorda tympani, in order to show that all the salivary nerves are not contained in the proper filaments of the trifacial (lingual), but that they perhaps proceed from its anastomosis with the facial (chorda tympani). ${ }^{4}$ These researches have been repeated, with the same results, by many authors. But it is to MI. Cl. Bernard that all the honour is due of having discovered the actual mechanism of certain local circulations, by showing that the vessels are placed under the control of constrictor and dilatator nerves which act upon them reciprocally. In 1856, he showed that by exciting the chorda tympani we could produce, in the

9 Stilling, Physiol. pathologische, und Med. pructische Untersuchungen über Spinal-irritation. Leipsig, 1840.

1 See the Comptes rendus de la Societe de biologie, Decembre, 1851. Guzette Medicale de Paris, 1852, p. 74. Comptes rendus de l'Académie des Sciences, séance du 29 Mars, 185̃2. Recherches expérimentales sur le grand sympathique et spécialement sur linfuence que le section de ce nerf exerce sur la température animale. Paris, 185 t.

'See 'Phila lelphia MIedical Lxaminer,' August, 1852, p. 489.

${ }^{3}$ See Comptes rendus de la Société de Viologie (Gazette Medicale, 18.53, p. 71).

* See Schiff, Ueber motorische Zungenlalunung (Archiv für physiologische HeilTiunde, von Wunderlich und Vierordt, 1852). 
organ to wlich it is distributed (the sub-maxillary gland), an overactivity of the capillary circulation, and a dilatation of the small arteries, such that the blood would circulate in the veins with all the appearances of arterial blood. He showed, also, that other nerves going to the arteries of the face had equally the power to dilate those arteries. ${ }^{5}$

B.-Opinion of M. Cl. Bernard in favour of the active ditatation of vessels.-Although treating of the therapentic influence of localized fararlization upon the activity of local circulation, I ignored the mechanism of this influence until our great physiologist, M. Cl. Bernard, after having contributed so largely to make known the proper action of the great sympathetic, entering upon a new path of discovery, had shown, especially by his experiments upon the chorda tympani, that, in opposition to the action of this nerve as a constrictor of the vessels, other nerres, when excited, produced dilatation of the vessels, and that hence they are dilatators of the arteries, and accelerators of the capillary circulation.

Althongh it is true that, in the hands of M. Cl. Bernard, experiment has as yet shown the two orders of vascular nerves (constrictors and dilatators) only on the arterioles of certain glands and of the face, yet it must be remembered that his genius has led him to believe that the active dilatation of ressels must be general. The following are the terms in which he expresses his opinion:-

"It is admitted, in general," he says, "that all the phenomena that follow section of the great sympathetic in the neck are the consequences of paralysis of the nerve and of the consecutive passive dilatation of the vessels. This explanation appears to me to be insufficient, to say no more. I am assured, indeed, after that experiment, that the blood which enters the venous system is warmer than that which traverses the arteries. There is then, among these phenomena, something more than a simple passive dilatation of the vessels: we are in the presence of an action of a different kind, and until now unknown. The contraction of the pupil, after division of the cervico-cephalic branch, is not a passive, but, on the contrary, is an active phenomenon; there is produced, after the section of the sympathetic, a predominance of action of the ciliary branches of the third pair.

"The variations observed in the size of the ressels appear to belong to the same class of phenomena, although here the contraction is produced when the sympathetic is excited, and the dilatation when it is divided, the opposite of what happens in the pupil. This

${ }_{5}$ See Cl. Bernarl, Leçons sur les pro- $\mid$ pathologiques des liquides de l'organisme, priétés physiologiques of les altérations $\mid$ tome ii., leçons des 4 et 9 Juin, 1858. 
is not a simple hypothesis ; the active dilatation of vessels is one of the most unquestionable verities which have been brought to our knowledge. I have proved by direct experiment that there are two orders of vaso-motor nerves, acting in opposite directions; and that their properties are, in the majority of cases, called into play by reflex influence." 6

MI. Cl. Bernard reiterated the same opinion, in 1864, in a more explicit manner. "The vascular system," he said, "is subject to the influence of two nervous systems, more or less distinct: the sympathetic system and the cerebro-spinal. Let us inquire what is the function of each of them; and what part may legitimately be assigned to each in the facts that we have to study. The former-the great sympathetic-has the function of a moderator of the vessels. When irritated, it produces a more or less considerable contraction of them, a contraction which forms a certain degree of impediment to the circulation, and hence diminishes its speed. On the other hand, by exciting the filaments of the cerebro-spinal system, we produce a dilatation of the same vessels. Such is the whole mechanism of the nervous influence. With these two modes of action alone-contraction and dilatation of vessels-the nervous system goverus all the chymical phenomena of the organism."7

But, admitting only what has been demonstrated by experiment, 1I. Cl. Bernard is careful to declare that the generalization of the phenomena of active dilatation of the capillary vessels is at present only a hypothesis.

C.-Necessity for the existence of dilatator vascular muscles, as moderators of the constrictor vaso-motors. - I venture to advance a new argument, showing the necessity for a system of nerves to moderate the force of the constrictors.

I am convinced that all muscular action should have its antagonist; and that this rule applies as much to unstriped as to striped fibre. Just, indeed, as wherever I have seen deficiency of antagonism in the muscles of animal life, the attitude and conformation of the limb or part set in movement by the muscles has been altered, so the preservation of the normal calibre of the vessels necessarily depends upon a certain equilibrium between the tonic force of the constrictors and that of the dilatators. Such appears to me to be the light thrown upon the question by my clinical observations.

If it be objected that this also is only a hypothesis, I reply that a lypothesis resting upon clinical observation is very near the

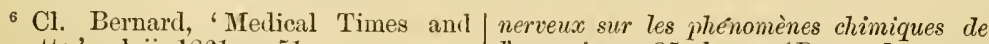
Gazette,' vol. ii. 1861, p. 51.

7 Cl. Bernard, Influence du système $/$ scientifiques, 28 Juin, 1864.) 
truth. How often, for example, in matters falling within my personal researches, has clinical observation elucidated the physiology of movement, and anticipated the anatomical fact. I will, therefore, in this place say briefly on what clinical facts my hypothesis reposes, as far as regards the vaso-motor dilatators.

I have said that the two orders of vaso-motors (constrictors or ganglionic vaso-motors, and dilatators or cerebro-spinal vaso-motors) as reciprocal moderators, appear to me necessary, not only to the functions of the organs on which they ramify, but also to the preservation of the normal calibre of the small vessels. Indeed, when one of the two sets is paralysed, the local circulation is profoundly altered. If the ganglionic vaso-motors are paralysed, the arterioles remain dilated, inert, and the venous blood becomes red; while the reverse happens if the cerebro-spinal nerves are affected.

Clinical observation seems to me to show that the force of the cardiac impulses (which moreover, as shown by M. Marey, are less felt in the vessels in proportion as their calibre diminishes) does not sufficiently moderate the power of the constrictor nerves of the vessels. In a certain number of cases indeed, in which it seemed established that the anterior cornua of the spinal cord had undergone rapid change, without lesion of the ganglionic system (as in infantile myelitis) I have observed, at a time more or less distant from the commencement of the disease, that the parts receiving morbid innervation were affected as follows:-The vessels were contracted, the calibre of the cutaneous veins diminished, the skin was discoloured or cyanosed, the temperature lowered, and at the same time the muscles were wasted. Having, then, found that the beats of the heart retained their normal energy, and that the pulse was not weakened, I have concluded that the power of the cardiac impulses was insufficient to neutralize the muscular force which tended incessantly to close the small vessels; that it was not enough alone to preserve the normal calibre; and, in a word, that the force which dilates the small vessels was paralysed.

I think then that, under these circumstances, the diminution of calibre of the small vessels is explained by the unrestrained or predominant action of their constrictor muscles, from which a local ischæmia results. Ordinarily, the lesions of the cord, and of the nerves which proceed from it, produce in the first place a neuro-paralytic hyperæmia, which, at a more advanced period, is replaced by the opposite disorder of the local circulation, that is, by the morbid phenomena described above. (May we not attribute also to these same disorders of the circulation the diminution or loss of electro-muscular contractility which supervenes so quickly in atrophic paralysis ?) 
D.-Therapeutic action of localized faradization upon the development of vessels. - If such is the mechanism of the disorder of local circulation that is observed consecutively to lesions, imflammatory, organic, or traumatic, of the cord and of the mixed nerves, we are able to explain one of the favourable results obtained by direct muscular faradization.

The first therapeutic effect of direct muscular faradization, in the treatment of these forms of paralysis, is, as I have said, slightly to redden the skin, in the excited region, during the passage of the current. We soon afterwards observe elevation of temperature, increase of sensibility, and at last, more slowly, the enlargement, almost visibly, of the cutaneous veins. I have often seen these phenomena continue after the faradization, and sometimes increase, so as to resemble an irritation, and to require the suspension of treatment and the use of emollients, baths, poultices, and the like.

It is certainly not the excitation of the ganglionic vaso-motors that produces these immediate effects. It, on the contrary, produces contraction of ressels, decoloration of the skin, and cooling of the excited region. Consequently, the hyperæmia that is witnessed during faradization, under such circumstances, must be considered as an active hyperæmia, produced by excitation of the dilatator vaso-motors.

Why do not the vaso-motor constrictors, that are influenced by the faradization at the same time as the dilatators, produce a consequent contraction of the vessels? For the present, I must admit, I do not know the reason. I am therefore bound to mention the fact.

E.- What is the mechanism of the active ditatation of vessels?

(a). According to MI. Cl. Bernard, the vaso-motor dilatators exert a paralysing action upon the raso-motor constrictors, by the intermediation of small peripheral ganglia, which are found in great numbers in the course of intra-glandular nerves; their irritation is propagated to these small controlling centres, the activity of which consists in checking function, in producing momentary paralysis of the ultimate nervous filaments in relation with the vascular muscles. With regard, for instance, to the chorda tympani, the excitation of this nerve proceeds, after the hypothesis of MI. Cl. Bernard, to rouse the activity of a sub-maxillary ganglion destined to moderate, or temporarily to paralyse, the vaso-motor constrictors that are distributed to the vessels of the sub-maxillary gland.

The hypothesis of MI. Cl. Bernard is supported by ingenious experiments; but, according to Schiff, this doctrine of a central 
office for peripheral ganglia, so often invoked for the purpose of explaining certain paralysing functions, rests only upon arbitrary hypotheses. The following is the most serious objection which Schiff opposes to the suggestion of Cl. Bernard. If it be sound, he says, with regard to the sub-maxillary gland, it should apply also to the ear of the rabbit, where we have equally the possibility of a vascular dilatation, produced by the direct irritation of nerves. But no one has discovered the smallest microscopic ganglion in the ear of the rabbit, any more than in that of the dog, or of man. $^{8}$

(b). M. Schiff has endearoured to simplify and to solve the question of mechanism now under discussion, by admitting the possibility of active vascular dilatation by longitudinal muscular fibres. But it may be objected anatomically, that such instruments of dilatation do not exist. ${ }^{9}$

At present, however, this plea seems to me to have lost its force, since M. Gimbert, whose admirable researches into the structure and texture of arteries I have already had occasion to quote, has discovered in some arteries the presence of numerous longitudinal and oblique muscular fibres, interlacing with the horizontal fibres.

"Their direction (the arterial muscular fibres), he says, "is most frequently horizontal, but it may be oblique, or even longitudinal." 1

He has shown the direction of these fibres in several figures, of which I have reproduced one only, fig. 42, which shows a

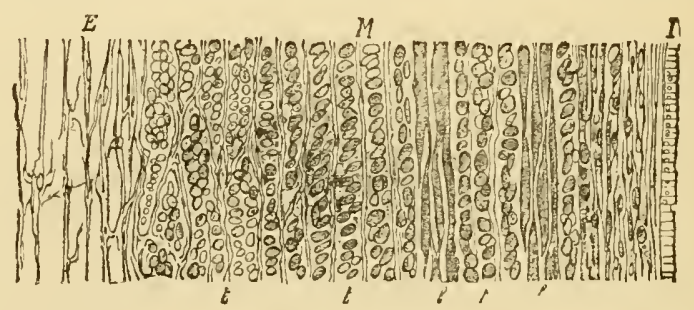

rig. 42.- - longitudinal section of umbilical artery at the base of the cord. Enlarged 700 diameters, after Gimbert.2

8 Schiff, loc. cit., tom. i., p. 271.

9 M. Schiff appears to have abandoned his hypothesis of the existence of longitudiual muscles, doubtless on account of this objection. He has written, indeed, in one of his lectures, "Although the instrument of active dilatation at present eludes our means of investigation, nothing has shown the impossibility of the process." All that we can say, provisionally, is that, while the mechanism of con- striction is directly explicable by microscopic examination of the vascular muscles, the active dilatation appears to be extraneous to the proper tissues of the vessels, and to be effected by the intermediation of extra-vascular tissues.

1 Gimbert. lcc. cit., p. 80.

2 l, l, Lougitudinal muscular fibres; $t, t$, horizontal muscular fibres; $\mathrm{E}$, outer coat; M, middle coat; I, inner coat. 
longitudinal section of the wall of an umbilical artery, at the base of the cord, magnified 700 diameters. We see at $l l$, says M. Gimbert, muscular fibres implanted in an amorphous matter, homogeneous and transparent, like grains of oats imbedded in a homogeneous and elastic mass, and also horizontal fibres cut transversely.

M. Gimbert has found this anatomical arrangement in several arteries, and he purposes to search for it in the capillaries. Manifestly, if it were general, the question of the mechanism of the active dilatation of vessels would be singularly simplified, and would be almost as firmly established as that of the vaso-motor constrictors.

The hypothesis of longitudinal muscular fibres destined to produce active dilatation is therefore verified, at least for certain vessels. And what other purpose could they serve than to shorten the vessels, and, consequently, to dilate them? Is it not an established fact in physics that the capacity of an elastic tube diminishes or increases, accordingly as it is extended or shortened longitudinally? The arteries seem to favour these changes of capacity by their structure, by the fact of their lengthening or shortening. May it not, indeed, be the case that the elastic elements are, under the form of fibres, the fundamental portion of the middle coat, and that they are arranged in all directions?

In conclusion, notwithstanding the obscurity and the differences of opinion which at present prevail with regard to the mechanism of active dilatation of local blood-vessels, the fact of the active dilatation of ressels, at least as regards some of them, is, even anatomically, perfectly established in my eyes. If it were not so, I should still say, with M. Schiff, "It would be bold to deny an observed fact, even although we may not understand its mechanism."

It is by admitting the generalization of this important physiological fact, brought to light chiefly by M. Cl. Bernard (the active dilatation of vessels), that I have been able to render an explanation not only of the active hyperæmia produced immediately by localized faradization, in the treatment of paralysis consecutive to lesions of the spinal centre, or of the nerves proceeding from it, but also of one of the principal therapeutic results, appreciable between the applications; namely, the augmentation of the activity of the local circulation, and of the tonic force of the vessels, appreciable especially by the reappearance, from development, of the cutaneous veins, and from the coloration and the normal temperature of the skin, without complication with hyperæmia. 


\section{III.-NERVES ACTING DIRECTLY UPON THE NUTRITION OF THE TISSUES.}

The increase of activity of the local circulation does not suffice to explain certain pathological states. This idea had taken root in some minds before it was confirmed, at least in part, by pathological anatomy.

A.-Theory of M. Brown-Séquard; opinion of M. Tirchow.1I. Brown-Séquard was the first to express clearly this important view, in the lectures which he published in 1858 , and republished in $1860 .^{3}$

"The nerrous system," he says, "determines an increase of the attraction exerted upon the blood by the living tissues, and, in this case, the phenomenon is attended by dilatation of the bloodvessels. The nervous system acts directly and originally upon the parenchyma of the tissues." Elsewhere he writes again, "The simple fact of an increase or a diminution in the quantity of blood that passes through a part of the body in a given time, assuredly suffices to explain the physiological changes, and some of the morbid modifications, which we habitually observe in secretion and in nutrition; but some other pathological states seem to require for. their production, something more than a simple change in the quantity of blood.

"An inflammation, for example, cannot be explained by a modification of this kind, because, after section of the cervical sympathetic, we see a considerable increase of blood occur in the eye, the ear, \&c., and continue during many weeks or months without producing inflammation. It is true that the morbid action (inflammation) developes itself much more easily in these parts than in others; but, I repeat, it does not manifest itself spontaneously, as a pure and simple consequence of increase in the quantity of blood. It is therefore necessary to admit that, when a nervous influence acts upon certain tissues so as to produce inflammation, the principal cause of the morbid process is not the increase in the quantity of blood, but rather the modifications which the tissues undergo, and which produce an unusual attraction for arterial blood. ${ }^{4}$

B.-Almost at the same time Virchow also declared that modifications of nutrition did not depend, directly, upon the larger or smaller quantity of blood that circulated through the part. But, resting upon the experiments of Cl. Bernard, who showed that

3 Brown-Séquard, 'Central Nerrous System,' Philadelphia, 1860, p. 172.

4 Ibid., loc. cit. 
section of the great sympathetic produced no other result than an increase of the local circulation and calorification, without participation of cells or morbid action, and withont inflammation, he argued against those (the neurists), who held that nutrition was under the control of innervation, and maintained that the properties of the cells themselves were the essential causes of nutritive phenomena.

"The increase in the nutrition of parts," he wrote, "should much rather be attributed to certain conditions of tissue (irritation); conditions which are able to modify the affinities of the tissues for the various principles of the blood, or to the presence in the blood of certain substances possessing a special attraction for certain parts of the body. ${ }^{5}$

It is well known that a distinguished pathologist, MI. Spiess, has maintained, in opposition to Virchow, that nutrition is always under the control of innervation. ${ }^{6}$

For myself, I have so often seen alterations of peripheral nutrition supervene consecutively to lesions of certain points of the nervous centres or of nerves, that it would be impossible for me to overlook the influence that innervation exerts upon utitrition. It was in reference to the difficulty of explaining these alterations of nutrition, consecutive to nervous lesion, that I found occasion to say, if there were no trophic nerves it would be necessary to invent them.

C.- Trophic nerves of Samuel.-Such were the ideas entertained in 1858 by Brown-Séquard and Virchow, upon the influence of the nervous system in the production of the phenomena of nutrition and secretion, when, in 1860, Samuel endeavoured to give shape to notions analogous to those of Brown-Séquard, by suggesting an matomical and physiological description of the nerves. which have for their function directly to support the nutritive activity of the tissues.

This learned observer has shown, in a highly interesting volume, the direct, and indirect, action of the nervous system upon nutrition; he has inquired what are the affections of this system, which occasion various disorders, and he has come to the conclusion, from experiments upon animals, and from clinical observation on man, that there are certain special fibres which he has denominated trophic nerves.

The following is a summary of the chief facts upon which he rests his doctrine. According to him, inflammation cannot be

5 Virchow, La Pathologie cellutuire, etc. $\mid$ Frankfurt-am-Main, 1857. srd edition, Paris, 1868.

${ }^{6}$ Spiess, Pathologische Physioloyie, 1 sig, 1860. 
produced either by excitation or by paralysis of the vascular nerves; but, when the spinal nerves are irritated experimentally, the parts supplied by them become inflamed; hence these nerves contain special fibres which may be called trophic. Irritation of these produces acute inflammation, with rapid proliferation of cells; their paralysis is followed by wasting of the tissues. They do not produce chemical changes, but excite only the nutritive activity of the cells and tissues. In the neuralgias attended by eruption, the inflammation attacks both the sensitive and the trophic nerves, whence the simultaneous occurrence of pain and of nutritive disturbance. The trophic nerves originate in the spinal ganglia, or in others corresponding to them,--the Gasserian, for example. They have a centrifugal direction. The foundation, the principle, the cause of nutrition is in the cells; its measure is in the trophic nerves.

D.-The clinical and anatomo-pathological facts observed by Bärensprung and Charcot realise only in part the theoretical ideas of M. Samuel. (a). Exposition of the facts. The hypothesis of the existence of trophic nerves began to be realised, in part at least, (so far as concerns the presence in the spinal ganglia of nervons fibres, exerting an evident action upon the nutrition of the skin), from the time when Professor Bärensprung discovered an alteration of the intervertebral ganglia, in a case of herpes zoster occupying a region that was in relations of innervation with them. ${ }^{8}$

Case X.-Summary.-A zona, occurring without known cause, in a tuberculous boy one year old, extended from the sixth to the ninth rib. More than two inches in width, it commenced posteriorly, not far from the middle line, from the sixth to the eighth vertebra, and forming a demi-cincture, it terminated exactly below the ensiform cartilage. The boy completely recovered from his zona, which followed a regular course, but died from phthisis six weeks after the zona commenced. The spinal ganglia corresponding to the sixth, seventh, and eighth nerves, were firmly adherent to the parietes of the intervertebral canal. The cellular tissue in their neighbourliood exhibited inflammatory redness, and the ganglia, as a whole, were increased in volume. The microscope shewed that the neurilemma also presented unquestionable traces of inflammation. There were, properly speaking, no changes in either the nervous elements of the ganglia or in those of the nerves.

8 Bärensprung, Case XXXVI. Alteration of many special ganglia and of the corresponding intercostal nerves. "Zona," Ann. Charité-Krankh, Berlin, vol. xi., 1863, p. 96.

This learned physician is the author of the most important treatise which has been published of late years, upon herpes zoster. At the close of his first memoir, in which he has brought together fiftyfive new and important cases, he states, for the first time, and before he had ascertained the anatomical fact mentioned above, his theory of Zona. "In conclusion," he says, "zona is an affection of the ganglionic portions of the nervous system; it follows especially from irritation of the spinal ganglia, or of the gasserian ganglion. However, an irritation of the peripheral portions of nerves which contain fibres proceeding from these ganglia, may also be followed by a resicular eruption." 
CASE XI.-The foregoing has been confirmed by another case seen by M. Charcot, in 1865, in a woman seventy-eight years of age, who was under his care at the Saltpêtrière. In this case the intervertebral ganglia and intercostal nerves, corresponding to the surface attacked by the zona, presented precisely the same appearance as in that last described. ${ }^{9}$

(b). Critical examination.-The following, I think, are the deductions that should be drawn from the anatomo-pathological facts of Bärensprung and Chareot. 1. Inflammation of spinal ganglia anatomically characterised by a well-marked vascular injection, and a proliferation of the perineurine, without lesion of nutrition, or with only a slight change of the nervous elements, produces, in the portions of skin which receive innervation from the parts affected, a special lesion of nutrition, of the nature of zoster. 2. The neuralgia which accompanies the zona is the result of excitation of the sensitive nerve-filaments which pass through the ganglion. 3. The spinal ganglia contain the trophic nerve-filaments of the skin. But the anatomo-pathological facts in no way confirm the general doctrine of Samuel and Bärensprung, who maintain that the trophic nerves originate in the spinal ganglia.

II. Chareot, a more strict observer, and one who never goes beyond his facts, has been too careful to draw any general conclusion from his own particular case.

The clinical facts do not prove, indeed, that other parts of the nervous centres may not contain trophic nerves of the skin. Clinical observation shows, moreover, that, under certain circumstances, lesions of the cord will alter the nutrition of the skin and of the subcutaneous cellular tissue. Of this I could cite many examples from my practice. Thas, in infantile spinal paralysis, the anatomical lesion of which is a wasting or destruction of the cells and a fibroid metamorphosis of the anterior cornua, ${ }^{1}$ whenever a muscle has been attacked in its nutrition and texture (granular or fatty degeneration), I have observed that the subcutaneous cellular tissue covering it was hypertrophied, and sometimes that the nutrition of the skin was altered in varions ways. These conditions will be more fully described in the chapter which treats of the spinal or fatty atrophic paralysis of infancy.

I should observe, also, that inflammation of the spinal ganglia produces no lesion of nutrition excepting in the skin. How, then, shall we explain the alterations of nutrition of the muscular and even of the osseous system, which have been observed consecutively

9 Charcot et Cotard. Altération (néurite) du plexus cervical et des ganglions correspundants des racines spinales posterieures, Zona du cou (Memoires de la Société de biologie, 1865, p. 47).

1 This anatomo-pathological fact, dis- vered in 1866 by M. Prevost, interne to M. Vulpian, has been confirmed by a recent autopsy in a case of infantile paralysis, communicated to me by M. Charcot. 
to certain lesions of the cord, if, as Samuel and Bärensprung believe, the trophic nerves take their origin only from the spinal ganglia. I shall hereafter describe cases which show, in the spinal disease of infancy, the muscular nutrition suffering more profoundly than the osseous, and vice versâ.

It is well known that peripheral nervous lesions produce various changes in the skin, which prove the presence of cutaneous trophic filaments in the peripheral nerves. This fact, first pointed out by the illustrions English surgeon, Mr. Paget, ${ }^{2}$ has been confirmed by other observers. I remember that I have myself often witnessed it; but I must confess that I did not perceive its importance, or, at least, that I did not investigate its mechanism.

I should observe, also, that in progressive locomotor ataxy (sclerosis of the posterior columus of the cord), and in secondary sclerosis of the lateral columns, consecutive to hemorrhage in the corpora striata, we sometimes have lesions of nutrition of the skin (eschars), of the articulations, and of the tendinous sheaths (arthropathies). These important facts have been discovered by MI. Charcot. $^{3}$

The foregoing facts and considerations seem to me to show that the origin of the anatomical elements of the spinal ganglia is subject to a general law of centralization; which it is the tendency of physiological experiment, and of clinical observation, more and more to establish. Thus, the great sympathetic was considered as a kind of nervous centre independent of the spinal centre; but observations and experiments have shown that lesions of the cord and of the medulla produce, in local circulations, disorders analogous to those which follow direct lesions of the sympathetic. These facts prove the existence of nerrous fibres of the great sympathetic, in the points of the spinal centre from which the nerves producing such disorders take their origin. In the same way it has been shown that the spinal ganglia shonld not be considered as the exclnsive centres of the trophic nerves in general, nor even of the cutaneous trophic nerves, which seem only to hold to them a relation of particular dependence.

15.-Anatomical considerations.-(a). According to Bärensprung, anatomy shows that the spinal ganglia are nerrous centres, the unipolar cells of which do not communicate with the spinal cord. He rests upon the opinion of Kölliker, who wrote: "As far as I

\footnotetext{
2 Taget, 'Surgical Pathology', Vol. i., pathologirque, tom. i., 1868, pp. 308-313).Mredical 'Times,' 1 sift.

${ }^{3}$ Chareot, Note sur la formation rapide t'une pscharre à la fesse du côté paralysé - dans lilémindégie réernte de canse cérésur quetques arthropathies qui paraissent dépendre d'une lésion du cerveau ou de la moelle épinière (Idem., pp. 161-178, 379400, Pl. (6).
} brale Archives de physiologie normale it 
have been able to observe, there is not in the ganglia any relation of continuity between the posterior roots and the ganglionic globules. It follous that each ganglion should perhaps be considered as a source of new fibres. In man and the mammalia, I have shown the existence of unipolar cells; and I believe I may even affirm that these cells are very numerous." ${ }^{4}$

This opinion of Kölliker's is in opposition to that of anatomists in general, who believe that most of the cells possess two poles, of which one is continuous with the spinal cord, the other with a peripheral nerve-fibre. It cannot, moreorer, be sustained, in the presence of my own icono-photographic researches. In the longitudinal sections of the spinal ganglia that I have photographed, I have found cells apolar, unipolar, and bipolar, the number of one or of the other predominating according to the chances of the section. Fig. 43, which represents a longi-

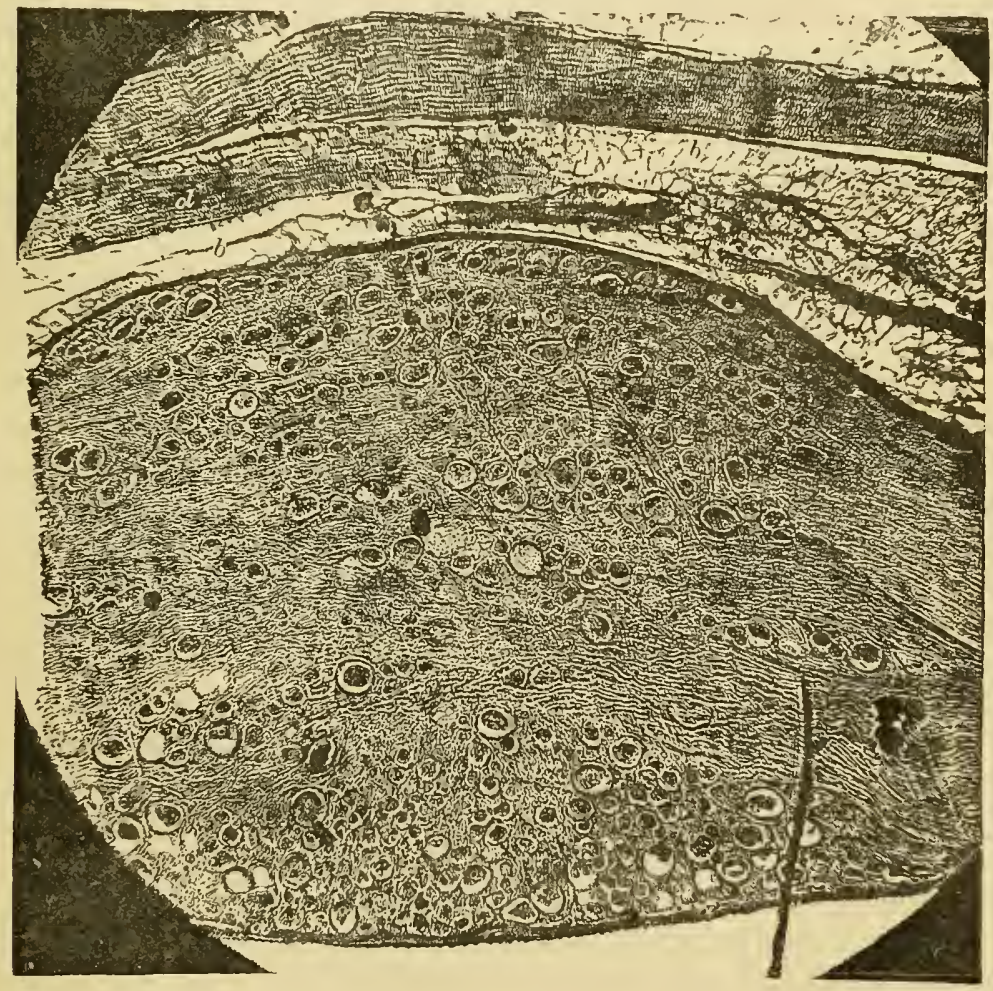

Fig. 43.-1.ongitudinal section of a spinal ganglion from the cervical region, photographed and heliographed to 21 diameters.

"A. Kölliker, Elénents thistologie humaine: "Stmeture des ganglions spinan.," P. $355,1856$. 
tudinal section of a spinal ganglion in the cervical region, and which has been heliographed after a direct photograph of 21 diameters, is an example. We see, in this figure, that the cells are collected in great numbers towards the walls of the ganglion (an anatomical arrangement that $I$ have found in all the sections of spinal ganglia, whether longitudinal or transverse, that I have made). It will be remarked that, towards the free margin $a a$ of this longitudinal section, nearly all the cells are apolar (their circular form is distinct), while on the opposite side, $b b$, in the vicinity of the posterior root, the cells are bipolar and unipolar, as shown by their pear-shaped or oval outline. Well, sometimes the reverse is the case; which proves that the appearances depend upon the chance of the section having been made through a greater or less number of such or such cells. It is difficult to say, even approximatively, what is the proportion of bipolar or unipolar cells, for sometimes the number of bipolar cells is the greatest, sometimes the contrary ; and it is, moreover, conceivable that cells which appear to be apolar in a given section, may be bipolar in reality.

Kölliker says also:- "Even where a cell gives off two prolongations, it does not follow that one of them must be centripetal, and the other centrifugal: on the contrary, both may be directed towards the periphery; at least this is what is seen in examining very small gauglia. Stannius himself found, in bipolar cells, the two prolongations uniting the one with the other." ${ }^{5}$

In not one of my longitudinal sections of spinal ganglia, have I met with this peripheral direction of the poles of bipolar cells. The poles always extend in opposite directions, one towards the cord, the other towards the surface, as shown in fig. 44, drawn from a photograph taken from nature to 200 diameters, at the point $c$ of fig. 43, which, as already described, represents a photograph, to 21 diameters, of a spinal ganglion. Fig. 44 shows an apolar cell, $a$, two small unipolar cells, $b, b^{\prime}$, and two large bipolar cells, $c, c^{\prime}$.

All my transverse sections of spinal ganglia have shown a certain number of cells that were each in communication with another cell, more or less near to them, by means of a lateral prolongation, the texture of which, at least, was in all respects like that of the central or peripheral prolongations; the lateral prolongation, arising from the middle portion of the cell, appeared to be a sort of nucleated sheath, which was continuous with the nucleated reticular envelope of the cell. I think I am entitled to conclude, 
from these anatomical facts, that the spinal ganglia, without doubt, contain tripolar cells, of which two poles are continued respectively

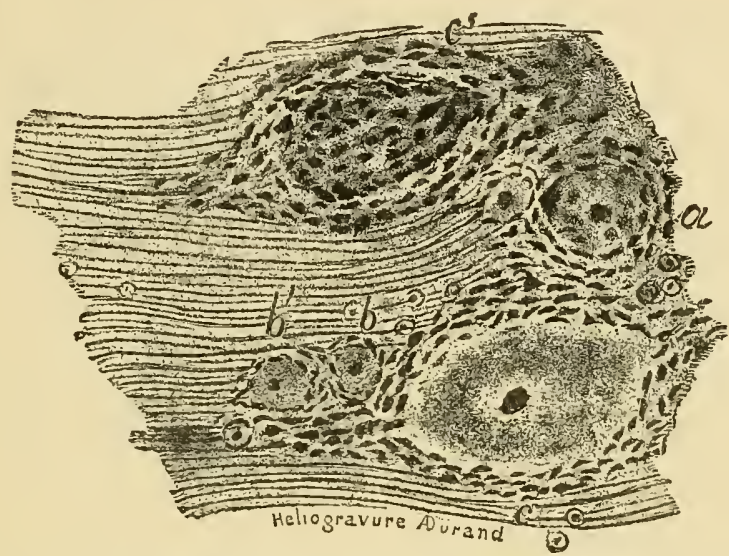

Fig. 44.-Cells plyotugraphed and helingraphed to 200 diameters, at the point $c$ of the spinal ganglion photographed to 21 diameters in Fig. $43.6^{6}$

in a central and a peripheral direction, like those of the cells $c$ and $c^{\prime}$ in fig. 43 , and the third, in a lateral direction.

If we suppose that, in endeavouring to isolate a tripolar cell, its central pole had been broken in preparation, we should have the appearance of a cell the two poles of which had a peripheral direction, as in the bipolar cells described by Kölliker.

In conclusion, it has been shown that the cells of the spinal ganglia communicate anatomically, in part at least, with the spinal cord; and that we should not consider these ganglia as the centres of the trophic nervous filaments. Are they, possibly, organs of re-inforcement for the trophic nerves of the skin?

From these data many clinical facts receive their explanation. Thus, inflammation of certain portions of the cord is not only followed by atrophy, with grauular or granulo-fatty degeneration of muscles, and by atrophy of bone, but also by a slight lesion of untrition of the skin, which becomes more or less wrinkled, scaly, and earthy, and, lastly, by decrease of temperature, with hyperplasia of the subcutaneous cellular tissue, in the locality of the wasted muscles. I have observed this assemblage of phenomena hundreds of times in the spinal paralysis of infancy; the anatomical cause of which, as shown by several autopsies, is situated in the

${ }^{6} a$, a polar cell ; $b, b^{\prime}$, two small mipolar cells; $c, c$ ', two large bipolar cells. 'The cell $c$, cut through the middle, displays its grannlar contents and nucleus, which are bidden in the cell $c^{\prime}$ by its reticular covering. 
anterior cornua (I shall hereafter describe its anatomical characters). To the above described disorders of nutrition will be added a more grave alteration in the skin, such as herpes or eschar, when the inflammation attacks not only the cord, but also the spinal ganglia, organs of re-inforcement of the trophic nerves of the skin. I have seen this happen a certain number of times, consecutively to fracs tures of the vertebral column, in which, at the place of injury, the cord and the ganglia had been entirely destroyed, either by the injury itself, or by consecutive inflammation. I do not doubt, that the same lesions of nutrition will be found in cases of spontaneous and simultaneous inflammation of the cord and the spinal ganglia, when it is no longer customary to neglect, as at present, to examine the condition of these organs. Lastly, when the inflammation is limited to the spinal ganglia, it will produce alterations of nutrition limited to the skin. 'This is what has been observed in the cases of zona published by Bärens]rung and Charcot.

(b). It follows, from the facts and the considerations above set forth, that the cerebro-spinal centre contains the nervous filaments which promote local circulation, and the nutrition of the tissues. What, then, should be the distinctive characters of these filaments? If this question be one day answered, it will be only by the concurrence of clinical observation with pathological anatony. At present I only attempt to call attention to it, in recording an

$a \times 200$

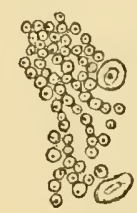

$6 \times 200$

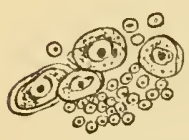

c $\times 400$
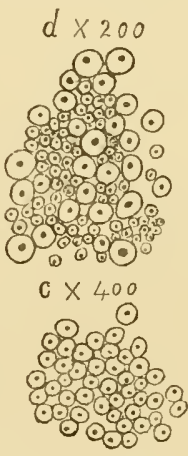

Fig. 45.-Transverse section of the nervetubes of a sympathetic ganglion, enlarged 200 diameters at $a$ and $U$, and 400 diameters at $c$; and of the anterior column of the cervical enlargement of the cord, enlarged 400 diameters, at $d$. from two such sections. ' They show a great number of these anatomical fact that my icono-photographic researches have brought under my observation.

In all the transverse sections that I have made, at different heights, through the cord, the medulla, and the anterior and posterior roots, I have found a multitude of nervous tubes, abont $0^{-\mathrm{mm}} .0033$ in diameter, scattered in groups among the motor or sensory tubes, of a diameter ranging from $0 . \mathrm{mm} .01$ to 0.02 and 0.03 . This anatomical fact has been brought to light by the photographs of the transverse sections of the several parts. The figures 45 and 46 have been drawn i I sent some of these photograplis to the Ararlémic des Sriences, in 1864, and called attontion to the number of small nervous tubes, of $0 \cdot \mathrm{mm} \cdot 00: 23)$, that were scattered through all points of the merlulla, the corrl, and the spinal ronts, and mixed with nerve tubes of $0^{\cdot m m} 01$, $(0 \cdot 0) \cdot 2$, and $0 \cdot 0: i$. 
small nerve-tubes placed betreen much larger tubes, and appearing in the transverse sections of: 1 . the anterior columm

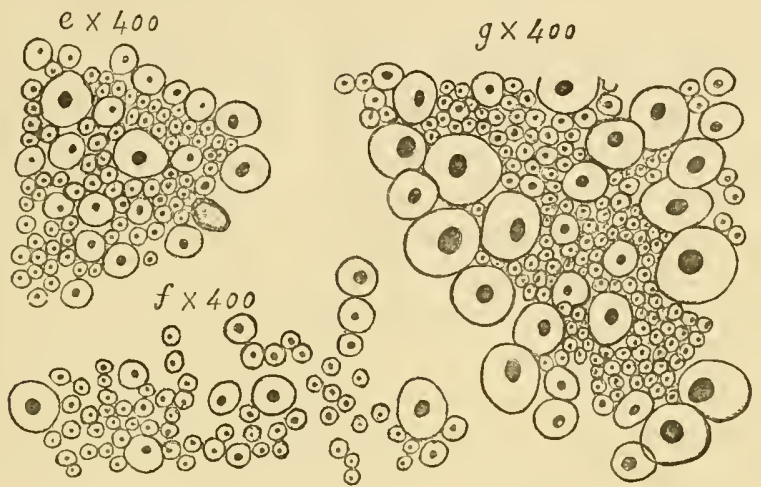

Fig. 46.-Transverse section of nerve-tubes at different heights in the medulla at $e$ and $f$, and in a posterior root at $g$.

at the cervical enlargement, at $d$, fig. 45 , to 200 diameters; 2 . the antero-lateral column of the lower portion of the medulla, at $e$, fig. 46 , to 400 ; 3 . at the level of the calamus scriptorius, at $f$, to 400 ; 4 . of a posterior spinal root, at $g$, to 400 .

All the groups of minute nerve-tubes in the columns of the spinal cord, and in the anterior and posterior roots, are far from being conclusive examples; and I hare selected those depicted at $d, e, f$, and $g$, in support of my thesis.

I should also remark that the minute nerve-tubes present the same diameter as those of the tubes represented at $a, b$, and $c$, in fig. 45, which have been drawn from different transrerse sections of sympathetic ganglia, photographed, at $a$ and $b$ to 200 , and at $c$ to 400 diameters.

Are they, in part, the origins of the great sympathetic, and do they contain, besides, the dilatator nerves of the vessels and the trophic nerves? The investigations I have made induce me to admit the hypothesis; but I repeat that I have no higher pretension than to raise the question.

\section{Part the Fourth.}

THE THERAPEUTIC VALUE OF GALVANIZATION BY INTERRUPTED CURRENTS, AND BY CONTINUOUS CURRENTS. DIRECT OR INDIRECT.

$$
\text { § I.-Historical (early experiments.) }
$$

The experiments that have been made upon the important question that forms the subject of this paragraph are so numerous, 
that they would furnish subject matter for more than one volume, if it were necessary for me to describe them in detail. Not wishing to exceed the limits that I have set myself, I am compelled to give only a brief summary of the principal facts that have been brought to light by these experiments.

At the commencement of my own researches, galvanic excitation of nerve-trunks by continuous currents appeared to me to be the method of electrization most applicable to paralysis in general. I knew that Alexander von Humboldt, in 1795, had opened the way to galvanic treatment, by an experiment made upon himself that is too well known to be related here, as well as by the general impulse which he had given to such researches. My reading had also informed me that, immediately after the discovery of the pile by Volta, galvanic currents had been applied, in Germany, to the treatment of some forms of paralysis and of nervous affection until 1804 or $1805,{ }^{8}$ and that these therapeutic experiments had been repeated in 1823 by Aldini, and afterwards by Nobili and Mattenci.

It seemed to me that the discoveries of Galvani, ${ }^{9}$ Volta, ${ }^{1}$ Ritter, ${ }^{2}$ Alexander von Humboldt, ${ }^{3}$ Jean Aldini, ${ }^{4}$ Valli, ${ }^{5}$ Lehot, ${ }^{6}$ and, since 1827, those of Marianini, ${ }^{7}$ Nobili, ${ }^{8}$ and Matteuci, ${ }^{9}$ were alone sufficient to furnish the elements of a rational electric treatment. Their works taught that, in animals, it was possible to destroy or

8 The following are the principal authors who, from 1797 to 1823 , have followed the expcriments of Humboldt, with regard to the therapentic action of galvanic currents:-Loder, Journal für Chirurgie, tom. iii.; Grappengiesser, Versuche den Galvanismus zur Heilung einiger Kranliheiten anzuwenden. Berlin, 1808. Augustiu, Versuch einer vollständigen Systemgeschichte; Struve, System der medizinischen Electricitatheher mit Rüclisicht auf den Galvanismus. Breslau and Leipzig, 1802.

${ }^{9}$ Galvani, Sur l'irritabilité hallérienne, Mémoires communiqués à l'Institut de Bologne, 9 Avril, 1772; Sur les mouvements musculaires des grenouilles, 22 Avril, 1773 ; Sur l'action de l'opium sur les nerfs des grenouilles, 1774; Commentaire De viribus electricitatis, 1791.

1 Volta, Collezione delle opere. Firenze, 1816.

2 V. Ritter, Preuves qui un galvanisme independant accompagne le processus vital dans le règne animal. Weimar, 1798.

3 A. von Humboldt, Versuche über die gereizte Nuskel und Nerven faser. Posen and Berlin, 1797, tom. i. 324. Expériences sur le galvanisme. Paris, 1793.

4 Aldini, Essai théorique et experimental sur le galvanisme. Paris, 1804. s Valli, Lettres sur l'électricité à Desgenettes et de Lamétrie, 1792.

' Lehot, Mémoire lu à l'Institut, 26 Frimaire, an. IX.

7 Marianini, Mémoire sur la secousse queprouvent les animaux au moment où ils cessent de scrvir d'are, au moment oic its cessent de servir de communication entre les pôles d'un électro moteur, et sur quelques autres phénomènes physiologiques produits par l'élertricité (Annales de Chimie et de Pluysique de Gay-Lussac et Arago. Paris, 1829, tom. xl.). Mémoire sur le phénomène électro-physiologique des alterzatives voltaiques, e'est-à-dire sur les plénomènes que présentent les muscles des animaux récemment tués, si l'on soumet longtemps ces muscles au courant électrique (Annales de Chimie et de Physique. Paris, 1834, tom. Ivi.)

s Nobili, Analisi sperimentali e teorica degli effetti electro-fisiologici della rana, con un" Appendice intorno alla natura del tetano, della paralisi, ed al modo di curare queste due malattie colla elettricita. Firenze, 1834.

${ }^{9}$ Matteuci, Traité des phénomènes électro-physiologiques des animaux. Paris, 1844. 
to diminish the excitability of a nerve, and at the same time to paralyse the limb under its control, by maintaining for a given time the passage through the nerve of a continuous current of a certain intensity; that, by an intermittent direct or inverse current, ${ }^{1}$ directed upon the nerve thus modified in its excitability by the continuous current, one could act upon the contractility, or upon the sensibility; and that it was possible to re-establish the excitability of the same nerves, by causing them to be traversed by currents in the contrary directions (alternative voltaic currents.)

From the principal facts established by a series of experiments upon animals, Mattenci thought himself able to draw electrotherapeutic deductions. Of these, I will only cite the more important. In speaking of the application of the various galvanic currents to the treatment of paralysis, this skilful physicist says :"We must admit that, in some cases of paralysis, the nerves of the limbs are altered in a manner analogous to the alteration which is produced by the continuous passage of an electric current. We have seen that, in order to restore to a nerve the excitability which has been lost by the passage of a current, it is necessary to expose it to the action of a current travelling in an opposite direction. In the same way, to remove a paralysis, we should cause the passage of a current in an opposite direction to that which would have produced it. We lience suppose that the paralysis that is to be subjected to electrical treatment is either of sensation or of motion only. Thus, for paralysis of motion, it is the inverse current that should be applied; and for paralysis of sensation, the direct current. In a case of complete paralysis, there would no longer be any reason for deciding upon the application of either one or the other.'

§II.-Physiological action of continuous currents, direct or inverse, applied to the nerves of man, upon sensibility, or muscular contractility.

\section{I.-MY FIRST EXPERIMENTAL RESEARCHES.}

I proceed now to the experimental researches that I undertook, in the hope of being able to determine the value of the foregoing therapeutical deductions.

1 A direct (centrifugal, or descending) current is produced by placing the rheophores over the course of a nerve, at a distance of two or three centimetres apart; the positive pole being nearer to the nervous centres, and the negative pole nearer to the extremities. For the inverse (centripetal, or ascending) current, the position of the poles is reversed. Such has been the method employed by experimenters.

${ }^{2}$ Matteuci, loc. cit., p. 266. 
Before arriving at conclusions, from experiments upon animals, with regard to the therapeutic action of continuous currents when applied to human patients, it seemed to me more rational to inquire whether the electro-physiological phenomena, observed in vivisections, would be produced in a healthy man. But how could a current be directed upon a nerve, without first laying it bare? Assuredly no one would venture to attempt such an operation in the human subject, for the purpose only of promoting scientific research. And electro-puncture, in which the needle traverses organs of different nature, and, as I have already shown, produces complex phenomena, would not serve to display the action of continuous currents upon nerve-trunks.

It is now perfectly demonstrated, after my researches, that, without puncturing or incising the skin, we may limit the electric action at pleasure, either in the skin or in subcutaneous organs; and that by certain methods of proceeding, the electric excitation will reach a nerve, without acting upon the skin that it penetrates. Certain, from this, that I could limit the electrical action to the chief nerve-trunks, I have studied, in a large number of subjects, the influence of continuous currents upon contractility and upon sensibility. I have principally selected for my experiments the median nerve, the erural, and the internal and external popliteal. Sometimes the rheophores have been placed on the course of a nerve at a point where it is subcutaneous, and two or three centimetres apart; and sometimes they have been separated as wirlely as possible, by placing, for example, one upon the brachial plexus, or upon the sacral plexus at the posterior wall of the rectum, and the other on the course of the nerve. The rheophores have been placed in such a manner as to be only in relation with the surface of the nerves.

It has been shown, by numerous experiments upon animals, that the continuous current manifests its special influence upon contractility or upon sensibility, according to the direction in which it passes longitudinally within the nerve, when the excitability has been previously diminished by the more or less prolonged action of a continuous current.

To discover the influence of a continuous current upon a healthy man, it was therefore necessary that the excitability of his nerves should be first modified by the continuous current. For this purpose, I employed, in the first instance, a trough battery (of Cruikshank and Wollaston) composed of sixty couples, and I caused a continuous current to pass through the nerves for from twenty to thirty minutes, by placing them in the circuit of this battery, and by using the method of procedure indicated above. 
This attempt having produced no result, I thought that a more powerful and more constant current might diminish the excitability of the human nerves; and I then had recourse to a battery composed of thirty of Bunsen's pairs, a little weakened by previous use. Afterwards I employed a Daniell's battery of from thirty to fifty elements. In all these experiments, I took care to avoid the occurrence of contraction on completion of the circuit, by proceeding in the way formerly described. (See note to page 12.) After twenty or thirty minutes of action of the continuous current from these batteries, in whatever direction, I was unable to discover that the sensibility of the nerves had diminished. The contractions of the muscles supplied by the nerves were quite as energetic, under the influence of an intermittent current, after, as before the experiment; the sensibility was not modified, and the power of voluntary movement was not diminished.

Although I was unable to diminish artificially the excitability of human nerve, I had at least occasion to observe certain instances of paralysis, in which the nerves and even the muscles had in part lost their excitability. In these cases again, the inverse continuous current acted no more upon the contractility, than did the direct continuous current upon the sensibility. Neither of these currents abolished the excitability of nerves already afiected, and through which they were made to pass for a considerable time.

To sum up, in a man in normal condition: 1. a continuous current passed through a nerve, during from twenty to thirty minutes, did not appear to me to diminish its excitability; 2 . when I directed a continuous current, centrifugal or centripetal, upon a nerve of normal excitability, or upon a nerve the excitability of which was diminished by certain forms of paralysis, I observed always the customary phenomena, namely, contractions and sensations; neither the one nor the other of the two (direct and inverse) currents ever acted, in an appreciable manner, upon either the sensibility or the contractility separately. ${ }^{3}$

These results caused me real disappointment: since I had expected to obtain, in man, the phenomena described by Marianini, Mobili, Matteuci, and their predecessors; and concerning which the experiments made upon animals seemed to me to be entirely conclusive.

It will be said, without doubt, that if I had directed upon human nerves a more intense current, I should have been able to diminish

3 I formulated almost the same conclusions in a memoir presented in 1848 to the Academie des Sciences. But at that time I had not endeavoured, in my ex- periments, to diminish the irritability of nerves, by subjecting them to the influence of a powerful continuous current. 
or destroy their excitability. But this objection might be made again, even when I had unsuccessfully experimented with a more powerful battery. And who would attempt such experiments upon man? Who would willingly submit to very painful experiments, capable of producing vesications, and even eschars, upon the skin?

It will also be objected, that I did not experiment under the same conditions, and that I should have caused the currents to pass through nerves previously laid bare. But it is demonstrated, I repeat, that I act with as much certainty through the skin, as I could do upon nerves exposed and isolated. My method seems to me to be even preferable to that employed by Matteuci and his predecessors; because the mutilation of a nerve, and its contact with the atmosphere, must inevitably exert a certain influence upon its vitality, or upon its excitability.

\section{II.-The experimental researches of Remak.}

The facts and the electro-physiological considerations, that I have stated above, were made the subject of a work that I published in 1852. But, since then, Remak has experimented-anew upon the action of continuous constant currents upon the nerves of healthy and of diseased men $;^{4}$ and he claims to have discovered electro-physiological facts, which to me do not appear to be demonstrated, and which, if they were real, would possess neither the weight nor the importance that he assigns to them.

We know that galvanic electricity possesses the property of producing a muscular contraction at the completion, and at the interruption of the circuit; the former being stronger than the latter. It has been stated that, during the interval between these two periods, the continuous current produces no contractions. I lave myself only observed the occurrence of fibrillar, oscillatory, and irregular contraction in the muscles of a limb that was traversed by a continuous current, powerful, and as constant as possible. I have also found that a galvanic current, directed upon the fifth nerve, produced an intense luminous sensation at the completion of the circuit, and a similar but less intense sensation at its interruption, and between these two periods, during the passage of the continuous current, a luminous sensation that was only appreciable in obscurity. (See Chap. I., p. 13.) When I have applied the moist rheophores over the median nerve, one by the inner border of the biceps, the other at the passage of the nerve over the bend of the arm, I have observed-besides the acute and

4 Remak, Galranotherapie. 
progressive cutaneous pain at the seat of the rheophores, so acute as to be almost unbearable, and besides the tingling and pricking experienced in the whole course of the nerve, down to its final ramifications in the fingers-similar oscillatory contractions in the muscles that it supplies.

Remak has gone further. He professes to have observed, in experiments analogons to my own, and made upon the median nerve of a healthy man, with a battery of thirty of Daniell's conples, the contractions continue, sometimes in the muscles subject to the median, sometimes, and commonly, in the muscles subject to the radial nerve, the antagonist of the median. Galvanization of the radial nerve, practised by placing the rheophores on two distant points of its comrse, produced, on the contrary, continued contraction of the muscles subject to the median nerve, the antagonist of the radial. These continued contractions have been called galvano-tonic by Remak-thus named, he says, to distinguish them from the tonic, or tetanic, contractions produced by induced currents, or by constant currents often interrupted. This phenomenon, on which Remak has built a new physiological theory (but, as I shall hereafter show, an imaginary one), forms the fundamental basis of that which he calls his method of galvanization.

I am sorry to have to declare that I have never been able to produce these singular phenomena in the galvanic experiments that I have made publicly upon human nerves in the course of my clinical teaching. What can be the reason? Is it that the acute and increasing cutaneous pain, at the place of contact of the rheophores, has produced involuntary movements of flexion and extension of the fingers, as if the subjects sought instinctively to escape from the suffering, and that these movements have perhaps concealed the galvano-tonic contractions, if thoy indeed occurred? Or were they not produced, in my experiments, because I have always avoided the contraction due to completion of the circuit, as I have already said, or because my rheophores were too near each other? Remak, indeed, applies one rheophore on the hand, and the other at the bend of the elbow; while, I, in general, have held them only tivo or three centimetres apart.

Desiring to test these electro-physiological facts announced by Remak, and to appreciate the worth of the explanation given by him, I have again many times galvanized the median nerve in the manner described by him. I have, moreover, not prod'uced the socalled galvano-tonic contractions, even after having allowed the current to pass through the nerve for more than a minute. Although I included, according to his precept, the greatest 
possible length of nerve in the circuit, and although I employed a powerful and very painful current, I must still declare, that I have seen nothing but the involuntary movements of flexion or extension of the fingers or the hand, and sometimes flexion of the forearm upon the arm, such as were produced in my former experiments, and which are due to the intolerable pain that is sometimes caused by the local action of the rheophores upon the skin. Remak knows so well that these instinctire movements occur during the experiment, that he has written, "It is, of a truth, difficult for many persons to abstain from calling into play their volition, and to leave the action exempt from the influence of such innervation, engendered at the moment of entrance of the current." 5

I wished, like other persons present at my clinical lectures, and who yielded themselves to the experiment, to nerve myself against the pain, and to prevent involuntary movement; but then we did not witness, in the muscles subject to the antagonist of the excited nerve, the so-called galvano-tonic contractions of Remak. On one occasion only, in one of these experiments, we observed that the contraction of the moment of completion was prolonged in the muscles supplied by the excited median nerve; but this phenomenon is not so new to science as has been said.

"The production of galvano-tonic contractions," says Remak, "is commonly promoted by the same circumstances which facilitate the contraction on completing the circuit,--that is, a sudden and prompt application of the rheophores over the nerves." I have observed, indeed, that the movements of the hand and of the fingers, which take place at the moment when the circuit is completed, sometimes continue, but only for a very short time. (This phenomenon had previously been observed by Ritter.) Under such circumstances there is certainly a complication of phenomena; that is, the contraction due to the completion of the circuit, and the contraction caused by the pain, or that which is produced, according to Remak, by the passage of the current.

In order to avoid the contraction at the completion of the circuit, I have caused the current to pass through a thick stratum of liquid, in such a manner that the completion produced no contraction; then I have progressively increased the tension of the current, until it attained its maximum; and, if I prevented the instinctive movements occasioned by the pain, I never perceived any contractions excepting those fibrillar or oscillatory ones already mentioned, and which are probably occasioned by those inequali-

semak, Galranotherapie, p. 56. 
ties of the current which are inevitably produced in even the most constant of the batteries themselves.

It follows therefore from my own experiments (made for the most part publicly), and from the foregoing considerations, that the production by continuous currents of the so-called galvano-tonic contractions has not at present been demonstrated.

Remak attributed these pretended galvano-tonic contractious, which occurred during the passage of a continuous current through a nerve-trunk, in the muscles controlled by its antagonist, to a reflex action of the cord; and he consequently thought that they were produced by reflex excitation of a sensitive nerve.

I crave pardon from my readers for stating the theories and hypotheses hazarded by Remak, in his wish to explain facts thus complex and irregular. It is, however, upon such facts, and such theories, that this physiologist has faucied that he has built up a new method of therapeutic galvanization! I shall have occasion to return to the subject.

No one, indeed, will contest the novelty of his theories; but in what consists the novelty of his manner of exciting the human muscles by a continuous current? We have already seen in this part (pages 176 and 177) that in my investigations long ago (and many others had practised them before me) I had caused a continuous current, as constant as possible, derived from twenty or twenty-five of Bunsen's elements, or from a Daniell's battery of from thirty to fifty elements, to pass through the continuity of human nerve. It is true that most commonly I placed my rheophores over the course of the nerve-trunk somewhat nearer together than was done by Remak. But does this slight difference in the procedure constitute by itself a new galvano-therapeutic method? And then, who can believe that so slight a difference between the procedure of Remak and my own could occasion such differences in our results?

$\S$ III. My latest researches on the therapeutic action of galvanization by interrupted or by continuous currents, in the treatment of paralytic or nervous affections, and as compared with localized faradization.

\section{Historical ANd Critical.}

A.-My first researches.-Considering the negative results of the electro-physiological experiments that I had made upon the action of direct or inverse continuous currents traversing the nerves of man (see $\S$ II.), it may be imagined that I no longer attached any weight to the electro-therapeutic deductions of Hattenci. In my 
first experiments on the therapeutic value of galvanization, I wished to obtain proof of the correctness of this estimate, by submitting cases of paralysis of movement to the influence of the continuous current, directed in the manner that he recommends.

In spite of the rigorous observation of the principles of muscular galvarization that he laid down, I did not succeed in modifying paralysis of movement more favourably than when I excited the nerve-trunks in their continuity, without any heed to the direction of the currents.

It was also shown by my researches that the direct current is entirely powerless against museular or cutaneous anæsthesia; just as, in the normal state, $\mathbf{I}$ had found that this current exercised no appreciable special influence upon the sensibility.

The critical consideration that I have given to the influence of the direction of the current upon the nerves of man, either in the physiological, or in a pathological condition, were the more necessary, since the doctrines of Matteuci and the physiologists in general had been re-echoed, and had exerted a considerable influence upon the practice of electrical treatment. Physicians, and especially those among them who were given to scientific research, had too long attached great importance, in the galvanization of man, to the direction of the course of the current in the nerves.

B.-Pemak's method; Hiffelsheim's method.-The therapeutic employment of continuous currents had been generally abandoned when, in 1858, Remak endeavoured to reluabilitate it by creating. a method of galvanization of which the following are the fundamental principles:-1. To cause the passage through the nerves or through the mascles of eurrents that were commonly labile and sometimes stabite, ${ }^{6}$ during a period of time that did not usually exceed fifteen minutes; 2. to produce, according to him, the reflex contractions that he denominated galvano-tonic.

Afterwards came the method of Hiffelsheim, ${ }^{7}$ by continuous currents described as permanent; meaning by that, that he caused the passage of a stabile current through organs for a long time, (from one to several hours).

Until the fundamental principles of these methods of galvaniza-

- A current is called stabile that is furnished by a battery of equal tension, and applied by rheophores each of which is kept stationary upon the same point of skin. A labile current is one furnished by the same battery, but applied by rheophores one or both of which are moved about upon the skin, without interruption of the circuit. In the same way, the contractions produced by the former method are a!so called stabile, and those produced by the latter method are called labile.

7 Hiffelsheim, Des Applications máticales le la pile de Tolta. Paris, 1861. 
tion are better defined, and more clearly formulated, it will be difficult to judge of their actual value.

(a). After Remak's method, the galvanic currents are applied with a kind of intermissions closely approximated, as I will proceed to show. In his experiments at La Charité, in 1860, every one saw that, when the current was made to pass through the muscular nerves, by placing the negative pole over the point of entrance of the muscular branch, and the positive pole over a point nearer to the spinal cord, he dwelt but a very short time (fifteen to thirty seconds), over the muscle to be electrified, and soon drew one or both of his rheophores over the surface, to fix them upon other points. In this way he produced what have been called labile contractions. But, as I have said above, these contractions are produced by a kind of intermissions, and only differ by their less intensity from those that are produced by actual intermissions. ${ }^{8}$ Such also is the opinion of MI. Rosenthal. ${ }^{9}$ Nay more, these labile contractions are produced by a veritable localized galvanization by intermittent currents, which is shown elsewhere by phenomena analysed by Remak himself: "When the excitability is sufficient," he says, "or "when it is artificially increased by the current, the labile contractions are produced by changing the place of one or both rheophores, without breaking the circuit, or interrupting the contact of their moist coverings with the skin. The muscular fibres that are touched by the rheophore contract, while those that the $r$ heophore leaves become relaxed. If the moving rheophore follows, over the muscle, the course of a nerve, all the fibres supplied by this nerve are called into action, when the sensibility is strong; those which are without the circuit in a much less degree, so that the local action on the muscular fibres might appear to be predominant." "

Who is there that will not recognise, in all these phenomena, those which are proper to localized galvanization? Remak himself has said as much in the lines that I have quoted, without understanding their significance.

It appears, then, that each of the séances, which we all witnessed at La Charité, was composed of a great number of weak (labile) galvanic intermissions, and of a certain number of continuous

8 Remak seems to me to have admitted this in the following passage:- "It is very important," he writes, "in relation to therapeutics, to be aequainted with the labile contractions, which I was the first to observe in man. These contractions are produced in the muscular nerve or in the muscles, witlout interruption of the current, by simple oscillations of its density; according to the law laid down by Dubois Reymond, that a muscle responds by a contraction, not only to an interruption, but also to an oscillation of the current. (Loc. cit., p. 120.)

${ }^{9}$ Loc. cit., p. 121.

1 Hiffelsheim, loc. cit., p. 12. 
currents of short duration, applied in the intervals between the labile contractions. This is what constitutes for Remak, or is called by him, galvanization by a continuous current. And it is in this manner that his pupils, or his adepts, have applied this method. The results obtained by such proceedings may as well be ascribed to the interrupted and localized galvanic currents as to the continuous currents. With regard to the so-called galvanotonic contractions, I shall shortly show that they have never existed except in Remak's imagination.

(b). Other experimentalists had caused continuous currents to pass through organs, or through paralysed limbs, in a permanent manner. I had commenced some electro-therapentic experiments by this method of galvanization, with my friend Aran, but unfortunately his premature death interrupted them.

The proceeding habitually employed for this purpose by Hiffelsheim ${ }^{2}$ is sufficiently original to be here described. Two moist sponges are kept fixed upon two points of the skin, such as to represent the extremities of an axis traversing the organs that it is desired to influence. A small stem of platinum, at least the fifth of a millimetre in diameter, is contained within each of the sponges, and these stems are in commmication with conducting wires leading to a battery of sulphate of lead, composed of a large number of elements of small surface, and of weak electrolytic action. The wires are coiled in spirals, and are long enough to allow the patient to change his place, or to walk a distance of some metres, without deranging the passage of the permanent continuous current.

The very prolonged application (for an hour or more), of a contimnous galranic current, exposes the skin in contact with the negative rheophore to changes more or less deep, of which I have seen several examples from the practice of Hiffelsheim himself. ${ }^{3}$ It is therefore necessary to guard against the inconveniences

2 Hiffelsheim, loc. cit., p. 12.

3 Case XV.-M. X., to whom I was called in consultation with M. Baillarger, ou account of hallucinations that had tormented him for several years, and to : whom I had refused to apply continuous currents for the pirpose of removing them, was placed under Hiffelsheim, who promised him a cure. Hiffelsheim kept the little sponges applied to each temple, with a band that encircled the head. These sponges communicated, as described, with a gal vanic battery of numerous (sixty) sulphate of lead elements of siuall surface. The permanent continuous current having been suffered to pass for twenty minutes or half an hour, or more, according to the advice given, the application was renewed each day, by the patient himself, for ten or fifteen days, each day on a fresh portion of the forehead. Subsequently M. $\mathrm{X}$. came to show me his forehead, adorned by two rows of indelible cicatrices. Instead of being cured, his hallucinations had increased in frequency, and tempted him to suicile. He complained bitterly of the treatment to which he had been subjected. "Hiffelsheim supposed," said M. $\mathrm{X}$. to me, "that by this method the current would pass through the brain." 
arising from the electrolytic action of the permanent continuous current, and these inconveniences may in part be avoided.

C.-The selection among these different methods.-Under what circumstances should we have recourse to voltaic currents of more or less rapid intermission (labile currents), or to permanent (prolonged) continuous currents? And in what dose, and for how long a time, should the permanent continuous currents be applied? Apart from surgical, electrolytic, or galvano-caustic applications, there is no rule on the subject that rests upon close or sufficient clinical research. It is certainly not thus with localized faradization, for which the various and clearly formulated methods have their precise indications, drawn from prolonged experiment or clinical observation.

For myself, who commenced my electro-therapeutic researches by the application of voltaic currents, and have never ceased to use them (a fact which certain persons do not know, or, rather, have forgotten),-for myself, I say, who have always continued to study the relative therapeutic worth of voltaic and induced currents, I have not confounded, in my researches, more or less intermittent voltaic currents, with voltaic currents circulating through the organs in a permanent manner. I even went so far when I applied the permanent (stabile) constant current, as to aroid the excitation produced by the completion of the circuit, by making the current pass throngh a column of water in a moderator tube, and by gradually diminishing the thickness of the column after the circuit was established. Neither have I neglected to experiment, in the way so much lauded by Remak, comparatively with permanent constant currents. I will, shortly, give a summary of the opinion I think may be formed of their therapentic value, from the researches that I have made since 1861 ; very insufficient ones, I know, but of this I have made no concealment.

\section{Apparatus AND inStRUMENTS required FOR THIS KIND OF RESEARCH. Methods OF Galvanization that I HaVE FOLLOWED IN MY COMPARATIVE INVESTIGATIONS.}

Before stating what deductions I have drawn from my experiments, with regard to the therapentic worth of galvanization as compared with faradization, it is necessary to state what iustruments I have used in the application of galvanic currents.

I have stated in the first chapter, that, in my latest researches, I had chiefly used a Daniell's battery, and a battery of bisulphate of mercury, and that, since 1861, I had used a battery of sulphate of lead, of from thirty to a hundred elements, the sinall surface battery of M. Alph. Mathieu, and, quite recently, the portable 
chloride of silver battery of Gaiffe. Having already described these batteries, their action and comparative worth, it is not necessary to return to them. It only remains to show the results of an inquiry that I have made quite recently, by the help of the voltameter, upon the comparative electrolytic action of the sulphate of lead battery, Siemens' battery (known as Remak's), and the portable battery of Gaiffe. I have found that the electrolytic action of the first is about one-third less strong than that of the other two, (MI. Gaiffe has endeavoured to give to his battery an electrolytic action equal to that of Remak's).

It is also necessary to describe here some instruments that have facilitated the use of the battery, and that are required for a comparative study of galvanization by continuous currents and faradization; they will form the subject of the next paragraph.

A.-Division of the high-tension battery into several sections in the intervals of the applications.-Coupling of these sections.-Distribution of the currents of the different sections, either isolated or reunited.-Reversal of the poles.

Galvanization sometimes requires the use of a battery of high tension, and of great electro-motor power; that is, composed of numerous elements. But it is well known that in all galvanic batteries, during a slightly prolonged interruption of the circuit, and especially when the battery is not often in nse, certain interior currents are produced, which increase in intensity in direct proportion to the number of elements, and the reactions of which produce rapid exhaustion of the battery. I was soon taught this by experience, after having for some months used a Daniell's battery of fifty pairs, to which I sometimes added another battery of twenty pairs, that was chiefly used for my induction instruments and electric clocks.

In order to exclude or to diminish this cause of rapid exhaustion of my battery, I had it divided into ten separate parts, in each of which the tension is too feeble to produce, during the interruption of the circuit, any reactions of importance; and I then constructed a small apparatus (manipulator), which being placed in communication with each of the ten piles of ten elements, was able, 1. to unite them all into a single battery; 2 . to distribute them at pleasure in circuits composed of $10,20,30,40,50,60$, and so on to 100 pairs, and consequently to graduate the electro-motor force of the battery; 3 . to replace them in their original state of isolation when the battery was not required in action. With the manipulator I could also reverse the poles, during the galvanization, without changing the place of the rheophores which com- 
pleted the circuit. These properties of the instrument justify me in calling it the divider, collector, and distributor of the battery. Finally, it is placed in my room, near other batteries of very small surface, and near induction instruments, in such a manner that they may all be applied successively and comparatively, withont loss of time. The following is the description :-

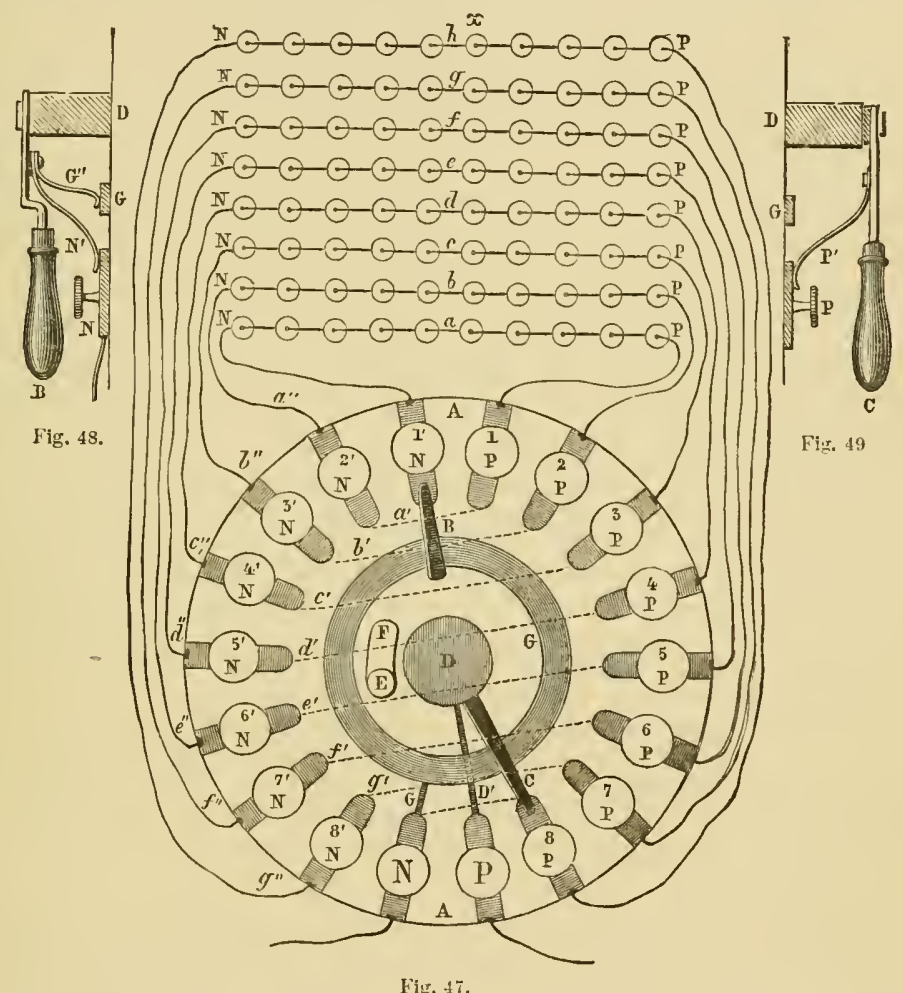

Fig. 47.-Divider, collector, and distributor of the battery. Figs. 48 and 49, handles of the divider, seen in profile. 4

1. A circular platform of wood, A A, ten centimetres in diameter, supports all the parts which form the mechanism of the apparatus. At the circumference of this platform, pieces of copper are screwed into notches cut to receive them. These pieces of copper are perforated in their longitudinal axes, and are furnished with the binding screws $1,1^{\prime}, 2,2^{\prime}, 3,3^{\prime}, 4,4^{\prime}, 5,5^{\prime}, 6,6^{\prime}, 7,7^{\prime}$, $8,8^{\prime}$, by which to fix the conducting wires that communicate with the negative poles $\mathrm{N} \mathrm{N}$, and with the positive poles $\mathrm{P} P$, of the several sections, $a, b$, $d$. , of the battery $x$. In this state each section of the battery is isolated.

2. The following is the mechanism by which the isolated sections (each one of which is an independent battery of ten elements) can be conpled together, so as to form a single battery of high tension, or of more or less great electromotor power. The support A A is groved on its posterior surface, to receive

4 Constructed, after ny designs, by A. Matthieu. 
a wooden disk that turns upon its axis; and to this disk are attached transverse brass springs, indicated by the dotted lines $a^{\prime}, b^{\prime}, c^{\prime}, d^{\prime}, e^{\prime}, f^{\prime}, y^{\prime}, h^{\prime}$; the first transverse spring, $a^{\prime}$, forms a communication between the knob that receives the positive pole 1 , and the knob 2 , which is connected with the negative pole; and so on, throughout the remaining springs. The disk is moved by the knob $\mathrm{E}$; and when this is pushed to $\mathrm{F}$ all the sections are united. In other words, $\mathrm{P}$, the terminal positive pole of the first section, communicates with the initial negative pole of the second section, by passing through $1^{\prime}, a^{\prime}, 2^{\prime}$, and $a^{\prime \prime}$; and so with the remaining sections. The sections should only be thus combined during the application of the current.

3. If, during the repose of the pile, the knob $\mathrm{E}$ is returned to its original place, the sections are again separated, the transverse metallic springs no longer connecting the terminal positive pole of each section with the initial negative pole of the next.

4. The apparatus serves, moreover, to distribute the currents of the battery $x$, either in fractions or as a whole, and thus graduates the electro-motor power. The distributors of the currents are the handles $\mathrm{B}, \mathrm{C}$, which conduct them in the following manner to the knobs $\mathrm{N}, \mathrm{P}$, which receive the conducting wires of the rheophores. The handle $\mathrm{C}$ carries a copper spring, shown in fig. 49 , which can be placed in contact with any one of the pieces of copper of the surface A A, and which communicates by its axis D, also of copper, and with the knob $\mathrm{P}$ by the line $\mathrm{D}^{\prime}$. The handle $\mathrm{B}$ carries a donble metallic spring, $N^{\prime}, G^{\prime \prime}$, one blacle of which communicates with the metallic circle, $G$, and the other with any one of the pieces of copper which receive conductors from each section of the battery.

5 . It often happens, in actual use of the battery, either for the therapeutic application of continuous currents, or to work induction instruments, that only a portion of the whole is required. If the same portion were always employed, the battery would be exhausted in an unequal manner. By a simple change of the handles, we may easily and quickly bring into alternate use the first, middle, or last third of the whole. If, for example, it were wished to use only the last twelre elements, the handle $B$ should be placed over the knob $7 p$, and the handle $\mathrm{C}$ over the knob $8 p$.

The apparatus lastly becomes, when required, a commntator or reverser of the poles. For this purpose it is only necessary to change the sides of the handles, the rheophores resting undisturbed orer the parts subjected to the current. In all the movements of the handles, the contacts are so disposed as to avoid intermissions of the current.

Graduation of galvanic currents.-The power to increase or diminish at pleasure, and rapidly, without any interruption of the circuit, by tens of elements at a time, the current from a large battery, may be considered as a graduation of its electro-motor force. This is obtained, as we have just seen, by the instrument above described.

But it is often also required to diminish gradually, at pleasure, the calorific and electrolytic action of the battery, the number of elements remaining the same. For this purpose, I cause the current to pass through a layer of liquid of greater or less thickness, contained in a glass tube that I have called a moderator, and that will be fully described hereafter. The liquid, which should be distilled water when the tube is used for the graduation of an inductive, and more especially of an induced current, should be replaced by water saturated with sea-salt when it is used to graduate voltaic currents, the tension of which is feeble, as com- 
pared with that of the induced, even when the battery is composed of a large number of elements.

By the aid of the voltameter, I have found that the electrolytic action of the battery diminishes in proportion to the leight of the column of liquid that it has to traverse; and I have also observed that the sensation of burning is proportionately diminished, at the points of contact of the rheophores with the skin.

The resistance opposed to the current by the liquid in the moderator diminishes also the electro-motor power, but much less than the electrolytic action. I have hence applied this method of graduation of the pile to its electricity of quantity.

Intermittences, in localized muscular galvanization by interrupted currents. - Intermittences of localized muscular galvanization by interrupted currents may be obtained by raising, at longer or shorter intervals, one or both of the moist rheophores from the skin. This is the proceeding generally employed; but I preferred to use the rheotomes which are ordinarily applied in muscular faradization, and to which I shall return when describing my induction instruments.

Wishing to study the comparative values of localized faradization and of localized galvanization by interrupted currents, I was bound to place myself, as far as possible, under identical conditions with regard to both; that is to say, as far as the intermittences were concerned, to produce them in localized muscular galvanization without shifting the rheophores, and by means of similar instruments.

(a). Pedal rheotome.-I habitually complete and break the circuit of the battery current with a pedal rheotome, which leaves my hands at liberty to manage the rheophores. I have elsewhere advised those, who, like myself, are in daily use of a batteryeither to work induction coils or for the physiological or therapeutical application of interrupted or continuous currents-to employ a pedal rheotome, in order to prevent the circuit from being left complete for a long time, as for an entire night, by any forgetfulness or accident. Such accidents, which were formerly of frequent occurrence with me, have not happened since I have been obliged to place my foot on the rheotome in order to complete the circuit.

(b). Wheel rheotomes. - I have also used rheotomes, which have allowed me to practise localized galvanization with intermissions nearly equal in speed to those of the trembler of an induction instrument. These iheotomes are furnished with several wheels, with teeth nearer or more distant, against which a spring impinges in such a manner as to obtain from two or four intermis- 
sions up to an extreme rapidity. At the time of my first researches, I had many rheotomes constructed which fulfilled these conditions, and which were moved either by the hand or by clockwork. These rheotomes are still in use for certain electrophysiological or therapeutical applizations. I use also habitually the wheel rheotome, called the distributor of the entrance or of the issue of the current, which is represented in fig. 40 , and described on page 123 .

B.-Distinction to be established between these different methods of gatvanization.-Having collected together, in my room and under my hand, the various instruments which are required for all methods or kinds of electrization, nothing has been more easy than this kind of comparative research. It has been chiefly carried out upon the patients of my clinique, patients suffering from all kinds of paralysis, atrophic, traumatic, saturnine, rheumatic, spinal, infantile; from various spasms and contractions, from chorea, and locomotor ataxy.

It is necessary to say, first, that a good number of these disorders will be improred or cured by electric excitations, of whatever kind they may be; by galvanic currents more or less intermittent, or continuous and permanent, or even by static elcetricity, as much as by induced currents. This fact will serve to explain the admiration that every specialist has constantly professed for the kind or mode of electrization that he has exclusively used, without knowing, or without having employed, comparatively, the others.

I have, howerer, seen forms of paralysis, neuralgia, neurosis, \&c., requiring a favourable prognosis on account of the ease with which they are habitually cured by induced currents, resist the action of intermittent or continuous voltaic currents.

I will not confine myself to generalities; but will say, in a few words, what are the various modes of galvanization that $I$ have used in my electro-therapeutic researches, and what deductions I have been able to form concerning them.

The distinctions that I have established between the different modes seem to me to afford the basis of a good method of galvanization. I divide muscular galvanization into-1. localized galvanization, which is performed by moving moist rheophores commected with the battery over all the points of the surface corresponding to the bulk of muscles, but at a distance from their nerves or fiom nerve trunks, and of which the varieties and the mode of physiological action have been already explained, both for interrupted and for continuous currents; and, 2. galvanization by reflex action. 
We shall see the importance of this fundamental division, if we recall the electro-physiological principles which distinguish direct localized muscular electrization from muscular electrization by reflex action, and which have been already laid down. In the former mode, indeed, all the anatomical elements of the organ acted upon are excited peripherally, as also its local innervation, or, in other words, its local circulation and its nutrition. In the latter, points of the nervous centres are chiefly and irregularly excited by the electrization of certain zones that stand in reflex relation to them. Every one will understand that these principles are perfectly applicable to localized muscular galvanization, and to muscular galvanization by reflex action.

\section{III.-Results OF My investigations into the therapeutic} APPLICATION OF DIRECT LOCALIZED MUSCULAR GALVANIZATION BY INTERMITTENT CURRENTS.

A.-Localized muscular galvanization is practised in exactly the same manner as localized muscular faradization. The same precepts that are set forth in Chapter II., and the same rheophores, are applicable to it.

I must, nevertheless, recall the fact that, unlike localized faradization, intermittent galvanization cannot produce contraction of muscle without at the same time exerting a calorific and electrolytic action upon the skin, and that the more energetically, the longer the rheophores remain in contact with the same point of the cutaneous surface. For this reason, I have been careful to change the place of the rheophores after each intermittence, by moving them over points corresponding to the masses of muscle to be galvanized. By thus proceeding, the cutaneous excitation is much diminshed.

B.-It is fitting to mention here the differential effects of the intermittences of induction currents, which appear to render difficult their study as compared with galvanization by interrupted currents; and I will explain how I have overcome this difficulty. We know that each intermission of the galvanic current produces a muscular contraction, at the completion and at the interruption of the circuit, but less strongly at the latter than at the former, without changing the direction of the current; and, on the other hand, it will be remembered that the contrary occurs, for indnction currents, at the completion and interruption of the circuit; the current going in an opposite direction, and producing an essentially different electro-physiological action, as far as the influence of the direction of currents is concernerl. It seemed therefore difficult, if not impossible, to compare the interrupted 
galranic currents, the course of which is always centrifugal or centripetal, with currents of induction which, with the usual rheotomes or with tremblers, would always be alternating.

It was for the purpose of obtaining only the entrance or the exit of the induced current, so as to cause the passage, at will, of a centrifugal or centripetal current through organs, that I constructed the distributor rheotome which has already been described (fig. 40, p. 123).

Faradization by centrifugal or centripetal currents, which I have been able to practise by means of this rheotome, has permitted me to observe, in frogs and rabbits, phenomena analogous to those which have been produced experimentally, under the influence of the direction of galvanic currents; but I must say that, in the treatment of paralysis, it has not exhibited any appreciable difference, as regards its action upon motility, sensibility, or nutrition, from faradization practised with ordinary rheotomes. I conclude, therefore, that muscular faradization, either with or without alternating currents, is perfectly comparable to muscular galvanization with intermissions.

C.-I have already stated (Chap. I.) that, between the completion and the interruption of a galvanic circuit, the current, which is then continuous, produces fibrillar oscillatory contractions. This is another character which distinguishes the intermissions of the galvanic current from those of the induced current. Therefore, when I have had to make a comparison between the therapentic action of these and those, I have always taken the precaution to diminish considerably, during the intermissions of the galvanic current, the duration of the intermediate action between its commencement and its cessation, even to the degree of almost entirely abolishing this period. For this purpose I have employed a rheophore, the teeth of which afforded only a small surface of contact, such that each intermission was necessarily very short, how slowly soever the toothed wheel might be turned.

T'he whole of the precautions indicated having been taken, it was possible for me to institute a comparison between the therapeutic action of intermittent localized galvanization and that of localized muscular faradization, in the treatment of paralysis.

D.-The result was, under all the foregoing conditions, and when the electro-motor power of the battery was sufficiently great, that localized muscular galvanization afforded nearly the same therapeutic results as faradization.

However, the benefits being equal, I have still preferred localized muscular faradization, which, exerting the same therapeutic in-. fluence over local circulation and nutrition, has none of the incon- 
veniences of galranism (electrolytic action, action upon the skin, and too acute excitation of the retina), and offers the advantage of acting sharply, according to the indications that are to be fulfilled, upon the muscular sensibility with the extra current, and upon the cutaneous sensibility with the induced current, by virtue of the properties special to each. If, besides these reasons, it is considered that induction instruments are of small bulk, easy of transport and of application, and inexpensive, it will be understood why $I$ have advised the use of muscular faradization in preference to intermittent localized muscular galvanization, which requires large batteries, only applicable, in general, in the rooms of specialists.

\section{IV.-Results of my investigations into the therapeutic}

APPLICATION OF REFLEX GALVANIZATION BY CONTINUOUS CURRENTS, MINGLED WITH FEEBLE INTERMITTENCES (labile currents), AS COMPARED WITH LOCALIZED FARADIZATION.

I will pass in review, as rapidly as possible, some of the principal maladies, or muscular affections, in which I have studied experimentally the therapentic action of continuous currents by Remak's method, as compared with localized faradization; reserving more complete details for the second part of the volume.

A.-Atrophic paralysis consecutive to traumatic lesions of nerves. - More than twenty years of electro-therapeutic research, conducted publicly upon a large scale, in the Paris hospitals and in my own clinique, tested and confirmed by a great number of observers in Europe and in America, have established, in an incontestable manner, the therapentic value of localized electrization by faradization, or by galvanization with interupted currents, and especially by the former, in the treatment of atrophic paralysis. The facts and considerations already brought forward cannot have left any doubt, upon this point, on the minds of my readers.

However, in presence of the claims of the promoters of Remak's method of galvanization, and especially after the assertions of young philosophers who, I regret to have to say, interpreting in a strange manner the results of their experiments on rats and rabbits, have come before the Académie des Sciences, and other learned bodies, to announce that induction currents paralyse innervation (a statement which would lead us to suppose that the therapeutic results had not happened), I wished to discover, in my clinique, whether experiment would explain such an aberration, if not fully, at least to some extent. For this purpose I have applied continuous currents, mixed with intermissions, exactly after the method of Remak, to the treatment of atrophic paralysis, con- 
secutive to traumatic lesions of nerves, in the cases that have come before me.

The results of these experimental researches have been far from fulfilling the pretensions of Remak and of his school. For improvement, in eases of any severity, I have had long to wait; and I have generally been unable to effect a complete cure except by localized electrization,-that is, by exciting peripherally all parts of the paralysed muscles (fibres, nerves, and ressels), by moving over all points of the surface the rheophores of my induction instrument.

It might be supposed, nevertheless, that the combination of the two methods of electrization might sometimes produce a more rapid cure, when the atrophic paralysis is symptomatic of a spinal lesion; because, by Remak's method, the reflex action stimulates the spinal centre, at the same time that the motor and sensitive nervous ramifications, and those that govern the local circulations, are peripherally excited by localized electrization.

For several months I have tried the two methods in combination, particularly in the spinal paralysis of infancy; but I am not yet able to pronounce upon their actual valne. I am not accustomed to form conclusions in so short a time, in my experimental researches upon therapentical questions.

B.-Lead-palsy.-It is especially with regard to lead-palsy that the exclusive partizans of the continnous currents, have been most unfortunate in their inconsiderate attacks upon the value of localized faradization. I have certainly treated by this method several hundred cases of all degrees, and more or less general in extent; and I have known few which have not been quickly cured.

With regard to the electric treatment of this form of paralysis, Remak has had the unskilfulness to write: "The kindness of many of my friends lias afforded me opportunities of treating a rertain number of patients, and has fully convinced me of the insufficiency of the induced current in many morbid states, especially in rheumatic and saturnine paralysis, which, according to II. Duchenne, ought to be cured by it." 5

To this attack I have already responded in the following manner: - "The facts which have been stated, and which, since their publication, now of old date (the first edition of 'Localized Electrization,' in 1855), have been supported by others too numerous to be reckoned, and by a vast electro-therapentic experimentation repeated by numerous observers; all these facts, incoutestable and 
publicly collected, enable us to appreciate correctly the strange and contradictory assertion of Remak.

"It is true that this experimentalist proposes to laud the therapeutic superiority of the continuous current, which, he says, cures lead-palsy in a marrellous manner. At the time when I write these lines, I have already tried this method of galvanization upon some patients with this form of disease. Out of four patients, two became tired of a painful treatment, which, after three weeks, had produced no amendment. A third begged me to employ the localized faradization, wbich he had seen applied with suecess to many of his comrades. The fourth, who has not at present been more fortunate than the other three, is willing to surrender himself to the continuous current for another fifteen days.

"Certainly such a result does not enable me to sing the praises of the continous current in the treatment of lead-palsy. Let us hope, nevertheless, that new and more extended experiment may rehabilitate the worth, so much vaunted by the German electrician, of the continuous current as applied to the treatment of this disorder." 6

'I'he strange assertion of Remak has been repeated by his adepts; and to refute it I have brought together a fresh series of cases, chosen from amongst those of my clinique, in which saturnine paralysis of rarious forms and severity has been cured by localized faradization. I should add that, after having continued to test, in my room, the therapeutic value of galvanization by Remak's method, as applied to the treatment of this form of paralysis, I have again found it inferior to localized faradization.

C.-Electro-muscular contractility.-The muscles which, in certain forms of paralysis, no longer contract under the influence of localized faradization, may sometimes still be thrown into contraction by the intermissions of a voltaic current, when these muscles or their nerves have been traversed, for one or two minutes, by a continuous current.

This phenomenon, which, for the first time, was described by Remak ${ }^{7}$ in pathological conditions, had been previously experimentally discovered in animals, by R. Heidenhain, ${ }^{8}$ who observed that on submitting a muscle to the shocks of induction, or on tiring it by stretching, or on plunging it into hot water $\left(28^{\circ}\right.$ to $30^{\circ}$ Cent.), it lost its excitability; and that this property (the faculty of contracting under the influence of electric excitation) was restored to it by the continuous current of a Daniell's battery of 25 or 30

${ }^{6}$ Duchenne, Electrisation localisée, $2^{\mathrm{e}}$ edition, p. 32S.

7 Remak, lor, cit.
8 Heidenhain, Physiologische Studien. Berlin, 1856, pp. 55-127. 
elements, in a manner more evident in employing the ascending than the descending current.

I have tried, a very large number of times, to prodnce this electro-muscular phenomenon, in traumatic paralysis of nerves, in saturnine paralysis, and in the spinal paralysis of infancy, but I have only seldom succeeded.

I should here point out the following source of error. When the contractility is considerably weakened, a time comes when the muscle no longer contracts un ?er the influence of the rapid intermissions of induction eurrents, while it still responds, in a very appreciable manner, to the more distant intermissions (from one to two seconds) of the same induction current. But, a great number of induction instruments giving none but rapid intermissions, it has happened that on applying them to the investigation of electro-muscular contractility this has been supposed to be abolished, while it would still have been called forth by the slow intermittences of an induction current, and eren of a galvanic current. It has been by proceeding in this way, there can be no doubt, that experimentalists have been deceived by appearances, and have been brought to believe in the frequency of the electropathological phenomenon now under consideration.

However this may be, I repeat that I have sometimes forind eases of paralysis in which the muscles no longer responded to the distant intermissions of an induced current, but in which they contracted more or less under the influence of the distant intermissions of a strong voltaic current, after having been traversed, during from sixty to eighty seconds, by a constant continuous r"urrent.

From this special action of voltaic currents upon muscular irritability, their exclusive partisans have argued to their greater therapeutic power in the treatment of paralysis. My own experience shows that these pretensions are unfounded. I have even seen a case of lead-palsy (for it is chiefly upon this form of disease that I have made such experiments) in which the paralysed muscles only contracted to the intermittences of a voltaic current, but in which they gained only a little under the influence of a sufficiently prolonged treatment by continuous labile and stabile currents, and were then completely cured by localized faradization alone. Nay, more, in the great majority of cases the lead-palsies in which the muscles no longer contract either to faradization or to intermittent roltaic currents, are none the less cured by the former method of electrization.

It is of little consequence, indeed, that one kind of electricity acts more energetically than another npon the excitability or 
irritability of muscle, when it is lirown, as I have demonstrated, that in paralysis the voluntary morements may be re-established by localized faradization, although the paralysis of electro-muscular contractility remain. It is this strange phenomenon that is expressed in the title of a memoir that I addressed to the Institut in 1846,- "The integrity of the electro-muscular contractility is not essential to the performance of voluntary movements."

D.- Paralytic contractions.-It is especially in the treatment, by the continuous current, of the secondary contractions observed in cases of hemiplegia consecutive to cerebral hæmorrhage, that Remak has made application of his theoretical ideas about the electro-physiological phenomenon which he has called galvanotonic reflex contraction; theoretical ideas which rest upon the experiments alrearly described and criticised.

(a). He has thought to discover a more complete demonstration in the influence of continuous constant currents, during their passage through certain nerve-trunls, upon paralytic contractions. The following is a summary of an example that he cites as a typical case, showing the reality of this special action of the continuous current.

CASE XIII. A woman, aged 35 years, was attacked by hemiplegic contraction of the right side consecutive to a cerebral hæmorrhage of eighteen months' standing (she was aphasic). Remak passed the continuous enrrent of from 20 to 30 of Daniell's elements through the right crural nerve, and he saw, after 30 or 40 seconds, the segments of the upper limb and the fingers of the hand of that side which had been strongly flexed, extend themselves completely, while analogous effeets were produced in the lower limb, by treating the median nerve in the same manner.

I have many times repeated this experiment of Remak's, under analogous conditions, upen the patients of my clinique, and I have very rarely witnessed the production of these so-called galvanotonic reflex contractions, which, according to him, should be the essential sigu of the therapeutic action of the continuous current. 'The following are the experiments that I made publicly at the time.

CASE XIV.-During several weeks I subjected to these expcriments, or rather to treatment by the continuous current after the method extolled by Remak, six patients of my clinique, who were suffering from secondary paralytie contraetions, conseeutive to cerebral hæmorrhage; four of them had lemiplegia of the right side, and were aphasie; the other two had hemiplegia of the left side, and their intelligence was unaffeeted. The paralytic contractions were of from one to two years' duration. In all, I caused the passage, in the median and erural nerves of the paralyzed side, of a descending (centrifugal) current, in a permanent manner in eaeh nerve during one or two minutes; each séance for each of them lasting from five to ten minutes, with a current of from fifty to a hundred elements of my sulphate of lead battery, and of a tension aceommodated to the degree of individual tolerance (tor it must not be forgotten that this kind of galvanization is painful). In one of 
the patients 1 s:veral times produced reflex contractions in the paralysed lower limbs, while causing the passuge of a strong continunus current, now through the median now through the crural nerve. The leg un extended upon the thigh, the fore-arm upon the arm, and at the same time the land was open:d. The patient (a woman 30 years of age) pointed out to me that the same thing happened nearly always when she yawned or experienced any emotion, especially upon awaking in the morning. In none of the other patients conld I prodnce reflex contractions, whatever might be the intensity of the continnous current or of its passage through the nerre. I remarked nothing but contraction of the muscles governed by the nerve excited. The patients, however, told me that their flexed and contracted limbs often became extended, and that their hands would open when they yawned or sneezed, or under the influence of any movement made with effort.

Let us admit that the reflex contractions described by Remak commonly occur under the influence of continuous currents traversing the nerve-trunks, and let us inquire their significance and their value.

Remak sees in these phenomena the proof of the electro-plysiological fact that he believes limself to have discovered,-- - the galvano-tonic contraction produced by the continnous constant curreut, and which he has made one of the fundamental bases of his method of galvanization. Thus, in his experiments (see (ase XIII.) the excitation of sensitive fibres, produced by the continnous current in the crural or sciatic nerve, should have reached the spinal origin of these sensitive fibres in the lumbar regions; and then, passing along the whole length of the spinal cord to the origin of the brachial plexus of the same side, the current has exerterl an elective action upon the motor fibres, which produce extension of the different segments of the upper limb, and principally upon the radial nerve.

For the clinical practitioner this experiment will not have the signification that has been given to it by Remak. Who, indeed, does not know that in the secondary contractions that supervene upon hemiplegia symptomatic of cerebral hæmorrhage, the side of the spinal cord, which supplies nerres to the paralyzed muscles, acquires in extreme excitability, and that the slightest impression from an external or peripheral cause may produce spasmodic contractions precisely lilie those which Remak considers as reflex contractions, specially prodnced by the influence of continuous currents upon certain sensitive nervous zones? Who has not seen in the hemiplegic paralytic contractions, the fingers, the hand, and the forearm, which remain in continuous flexion, sometimes extend themselves completely during yawning, sneezing, or under the influence of any impression or cmotion whaterer (see Case XIV.).

Sometimes the same phenomenon is produced during a voluntary movement of the lower limb; thus, I have then seen in hemiplegies, with contracted "pper limbs, sometimes the hand open 
and the arm extend itself, or sometimes, on the contrary, the spasm show itself in the flexors. I have sometimes even seen localized faradization, in these conditions, whether it was cutaneous or muscular, produce analogous spasms if it were practised painfully.

In fine, it follows from the facts set forth above: 1 . that, in the morbid conditions under cliscussion, the power to produce reflex spasm in the antagonists of the contracted muscles, and often even in the latter themselves, is a property common to most excitants, (an acute and sudden excitation of the skin, an emotion, a yawn, a sneeze, and lastly the excitation produced by the passage of a continuous current through a nerve-trunk, especially if the operation is painful); 2. consequently, that to call these reflex galvano-tonic contractions has no foundation in reason.

(b). Is there, then, any occasion to seek a way so circuitous, so imaginary, in order to explain the therapeutic action of reflex and continuous galvanization of nerve-trunks, in the treatment of contractions? The paralyzing or hyposthenizing action of descending continuous currents upon nerve-trunks, discovered by Eckhart, ${ }^{9}$ would afford a better reason for their effect against contractions. It was this paralyzing property of the continuous descending currents which originally suggested to Remak the first idea of their application in pathological conditions. The idea was rational and happy, but it has been wholly spoilt by his imagination.

With regard to the therapeutic influence of the continuous currents against the paralytic state which accompanies the contractions, it may be explained by the reflex excitation of the nervous centres that is produced by this kind of galvanization.

$(c)$. The therapeutic theory of this method of galvanization would here be of little consequence, if it really cured the paralytic contractions consecutive to cerebral hæmorrhage. Is it true, is it possible, that it does cure them? Into this it has been necessary for me to examine, and I have made it the subject of experimental research.

Every one knows, or in the present day should know, after the artmirable researches of MII. Charcot and Bouchard, ${ }^{1}$ that these contractions are symptomatic of the inflammatory state of the secondary sclerosis of certain nervous bundles which, when the optic thalami or corpora striata are the seats of hæmorrhage,

Eckhart, Beitrïge zur Anatomie und Physiologie. Giessen, 1855.

1 I should say here that M. C. II. Bouchard, in his excellent memoir upon the secondary degtnerations of the spinal cord (Archives gênérates de Mêlecine, 1866 et 1867), was the first to consider the permanent contractions consecutive to cerebral hæmorrhage as symptomatic of secondary sclerosis of the cord. 
is propagated to the lateral columns of the opposite side, and which begins from the tenth to the fifteenth day. Surely no one would pretend to cure such an anatomical lesion, especially when it had existed six months or a year. If Remak had lived, he would probably regret to-day the therapeutic announcement that he formulated in a manner so absolute, and of which he made so much.

I shall, therefore, surprise no one by saying that, in tne applications I have made of continuous currents, to the treatment of the paralytic contractions of cerebral hemiplegia, I have in general obtained no appreciable result. I should add that, in certain conditions that will be specified when I speak of cerebral paralysis, this method of galvanization is not free from danger, and that hence it is necessary to be very circumspect in its employment.

I make one reservation to my statement that, in general, I have obtained nothing by Remak's method,-riz, in some cases I have observed, after the application of his mode of galvanization, a diminution of the contractions and an improvement in the motility; but to this I must arld, that I have sometimes obtained the same by faradization of the antagonists of the contracted muscles. How are such results to be explained? I also might advance my theory; but I declare, beforehand, that I attach little importance to it.

Whatever it may be, the general conclusion to be drawn, as regards therapentics, is that the two methods may or ought to be employed concurrently or alteruately in the treatment of paralytic contractions arising from a cerebral cause. Such, at present at least, is what I practise habitually.

I have tried, during several months, the hyposthenizing action of continuous currents upon the nerve-trunks, in cases of cerebrospinal or chronic-spinal meningitis. I am not at present in a position to express an opinion upon the therapentic value, having received but two cases. In these, lowever, I have not obtained any appreciable modification.

E.-Functional spasms (Scrivener's palsy, \&c.).-Neuralgic contractions called rheumatic.-Reflex ascending contractions from articular injuries.-Chorea.

(a). Functional spasms.- When I come to treat of functional spasm, in the second Part, I shall have to say that localized faradization has appeared to me to be most frequently powerless against this nervous affection. It will, therefore, be understood that I have not neglecter the application of those continuous currents which Remak; and some of his alepts, say that they have applied 
successfully against writer's spasm. I have frequently had occasion to see this local affestion among my patients; and it is not a single case only that I have treated by the method so much extolled by him. I regret having to say that $I$ have yet to wait for a cure. I have not seen even one success obtained by those who have praised the treatment: on the contrary, the majority of the cases that during several years have presented themselves for consultation have been treated without benefit by the continuous currents, and the number is now large. I will mention, as an example, a very remarkable case that was sent to me by Professor Germain Sée, in 1869, upon which M. Onimus had applied continuous currents for about a month without any appreciable result. (The patient still presented the marks of the electrolytic action of the rheophores.) It is useless to add that the faradization tried by myself failed like the continuous current. I nevertheless advise its application in the treatment of writer's cramp, and shall myself continue its employment in this affection, sinee conscientions observers declare that they have obtained from it good results.

(b).- Indolent tic.-I have applied the continuous current to three cases of inclolent tic of the face, one of which was recent (of about a month's standing), and limited to certain muscles (the zygomaticus minor, levator labii superioris alæque nasi, and orbicularis palpebrarum). In this case the contraction gradually diminished after the third séance, and was cured in abont twelve séances. Several months after the treatment, I was told that the tic had not reappeared, but I lave had no later intelligence. The two other cases appeared to me to be rendered worse by the same treatment.

Localized faradization, which I have applied sufficiently often in indolent tic, has sometimes produced a temporary amelioration, but not a single cure. Hence continuous currents appear to me to merit the preference in the treatment of this disorder.

(c).-Rheumatic contractions.-The local or partial contractions produced by emrents of cold air (rheumatic), or without known cause (nervous), the cases of torticollis from contraction of the sterno-mastoid, the splenius, the clavicular portion of the trapezius, or of some deeper muscle, such as the rhomboidens, are in general cured by faradization, with rapid intermissions, of the antagonists of the contracterl muscles.

I have endeavoured to ascertain, experimentally, whether the hyposthenising influence of continnous currents upon the contracted muscles would be preferable to faradization of the antagonists. The continuous currents have, in general, produced only an improvement; and I have nearly always been compelled to have 
recourse to faradization of the antagonists, in order to obtain a complete cure.

(d).-Ascending reflex contraction from articular injuries.-The reflex ascending contraction, in which $I$ have comparatively studied the therapeutic action of the continuons current, and of localized faradization, has not at present, to my knowledge at least, been described or mentioned by authors. This kind of contraction generally follows after violence exerted upon certain articulations, principally upon the artieulation of the wrist, in falls upon the back or the palm of the hand, violence which produces an arthritis more or less intense, or a simple articular pain of short duration. The contraction which sometimes appears a little while after the joint has ceased to be painful, and when it appears to be entirely rured, has its seat in a larger or smaller number of the motor muscles of the joint affected; and at length may extend to the motor muscles of other joints on the same side of the limb. The pain, limited at first to the contracted muscles, is moderate; but it afterwards attacks the other muscles, although always remaining most acute in those which were originally affected; and at length it attacks the nerve-trunks which supply the muscles, and, finally, their origin in the brachial plexus. Often, even when the contractions have disappeared, the patients are still (sometimes eren for years) sufferers from continual pains towards the origin of the nerres of the affected limb, pains which seem to be symptomatic of a morbil condition of the spinal cord. Lastly, the strength and sensibility of the limb in which the contractions are seated, are generally found to be diminished.

I have cured many of these reflex ascending contractions by faradization practised in a painful manner (with the extra current, and with rapid intermissions). I shall here relate a single case that was remarkable for the reason that, being of a certain severity, it was for more than a year rebellions to various kinds of treatment, and even to a long application of constant currents, according to the method of Remak, the direction of which were centrifingal during the first two thirds of the treatment, and centripetal during the remaining third, and that it was nevertheless cured in a few séances by the fararlization, in strong dose and with rapid intermissions, of the antagonists of the contracted muscles. I give the ease as a typical one, or rather as a picture of this kind of contraction.

CASE XV.-Summary. Reflex contraction of a large number of the motor muscles of the right upper extremity, existing for two yeurs, consecutive to an arthritis of the wrist of that side, produced by i full upon the back of the hand, rebellious to varial malical treatment, and to the application during thirty séances of constant continuous currents (according to hemal's method), eventually curcd in a few sémas by energetic furudization with rapid intermissions of the 
antagonists of the contructed muscles. Continuance of spinal pains in the cervicodorsal region.

Mademoiselle $\mathrm{X} * * *$, of Saint Brieux, sixteen years of age, born of healthy parents, of a good constitution, had menstruated regularly since twelve years of age, and had never suffered from serious illuess or from hysterical troubles.

When eleren years old (in 1861), she fell upon the back of her right hand, and afterwards complained of pain in the radio-carpal articulation, with swelling and weakness of its morements; she was compelled to keep the arm in a sling for two months, after which there remained only a little weakness. When she wrote, this weakness rapidly increased, and pains were felt in the wrist, returning at regular intervals, and contimuing for one or two homs.

Towards the age of thirteen years and a half, after having danced one evening with much animation, the pain in the right wrist, which had been pulled a little roughly, retumed with such intensity that all use of the hand beeame impossible. There was neither redness nor swelling to be seen. The pain continuing, Jobert de Lamballe, who was consulted two months afterwards, advised the application of flying blisters to the painful part, which produced no result. In December, 1861, the pain still coutinued; although limited to the wrist, it forbade all movement or use of the hand, and occasioned loss of rest. At this period MI. Nélaton was consulted, and he applied an immovable compressive apparatus, which was renewed every ten clays, and frictions of tincture of iodine.

This apparatus was employed for four months, but without result; the pain, on the contrary, became more severe, and after two months a contraction appeared in the radial and pronator muscles of the injured side. During the year 1865, by the alvice of Dr. Regnanlt of Remmes, recourse was had in succession to six flying blisters, to sea-bathing, to the application of hot sand, to aromatic baths, and, finally, to superficial canterization over the affected muscles, and all without modifying the pain or the contraction.

In 1866, Mrademoiselle $\mathrm{X} * * *$ returued to M. Nélaton, who sent her to me. I then found the following condition: permanent flexion of the hand mpon the forearm, with pronation; the patient being unable to extend the wrist or to supinate the hand. With some effort, however, I was able to restore the position of the limb, producing cries of pain from the patient; aul, when left alone, it returned to its former position. The use of the hand was lost; there was perfect movement of shoulder and elbow ; the limbs had preserved their natural size; the general health was satisfactory; the appetite was good; there was no lesion of the circulation, and no appearance of anrmia. I advised at that time the application of continuous currents, and a ferruginons treatment. The first part was postponed on account of an epidemic of cholera that then prevailed in Paris. Mademoiselle $\mathrm{X} * * *$ had scarcely returned to her home when the contraction extended to other muscles: the flexors of the fingers kept the hard constantly shut, so that the nails penetrated the skin; deep pains were felt towards the origin of the brachial plexus, and descending to the dorsal region; then fever supervened, with insomnia. Cupping was practised three or four times over the painful part, then flying blisters were applied to the same region, but without any improvement.

On her return to Paris, April 18th, 1866, Mademoiselle $\mathrm{X} * * *$ came to me again. I found, in the right upper extremity, contractions of the superficial and deep flexors of the fingers, of the biceps brachialis, and of most of the muscles which move the arm upon the shoulder. These contractions called forth pains in the whole limb, and in the cervico-dorsal portion of the spine, pains which were increased when an attempt was made to overcome the contractions. The continuous flexion buried the nails in the palm of the hand, which was inflamed, and threatened to nlcerate; there was the greatest difficulty in extending the fingers. The thumb, strongly compressed between the forr fingers and the paln of the hand, was as if atrophied by this compression, and was more difficult to extend than the fingers; the size of 
the contracted limb was preserved. The sleep was interrupted, moreover, by the spinal pain in the dorsal region; there had neither been headache, nor fever, the menstruation was regular, and the appetite and general health were good.

Galvanic treatment, by continuous and reflex currents, was commenced immediately. I employed a pile composed of 80 sulphate of lead elements, so graduated as at first to give only a force of 10,20 , or 30 elements, according to the maximum of tolerance, which was different on different points on which the rheophores were placed. Sometimes the negative rheophore was piaced over the course of the median nerve, at the lower part of the forearm, and the positive at the bend or the middle part of the arm, over the course of the same nerve; sometimes the contracted hand was immersed in water that was in communication with the negative pole; while the positive pole was placed either at the origin of the cervical and dorsal nerves, or over the brachial plexus, or orer the course of the nerves that proceed from it.

After about ten sérnces, the patient was able a little to correct the position of the wrist during the passage of the current; but this power ceased with the operation. From this time the hand progressively opened, and remained open at first for some minutes, and, after further applications, for some hours after excl galvanization. Then the patient was able to open and shut the hand a little at will; but a few days later menstruation set in, and the contraction of the flexors of the fingers became as complete as before.

After a repose of six days, the application of the continuons current was renewed as before; and, at the end of three séances, the contraction of the flexors of the fingers and thumb had disappenred. But the spasms continued in the other muscles, and increased in the biceps, and even in the radial muscles, until, although Mademoiselle X *** could open and shut the hand freely enough, it remained in a state of supination and forced extension, which rendered it useless.

The continnous eurrent, employed for twelve days longer in an ascending direction, produced no improvement in the state of these contractions.

I then determined, before abandoning electric treatment, to try localized faradization with rapid intermissions. I applied it very energetically to the antagonists of the contracted muscles, to the pronator teres, the triceps brachialis, the interossei, and to the extensors of the fingers. To my great surprise, in the course of a few minutes all the contractions had completely disappeared. The result obtained so rapidly was maintained, and lialf an hour after, Mademoiselle $\mathrm{X} * * *$ could write, and, in my presence even played scales upon the piano, which she had been unable to do for more than two years. The localized faradization was continued for twelve days longer, in order to confirm the cure, and to increase the strength and sensibility of the hand. After twelve séances, the force of the flexor's of the fingers which, measured by my dynamometer, at first scarcely equalled four kilogrammes, had monnted to eighteen.

Retumed to her home, Mademoiselle $\mathrm{X} * * *$ preserved the full use of the light hand and arm until December 1866, but with the first access of severo cold her forearm was rendered supine by contraction of the supinator brevis: the hand preserving full liberty in all its movements. The pain, which had extended from the last cervical vertebra to the middle of the dorsal region, returned more acutely. Blisters, dressed with morphia, dry cupping, and opium internally, were used without success.

On the 29th of September, 1867, Miademoiselle $\mathrm{X} * * *$ was brought to me in this condition. In a few séances I again mastered the contraction of the supinator brevis, by energetic faradization of the antagonists with rapid intermissions. I was afterwards able notably to diminish the spinal pains by electro-cutaneons excitation over their seat. Since then, the contractions have not reappeared; but, some wceks after, the spinal pains returned, more acutely than ever, with exacerbations during the night; and they still continucd in 1 s 69 , notwithstanding all the medication that was employed. 
I purpose to return, in the second Part, to the patloological questions that underlie the foregoing record; and I confine myself for the present to remark on the evidence that it affords towards a therapeutic conclusion with regard to the respective usefulness of continuous currents and of localized faradization.

Although I have generally obtained a cure of rheumatic contraetions by energetic faradization, with rapid intermissions, of the antagonist museles, it has been seen that, in the foregoing ease of reflex ascending contraction from articular injury, I gave the preference to reflex galvanization by a continuous eurrent. This seemed to me to be the more rational course. It was manifest that the contractions had been caused by the articular irritation consequent upon the fall on the wrist, and henee that they were reflex. Believing that they were symptomatic of a hyperæmic condition of a point in the cerrical region of the cord. eorresponding to the origin of the brachial plexus, the application to the upper limb of that reflex galvanization by a descending continnous current, that is considered to be an hyposthenisant, seemed to be entirely indicated, and to offer, in a rebellions ease already of some duration, a better prospect of suecess than localized faradization of the antagonists of the contracted muscles. It has been seen also that I was deceived in my liope ; that the treatment, applied for a long time, and in aceorlanee with the rules laid down, showed itself to be powerless against this kind of contraction; that before abandoning electricity I wished to try, although suecess appeared improbable, faradization of the antagonist museles; and that then, to my great surprise, I obtained a cure of the eontraction with great rapidity.

The action of this mode of faradization of the antagonist muscles was so heroic that, notwithstanding the persistence of spinal pains, it rapidly conquered for the second time the contraction of the supinator brevis, which had returned and had continued for several months.

I shall show hereafter that the spinal pains were symptomatic of a secondary morbid condition of the spinal cord (selerosis) which still existed in November, 1869, and which is the ordinary termination of a reflex ascending contraction from articular injury; and that their long persistence gives to the ease a certain degree of gravity.

\section{(e). Chorea.}

CASE XVI.-I have submitted to the action of the continnous currents, of from 30 to 50 elements of my sulphate of lead battery, two cases of chorea, in young subjects aged respectively fourteen and sixteen years; the current traversing the affected limbs and the vertebral column, in a direction 
sometimes ascending and sometimes descending, and each séance having lasted from ten to fifteen minutes. In one of these cases, both the upper and one of the lower limbs were affected by the partial muscular contractions. The patient was cured in fifteen applications; a progressive improvement having: been obtained after the first few. The other case, in which the muscular spasms were less general, but of more than a year's duration, derived no benefit from the continuous current, which was applied in the same manner.

These two cases afford confirmation of what had been already established by others (Remak, Hiffelsheim, Benedikt,) that (chorea may sometimes, but not always, be cured by the continuous current.

I should remark that localized faradization, especially by reflex action, may also comnt its cures of chorea. I have myself obtained them, although I have only treated a very small number of cases. Some have even been cured by static electricity.

Since chorea may thus be cured by all kinds of electrization, it behoves us to seek in the future what proceeding or method, whether faradization or galvanization, deserves the preference in its treatment.

MI. Onimus says that, in the treatment of chorea, "induction currents are rejected by all physicians who are engaged in electrotherapentics," and then he describes a treatment by continnous currents, which, he says, "has always succeeded with him in this affection, and uhich consists in causing the passage of an ascending contimuous current through the limbs or through the vertebral column during from thirty to fifty minutes." 2

What I have already said about the therapentic influence of induction currents, especially when applied by reflex action, in the treatment of chorea, proves that this writer is deceived when he states that they are abandoned by all who practise electrotherapentics.

I cannot believe that he thinks that the method proposed by lim, of galvanization by an ascending continuous current, would always succeed in the treatment of chorea, for he has only applied it in a few cases. Moreover, every experienced practitioner knows that, in order to judge of a therapeutic question, we require numerous facts, and the test of time.

Is it thoroughly demonstrated, as M. Onimus affirms, that the continuous ascending current has an advantage over the descending for the cure of chorea. It seemed to me that an experimental counter-proof was necessary. I proceeded to apply it, and the result is shown in the following case, which is given only in ontline.

2 Onimus, De l'action des rourants continus dans le traitement de la chorée (Gaz. des IIôp., is Srpt., 1869). 
Case XVII.-On the day on which the discovery of this eurions therapeutic property of the ascending eurrent was ammonnced by M. Onimus in the Gazette des Hópitaux, an opportunity for the required counter-proof was afforded me by the ease of a young lad, who had been suffering from chorea five months. I caused the passage of a descending current from 30 elements of my sulphate of lead battery, during five or six minutes, through each of the limbs agitated by choreic contractions. The hands were placed alternately in a basin of water in relation with the negative pole; and the rheophore of the positive pole was placed on a point corresponding to the origin of the brachial plexns. After the second application, the agitation of the npper limbs had notably diminished. I then passed a descending current through the lower limbs and (following the direction of the spinal cord) through the trunk, which was the seat of some isolated convulsive contractions. From that time the improvement was progressive, and after fourteen applications the young patient was cured.

It is possible, and even probable, that a greater or less number of eures of chorea, obtained by other observers, have also been by the agency of the descending continuous current. Bnt the case I have recorded, even if it were solitary, proves, contrary to the assertion of MI. Onimus, that the descending continuous eurrent possesses, like the ascending eurrent, the power to eure chorea.

Is this therapeutic action of the descending equal to that of the ascending current? I reserve this question for future inquiry.

(F).-Progressive muscular atropluy. Sclerosis.-Do the continuons currents, by reflex action, exert upon the nervous centres a more favourable influence than localized induction currents, in modifying the conditions of local cireulation or nutrition; for example, in progressive muscular atrophy, or in sclerosis of the cord. The affirmative is maintained by Remak, and by the exclusive partisans of continuous currents.

I shall show, in the second Part, that localized faradization is one of the best means of immediately encountering the disorders that are symptomatic of the maladies in question. Thus, for example: 1. in progressive museular atrophy, museular faradization will sometimes arrest the progress of the wasting, and will even develop the muscles that are on the way to destruction; 2 , in glosso-labio-laryngeal paralysis it improves for a time the articulation of words and the deglutition; 3. in locomotor ataxy, electro-cutaneous faradization often cures local anresthesia, and consequently much diminishes the functional disorders that it oceasions when seated in the extremities; it sometimes causes the disappearance of fixed eutaneous hyperesthesia; faradization of the eye may arrest atrophy of the papilla of the optic nerve; and, lastly, faradization sometimes cures or removes paralysis of the motor-muscles of the bladder, the rectum, and other viscera.

In the presence of such results, and especially when, after having applied faradization in a general manner, I hat seen the 
disappearance of the symptoms of progressive locomotor ataxy, I conceived the hope, at the commencement of my researches into this disease, and prior to describing it, that faradization would be one of the best means of treatment by which it could be opposed.

But, unfortunately, experience soon taught me that the results obtained locally-all incontestable as they were-were still but teniporary; that the cure of the disease was only apparent; that, in its normal course, ataxy is essentially remittent; that it was not uncommon to see the occurrence, even spontaneously (among unfortunates who, for want of means, were unable to pursue any treatment) of remissions of even long continuance (sometimes of a year or more), during which all the symptoms of the malady had disappeared, so that it was possible to believe it to be entirely cured. I must, in one word, acknowledge that, perchance, the faradization may have been applied coincidently with one of these periods of remission; but the ataxy none the less resumed, after a time, its progressive course. The same may apply to glosso-labio-laryngeal paralysis.

Remak and his adepts have, however, announced that constant continuons currents have power to cure the profound lesions of the nervous ecntres which are characteristic of progressive locomotor ataxy. I accepted, as a great benefit, the promise of a medication that should be efficacious in the treatment of this malady, the march of which has appeared to me to be so fatal that, in describing it, I gave it the name " progressive," and I hastened to test the value of the plan proposed.

I do not yet venture to formulate my opinion of the actual therapeutic value of continuous eurrents in these organic lesions of the nervous centres. I cannot so soon forget the lesson that I drew from my illusions about the seemingly happy results of my first applications of faradization in similar cases. I even fear that those who have published cases of the cure of locomotor ataxy by continuous currents may have been too hasty in their conclusions. They possibly may not be sufficiently aware of what sometimes occurs in the natural course of the disease, without any treatment whatever, - of the long remissions that I have described, during which the symptoms may so far disappear that the patient may seem to be definitely cured.

\section{V.-Galvanization by Permanent Continuous Constant Currents.}

Galvanization by permanent continuous constant currents, consists in cansing the passage of these currents throngh organs, 
without intermissions, for as long a time as possible (from thirty minutes to several hours), and in reducing to a minimum the action of the completion and of the interruption of the circuit.

It is in this lind of galvanization that we have most to fear the electrolytic action of the battery. Hiffelsheim, who has most extolled, as I have already stated (see p. 184), the therapentic application of permanent continnous currents, has constructed a small sulphate of lead battery, formed of 80 or 100 elements, which are each 5 or 6 centimetres in length by $S$ in width. Although this little battery, the current of which is very constant and the tension sufficiently great, exerts a comparatively feeble electrolytic action, yet we have already seen that the permanence of the current, and its long passage at the same point, will affect the cutaneous surface more or less deeply (Case XV. p. 1S4.) It is therefore necessary to know exactly, under such circumstances, the electrolytic power of the battery that we are abont to use, and to know how long the rheophores of its permanent current may remain in contact with the same points of skin, without producing vesications or eschars.

It is hardly necessary to point out that galranization by permanent continnous currents should also be divided into reflex and localized; that the former acts specially upon the nerrous centres, and the latter upon the periphery.

Does reflex galvanization by stabile permanent continuous currents of long duration (that is to say, for séances of from half an hour to several hours in length, and necessarily of a dose comparatively feeble) exert upon the nervous centres a more powerful therapentic influence than that of reflex galvanization by continnous (stabile) currents, mixed with intermissions? I must reserve my reply to this question, which I am now engaged in studying experimentally.

I have seen localized galvanization by permanent continnous currents exert a resorbent action, that Remak has called catalytic, in chronic articular affections with nodosities, in ganglionic tumours, in one case of muscular tumour of obscure character (I regret not to have treated in this manner an analogous case to which I was called in consultation with M. Nélaton), and lastly in atrophy of the deltoich produced by rheumatism. I have not the time to devote myself, on a sufficiently large scale, to investigations of this kind; interesting, it is true, but which would take me too much away from my habitual clinical study of maladies of the nerrous centres, of the nerves, and of the muscles. In the cases in which I have been obliged to apply or to cause the application of this treatment, I have covered the greater part of 
the cutaneous surface corresponding to the diseased organ or region with a moist rheophore (a plate of platinized copper, covered with wet leather), and I have placed a second moist rheophore higher up, at a point as near as possible to the former. Then, for half an hour or an hour, aceording to the tolerance of the individual, I have cansed the passage of a permanient continuous current from 10, 15, or 20 elements (in proportion to the length of the séance), 7 centimetres in height, of a sulphate of lead battery. I have only habitually assisted at the one or two first applications; and the patients, whom I have visited from time to time, have themselves continued the treatment; with the precaution, well understood, to avoid the production of blisters or eschars. I have had no occasion but to praise, in their way, the catalytic effects of this mode of galranization; and althongh they are usually very slowly produced, I have obtained a complete cure on several occasions. Remak has had the great merit, first of suggesting, and then of demonstrating, the valuable catalytic effects of continuous constant currents.

\section{VI.-The therapeutic action of galvanization of tile SEIN, AS COMPARED WITH CUTANEOUS FARAdization.}

Intermittent cutaneous galranization is practised by applying dry metallic rheophores to the skin, previously dried by an absorbent powder; and by the aid of a rheotome set in motion by clockwork, the intermissions of which are nearly as rapid as those of the trembler of an induction apparatus.

The tension of a battery of good electro-motor power, such as that of my sulphate of lead battery of 100 elements, is not sufficiently great to excite acutely the sensibility of the skin; if it should do so in the same degree as cutaneous faradization, as when the skin is slightly humid, it would be by disorganising it by the electrolytic action of the galvanic current. Cutaneous galvanization by continuous currents is almost null when the skin is dry; and, if the skin be moist, it is still less acute than intermittent cutaneous galvanization.

Consequently, in cases where it is wished to produce upon the skin, either for a moment, or during several hours, a more or less powerful revulsion, which may be gradnated from a simple tingling to a sensation greater than that produced by fire, without producing the least electrolytic or calorific alteration, cutaneous galvanization would be either powerless or inconvenient.

Nothing but cutaneous faradization will correspond to these requirements. The clinical facts which I shall adduce, in the 
second Part, will show that at its maximum, or in a sufficient degree of force, I have seen it cure angina pectoris, and very painful sciatic neuralgia which had resisted all medication; while by lesser degrees I have successfully treated more or less grave neuroses and nervous maladies, or certain dynamic conditions (as in diphtheritic poisoning) by exciting the precordial cutaneous zone that is in reflex relation with the origin of the pueumogastric.

\section{Genelial Conchusions.}

It is not my intention to attempt a complete report on the therapeutic application of continnons currents. I believe them to be clestined to render great services in practice. In summing up in this chapter my personal experience of the applications that I have made of them, I am endeavouring to appreciate them at their proper value; and at the same time to guard the public against the exaggerations of their exclusive partisans. On the other land, in showing the admirable results of the employment of localized faradization, and in proving by facts that, in a large number of cases, it should not be supplanted by continuous currents, I have wished to retain in medical practice an excellent therapentic agent, however unjustly it may have been depreciated by persons who have not known how to use it, or who have not used it comparatively; an agent, finally, the application and management of which are easy and may become general. If I attain my object, I shall have the satisfaction of having avoided the great errors of electro-therapeutics, of having preserved faraclization from the disregard in which an endeavour has been made to place it; and of having largely contributed to its diffusion in medical practice.

I sum up, in the following conclusions, the principal facts that have been stated or demonstrated in this chapter.

I. The exclusive partisans of the therapentic application of continuous currents, who, with the object of obtaining the prevalence of their method, have charged localized faradization not only with being powerless in the treatment of paralysis, either with or without atrophy, but also with being hurtful by exerting a paralyzing action upon the vaso-motor nerves, these galvanotherapeutists, I say, have ignored or misunderstood the admirable results of the employment of localized faradization, in the treatinent of these affections, upon a great scale, for more than twenty years (see $§$ I. p. 147); in consequence, if they have not obtained the same results, they have but to blame themselves for not having followed the precepts that I have established after long: 
clinical experiment. It is shown elsewhere that the majority of them have not made any comparative use of faradization and of continnous currents.

II.--The electro-physiological experiments, made by many of them, and which have served as the basis of the attacks recently directed against the therapentic nse of localized faradization, have been badly interpreted, or are inexact. Contrary to certain assertions, the continuous constant eurrents and the induction currents, in equivalent doses, exert an identical action mpon the raso-motor constrictors. (See $\$$ II., p. 149.)

III.-The therapentic action of localized faradization is exerted peripherally, especially in promoting local circulation, and probably in exciting the trophic nerves which govern nutrition. (See § III., p. 153.)

IV.-The fundamental principles of the different methods of galvanization which have been lander in their turn, have not at present been expressed with sufficient clearness; whence may be explained the disagreement of experimenters with regard to the results of the therapentic application of galvanic currents. In the clinical researches that I have marle npon the comparative therapentic value of galvanization and faradization, I have divided the former into localized galvanization by intermittent currents, localized galvanization by permanent continnous constant currents, and reflex galvanization by labile and stabile continuous coustant currents. (See $\$$ III., p. 191 et seq.)

V.-A considerable number of paralytic affections-I mention this in the present place before making known the result of comparative researches-may be cured by any kind of electric excitation (faradization, galvanization, static electricity), in whatever manner it may be applied. (See p. 190.)

VI.-Localized muscular galvanization by interrupted currents, applied with a battery of sufficient electro-motor power, has appeared to me to exert nearly the same therapentic action as localized muscular faradization; but the latter is to be preferred, becanse, unlike the former, it exerts no painful electrolytic action; and because the instruments which furnish the induction current are of small bulk, portable, and inexpensive, while the batteries which, in these cases, should be composed of a large number of elements (about a hundred), are difficult of management, and only applicable in the reception-room of the specialist. (See p. 192.)

VII.-The electro-physiological phenomenon which forms the principal basis of the method of reflex galvanization by continuous currents extolled by Remak,-a phenomenon which consists in the production of tetanoid contractions in muscles supplied by the 
nerve antagonistic to that throngh which a strong constant current (of thirty of Daniell's couples at least) is passing and which he has called galvano-tonic contractions or shortenings, this electro-physiological phenomenon, I say, is imaginary. I have never seen it produced in any of the numerons experiments which I have made publicly upon healthy men. (See page 179.)

VIII.-It is true that reflex contractions are sometimes produced in certain pathological conditions; that we may see, for example, in paralytic contractions of the muscles supplied by the median nerve, consecutive to cerebral hrmorrhage, the hand open, the wrist and fore-arm become extended, during the passage of the continuous current through the nerve; but this phenomenon in no way confirms the theory, imagined by Remak, of the so-called galvano-tonic contractions. In fact, in these morbid conditions the spinal cord, being in a state of extreme excitability, any emotion whatever, as well as the act of sneezing or yawning, is sufficient to provoke the same reflex contractions.

The power to produce, in these morbid conditions, reflex spasms in the antagonists of the contracted muscles, is therefore a property common to many excitants; and, consequently, the denomination of galvano-tonic, given to these spasms, camnot be justified. (See a, page 197).

IX.-The hyposthenizing or paralyzing action (discovered by Eckhart) of continuous currents upon the nerve-trunks, may perhaps account for the therapeutic action which they sometimes exert on paralytic contractures. (See $b, \mathrm{p}, 199$ ).

X.-Contrary to the assertion of Remak and his followers, contractures consecutive to cerebral hemorrhage are seldom cured or improved by continuous currents applied after Remak's manner; because such contractures are nearly always (a fact which Remak ignores) symptomatic of secondary sclerosis of certain fibres of the cord. (See $c$, p. 199).

XI.-Rhemmatic contractures, and ascending contractures, after articular injury (an affection not hitherto described), can be better treated by energetic firadization of the antagonists of the contracted muscles than by continnous currents. (See $c$ and $d$, pP. 201, 202).

XIl.-The atrophic paralysis consecutive to injuries of the nerves is, in general, less quickly and less completely cured by continuons eurrents (according to Remak's method) than by localized muscular faradization; but the combination of both methods has appearel to me to yield the most satisfactory results in the treatment of these affections. (See A, p. 193).

The same statement will apply to load-palsies; although, 
according to Remak and some of his followers, they are not even susceptible of amelioration by the influence of induction currents. (See B, p. 194).

XIII.-It seems to me, however, to follow from some of uny experiments that, in the affections referred to above (XI. and XII.), a combination of the two methods of electrization, applied alternately, produces the most prompt and most satisfactory results; and that, in any case in which localized faradization has failed, we should have recourse to reflex galvanization by continuous currents.

XIV.-The trial of the therapeutic action of reflex galvanization by continuous currents is indicated at an early period of those chronic affections of the nerrous centres which auatomically form a great class of scleroses and atrophies of the structural elements of the cord or of the merlulla (progressive locomotor ataxy, glosso-labio-laryngeal paralysis, scattered sclerosis). I am unable to arrive at any conclusion from my own experiments with galvanism in these lesions. (See $f$, p. 207).

$\mathrm{XV}$.-Localized galvanization by permanent continuous constant currents exerts a therapentic influence, by virtue of its electrolytic action, which faradization is unable to replace. (See V., p. 208).

XVI.-An induction current passing from the anus to the mouth (operating by reflex action upon the spinal cord) - which has been charged with hastening, or even with occasioning death, when applied to the treatment of asphyxia-re-establishes, on the contrary, if it is applied in a slight degree, the respiration and the cardiac circulation, as perfectly as the continnous current administered in the same manner. (See C, p. 130).

XVII.-Cutaneous galvanization cannot be practised in any degree of intensity without producing alterations in the skin. It can neither equal nor supplement fiaradization of the skin, by which we can instantly produce an effect on the sensibility, ranging from a simple tingling to a pain greater than that which would be caused by burning, without producing the slightest organic change, however prolonged the application. The therapentic employment of cutaneous faradization is very frequently indicated; and has cured the most grave nemralgias and neuroses, among others, cases of angina pectoris, of asphyxia (see I., p. 134), and of disorder of the respiration and of the cardiac circulation. (See II., p. 137).

\section{Bibliographical Index.}

I have abstained in this chapter from entering into listorical details of the numerous experimental electro-physiological researches upon the action of continuous currents traversing longi- 
tudinally the nerves of man, that have been made from the time of Galvani to our own day; because these details are to be found in special treatises, and because I have not space even for an analysis of them. Moreover, I have wished to place before my readers, as far as possible, the results of my own researches, and only to eite those of others when I wished to be confirmed by them, or to refute them as being erromeous and in olposition to iny own. I have already brought together, in a bibliographic note, and in chronological order, the principal experimental and physiological researehes upon the action of eurrents traversing the nerves, which have been made from the time of Galvani to that of Mattencei (from 1791 to 1814 ).

It still remains for me to mention, in ehronological order, the most important subsequent works which have treated of the same subject, and especially those which have dealt with therapentic questions : ${ }^{1}$ -

SEchner, 'Lehrbuch des Galvanismus und der Electrochemie.' Leipzig, 1844. Asson, 'Rapporto della commissione che ha fatto gli sperimenti sull' elettroponetura come mezzo congelante il sangue nelle arterie e sull' obliterazione delle vase,' in Annuli unicersali di Medicina, 1847.

Duchenne (de Boulogne). 'De l'électrisation localisée et de son application à la thérapentique.' Ist edit., Paris, 1855. 2nd edition, 1861.

Heidinieich, 'Pliys.-chem. Untersuchungen des Blutes duch die electrische Säule,' in Neue med. Zeitung, 1847.

Du Bors-Reymond, 'Untersuchungen iiber thierische Electricität.' Berlin, 1848-49.

JALlabert, 'Expériences sur l'électricité appliquée à la médecine.' Paris, 1819.

Gurl (IV.), 'On the Value of Electricity as a Remedial Agent,' in 'Guy's Hospital Reports,' 1852.

Baierlacher, 'Die-Inductions-Electricität.' Nürnberg, 1853.

Eckinard, 'Grundziige der Plyssiologie des Nervensystems.' Giessen, 1854.'Beiträge zur Anatomie und Physiologie.' Giessen, 1855.

Schultz, "Die Reflexwirkungen der Inductions-Electricität als Heilmittel gegen Abuormitäten der Menstruation,' in IIiener med. Wochenschrift, 1855 .

Hilinenifan, 'Physiolog. Studien.' Berlin, 1856.

Risask, 'Uuber methodische Electrisirung motonischer Nerven.' Berlin, 18555-56. - 'Galvanotherapie der Nerven- und Muskelkrankheiten.' Berlin, 1858.

Fıck, 'Die med. Physik.' Berlin, 1856.

Eridunn, 'Die örtliche Anwendung' der' Electricität in Bezug' auf Physiologie, Pathologie und Therapie.' Leipzig, 15 õ6.

Trewnen, 'Die Electricität in der Medizin.' Berlin, 1857.

Becquerel, 'Traité des applications de l'électricité à la thérapentique.' Paris, 1857.

- Von Wittich, 'Teber den Einfluss des gaivanischen Stromes anf Eiweisslösungen und Eiweissdiffusion,' in Jon 'n. $f$. pralit. C'hrmie, Bd. Ixxiii., I857.

Rosenthal, 'Physikalische und pliysiologische Bemerkungen uiber Electrotherapie.' (Deutsche Klinik, 1858.)

1 This bibliographical index, as fur as Jaccoud, Nouv. Dict.de Mced.et de Chirnryie relates to works published in Germany, pretique. Paris, 1870 , tom. xii, int. has been in great part printed by Mi. | ÉLectriciś. 
Regnauld (Jules), 'Recherches électro-physiologiques.' (Journ. de Physiol. 1858.)

Moutard-Martin et Lardeau, Gaz. des Hópitanx, 1859.

Chauveau, 'Théorie des effets physiologiques produits par l'électricité.' (.Journul de Physiol., t. ii., 18\%9, t. iii., 1860.)

Pfldger, 'Untersuchungen über die Physiologie des Electrotomes.' Berlin, 1859.

Faxo, 'Flexions de l'utérus.' (L'nion méd., 18599.)

Deвout, bull. de Thiérup., 1860.

Tripier, 'Manmel d'électrothéraphie.' Paris, 1861.

BenedikT, 'Die Resultate der electrischen Untersuchung und Behandlung.' Wien, I861.- ' Nervopathologische Beobachtungen an Geisteskranken,' ete., in Archiv für Heillumde, 1867.- 'Electrotherapie.' Wien, 1868. - 'Ueber' die physiol. und pathol. Wirkungen des constanten Stromes.' (Wiener med. Hulle, 1861.)

Clenens, 'Die angewandte Heilelectricität,' in Deutsche Klinik, 1860.

Ton Holsbeck, Annules de l'Electricité médicale. Bruxelles, 1860-69.

Kistiakowskr, 'Ueber' die Wirkumg des constanten und Inductionsstromes auf die Flimmerbewegung,' in Moleschott's Untersuchungen, 1866.

StraderLi, 'Sulla contrattilita elettrica e volontaria.' (Gơz. med. Lombardu, 1866.)

Hernann, 'Weitere Untersuchungen zur Physiologie der Muskeln und Nerven.' Berlin, 1867.

Meyer, 'Beiträge zur Lehre von der' electrischen Nervenreizung:' Ziirich, 1867.

ALbY, 'Die Reiznng der quergestreiften Muskelfaser' durch Kettenströme,' in Archiv für Anutomi, 1867.

Syctanke, 'Ueber die Wirkung des galvanischen Stromes auf das Gehörorgan,' in Areh. für kilin. Med., 1867.

Lamarsky, 'Erregung motonischer Nerven durch den kurzdauernden Strom,' in Centrulblatt der med. Wissenschuften, 1867.

ERB, 'Ueber electrotonische Erscheinungen am lebenden Menschen.' (Archiv fiir klimiche Med.'’ 1867.)

Garnadit, 'Lecons élémentaires d'électricité.' Paris, 1866.

Wintrebert, 'Des colurants continus et de leur action sur l'organisme.' Thicse cle l'uris, 1866.

RANKE, 'Ueber die krampfstillende Wirkung des constanten Stromes.' (Zeitschrift für' Biologie, 1866.)

Poggroli, 'Sur le traitement des maladies par l'électricité statique.' (Bulletin de l'Aculémie de Médecine, 1866.)

Schrvard, 'Frammenti di clinica ellettrojatrica.' (Gaz. med. itul. ali Lombardia, 1866.)

Namas, 'Sui principi regolatori delle cure elettriche.' (Giornale J'enuto, 1867.)

Erd, 'Galvanotherapentische Mittheilungen.' (Archiv. für Klin. Med., 1867.)

Hiszig, 'Ueber die Anwendung unpolarisirbarer Electroden in der Electrotherapie,' in Berliner klin. Wochenschrift, 1867.

Eulenburg, 'Ueher die electronisirenden Wirkungen bei percutaner Anwendung des constanten Stromes.' (Archiv fïr lilin. Med., 1867.)

Semlmü̈liLer, 'Ueber Anwendung der Electricitat bei Krankheiten.' (Correspondenzblutt des leveins der Aerate in Mersebuing, 1867.)

Beard and Rockwelus, "The Medical Use of Electricity," in "New York Med. Review,' 1867.

Erdusns, 'Beitrüge zur Electrotherapie.' (Arch. für klin. Mecl., 1867.)

LANGES, 'Ueber Anwendung del' Inductions-Eleetricität gegen paralytiseho Contracturen,' in I'iener med. Wochenschrift, 1867.)

Alvarenga, 'Ascite con anasarca,' etc. (Giaz. med. de Lisbou, 1867.)

Oninus, 'De l'emploi des comrants constants.' (Guz. des Ilôp., 1868-69).

BENEDI Kт, 'Electrotherapie.' Wien, 1868.

Bærinkel, 'Zur electrotherapeutischen Casuistik.' (Arch. der Ileill:, 1868.)

Jaccoud, Toue. Dict. de méd. et de chirurgie pratiques. Paris, 1870, t. xii., art. 'Électricité,' 


\section{On the Portable Constant-Current Battery of M. Ruhmiorff.}

MI. Ruhmkorff informed me, a little tardily, that he had constructed a portable constant-current battery, with bisulphate of mereury, ox with chloride of silver. He has placed at my disposal a portable battery of bisulphate of mereury (carbon, zine, and solution of bisulphate of mereury), in order that I might test its merits. Having done so, I hare been able to judge of its value, and to point out certain imperfections, which this skilful mechanician has applied himself to remove at my request.

The portable battery, thus modified, seems destined to be of great service in the application of continuous currents; for which reason I have determined to give a description of it in this note.

The battery (see fig. 50) is enclosed in a box, $\mathrm{A}, \mathrm{A}^{\prime}, \mathrm{A}^{\prime \prime}$, containing forty-

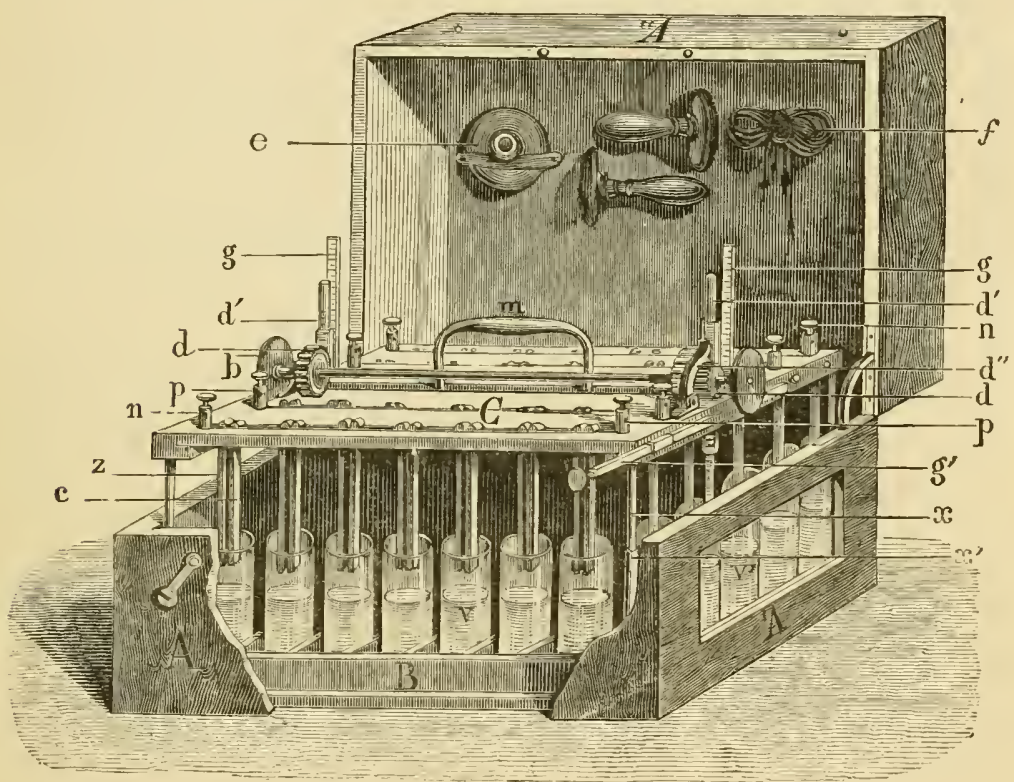

Fig. 50.-Ruimkori's portable battery, modified after the suggestions of M. Duchenne.

two small elements, the glass cells of which are placed in divisions at the bottom of the box B, by which they are separated one from another. These divisions are arranged in six rows of seven. The zine and the earbon of each element ,z, c, are fixed to a donble movable platform, C, of hard vulcanite, placed above the glass cells containing a solution of bisulphate of murcury, into which they can be lowered, more or less, at pleasure, by turning the milled head, $b$, which acts upon the pinions and racks, $d, d^{\prime}$, and Iy, $g^{\prime}$, which elevate or depress the double platform, and consequently the zinc and earbon of every element. When the battery is not in nse, the platform, $\mathrm{C}$, is so lifted as to take the elements entirely ont of the solution.

'I'he following are the modifications that I have requested MI. Tuhmlrorff to introduce into a battery for my private use.

1. In the battery sent me for experiment the carbons were cylinlrical, nine centimetres in height, and conld be immersed for five centinetres with 
sixteen millimetres of section. In the centre of each carbon was a eylinder of zine of the same height, a centimetre and a half in diameter. This battery gave too great a quantity of electricity, in direct proportion to its extent of surface.

Its electrolytic action was such that, with forty-two elements immersed scarcely a centimetre, its rheophores produced resication of the skin in two or three minutes. I therefore requested M. Ruhmkorff to diminish the surface by two-thirds, and he has done so by constrneting the zine and carbon as shown in the figure. They measure nine centimetres by eight millimetres, and act only by the surfaces that are mutually opposed. I have also replaced the solution of bisulphate of mercury by a weak solution of the protosnlphate.

As thins modified, the battery may be used for all electro-physiological or therapentic applications of contimnons currents. It has also been rendered less heary and less costly by diminishing the bulk of the carbons. The greater or less degree of immersion of the elements in the solution is obtained, as I have said, by raising or lowering the donble platform C. To raise this platform, with the elements that are fixed to it, it may be lifted more or less by the handle, $m$, and its descent is prevented by the eatch, $d^{\prime \prime}$, which falls betwcen the teeth of the pinion, and which must be lifted in order to lower the platform. The teetl of the pinion are so arranged that each one allows the platform to descend a single millimetre.

The mechanism of the system of gradnal immersion or emergence of the elements is assuredly simple and good, but it leaves something to be required with regard to the exact gradnation of the electrolytic action of the battery. The indicator does not show precisely the degree of immersion of the elements, except on the condition that the liquid in the cells shonld stand always at the same level. But, as the cells are not closed, the height of the liquid is constantly diminisled by evaporation. I have therefore had a line marked on the walls of the eells, to show the height at which the liquid shonld be kept, and a large opening made in the side or front of the box, and filled with glass, throngh which the height of the liquid may be seen.

2. The battery is divided into six jows, each composed of seren clements. The serews of the first row, which fix the zinc and carbon to the platform $\mathrm{C}$, are furnished with openings in which to place the conductors; so that the current may be increased or diminished by from one to seven elements.

This graduation is particularly applicable to delicate physiological experiments. 'The metallic stem $g$ ' (graduator' of the tension), which M. Ruhmkorff has added to the apparatus at my desire, is so constructed as to gradnate the tension of the currents, withont producing intermissions. When it is pushed in the tension of the battery is at its maximum, and as it is withdrawn the number of elements is diminished by serens, as shown by the divisions marked upon the stem, without its being necessary to change the enductors fixed to the knobs $n$ and $p$.

WThen the battery is exhansted, the platform $\mathrm{C}$ is entirely remored from the box by the handle $m$, and the cells are refilled with fresh solution. For that purpose, water is poured into them to the level of the horizontal line already mentioned, and a single gramme of protosulphate of meremry is added to each, by means of a little measure which is attached to the apparatus. This manipulation is easy, and can always be exactly done by the means indieated abore.

One of the principal adrantages of this battery is the facility of graduating the degree of immersion of the elements; or, in other words, of measuring the electrolytic action while preserving the force of the tension. The possibility of obtaining from it a weaker or more powerful electrolytic action has the great advantage of rendering it equally applicable to cases for which the use of the continuous current by reflex action is required, and to those in which the surgeon wishes for electrolytic effects.

In M. Rulimkorff's chloride of silver battery the carbons are replaced by eylindrical stems of chloride of silver. 
Several questions in regard to the therapeutic and diagnostic uses of the galvanic current have enlisted the attention of medical men in England, to which Dr. Duchenne has only incidentally referred in the preceding chapter. Some of these questions were originated by Remak; others refer to the effects of interrupted currents of low tension in certain forms of paralysis. These questions have been so ably discussed by Ziemssen ${ }^{2}$ in an examination of the therapeutic uses of galvanic currents, that I have not hesitated, as the most instructive mode of dealing with the subject, to append a condensed translation of so much of his observations as may serve for their elucidation. $-H$. $T$.

\section{ZIEMSSEN ON CERTAIN QUESTIONS WITH REGARD TO THE VALUE OF GALVANIC CURRENTS IN MEDICINE.}

With regard to the effects of the continuous current upon diseased muscles and nerves, and of certain indications for its use in therapentics, it will be necessary first clearly to describe the facts that have been ascertained by competent observers; and, secondly, to point out the questions on which further investigations are required. Manifestly for this purpose we must set forth from the fundamental doctrines laid down by Remak from his own observations and experience.

Remak classifies the most conspicuous curative effects of the continuous current under the three following heads:-

\section{I.-Catalytic actions.}

Remak calls those effects catalytic, or antiphlogistic, which are procluced by the continnous current upon inflammatory conditions and their consequences. He gives an analysis of this action, and calls it catalytic because "the action of the current where it meets with an abnormal state of the tissues, produced either by failure of the blood circulation or by morbid effusion, is not limited to an electrolysis in the narrow sense, that is, to a change in the tissues analogous to a watery solution, but performs an important part, by dilatation of the blood ressels, in promoting circulation and absorption."

The continuous current, according to Remak, exerts this "catalytic" action in the following states:-

1. In inflammatory conditions.

2. In effusions left behind by any of the foregoing inflammatory conditions; e. $\%$. in watery effusion into a joint.

3. In painful and inflammatory swellings.

As regards the manner of proceeding, Remak insists that it is necessary to proceed from the assumption that a passage of fluids occurs from the positive to the negative pole ; and hence, to place the negative electrode in nnion with the inflamed spot, and the positive as near to it as possible. This direction of the current.should, however, we reversed, when there is evidence of watery exudation together with the inflammation. Since also the positive pole diminishes and the negative increases the excitability of the nerves, the operator must take heed to the character of the inflammation, and in erethismal conditions must place the positive pole, in torpid conditions the negative pole, exclusively or alternately in union with the inflamed parts. The strength of the current must be moderate (about twenty elements), but the time of application considerable (at least several minutes). The benumbing of sensibility in the nerves affected by the current may serve as a limit for the length of application.

2 Ziemssen, Nie Electricität in der Medicin. 
The experience of those observers who have tested Remak's assertions, has been generally very favourahle to the catalytic action of the electric current; especially in chronic glandular swellings and firm exudations into and around joints. The observations hitherto pullished do not afford materials for judging whether a solvent or absorption-producing action is exerted in a ligher degree, as Remak belieres, by the continuous than by the induced current.

\section{II.-Antispastic actions.}

These depend partly npon this, "that the current increases the power of the will orer the muscles that are seized with spasm or tremor, or ly its catalytic action removes the irritation by which spasm had lieen exeited," and partly that 'an increased excitalility of nerves or muscles, which appears to be a cause of local spasms, is diminished by a proper application of the current."

These antispastic actions, which Remak professes to have olserved, have justly excited among practitioners great doubts of the accuracy of his observations; and are wholly unconfirmed by others.

In the discourses, delivered in Paris hy Remak in 1S64, the antispastic actions are not generally mentioned; but he speaks of "tranquilizing" actions in contractures and hyperasthesiz. The latter, whether of inflammatory or simply of nenralgic origin, yield, according to Remak, to the weak and painless currents of $15-25$ elements, in stabile application; that is, with jmmovably held electrodes, so that the magnetic needle of the galvanoscope, the deriation of which need not exceed $20^{\circ}$, may lemain stationary. The positire electrode, in the form of a large plate, should be laid on the painful part; the negative applied to any distant point of the surface. After the current. has heen passing for from ten to tifteen minutes, the pain should be considerably climinished. The tranquilizing action should also be displayed by placing the positive electrode upon the nerve-trunk, the branches of which are distributed to the painful part, as far from this part as possible; while the negative is placed on any distant point, and contact with the seat of pain is wholly a roided.

For the treatment of contractures, Remak found that intermissions of the contimnous current were more efficacions than the 'stabile' action, and he is generally disposed to ascribe to intermissions a favourable operation.

\section{III.-Antiparatytic actions.}

These were observed by Remak under the following conditions:-

1. In secondary pareses and atrophies with or withont coutraction, remaining after articular and muscular rhemmatism, or after false anchylosis.

2. In the first stages of primary musenlar atrophy, even when progressive.

3. In traumatic paralysis, produced by contusion or stretching of nerves and muscles.

4. In lemiplegia, within the limits determined by the nature of the central lesion.

5. In paraplegia, and in certain forms of the so-called tabes dorsalis; according to the extent of the atrophy of the cord.

6. In annesthesia either as an independent disease or as a complication of motor paralysis.

Subsequently, Remak has designated the antiparalytic influence of the current as an "exciting" and "revivifying" action. To obtain this he employs both the "stabile" current (application du courant en repos); and the so-called "labile" (application du courant en mouvement). The latter he obtains by gliding the electrodes to and fro orer the skin. It is manifest that, in these morements of the electrodes, the tension of the current cannot remain unchanged, but must undergo oscillations, dejendent mpon the variations of 
resistance as the electrodes more their places on the surface. Remak assigns it as a difference between his "labile" galvanic and the common induced currents that, in the latter, the fall of the current is from its maximum to nil; but that, in the 'labile,' although the current certainly falls from its height, it still remains always consilerably more than nit. This distinction is illusory. In the 'labile' application we can no longer speak of constant currents; but only of contimuous currents of irregularly oscillating strength. After Rosenthal, Oppenheimer, Sinsteden, and others, had confirmed my opinion upon this point, lemak confessed himself to have bcen in error.

Disregarding only these obscurities abont the methods of galvanizing, Remak's doctrines, with regard to the antiparalytic curative effects, contain yet many other points that justify considerable hesitation. This especially applies to the centripeful action of the currents, which Remak considers to be partly dne to a direct action on the brain and spinal cord, and to be best explicable by an action on the raso-motor nerves; partly to reflex action.

With regard to the first, Remak deals entirely in hyputheses of the boldest kind. He not only believes in producing a regulation of the circulation in the brain by galvanizing the plexuses or ganglia of the sympathetic (preferably the superior cervical ganglia); but also in diminishing, ly the same procedure, the irritation of the cercbral snbstance around an apoplectic clot, and in lrastening the absorption of the blood, so as to prevent the ocenrrence of contractions in the paralysed half of the body; and this the more easily and certainly, the more recent the paralysis. Remak's method, in such cases, consists in the 'labile' application of the electrodes over the cervical ganglia of the sympathetic, with the current of from 20 to 30 elements.

Remak also believes that he has obtained a curative action ripon the spinal cord, by the labile application of a current from 20 to 30 clements in the neighbourhood of the solar plexus, and of the lumbar plexuses of the sympathetic, in cases of ascending spinal paralysis, motor ataxy, hysterical paraplegia, and other disorders of motion due to changes in the spinal cord.

Even in the most superficial view, it excites suspicion to hear such proforund anatomical lesions described as being generally curable; especially when no clinical evidence is fortheoming. It must, moreover, appear highly improbable, $\grave{a}$ miori, that it is generally possible, by the method described, to produce excitation of the sympathetic ganglia and plexuses. The tension of an electric current entering the body, as soon as it has passed through the skin, undergoes such rapid diminntion, that muscles or nerves, separated from the surface by a moderately thick layer of well conducting tissues $(e, g$, of muscle), are no longer acted upon, unless extremely powerful currents are employed. How much less is it to be expected that the comparatively feeble current of a battery of twenty or thirty elements, in labile application, can be made to reach the superior cervical ganglion, or still more the coeliac plexus, with the tension necessary to produce any excitation.

But, even assuming it to be proved, that a direct action of the current upon these nervous organs is possible, there still remains the larger question, whether excitation of the plexuses and ganglia of the sympathetic does actually have, or conld have, the action upon the central organs that Remak professes to have observed. Of the functions of the ganglia, we know what amounts to nothing; and of the cervical sympathetic we know that its division, in animals, or its paralysis from other canses, is followed by a dilatation of ressels and increase of temperature in the corresponding half of the face, and especially in the car. Its irritation, on the contrary, produces contraction of the vessels, diminution of the gruantity of the blood and of temperature in the same soft parts. If, therefore, Remak obtains by galvanism a dilatation of the intra-cephalic and intra-spinal bloodvessels, as he in various places declares, this action mnst be considered evidence of a paralysing, not of an exciting, influence of the current.. ${ }^{3}$ Such a paralysing,

3 [Upon this point, compare Duchenne upon the active dilatation of vessels, p. 161, et seq. $-H . T$. ] 
or, more correctly, conduction checking or delaying influence, has been now shown by physiological experiment to attach to the positive pole. This is the state of anelectrotonus. But in mankind only vain endeavours to produce the anelectrotonic condition have been made; and especially the experiments of Fick and myself in this direction have furnished wholly negative results.

We see into what a chaos of hypotheses the dogmas of Remak conduct us.

Not less insecure is the ground of his statements upon the galvanotonic reflex contractions. These occur, according to him, throngh the intermediation of the central organ; and during peripheral excitation of a cerebrospinal or sympathetic nerve. In excitation of a motor nerve they do not occur in its own muscles, but either in their antagonists (e.g., in the extensors of the forearm when the median nerve is excited), galvanotonic antagonistic contraction; or in corresponding museles of the opposite side of the body, errssed galvanotonic contraction; or, lastly, through irritation of two points, on the opposite side of the borly, remote from the muscles to he exeited (diplegic reflex contrartion). As the most important point at which to mndertake this irritation with the positive pole, Remak mentions the anriculo-mastoid fossa, between the ascending ramus of the lower jaw, the auricle, and the mastoid process, at the lieight of the superior cervical ganglion. In a case, for example, of progressire atrophy of the muscles of both upper extremities, Remak places a positive electrode, furnished with a knob at its extremity, on the spot ahove specified, on the right side, and completes the cirenit by a negative electrode with a larger surface of contact, placed on the left margin of the sixth dorsal vertebra. Then, the electrodes remaining stationary, with a current from thirty to thirty-six of Siemens' elements, a contraction will oceur in the paralysed muscles of the hand on the left side; or of the same muscles on the right side, when the positive electrode is placed in the left mastoid fossa, the negative remaining as before. The latter, however, withont diminntion of the contraction in the hand, can be moved down to the lumbar region; but the contraction ceases as soon as the negative electrode is moved above the fifth dorsal vertebra, or above a horizontal line passing throngh it. ${ }^{4}$

It would lead us too far, to go more exactly into the details of this wholly unintelligible reflex contraction of Piemak's. He himself admits that no explanation of it can he given in the present state of our knowledge; since he says that the facts stand opposed to the formule of physies and of physiology, according to which the effects are more energetic the nearer the electrodes to the parts influenced; while here the contraction ceases as soon as both electrodes are above the lerel of the fifth rlorsal vertebra. Remak suspects that he produces simultaneous excitation of two distant sympathetic ganglia, and that the excitation is conveyed from these to the spinal cord, and thence to the motor fibres of the same or of the opposite side.

Remak describes the therapentic effect of this diplegic central excitation as very considerable. The atrophied muscles, which no longer display any reaction to the direct influence of the constant current, not only contract, but swell out, and recover their lost strength.

Remak found, moreover, snch diplegie contractions in the muscles of the hands in some cases of arthritis nodosa, in its early fehrile stage. In these eases the diplegic application produced a diminution of the pain and the articular swelling, inerease in the cireumference of the muscles, diminished quickness of the pulse, and decrease of temperature. Moreorer the production of contractions was not always necessary. Without them, a gentle, continued action was curative, when there were no profound lesions of the spinal cord, or of the sympathetic ganglia. The internal administration of

${ }^{4}$ [As yet, after the most careful observation, and equally careful and very necessary endearour to cxclude sources of error, "diplegic reflex contrartion." so- called, has never been observed in the practice and experimental researches at the National Hospital for the Paralysed and Epi'eptic.-H.T.] 
strychnia rendered the diplegic contractions more perceptible, where they were at first wanting.

It should not be supposed that Remak has not actually witnessed these phenomena. In hysterical people-and of such he treated great numbersfar more wonderful things occur. It may be remarked, however, that the observations are at present wholly without corroboration from trustworthy persons.

The more dissatisfied we leave this region of obscure observations, insufficiently founded diagnosis, and hypothetical explanations of doubtful curative actions, the more contented shall we be to return to the basis of facts, which, gained by means of sober inquiry, are already recognized as valuable scientific property.

These observations refer to the changes produced by the electric current in the irritability of paralysed nerves and muscles with regard both to volition and to the electric stimulus.

Between 1840-50, it was established by Duchenne, that induced currents usually quickly improved the excitability of enfeebled or completely paralysed rouscles, when their electric contractility is retained or only slightly enfeebled,

At the same time he conchuded that complete loss of electro-muscular cont netility was a most unfavourable symptom with regard to paralysis in general; indicating long duration of the condition, and great resistance to the action of remedies. In traumatic paralysis of mixed nerves, in which total loss of electro-muscular contractility was present, Duchenne declared the application of induced currents to be useless during the first six to ten 'months, since the nerve injury was not yet repaired. Afterwards benefit might be expected from faradization.

Duchenne subsequently obserred the remarkable phenomenon that the increase of excitability of motor nerves or muscles, produced by the induced current, was often only displayed in response to the stimulns of the will; so that therefore the motility returned while the absence of excitability by induced currents contimued. In lead-palsy, traumatic paralysis, and paralysis of the facial nerve, Duchenne found that muscles might contract faultlessly in response to the will, and perform designed movements, while they were perfectly insensitive to the excitation of induced currents.

These facts have since been frequently observed. We may now consider it established:-1. That paralyses, affecting the same nerves or muscles, and proceeding from simitar lesions, may display either a normal degree, a diminution, ol a complete loss of faradic contractility.

2. That the increase of excitability by the will, produced by the application of faradic currents, occurs quickly or immediately in the two first cases; slowly, or not at all, in the third.

3. That the excitability of the paralysed nerves and muscles by the will, under the application of induced currents, completely returns, in many cases; while the excitability by the induced current is still absent, and returns only subsequently to the former.

Recent years have much enriched our knowledge upon this subject, by many interesting and practically important facts, gained by Remak's application of battery currents in paralysis.

Remak, in the course of his experiments, came first to the conclusion that constant "labile" currents increased the irritability of motor and sensitive nerves both against the same currents and the induced, while at the same time the mechanical power of action was increased; but that induced currents which certainly somewhat increased the irritability, diminished the power of muscular action.

These experiments were performed by Remak preferentially upon diseased but also upon healthy muscles and nerves. Unfortunately his accounts of the results are inexact and confusing, by the strange style of description. I therefore only extract what is for the present question important; that, according to Remak, "the irritability of muscles and nerves for induced currents, and for the entrance and exit of constant currents, remains, as a rule, uniform, so 
far as it can be judged of; but that, in some healthy persons, and eertainly more clearly in some paralysed limbs, the irritability for one or the other kind of current unequivocally preponderates."

This difference between the actions of indnced and eontinuous currents, very generally stated by Remak, has since become the subject of numerous and searching inquiries.

The great and general result of these inquiries is the following. In completely paralysed mascles and neries the excitability for battery currents is sometimes retained or even increased, while the excitability for induced currents is completely lost. In such cases the curative action of the continuous current is superior to that of the induced. With the return of motility the excitability for both Finds of current commonly clanges.

The first observation hearing upon these points was published by Baierlacher, in the beginning of the year 1859. To him, therefore, undoubtedly belongs the merit of having heen the first to notice this interesting and important faet. I give a brief abstract of the ease.

II. B., agerl 28, workwoman in a factory, came under treatment for paralysis of one side of the face of eight weeks' duration. Induced currents of great strength produced only the very smallest action. After three weeks' use of the induced curests there was no change. A galranic eurrent from fifteen elements was then applied, and produced strong contractions in all the muscles. After only three applications of the continuons current to the nerve-trunk and muscles considerable improvement was manifest, aud after four more applications the paralysis had almost entirely disappeared.

There next appeared eommunications from Schulz, upon several eases of facial paralysis, in which indnced currents produced no action on the paralysed side. Schulz obtained, by testing with the continuons current, the following results. On the paralysed sites a eurrent from eight Daniell's elements produced in all the muscles a manifest contraction at opening and closing, whether the direction of the current were ascending or descending, and although the same current on the sonnd side prodnced no contraction. By increasing the number of elements up to twenty, eontractions on the sound sicle were produced at opening and closing; but these contractions were mueh exceeded by those of the paralysed side.

This condition changed during the course of treatment. The increased excitability of the paralysed muscles to the continuous current gradually decreased, bccame more feeble from sitting to sitting, and at last disappeared entirely, as upon the healthy side. unless when the number of elements was gradually increased. This diminution of the exeitability for the continuous eurrent is, according to Schulz, a result of treatment and a sign of improvement; since the restoration of the excitability by the will proceeds with it pari pass". The earlier or later occurrence of this combination of symptoms affords the most sure basis for a judgment with regard to the duration of the paralysis. The sooner the excitability by the continuous current diminishes the shorter will be the general duration of the paralysis, and vice versâ. With the disappearance of the paralysis the excitability by induced currents returns, and becomes the same as that of the museles on the healthy side.

To these observations by Schulz may be appended one by M. Meyer.

The case was that of a woman forty-eight years old, with paralysis of the left side of the face, of fourteen days' duration. There was no trace of reaction to the induced current, but a strong contraction of the paralysed muscles was produced by elosing the circuit of a battery composed of six of Bunsen's cells. Under the treatment by the continuons enrrent, improvement occurred slowly but still manifestly from sitting to sitting.

This ease (reported at great length by Ziemssen) acquired much interest, from the long time that it was under ohservation. While the branches of the left facial nerve, and the muscles supplied by them, had completely lost, three weeks after the commencement of the paralysis, their excitability either by volition or by induced currents, their excitability by ontinuons currents was 
not only retained, but by comparison with the sound side eren heightened. From the tenth week the excitability by the continuons current, in almost the whole territory of the facial nerve, markedly diminished, while the distortion of the countenance improved. In the twelfth week the reaction to battery currents wholly ceased, and in the forrteenth reek not only was the normal position of the face quite restored, but the mastery of the will over the paralysed muscles was regained. At the discharge of the patient in the seventeenth week the facial morements on the left side were almost faultless, while the excitability for either induced or continuous currents was wholly wanting.

Two years later, with completely normal motility, the excitability for electric currents was restored, and for both currents in the same proportion. But the excitability displayed on the right (sound side), and the sensibility of the left, were both far below the normal.

To this case Ziemssen has added another, also affecting the facial nerve, (which he also reports at length), and which was in many respects clearer and more transparent than the first, and is of especial importance with regard to the significance of many of the phenomena in question. It was a purely trammatic facial paralysis, produced by a surgical operation, in which division of the facial nerve, immediately after its exit from the canal of Fallopius, conld not be avoided.

The details of this observation were unmistakeable and convincing. The trunk of the facial nerve was certainly completely clivicled. At the end of the third week after the division, the excitability of the motor nerves by the will, by the induced current and by the galranic current, was lost, and was only retained in a small degree in one branch for the galvanic current. The paralysed muscles, on the contrary, had preserved their irritability to the continnous current, and responded to direct galvanizing by a slow contraction which, with a stronger current, passed into tetanus. The entrance contraction at closure of the cireuit was lost, and the slow and continuing contraction, that Wundt and Fick had determined experimentally to be due to the muscular tissue without the agency of nerve; was retained.

M. Meyer found that those cases of facial paralysis in which the reaction to the intemupted current, although diminished, was not wholly lost, showed the same diminution of reaction to the constant current also; and that such cases admitted of a favourable prognosis, inasmuch as they usually ended in recovery within a few weeks. Cases, on the contrary, in which, eight days after their commencement, no reaction was produced by the intermitting current, but in which a weaker battery current produced active contractions, were of far less favomrable prognosis, in so far as their recovery was delayed for months, or remained always imperfect.

Meyer supposed the seat of the cause of the paralysis in the latter cases to be in the trunk of the facial nerve, within the petrous bone, and held that the muscular contractions produced by feeble battery currents were reflex, with the geniculate ganglion for their centre. Meyer sought the basis for this hypothesis in the spasmodic character of the contractions as opposed to their slower character on the healthy side, in the disproportionate measure of the contraction, and lastly in the fact that it is necessary gradually to add more elements in order to obtain a contraction, which allows of the conclusion that the track is free.

In respect of curative action, Meyer recommends the current which produces contractions with the least intensity as the best: that is, in the slighter cases, the interrupted; in the more severe, the constant. Meyer saw contractions cured, not only by the application of the induced, but also by the use of the constant currents; but they were generally removed surprisingly quickly by the latter, eren after they had existed for years.

Ziemssen next reports an important case of facial paralysis reported by Neumann, and differing in many points from the foregoing.

Herr H_-, 60 years old, contracted a paralysis of the left side of the face from exposure to colil, at the end of Scptember, 1863. It was at first attended by acute 
neuralgia, afterwards by diminished sensitiveness to the electric exeitation. The paralysis of motion was complete, the sense of taste was lost on the affected side of the tongue, and the condition of the soft palate was normal. From the end of Oetober to the 4 th of December the induced eurrent was applied daily for half an hour without result. Neumann then had recourse to the continuous current, which by the 24th of January had prodnced considerable improvement. The induced curreut at first produced no reaction, in whatever strength it was applied or wherever the electrodes were placed. With the beginning of the return of volitional control over the muscles there appearesl also a trace of reaction to faradism. This appeared only on direct muscular excitation, and was much less on the cliseased than on the healthy sile. The continuous current, on the contrary, prodnced stronger contractions on the diseased side than on the healthy. By the application only of from six to eight of Siemens' elements contraction was produced on the former, but not with less than from ten to twelve elements on the latter. On the healthy side, with from fourteen to sixteen elements, reaction could be produced either by intra-muscular or extra-musenlar excitation, while on the diseased side it was only produced by the direct method.

In this case Neumamn instituted experiments calculated to afford a physical explanation of the difference of action of the two emrrents. He finds the physical peculiarities of the induced currents, as compared with continuous currents, to be in the rapid succession of currents separated by intervals, in their alternating direction, and in their momentary individual duration. A fourth possibility, that the strength of the single induced currents may he somewhat less than the strength of the continuons battery current, and that this may explain the inefficacy of the former, may at once be set aside by the foregoing; since a current from six of Siemens' elements, when the human body is included within its circuit, produces a scarccly appreciable reaction, and induced currents from superimposed coils produce an unbearably strong reaction.

In order to test the first three points of difference, the hammer of an induction apparatus was scenred; and a cup of mereury, with wires dipping into it, arranged so as to form part of the circuit of the induced current. By raising and again dipping the wires, completion and interruption induction shocks were obtained, and it was found that neither the stronger interruption shock, nor the weaker completion shock, produced contractions on the paralysed side, although the completion shocks in the same experiment acted powerfully upon the sound side. Hence it appeared that neither the rapid sncecssion of the individual induction shocks, nor their alternating direction, could be the cause of their inefficacy, and hence only their brief duration remains to he considered. The following experiment may serve for proof. Neumann reduced the duration of the battery current to the smallest appreciable time by introdneing into the circuit a mechanism in which a fine platinum wire was drawn quickly orer a little plate of wood in which a thin plate of platinum was inserted, with its edge level with the surface. The current only passed whilst the wire and the plate of platinum were in contact, and the electrodes being first put in position, the circuit was thus completed. $\mathrm{He}$ found that the strongest battery current was thus rendered almost ineffectual on the paralysed side, although on the sound side it produced active contraction. If one electrode was placed ou the paralysed cheek, the other on the chin exactly in the median line, and then a current from fifty elements was allowed to pass through the abore described apparatus, the paralysed side showed only a trace of contraction, while the sound side, although lying outside of the proper course of the current, was more strongly mored. The principal current acted with less power on the paralysed side than the side current on the sound side, and Netumann has observed the same thing with strong induction shocks in the same position of the electrodes.

Neumann replies as follows to the question as to the nature of the physical difference of the induced and of the battery currents in their action upon paralysed nerves. The excitability of the paralysed nerves and muscles as against momentary currents - even when of considerable strength-is lost; but their excitability as against currents of greater than momentary dura- 
tion, even of a less degree of strength, is retained, and even somewhat increased.

Neumann has since observed the same phenomena, in the nerves and muscles of frogs after the deatl of the animal, as in the paralysed muscles of the face. Before the total loss of excitability, he has seen a period of diminution, often lasting for several hours, during which the strongest induced currents of the slide apparatus, with its eylinder pushed in, were ineffectual to excite contractions, while the completion and interruption of battery eurrents from four or six of Siemens' elements produced a marked reaction, both through the motor nerves, and in direct excitation of the muscles. Nenmann applies to this phenomenon the above explanation; namely, that under the conditions present, a stronger momentary current produces a less effect than the interruption and completion of a weaker one of longer duration; and he refer's to the researches of von Bezold and Fick, according to whom, next to the oscillations of tension, the time during which the current passes at a constant height throngh nerve and muscle, forms an important factor of the stimulation.

In succession to these observations of Neumann, Briuckner has instituted experiments on sereral patients suffering from fatty atrophic paralysis, and has confirmed Nemmanm's view. On the paralysed muscles an induced current had 110 action; but a battery current of twenty elements produced a contraction at closing. Brïckner observed that this contraction at closing did not take place immediately, as it does in sound or only slightly paralysed muscles, but that a very small period of time intervened; also that in slow succession of completion and interruption (from halt to one second) the contractions were greater than in quicker succession (four or fire in a second); that in changing the current by the commutator, the contractions werc yet more considerable than in slow interruptions and completions; and, lastly, that in approximating instantaneous interruptions of a continuous current, the contraction wholly ceased. Briickner tosted these facts on the peroneal, crural, sciatic and tibial nerves, in this manner. The current passed through a cirenit completed by an electric key; and this eirenit, by means of a short gentle tap on the heal of the key, was opened and instantly closed again. During this momentary interruption, the contraction of the muscle remained unaltered; but in a longer intermption it always became stronger. That an interruption actually took place, is rendered evident by Brückner's test experiments. With how many elements he worked on the individnal muscles and nerves, and whether he went on to employ currents of considerable strength, are not stated. He concludes by saying that, so far as any conclusion can be drawn from his few experiments, a certain tardiness of the nerves with respect to the electrotonic state is not to be mistaken, since they require a longer duration of current in order to be brought into this state, and, when so brought, a longer interruption in order to lose it, and in a momentary interruption they contimue in it.

For the last observations bearing upon this question we are indebted to $\mathrm{A}$. Enlenburg. He saw the case of a girl, twenty years old, who had suffered for eight weeks from complete facial paralysis of the right side, of rheumatic or traumatic origin. With normal cutaneons sensibility the affected side had completely lost its excitability by intermitting currents, either intra- or extramuscular, either with slow or rapid interruptions, or with changing the direction of the current. Faradization, and the subcutaneous injection of strychnine, in the course of forty-one days, produced no alteration whatever. A galvanic current from eighteen defective Daniell's elements was then employed; this, althongh so weak that it produced no trace of effect upon the sound side, either motor or sensory, produced strong contractions on the diseased side, especially when both poles were placed upon the face. After seren sittings, the paralysis was decidedly improved, but the difference in the action of the two kinds of current was unaltered. After nineteen sittings (from February 27 to March 17) the deformity was removed, and the power of voluntary movement was restored. Notwithstanding this, the reaction to 
the two kinds of current remained unaltered from the first condition; and, especially during the recovery, the susceptibility to the galvanic eurrent did not diminish, nor that to the induced current increase, as might have been expected from former observations.

Enleuburg-who contests Meyer's doctrine, that the contractions obtained through the facial are of a reflex character-rightly places stress upon the following points in his observations, as incompatible with that doctrine. 1. 'The stationary character of the galvanic stimulating effect. 2. The alsence of any considerable irritation of the sensitive or sensory (special) nerves. 3. That the contractions had no spasmodic character. 4. That on localizing the current in any muscle, this and no other would react. 5. That slow intermission of very painful induced eurrents produced no contraction.

Enlenburg thus points ont a modified action of the current mpon the facial; since, in such a case, the abiding loss of sensibility to the induced current remains unexplained. He is inclined to suppose the existence of different specific energies of the motor nerves, namely, for the galranic, the faradic, and the volitional stimulus. He thinks it possible that, under certain unknown differences of molecular condition, one or other of these energies, or even two of them, may be wholly lost, without injury to the thircl. He recalls the hitherto little studied cases in which, while the motility remains intact, the galvanic and faradic irritabilities are lost; and also the occurrence of loss of sense of pain, and loss of sense of temperature in certain cerebral derangements, while the sense of tonch remains.

All the preceding observations, given in abstract, with the exception of Brickner's, refer solely to paralysis of the facial nerve; and Ziemssen adds two more, which belong to the same order' with Brickner's, and which prove that the difference in the excitability for the two kinds of current affects other nerves also, in the same way as the facial. The first of these observations lias reference to a paralysis of the parts supplied by the ulnar nerve; the second, to paralysis of the parts supplied by the radial.

Ziemssen next cites two cases which afford proof that the control of the will over paralysed muscles may be restored, without any restoration of their excitability to either faradic or galvanic stimulus. He then attempts to classify the very numerons phenomena described in his previous observations, and displayed in cases of peripheral paralysis when the paralysed nerves and muscles are tested by inducel and continuous currents, and obtains the following series:-

I. Paralysis in which the affected muscles and nerres have entirely lost their excitability, either for the will, or for both kinds of electric current.(Ziemssen.)

II. Paralysis in which the motility is partly retained or regained; while the affected muscles and their motor nerres are not susceptible of the influence of either kind of electric current.-(Eulenburg, Ziemssen.)

III. Paralysis in which the motility is entirely lost; but in which the excitability by both electric currents is not lost, although much diminished. -(M. Meyer.)

IV. Paralysis in which the affected muscles and nerves have entirely lost their excitability by the will, or by the induced cmrrent; while their excitability by the continuons current remains.

In this class the preceding cases have displayed the following phenomena:-

(a.) During the absence of motility.

1. The excitability for the continuous current is increased; so that feeble battery currents, which produce no trace of effect upon the healthy homologous muscles, excite the paralysed muscles to active contraction.-(Baierlacher, Schulz, Neumann, Eulenberg, Ziemssen.)

2 . The excitability for the continuous current increases greatly during treatment with it; rapidly attains its maximum, and then diminishes.(Zirmssen.) 
3. The excitability for the continuous current is not always simnltaneously equally increased in all branclies of the paralysed nerves. In the less excitable muscles and nerves the excitability increases, and then diminishes, later than in those that were more excitable at the first.-(Ziemssen.)

4. The contractions of the paralysed muscles, occasioned by battery currents, do not occur with the same precision as in healthy muscles, but appear somewhat drawn out.-(Brückner, Ziemssen.)

5. The contractions are prodnced in many cases only by direct excitation of the muscles; and not by excitation of the corresponding motor nerves. (Neumann, Ziemssen).

(b.) With the return of motility.

6. The excitability by the continuous current is gradually lost with the return of voluntary movement; and the excitability by induced currents gradually returns at the same time.-(Selulz, Zicmssn.)

7. The excitability by the continuous current is gradually lost with the returu of motility; but, in spite of the complete restoration of motility, the excitability by the induced current does not return. The excitability for both currents, first after the lapse of months or years, returns gradually and uniformly to its normal standard.-(Ziemssen.)

8. The excitability by the induced current does certainly return, but remains less than in the lomologous muscles of the healthy side, and is only manifest on direct muscular excitation. The contimuous current permanently produces stronger contractions in the paralysed than in the lomologous healthy muscles, but also only by direct excitation.-(Neumann.)

9. The excitability by the continuous current remains undiminished; but the excitability by the induced current (for the present at least) does not return.-(Eulenbuig, Ziemssen.)

V. From a therapentic poiut of view, in peripheral paralysis (smpposing it to be curable), that current is the most useful to which the muscles preserve their excitability. Those paralyses, therefore, in which faradic contractility is wholly or in part retained, shonld be treated by induced currents; and those by galvanic eurrents in which the faradic contractility is lost, but the galvanic contractility retained.

In a further examination of the physical and physiological questions arising out of the phenomena described, Ziemssen thinks (1), in accordance with Neumann, that the physical difference between the action of continuous and induced currents may be expressed as follows:-Paralysed nerves and muscles lose, under certuin eircumstanes, their excitability by electric currents of momentary duration, evcn when these are applied in rapid succession and of great strength. On the other hand, they retain their excitability, and it may even be inereased above the normal foi currents of longer duration, even when the latter possess very little intensity. (2.) Further, he concludes that in peripheral paralysis, the disturbance of nutrition in the affected nerves affords the most important impulse for the genesis of the consecutive changes of excitability to the electric currents; and from the cases cited he thinks there is a clinical basis, especially from the testing with electric currents, to distinguish several degrees of lesion.

First (Slightest) Degree.-Motility diminished or suspended. Excitability for intermittent and constant currents normal. Inconsiderable disturbance of nutrition, followed by speedy return of motility. The same phenomena are present during the first week after severe injuries, so long as degeneration has not yet occurred.

Second Degree.-Motility suspended, faradic and galvanic contractility diminished. Slight disturbance of nutrition, early return of motility, and improvement of the electro-muscular contractility under the employment of the induced current.

Third Degree.-Motility suspended. Excitability for the induced enrent lost, for the continuous current retained both by nerve and musele. Serpre 
disturbance of nutrition, commonly followed by gradual loss of galvanic irritability; but in which, in favourable cases, the motility, and frequently also the faradic excitability, quickly retırn. The most effectual treatment is by continuous currents.

Fourth Dearee. - The excitability of the nerves by volition, and ly electric currents of both kinds, is lost; but the irritability of the muscles for the continuous current is retained. Complete degeneration of the nerres to their terminations in muscles. Results of treatment by continuons current doubtful.

Fifth Degree.-For nerves and muscles alike, the excitability for the will or for both kinds of electric current, is wholly lost. Very severe disturbance of nutrition both in nerves and muscles. Prognosis unfarourable.

It is self-evident, Ziemssen adils, that this division is only superficial; and no one can suppose that a number of apparently paradoxical phenomena can receive their explanation in this mamner.

For the present, the different condition of the excitability for the will, and for the electric current in the third degree of disturbance of nntrition, remains not understood; especially the complete return of the volitional influence during the complete absence of excitability by the electric current ; further, the opposite action of the two currents in the course of the paralysis, and the want of coincidence in the phenomena observed in the recorded cases. Possibly inequalities or differences may exist in the changes in the anatomical, physical, and chemical condition of the nerres, and may cause the various phenomena, while they themselves escape our perception. Possibly, also, as Eulenburg assumes, the motor nerves may possess separate energies for the different stimuli.

Ziemssen also gives the notes of a case which affords cridence that the difference of excitability for galvanic and induced currents, is displayed also in diptheritic paralysis of the relum, and that it affords as little ground for unfavourable prognosis in these as in other muscles, since the paralysis was eured by galranization in fourteen days.

A like difference of reaction to the induced and galvanic currents has heen ohserved by my predecessor at the National Hospital for the Paralysed and Epileptic, Mr. J. Netten Radcliffe, and hy Professor Hammond, of New York, in certain forms of infantile paralysis; and by Mr. J. N. Radeliffe in other forms of local paralysis than those described by Ziemssen. The therapentical effect of the interrupted galvanic current in the treatment of infantile paralysis, as observed in the National Hospital and by Professor Hammond, will be discussed in the chaptex devoted to this disease. The following remarkably interesting case of successful treatment of paralysis of the deltoid is recorded from the practice of Mr. Radclifte, in the 'Lancet' for 1866 (vol. ii. p. $57(i)$.

The patient was a blacksmith, aged 25 years. After several days' suffering from severe "rheumatic pains," so-called, in both shoulders, but particularly in the left, he lost suddenly, while workmg with an ordinary sized hammer one morning, the power of raising his right arm. When first seen, five weeks after this occurred, the deltoid and infra-spinatus muscles of the right side were found to be completely paralysed, and there was some wasting of the former muscle. The contractility of both muscles under the induced current was annihilated; the electric sensibility was diminished crer the infraspinatus, and this form of sensibility, as well as the sensibility to heat, cold, tickling, and touch, were absolutely wanting in a triangular space (the apex pointing downwards) over the deltoid, measuring two inches and a-half at the base, and five inches from the base to the apex. Under powerful faradization, seven times repeated at intervals of three days, not a trace of contraction conld be excited in the paralysed muscles, and the wasting eviclently increased. Electro-puncture was had recourse to several times, but a dubious action of some superficial fibres was the only result, Mr. Radcliffe then tried the interrupted galvanic eurrent. With thirty-five cells 
of a Becker-Muirhead battery, which produced no effect on the healthy left deltoid and infra-spinatus, a marked contraction of both paralysed muscles was excited: with forty-five cells (also ineffective upon the healthy muscles) an energetic contraction. Mr. Radeliffe from this time used the interrupted galvanic current alone, thrice reekly, and each time about ten minutes, until it had been applied thirty times. At the end of this period, serenty-five cells were required to produce the amount of contraction formerly caused by forty-five; and under the full force of the current from the primary coil of a large Stöhrer's induction apparatus, slight contraction was produced in the deltoid. The further wasting of the muscles appeared also to have ceased, althongh they did not gain in bulk.

At this time treatment was suspended for two months, the patient being compelled to leave town. In the interval, not only was the little ground which had been gained by electrical treatment lost, but the paralysed muscles had become more and more wasted, and it was now clear that the supraspinatus also suffered. The wasting, indeed, was so great, that of the deltoid barely a filmy layer of fibres could be presumed to remain. Faradization over the affected muscles did not excite a trace of contraction, and electropuncture yielded only doubtful action. But again the interrupted galvanic current caused marked contraction; and, pursuing the same course that he had done before, Mr. Radcliffe used daily for about a dozen times this form of current only. At the end of the twelve applications, on using the full force of the current from the primary coil of a large Stöhrer's induction apparatus, distinct but slight contraction of the deltoid and infra-spinatus occurred. From this period faradization of the paralysed muscles was persisted in form times in the week.

The history of this case was not completed in the account given by the 'Lancet' reporter. I am now able to give the result. The affected muscles steadily increased in bulk, their electro-contractility improved, and sensibility to the electric current, touch, tickling, heat, and cold returned, and at the end of four months, the patient was enabled to use his right arm freely, and return to his ordinary occupation. At this time no difference could be distinguished between the right and left deltoid, and voluntary control was as complete over the one muscle as the other; but the electromotility of the right muscle was not equal to that of the left.

In the antumn of 1869 , this case came under my observation. During the interval the man had followed his occupation, using with freedom even the large hammer. But, about four weeks before placing himself under medical care again, he had begun to suffer from severe erratic pains in the right ar'm and forearm, and an inability to wield the hammer freely. In bulk of muscles and voluntary action, no difference could be detected between the right and left arms. The right deltoid was apparently as fully developed as the left. 'There was no alteration of sensibility of any form over the right arm, and all the muscles responded to an induced current of ordinary strength, an interrupted galvanic current producing no contraction until its force was raised to a point that it would act upon healthy muscles. But the motor effect produced on the right deltoid by induced currents of equal strength was much less than on the left. The case unfortunately was seen only three or forr times, and its further history is unknown.- II. I'] 


\section{CHAPTER IV.}

ELECTRO-MEDICAL INSTRUMENTS, WITH REGARD TO THEIR APPLICATION IN PHYSIOLOGY, IN PATHOLOGY, AND IN THERAPEUTICS.

THe choice of a good apparatus is of great importance in the practice of localized electrization. To what purpose, incleed, should we be skilful in the art, if the apparatus at our command were unable to respond to the requirements of physiological, pathological, and therapeutical research?

I therefore purpose, in this chapter, first, to examine the properties that should be possessed by electro-medical instruments; secondly, to describe the instruments that I have myself contrived, and that appear to me to correspond with the progress of medical electricity; thirdly, to examine whether the instruments in common use possess the properties that are required.

\section{Part the Finst.}

THE PROPERTIES WHICH INSTRUMENTS SHOULD POSSESS.

\$ I. Any faradic apparatus intended for medical practice, but not possessing primary and secondary coils, or in which these coits are not constructed after certain proportions of thickness and length of wire, cannot fulfil all the requirements of therapeutics.

The foregoing proposition is but a deduction framed from the electro-physiological experiments, and the facts detailed in $\S \mathrm{I}$., p. 22 et seq., and which it would be superfluous to reproduce, or even to recapitulate. The proposition cannot be disputed unless the facts on which it rests can be disputed; and these have been submitted to the test of prolonged and public experiment, have been sanctioned by many academic commissions, and have been (hecked and witnessed by a large number of observers, both French and of other countries. They therefore form part of science, in spite of some ill-wishers, and of conduct that can only be explained by ignorance, or bad faith, or malevolence.

I have defined the conditions under which I have worked, in order to obtain the electro-physiological results that I have described. I have stated what, approximatively, should be the diameter and the length of wire of the two coils for double induction, in order to endow them with the maximum of their differen- 
tial properties. It is evidently necessary that any other experimenter should place himself under the same conditions of experiment, if he would judge correctly of the value of my electrophysiological researches and of the deductions drawn from them, or if he desires to apply them in practice. From this point of view, at least, electro-medical instruments should be constructed in the manner that I have laid down.

\$II. The slow or rapid intermissions of the electro-medical instruments produce special physiological effects, and cannot replace one another in practice.

In order to make clear the utility or the necessity, the inconveniences or the dangers, of faradic currents with intermissions more or less slow or rapid, it is sufficient to describe the physiological phenomena that are produced under their influence; and to frame from these, immediate practical deductions, which - will be fully borne out by the facts stated in the course of this volume.

\section{Action of the Rapid Intermissions of Currents.}

A.-Upon electro-muscular contractility.-A muscle, which receives the stimulus of a single intermission of an induced current, contracts, but immediately falls back into complete relaxation. If this intermission be followed by many others, sufficiently near together, the muscular contractions follow one another; and the muscle becomes less completely relaxed between the intermissions in proportion as they are less distant. It follows that the muscular fibres contract more, the more rapid is the induced current.

It is necessary to guard against the supposition that a current of rapid intermissions causes more energetic contraction of muscle than a current of slow intermissions. That such a belief would be erroneous may be shown by the following experiment. If we apply a rapid and a slow current alternately to a paralysed muscle, the irritability of which is undiminished, for example, to the flexor communis digitorum, and if we attach a weight to the fingers moved by the muscle, we shall see that the rapid current does not give the muscle power to sustain a heavier weight than it would sustain under the influence of the slow current. If, under a rapid current, the extent of the muscular contraction is increased, and if therefore the movement of a finger is more extensive, this is because, the fibres relaxing little or not at all during the brief intervals, each excitation shortens them more and wore. 'To 
obtain a more energetic contraction, it is necessary to use a current that is more intense.

The muscular shocks produced by the intermissions diminish as the latter become more rapid; and at a certain rate of speed the contraction appears to be continuons, as if it were produced physiologically by the nervous current, that is, by the will. For this it is necessary, however, that the rapility should be extreme; since otherwise the resulting movements will resemble a kind of tremor.

B.- Upon muscular sensibility.-I have just shown that the force of the muscular contraction is not augmented by increasing the rapidity of the intermissions. The case is different as regards the muscular sensation produced; for this is always augmented by increasing the rapidity. When a high degree of speed is attained the sensation becomes very painful, almost tetanic, and is attended by cramps.

C.-Upon muscular tonicity.-I have found by experience that currents of rapid intermission augment the tonic power of muscles, not only when this power is diminished, as we see it in certain conditions of disease, but also when the muscles are in their normal state. This special effect of rapid intermissions, when it is long continued, may even produce shortening; and facts on which this statement rests will appear in subsequent pages. It will then be seen how I was led to observe this phenomenon, and also that, in order to produce it, the currents must be of extreme rapidity.

D.-Upon muscular nutrition.-The nutrition of muscles sometimes becomes very much increased under the influence of currents of rapid intermission. The cases of muscular atrophy or of atrophic paralysis, in which I have compared the effects of currents of slow and of rapid intermission, place this statement beyond doubt. However, it is necessary to use the latter with circumspection; because, in certain morbid conditions, they may destroy the remaining innervation of the muscles.

E.-Upon electro-cutaneous sensibitity.-It is enough to experiment upon one's self in order to be convinced that the electrocutaneous excitation increases in direct proportion to the rapidity of the current rather than in proportion to its intensity.

To complete the account of the influence of the rapid intermissions of an electric current upon the sensibility, I should add that, generally, every organ that receives the nerves of animal life is excited by any current, the more painfully the more rapid are its intermissions.

Finally, the muscles of organic life-of the intestines, for example - contract more porrerfully if the intermissions are more rapid. 


\section{Indications and Contra-indications for Rapid INTERMISSIONS.}

A knowledge of the foregoing facts enables us to foresee the numerous indications or contra-indications for rapid intermission of the currents; and I proceed to set forth what a long and daily experience has taught me upon this subject.

A.-Cases in which the employment of rapid intermissions is indicated.- $(a)$. The property possessed by rapid intermissions, of producing artificial contractions which perfectly imitate voluntary morements, enables us to employ them in the study of the individual actions of muscles. But it will be seen that such researches require the employment of an internittent current of extreme rapidity, especially for the muscles of the face. The magnetoelectric instruments, the intermissions of which are scarcely of sufficient rapidity to prodnce contractions without tremor of the muscles of the limbs and of the truuk, have been insufficient for my researches upon those of the face. 'The constant quivering of the fircial muscles, when excited by the currents from such instruments, forbid one to observe exactly the individual influence of each one upon expression.

(b). The study of the physiological action of slow and of rapid intermissions has shown that we can, by the aid of the former, act with more or less eneroy upon the contractility or the sensibility of muscle. For, since every electric excitation of a muscle necessarily produces at once a sensation and a contraction, we may diminish at pleasure the force of the contraction, and may increase the sensation, by combinining the current of the primary coil, the special action of which upon muscular sensibility is known, with rapid intermissions, or vice versî.

The power of energetically arousing the muscular sensibility by the aid of rapid intermissions finds its most useful applications in cases in which the muscles have lost their sensibility. Such cases, as I shall have occasion to show, are not infrequent; and I shall hereafter. cite numerous examples.

I have often found muscles which, whether deprived or not of the power of voluntary movement, were completely insensitive to the electric excitation; so that I was able to produce very energetic contractions by the application of the most rapid currents, without the patients being conscions of them. The skin covering such muscles was also in some cases insensitive; but in others it retained its normal sensibility.

Patients who are deprived only of the sensibility of the skin (anæesthesia or analgesia) preserve their consciousness of the move- 
ments which they execute or which are impressed upon their limbs. 'Ihey feel blows which affect the deeper tissnes; and their feet, when resting upon the ground, appear to them as if placed upon a soft surface, such as that of a carpet. We also see them stamp upon the ground in walking, so as to feel it better. If they are at the same time deprived of the cutaneous, the muscular, and the articular sensibility, and if the bones are also insensitive (a condition the existence of which I have ascertained by electric excitation of the osseous surfaces), they do not feel even the most violent blows, they have no consciousness of their movements, they cannot maintain an upright posture, except by looking at the ground which they have ceased to feel; and, in bed, they are unconscious of their limbs, unless they see them.

rase XVIII.-I have seen, in La Charité, a patient completely anæsthetic and analgesic, who felt as if she were suspended in the air when she ceased to see the bed on which she was placed. 'Tlis cansed her continual terrors at the moment of falling asleep; for, not seeing the bed, she believed herself in danger of falling, and could not be re-assured mutil she had ascertained by sight that she was actually lying upon a solid bed.

The most intense induction currents are powerless against these profound lesions of sensibility, unless their intermissions succeed each other with great rapility. The patient above referred to had suffered from general insensibility for many weeks; and was very quickly cured by currents of rapid intermission, the re-composition of which was effected in the muscles, the nerves, and the bones. I should add that I had previously faradized her with slow intermissions, but with no benefit, the power of the instrument being at its maximum.

It is especially in that common affection, cutaneous anæsthesia, that rapid intermissions are necessary in order to obtain the therapentic influence of the induced currents; withont them we should fail nearly always, notwithstanding the use of the current of the second coil, graduated to the maximum.

(c). I have employed, in therapeutics, the singular property possessed by rapid induced currents of increasing the power of muscular tonicity, a force which never sleeps. It is this tonic force which, in the absence of voluntary contractions, forms the physiognomy, and maintains the natural attitudes of the limbs; and which, if diminished or increased, will disarrange their admirably combined mechanism, as happens in muscular pathology. This, indeed, shows us the correctness of these assertions; we then see the muscles contract, or lose more or less of their tonic force; they are springs which have either become tense or have relaxed themselves; if I may be pardoned the use of a comparison that is 
trivial, but accurate. The physiognomy then loses its stamp of individuality; the limbs assume vicions attiturles. It is under such circumstances that, in order to re-establish the harmony of the visual traits, or to correct certain deformities of the limbs, I have rendered tense, so to speak, these muscular springs, sometimes by acting upon the relaxed muscles, sometimes by opposing artificial contraction of the antagonists to the contraction caused by disease; in either case by means of induced currents of extreme rapidity. This new and very important subject will be fully treated in a subsequent portion of the volume.

(d). Lastly, experience has shown me that the use of currents of rapid intermission is necessary in the treatment of certain forms of muscular atrolhy, whether they may be complicated with paralysis or not. When, however, a muscle has in great part undergone fatty, or granular, or granulo-fatty degeneration, and when there only remain a very small number of its fasciculi or fibres, I have observed that the rapid intermissions have hastened its destruction.

\section{B.-Conditions in which rapid intermissions are contra-indicated.}

The utility and even the necessity of induction currents of rapid intermission has been perfectly established by the foregoing considerations. Unfortunately, every medal has its reverse; and the very properties of these rapid currents render their employment dangerous or lifficult in certain cases.

(a). There is a period at which cases of paralysis of cerebral origin may be advantageously treated by local furadization; and it is when the primary lesion has disappeared or is diminished. The hæmorrhagic effusion, for instance, will be absorbed, entirely or in part, after the lapse of six months or a year. There will only be left, in the brain, a cicatrix, or a cyst of small dimensions. The influence of the brain is transmitted freely, or with diminished difficulty, to the muscles; but these no longer react to the influence of voluntary excitation. In such a case, the paralysis may be considered as limited to the muscles; and then localized faradization, as I have shown, will restore their aptitude for motion. But how can we determine exactly the state of the brain? It is never possible to say at once that there is not, around the eyst, some remnant of inflammatory action, undergoing resolution, but which a spark may rekindle into flame. We know that the occurrence of a cerebral hremorrhage predisposes to a second, in consequence of the pathological conditions which exist in the vaseular system or the heart; conditions which produce either 
arterio-sclerosis, or miliary aneurisms, or embolisms. We know, also, that any excessive general excitement may recoil upon the nervous centres, and may produce accidents of the same kind. The employment of an apparatus with rapid intermissions, and that acutely excites the sensibility, is therefore very dangerous in the treatment of cerebral paralysis. Of this fact I had melancholy proofs at the commencement of my researches.

It follows, consequently, that in forms of paralysis of cerebral origin it is necessary to be very circumspect in the use of localized faradization, and to avoid exciting too acutely the general sensibility, under pain of exposing the patient to serions mischances. And, as we know, fararlization by rapid intermission does excite the sensibility acutely.

Because currents of rapid intermission might be dangerous or inapplicable in the treatment of cerebral paralysis, it does not follow that such cases onght not to be subjected to localized faradization. We must remember that it is possible to produce by faradization very energetic contractions, without causing any severe pain, if only the intermissions are sufficiently far apart (one or two in a second, for example). Practised thus, with slow intermissions, the faradization exposes the patient to no danger, and may on the contrary oiten be of great utility.

Rapid muscular faradization is only required when the muscles have in various degrees lost their sensibility, or when their nutrition is enclangered. But their sensibility is rarely affected in cases of cerebral paralysis; and, as regards their nutrition, I shall show hereafter that the muscular fibre remains intact in such cases. ITe cannot consider as a lesion of nutrition the wasting that sometimes occurs, and that is dne solely to prolonged inaction.

(b). Even when the state of the nervous centres in no way contraindicates the use of rapid intermissions, it is never a matter of indifference whether faradization is practised with or withont pain. . There are patients who, either from want of courage, or from nervous excitability, cannot bear pain. Such, for example, are women and children.

If, on the one hand, the power of currents of rapid intermission to increase the tonic force of muscles, and frequently to occasion their contraction, is sometimes useful in therapeuties; yet, on the other hand, it may occasion great mischief. I shall show by examples, that when applied to the face it has produced, in some instances, contractions that have occasioned permanent delormity.

(c). Lastly, there are a great number of instances in which we desire only the application of a very small number of isolated 
intermissions; as when we endeavour to excite the membrana tympani, in the treatment of deafness.

T'o sum up, such considerations show abundantly that faradization by rapid intermissions has numerous useful applications in physiology and therapentics; but that in certain cases, when applied to the excitation of muscles, it is attended by dangers arising from the accidents or the deformities which it may occasion, and that then it may be advantageously replaced by currents of slow intermission.

\section{\$III.-In a great number of cases the apparatus used for faradiza-} tion cannot be too powerful.

What should we unclerstand by the power of an induction apparatus? and how should this power be measured?

An apparatus is powerful when it acts with energy upon the muscular contractility, upon the sensibility of the skin, of the muscles, of other subcutaneous organs, and of the retina; and when it is able to traverse a considerable thickness of tissue, so that its recompositions may occur in deeply seated organs.

We are now aware, from the facts and considerations already set forth (Art. III., Chap. I.) that the different actions of induction currents that are applicable in practice are only found combined in an apparatus of double induction, the coils of which are constructed in certain proportions, and that the energy of these different actions, specially exerted, some by the first coil (the extra current), the others by the second coil (the induced current), are in direct ratio to the length of the wires. It therefore follows that the power of an induction apparatus is necessarily proportimate to its size and weight. Unfortunately we have as yet discovered no means of producing a powerful induction by means of a short length of copper wire.

We may, however, be deceived by appearances if we judge of the power of an apparatus by its size and weight alone, and without actually testing it. And, for the latter purpose, the time has passed by at which it was sufficient to take a rheophore in each hand. It is necessary, if we wish to know the exact degree of power exerted upon this or that function, to direct and limit one or the other current of the apparatus upon its organ, and to proceed with such experiments in the manner that I have already laid down.

I now proceed to prove, by a few examples, the truth of the statement which forms the lieading to this Section, that in certain cases the induction apparatus camnot be too powerful. 
I have shown in the second chapter that when electric excitation is localized in organs or tissues situated beneath the skin, the recomposition of the current occurs at a greater depth, the greater the degree of its tension. It follows that a current, the tension of which is too feeble, will not penetrate to the muscles if the subcutaneous areolar tissue opposes to it too great a thickness, either from the deposition of fat or from serous infiltration. Indeed, the instances in which the thickness of the areolar tissue opposes a great resistance to electric currents are not rare, especially in the lower limbs. It has even happened to me that the tension of my most powerful faradic apparatus has been insufficient to overcome this resistance, and that I have been compelled to call to my aid the more penetrating tension of a Leyden jar. It is on account of such cases that a faradic apparatus camnot be too powerfinl. It must be remembered that, whatever be the degree of tension, the apparatus may always be used without too much pain, if only care be taken that the intermissions are sufficiently distant.

There are also pathological conditions against which the apparatus camnot be too powerful. Among the forms of paralysis caused by injury to the nerves there are some which deprive the muscles of all their properties, so that they have completely lost both contractility and sensibility. The skin, and even the bones, are equally rendered insensitive, and the paralysed limb becomes shrivelled and as if death-stricken. In such cases, of which I have seen many, and which I have marle the subjects of special inrestigation, it has been necessary to have recomrse to an apparatus of enormous intensity, and I hare even had to regret the want of one still more powerful. It was only after I had recalled some life to the limbs that I was able to diminish the intensity of the current, and that an apparatus of medium power became sufficient. Other muscular affections also, of a different lind, of which there will be due notice in the course of the volume, require the use of very powerful instruments.

Hysterical anæsthesia of the skin may be cured, in general, with an apparatus of ordinary power, provided that the intermissions are extremely rapid. But I have met with a certain number of such instances, and also with cases of cutaneous anresthesia of a different kind, which have resisted currents of medium intensity, and have only yielded to an apparatus which acted in the most energetic way upon the sensibility of the skin. 
\$ IV.-Every apparatus for faradization should possess a means of graduating the dose of electricity exactly, and proportionately to the degree of excitability of the organs; a degree which will vary with the state of health and with the nature of the clisease.

The considerations already set forth in the second chapter, with regard to the special excitability of different organs, of the muscles, of the nerves, and of different regions of the skin,-an excitability varying with the state of health, and with the nature of the disease, - these considerations should suffice to show the importance and the eorrectness of the proposition that is formulated above.

How, indeed, would it be possible to limit the electric force to any given organ, unless the apparatus were so constructed as to allow us to apportion its proper dose to each? I will make this more clear by examples. In the normal condition, the cervix uteri, the bladder, and the rectum, are but little sensitive, while, on the other hand, the weakest currents act energetically upon the sensibility of the muscles of the face. Between these extremes there exist intermediate degrees proper to other organs. To be convinced of this, we need only compare the differences of sensibility and contractility which exist between the muscles of the face themselves, although the least excitable of these muscles is infinitely more sensitive than those of other regions. We shall find that each facial muscle is endowed with a special degree of excitability, which requires an electric dose that cannot be exceeded, if we wish to obtain only partial contractions, without the production of too acute pain. (I need not recur to this sulject, after what I have already said in the second chapter upon the degrees of individual excitability of the muscles of the face.) We shall understand the absolute necessity of giving to induction instruments a means of exict graduation, upon a suffieiently extended scale, if we bear in mind that the degree of excitability varies in each of the regions, and even in each of the points, of the face. Besides the differences in the excitability of organs, we must consider also the differences which exist in the anatomical state of the tissues on which we act (the greater or less thickness of the skin, of the areolar adipose layer, and of the aponeuroses to be traversed, and of the muscular layer in which it is desired to localize the excitation), - an anatomical state that must be known in order to produce, with any certainty, the electric recomposition at any stated depth.

But the graduation will not be exact, unless there be an arithmetical proportion between the divisions of the graduator and the progressive force of the apparatus. 
The graduation should be made upon a scale sufficiently extensire to measure every degree of intensity of the current, in proportion to the degree of excitability of every organ.

It is, therefore, necessary that a faradic apparatus should admit of being rendered very powerful or very feeble, according to the indications to be fulfilled, and by the aid of an independent system of graduation, calculatel exactly to measure and to distribute either powerful or feeble currents.

Lastly, it is necessary, in a volta-electric apparatus, that the initial force of the battery should always act with the same degree of intensity, or, at least, that its intensity shonld be known to the operator. But the battery which works a volta-faradic apparatus will be more or less constant, accordingly as its acids are more or less concentrated, accordingly as it polarises or exhausts itself more or less quickly, or accordingly as it becomes more or less choked by imperfectly conducting crystallized salts. We may see that, with such variations in the initial force, the measurement of the electric doses becomes illusory, and that hence it is impossible exactly to formulate the loses of electricity which may properly be administered to every organ.

Such are the principal properties which shonld be found united in every faradic apparatus intended for electro-physiological or pathological study, or for therapeutical employment. There are yet others which it is very important to obtain. Thus, the instruments should be portable, easy of management, and moderate in price. But, as these conditions involve no scientific question, I have not thought it desirable to discuss them in this place, and shall return to them hereafter. Although secondary, they have very great bearing upon the practice of medical electricity.

In the first edition I added to the account of the different properties of the several currents of the induction apparatus, a critical examination of the instruments then in use in medical practice; and showed that they did not combine the whole of the conditions necessary for localized faradization, or for the application of this kind of electricity to my electro-physiological researches; and that they did not fulfil the requirements of electro-therapentics.

This critical examination has borne its fruits. In fact, the utility of the employment of localized faradization in the study of muscular physiology and pathology, and the general diffusion of electric treatment, have produced great activity in the commercial manufacture of induction instruments. Hence it has followed, that physicists and manufacturers-the former to aid in the growth of knowledge, the latter to obtain the patronage of physicians-have alike striven to improve the instruments and to bring them to 
perfection. The number of induction instruments produced, and variously improved or modified, has so increased, both in France and in other countries, that to describe them would require me to enter into details which would carry me far beyond the limits I have set to my task. The laudable efforts referred to have produced some highly ingenious forms of apparatus. Unfortunately, the inventors have chiefly sought to diminish size and price, and have not always been sufficiently impressed with the importance of the most recent requirements of science and of therapeutics; requirements arising from the method of localized faradization, and from the discovery of the several properties of the currents of the different coils.

The critical observations that I made upon the instruments in general, were founded upon long experience of those which then were chiefly used. It seemed to me that the very severity of my criticism imposed upon me a new duty, - that of endeavouring to produce instruments that should be better. For this reason, I devoted myself with ardour to a new order of researches, in little harmony with my habits, and which would even have been very irksome, if I had not possessed some aptitude and taste for mechanics, and if I had not kept constantly before my mind the realization of a single idea,-the localization of the electric force in organs, and its application to medical science.

I therefore set myself to frequent workshops, in which I acquired many of the secrets of manufacture; and, after several trials, I was able to produce instruments made by my own hands, although I had previously never used a file or a hammer. From these models I have drawn the designs for the volta-faradic and magneto-furadic instruments which have been skilfully manufactured by MMI. Charrière and Deleuil. Since then, they have undergone new and important modifications, all of which will be described in the next part of this chapter; while, in a subsequent part, I shall pass in review the instruments that are chiefly employed, and subject them to critical examination.

\section{Part the Second.}

\section{THE AUTHOR'S LARGE DOUBLE-CURRENT VOLTA-FARADIC APPARATUS.}

My double-current volta-faradic apparatus was, in its original form, somewhat complicated; and it underwent the fate of every new mechanical contrivance. The number of its connections rendered a description difficult to understand, and, although the method of setting it in action was very simple, occasioned frequent derange- 
ments. The use of this primitive instrument having shown me all its faults, I was led to make successive important modifications, which have produced perfect simplicity of construction, and have consequently diminished the frequency of accidents capable of arresting the course or diminishing the power of the current.

Noreover, the modified instrument presents a more perfect system of graduation, and more varied methods of producing more or less rapid intermissions.

In order better to explain the importance of the alterations, I have represented the original instrument in fig. 51 ; and the

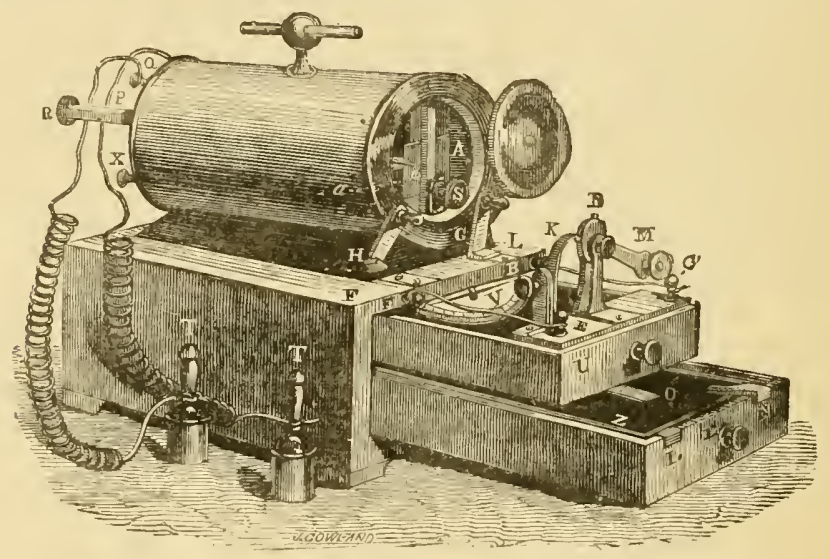

Fig 51. - Large double-current volta-furadic iustrumeut. Duchenne's original model.

perfected instruments in figs. 52 and 55 . In the former, the coils are concealed by a covering; in the latter, they are exposed to view.

\section{$\S$ I.-Description.}

\section{A.-Large volta-faradic apparatus closed (latest model.)}

The first model of the volta-faradic apparatus (fig. 51) ${ }^{5}$ was composed-1, of a flat battery, $0 ; 2$, of two superposed coils; 3 , of a graduator formed by the external movable coil, which could be moved upon the internal coil by means of the stem $R$; 4 , of a magnetic rheometer, V; 5 , of a toothed wheel, D, fixed to a little plate shut within the drawer $\mathrm{U}$, and which, when required for use, could be lifted up, as in fig. $51 ; 6$, of a trembler, A.

The last model (figs. 52 and 5.5), ${ }^{6}$ differs from the foregoing, 1 , by

5 This apparatus was presented to the ${ }^{6}$ This modified apparatus was preAcademy of Sciences of France, in 1848, sented to the Academy of Medicine of by M. Despretz. 


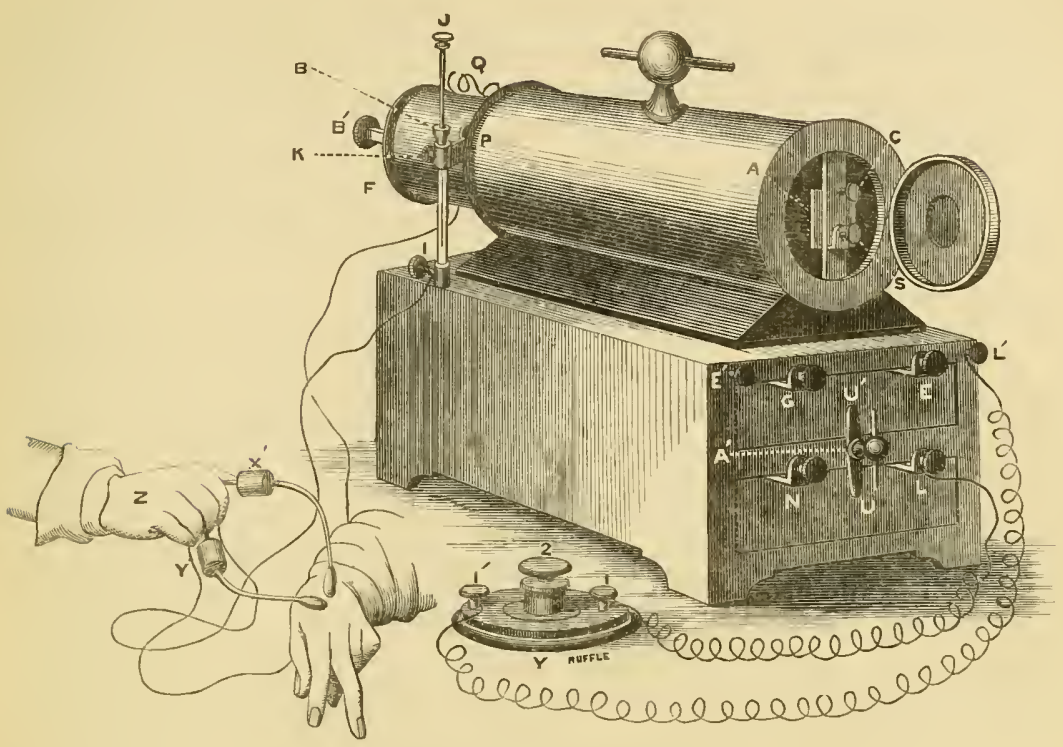

Fig 52.

the suppression of the toothed wheel I), fig. 51, which is replaneel by a pedal rheotome $\mathrm{Y}$, fig. 52, the mechanism of which will be explained hereafter, and which has rendered it possible to dispense with the numerous comnections of the first instrument, and much to simplify the construction; 2 , by a graduation, acting by means of a tube of copper, $\mathrm{B}$, which covers more or less of the two coils which afford the double induction; 3 , by the use of a tube, F, containing water, and called the moderator, which, in combination with the graduator tubes, renders it possible to measure the smallest doses of electricity, as well as those that are larger; 4 , by the nse of a commutator of the coils, $\mathrm{E}$ (fig. 55), the use of which will be explained; 5 , and last, by a considerable modification of the battery.

I proceerl to the description of the apparatus as modified by these changes, and with reference to figures $52,53,54,55$, and 56 , for illustrations of the several parts.

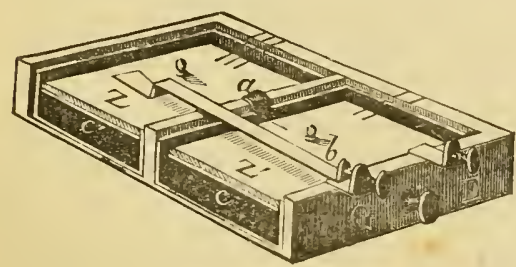

Fig. 53 .

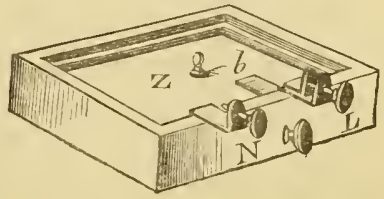

Fig. 51 . 
The apparatus is set in action by a battery composed of three pairs of elements. These are enclosed within the drawers, $U$ and $U^{\prime}$ (fig. 52). 'There are two pairs in the upper drawer, and one in the lower (fig. 54.)

Each pair is composed of a carbon plate, $\mathrm{CC}^{\prime}$ (fig. 53), fixed to a cell of hard caoutchouc, and of a zinc plate, $Z_{Z^{\prime}}^{\prime}$, of the same surface as the carbon, and separated from it by a cloth diaphragm.

The platinum wires which form the carbon contacts are arranged as in the small instrument (p. 267), while the zinc contacts differ in each of the pairs:-1, in the lower drawer, $U$ (fig. 52), a strip of iron, $b$ (fig. 54), rivetted and soldered to the zinc $\mathrm{Z}$, is bent upwards at a right angle at its anterior extremity, in such a way that, when the drawer is closed, the iron comes in contact with a small plate of platinum fixed in the front of the apparatus on a level with the knob $L ; 2$, in the further compartment of the upper drawer, the zine $Z$ (fig. 53) is also prolonged by a strip of iron, $b$. This strip is also bent upwards at a right angle at its anterior extremity, and, when the drawer is closed, can be brought in contact with another small plate of platinum fixed in the front of the apparatus on a level with the knob G; 3, lastly, the zine Z' (fig. 53), of the pair in the front compartment of the upper drawer, rests on a platinum wire which winds over the posterior wall of the cell, and comes in contact with a double spring, $a$, fixed to the partition of the drawer, which spring, in the farther compartment, rests by a platinized surface upon the carbon C'. The left-side drawer is absent in the figure, in order to display the arrangement of the parts forming the two pairs.

2. The two superposed coils forming the system of induction are composed of two copper wires, differing in diameter and in length, and covered with silk.

The thicker and shorter of the two wires (half a millimetre in diameter, and 200 metres in length) is rolled around a bundle of soft iron wire, so as to form a coil. The extremities of this copper wire, which produces the current of the first coil (the extracurrent of authors), terminate upon two small plates of platinum, fixed to the apparatus at the level of the knobs E, L (fig. 52), which are the positive and negative poles of the battery enclosed in the drawers $U$ and $U^{\prime}$.

The finer and longer wire (one tenth of a millimetre in diameter, and 1000 metres in length) is rolled around the one preceding. It gives origin to the current of the second coil (current of the first order, of authors); its extremities terminate at the two springs of the commutator of the coils. 
3. The commutator $\mathrm{E}$, represented in fig. 55 , but not shown in fig. 5:2, is intended to transmit, rapidly and alternately, the current either of the first or of the second coil to the conductors attached to the knobs $\mathrm{P}$ and $\mathrm{Q}$. It is unuecessary to describe it here; and it is enough to say that, by turning the needle $\mathrm{F}$ (fig. 55) to the right or the left, it conducts one or the other current to the rheo phores; as, indeed, is shown upon a plate sitnated above the needle.

4. The graduator B (fig. 52) is a cylinder of copper, which surrounds the coils, and which has a scale marked upon its upper part. The knob $B^{\prime}$, fixed to its extremity, is a handle by which it can be drawn out or pushed back.

5. The moderator is a glass tube, $\mathrm{F}$ (fig. 52), terminating below in a metallic basis to which is attached a knob, I, and above, in a collar, K, from which proceeds a hook, which serves to connect the moderator with one of the knols, $\mathrm{P}$, which receive the conductors to the rheophores, and in which the poles of the coils terminate. In the collar is a small opening, traversed by the stem of the moderator $\mathrm{J}$. The tube is filled with water.

6. 'The trembler is composed of a piece of soft iron, A (fig. 52), and of a platinized screw, $\mathrm{S}$, against which the soft iron is pressed by a small spring.

The pedal Y (fig. 52), which replaces the toothed wheel, D (fig. 51 ), is to allow slow intermissions to be produced with the foot; an arrangement which leaves the hands of the operator free, either to hold the rheophores or to graduate the currents.

8. The magnetic rheometer measures the intensity of the initial current. It is a compass divided into four parts, of which each is subdivided into ninety degrees. The rheometer no longer forms part of the apparatus.

\section{B.-Large uncovered volta-faradic apparatus.}

I have represented in fig. 55 an instrument which has its coils exposed. It differs from the common form (fig. 52) in the manner following:-1. It has no external covering, so that we may see the arrangement of the coil $\mathrm{A}$, and the movement of the cylinder $\mathrm{B}$ over it. 2. It possesses two graduator tubes; one, $\mathrm{B}$, which acts upon the second coil; and another, $\mathrm{C}$, which disables the primary coil. 3. Its core of soft iron, D, is movable, and may be withdrawn (fig. 56), so that we may study the influences of the tubes . B and $\mathrm{C}$, independently of temporary magnetization. 4. Besides the commutator of the coils, $\mathrm{E}$, it possesses a commutator of the poles, $\mathrm{H}$, forming part of its structure, and by which the direction of the currents may be rapidly changed without displacing the 


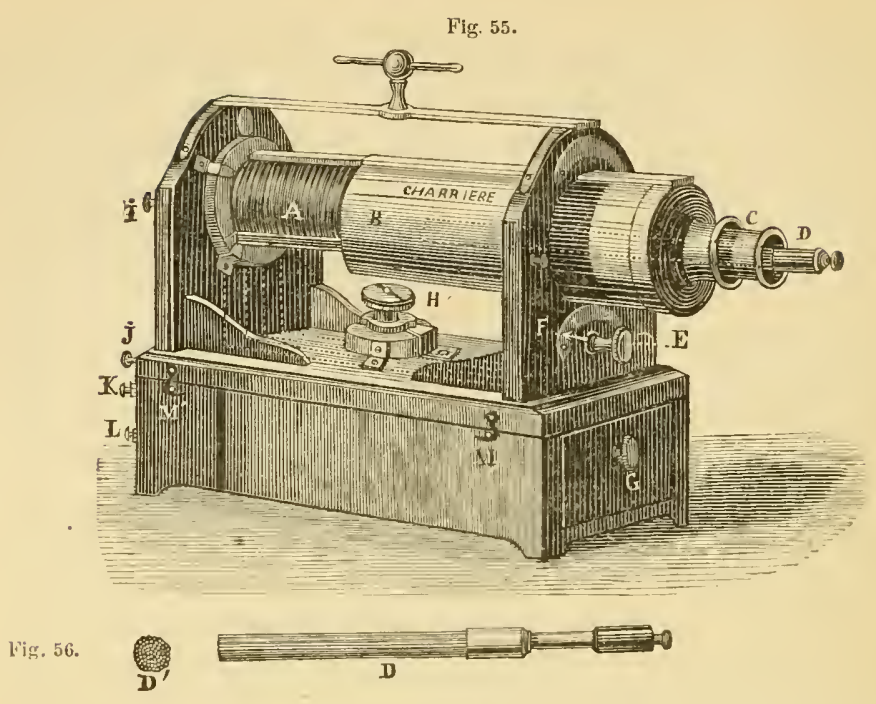

Fig. 55.-Large uncovered volta-faradic apparatus.

Fig. 56.-Bundle of soft iron wire.

rheophores. This eommutator has also other advantages, which it is unnecessary here to describe. 5. The trembler (fig. 57) is so constructed that the rapidity of the intermissions can be progressively increased, from four or eight in the second, to an almost incalculable number in the same period of time.

The battery is independent of the coil, and ean be fixed to the latter by the hooks II MI (fig. 55). It is formed as already deseribed, but of four elements instead of three. The coil, like the former one, can be worked by any battery whatever.7

\section{§II.-Manner of setting the apparatus in action.}

1. Since 1861, as I have already stated, I have entirely abandoned the use of sulphurie acid for my flat batteries; and have used only the bisulphate of meremry. In order to charge the battery, water must be ponred over the carbon until it is well saturated; we then spread over its surface the bisulphate of mercury, in sufficient quantity to form a layer about half a millimetre in thickness, and we moisten this with a little water. We next place upon the bisulphate the cloth diaphragm, first wetted with water, and over this the plate of amalgamated zinc, which then comes in contact with the platinum wires. Thus prepared, the

7 The large unenvered apparatus at my $\mid$ source of electricity is not necessary; but own house is worked by a sulphate of leal battery of twenty pairs. So powerful a eleetric clocks and chamber telegraph. 
elements are placed in the compartments for which they are destined; and which are distinguished by numbers marked on the sides of the caoutchouc cells (see figs. 53 and 54). Lastly, the drawers must be shut (U U', fig. 52) and the button $\mathrm{A}^{\prime}$ turned in such a manner that its bar assumes a vertical direction and prevents them from opening. The communications of the elements of the battery fith each other, and with the circuit of the primary coil, are then established in the following manner.

The zinc of the pair of elements in the lower drawer, which is the negative pole of the battery, corresponds to the knob $\mathrm{L}$ (fig. 52 ), and the platinum contact of the carbon of this pair commmicates with the spring of the piece of copper by which it is serewed to the knob N. But, as the knobs N and G are opposite the two small platinized plates that are seen on the right side of the front of the apparatus above the drawers $\mathrm{U} \mathrm{U}^{\prime}$, and as these two plates are connected by a copper wire, if we turn the knobs $\mathrm{N}$ and $\mathrm{G}$ from left to right into contact with the plates, they comnect the carbon of the pair in the lower drawer with the zinc Z (fig. 53) of the farther pair of the upper drawer. Lastly, the carbon of the latter pair being placed, as I have said already, in communication with the zinc $\mathrm{Z}^{\prime}$ of the front pair, by means of the double spring $a$, and the platinum wire of the carbon of this last pair being in contact, through a spring, with the knob $\mathrm{E}$, this becomes the positive pole of the battery. The small plates of platinum, which are fixed to the front of the apparatus on the left, receive the extremities of the wire of the primary coil; so that, by turning the knobs $\mathrm{E}$ and $\mathrm{L}$, which are the poles of the battery, from left to right into contact, the current of the battery passes through the wire of the primary coil.

It therefore suffices to place in their respective compartments the three pairs of elements, charged with bisulphate of mercury as described, to shut the drawers $U$ and $U^{\prime}$ (fig. 52), to turn the knob $\mathrm{L}^{\prime}$ from left to right (for a reason that will be declared hereafter), and lastly, to turn into contact the knobs $\mathrm{E}, \mathrm{G}, \mathrm{L}, \mathrm{N}$, in order that the battery current may pass through the wire of the primary coil, or, in other words, that the circuit formed by the battery and the primary coil may be completed.

The apparatus may be worked with only the two elements of the upper drawer U' (fig. 52); when it will still possess very considerable power, and will fulfil the ordinary requirements of practice. We must then take care to turn the knob L' (fig. 52) from left to right into contact, in order to suppress the elements of the lower drawer $\mathrm{U}$, which may be used as a place for the rheophores and the conductors. It is unnecessary to say that when we wish to 
use the three elements of the battery, for cases in which great power is required, the knob $\mathrm{L}^{\prime}$ should be turned back in the contrary direction.

2. If it be desired to obtain rapid intermissions of the trembler A (fig. 52), we turn from right to left the knob $\mathrm{C}$ which fixes the movable plate, and then turn froin left to right the buttons $\mathrm{E}, \mathrm{G}$, $\mathrm{L}, \mathrm{N}$, so as to bring them into contact with the pieces of platinum to which they correspond. When this is done, the plate oscillates rapidly between the screw $S$ and the temporary magnet of the central coil.

If intermittences more or less separated from one another are desired, the arrangement is made as before, except that the button $\mathrm{C}$ is turned from left to right, so as to render the plate $\mathrm{A}$ of the trembler immovable, and the button $\mathrm{L}$ from left to right, so as to separate it from its platinum comnection. We then connect with this button one of the conductors fixed to one of the knobs, 1 , of the pedal rheotome $\mathrm{Y}$, and the other conductor, from the knob $1^{\prime}$ of the rheotome, is attached to the button $\mathrm{L}^{\prime}$. It is then only necessary to govern with the foot the spring contact 2 of the pedal rheotome, in order to make and break contact at pleasure. It is, therefore, perfectly easy to obtain intermissions separated by any desired interval.

3. When the graduator $B$ is entirely contained within the instrument, the currents are at the minimum of intensity; and, in order to increase them, it is only necessary to draw out this tube, either by millimetres or by centimetres.

4. The apparatus being in action, the conductors must be fixed to the knobs $\mathrm{P}$ and $\mathrm{Q}$, to which, according to the indications to be fulfilled, we bring the current of either the primary or of the secondary coil, by turning to the right or the left the needle of the commutator. To the free extremities of the conductors are attached the rheophores, cylinders, metallic brushes, \&c. \&c.

5 . If it be desired to faradize very excitable organs, or to measure infinitely weak doses, the upper part, $K$, of the moderator $\mathrm{F}$ must be connected with one of the knobs $\mathrm{P}$ or $\mathrm{Q}$, and one of the rheophores with the lower part of the moderator I. Then, the more we draw up the stem $J$ of the moderator, the greater will be the thickness of the water traversed by the current, and the more the latter will be weakened. The current may then be divided and measured by the tube graduator B.

6 . In the intervals between faradizations, we interrupt the current by turning back the screw $\mathrm{E}$, so as to preserve the force of the pile. At the same time, plates of hard caoutchone must be placed between the cloth and the zinc, care being taken to wipe 
the latter, if the apparatus will not be required again for some hours. It will be observed, after being wiped, that the zinc has become amalgamated by the action of the battery itself.

A battery of sulphate of mercury, thus charged, will last for two or three weeks, and may be used for several hours a day, without any other care than to renew the moisture of the eloth, and to move a little, every day before use, the paste of mercury upon the carbon. It is also desirable to maintain the platinum connections in a state of cleanliness.

When the mercurial paste is quite decomposed (its colour, originally yellow, will then be green) the carbon and the cloth must be washed, and the battery charged with fresh dento-sulphate as before. If the battery belonging to the apparatus has undergone deterioration, or is in need of repair, the coil may be put in action by any other (either a Daniell's, a Bunsen's, or a sulphate of lead). It is only necessary to connect the poles of the substituted battery with the knobs $\mathrm{E}^{\prime}$ and $\mathrm{L}^{\prime}$ (fig. 52), which receive the terminations of the wire of the primary coil.

\section{\$ III.-Theory of the anthor's volta-faradic apparatus.}

If the battery be inclosed in the apparatus, with no interruption either by the pedal or the trembler, the battery current, which is transmitted by the buttons, L, E (fig. 52), to the wire of the central bobbin, is continuous, and occasions none of the phenomena of induction, although it magnetises the central core of soft iron.

In this state of things, if we set at liberty the plate $A$ of the trembler, by turning back the screw $\mathrm{C}$, that plate is attracted by the magnet of the bobbin. At the same instant, the current being interrupted, the magnetization ceases, the soft iron of the trembler is driven back against the platinum point of the screw $\mathrm{S}$, by a spring, and restores the current, which produces a fresh magnetization. These temporary magnetizations, and these breaks in the current, succeed each other with extreme rapidity, in the covered (fig. 52) apparatus; and with more or less rapidity, at the pleasure of the operator, in the uncovered apparatus (fig. 55). Although the theory of the trembler is well known, it is desirable briefly to state it, in order to explain the mechanism of its several portions.

At the moment when the current of the primary coil is interrupted, we may observe the physical and physiological phenomena that are produced by virtue of two combined forces; namely, the inductive action of the current upon itself, by the mutual influence of its coils, and by the reciprocal influence of the current and of the magnetized soft iron of the bobbin.

If the extremities of the fine wire, that forms the outer coil, are 
united by any conducting body, a current will be produced in this outer coil, and will be displayed, at each interruption to the current of the primary coil, by the physical and physiological effects that will be developed in bodies placed within its circuit.

For an account of the theory of the graduation by the metallic tube B, see further, § IV., C.

\$ IV.-Exposition of the principal properties of my large doublecurrent volta-faradic apparatus, and of the principles which have guided me in its construction.

\section{A.-The Coits.}

The two eoils of the volta-electric apparatus, just described, possess the maximum of their differential plysiological properties. by virtue of the proportions of length and thickness that I have given to their respective wires-proportions that I have learnt by experience are the best for this purpose.

I have laid down that the power of the coils ought to be very considerable under certain cireumstances, aud experience, or rather the requirements of therapeutics, have compelled me to increase this power more and more. For this reason, the length of my coils, which was at first but eight centimetres, has now been nearly doubled, and the length of their wires has been increased in proportion (to 1000 metres for the fine, and 200 metres for the coarse wire). It would be easy to go beyond the power thus given, but I have not at present seen that it would be useful, and it would perhaps be imprudent.

Such an increase of strength has necessarily increased the weight of the instrument represented in figs. 52 and 55 ; but without rendering its bulk more considerable.

\section{B.-The commutator of the coits.}

In my original instruments, the ends of the wire of each coil terminated in the metallic knobs (two below for the primary coil, and two above for the secondary coil) to which the conductors for the rheophores were affixed. It was therefore necessary to change the attachment of these conductors every time that it was wished to change the coil. Such an arrangement rendered certain experiments difficult, and was very inconvenient in practice. It is much more easy to compare the differential properties of the currents of the two coils when we can pass rapidly from one to the other; and it often happens that it is desirable to employ them, by turns, for the same patient. Nothing is more tiresome than to have to change, each moment, the attachment of the conductors, 
and to do so wastes a considerable amount of time. Upon such considerations is founded the usefulness of the commutator of the coils.

\section{C.-Graduation of the induced curvents.}

Although, as I have shown, a very powerful apparatus is necessary for the treatment of certain diseases, yet such an apparatus would be unfitted for physiological and pathological investigations, and would even become dangerous, unless we were able to apportion electricity to every single organ in accordance with the degree of excitability that it may possess.

We owe to Dr. Rognetta a method of graduation which he applied to the electro-dynamic apparatus of MII. Breton, frères, and which permits us to control exactly the dose of electric excitation. It is effected by pushing in or drawing out the central coil within the external one, so as to produce induction in a greater or less number of the turns of the latter. I had adopted this method of graduation for my first volta-electric apparatus (fig. 51), presented to the Academy of Sciences in 1817, with the single difference that I made the external reel movable, while the intermal one was fixed to receive the system of the trembler. ${ }^{8}$ I soon discovered, however, that this method of graduation was not free from inconreniences; for the movable coil necessitated the employment of morable connections, which were frequently out of order, which complicated the manufacture of the instrument, and either diminished, by oxydation, the intensity of the currents, or even intercepter their passage.

A fortunate accident led me to discover a means of graduation much more simple, and which I applied to my instruments without delay, instead of the method by the mutual influence of the coils. I made this important discorery in the following manner:-

At the beginning of my investigations, I had covered a powerful coil with copper in order to protect the wire. To my great surprise, I found that the coil had lost nearly all its power. I observed also, that, in removing the covering, the more the coil was exposed the more its current increased. The fact was a ray of light; for, from that moment, the method of graduation by the metallic tube was discovered. I found, after some experiments, that the last half of the tube measured the intensity of the current as exactly as the first; and that the current increased or diminished, as the tube was replaced or withdrawn, in exactly arithmeticil proportion.

8 MI. Dubois-Reymond, of Berlin, has adopted the latter system for the graduation of his induction apparatus. 
A second accident led me to the discovery that a slit tube lost its influence upon the current of the coil that it enclosed. The metal cap of the little instrument, mentioned above, was too tight to glide easily over the coil, and I split it down its entire length. I found that it no longer graduated the current. I paid no attention to this phenomenon, which I attributed solely to the vicinity of the metallic plate; but I soldered to one of the extremities of the tube a very fine copper wire, which reunited its edges, and its properties were immediately almost entirely restored.

I made several other experiments, from which I found that, the greater the distance between the coil and the walls of the tube, thus mended by the wire, the less was the influence of the latter. I concluded that, in order to obtain a maximum of diminution by the metallic tube, it must be as nearly as possible in contact with the coil.

The external tube, howerer, did not completely neutralise the induced currents; and, indeed, when it covered the coil entirely, the latter still possessed a very notable force, such that, if the instrument was one of great power, this force would still be very considerable. It was a matter of necessity to graduate this remaining force, on which the extemal cylinder exerted no action.

It then occurred to me to try the effect of a second tube of copper, made to slide in the interior of the coil, and covering or uncovering the bundle of soft iron wire. The excess of induction current which had not been neutralised by the external tube was considerably diminished, in this experiment, by the influence of the internal tube; and, when I withdrew the latter slowly, I felt the power of the current increase in proportion. To discover which of the two tubes exerted the greatest nentralizing influence, I used them alternately, and compared their effects upon the power of induction. I found that the inner tube diminisled, much more than the outer one, the physiological powers of the currents of both the primary and the secondary coil. It might have been expected that when the inner tube was once completely inserted, the outer tube would no longer influence the induced current; but this was not so, for I found that the considerable power possessed by the current when the internal tube was in situ, was very sensibly diminished, in proportion as the external tube was pushed over the coil.

In all these experiments I had not observed that the magnetization of the bundle of iron wire forming the core of the coil had been diminished in proportion to the diminution of the physiological power of the induced currents. Thus, a compass being placed at a given distance from the extremity of the coil, in such 
a manner that the axis of the latter was perpendicular to the magnetic meridian, the needle was seen to make a considerable deviation at the moment when the core became magnetic as the circuit was closed. It underwent no further movement, not even the slightest oscillation, when the cylinders were pushed home, either together or alternately. This experiment proved that, if the magnetization was at all diminished by the action of the covering tubes, the diminution was, at most, extremely slight. Neither could I discover any diminution of magnetism by testing the power of the electro-magnet to carry different weights, when the coil was under or exempt from the influence of the tubes. It was only by the more or less rapid and noisy vibrations of the trembler, and by the diminished brilliance of the passing spark, that I could determine that the magnetization of the central iron core was really diminished by the tubes. This influence is extremely small on the part of the external tube, by which the noise of the trembler is very little diminished, even when the coil is entirely covered; but the internal tube, on the contrary, diminishes the magnetization sufficiently to impede the motion of the trembler, and to slacken the rapidity of its beats.

Since I have shown that the copper tubes, placed within or without the coil, influence so feebly the magnetization of the soft iron at its centre, it follows that their neutralizing power must be exerted directly upon the induced currents. This view, in fact, is fully confirmed by experience; and its accuracy may easily be shown by repeating the foregoing experiments with a coil from which the central core has been withdrawn. We may then see that the tubes act upon the induced currents precisely as when the core is in its place; that is to say, they neutralise them precisely in proportion as they are pushed onwards into or over the eoil. It is not only the physiological power of the current that is then diminished, but also the brilliancy of the sparks produced by the intermissions, that is, by the calorific action of the current. Evidently also, in this instance, the tension of the induced current is diminished, as may be shown by various experiments, and, amongst others, by the thickness of the layer of water through which it will pass, and of which it will overcome more or less of the resistance, according to the position of the tubes. (It is unnecessary to say that, from the fact of the absence of the central core, the intensity of the currents to be graduated by the tubes is much less great.)

Each of the tubes exerts a special influence upon the current to which it is nearest; thus, when the central coil is deprived of the soft iron core, the action of the inner tube is very powerful 
upon its current, but is scarcely appreciable upon that of the second coil. The outer tube, on the contrary, acts chiefly on the currents of the second coil. This experiment affords an explanation of the difference in the influence exerted by the two tubes upon the rapidity and energy of the beats of the trembler. $\mathrm{IVe}$ ean understand that, the magnetization of the iron core being produced by the current of the first coil, this magnetization must be more enfeebled by the inner tube, which acts specially upon that current, than by the outer tube, of which the influence upon the primary current is almost nit. I shall hereafter point out the importance of knowledge of this phenomenon.

The apparatus with a double graduator tube, and with a double movable iron core (fig. 55) will serve to repeat the whole of these experiments.

If it be desired to study the influence of the outer tube B upon the currents, the inner tube $\mathrm{C}$ is drawn completely out from the coil, as in fig. 55. The outer tube is similarly drawn off when it is desired to experiment ouly with the inner one.

A very simple mechauism suffices to unite the two tubes so that they move together, and cover or uncover simultaneously the internal and external surfaces of the coil. It follows that the induction currents are then more completely neutralised than when the tubes act separately. Lastly, the iron core itself being movable, it is easy to experiment on the action of the tubes alternately or simultaneously, either with or without the assistance of the magnetic force.

To recapitulate: 1. A tube of copper, sometimes called a diaphragm, placed in the interior, or at the exterior, of a reel, formed of a primary and of a secondary superposed coil, and within which there is no core of soft iron, neutralises the currents in proportion as it covers more and more of the spirals, and operates as a closed conductor. 2. The external tube acts specially upon the current of the second coil, while the internal tube affects principally that of the primary coil. 3. The tubes diminish in the same degree the intensity of the currents of the primary and secondary coils, when moving together in the same direction, as when acting separately. 4. The presence of a core of soft iron within the reel in no way modifies the power of the tubes to neutralise the intensity of the currents; for if, on the one hand, the magnetic influence of the iron upon the coils angments this intensity, on the other they neutralise it proportionately, and as well as when the iron core is absent. 5. The magnetization of the iron core is very little diminished when the tubes cover the coils; from which we may conclude that the tubes act directly upon the currents of the coils, 
as is also proved by the experiments related above, and that it is not a diminution of the magnetization of the core that puts a check upon induction. ${ }^{9}$

These facts being completely established, I proceed next to their application. I have no necessity to enter into the most recent discoveries, in order to display the advantages of the application of metallic tubes as graduators. It is enough to mention them, in order to render intelligible their ntility and importance in the practice of localized faradization. The double graduation by the double internal and external metallic cylinders is certainly preferable to graduation by one alone, since the neutralization of the currents is more complete when the tubes move together, and when they cover the coils successively one can use at pleasure a scale for graduation of double extent.

\section{D.-Moderator.}

The graduator tube does not completely destroy the current upon which it acts, so that the scale produced by graduation does

9 Prior to the publication of 'the important work, Traité de l'électricité théorique et appliquée, in which MI. de la Rive las set forth the state of the science with mueh learning and lueidity, I was not aware that Herr Dove, a physicist of great merit, and professor of meteorology at Berlin, had instituted, in 1842 , rescarches into the influence exerted by a copper tube placed within a coil, and containing a bundle of rods of soft iron. I believed, when I laid before the Academy of Ielicine the inrestigations which form the subject of this chapter, that I was the first who had studied the influence of metallic tubes upon the phenomena of induction. I trust I may be forgiven for my ignorance about this point in the history of discovery in physies, when I repeat that I am not a physicist. MIoreover, the physicists who have witnessed my labours, and whom I have consulted upou the point, have supported me in my belief. After having read in the treatise of MI. De la Rive an account of the researches of Herr Dove upon the influence of metallic masses introduced into a coil, and having male myself acquainted with the original work of Dore, I do not now think it requisite to modify the terms in which I described my own results in a memoir addressed, in 1851, to the Academy of Medicine. 1. Indeed, Herr Dove has not studied the influences exerted by metallic tubes upon coils in the interior of which there is no core. 2. If it be true that this experimenter discorered that a metallic tube, introduced into the axis of $\mid$ a coil, and containing a core of soft iron, diminished the foree of iuduction, it is incontestable that he never said that the influence of the tube increased in direct ratio to its advance within the coil; and, if he had been aware of this, he would doubtless have plainly stated it, on account of the praetical importance of the fact. 3. Herr Dove has not tried the effeet of an external metallic tube, covering the current of the secondary coil; and, in consequence, has not compared the different action exerted by an inner and an onter tube, either upon the currents or upon the magnetizition of the iron core. 4. Lastly, this celebrated physicist appears not to have arrived at the same results with myself, in the experiments which he has made with magneto-faradic instruments; for, according to him, we do not see the intensity of the inducad current diminished by introducing a metallic cylinder within the induction coil, ami over the bundle of iron wires in its axis, when we magnetize these wires by the approach of a magnet, instend of by $a$ roltaic current circulating in the coil. $\mathrm{My}$ owu experiments have convinced me that metallic tubes neutralise the currents of the primary and secondary cuils of magneto-fararlic instruments, when they cover their reels, just as completely as they do the currents of volta-faradic instruments. In the succeeding Part will be set forth a demonstration of the facts, applied to the graduation of my magneto-faradic apparatus. 
not commence at zero. The feeble current which remains is insignificant, when applied to the muscles of the trunk or of the limbs. But it is no longer so when used for delicate operations, as for faradization of the facial muscles, or of the membrana tympani, and for these I use the water moderator already described. After having diminished the intensity of the current, it becomes possible, by making this weakened current pass through a stratum of water more or less thick, to divide it still further into infinitely feeble doses. The combination of graduator and moderator has even enabled me to measure the degree of excitability of each of the muscles of the frog.

\section{E.-MIagnetic Rheometer.}

The graduator tube divides into exact doses the current given by either the primary or the secondary coil; but it does not measure the force of the initial current,-a force that is liable to variation from numerous causes, and that therefore produces inevitable differences in the force of the induction. To endeavour to apportion the electricity by the aid of the graduator alone, seems to be as absurd as to weigh medicines in an accurate balance, but with false weights.

I employ a very simple means by which to ineasure the power of the initial current, and the procedure also indicates the degree of magnetization of the soft iron of the core. The action of that magnetization upon a magnetic needle forms the basis of my rheometer. The soft iron is differently magnetised at its extremities, according to the direction of the current. Let us suppose that the extremity nearest the rheometer becomes the north pole. The needle, placed as already described, will be made to deviate from the magnetic meridian by the attraction between its south pole and the magnetised core, and as the magnetization of the core is directly as the intensity of the initial current, the degree of deviation of the needle furnishes an index of both.

I employ the rheorneter to measure the force of the initial current, and to keep it nearly at the same degree. The causes which may weaken a pile are so various that no form of pile can be called really and absolutely constant. The rheometer, therefore, not only measures the initial force, but renders it possible always to' operate under the same conditions. The combined action of the rheometer and the graduator enables us to administer electric excitation in precise doses. It seems sufficient to enunciate this proposition, and unnecessary to enter at large into considerations which show that, although the graduator alone measures nothing, yet its combination with the rheometer affords all the 
conditions of exactitude necessary to formulate the physiological and therapeutic applications of electricity.

\section{F.-Pedal Rheotome and Trembler.}

As I have shown at the commencement of the chapter, the intermittences during faradization should be few or frequent, slow or quick, according to the physiological or therapeutical indication that is to be fulfilled. Any apparatus that does not provide for this requirement is incomplete, and may even be dangerous. In order to meet all wants, I have adapted to my instruments two varieties of rheotome,-the pedal and the trembler. I have also described already (page 123, fig. 40) a rheotome that I have contrived in order to avoid the alternations of an induced current, and to render it either centripetal or centrifugal.

(a). Pedal. With the pedal (Y, fig. 52) the operator obtains, at pleasure, intermissions of greater or less rapidity.

For this purpose, I was for a long time in the habit of employing a toothed wheel attached to the instrument (D, fig. 51), but to turn this wheel occupied the hand which should be reserved to control the graduator, the moderator, the commutators of the coils and poles, \&e. For this reason, I have long abandoned the wheel in favour of the pedal; more especially as the former complicated the apparatus, and sometimes became a cause of its deterioration.

I attach little value, as a rule, to any system of intermittence that cannot be regulated in accordance with the requirements of experimentation or of treatment. I have made sereral small mechanical rheotomes, in which the motor power was furnished by. clock-work, which would maintain more or less rapid intermissions for a considerable time. Expcrience soon taught me that such movements could not replace those that were subject to the will of the operator. Notwithstanding the convenience of the mechanical rheotomes, I returned to the toothed wheel, and eventually to the pedal.

On occasions where great precision was required, I have, however, employed a clock to which a rheotome was adapted, so as to give exactly, at pleasure, one, two, three, or four intermissions per second. ${ }^{1}$

(b). Trembler:-However rapid we may be able to render the intermissions by means of the toothed wheel or the pedal, they yet never reach the speed given by the trembler,-a speed such that

1 This rhentome, fitted to an electric rupting the circuit by a common metroclock, was made by MI. A. Mathien. The nome.

same effect has been produced by inter- 
the muscular contraction which it produces appears to be continuous. The special properties of this very rapid movement, and the energetic influence that it exerts upon sensibility, have induced me to adopt it as a valuable resource in the investigation of certain electro-physiological phenomena, and in the treatment of certain dynamic lesions. But the necessary mechanism cannot always replace the toothed wheel or the pedal, because it may, in some cases, produce even serious accidents. I hold, therefore, that every instrument should be furnished with both a pedal and a trembler.

The common forms of trembler-all of. which I have studied-so often become ont of order, and, so to speak, stop so capriciously, that it would be necessary to abandon their application to induction instruments, if these imperfections could not be overcome. It has often occurred to me, with certain instruments, to be unable to proceed with an experiment, or a therapentical application, on account of the stopping or the irregular action of the trembler,an apparent caprice, the cause of which I could not immediately discover.

By increasing the power of the magnetization of the soft iron of the core, and of the movable piece of iron which produces the intermissions, and by rendering the vibration more simple and easy by a proper adjustment of the force of the spring to the weight of the movable iron, I have now so well regulated the action of the trembler that it never fails, in my instruments, if the power of the pile be sufficient.

Tremblers may be made with or without vibrations. The tremblers with vibrations are those which, between the intermissions, undergo rapid recoils or rebounds, which render faradization very unpleasant.

The more painful sensation produced by the trembler with vibrations renders certain experiments on the muscles more difficult, eren when only a moderate dose is employed. This trembler, however, may be very useful in cases in which it is desired to excite sensibility and nutrition very acutely.

I have contrived a trembler of which the beats may be quickened or retarded at pleasure, so that it may give as few as four intermissions in a second, or an incalculable number, passing through all intermediate degrees of rapidity. I have adapted this trembler to my uncovered instrument (fig. 56).

Fig. 57 offer's a front view of this apparatus, showing the trembler. One of the extremities of the wire of the primary coil communicates with the knob E, the other with the screw C. The movable piece of copper, $A$, which is pressed back by a spring 
placed in front of it against the screw $\mathrm{C}$, communicates with the knob F. As soon as the knobs $\mathrm{E}$ and $\mathrm{F}$ are placed in communication with the poles of a pile, the current passes through the wire of the primary coil, and magnetises its core of soft iron, which attracts the movable piece of soft iron G. This draws with it the piece of copper A, which was resting against the screw C. A solution of continuity is produced, demagnetising the core, and causing the return of $\mathrm{A}$ to $\mathrm{C}$. The current is then re-established, and the same order of events continues.

So far it will be seen that the trembler differs but little from that of the instrument represented in fig. 52 , from which, however, it is dis-

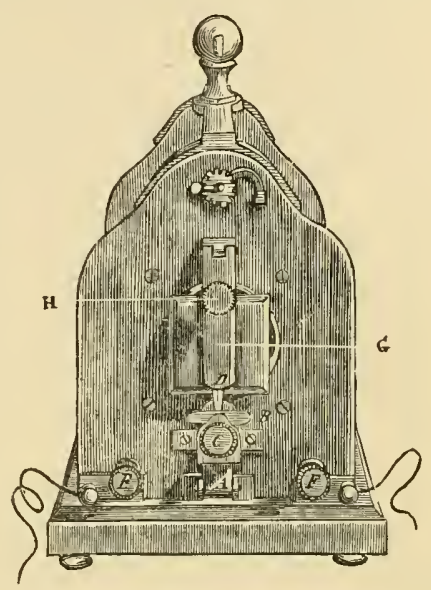

Fig 57.-Front view of the uncovered apparatus shown in fig. 55 . tinguished by the following modifications. The movable soft iron, G (fig. 57), is sufficiently remote from the core to have a much longer distance to travel when attracted by the temporary magnetization of the latter. In this condition it will beat only four times in a second; but, by gradually turning the screw $C$ from right to left, the distance is progressively diminished, and consequently the rapidity of movement of the trembler can be progressively increased at the pleasure of the operator, who is able, by this simple means, to regulate the speed according to the effect that he desires to obtain. In order that the magnetic force may exert its attraction when the distance is at the greatest, it is necessary to increase the bulk of the movable soft iron. For this purpose, I have attached to the latter an additional bar of soft iron, fourteen centimetres in length, and which extends over the surface of the end of the coil, as shown in fig. 57. The magnetization of the plate $G$ by the iron of the core is then considerably increased, since the plate forms but one mass with the piece added to it. When the screw $\mathrm{C}$ is tightened, the spring which presses the piece $A$ against it is sufficiently tense to cause the intermissions to occur without vibrations; but, when the spring is relaxed by loosening the screw, each beat or intermission is followed by a vibration, the special action of which upon the sensibility I have mentioned above. These vibrations are such, when the spring is completely relaxed, that even the slowest action of the trembler (four intermissions per second) produces muscular contractions that are 
painful, although infrequent. These slow intermissions with vibration are highly useful when it is desired to act at once upon the contractility and the sensibility or the nutrition of muscles, and when a current with rapirl intermissions cannot be borne by the patient. For example, when rapid intermissions produce cramps, and therefore cannot be used, I replace them by slow intermissions with vibration, which, without being attended by inconvenience, act with sufficient power upon sensibility and nutrition.

The slow intermissions with ribration are contra-indicated whenever it is dangerous to over-excite sensibility. Hence the apparatus that is provided with a trembler for producing them should have also a rheotome to give slow intermissions without vibration.

Mr. F. P. Le Roux, professor agrégé of physical science, has shown, in an excellent thesis, the importance, in certain conditions, of the study of thie differences between tremblers with and withont vibrations. It will be seen that the electro-physiological phenomena produced by tremblers, and that I have stated above, are in accord with his experience.

"The necessity," he says, "to determine in an absolute manner the number of interruptions has been well stated by MI. Duchenne, who has been careful to furnish his volta-faradic apparatus with a trembler, the beats of which can be slackened or accelerated at pleasure, so that it will give no more than four intermissions in a second, or will produce a very great number in the same time, affording also all intermediate degrees of rapidity.

" II. Duchenne makes a distinction between tremblers with and without vibrations. The tremblers with vibrations are those which, between each intermission are agitated by several rapid recoils or rebounds, which render electrization very disagreeable. Save in certain cases, one should avoid the arrangements of a trembler which produce these effects, because they render certain electromuscular experiments more difficult.

"We know that very closely approximated electric shocks produce a tetanic condition instead of isolated contractions; the vibrations in the trembler produce in reality a series of tetanic convulsions of more or less duration and separation.

"The facts of a purely physical kind that I have observed confirm those physiological ones that have been stated by MI. Duchenne. I have had occasion to observe that the mamner in which the extra current is produced, may give rise to a division of the induced spark, of which the following fact may convey an idea. In experiments upon the rapidity of the transmission of 
sound, ${ }^{2}$ I used as a means of chronoscopy the trace left upon a plate of iodized silver by an induction spark proceeding from one of Ruhmkorff's coils. The interruption of the inducing current was produced mechanically; but under certain circumstances, instead of a single trace, I observed as many as fifteen traces, corresponding to as many small sparks into which a single discharge had been subdivided. I conld distinguish one from another becanse the plate was in rapid rotation, and the small sparks, being successive, left their marks on different points; but the fifteen traces were separated only by the length of a millimetre, which corresponded to a time notably less than the fifty-thousandth part of a second. The sparks were unquestionably successive, because they were all aligned in the direction of the movement of the plate.

"There is no room to believe but that each of these partial sparks corresponded to a vibration produced in the interrupter, for it is manifest that, according to the law of the movement of interruption, the induced discharge, which most commonly manifests itself in a single spark, may divide itself into a certain number of partial discharges. These phenomena were observed with a Ruhmkorff's coil, of moderate dimensions." ${ }^{3}$

In the tremblers, the point of the screw is platinized, and comes into contact with a plate also platinized, and soldered to the piece which beats against it, so that perfect contact is as little as possible interfered with by oxydation. In time, however, the platinum, burnt by the electric spark, does undergo oxydation; and the oxyde deposited on its surfaces checks the passage of the current, and weakens the power of the apparatus; the play of the trembler being either hindered or altogether stopped. I have remedied this fault by increasing the thickness of the piece of platinum, and by making it movable, so that when, after working some hours a day for many months, it becomes oxydized, it may be shifted a little to the left or right, and a new place brought into use. It is also necessary to clean the point of the screw from time to time, by rubbing it with a piece of cloth or linen, so as to remove the oxyde. Finally, in order to diminish the oxydation of the platinum, I have increased the contact surface, giving to it from two to three millimetres of transverse diameter.

2 Le Roux, Détermination expérimentale de la vitesse de propagation d'un ébranlement sonore dans un tuyau cylindrique (Annale de Chimie et de Physique, $t^{\mathrm{e}}$ série, tome xii. p. 345 ).

3 F. P. Le Roux, De linduction et des appareils electro-médicaux, p. 46. Thèse présentée à l'Ecole Supérieure de pharmacie pour l'aggrégation des Sciences physique. 


\section{G.-The Battery.}

From the beginning of my researches and until 1858, I had employed Bunsen's battery in my volta-faradic instruments, because it combines with sufficient power a considerable degree of constancy. I modified it only by arranging it in a flat form, so that it could be contained in a drawer. Instead of immersing the carbon in nitric acid, I poured the acid over a plate of carbon so as to saturate its pores, and then put this plate into a zinc cell containing a small quantity of solution of sea-salt. My only diaphragm was thus a thin layer of saline solution; by which arrangement the resistance of the battery was much diminished, and its power proportionately increased.

Such a battery as this was perfectly in accord with the force of my first instrument. But, since I have fully doubled the original length of the coils and of their wires, it has been insufficient to develop the full power of the apparatus. I have therefore been compelled to seek a battery yielding electricity of higher tension. For this purpose I have diminished by one-half the surface of the elements, and have increased their number to three or even four.

The inconveniences due to the employment of nitric acid, that is to say, the inevitable liberation of nitrous gas, which was a cause of the rapid deterioration of the several parts of the apparatus, induced me to replace the nitric by sulphuric acid. For more than a year I had employed this new arrangement, when MI. MariéDavy made known his battery with bisulphate of mercury, which, with some modifications, I adopted for my instruments. It is to this source of electricity that $I$ have now for six years given the preference for my portable forms of apparatus, and a description of it has already been given to the reader. It possesses the advantage that it may be carried from place to place, without fear of spilling any liquid. It is sufficient to place a piece of hard caoutchouc between the cloth and the zinc, carefully cleaned, in the intervals between the applications; and when we wish to put the battery in action, to pour a little water upon the cloth, and to remove the plates of caoutchouc. The same bisulphate of mercury will last from fifteen days to three weeks.

The pieces connecting the system of induction with the carbon, composed originally of copper, platinized at its contact with the carbon, were rapidly destroyed by oxydation. I have replaced them by platinum wires, and they are now unalterable. The two or three small elements which form a part of my volta-electric apparatus, allow me to have, at the bedside of the patient a power of 
induction that is always sufficient; but the induction of my apparatus, is not, so to speak, saturated by these three elements; for I have obtained from it a much greater foree, with the fifteen or twenty elements of bisulphate of lead that I can adapt to it at my own house.

Although M. Marié-Davy has arvised the use only of the protosulphate of mercury, I have substituted the bisulphate in order to obtain greater power. I have only employed the proto-sulphate, as I have said, for the formation of a battery composed of from thirty to a hundred elements, for the application of continuous currents.

Manufacture of the carbon plates.-After numerous experiments with regard to the best kinds of coal and coke, to the proportions in which they should be mixed, to the manner of setting the mixture in the moulds, to the form of the latter, and to the amount of burning required, I succeeded in manufacturing carbon plates that were hard and free from fissures. The manufacture of these plates being, however, both difficult and costly, I sought to replace them by carbon sawn out of gas-coke.

The coke deposited on the interior of the retorts used for making gas is formed, as is known, from the bituminous matter called petroleum. This petroleum becomes carbonized, and deposited, layer upon layer, on the inner surfaces of the retorts in which the coal is distilled. It forms at length a thick erust, which it is necessary to remove from time to time. Unfortunately, the carbon plates formed from gas-coke, which are excellent when immersed in nitric acid, and which, being very pure and free from earthy matter, seem then to increase power, do not succeed for the system that. I have adopted in my instruments. In a few minutes (two or three) the current furnished by them diminishes by more than three-fourths, when the circuit is closed. We then observe the singular phenomenon-which is produced also in piles of zinc and copper-that the current recorers its intensity when the circuit has been for a short time interrupted. I attribute this rapid weakening of the current to the great density of the gas-coke, the interstices of which become obstructed by the salts that are formed by the chemical action of the pile, and that are rapidly dissolved when the circuit is left open. 'The carbon plates that I have had manufactured, without being too soft, which would interfere with their durability, are yet hard enough. It is not until after some time (five or six months of frequent use) that the salts resulting from the interior chemical action of the pile are deposited in the interstices in sufficient quantity to diminish the power of the apparatus by resisting the passage of the current. It is important 
to be aware when this occurs, becanse the power may be diminished considerably.

\section{H.-The Commutator of the Poles.}

It is useful to be able rapidly to change the direction of the clirrents, without displacing the rheophores from their position on the patient. For this purpose, I have fitted to my covered instrument a commutator of the poles ( $\mathrm{H}$, fig. 55$)$, which, by turning to the right or to the left, changes their direction. Its mechanism is the same as that of the commutator of the currents.

\section{Parti tile 'Third.}

SMALL DOUBLE-CURRENT VOLTA-FARADIC APPARATUS.

This instrument, with medium force, possesses all the properties required in a medical apparatus. It is a reduction of my large double-current volta-firradic instrument; and is generally capable of replacing it in practice.

\section{A.-Description.}

I lare given to this apparatus the form of a flat oblong box, or of an octavo volume, as shown in fig. 58 . When it is open, as in
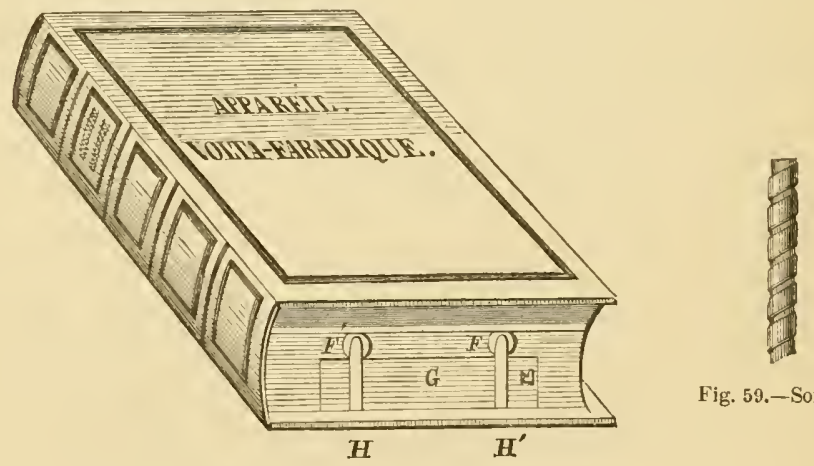

Fig. 59.-Soft irou.

Fig. 58.-Author's small volta-faradic apparatus (closed).

fig. 60 , it is seen to be divided into two parts. To the right is placed the induction apparatus properly so called. To the left is the battery, in the lower compartment A ; and the rheophores, with their conductors, are in the upper compartment B.

The induction apparatus is composed :-

1. Of a reel, six centimetres in length, formed by two superposed coils, the wires of which are of different length and diameter (the wire of the primary coil is sixty metres in length and a half 
Fig 6).

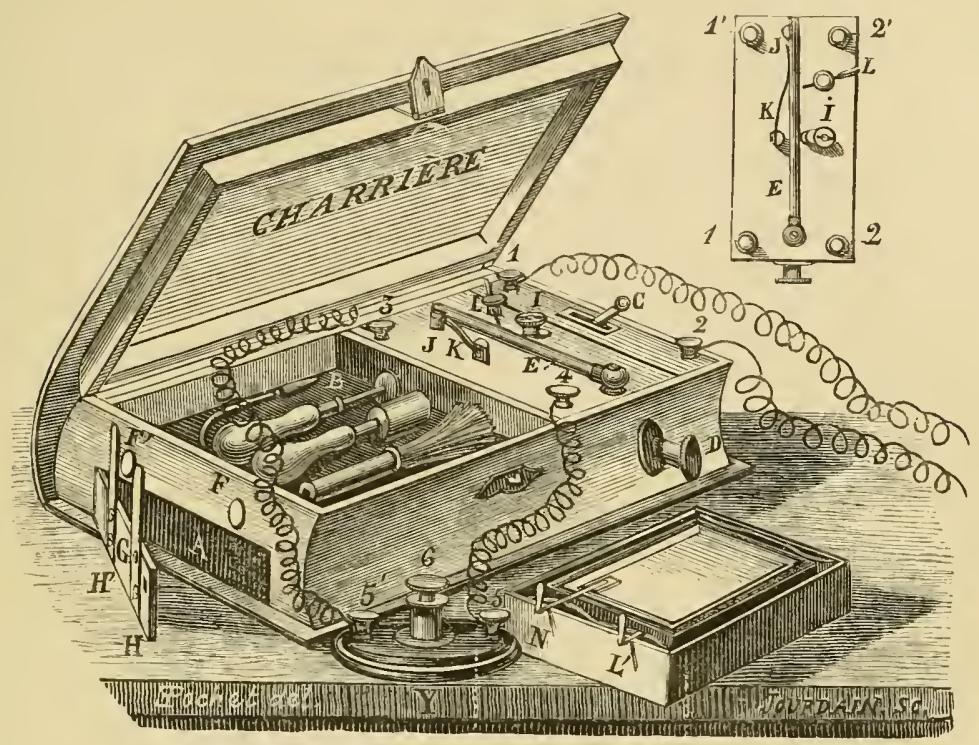

Fig. 62.

Fig. 63.

Fig. 60.-Author's small rolta-faradic apparatus (opencd).

Fig. 61.-Details of the cover of the compartment to the right.

Fig. 62.-Pedal

Fig. 63.-Pair of elements with bisulphate of mercury.

a millimetre in diameter, and the wire of the secondary coil is three hundred metres in length and a sixth of a millimetre in diameter); 2 , of a core formed by a broad band of soft iron rolled into a helix, as in fig. 59, so that it can be placed in the centre of the reel; 3 , of a commutator of the coil, $\mathrm{C}$ (all the foregoing portions are concealed in the compartment to the right); 4 , of a graduator tube D; 5, of a trembler E, and of a rheotome $\mathrm{L}$ for slow intermissions.

\section{B. - Manner of putting the apparatus in action.}

A pair of elements with bisulphate of mercury (fig. 63), like one of the pairs of my large apparatus, is introduced into the compartment $A$. Then if the door $G$ of this compartment be closed, as in fig. 58, the small platinized plate $\mathrm{L}^{\prime}$ (fig. 63), which communicates with the carbon contained in the cantchouc cell, comes in contact with the spring $\mathrm{H}$, which rests against the platinized termination $\mathrm{F}$ of one of the extremities (poles) of the primary coil, while the zinc $\mathrm{N}$ (fig. 63) is brought into communication with the other extremity (pole) of the primary coil, by the spring $H^{\prime}$. The separations of this electro-motor from the coil are produced by the separation of the horizontal bar $\mathrm{E}$ (fig. 60) from the knob I. 
If it is wished that the intermissions should be made slowly, the knob L (fig. 61) must be turned till the line traced upon it is directed transversely, since in drawing to itself the stem of the rheotome until the bar $\mathrm{E}$ of the trembler is in contact with the knob I, it completes the circuit formed by the battery and the wire of the primary coil, which is broken by pushing back the stem of the rheotome L. In this way an intermission is obtained, and we may thus obtain a series more or less quickly. If rapid intermissions of the trembler are desired, the button I must be turned from right to left until the bar E, pushed forward by a platinized eccentric fixed to the button, is sufficiently near to the temporary magnet $J$, which is in contact with the soft iron in the centre of the coil. The approximation is sufficient when the noise of the trembler can be heard. It is necessary to regulate the movements of the trembler carefully; for if they are too rapid, in consequence of too near an approach of the bar E to the magnet $J$, the separations will be less complete, and the contact of the portions of the trembler will be less perfect. It follows that the power of induction will be diminished. We may also produce small intermissions with the pedal rheotome $\mathrm{Y}$ (fig. 62), which has been described (p. 259), and which is applied to the large instruments. For this purpose, its conducting cords must be attached to the knobs 3 and 4 (fig. 60), and then, after having turned the button $\mathrm{L}$ from left to right, until the bar $\mathrm{E}$ is in contact with the magnet $\mathbf{J}$ (in this position the battery current is interrupted), the intermissions are to be made with the foot in the manner already described. (By pressing on the knob 6, fig. 62, the battery current is completed.)

The electrode knobs, 1 and 2, receive the currents of each coil. To them are fixed the conducting cords to which the rheophores are attached. We can bring to these knobs the current either of the primary or of the secondary coil, by pushing to the left or to the right, as far as it will go, the stem of the commutator of the coils C. The figures engraved upon the small plate of copper traversed by this stem, point out the side towards which the stem should be pushed in order to bring one or other of the currents to the knobs 1 and 2. Lastly, the graduation is effected by the tube $\mathrm{D}$, as in the other instruments. In the intervals between the applications, the battery current is interrupted by pushing back the stem of the rheotome $\mathrm{L}$, until the bar $\mathrm{E}$ is in contact with the soft iron J.

The small apparatus just described is that to which $I$ give the preference in the great majority of cases. I have recourse to the large instruments only for the purposes of diagnosis, or for electro- 
physiological and pathological experiments, and for treatment in a few cases in which contractility or sensibility are either abolished or sensibly diminished.

The power of this small instrument is very considerable, regard being had to the small size of its coil. This is not only due to the excellent proportions of length and diameter of the wires, and to good mannfacture, but also and chiefly to the powerful magnetization of its band of soft iron rolled into a helix, which thus offers a considerable extent of surface. ${ }^{4}$

\section{Part the Fourth.}

\section{THE AUTHOR'S DOUBLE-CURRENT MAGNETO-FARADIC APPARATUS.}

The volta-faradic instruments, the construction and properties of which have been described in the preceding Part, have been employed in my numerous researches in electro-physiology, pathology, and therapentics. They have endured this test satisfactorily, and their usefulness is now perfectly established. Unfortunately, the preparation of the battery, simple though it be, necessarily requires some amount of manipulation. This single inconvenience has presented a difficulty to some physicians, and has often induced them to prefer the common magneto-electric instrument.

For this reason, I have endeavoured to give to a magnetoelectric instrument, as far as possible, the properties that I believe to be indispensable for the practice of localized faradization.

\section{§.-Descripion of the Apparatus.}

My magneto-faradic apparatus (see fig. 64) is composed of a magnet, of an armature, set in movement by a peculiar mechanism, of a regulator of the armature, that is at once a moderator of the currents and a magnetic tensor; of two coils of copper wire, of unequal length and thickness; of a rheotome; of a regulator of the intermissions; of a graduator of the currents ; and, lastly, of a commutator of the coils.

\section{A.-The Magnet.}

The magnet is formed of two parallel cylindrical branches,

${ }^{4}$ I have experimented comparatively, with different forms of core, with regard to their respective physiological effects; using iron wires (after Dove's method), split tubes of tin, enclosed the one within the other, and each insulated by a paper covering, then a broarl band of tin rolled into a helix, and with its internal surface insulated by a sheet of paper. I have fonnd that the helix of soft iron has given the best results, both as regards the powcr of physiological effects and as regards the force of the magnetization. 


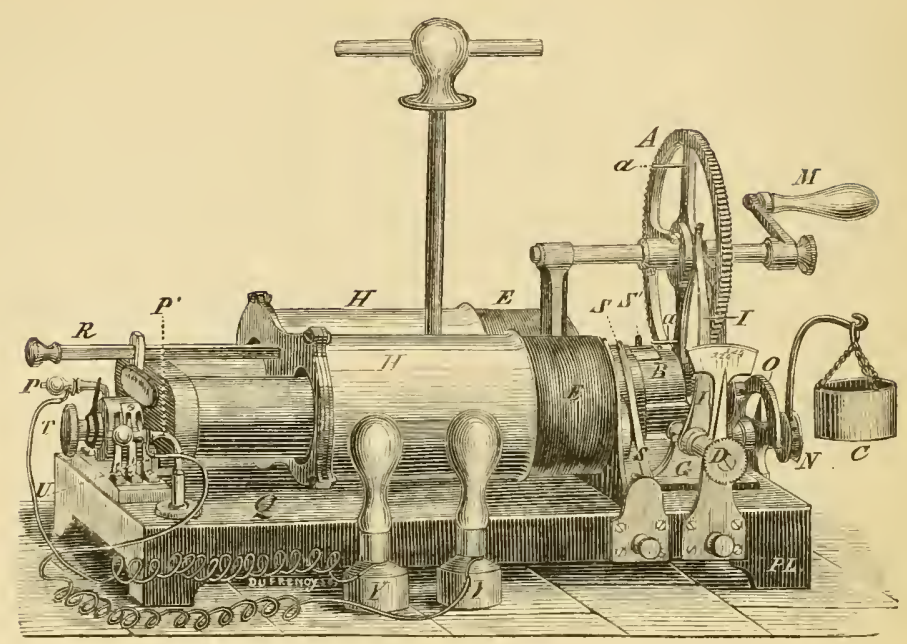

Fig. 64,

connected together at one of their extremities by a transverse bar of soft iron.

It is placed on the flat, and horizontally upon a support fixed to the posterior part of the case of the apparatus. Its anterior extremity rests on two other supports.

\section{B.-The armature and its motor system.}

The armature, which by its movement of rotation produces the intermissions of the magnetic current, is traversed in its middle part by a horizontal axis, terminated at its extremities by a very hard steel point, received in steel sockets, which are screwed into two copper mounts. These mounts are firmly fixed to a square movable plate of copper, $G$, which rests on the base of the apparatus. Between these two mounts, and at their superior extremity, is fitted a large wheel, $A$, the axis of which traverses on one side the anterior mount in which it rotates, while the other extremity terminates in a point, and is received in a piece fixed to the posterior mount. The handle M, which puts the large wheel in motion, can be removed at pleasure. The circumference of the large wheel $\mathrm{A}$ is divided into sixty-four teeth, which set in action a small wheel of eight teeth, fixed upon the axis of the armature in such a manner that at every revolution of the great wheel the armature tums eight times upon its axis, and consequently produces thirty-two intermissions of the current of induction. As it is possible to make the large wheel revolve twice in a second, we may obtain sixty-four intermissions in the same period of time. 


\section{C.-The regulator of the armature.}

The movable plate $G$, upon which are placed the armature and its motor system, is approximated to or remover from the magnet, by means of a strong screw $\mathrm{N}$, callerl the regulator of the armature. This screw, turning in a nut fixed to the apparatus, acts upon the base of one of the momts which forms part of the plate $\mathrm{G}$, which it moves backwards and forwards.

In order that the armature may exercise a teusion upon the magnet, the screw $\mathrm{N}$ is turned in such a manner that the needle is brought to the middle part of the are of a circle $O$, which is called the indicator. Then the armature, placed transversely, is brought near to the magnet, and is in contact with its extremities.

We shall see hereafter in what way the regulator of the armature serves also to weaken or to molerate the induction currents.

\section{D.-The coils.}

A reel nine eentimetres and a half in length, and upon which twenty-four metres of copper wire, covered with silk, and half a millimetre in diameter, are rolled spirally, is fixed to each arm of the magnet, in such a manner that its anterior borler is level with the free edge of the magnet. The rounded anterior extremity of the reel rests in a support which maintains the magnet in a position parallel to the plane of the apparatus.

A second copper wire, the sixth of a millimetre in diameter, six hundred metres in length, and covered with silk, is rolled over the thicker wire, and forms a second coil. The thick wire is inductive relatively to the thinner wire, which is induced by it. I shall demonstrate this hereafter.

The two superposed copper wires are rolled in the same direction, and the extremity of the one is soldered to the commencement of the other, while the other ends are brought, that of the thick wire (central) to the springs $\mathrm{S}$ and $\mathrm{S}^{\prime}$, which produce the intermissions on the reel B, and that of the fine wire (external) to the right side, $\mathrm{U}$, of the commutator of the coils ' $\mathrm{T}$, which conveys the current of the primary coil to the knobs $\mathrm{P}$ and $\mathrm{P}^{\prime}$, to which are attached the conductors of the rheophores. 'The spring $\mathrm{S}^{\prime}$ gives origin to a conducting wire which proceeds to the left side of the commutator T.

\section{E.-The Rheotome.}

The rheotome is composed of a small wooden reel, B, and of two springs, $\mathrm{S}$ and $\mathrm{S}^{\prime}$. The reel is fixed npon an axis of soft iron. One of the springs, $S$, in relation with one extremity of the central 
wire, rests upon a metallic ring fixed upon the reel. This ring is divided into four teeth, two of which are very short. The second spring of the rheotome $S^{\prime}$, which communicates with the other extremity of the central wire, is brought in contact with the four, or with the two teeth, by means of the regulating knol- of the commutator (not shown in fig. 64), which is fixed to the right of the apparatns, and which turns from right to left, and vice versâ.

\section{F.- The regulator of the intermissions.}

A piece of copper is fixed to the base of the apparatus, to the left of the large wheel. It is traversed by a screw, D, to which is soldered a brass spring, I. By means of the screw D the spring I can be made to perform a to-and-fro movement, which brings it in contact sometimes with the pins $a$, upon the posterior face of the great wheel $A$, and sometimes with the plate $G$, which supports the latter. One of the extremities of the inner (thick) wire of the coil, and of the outer (fine) wire of the coil, commmicates with this plate $G$. The brass spring $I$ is in relation, by a copper wire, with the left side $\mathrm{U}$, of the commutator of the coils $\mathrm{T}$.

\section{G.-Graduator of the currents.}

Two tubes of copper, $\mathrm{H}, \mathrm{H}$, covering the reels which are placed under the arms of the magnet, and connected by a cross-piece at their posterior extremity, are pulled ont or pusherl in, gliding over the reels by means of the stem $R$. The latter, fixed to the crosspiece, and terminating in a handle the length of the reels, is divided into centimetres and millimetres. When pushed entirely within the apparatus, the cylinders cover the reels, and the currents are at their minimum of intensity. The currents are, on the contrary, at their maximum when the stem is drawn completely ont of the apparatus. Their intermediate degrees of force are in direct proportion to the position of the stem. This stem, therefore, merits the denomination of a graduator of the currents.

\section{H.-Commutator of the coils.}

The commutator of the coils, $T$, is constructed like that of my volta-faradic instrument.

\section{§ II.-Theory of the Magneto-faradic Apparatus.}

The simultaneous intermissions of the magnetic current, and of that of the central coil produced by the rotary motion of the wheel $A$, which puts in action the armature, and the rheotome, $B$, 
develop the phenomena of induction in the thick wire of the central coil. The theory of these phenomena being the same as that of induction in all magneto-faradic instruments, it is unnecessary to describe it here.

At the instant when this induction is produced in the thick wire of the central coil, if union of the two ends of the fine wire of the second coil is effected by a complete conductor, a counter current will be developed in this fine wire, in a direction opposite to that of the current of the primary coil. (The possibility of this inductive action of the central upon the secondary coil, in electro-magnetic instruments, has been contested, on theoretical grounds, by MII. Becquerel. I will hereafter show, by experiments, that it takes place in my apparatus.)

As one of the terminations of the wire of the primary coil and of the secondary coil proceerls directly to $\mathrm{P}$, the knob of the rheophore, and as the other extremity of these wires communicates with the metallic plate $G$, which supports the large wheel, it becomes necessary to bring this plate into relation with $\mathrm{P}^{\prime}$, the knob of the second rheophore. The regulator of the intermissions, D, fulfils this condition completely; because it communicates with the knob $\mathrm{P}^{\prime}$ by a copper wire, and it is brought in contact either with the plate $\mathrm{G}$, or with the pins $a$ of the great wheel $\mathrm{A}$, fixed to that plate, by means of the to-and-fro movement effected by the knob $\mathrm{D}$.

If it be wished to pass rapid currents through the conducting cord of the rheophore attached to the knob $\mathrm{P}$, it is evident that the knob D should bring the spring I into relation with the plate $G$, which is itself in relation with the other extremity of one of the two wires. But if the spring I only communicates with the plate $G$ by the pins $a$ of the large wheel $A$, the current will only reach the knob $\mathrm{P}^{\prime}$ four times, or twice, or even once, for each revolution of the large wheel. Consequently, the conductors of the electrodes attached to the knobs $\mathrm{P}$ and $\mathrm{P}^{\prime}$ receive only one, or two, or four intermissions per revolution, when the spring touches the pins; while they receive the rapid current (thirty-two, or sixty-four intermissions per revolution) when the spring touches the metallic plate G. I therefore call the knob D, which moves the spring, the regulator of the intermissions.

The commutator of the coils acts in the same way as that of the volta-faradic apparatus.

For the theory of the graduation of the two currents, I must refer to what has been said on the same subject, with regard to the volta-faradic apparatus. I shall hereafter explain how to administer the most powerful or the most feeble doses with my magneto-electric instrument. 
§ III.-Explanation of THE PRINCIPAL PROPERTIES OF THE Double-current Magneto-faradic Apparatus and the DIFFERENT COMBINATIONS WHICH HAVE GOVERNED ITS CONSTRUCTION.

Although double induction had not previously been applied to magneto-faradic instruments, I had no doubt of the possibility of obtaining it. I have described how, and by what series of researches, I was led to its realization.

The MII. Becquerel, however, physicists of great scientific authority, have disputed the possibility of producing double induction with my magneto-faradic instrument. I may be permitted to endeavour to show, by experiment, that I am not, on this point, under an error.

I must repeat, that, in my volta-faradic instrument, the initial force is mixed, that the induction of the primary coil results from the modification produced: first, by the battery current circulating through the coils of its helix in an intermittent manner; secondly, by the influence of the temporary magnet.

I have already stated (Chap. I., Part III. $\S 1$, page 30 ) that MI. E. Becquerel attributes the special physiological phenomena of the current of the secondary coil to the direct influence of the temporary magnet. It will be remembered that, in order to prove how unfounded was this hypothesis, I made experiments without placing the soft iron core in the centre of the inductive helix; and that the phenomena peculiar to the current of the secondary helix were, none the less, very powerfully produced (see experiment, page 29).

A physicist, celebrated for his discoveries, and of no less scientific authority than MIM. Becquerel-M. de la Rive-has thus written, with reference to this experiment, which I repeated in his presence :-

"I must admit, with M. Duchenne, that the current which circulates through the primary coil must certainly act upon the wive of the secondary, since we know that, even when there is no soft iron core, induction is produced." But he is careful to add, "The presence of the soft iron increases the induction in an enormous degree, and the two actions, coinciding to produce the same effects, greatly increase them. The current induced in the second wire is therefore precisely like that uhich induces the direct action of the magnet, and hence I am unable to discover in this anything but a current of the first kind, and not a current of the second kind, as believed by M. Duchenne." ${ }^{5}$

${ }_{3}$ De la Rive, loc. cit. t. iii, p. 581. 
I am willing to bow before the great authority of M. de la Rive, but I must nevertheless ask permission to remind him of an experiment which he witnessed in my laboratory. ${ }^{6}$ He has, withont doubt, forgotten that I showed him, with the primary coil of my double-induction instrument, the physiological power of the extra current, comparatively, with and without the soft iron core. We then saw that, under the influence of the magnetization of this iron core, the power of induction of the extra current was increased in a considerable proportion. Is it not reasonable to conclude that, under these circumstances, the greater part, if not the whole, of the power of the temporary magnetization was employed to influence the primary coil, and thus to increase the energy of the extra current? But in order to admit, with ML. de la Rive, that the induced current, which is then developed in the completed circuit of the superposed secondary coil, is precisely like that which induces the direct action of the magnet, it would be necessary, I think, to prove that the magnet then ceases to exercise its influence upon the primary coil, which is the nearest to it, and removes its action to the secondary coil, which is the most remote from it. But I am not aware that this, which to me seems improbable, has ever been demonstrated.

It is after having made the foregoing experiments, and after having interpreted them in this manner, that I think I am authorized to formulate the following conclusions:-

1. In the volta-electric instruments, the temporary magnetiza-

6 Before forming an opinion of the value of my electro-physiological and theripeutical researches, and especially before criticising them, this pliilosoplier has not only thoroughly studied what I have written, but has also done me the honour to seek from me explanations with regard to my instruments. Then, experimenting upon hinself, I have been able to show him the reality of the chief special or differential pliysiological actions of the currents of the primary or of the secontary coils of these instruments.

'To the opinion of M. E. Becquerel, who writes, "the extra current and the current of the first lind do not possess elective pro. perties over this or that function, but they lave an action more or less energetic, by reason of their tension," it is interesting to oppose that of M. de la Rive, who has endeavoured to explain the difference between the physiological properties of the two currents-a difference such that they cannot be substituted for one another in practice, and that depends, according to him, not only upon the inauced current possessing greater tension than the extracurrent, but also upon its being in general more momentary, aud, lastly, apon its being composed of two currents passing alternately in contrary dircetions; while the extra current is composerl of one induced current only, passing always in the same direction.

" We must here remember," says M. de la Rive, "that we may have, between tlie extra current and the current induced in the second wire, other physical diflerences than those relating to quautity or intensity; and that hence, although we may construct coils, in which, as regards tlie last-named qualities, the currents slall be alike, they may still difier from one another. In the first place, the extra current is never composed of more thau a single induced current, which always passes in the same direction with the battery current, while the induced current properly socalled, is composel of two instantaneous currents, passing alternately in opposite directions. This difference may well produce a difference of physiological action." 
tion acts as a second initial source, nearly in the same manner as the battery, upon the wire of the primary coil, the current of which it reinforces, and which, in its turn, reacts upon the current of the secondary coil, so that the power of this last current is proportionately increased. In the magneto-faradic instruments, the initial force is more simple; it is the intermittent changes in the state of the fixed magnet which produce induction in the wire coiled immediately around it. But the power of this induction is very feeble, if exerted by that influence alone, or, in other words, only by the rotary movements of the armature. In order that the induction should acquire power, it is necessary that the solutions of contact should take place in the wire of the coil, at the moment when the modifications of the magnet are at their maximum (a little before the contact reaches the vertical or horizontal position). Such are the arrangements of the primary coil of my magnetofaradic apparatus; the solutions of contact, which are produced by the rheotome, occur only in the thick wire which immediately surrounds the fixed magnet; the long fine wire, which is rolled round the thick wire, has no communication with the rheotome. If we suppress, in the thick wire, the solutions of contact produced by the rheotome, the current in the fine wire of the outer coil immediately becomes extremely weak, just as if we produced, by the same rheotome, solutions of contact in the fine wire. The intensity of the current of the last then increases a little, but remains very feeble, as compared with that which is developed when the solutions of contact ocenr in the thick wire. The following is the proof :-

Experiment.-The power of the current dereloped in the fine wire of my magneto-faradic apparatus is considerable. But if the solutions of contact of the wire of the primary coil, produced by the rheotome, are suspended (which may be effected in my instrument by turning the knob Q, fig. 64 , from right to left), that is to say, when the current formed by the wire of the primary coil remains interrupted, the power of the current of the secondary coil is so diminished that it can scarcely be felt, even by placing the rheophores upon the lips. Even when the solutions of contact are produced by the rheotome in the wire of the secondary coil itself-an effect which I have obtained by a simple mechanism, in an apparatus constructed purposely for these experiments-its current is still infinitely more feeble than when the same coil is induced by the primary one.

I have thought, aud the idea has, without doubt, presented itself to the minds of my eritics, although they have not succeeded in expressing it very clearly, ${ }^{7}$ that possibly my primary coil acted

; I here quote, precisely, the criticism which one of my learned opponents, Mr. A. Beeqnerel, has bestowed upon my magneto-faradic instrument, and npon the

properties of its two coils: "In the mag-, neto-electric apparatus of M. Duchenne," he says, "two wires are coiled, one over the other, around a fixed magnet, the first 
upon the second coil like a copper tube, and masked, or neutralised its power; and that the sudden interruption produced by the intermissions permitted the physiological action produced by the influence of the current of the secondary coil upon the magnet, to display itself in all its force. If such were the influence exerted by the primary upon the secondary coil in my electro-magnetic instrument, no one could regard it as an induction phenomenon. But it has been shown, by experiment, that this is not the case. I

thicker and shorter, and the second finer and longer. According to M. Duclienne, the current produced in the former wire is an induced current of the first order (of the primary coil), and the current produced in the latter wire is determined by the influcnce of the former, and is an induced current of the second order (of the secondary coil). M. Duchenne is deceived. The currents produced in the two wires are both under the infuence of a magnet which destroys the cffert produced by the first wive upon the second, or rather reduces it to something extremely smull. The current prolnced in the first, and that produced in the sccond wire, are both induced currents of the first lind; only the former, produced in the shorter and thiclier wire, is a current of less tension than that producen in the longer and finer wire, and consequently produces less energetic effects upon the slitin and upon the retina."

Is there occasion to show the small value of the following experiments, which, according to my opponent, prove incontestably the truth of his assertion?

I. "Around a fixed magnet," he says, "roll, by superposition, two wires of equal diameter and length, and cause the armature of the soft iron to act by rotation; an induced current of the first order will be produced in each wire."

Yes, if the circuit of the first coil is neither completed nor interrupted by solutions of contact produced in its wire by a commutator, as in my double-coil instrument; but then the experiment proves nothing against my theory. But if, on the contrary, my learned opponent were placed in the same conditions that obtain in my magneto-electric instrument, after the explanations of its mechanism and of its action that I have given above, he would doubtless abandon his opinion as untenable. In his experiment, then, the current of the second wire evidently receives the influcnce of the current of the first wire, resulting from the simultaneous action of the magnet, and of the solutions of contact in this wire, as I have alrcady explained.
II. After liaving related the foregoing experiment, M. A. Becquercl adds, "the current of the first wire will be more intense than that of the sccond, the cmrent of the second less intense than that of the first, by reason of its greater distance from the magnet. The current of the second wire will no longer produce the special eflects of the so-called currents of the second kind."

From the last point of view, this experiment pioves absolutely notling, for many reasons: I. Why cliange the ordinary conditions of the clectro-medical instruments of which we have to study the special properties? How is that an instrument of double induction the wires of which are of equal diameter? 2. There is no maker of volta-faradic instruments who does not know that one of the principal conclitions for obtaining great physiological power in the secondary (induced) coil is, that its wire should not only be much finer, but also murh longer than the inductive wire. For whiat reason should it not be the same in a donble-current magneto-faradic apparatus? Let us lieep to the question, which is, to determine the properties of the current of the secondary cuil, developed in a wire that is fine and long, as compared with the properties of the current of the primary coil (extra current) produced in a wire that is thicker and shorter. I liave already proved (see Chap. I., Part III., b, page 30), with regiard to volta-faradic instruments, that the plyysiological properties of the same coil of fine wire differ accordingly as its current, in common language, is an induced or an extra current.

It is hard to understand why it should be otherwise with regard to electro-magnetic instruments; and I therefore read with much surprise the following assertion by AI. A. Becquerel. "If a fixed magnet be surrounded by only a single wire, very fine, and very long, it will yield a current of great tension, which possesses all the properties of the so-called currents of the second kind." This assertion is in direct contrarliction to the facts established by my experimental researehes. 
have ascertained that when, by suppressing the solution of contact of the thick wire, and leaving its circuit interrupted, there remained only the direct influence of the magnet upon the second wire, the physiological effects produced by this influence are reducerl almost to nothing.

To sum up. In my double-current electro-magnetic apparatus, it is the current dereloped in the primary coil, under the simultaneous influence of the temporary modifications of the magnet, and of the interruptions produced in the circuit of the thick wire, which reacts upon the secondary coil.

Although, until quite recently, the above proposition was considered inadmissible by eminent physicists, I do not the less maintain my belief that it rests upon fact and experiments that are incontestable. It has been demonstraterl, in a more scientific manner, and in more technical language, by II. F. P. Le Roux, who, in his thesis presented to the upper school of pharmacy, which I have already had occasion to quote, has maintained that my idea is in great part correct, at least in the case of my magnetofaradic apparatus.

The following is an extract from the thesis, remarkable in many other respects, in which this important demonstration is to be found. (The reader will easily understand this demonstration, if he is acquainter with the theory of magneto-electric instruments which M. Le Roux has laid down in his work, with reference to the apparatus of Pixii, and which I have reprinted on page 287 ) :-

"MI. Duchenne has attributer the particular effects which he has observed in his second coil, to the action of currents, the distribution and intensity of which are different from those of the currents which are induced in the primary coil. Others, on the contrary, have thought that the two coils differ only in the length of their wires. I am induced to believe that what is wanting to the idea of MI. Duchenne is to be supported by precise reasoning, and that it is in the main correct, at least in the case of his magneto-faradic apparatus.

"It may be regarded as proved that, in all magneto-electric instruments of small dimensions, the alternations of magnetization and demagnetization are insufficient to produce currents capable of causing shocks, whatever be the speed of the armatures or the reels, or whatever the length of the wires; while the interruption of the current, near the time of its maximum intensity, causes them energetically. The reason of this is, that the diminution of intensity which takes place at the moment of an interruption is much more rapid than the most rapid demagnetization that can be pro- 
duced by movements of approximation or removal operating mechanically." 8

This understood, when we interrupt the current at the moment of its maximum, it is as if we should interrupt a constant current, and it produces an extra current, the curve of which lias been already given, and which we here will reproduce (fig. 65 ).

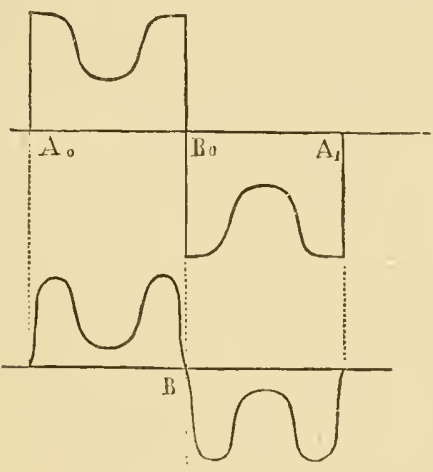

Fig. 65.

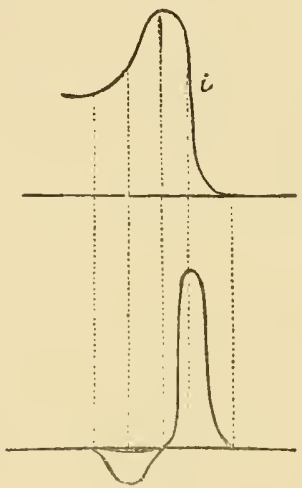

Fig. 66 .

Fig, 65.-Above: approximative representation of the current induced by the movement of the reels of a Clarke's machine; below : a more exact representation.

Fig. 66.-Above: representation of the extra current of interruption; below: representation of the current induced by this extra current.

"It would seem that the arcs, which intercept at the point $B$, should be in excellent condition of form to give rise to intense induced currents. That is true, but it must be remembered that unity of time is here represented by a very short space, so that, if we wish to represent the interval of time $A_{0} B_{0}$ with the same unity that has been employed,-for example, in fig. 65, to represent the cessation of an interrupted current,-it would be necessary to give to $A_{0} B_{0}$ a length several hundred times greater. It is manifest that, when thus extended, the curve of the current of the reel would present only an insensible sinuosity, when compared with that of the current of interruption.

$s$ It may be inquired how it happens that an extra current is not produced at the moment when a Clarke's reel, for example, passes before the pole of a magnet, since at that moment the current suddenly changes its direction, as has been stated above; and it would seem that a change of direction of this kind should be even more efficacious than an interruption. But, in fact, the sudilen chauge represented by the curve is only a fiction; in reality, the change is relatively rapid but not sudden, because we have not mathematical points which pass one before the other; and the relatively considerable dimensions of the reels of the magnets cause the action to diminish from a certain movement, and to increase again. The decrease which really occurs in the vicinity of $\mathrm{B}_{0}$ (fig. 65), however rapid it may be, is still very slow as compared to the return of the natural state in a circuit that is interrupted.

Fig. 65 shows, by its Iower curve, what would be the actual distribution of the intensity of the currents in one of Clarke's reels. 
"We may therefore easily understand that the mechanical variation can only give rise to induced currents that are scarcely appreciable when compared with those that are produced by interruption of a current by solution of the circuit.

"If, on the contrary, we place this extra current in relation with the circuit npon which it can act inductively, it will determine the induced current represented in fig. 65. Its negative part is slightly marked, but it is not the same with its positive part, which may possess a greater intensity, and may present a more sudden variation, than the extra current itself, so that it may also differ from the latter in its effects upon the organism.

"Such a conclusion would be evidently ont of the question, if the circuit of the second coil were only subject to the influence of the first. But would it be the same when the second coil, like the first, is in the presence of a magnet, the magnetization of which is determined by the variations of intensity?

"We commenced by observing that, as the second coil included in its circuit the human body, the resistance of which is very great, the inductions due to variations of the intensity of the magnet could develop but a feeble current; and that, moreover, this current not being subject to interruption by the rheotome, there will be in the wire no sensible extra current, so that I cannot see any condition capable of causing an appreciable disturbance in the effects of the induction prorluced in the second coil by the extra current of the first.

"Such is, in my judgment, the proper manner of regarding the phenomena exhibited by electro-magnetic instruments; the inductive effect of the extra current of the first coil should be the chief cause of that which passes through the second."

\section{B.-The Rheotome.}

In my instrument, by means of a special mechanism, the intermission can be obtained either twice or four times for each revolution of the soft iron, according to the indications. In order properly to appreciate the importance of this arrangement, from a practical point of view, it is necessary to recall the theory of those phenomena which are produced in the common magneto-faradic instruments.

This theory has been stated in the first chapter. It has there been shown:-1, that two intermissions of the rheotome, for each revolution of the soft iron, produce four inductions, of which two, powerful ones, occur at the moment of the intermissions; 2 , that

\footnotetext{
${ }^{9}$ F. P. Le Roux, loc. cit., p. 36.
} 
four intermissions of the rheotome, for each revolution of the soft iron, produce four inductions of equal force; 3 , that the currents which arise from these different inductions proceed alternately in contrary directions.

If, then, we faradize with two intermissions for each revolution of the armature, we should obtain two powerful actions, and the current will travel each time in the same direction. The two other actions, during which the current passes in a contrary direction, are so feeble, that in man they give rise to no appreciable phenomena, unless directed upon the retina, when they produce a phosphene. Hence, by the aid of its regulator, the spring of the rheotome must be so placed as to give two intermissions, when it is desired that the currents should pass always in the same direction.

We may therefore apply my magneto-faradic apparatus, the intermissions of which occur but twice for each revolution of the armature, to the study of the influence exerted by the direction of the currents upon the contractility or the sensibility. ${ }^{1}$ The same study cannot be pursued with four intermissions to each revolution of the armature; because the four inductions act with equal force, and alternately in opposite directions.

The four intermissions per revolution produce a current of double rapidity. Their employment therefore fulfils numerous indieations; as when we wish to act specially upon the sensibility, or to study the muscular functions by the aid of faradization. But although, with four intermissions, the current is twice as rapid as in the instrument of Clarke, which gives only two intermissions per revolution, its rapidity is still insufficient for the study of the muscular functions of the face. The same condition of the rheotome renders the action of the magneto-electric instruments upon the cutaneous sensibility much less powerful than that of the voltaelectric instruments, the intermissions of which may be made by the trembler with a rapidity that is incalculable.

\section{C.-The Regulator of the Intermissions.}

No magneto-faradic apparatus is applicable to all the requirements of therapeutics, unless it possesses a mechanism by which to obtain one, two, or four faradic excitations in the half-second; instead of very numerous intermissions during the same period of time. I have contrived such an arrangement for my magueto-

1 It is well known that the electrophysiological phenomena produced according to the direction of currents traversing longitudinally the nerve-trunks of animals, have been used by Mattencei as the basis of therapentic deduction applicable to man. 
faradic apparatus; and have thus rendered it available in every case. The mechanism is extremely simple, and need only be described, to show its utility and necessity.

The action of the regulator of intermissions, D (fig. 64), should be combined with that of the regulator of the rheotome $B$. The former is so arranged as to produce a very rapid intermittent current, and the second so as to touch the four teeth of the rheotome.

If, on the contrary, the intermissions are to be produced but slowly, the spring of the rheotome should touch only the two teeth of the rheotome $B$. The following is the reason. While the spring touches one of the pins of the large wheel, the contact sometimes lasts a little too long; and it may happen that two or four inductions are produced, in rapid succession, at the moment of contact, according to the position of the rheotome: if the latter touches the four teeth, the four inductions which are then produced excite a more powerful sensation; if, on the contrary, the spring tonches but two teeth, the inductions will not be sufficiently near together to occur at the moment when contact is produced with one of the pins of the large wheel. The slow intermissions, and the sensations which they produce, will then be more regular.

\section{D.-The Graduator of the Currents.}

The graduating tube of my magneto-faradic apparatus measures the electric doses as exactly as in the case of the volta-faradic apparatus. The details which I have already given with regard to the latter, render it unnecessary to enter into the question anew.

This system of graduation is the only one by which we can administer to all organs electric doses, proportionately to their degree of excitability, which, as I have shown, is extremely variable. It may be asked, however, whether the initial force of magnetofaradic instruments does not itself vary so much as to render fruitless the employment of any equal measure? It is established that a magnet, after being subjected to much traction or friction, almost completely loses its power to sustain a given weight. Does this weakening diminish also, in the same proportion, the power of induction? I have related, in the earlier editions, experiments which seemed to answer both questions in the negative.

\section{E.-The Moderator of the Currents.}

In the magneto-faradic instruments, as in the rolta-faradic, the graduating tubes do not entirely neutralize the currents of the coils they cover. It follows that they would be inapplicable to 
very excitable organs, or for very delicate experiments, unless we could employ a moderator of the currents, analogous to that of the volta-faradic instruments.

I have found in the apparatus itself the elements of an excellent morlerator.

(a). The armature and its motor system can be moved away from the magnet over a range of two centinetres and a half, by means of the screw $\mathrm{N}$, called the regulator of the armature, and, at this distance, the influence of the soft iron is considerably diminished. This simple moderator suffices for all operations upon man, and, by combining its action with that of the graduator, permits the administration of extremely feeble doses.

(b). But there are researches or experiments still more delicate, - such, for example, as may be practised upon the frog,-which require the employment of doses that are still smaller, on account of the degree of excitability of the organs of that animal. I have contrived the fullowing arrangement for bringing the electromagnetic apparatus infinitely more under control. By turning from right to left the small screw placed over the base of the spring $\mathrm{S}^{\prime}$ (one of the springs which produce the intermissions of the current of the central coil upon the teeth of the rheotome B), I prevent the current of this coil from passing through the commutator. It will be understood that induction can then only take place by the influence of the soft iron upon the magnet. I do not know whether this experiment has ever been made under the same conditions; but I can say that the induction of the magnetofaradic apparatus, thus deprived of the simultaneous intermissions occurring in the wire of the coil, which immediately covers the magnet, is so feeble that the current of my powerful instrument is only appreciable by placing the rheophores between the lips. If, then, after having placed the soft iron at its greatest distance from the magnet, we suspend, by the above described method, the intermissions which occur through the rheotome $B$, the instrument will produce only an induction infinitely feeble, which may still be divided in degrees smaller and smaller, by stiding the graduator tubes over the coils. It is thus that the same instrument, which is capable of affording currents of great intensity, may be used to test the relative excitability of the muscles of the frog.

\section{§ IV.-The MaNNer OF SETTING THE INSTRUMENT IN ACTION.}

The employment of the magneto-faradic apparatus is, fortunately, as we shall see, a matter of extreme simplicity. Without knowledge of its construction or of its theory, it is enough for the plysician, in order to use it, to understand the brief precepts that 
I am about to lay down. I must therefore ask, above all, for an attentive perusal of the following lines.

In order to put the apparatus in action, the regulator of the armature, $\mathrm{N}$, must be turned from left to right, until the soft iron no longer comes in contact with the magnet during its rotary motion.

If the employment of a rapid current is indicated, the regulator of the intermissions, $\mathrm{D}$, must be turned from right to left, until it reaches its point of arrest. If, on the contrary, it is necessary to produce distant intermissions, the same regulator, $\mathrm{D}$, must be turned in the opposite direction, and stopped when the needle of the indicator points to the number of intermissions which it is desired to obtain for each revolution of the large wheel. The handle, turned from left to right, shonld always be moved very quietly, making perhaps two revolutions in a second.

In order to graduate the eurrents, it is sufficient to remember that when the stem $R$ is pushed within the apparatus the eurrent is at its minimum. The graduation of this stem in millimetres renders it possible to divile the electric doses in fractions, in proportion to the excitability of the organs, and to the requirements of therapeutics.

If the region operated upon be highly excitable, or, in other terms, if it is necessary 10 administer very feeble doses, the armature must be moved farther away from the magnet, by turning the regulator $\mathrm{N}$ from left to right.

If it be desired to experiment upon the frog, the armature is moved away from the magnet as far as possible, and at the same time the current is cut off from the commutator, by turning back, from right to left, the small serew at the base of the spring $S$.

After having thus diminished the power of the apparatus, infinitely small doses can be measured with the same exactitude as powerful ones.

Lastly, the knob $\mathrm{T}$ of the commutator of the coils must be turned from right to left, when we wish to bring the current of the primary coil to the knobs $\mathrm{P}$ and $\mathrm{P}^{\prime}$, to which the conductors of the rheophores are fixed. We turn, on the contrary, the knob $\mathrm{T}$ from left to right, in order to bring to the rheophores the current of the secondary coil.

\section{$\S$ V.-Recapitulation.}

The description of the properties possessed by my magnetofaradic apparatus has shown that its invention constituted a real progress, not only with regard to its applications in medicine, but also from a purely physical aspect. 


\section{A.-In its practical application.}

It has been shown that it possesses-1. Two currents, the one an extra current, the other an induced current, furnished by two superposed coils, each having special properties. 2. Intermissions that are slow or rapid, at the pleasure of the operator. 3. Currents that are either alternate or in the same direction. 4. An exact and truly medical graduation, which measures the largest and the smallest doses with equal exactness, thanks to a combination of graduator tubes with the mechanism which I have called the moderator of the currents.

\section{B.-Under a pluysical aspect.}

(a). My apparatus shows that the metallic cylinders are induced by the coils as much in a magneto-faradic as in a voltafaradic instrument, which has been disputed by the celebrated physicist of Vienna, Herr Dove; and, further, that the reciprocal influence of the coils and the cylinders is only exerted in the parts of the coils that are covered by the cylinders, whence it follows that the action of the latter occurs in a progressive manner, and in arithmetical proportion.

When, from lapse of time and by employment, the magnet becomes weakened, its power may be restored without dismounting the apparatus. The turns around the arms of the magnet of the thick wire, which supplies the current of the primary coil, enable us to restore to the magnet its original power, by passing the current of a voltaic battery through the spirals formed by the wire. The proceeding is thus accomplished:-the armature is placed in contact with the magnet, and then the current from a battery of eight or ten of Bunsen's elements is made to pass through the primary coil, by placing the positive pole upon that one of the springs of the intermissions which corresponds to the north pole of the magnet, while the negative pole is brought into contact with the spring to the opposite side. While the voltaic current passes through the primary coil, the magnet gains considerably in power (it will support a weight of forty kilogrammes), and, when the current is interrupted, although the magnet loses a great part of the force which it received from the voltaic current, it yet preserves the maximum of magnetic intensity which it received at the hands of the manufacturer. If the experiment be repeated, with the poles of the battery current counterchanged, the magnet is almost entirely demagnetised, but can be immediately restored to its original power by acting as on the first occasion. I have often repeated these experiments, and can at any time magnetize or demagnetize my apparatus. 
(b). Thanks to the combination of two superposed coils, formed of wires of different dimensions, we have not only the advantage of obtaining currents of different kinds, with special physiological properties, but we are also able to exhibit the phenomenon of decomposition of water by the current of the primary coil, the wire of which is of sufficient size to produce this chemical action, without requiring to change the reels, as in the case of Clarke's apparatus. I should add that the commutator of the coils, which enables us to pass rapidly from one current to the other, without displacing the conductors, renders this experiment more easy.

(c). Lastly, my apparatus serves to show the great difference which exists between the induction produced in the magnetofaradic instruments, by the sole influence of the armature on the magnet, and that which is the result of solutions of continuity of the circuit of the primary coil, at the moment when the armature leaves or approaches the magnet. The mechanism which permits these experiments is rery simple, since it is sufficient to turn in opposite directions the screw of the rheotome B (fig. 64), placed at the base of one of the springs, in order to transmit or to arrest the current, the solutions of which are made between the teeth of the ring placed upon the small reel.

These results form, I repeat, an incontestable progress. And yet, how is it that the apparatus has been disregarded, to say no more, by the physicists whose electro-physiological theories, which to me seem erroneous, I have so often had to dispute? It is because these physicists, ignorant of the requirements of those physiological, pathological, and therapentical researches for which the instrument is adapted, are unable to form an idea of the utility of the properties that it possesses; it is, that they have not even seen the instrument in action, and that they only know it by a description which they have read withont being able to understand. A great number of physicists have examined it, and I have found that once showing it to them has enabled them perfectly to understand its mechanism, which they have afterwards explained in their lectures or in their works.2 I can affirm, indeed,

2 I may mention, anong others, MM. De la Rive and Jamin, before whom I have exhibited electro-physiological experiments which show the different physiological action of the coils of my magneto-electric apparatus. I may also quote the opinion of M. Le Ronx upon its merits :-

"This apparatus is unquestionably more complete than any other of the kind. The weans which it offers of graduating and varying its effects are numerous; and some of them may even appear superfluous; but it would not be wise to pronounce, withont much eireumspection, upon the identity of the intimate cconomy of complicated phenomena which we see only in the aggregate. Morenver, it is necessary to recognise that the labours of this author have not been without intluence in perfecting the small instruments which are now at the disposal of medical practice, and of which there are numbers presenting almost equal advantages." 
that it is not more difficult to understand than other forms of apparatus.

\section{Part the Fifth.}

\section{HISTORICAL AND CRITICAL ACCOUNT OF INDUCTION INSTRUMENTS.}

In order better to appreciate the value of my induction instruments, the description and properties of which have been set forth in the preceding Parts, and to judge of the progress of the art of fabricating electro-medical instruments, and also in order that the physician may exercise an enlightened choice among these instruments, I procecd, in the following pages, to pass in review the principal forms of induction apparatus, from their origin to the present day. I shall reprint the figures and description of two learned physicists, and especially of one of them, who, in a work I have had occasion to quote, ${ }^{3}$ has profoundly studied electromedical instruments. In the second place, I shall enter upon certain critical consilerations, that $I$ have already in great part set forth in 1855, upon the electro-medical instruments then in use, and I shall point out their improvements, as well as the desiderata which still remain to be attained.

\section{§ I.-Historical Review.}

\section{1.-Magneto-electric instruments.}

A._Faraday's instrument._-"The first magneto-electric machine," says M. de la Rive, was constructed by Faraday (fig. 67). It consists of a copper disc, $c$, movable in a vertical plane upon a horizontal axis, and which is made to turn between the two opposite poles of a magnet, $n$. We have seen that if we connect the two ends of the galvanometer $g$, the one, $w$, with the axis of the disc, the other, $w^{\prime}$,

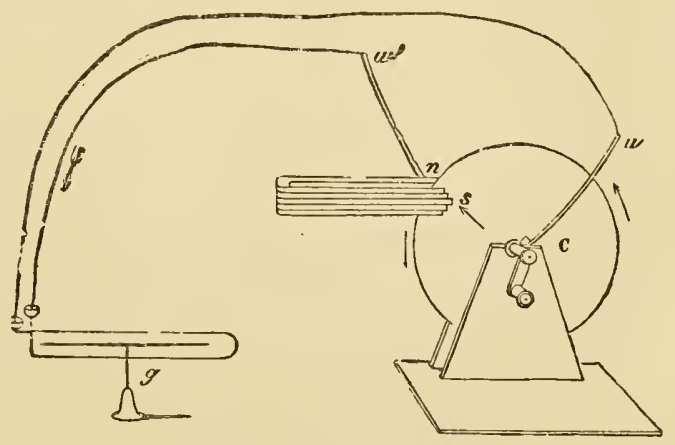

Fig. 67.-Faraday's instrument.

with a point on its circumference, the deviation of the needle which takes place in one or the other direction, according to 
the direction of the rotation, indicates the liberation of a constant current in the disc. But this current is of very small intensity; it is incapalle of producing chemical decompositions, contractions, \&c. It is the same with the induction currents, which are occasioned by the terrestrial magnetism. ${ }^{4}$

B.-Instrument of Pixii.- " To obtain induced currents of somewhat marked intensity, it is necessary that they should be

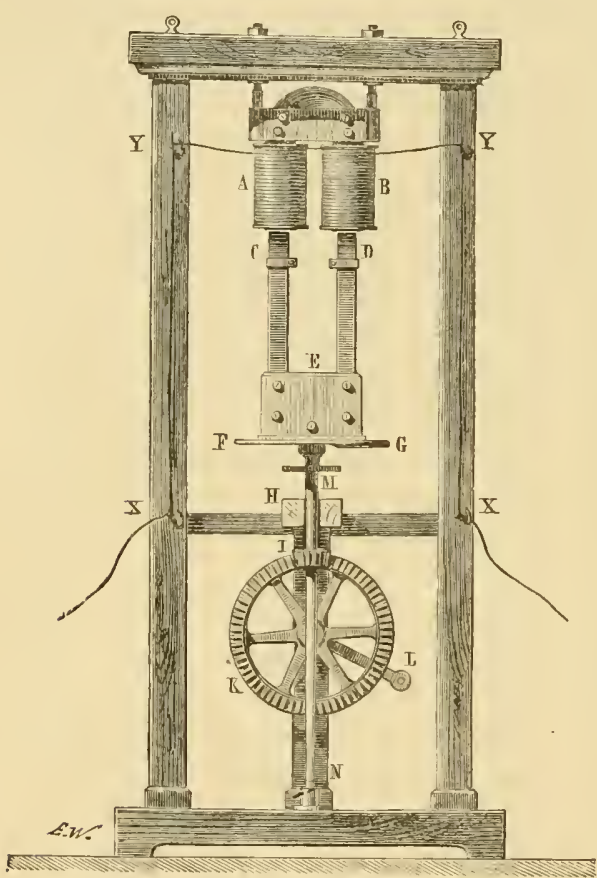

Fig. 63.-Apparatus of Pixii. developed in wires of some length, in such a way that the conductor which reunites the ends of the wire should be moderately good, or at least not much inferior to the wire itself, so that the current may traverse it, instead of passing back again by the same wire in which the induction took place. II. Pixii was the first to construct an instrument on this principle." 5

"The instrument of Pixii is shown in fig. 68 . $A B$ is a bar of soft iron, curved in to the form of a horseshoe, and round it is rolled an insulated copper wire, the extremities of which are

shown at $\mathrm{X}, \mathrm{Y}$; this electro-magnet is fixed. Opposite to it is placed a permanent magnet, the poles of which are seen at $\mathrm{C}$ and $\mathrm{D}$. This magnet is supported by a vertical axis, $\mathrm{M} \mathrm{N}$, on which is mounted a pinion, I, to which a wheel, K, communicates movement; a handle, $\mathrm{L}$, serves to put the instrument in motion.

"As will be seen, the poles $\mathrm{C}$ and $\mathrm{D}$ of the permanent magnet alternately approach and recede from the extremities of the electro-magnet; there is, then, induced, in the wire covering the latter, a current which changes its direction with each half turn. In the original instrument of Pixii a sort of cam was placed at G,

4 De la Rive, Traité d'elestricité théorique et appliquée, 1854, tom. i., p. 378.

5 Ibid., loc. cit., p. 79. 
in order to work a commutator, which, on account of its complexity, has not been represented in the figure. It is enough to say that this part of the apparatus had for its office to conduct the induced current always in the same direction. Clarke's instrument will presently furnish us with an example of a contrivance which is more simple, and better adapted to its purposes.

"We can produce, by the aid of Pixii's instrument, all the ordinary effects of induced currents,-heat, light, chemical decompositions, \&c.

"In order to explain the effect of the arrangement which has been described, let A and B (fig. 69) be the projections of the reels, and $\mathrm{N}$ and $\mathrm{S}$ the poles of the magnet, on the plane described by them in their movement of rotation, which is supposed to occur in the direction indicated by the arrows.

"The pole $\mathrm{N}$ retires from the wheel $A$; the pole $S$ approaches it; as the two poles are of contrary names, their actions upon the reel A concur.

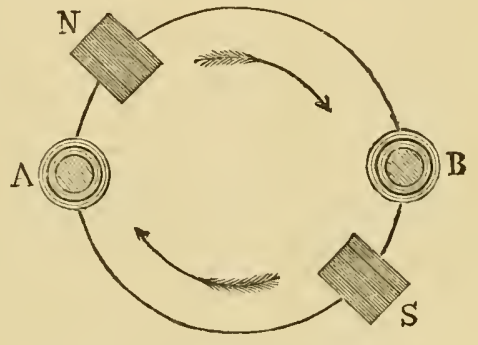

Fig. 69.-Theory of Pixii's arrangement.

": They concur also upon the reel $\mathrm{B}$, but the current originating in this reel is the opposite of that which originates in the reel A; and for these currents to combine it is only necessary to connect, in a convenient manner, the wires which are rolled round the reels; and it is sufficient for this, that, if we suppose the horseshoe-shaped iron to be set upright, the wire should be wound in the same direction rom both its branches.

"The inductive action is at its minimum when the poles are equi-distant from the reels, but it does not cease. At the moment when the poles are very near the reels, the action is much greater, and it attains its maximum when the poles are opposite the reels; but at this moment the induction changes its direction, because the movement which approximated each of the poles to the reel before which it passed, then removes it."

After having described the apparatus of Pixii, M. Le Roux has stated the following theory of magneto-electric instruments, which, by reason of its novelty and importance, merits to be here reproduced:-

"The curve of the intensities of the current may be exhibited as in fig. 70 , where the distance $A_{0}$ and $B_{0}$ represents the time in which each pole passes from the reel $A$ to the reel $B$, and so onl.

"In reality, the passage from the positive arc, $a m b$, to the 


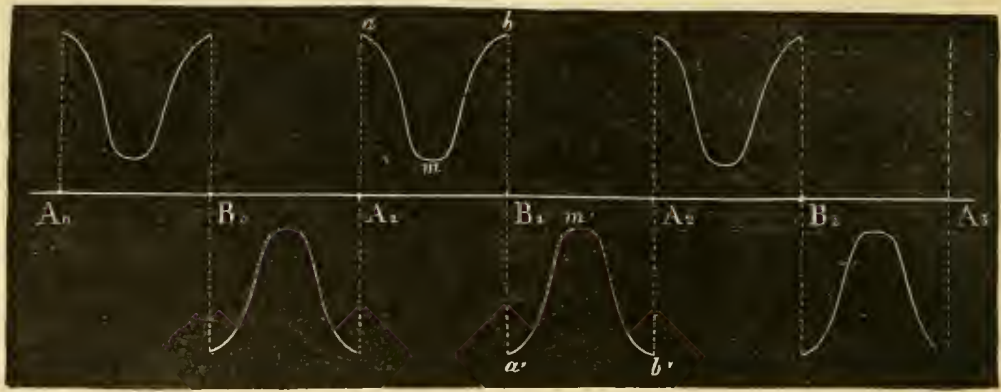

Fig. 70.-Theory of the arrangement.

negative arc, $a^{\prime} m^{\prime} b^{\prime}$, does not occur so suddenly as is here indicated, because the extremities of the reels and of the magnets are not mathematical points, but possess considerible transverse dimensions. We shall return hereafter to this question.

"We may say at once, although it is foreign to the subject of Pixii's apparatus, that, if we cause the current engendered to act upon a second cirenit, we obtain an induced current, which will be an induced current of the second kind, caused by magnetic induction, and the distribution of which is shown in fig. 71 .

"In this figure the upper curve is an ideal representation of the intensity of the current consequent upon the variations of distance between the reels and

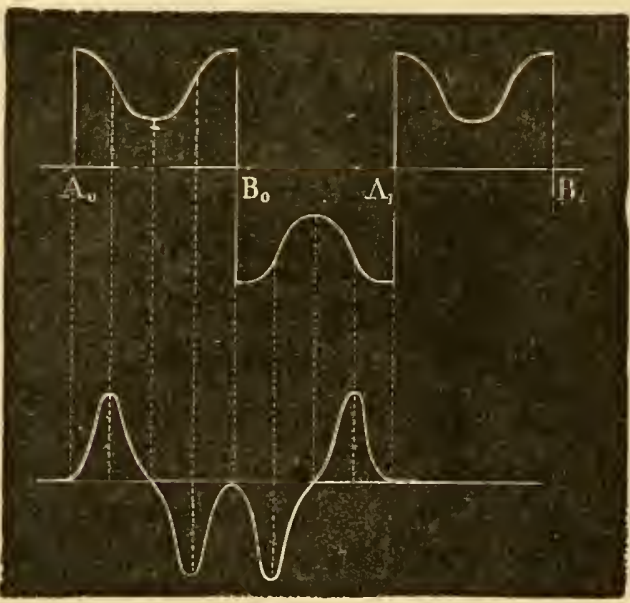

Fig. 71.-Theory of the arrangement. ${ }^{6}$ the magnets, and is such as would be obtained by taking nothing but these variations into account; it presents a perfectly symmetrical outline. But this variable current, being able to indnce a second cireuit, will induce its own in such a manner that we shall have very nearly the truc curve of the intensities of the current of the reels by

B The npper trace shows the current | The lower trace, the induced current, induced in the reels of Pixii's instrument. $\mid$ caused by a second circuit. 
adding algebraically the ordinates of the superior curve to those of the second, reduced to a certain ratio. The final result would be to destroy the symmetry, and to displace the minima, as if there were some impediment.

C.-Apparatus of Saxton.- " IVe shall readily perceive that it was not desirable, and that it was perhaps even objectionable, to have so massive an electro-magnet as that of Pixii; while, at the same time, it was desirable to increase, as much as possible, the power of the permanent magnet. It therefore became more convenient to fix the latter, and to render the former movable. 'This was done by Saxton, who made Pixii's instrument approach nearer to portability by placing a fixed magnet horizontally (see fig. 72), and by causing the electromagnet, or induced reel, to turn upon an axis parallel to the plane of the magnet.

"It is therefore still the principle of Pixii that we find in this instrument; which, although presenting an arrangement less

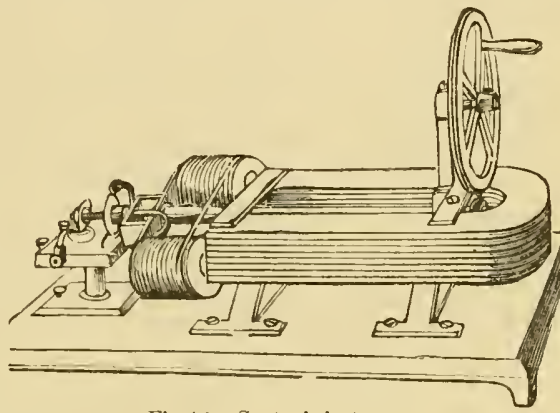

Fig. 72.-Saxton's instrument. desirable, in a theoretical point of view, has none the less prepared the way for the employment of magneto-electric instruments in medicine." 7

In this instrument; the handle causes the rotation upon its axis of the armature of soft iron which carries the reels.

D.-Clarke's apparatus. - There was yet a means of rendering. the instrument still less cumbrous, without occasioning any notable diminution in its power: this was, to render the magnet $A$ rertical, and to cause the electro-magnet to more round a horizontal axis $\mathrm{X} \mathrm{X}^{\prime}$ (fig. 73) in such a manner that the extremities of the electro-magnet revolved in a plane parallel to the branches of the permanent magnet.

"Such an arrangement was realized by Clarke, and is shown in figure 74 . A represents the permanent magnet; and $H$ is the axis on which the electro-

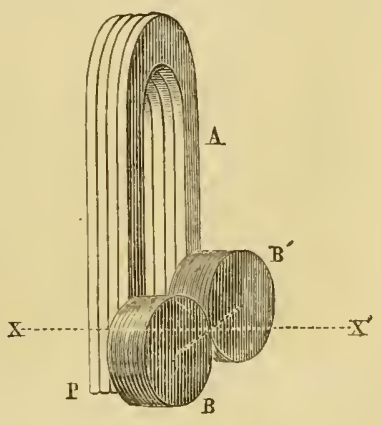

Fig. 73.- Clarke's instr?mur 
magnet turns. This axis carries a pinion, over which passes a Vancanson's chain, carried by the large wheel $\mathrm{E}$, which is itself turned by a handle.

"The electro-magnet is no longer, as in Pixii's instrument, a single bar of soft iron curred into horse-shoe shape; but,

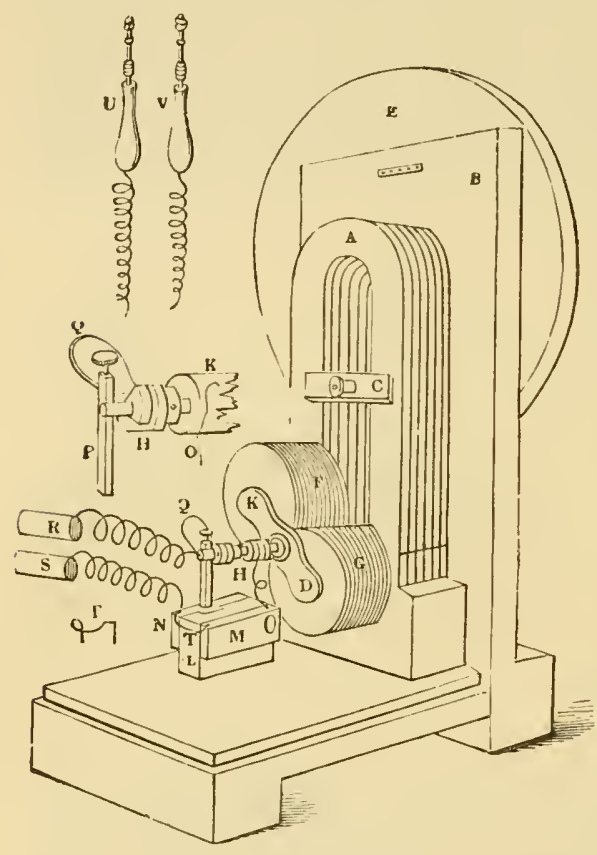

Fig. 74.-Clarke's instrument. consists of two distinct reels, $\mathrm{G}$ and $\mathrm{F}$, the softiron centres of which are mited, on one side, by a traverse of the same material, and on the other sicle by a traverse of a material that is not magnetic. 'This arrangement is equivalent to an electromagnet formed in a single piece, but it is more easy of construction, and less cumbrous.

"The analysis of the currents produced by this last apparatus is the same as for that of Pixii's; the currents changing their direction at each halfrevolution of the reels. When it is desired to have a current al ways in the same direction, it is necessary to interpose, between the reel and the place where the electricity is to be used, a contrivance which has received the name of the Redresser of the currents or the Commutator.

"The following is a description of this contrivance, in Clarke's instrument:-A collar, $\mathrm{H}$, formed of non-conducting material, is mounted on one of the extremities of the axis on which the reels turn; and two metallic half-cylinders, separated from each other by two small intervals at diametrically opposite points, are placed upon the collar. Each of them is permanently connected with one of the ends of the wire of the reels; and two springs are constantly applied over the half-cylinders. By the rotation of the axis, each of the half-cylinders comes successively into contact with each of the two springs.

"When we wish to display physiological effects, we can employ he current without the commutator; but the effects will then be 
of slight intensity. The power of the shock may be increased by utilizing a final extra current that may be produced by the following contrivance.

"The metallic plates $\mathrm{M}$ and $\mathrm{N}$, communicate with the conductors $R$ and $S$, which are represented in fig. 74 , and the circuit being completed by any part of the human body, if we then make a communication by a metallic are between the two half-cylinders, the current will take, by preference, this direction, in which the resistance is extremely weak, rather than pass through any part of the body, so that the latter will receive scarcely anything; and, moreover, the current of the reels will have its maximum of intensity for the given speed of rotation of the instrument, since the external resistance is almost nil. If we then interrupt the metallic communication between the two half-cylinders, the intensity of the current will be reduced almost to zero by the great resistance of the human body, and it will induce in the reel a current of interruption, of which the intensity is very great, as its electro-motor force is considerable; and which, during its short duration, will possess a new physiological quality.

"This extra current will be stronger, in proportion as the current, by the interruption of which it was engendered, was itself more powerful; and it ought, therefore, to be produced at the moment of coincidence of the poles with the reels." 8

E.-Page's instrument.- "When we approximate, or remove, a piece of soft iron to or from the polar extremities of the permanent magnet, we produce in its branches variations of magnetism which may be used for the induction of a circuit. This lias been done by the American physician, who has given his name to the iustrument next to be described.

"Page has surrounded the extremities of the branches of a permanent magnet with coils of fine and long wire; and by means of a screw, worked by the handle D (fig. 75), this magnet can be moved nearer to, or farther from, a bar of soft iron, E F, which is mounted perpendicularly upon an axis, carrying a pinion to which rapid movement can be communicated by a large toothed wheel. 'The movement of the magnet is a meaus of graduating the intensity of the currents that are produced. These currents are connected, as in Clarke's machine, by a commutator mounted on the same axis as the soft iron; to this is added an interruptor, $K$, of the same kind as has already been described, and which is arranged for the production of an extra current, required for physiological action.

"In the instrument shown in fig. 75, the position of the commu-

${ }^{8}$ Le Roux, p. 30. 


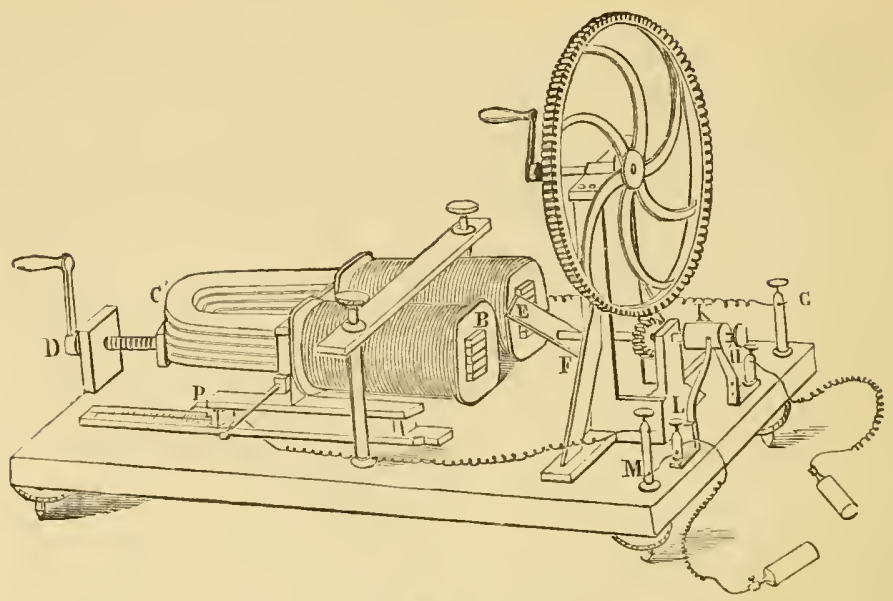

Fig. 75.-Page's instrument.

tator may be regulated relatively to that of the soft iron, in such a manner as to obtain the maximum of effect, and to exhibit many circumstances connected with the production of induced currents.

"It should also be remarked that, in this instrument, a complete revolution of the soft iron produces four induced currents, changing their directions alternately; while only two are produced by instruments of the type of that of Pixii.

F.-Instrument of Dujardin.- "Construeted on the same principles as the foregoing.

G.-Instrument of $M M$. Breton, frères.- "Its principle is also the same as that of Page's instrument; but it was one of the first

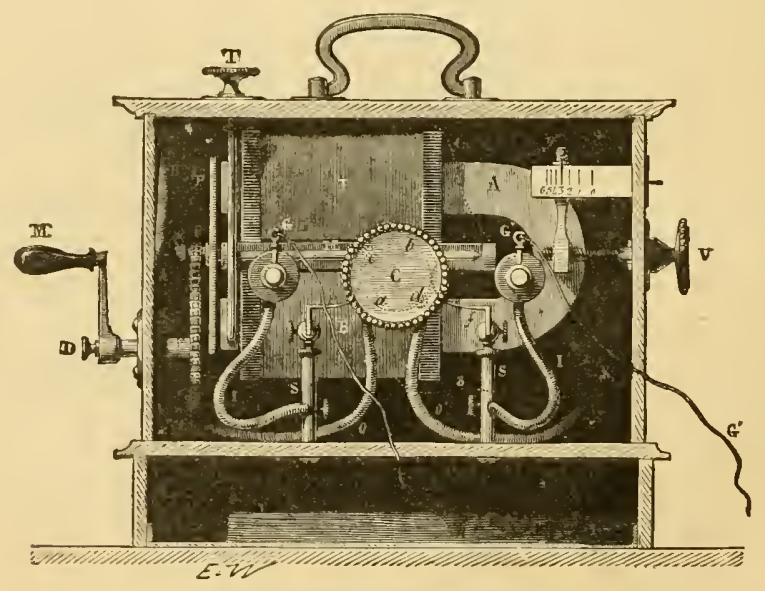

Fig 76.-Iustrument of Breton, frères. 
made easily portable, and adapted for use in practical medicine (fig. 76 ).

"The means of graduation are the same as in Page's instrument.

" MIM. Breton, following the example of MI. Duchenne, wound two coils of wire round the branches of their permanent magnet; ${ }^{9}$ the external is formed by a finer and longer wire than the internal, and they can, at pleasure, utilize the induced currents that are developerl in one or other of these cuils, in the effects of which they can discover certain differences. We shall examine this question more fully, with reference to the instrument of MI. Duchenne." I

H.-Gaiffe's instrument.- "This is at once the instrument of Saxton and of Page; or it may be described as Page's instrument,

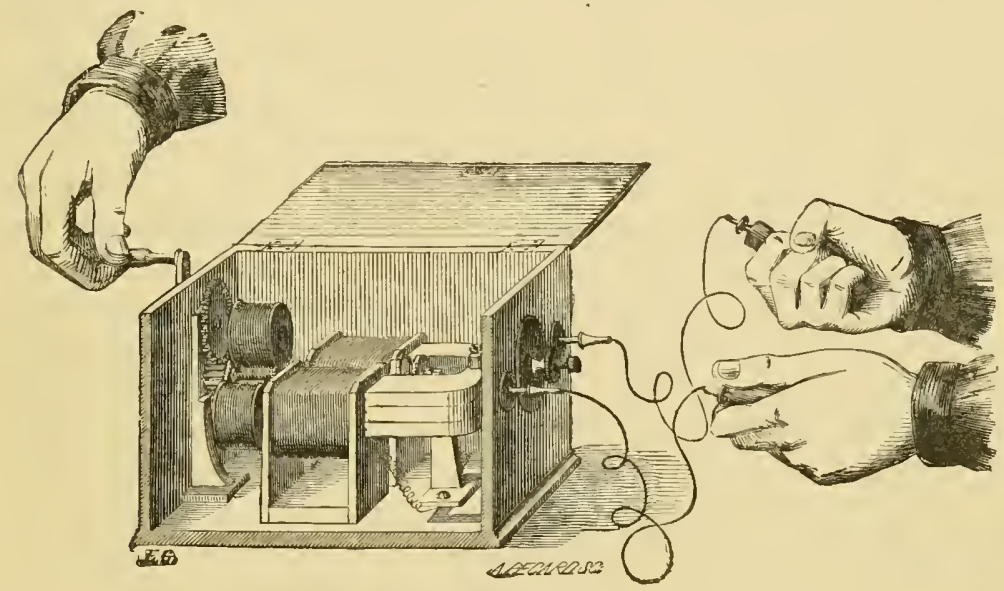

Fig. 77,-Gaiffe's instrument.

in which the bar of soft iron has been replaced by Clarke's electromagnet (fig. 77).

"The chief merit of the maker of this instrument has been to understand that, as magnets have a force relatively greater in proportion as their weight is less, while the induced forces rapidly increase as the distances diminish, there was every advantage in reducing the size of the apparatus, when tension, rather thait quantity, is desired.

"Thus, the magnet of the instrument, represented above, weighs only half a kilograinme, and will support about five kilogrammes.

9 Le Roux, p. 31.

1 'This arragement of the magnetoelectric apparatus of the MII. Breton is quite rocent. Their original instrument had only one coil ; and it is smaller and weaker than the present one, The former is that which is generally to be found in the Paris lospitals, although the latter is much to be preferred on accomt of the recent improvement. 
The coils of the armature are formed by a wire one-tenth of a millimetre in diameter, and about $\mathbf{1 7 0}$ metres in length; those of the magnet are formed by 280 metres of the same wire.

"M. Gaiffe considers that the coils of the armature furnish about two-thirds of the effect, and those of the magnet one-third only.

"The currents of the armature change their direction twice during one revolution of the axis on which they are carried, while those of the coils rolled round the magnet cliange four times,- - the maxima of the two first being considered as coinciding with two of the second; and it may be said that, by an arrangement of the wire, the directions of the currents are the same. As the currents change their direction in the reels of the magnet before they change in those of the armature, their effects are at one moment in part destructive of each other, unless controlled by a commutator arranged to correct them singly in proper time; but this addition would be an extreme and perfectly useless complication, because the currents that are produced are utilized by an interrupter of the kind that has been described with reference to the machine of Clarke.

"This interrupter acts only at the noment when the currents of the armature are at their maximum; becanse that is the time at which is established the additional action of the two currents.

"The apparatus also possesses a redresser of the current analogous to that of Clarke, but with no other office than to give the same direction to two discharges which are produced at each revolution. This direction is indicated by marks on the binding-screws which serve to give attachment to the conductors; and the furnishing of such marks is a care that cannot be too strongly recommended to manufacturers. The direction of the discharges is not a matter of indifference, and manufacturers should undertake to furnish to practitioners every possible facility for determining it." ${ }^{2}$

\section{2.-Electro-Dynamic Instrunents.}

A.-Ruhmkorff"s Induction Coil.- "In the cases, although they are somewhat rare, in which sparks of high tension are required, there is no better source of them than the instrument which has gained for M. Ruhmkorff a well-merited celebrity. WV give (fig. 78) an illustration of a small model, which produces sparks eight or ten mivingetres in length, and which can be borne when they do not succiad one another too quickly. These coils of relatively strong tensiou may repiace the electricity of the ordinary friction

2 Le Roux, lnc. eit., p. 31. 
machines, especially when they are furnished with an interrupter for giving isolated discharges at the pleasure of the operator.

(a). Ruhmkorff's hospital apparatus. - " MI. Ruhmkorff has recently arranged a portable apparatus, designed especially for hospital use, and in which are combined the conditions most essential to secure the regular employment of induction currents.

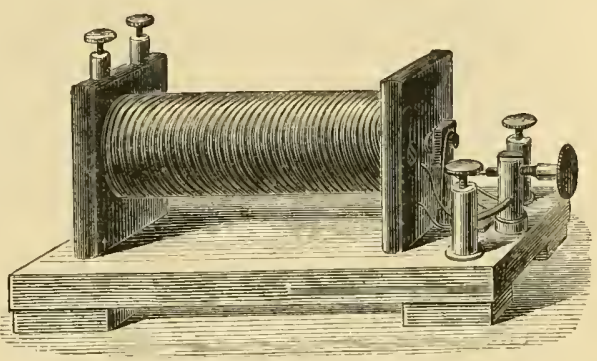

Fig. 78.-Puhmkorff's induction reel.

"This apparatus (fig. 79) contains two elements of zinc and carbon with bisulphate of mercury; the zinc can be more or less deeply immersed in the liquid, as a means of graduating the inductive effect. One of these elements suffices for ordinary cases; the second is especially as a reserve. The mode of graduation is by the greater or less insertion of one coil within the other, by which the effect can be reduced, if needful, almost to zero. The trembler is that of Neef; and the frequency of its inter-

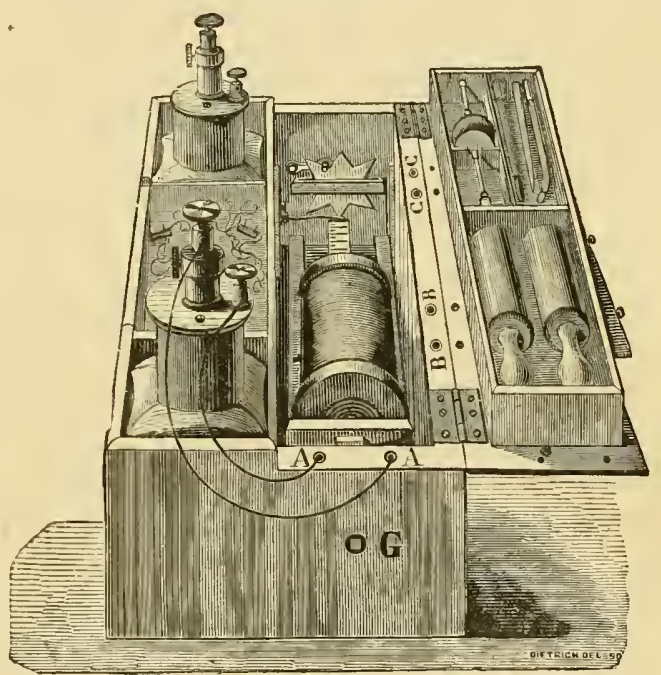

Fig. 79.-Ruhmkorff's apparatus. ruptions can be regulated by means of a screw. A star, moved by a small handle, enables the operator to produce intermittent effects.

"This instrument gives either the extra current, or the induced current of the first lind."

(b). Ruhmliorff"s portable apparatus. - "The same manufacturer has also long made a portable apparatus specially intended for private practice, and which is of much smaller bulk than the foregoing.

It contains two elements of zine and carbon with bisulphate of 


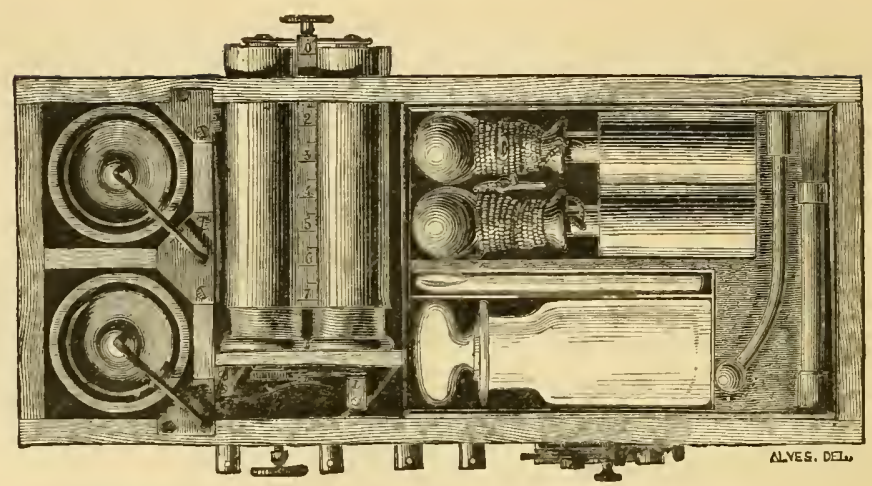

Fig. 80.-Ruhmkurff's portab:e apparatus.

mercury; and the elements are extremely simple. The carbon is obtained from the inside of gas retorts, and is in the form of little cups, in which are placed thin plates of zinc. The monnting of this battery is very easy; and its action contiuues sufficiently long for most applications. It must, it is true, be mounted and dismantled at each time of using.

"There are two coils, which act precisely in the same manner, and are, indeed, one coil diviled into two. The graduator is a copper covering external to the coils.

"The interrupter is.the trembler of Neef; and a star outside the box permits the production of intermissions.

"The apparatus gives, at pleasure, the extra current or the induced current.

"It is represented in fig. 80 at one-third of its natural size." 3

(c). Dr. A. Tripier's apparatus.-This is a non-portable apparatus with a detached battery. I have reproduced the author's illustration, and part of his description. ${ }^{4}$

"In this figure (fig. 81 ) the induction circuit is that of the reel of fine wire, B; the commutator $\mathrm{C}$, and the hanlle $\mathrm{D}$ are so arranged as to convey to it the battery current, the electrodes of which terminate at $a$ and $a^{\prime}$. The rheophores attached to the polar extremities of the circuit $B$ receive, by two han lles, the extra currents of high tension that are leveloped in the circuit. The rheophores attached to the reel $B^{\prime}$ proceed each to one of the branches of a forceps for experimental purposes, and afford induced currents of low tension.

"The endeavour of M. Tripier is certainly praiseworthy, and it

3 Le Roux, loc. cit., p. 50.

4 Tripier, Manuel d'électro-thérapie, l'aris, 1861' (which see, for the different parts of his apparatus). 
is a matter of great interest to be able to compare the effects produced inder circumstances that are very different, and moreover well defined. But it was, perhaps, not wise to seek the means of this comparative study in a complex apparatus, in which the building up of different parts above one another might create a diversity of conditions diffcult to estimate,-such as differences of distance, of intensity, \&c. In the performance of chamber experiments it is much more simple, more exact, and also more economical, to have a number of reels in which a single condition varies from one to another, and all supplied by a single interrupter in which the motor is independent of the active current, as arranged in some models of the interrupter of Foucalt."

(d). The apparatus of Du Bois Reymond, or. of Siemens and Halske. - "The battery current is brought to the

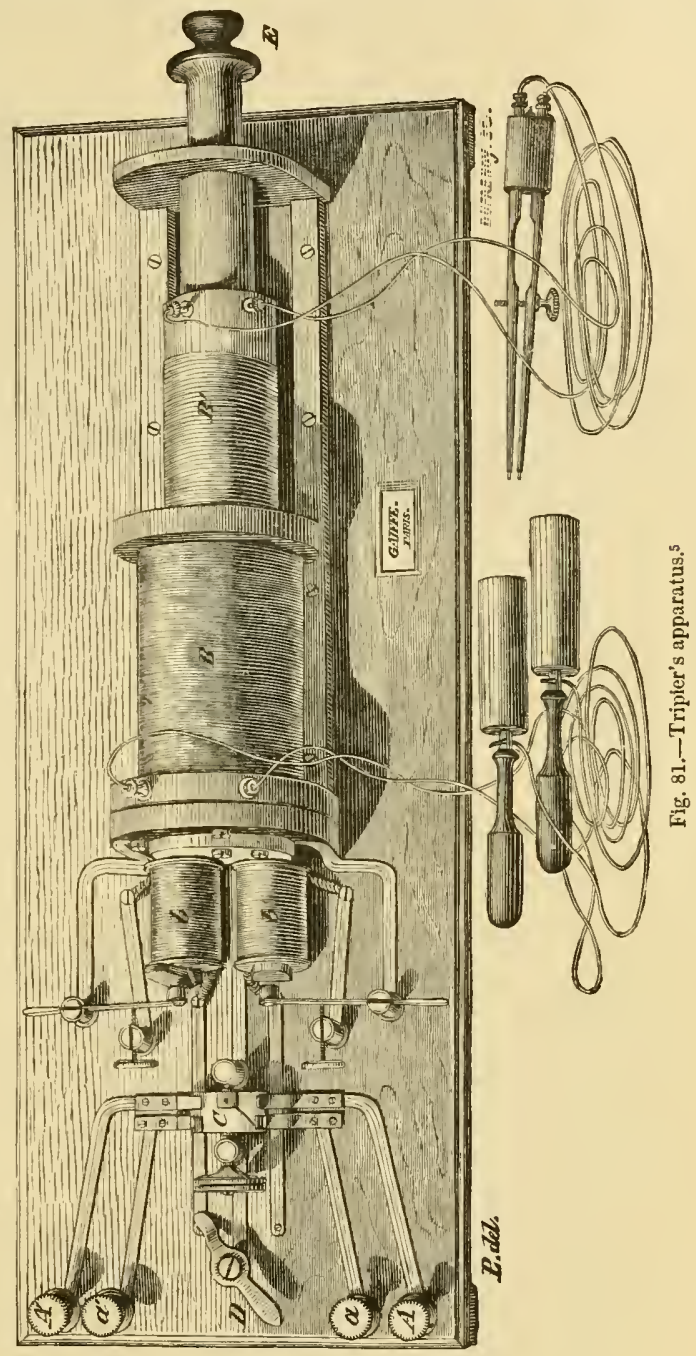

5 A, A', binding-screws, to receive the electrodes of one of the batteries: $a, a^{\prime}$, binding-screws to receive the electrodes of the other battery. B, reel on which is coiled the fine wire; $b$, small reel of fine wire containing within it an electromagnet, which regulates the circuit of the preceding. B', reel on which is coiled the thick wire; $b^{\prime}$, a small reel of thick wire, containing an electro-magnet, which regulates the interruptions of the circuit of the reel $B^{\prime}$, when this is traversed by the battery-current. $\mathbf{E}$, head of the stem which continues the central electromagnet, and forms a handle for it. C, commutator. D, handle, placing the apparatus in communication with one or other of the batteries. 
two binding-screws $\mathrm{A} \mathrm{A}^{\prime}$ (fig. 82), and traverses the electro-magnet $\mathrm{D}$, and the inductive coil $\mathrm{B}$; the pieces $\mathrm{E}, v, a$, constitute a Neef's trembler. The instrument furnishes an extra current. ${ }^{6}$

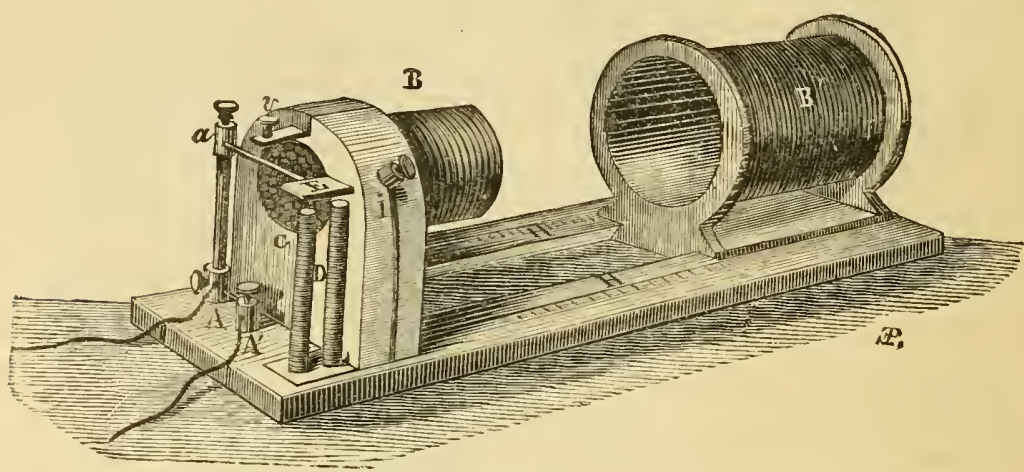

Fig. 82.-Apparatus of Du Bois-Reymond.

" $A$ second reel B', wound with a fine wire, may either cover the first completely or be removed to a distance from it. This is the seat of the induction.

"The inductive coil contains wires of soft iron, the number of which may be varied so as to graduate the effects in another manner.

"The apparatus is very simple, and very well contrived with regard to experimentation, and for all that regards the graduation of the induced currents." 7

(e). Morin's apparatus. - "This instrument was one of the first to fulfil the conditions of convenience required for the carrying out of electro-therapentics in private practice; and among the most useful of MI. Mlorin's arrangements may be reckoned the close combination of the battery and the induction coil. The combination was the more difficult, as it was accomplished under the condition of employing a Bunsen's battery, only slightly modified.

"Fig. 83 gives a general view of the apparatus. The battery and its accessories are placed in the division to the right. $\mathrm{A}$ is a planchette, to which is fixed the induction apparatus properly so called; and at $A^{\prime}$ the same system is shown uncovered.

Fig. 84 exhibits a section of the coil. D is a tube of tin, which can be withdrawn at pleasure, together with rarious rods of iron wire; $G$ is a movable copper cover, which acts as graduator; then

${ }^{6}$ Le Roux, loc.cit., p. 53. The original influence of the coils, of which the outer instruments of my own, presented in 1818 one was movable. This disposition has to the Academy, had a novable arrange- been imitated by MI. Du Bois-Reymond; ment of the coils, the thick wire being I shall presently explain for what reason I intermal and fixed; and were graduated in the same mamner by the reciprocal myself abandoned it.

7 Ib., p. 54 . 


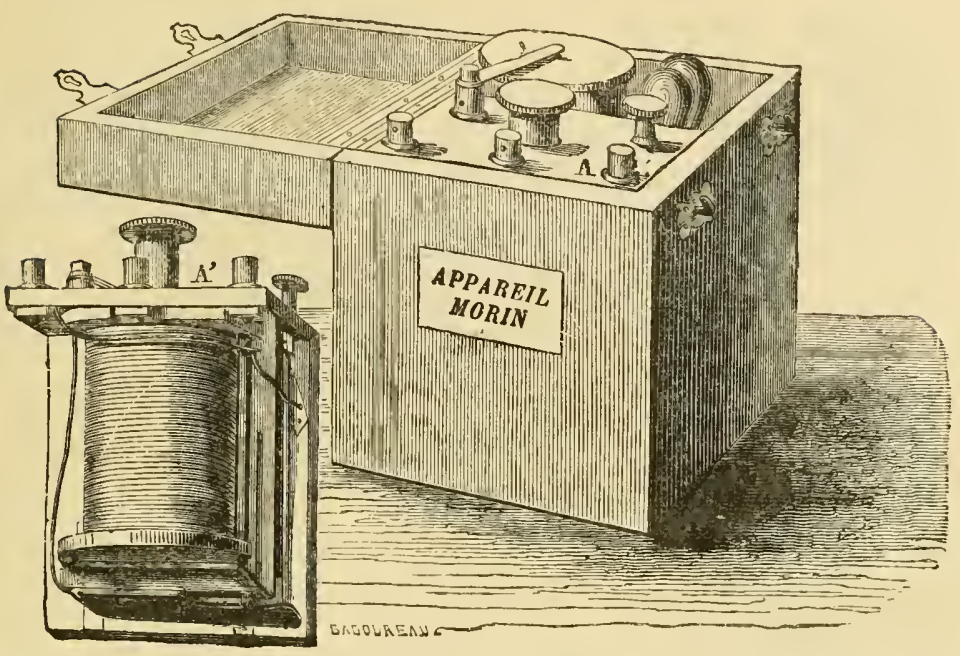

Fig. 83.-Morin's apparatus.

comes the fine wire that is the seat of the induction; and the thick or inductive wire is placed external to it. At $e$ is a small piece of iron screwed into the wood of the reel; it becomes magnetic under the influence of the current, and attracts the contactmaker $d$. This is formed by a plate of soft iron jointed at its extremity to another plate of the same metal, $a$, placed at the side of the coil in such a manner as to be magnetized by the inductive current and to increase the attraction. It has no antagonistic spring, -its weight rendering one unnecessary. The contactmaker $d$ carries a small plate of platinum, which strikes upon another plate of the same metal, the distance of which is regulated by means of the stem terminating in the knob $h$. This knob, after it has been turned a certain number of

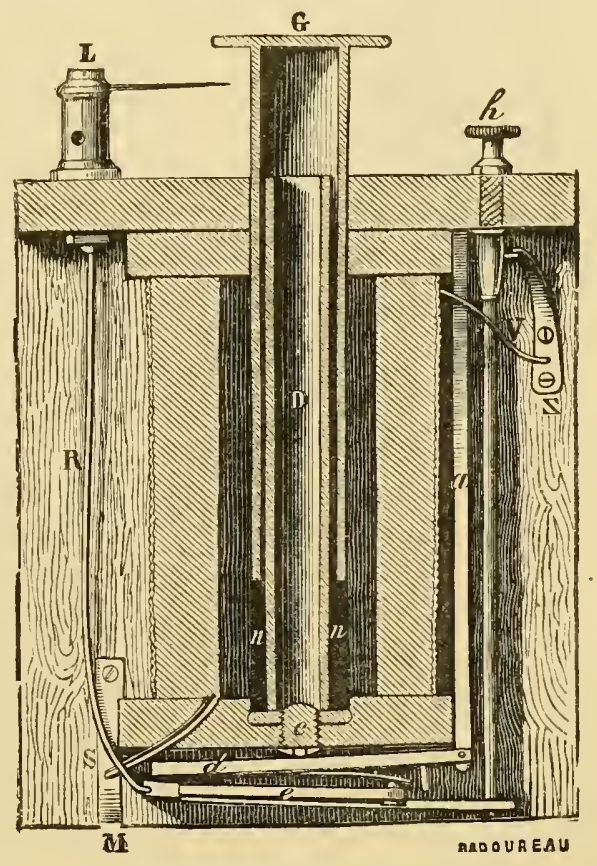

Fig. 84.-Apparatus of Legendre and Morin. 
times, serves to produce more or less rapid intermissions, at the pleasure of the operator.

"At $\mathrm{L}$ is a movable and flexible lever, which serves to establish communication between the coil and the battery. For this purpose it carries a stem which proceeds to the platinum, I, that is fixed in the carbon of the battery.

"'The battery (fig. 85) is composed of a copper cyliuder, A, lined by thick zine, $\dot{\mathrm{E}}$, and within this a porous vase, $\mathrm{C}$, containing a bar

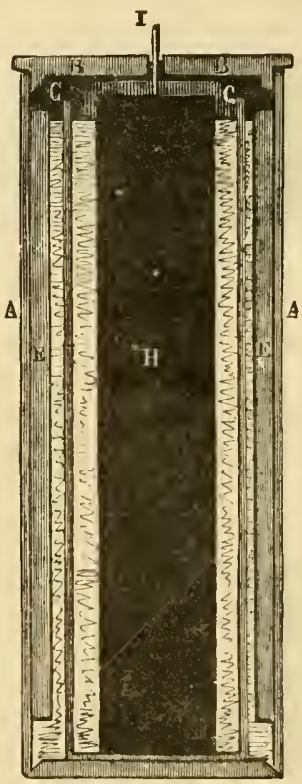

Fig. 85.-Battery of Legendre and ilorin's instrument. of gas carbon, H. Within the porons vase is put nitric acid or bichromate of potash, and pure water ontside it. A lid of gutta percha, B, closes the whole system almost hermetically.

This battery presents the inconveniences inseparable from the use of nitric acid, but it has the alvantage of yielding a notable quantity of electricity, with that of being prepared beforehand, of remaining many hours in action, and of being dismantled at leisure; while the small batteries of bisulphate of mercury should be prepared at the bedside of the patient.

"The apparatus of Norin may be regarded as arranged to furnish currents for purposes in which quantity is one of the elements to be regarded; while most of the other portable instruments have been constructed chiefly with a view to tension, and in order to attain the mean limit of effect fixer by the sensibility of our organs. The inventor was desirous, according to his own expression, not merely to construct an apparatus for giving shorks, but one that should be truly physiological.

"The apparatus gives the extra current, the indnced current, and the two together. Mr. Morin appears to have been the first to employ this combination." 8

(f). The instruments of $M$. Gaiffe.-This ingenious maker long ago constructed a small and very portable apparatus, represented at one-fourtls of its natural size in fig. $S 6$.

"The battery and the induction coil occupy two separate compartments, opening independently of each other. 'The battery is composed of two elements of zine and bisulphate of mercury, con- 


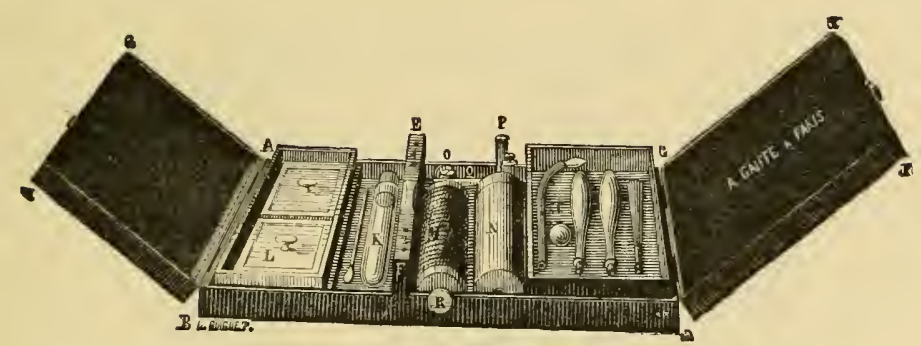

Fig. 86.-Gaiffe's apparatus.

tained in a cell of hard caoutchouc, at the bottom of which are fixed two plates of gas-carbon. The platinum wires that establish the communication are imbedded in the mass, and project beyond it; the two zinc plates lie flat in the place prepared for them; and the apparatus requires nothing beyond the establishment of the communications, - nothing can be more easy of manipulation.

"The apparatus furnishes the extra current, the indnced current, and, if needed, the combination of the two. The communcations are easy to make: since M. Gaiffe has taken the precaution to mark the direction of the currents upon the binding-screws that receive the conductors to the rheophores.

"In the figure, $\mathrm{L}$ is the small cell of caontchouc, containing the two elements of the battery. $K$, a tube containing a provision of bisulphate of mercury; MI, induction coil; $R$, knob of the graduator tube ; $\mathrm{O}, \mathrm{Q}$, pieces of the trembler ; $\mathrm{P}$, knob that is pressed upon to produce isolated interruptions; $\mathrm{N}$, cylinders; $\mathrm{T}$, various exciters.

New-pattern chloride of silver battery.-" MI. Gaiffe has arranged a new pattern, the felicitous combination of which practitioners will not fail to appreciate. He has been stimulated by the example of M. Trouvé, whose hermetically sealed pile will presently be described; lut he has known how to avail himself of the valuable properties of the fused chloride of silver, ${ }^{9}$ to which Mr. Warren de la Rue has recently called attention. Each of the elements of the battery contained in Gaiffe's apparatus is composer

${ }^{9}$ A battery of salt water, chloride of silver, and iron or zinc, had been employed, nearly thirty yea!s ago, by M. Beeqnerel, in his researches upon the elictro-chemical treatment of the ores of silver (Sec Bte. nerel and E. Becquerel, Traité d'électricite, tom. ii. Paris, 1855). II. Marié-Davy has construeted a battery of zine, pure water, and fused chloride of silver Comptes rendus de l'Acallmie des Seiences, tom. xlix., 1855). M. E.
Beequerel (Mémoire sur la pile voltaique in Annales du Consercatoire des arts $e t$ métiers, tom. i., p. 295,1861 has determined the electru-motor force of a pair in which the depolarization of the negative electrode was obtained by precipitated chloride of silver placed upon a diaphlragm of the battery. He found it a little inferior to that of a pair with sulphate of copper; that is to say, very little more than half that of a pair' with nitric acid. 


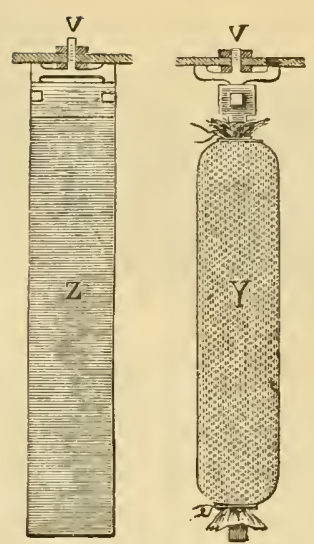

Fig. 87.

Part of the chloride of sliver battery.

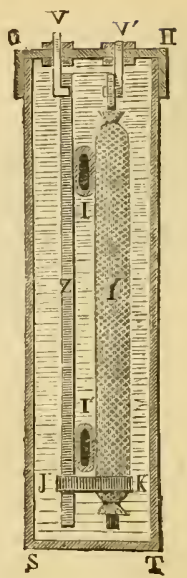

Fig. 88.

Internal view of the chloride of silver battery.

(figs. 87 and 88 ) of a plate of zinc, Z, and a plate of silver, Y, over which is fused a certain quantity of chloride of silver. It is then inclosed in a covering of fine tissue, to prevent the reduced silver being precipitated to the bottom of the cell. The whole is contained within a sort of box of hard caoutchonc, closed by a screwed stopper, and filled up with water containing five parts of sea-salt in every hundred. 'The projections of pure silver, V, V', to which the plates $\mathrm{Z}$ and $\mathrm{Y}$ are secured, convey the current outwards. $J, K$, is a caoutchouc band, which holds the plates together, while the interrening caoutchouc cushions, I I', maintain them at a constant distance.

"The quantity of chloride of silver is such that the battery will continue in steady action for ten hours with constant intensity, the circuit being completed by the induction coil.

"When the battery is exliansted, it is sufficient to unscrew the cover, and to attach a fresh plate, $\mathrm{Y}$, of chloride of silver, with which one should be furnished in advance. The reduced silver being all recovered, the expense consists only in manipulation, and in converting the reduced silver into chloride.

"This battery affords the inestimable advantage of remaining always ready, and in its place in the apparatus, for any length of time without alteration, as the chemical action which determines the reduction of the nitrate of silver does not occur unless the circuit is complete.

"According to the estimate given in the note above, we may say that MI. Gaiffe's two elements are equal, in electro-motor power, to one element of Bunsen's. 
"Lastly, the apparatus affords a valuable source of graduation in the power to use either one or two elements, as they are independent of each other.

"Fig. 89 affords a general view of the apparatus; it is entirely contained in the box $\mathrm{A}, \mathrm{B}, \mathrm{C}, \mathrm{D}$, which is divided into two parts.

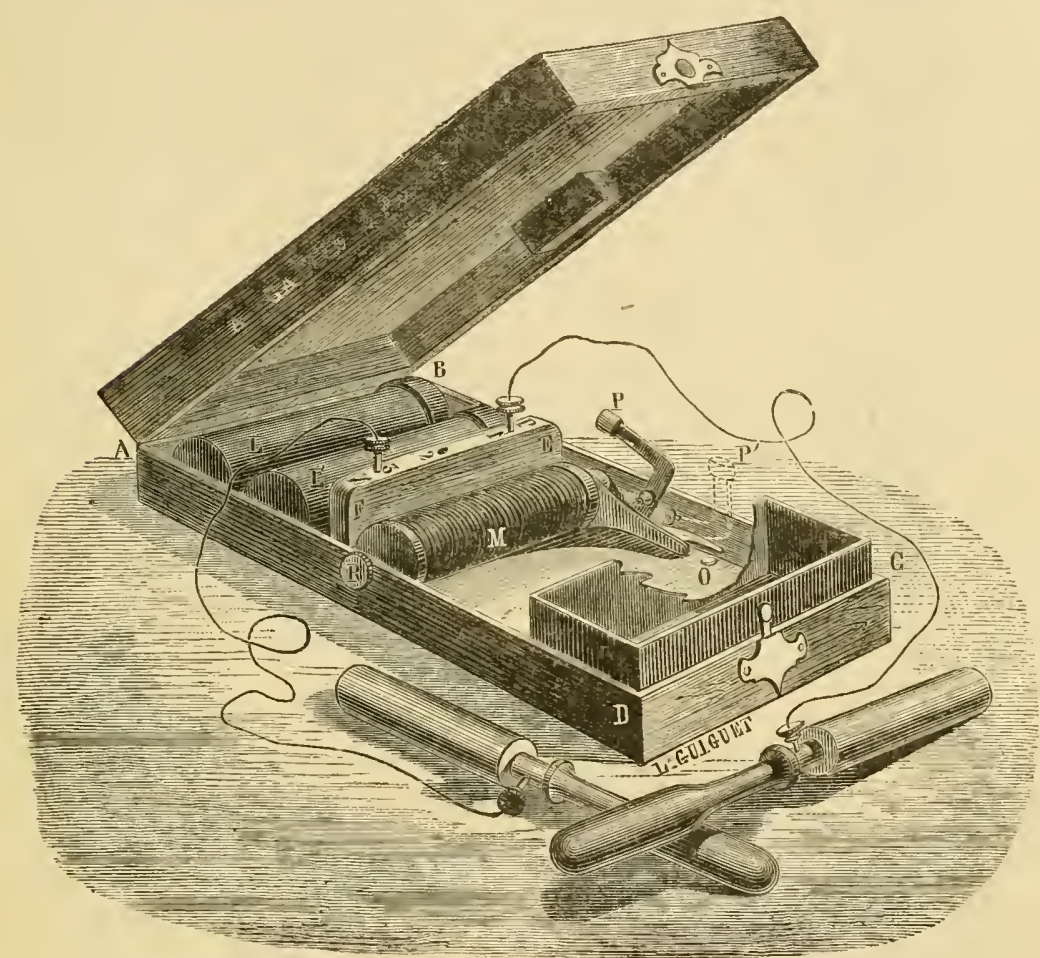

Fig. 89.-Chloride of silver battery.

The first comprartment contains the two elements which have been described, L L', fixed between the sides of the box, by the springs which serve to establish the communications. The second compartment contains the coil $\mathrm{lI}$, and its accessories. At one end of the coil is the knol) $\mathrm{R}$ of the graduator tube; at the other, a trembler, which MI. Gaiffe has ingeniously modified. Instead of its being regulated by a screw, which, in inexperienced hands, often strains the spring, it is under the control of a bent lever, $P$, which may be depressed to the position $\mathrm{P}^{\prime}$, where it serves as a pedal for the production of slow interruptions, when pressed down with the finger, so as to touch momentarily the small screw $\mathrm{O}$."

'Le Roux, p. 5!, loc. cit. 
(g). Trouvés electric roll.- "The small induction coils constructed by this skilful mechanician are chefs d'cuvre of in-

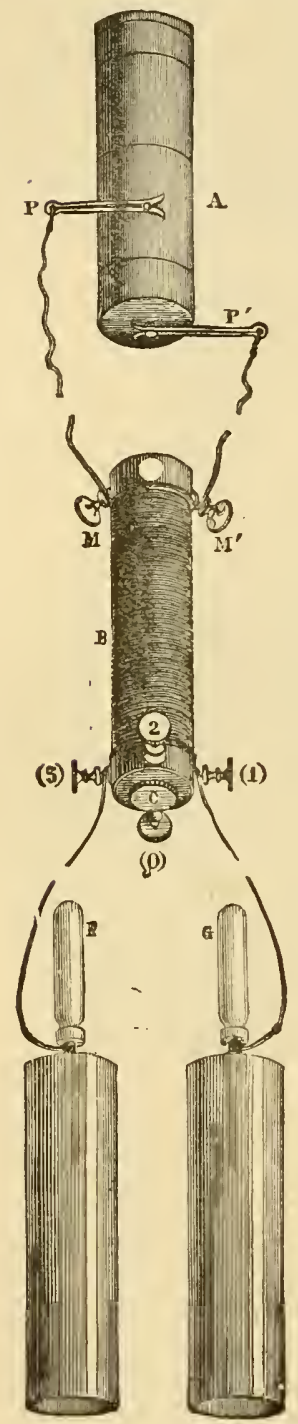

Fig. 90.-Trouvé's electric roll. genuity and precision. One of these coils is shown at B, in fig. 90, at half the natural size.

"We have already described the interrupter employed by iI. Trouré.

"His coil is composed of a very delicate plate of soft sheet-iron, rolled into a helix, and surrounded by a copper graduating-tube, the end of which is seen at $\mathrm{O}$; the inductive wire and the induced wire are placed endwise; the first forms six layers, the second eighteen.

"The knobs for the rheophores are placed at 5 and 1. 'The extra current of the two layers, the complete extra current, the induced current only, and the combination of both currents may be obtained.

"Single intermissions are prodnced by interrupting with the hand the contact between the battery and a small knob attached to the coil for the purpose.

"The battery, which is shown at $A$, is a box of hard caoutchouc, closed by a screw lid, which carries a cylinder of zinc, a little less than half the length of the box. The latter is laalf filled with water, to which is added three or four grammes of bisulphate of mercury. It follows, from this arrangeinent, that the battery only acts when the box is placed with its lid downwards. Thus charged, it will work for about an hour and a half.

"The effects of this small apparatus are comparatively remarkable, and are to be explained by the care sliown in its construction, by the complete insulation of all its parts, and by its perfect combination of the conditions most farourable for producing the results that are desired.

"It is only just to add, that M. Gaiffe long since prepared the way for the manufacture of small induction coils; but M. Trouré 
appears to have reached the farthest limits of possibility in this direction." 2

\section{§II.-Critical examination.}

The account of the principal electro-medical instruments, in their chronological order, which has been given in the preceding pages, and which has been in great part quoted from the excellent memoir of M. Le Roux, has brought sufficiently into relief their successive improvements, at least from the physical point of view. But do they fulfil the requirements of localized faradization, as applied either to the electro-muscular experiments (which, in my researches, have thrown so much light upon the physiology of locomotion), or to electro-muscular testing (which often decides the diagnosis of so many muscular affections), or to the treatment of disorders of the central or peripheral innervation, of sensibility, of motility, or lastly, of nutrition? To decide snch a question, it is necessary to possess some anatomical and physiological knowledge, and to have acquired, at the bedside of patients, sufficient practical experience.

The physicist whom I have quoted (II. Le Roux) has wisely kept silence upon the point; thus implicitly declaring himself incompetent to deal with a purely physiological and medical question. In order to ascertain whether all induction instruments possess the whole properties which would render them applicable to the electro-physiological and clinical studies which I have described, and to the fulfilment of certain therapeutical indications, I must inquire whether they have been constructed with the physical and mechanical conditions necessary for the attainment of these properties; or, that is, whether they possess,-1. a double induction; 2. an exact graduation; 3 . intermissions, rapid or slow at pleasure ; 4 . a sufficient degree of force? The considerations which I have already stated with regard to the utility of these conditions, which all electro-medical instruments should combine, renter it unnecessary for me to enter upon the subject anew. It will be enough to state briefly the principal facts upon which my critical examination will be based.

\section{Magneto-electric instruments.}

(A). Double induction.-Whatever may be the physical theory of the phenomena of double induction in magneto-electric instruments-a theory on which physicists will, I hope, before long agree,-it cannot now be denied that the currents which proceed

${ }^{2}$ Le Roux, p. 63. 
from each of the two coils exert, as in the electro-dynamic instruments, physiological actions which distinguish them apart, and which severally fulfil special therapeutical indications.

It follows from the facts, the experiments, and the considerations, which I have already stated (Chap. I.) :-

1. That the current of the second coil excites the retina more acutely than that of the first coil, when it is applied by moist rheophores to the face, or to the eyeball (see A, p. 23).

2. That the current of the second coil excites the cutaneous sensibility more acutely than the current of the first coil (see B, p. 24).

3. That the current of the first coil excites, more acutely than that of the second coil, the sensibility of certain organs situated more or less deeply beneath the skin (see C, p. 24).

4. That, in faradization of the nervous extremities by reflex action, the current of the second coil produces more energetic muscular contractions than the current of the first coil (see D, p. 26).

5. That when the moist rheophores are applied to the cutaneous surface, the current of the second coil penetrates more deeply into the tissues than that of the first coil (see E, p. 26).

6. That the different physiological actions of the currents of the first and the second coil cannot be applied indifferently in therapentics (see A, p. 33).

As a corollary to the foregoing propositions, we may conclude that any magneto-electric apparatus intended for medical practice, which does not possess, first and second coils, constructed with certain relative proportions of length and diameter of wive, will not fulfil all the requirements of therapeutics.

The foregoing considerations have not failed to exert a great influence on the manufacture of magneto-electric instruments. Indeed, either from understanding the importance of the matter, or in order to supply the demands of physicians, the makers of the magneto-electric instruments most generally found in medical practice (MM. Breton frères, and Gaiffe) lave applied double induction to them. It is, doubtless, from forgetfulness that MI. Le Roux has failed to mention this important feature of Gaiffe's instrument, on which, in other respects, he has bestowed a merited eulogium. The omission is more to be regretted, since this skilful manufacturer has endeavoured to find those relative proportions of diameter and length of wire, with reference to the power of the magnet, which will give to the coils the maximum of their different physiological action. M. Gaiffe manufactures a small, a medium, and a large magneto-electric instrument, and it is to the last two 
that he has adapted the double induction. It is to be regretted that the Paris hospitals are supplied only with the first or small model, which is insufficient, not only on account of the weakness of its current, but also from the absence of the current of the second coil.

MIII. Breton frères have manufactured a large and a small magneto-electric instrument. According to MI. Le Roux, they have endeavoured to add the double induction to the former.

I have had occasion to examine the differential action of the two coils in one of two of these instruments which I found in one of the Paris hospitals, and, to my great surprise, the distinctive characters of their differential action were scarcely appreciable.

What could be the reason of this? Have MLI. Breton observed those proportions of length and diameter, which the wires of the first and secoud coils should possess, with regard to the initial foree of the magnet, in order that double induction may be produced? Are the two coils perfectly independent of each other? Not having unwound them, I am unable to explain the actual reason. It would be easy for these skilful manufacturers, who, by having been the first to construct portable magneto-electric instruments, have so much contributed to popularize the application of induction currents, to fulfil the desideratum which it has been my duty to point out.

B.-Slow or rapid intermissions. - In the part (Chap. I., Part I.) in which I have described the properties and the particular arrangements which every induction instrument intended for medical practice should possess, I have made known the special action which slow or rapid intermissions exert,-1, upon electromuscular contractility; 2, upon muscular sensibility; 3, upon muscular tonicity; 4, upon muscular nutrition; 5, upon electre cutaneous sensibility.

Lastly, I have passed in review the chief pathological conditions in which rapid intermissions should be preferred; and also those in which they are formally contra-indicated, and in which they should be replaced by intermissions more or less distant, on pain of sometimes occasioning accidents. It follows that any magnetoelectric apparatus which is not so constructed as to yield currents with slow or rapid intermissions, according to the therapeutic indications to be fulfilled, will be inapplicable in a great number of cases.

This has been well understood by M. Gaiffe, who, in imitation of my magneto-electric instrument, allows the electrodes to receive only one or two intermissions for each revolution of the large wheel. 
It is to be hoped that all other manuracturers will follow an example that is easy of imitation. It would be a great step in advance.

C.-Graduation.-Every medical induction instrument should afford a means of distributing its currents in doses proportionate to the very different degrees of excitability (which may depend upon the state of health or upon the state of the disease) of the sensitive or motor nerves, of the muscles, and of other organs more or less deeply situated (see $§ I V .$, p. 241). Moreover, the graduation should be exact, and should range over a sufficiently extended scale.

Without these conditions, magneto-electric instruments are often inapplicable to medical practice.

'The instruments of Pixii, of Clarke, and of Saxton are entirely without graduation. Page was the first who endearomred to obtain it, by rendering the magnet of his apparatus movable (see fig. 75), so that it could be placed nearer to or farther from the soft iron. The idea rested upon the principle, that, in a magneto-electric instrument, the further the magnet is moved from its soft iron or its electro-magnet, the more the inductive influence of the alternate changes of state of these magnets will be weakened, and vice versâ.

Since then, this method of graduation has been imitated by most makers. But it is very defective, because there is no proportion between the divisions of the graduator and the diminution or augmentation of the intensity of the current, when the magnet is moved farther from or nearer to the soft iron.

The graduator is, in general, a small metallic stem, which serves to draw back or to push forward the magnet. It is about a centimetre and a half in length; and its action only becomes sensible in its second half, because, in the first half, the soft iron does not exert on the magnet any physiological influence that is appreciable in man. From the commencement of the second half, or thereabouts, of the graduator, the current increases in enormous proportion (in geometric ratio, or nearly as the square of the distance). It is impossible exactly to apportion the power of faradization by means of a system that is so irregular.

Moreover, even if the movement of the graduator and the increase of the force of the current were in exact relation, or, in other words, if the increase and diminution of the intensity of the current were in arithmetical proportion to the movement, the system would still be inapplicable to localized faradization, either for physiological, pathological, or therapeutical purposes, because it is not made upon a scale of sufficient extent. 
It has been seen, however, that I have applied the system of Page to my magneto-faradic instrument (with the difference, that instead of the magnet, it is the soft iron that I have made movable), but that I have employed it only as a moderator of the currents (see $\mathrm{E}, \mathrm{p} .282$ ), that is to say that its purpose is to convert a very powerful magneto-electric apparatus into one that is more or less feeble, the stronger or the weakest doses of which I measure by metallic gradnated cylinders (see D, p. 2S2). This system of graduation by cylinders is only applicable to coils rolled around the magnet.

\section{2.-Electro-Drnamic Instruments.}

A.-Double Induction.-The differential electro-physiological action of the currents of the primary coil (extra current), and of the secondary coil (induced current), is incomparably more marked in the volta-electric than in the magneto-electric instruments. MIoreover, we obtain from the former, in small bulk, a much greater power. These are the chief reasons which render the volta-electric instruments the most useful for medical purposes, and which have gained for them the preference in practice, and especially in the Paris hospitals, where they are more common than magneto-electric instruments.

When I commenced my researches (towards $18 \pm 6$ ), the electrodynamic instruments had usually only an extra current; and those which possessed double induction, like the apparatus of Rognetta, constructed by MIII. Breton frères, used only the induced current. But, since I liave shown that the extra current and the induced current exert different actions (which compelled me to write that they possessed different physiological properties, an expression so much and so unfairly criticized by the MIM. Becquerel), the makers have endeavoured to construct instruments which should yield one or the other current, at pleasure, according to the indication to be fulfilled.

It is necessary to know that, in order to obtain an extra current of sufficient intensity to be useful, the wire of the coil must be of sufficient length. I have not found in any electro-dynamic apparatus, even the most bulky, a sufficient differential action between the currents of the two coils, which evidently depends upon a fault of construction (a defect in proportion between the length and the diameter of the wire) ; in some instruments, I have even found no difference-which is to be explained by a fraud that I proceed to describe. These instruments have but a single coil, composed of a thick wire (about half a millimetre in diameter), of sufficient length, and continued by a wire that is relatively very fine (from a sixth or a seventl of a millimetre in diameter). Two 
electrodes receive the current from the thick wire, and two others, which communicate with the extremities of the fine wire, receive the current of the entire helix, which is then composed of a thick wire in its first part, and of a longer and very fine wire in its second. Every one will understand that these electrodes can only give an extra current proceeding from the same coil, and that the current of the entire coil will be more intense than that of its first portion; but that these currents cannot possess the differential properties of the extra current and of the induced current. Such instruments are sent into the market as if they possessed both. Their manufacture is, as may be imagined, more simple and less costly than that of double-induction instruments; but the purchaser is deceired. It is fortunately easy, when forewarned, to discover the frand, by the absence of the differential properties of the currents.

B.-Intermissions.-Formerly, the elertro-dynamic instruments uade only rapid intermissions, oltained either by wheels analogous to that of Masson, or by tremblers constructed on the principle of the apparatus of Neeff.

At present, most of the instruments afford, at pleasure, either slow or very rapid intermissions. But each of the slow intermissions should be perfectly isolated, distinct, and without vibrations.

The slow intermissions of the large electro-medical apparatus of Ruhmkorff (see fig. 79) are deceptive; each tooth of his starshaped wheel receives a series of very rapid intermissions from his trembler. I have pointed out this fanlt of construction to this skilful maker, who has undertaken to correct it, and to produce on his wheel defined and isolated intermissions.

The intermissions produced by the contact of a metal plate, forming a spring, are not always well defined. The friction gives one strong intermissioni, followed by a series of extremely rapid small vibrations, which acutely excite the sensibility, and render muscular firadization painful.

The rheotomes of slow intermission have the inconvenience that they either occupy one of the hands of the operator or require the presence of an assistant; with the pedal rheotome, such as I use myself (see Y, fig. 52), this inconvenience is avoided. It is to be desired that all makers would attach this accessory to their electrodynamic instruments.

The greater part of the tremblers of electro-dynamic instruments give vibratory intermissions. M. Gaiffe has especially endeavoured to remedy this defect.

C.-Graduation.-When I commenced my researches upon the improvements that could be made in induction instruments, MIM. Breton fieres had already constructed, under the direction of 
Rognetta, an electro-dynamic apparatus of double induction, in which the graduation of the induced current was obtained by gliding the coil of thick within the coil of fine wire. While gradually placing the first within the second, the induction only occurred in the spirals that were brought into mutual relation, and hence the intensity was proportionately increased. The reverse occurred as the first coil was withdrawn. This method of graduation was perfect; and I originally adopted it for my volta-electric and magneto-electric instruments (see fig. 51); modifying it, however, in an important manner, and applying it to the graduation of both coils (which Rognetta had neglecterl becanse he did not utilize the extra current,--not being aware of the difference of its action from that of the second coil).

Instead of the internal coil (of thick wire), as in the apparatus of Rognetta, it is the external coil of fine wire which I have rendered movable, and which, in covering or uncovering more or less of the internal, glides upon two metallic conducting stems, which are in communication with the extremities of its wire and with the knobs of the electrodes. In order to obtain the graduation of the extra current by the second coil, I completed the circuit of its wire, and caused it to move over the first coil. It is needless to say that the graduation was the same as in Rognetta's instrument.

This system of graduation was excellent, but I have abandoned it, since I discovered that metallic cylinders moving over the interior and the exterior of the coils graduated equally well the intensity of the currents. Then, instead of constructing two independent coils-the external to receive the internal which remained fixed,-I wound the coil of fine wire directly over the thick wire (each layer being insulated, it must be understood, by varnished paper). The result was, an increase of the intensity of the induced current, by the approximation of the two coils, so that it equalled one produced in two thousand metres of wire; and a simplification of manufacture. My instrument had also gained in solidity.

Graduation by the mutual influence of the coils (which, it will be seen, is of French origin) has been adopted by II. Ruhmkorff, who has constructed what is known as the carriage apparatus of M. Du Bois-Reymond.

It was, doubtless, in order to please some admirers of everything that is done at Berlin, that MI. Ruhmkorff has imitated the apparatus of Siemens in his large medical instrument. (I shall hereafter show the inconveniences of this system of graduation, especially in Ruhmkorff's instrument.) The preference given by most of the Paris manufacturers to my system of graduation by metallic cylinders, proves that I had reason for my choice. 
The electro-dynamic instruments of moderate force, even when they are gradnated to a minimum, are still too powerful for certain delicate applications, such as faradization of the chorda tympani. I have desired the makers to add to these instruments a tube containing water, which I have called a moderator (see F, fig. 52), which is designed to reduce the most powerful apparatus to one of extreme feebleness, which can then be graluated by means of the metallic cylinclers.

D.-Battery.-The inconveniences connected with the use of the battery, in the electro dynamic instruments, are such, that they have induced many to prefer the electro-magnetic instruments. Practitioners recoil from the more or less tedious and disagreeable manipulation that is required; and the least neglect or awkwardness may occasion more or less serious injury to the instruments. For this reason, makers have endeavoured to remore or to diminish these inconveniences; and, on this part of the subject, I have to record some progress.

(a). The numerous disadvantages of nitric acid, principally on account of the disengagement of nitrous gas, which attacks the metallic portions of the instruments, have caused the rejection of Bunsen's battery by the majority of makers.

MLI. Legendre and Morin have continued its employment for their instrument, because it is much more powerful than other batteries (such as Daniell's, or the bisulphate of mercury of Marié-Dary), and, while giving a notable quantity of electricity, it may be prepared several hours in advanee, will remain several hours in action, and, lastly, may be dismantled at leisure. These makers have, indeed, removed the inconveniences due to the disengagement of nitrous gas, by adopting to their battery (fig. 85) a cover of gutta percha, B, which closes the whole system almost hermetically. Notwithstanding this improvement, MII. Legendre and Morin should abandon the Bunsen's battery, or they will see their little instrument lose, especially in hospitals, the favour which it has hitherto enjoyed, and which it, in other respects, merits.

(b). The battery of Marié-Davy (carbon, zinc, and solution of bisulphate of mercury) may advantageonsly replace that of Bunsen, as being free from the inconveniences which I have described. It is for this reason that I have employed it for the flat battery of my volta-faradic apparatus.

The majority of makers have followed this example, and they have constructed the elements in a flat form, similar to that which I lave used during many years in my instruments; the carbon elements being below, covered with powdered bisulphate of mercury, 
over which a little water is poured, and the zinc covering all. (It will be remembered that, before the employment of bisulphate of mercury, the elements were arranged in the same manner, and that, instead of the solution of bisulphate of mercury, I employed, originally, nitric acid, and subsequently diluted sulphuric acid, with which to saturate my carbon.) But the mode of preparation of their batteries does not appear to me to give results equally advantageous with mine.

The powder of bisulphate of mercury, that I strew upon the surface of my carbon plate, is sufficient in quantity to form, when moistened, a stratum half a millimetre in thickness. The water which I pour upon this paste, when I am about to use it, saturates it completely; and the thick cloth, which is placed between it and the zinc itself, is well soaked with water. Thus charged, my battery will continue in action for twelve hours, with rapid intermissions; and for several days, with the slow intermissions that I habitually employ. In the intervals between the applications, the zinc only is removed and wiped (so that its bright surface is always amalgamated) ; and, in order to avoid the evaporation of liquid by contact with the air, a plate of hard caoutchonc replaces the zinc.

With this mode of preparation, a battery is for a long time always ready for action before $I$ am called upon to renew the bisulphate of mercury; it preserves its force until the entire decomposition of the salt; and there is only occasion to pour, from time to time, a little water upon the cloth.

The bisulphate of mercury batteries, in the small instruments of Gaiffe, Ruhmkorff, and Trouré, contain only the small quantity of bisulphate which they can hold in solution; so that they cannot be used for more than an hour at a time, withont recharging the battery. After using them only for a few minutes, it is necessary each time to throw away the liquid, to clean the zinc, and to renew the preparation for the next patient.

Lastly, I have observed that my battery polarizes less, which I attribute to the application, immediately against the zinc, of the wet cloth, which forbids a great number of the bubbles of hydrogen gas produced by the action of the battery to adhere to its surface. In other batteries there is nothing to prevent these bubbles of hydrogen from sticking to the zinc, and shielding the greater part of its surface from the chemical action of the battery. I therefore advise maker's who employ flat and unclosed batteries of bisulphate of mercury, to prepare them like mine.

The flask battery of bisulphate of mercury, which M. Ruhmkorft las added to his hospital induction apparatus (see fig. 79, page 297 ) is well conceived. 
It recalls, indeed, by its form, its disposition, and its mechanisin, the flask battery of M. Grenet, from which it differs by the employment of a saturated solution of bisulphate of mercury, in place of chromate of potash, and by being mounted in hard caoutchouc, instead of in metal, which inevitably undergoes amalgamation by the mercury, and becomes brittle.

This flask battery of MI. Ruhmkorff will work for a long time without requiring to be recharged. When not in use, the zine is drawn up out of the solution of bisulphate of mereury, by means of a metallic stem; and when it is required, it is pushed down again into the liquid. This may be done gradually, in such a way as to increase by degrees, and at pleasure, the intensity of the current. The employment of this battery will be a source of great economy in hospitals, because it oceasions no damage to instruments, and because its management is so simple.

But the flask of this battery is itself seven or eight centimetres in height, without reckoning six centimetres, or more, for the stem, which, when it is not in use, serves to keep the zine raised out of the solution. In other words, its size fills a considerable space in the apparatus, and its leight prevents it from being enclosed in the same box. This is the only fanlt which can be found with it; but its bulk is unquestionably a real inconvenience, and renders the apparatus less portable.

Other manufacturers, endeavouring, like II. Ruhmkorff, to render the manipulations of the batteries more easy and less frequent, have particularly applied themselves to diminish their bulk, and to make them more portable. They have enelosed them in a box of hard caontchouc, by the aid of a screwed lid, to which the solid parts of the battery are fixed. These small hermeticallyclosed batteries were at first employed as sources of anusement, on account of their small bulk. Conjurors earried them in their pockets, and, conveying them adroitly into their hands, set in action from a distance, the electro-magnets which communicated with them. Notwithstanding the trivial origin of these little batteries, their application, as medical induction instruments, has nevertheless been highly useful.

It is, however, just to say that MI. Trouve appears to have been the first to construct a battery in a closed caoutchouc box, which he called an hermetic battery, and which has already been described.

II. Gaiffe has given to his batteries the same external form as those of M. Trouré. But their composition is essentially different, since they consist of ehloride of silver (see figs. 87 and 88). II. Trouvés bisulphate of mercury hermetic battery will only work for an hour and a half, after which it requires to be recharged. The 
chloride of silver hermetic battery of MI. Gaiffe would originally furnish continuous action of constant intensity for about ten hours, the circuit being completed by an induction coil ; but the manufacturer now informs me that his battery will work for twentyfour hours, at least, in consequence of a modification that he has made in it. ${ }^{3}$

When this battery is exhansted, it is necessary to recharge it with a new piece of chloride of silver (see description, fig. 87). This is easy of execution.

One of the principal merits of these batteries in boxes is, according to their makers, that they close hermetically, so that there is no fear that any liquid will escape from them to injure the instrument, or the clothing, when carried upon the person. 'This is an error'; for the hydrogen-a greater or less development of which is the inevitable result of the chemical action-never fails to make its way by a little fissure, which generally exists around the metallic wires, which pass through the lid. Even if the mastic, which surrounds the metallic wires, resists the pressure of the gas, so that it cannot escape, there will still be a risk of explosion if, under any circumstances, there should be a rapicl production of gas in any large quantity.

We are chiefly exposed to the same accident with the bisulphate of mercury battery, the chemical action of which produces hydrogen by the decomposition of water. The chemical action of the chloride of silver battery neither disengages hydrogen nor any other gas. ${ }^{4}$ But if its chloride of silver is exhausted, that is, if it loses its proper action, and forms part of a circuit of a battery, composed of a sufficient number of elements, it fulfils the office of a voltameter, and then the current, in traversing it, decomposes its water, and produces a great quantity of hydrogen. It is under such circumstances that a chloride of silver element, inclosed in an impermeable case, may explocle. ${ }^{5}$

3 "The chloride of sodium," MI. Gaiffe writes to me, "being only employed to render the liquid a conductor, and the product of the battery being ehloride of zine, I have replaced the former by an equal quantity of the latter, in order to have one salt only, and to avoid the action which the two chloriles exert upon each other; an action which was injurious to the battery, since, charged in the new way, it will work regularly for a much longer time. Another aidvantage of the chloride of zine is that, by its employment, we avoid the production of gas, even when the charge of chloride is exhausted."
4 There is no production of gas in the chloride of silver battery, becanse there is only a displacement of chlorine, which leaves the silver to combine with the zine; so that before the battery begins working we have: $\mathrm{AgCl}, \mathrm{Zn}, \mathrm{HO}, \mathrm{NaCl}$, and, after its action: $\mathrm{Ag}, \mathrm{ZnHO}, \mathrm{NaCl}$. The chlorine only, therefore, has changed its place; the water and the salt have only acted by their presence.

5 'This has happened to myself, when holding in my hand one of these small closed batteries of hard canntchouc. The case burst, and some of the contained liquid was thrown in the face of a person standing npposite to me. 
This, then, being established, it is more simple and prudent to make a capillary opening through the caoutchouc corering, by which the gas may escape if the pressure becomes too great.

E.-Pover and size of the electro-dynamic instruments.-In certain cases, as I have said, the induction instruments should be very powerful (see III., p. 239). We have here to consider their physiological action, and from this point of view to determine their power. This, therefore, is not so simple a matter as was thought by III. Becquerel, when they wrote:-." The extra current, and the current of the first kind, do not possess an elective action over this or that function, but they have an action more or less energetic, by reason of their tension." ... It was a mere matter of inexperience, at the time when Mr. Becquerel the younger maintained this position in defence of a physical theory. It was no longer a matter of inexperience alone, when it was maintained before the Institute, eight years subsequently, by MI. Becquerel the elder; because it would have been easy for him to have acquired a personal knowlerlge of the subject, by submitting to my experiments, as so many other physicists of equal position had done. But, fortunately, he has been unable to arrest the progress of science, and it is now perfectly established-1. That the extra current, which has very little tension, or infinitely less than the induced current, excites acutely, and more acutely than the latter, the sensibility of the greater number of the organs situated beneath the skin (the nerves, the muscles, the bladder, the uterus, the testes). 2. That its therapentic application, in full dose, is indicated in the cases where these organs are deprived of sensibility, in eases where it is necessary to act upon the nutrition (which I have observed that it seems most to stimulate, perhaps by producing less contraction of the capillary vessels than the induced current). 3. That, by reason of the weakness of its power to penetrate to deeper tissues (in other words, the weakness of its tension), the extra current is the only one that is adapted for cases in which it is indicated strongly to excite the contractility and sensibility of superficial muscles, without acting upon those that are beneath them. 4. 'That, in order to obtain this special physiological action of the extra current, its wire should have a certain diameter and a sufficient length, so as to have an initial force proportionate to that of the magnet and of the battery. 5, and lastly, That this extra current becomes powerless, when we wish to penetrate a great thickness of tissue, so as to excite deeplysituated organs, such as muscles of the deeper layer in very fat or ndematous persons, or when we wish to excite very acutely the sensibility of the skin, as in profound anæsthesia. It is then 
necessary to have reconrse to a strong induced current of powerful tension.

These facts show the insufficiency of the very small induction instruments of Gaiffe, Ruhmkorff, and Trouvé,-instruments the extra currents of which are almost powerless, and of which the induced currents have not sufficient tension to reach the muscles, or to pass through any depth of tissue. (It is especially with slow intermissions that we can appreciate the weakness of these small instruments.)

Instruments of very small size and of easy transport can therefore only be utilised for therapeutical purposes in a very small number of cases. I regard them as pretty toys, which may be relegated to the domain of drawing-room physics. MM. Gaiffe, Legendre and Moriu, and Ruhmkorff, have fortunately constructed instruments of medium force, almost equal to that of my own small instrument in the form of a book (see figs. 58 and 60), and which fulfil nearly all the requirements of practice.

That of M. Gaiffe preserves the shape of the small instrument represented in fig. 86. Its size being only increased one-fourth, it is still very portable, and its flattened shape allows it to be carried under the arm like a book. It is not the same with the instruments of MI. Ruhmkorff, or of Legendre and MLorin, which are contained in boxes that must be carried by the hand. The instrument of Rulumkorff is twice the size of that of Legendre and Morin. It might be easily reduced by about a third, if the maker would substitute graduation by a metallic tube for graduation by the mutual influence of the coils. The former has, over the latter, advantages which have induced me to give the preference to it (see p. 253). The modification which I propose would simplify the construction of Ruhmkorff's apparatus, and would consequently diminish its size and its price.

These instruments of medium power are far from attaining the force of my large volta-faradic apparatus (see figs. 52 and 56), which has rendered me such services in cases in which the former would have been insufficient or powerless, and which, notwithstanding its greater size and greater weight, is still portable. It usually serves me for my own chamber apparatus, and it is worked by a fraction of my sulphate of lead battery.

I would advise manufacturers to take it as their model, 1. as regards its power, which could not be exceeded, I believe, without danger to patients; 2 . as regards its graduation by internal and external metallic tubes; and, lastly, as regards its commutator of the coils, and of the poles. They should not forget to attach to it a water moderator (F, fig. 52), which converts, at pleasure, this 
powerful apparatns into one that is more or less weak, in such a manner that, reduced to its minimum, its graduating tubes will measure its weakest doses as exactly as its most powerful ones.

[Stöhrer's induction apparatus.-Probably the greatest obstacle to the use of induced electricity as a therapeutical agent in England, has been the want of an efficient portable induction apparatus, in which the trouble commonly connected with the regulation of the voltaic elements shall have been reduced to the smallest point, which should be at all times immediately available for use, and which should not prove too costly. This obstacle has been most successfully overcome by Emil Stöhrer, Ph.D., of Dresden. He has constructed an induction apparatus, that fully meets the requirements of daily practice. The peculiarity of this apparatus consists in the arrangement of the zinco-carbon cell, already described (p. 57, fig. 15). The simple mechanism by which the elements can at once be immersed, or removed from the exciting liquid, and the length of period over which, with ordinary care, these elements retain their activity-without need for re-amalgamation of the zine, or remoral of the acids (not less, indeed, than from six to eight wecks); these are invaluable advantages in active practice. They render the use of induced electricity, for all purposes of diagnosis or treatment to which it may be applied, as facile as the test of a speculum, an ophtlalmoscope, or a laryngoseope. The readiness with which this induction apparatus can be placed in action, makes it highly desirable that one should be kept at hand in all hospital operation rooms, and, indeed, in all institutions where chloroform is used; for it affords an instant and most valuable menns of exciting artificial respiration in asphyxia, by faradization of the phrenic nerves.

Stöhrer constructs a smaller (fig. 91) and a larger (fig. 92) induction appar-

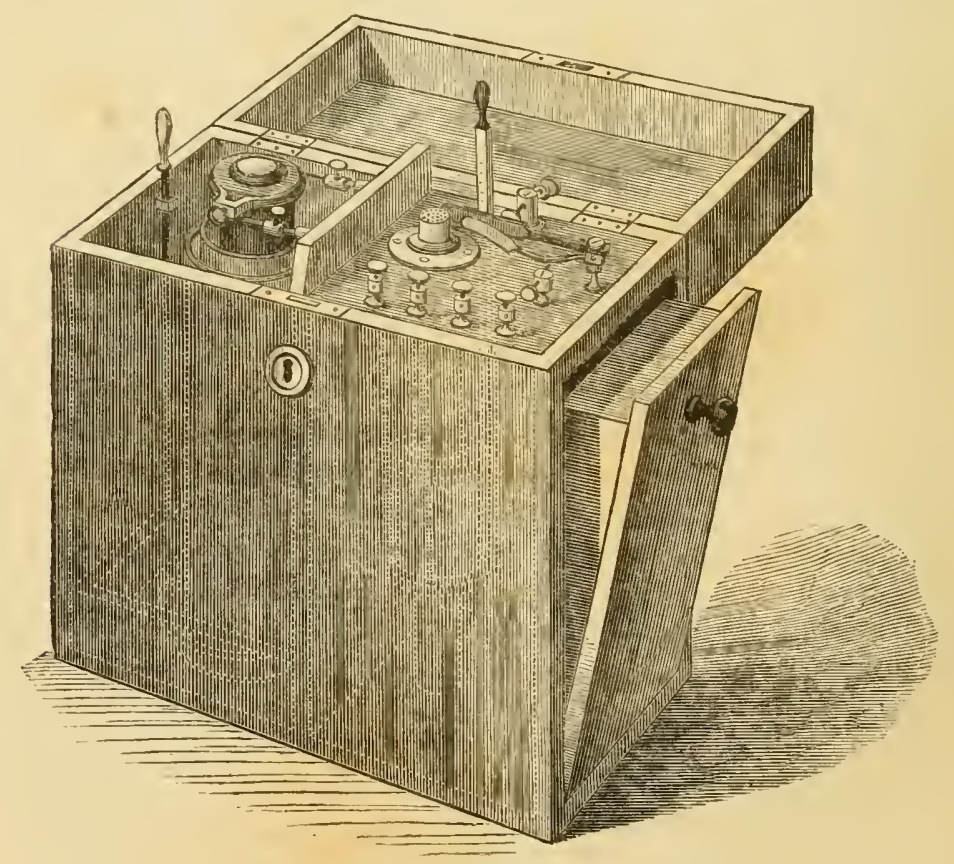

Fig. 91.-Stöhrer's smaller induction npparatus. 


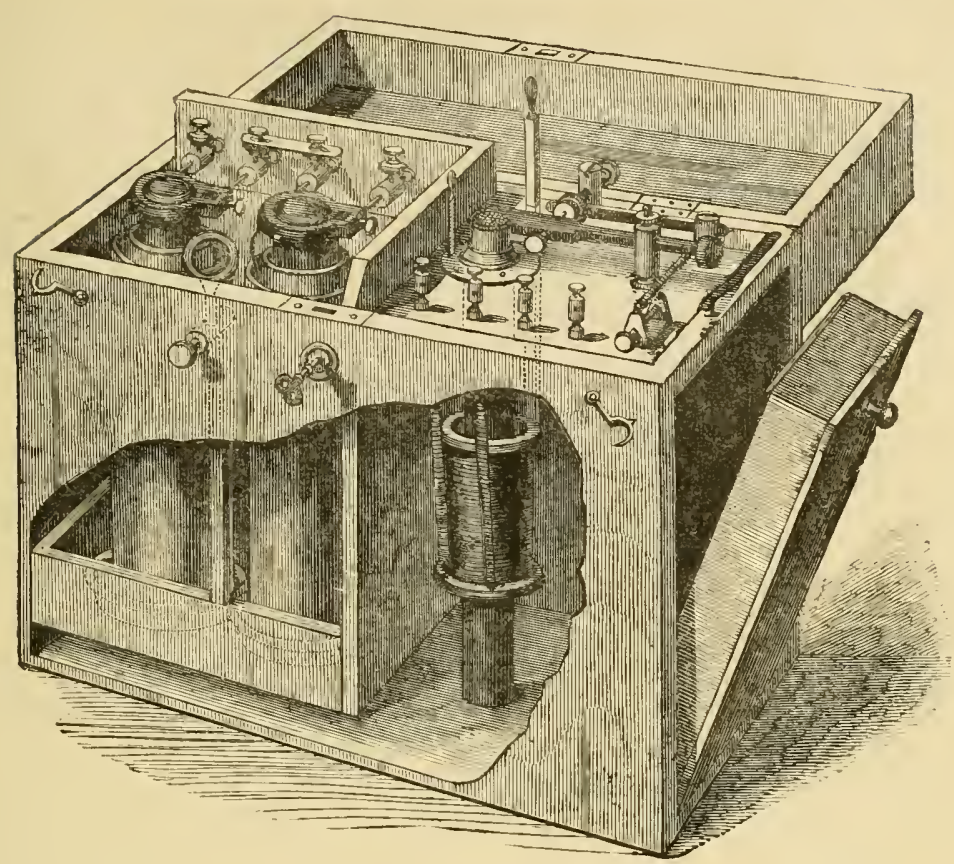

Fig, 92.-Stöhrer's larger induction apparatus.

atus. The battery of the former is constituted by a single cell; of the latter by two cells, which may, however, be arranged either as two pairs, or as a single pair of elements. Both possess a primary and a sccondary coil, the currents of each of which can be made use of separately; and, in both, the currents have a definite direction, positive electricity being sct free at one terminal, and negative at the other, of each of the coils. 'The terminal from which the positive current proceeds may be ascertained easily by the decomposition of iodide of potassium. The larger apparatus differs from the smaller, in having a much greater range of power, more thorough means of graduating: the currents, and a more elaborate arrangement of the interrupting hammer. With practice, however, the force and rate of interruption of the smaller apparatus may be regulated with much nicety. To neither instrument is a water-graduator attached; but, if needed for any special nicety of application, one can readily be added, and it would be best carried loose in the drawer for accessories, to be attached only when required. Graduation of the strength of the currents is effected by the degree of immersion of the voltaic elements in the exciting acid, and by the arrangement of the coils. The primary coil is fixed upon a pedestal; the secondary is movable, and is brought into or placed out of action by being lifted over or thrust away from the primary coil. The degree of action in the secondary coil is proportionate to the extent to which it is brought under the influence of the primary coil. The action of the primary coil is regulated in the smaller instrument by the degree of immersion of the voltaic elements, and by the extent to which the coil is masked by the secondary coil,-- the latter coil acting upon it, when the primary coil is alone in use, as a metallic sheath would do. In the larger apparatus, a special copper sheath is provided for the graduation of the current of the primary coil. It is to be regretted that a similar arrangement has not been adopted in the smaller apparatus. 
Although, of all indnction instruments for daily uso, Stöhrer's involves the least difficulty and care, still, to the perfection of its use, as of all delicate instruments, care is necessary. It is especially important that the zine should be thoroughly amalgamated, and too great pains cannot be given to secure this end. It is necessary also that all the points of connection should be kept thoroughly clean, and all the accessories in good order. The zine should be amalgamated whenever, in use, effervescence of the acid is perceived.

Duchenne's large volta-faradic apparatus is unquestionably the most perfect for medical purposes; Stöhrer's apparatus is the best adapted for the ordinary exigencies of active practice, $-\dot{I}$. ' $T$.'] 
LONDON :

PRINPFI BY WILLIAM CIOWES AND SONS, STAMPORD STREET, AND CHARING cROSS 



\section{CATA I O G U}

$\mathrm{OF}$

\section{WORKS ON MEDICINE, SURGERY, DENTISTRY, AND THE COLLATERAL SCIENCES.}

PUBLISHED BY

\section{LINDSAY \& BLAKISTON, PHILADELPHIA.}

\section{NEW BOOKS AND NEW EDITIONS, Preparing for Publicution.}

Rindelisisch's Pathological Histology, translated from the German. 208 Illustrations, 1 Volume, Octaro.

MaCrenzie on LARYNGeAL Growths, with Colored and other Illastrations. Swerrafan's Pharmaceutical Lexicon, a Dictionary of Pharmaceutical Science. 1 Tolume, Octavo.

Beale on Diseises of tile Liver and tueir Treatient. A New Revised and Enlarged Erlition.

Fuller on Diseases of tiff Heart and Great Tessels. 2d Edition.

'Trousseat's Lectures on Clinical Medicine, Tol. 4.

Wedi's Dextal Patuology, translated from the German, with Illustrations.

Duchenne's Localized Electrization, with Notes and Additions by the Translator, and Numerous Illustrations.

Fulier on Rheumatisi, Rineunatic Gout, etc., etc. A New and Enlarged Edition.

Pereira's Prescription Book. A New American, from the Fifteenth: Loulon Edition.

Tanner and Meadow's Practical Treatise on the Diseases of InFancr and Chumbioon, Secoml Edition, Revised and Enlarged.

Lindosay and Blakiston's Physician's Tisiting List for 1872. Various Sizes and Styles of Binding.

Hood's Treatise on Gout, Rueumatisi, and the Allied Affections.

\section{Sydenham Society's Publications to be Issued during 1871.}

Wunderlich on tue Use of the Thermometer in Disease. Translated, with Notes, by Dr. Woodman.

A Biennial Retrospect of M edicine and Surgery for the Years 1869 And 1870. Erlited by Mr. Power, Dr. Shepherd, Mr. Warren Tay, Dr. Barnes, Mr. Carter, and Dr. Stevenson.

Trotssaju's Lectures on Clinical Medicine, Vol. 4. Translated by IIr. Rose Cormack.

The Eleventh Fasciculus of the Atcas of Portraits of Skin Disrases. Also a Second Part of Tol. 40, heing a Descriptive Catalogue of the Portraits of Skin Diseases, compiled by Mr. Hutchinson.

Dor ORDER for any Medical Books, whether published in the United States or elsewhere, and not contained in this Catalogue, will be executed promptly, and at the lowest prices. 


\section{Itedical Publishers a Booliseller's,}

No. 25 South Sixth Street.

\section{The Change of Life in Health and Disease.}

A Pratical Treatise on the Nerrous and other Affections incidental to Women at the Decline of Life. By Edwand John Tilt, MI.D. From the Third London Edition. In one volume, Octavo. . . . \$3.00

The work is rich in personal experience and observation as well as in ready and sensible refleetion on the experience and observation of others. The book is one that no practitioner should be without, as the best we have on a class of diseases that makes a constant demand upon our eare, and requires very judicious management on the part of the practitioner. - London Lancet.

The great abilities of Dr. Tilt, his extensire knowledge, and his literary power are well shown in this book, and we are pleased to say that it has substantial and peculiar merits, It contains many sage, practical reeommendations, and will amply repay pernsal. - Edinburgh Medical Journal.

Dr. Tilt has been a very earnest and a very faithful worker in the physiology and diseases of women, and has nade contributions to the literature of the subject which are all of accepted value. - British Medical Journal.

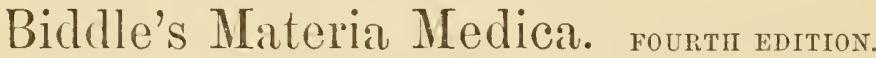

For the Use of Students. With Illustrations. By J. B. Bidder, M.D., Profcssor of Muteria Medica and Therapeutics in the Jefferson Medical College, Philadelphia, \&c., \&c. Revised and Eularged.

This is a thoroughly revised and enlarged edition of Prof. Bidlle's work on Materia riediea. It is designed to present the leading faets and principles nsually eomprised udder this head as set forth by the stanclard authorities, and to fill a vacuum which stems to exist in the want of an elementary work on the subject, The larger works usually recommended as Text-books in our Medical Schools are too voluminous for eonvenient use. 'This will be found to contain, in a condensed form, all that is most valuable, and will supply students with a reliable guide to the conrses of lectures on Materia Mediea as delivered at the various Medical Schools in the United States.

DR. BEALE'S NEW YOLUMES.

DISEASE GERMS : THEIR REAL NATURE. Twenty-four Illustrations.

An Original Investigation. Including the minute Pathology of Contagious Diseases. Price, . . . . . . . . $\$ 4.00$

DISEASE GERMS : THEIR SUPPOSED NATURE. With Colored Plutes.

An Original Investigation with the Aid of the Higliest Powers yet made. 'I'his work contains a critical examination of Dr. Tyudall's statements concerning "Dust and Disease." 12mo. Cloth. . . . \$1.75

THE ARCHIVES OF MEDICINE. Part IVII. now Ready. . . \$1.50

A Record of Practical Observations, and Anatonical and Chemical Researches connected with the Investigation and Treatment of Disease. With 16 Iliustrativns,

THE MYSTERY 04 LIFE. \n Essay in Reply to Dr. Gull's Attack on the Theory of Vitality in ws Harverian Oration for 1870. With Two Colored Plates. Price, 
THOMAS HAWKES TANNER'S WORKS.

"The leading jeature of Dr. Tanner's books is their essentially practical character." LoNDON LANCET.

\section{The.Practice of Medicine. just ready.}

\section{FIFTH AMERICAN, FROM THE SIXTH LONDON EDITION.}

Fievised, much Enlarged, and thoroughly brought up to the present time. With a complete Section on the Discases Peculiar to Women added; also an cxtensive Appendix of Formule for Medicines, Baths, Mineral Waters, Climates, \&e. By Thomas Hawkes Tanner, M.D., Fellow of the Royul College of Physicians, \&.c. One Volume. Royal Octavo, containing over 1100 pages. Price, handsomely bound in Cloth, . . \$ \$6.00

$$
\text { " " " Leather, . \$7.00 }
$$

There is a common character about the writings of Dr. Tanner-a character which constitutes one of their chief values: they are all essentially and thoronghly priatical. Dr. Tanner never, for one moment, allows this utilitarian end to escape his mental view. II aims at teaching how to reeognize and how to enre discase, and in this he is thoroughly suceessful. . . It is, indeed, in wonderful mine of knowledge.—Medical Times.

Dr. Tanner has always shown in his writings that he possesses a peculiar faculty of committing to print just that kind of information which the practitioner most needs in everyday practice, and of rejecting useless theories or hypothetical statements.-Lancet.

The author has, in the maner in which he has dealt with the subject, given another evidence of that happy facility which he possesses of giving the essential points of a mass of information in a well connected and instructive form. -- Lrit. Med. Journ.

The student will find the work the best text-book on the practice of medicine, whilo the practitioner will possess in it a thoroughly safe guide at the bedside. - Dub. Med. Quarterly.

\section{Tanner's Practical Treatise on the Diseases of Infancy and Childhood. mearly ready.}

\section{THE SECOND EDITION, REVISED AND ENLARGED.}

By Alfred Meadows, M.D., London, M.R.C.P., Plyysician to the Hospital for Women and to the General Lying-in Hospital.

As it stands, the book is one of the most complete in our language. It no longer deals with the diseases of children only, but also with the peculiar conditions of childhood, both normal and abnormal. - Hed. Times.

The book will be an invaluable work of frequent reference to the practitioner.-The Lanct.

Recently Published.

\section{Tanner's Index of Diseases and their}

'Teatment. With upuards of 500 Formule for Medicines, Baths, Mineral Wuters, Climates for Invalids, \&c., \&c. Octavo. . . \$\$3.00

To the busy practitioner it must be gn advantage to see at a glance on a quarter or balf a page the principal point in any rlisease about which he may wish to hare his memory refreslied or his mind stimulated. It will be found a most valuable companion to the judicious practitioner. - The Lancet.

\section{'Tanner's Memoranda of Poisons.}




\section{Byford on the Uterus.}

On the Chronic Inflammation and Displacement of the Unimpregnated Uterus. A New, Enlarged, and Thoroughly Revised Edition, with Numerous Illustrations. Now Ready. One volume. Octavo. \$3.00

From Fordyce Barker, M.D., Professor of Obstetrics and Diseases of Women and Children in Bellevue IIospital Medicul College.

Some weeks ago I received a copy of your work on the Uterus. I lave delayed ackrowledging the faror until $I$ coull give the book a careful perusal. I have just finished a thorough reading of it. I feel personally indebted to you, not merely for the coly - which, of course, I should have bought - but for writing the book; and I think you have laid the Profession in this comntry under a load of obligation by giving them such a clear, concise, anil practical treatise on a class of affections that even now is very little understood by the greater majority.

From R. A. F. Pexrose, M.D., Professor of Obstetrics in the University of Pennsylvania.

Accept my thanks for the copy of your new work which you so kindly sent me. I have, as yet, not had time to give it the careful study it merits; but from the superficial iuspection I have made, I find much that is most valuable.

From S. G. Ifubband, M.D., Professor of Oustetrics in Yale College, New IIaven.

I was gratified by the receipt of your new work on the Uterus, and I thank you sincerely for it. I have spent all my leisure in its examination, and have derived hoth pleasure and profit from its perusal. It is commendable for its clearness and definiteness as well as for the great practicnl common sense which pervadesit. I am sure that it will prove a rery usetul treatise, not only to junior practitioners, but to those also among us who, from not having devoted themselves to the treatment of uterine lisease, as a specialty, have neither time nor opportunity to make original investigations in this department, and are not, therefore, perfectly at home in its practice.

From James P. Wните, Professor of Obstetrics and Diseases of Women and Children in the University of Bufjulo.

I have had time, as yet, to run over but few of the chapters of your work on the Uterus; I am most happy, however, in being able to say that, so far as I Lave real, it does credit to American authorship. It is concise and brief, and eminently practical. The work was certainly a lesideratum. and will be especially useful to practitioners who can illy afford to purchase all or most of the works referred to in your preface. I shall examine it with much interest, and, no doubt, often consult it with profit.

From G. S. Bedford, AI.D., Professor of Olstetrics and Diseases of Women aud Children in the University of New Tork.

On my return to the city I found on my table "Byford on the Uterus." The next day I commenced perusing it, and have read it from cover to cover. I need not say that, in uy judgment, the book enhances your deserved reputation. You have, if my opinion be worth anything, given the Profession an excellent work, and one that is sensible and practical. Go on, my dear Doctor, and give us more of your experience. It is what the Profession most needs - the expericnce of good and ripe minds.

\section{RECENTLT PUBLISHED, THE SECOND EDITION OF}

\section{Brford's Practice of Medicine and Surgery.} Applied to the Diseases and Accidents Incident to Women. By W. H. ByFord, A.M., MI.D., Professor of Obstetrics and Diseases of Women and Children in the Chicago Medical College, \& c., \&.c. The Second Edition, Rievised and Enlarged, with Additional Illustrations. One volume. Octaro. . . . . . . . . $\$ \$ 5.00$

The rapid sale of the first edition of this book, which was exhausted in a little more than a year, has euabled the author to carefully revise the whole work, add many improrements, and to make a large addition of uew matter, without, however, materially increasing the size of the rolume.

This work treats well-nigh all the diseases incident to women, diseases and accidents of the vulva and perineum, stone in the bladder, inflammation of the vagina, menstruation and its disorders, the uterus and its ailments, ovarian tumors, diseases of the wamma, puerperal convulsions, phlegmasia alba dolens, puerperal fever, \&c. Its scope is thus of the most extended character, yet the observations are concise, but convey mucb practical information. - London Lancet. 
"The Representative Book of Medical Science." - Londov Lanckr.

\section{Aitken's Science and Practice of Medicine.}

\section{SECOND AMERICAN FROM THE FIFTH LONDON EDITION,}

Containing Adpitions equal to 500 pages of the English Edition, prepared by the Americas Eoitor with Special Reference to the wants of the Ayerican Practitroner.

The Science and Practice of Medicine. By Wildia A itken, M.D. Second American from the Fifth Enlarged London Edition, with Adlitions by Merediti Clymer, M.D., late Professor of the Institutes and Practice of Medicine in the University of New York, Physician to the Philadelphia Hospital, etc. In 2 Tolumes Royal Octaro.

With a COLORED MAP, a LITIIOGRAPHIC PLATE, and ONE HUNLRED AND THIRTY ILLUSTRATIONS ON WOOD.

Price, bound in Cloth, bevelled boards

$$
\text { " " Leather, . }
$$

Filteen montlis hare been spent by Dr. Artses in thoroughly revising this Great Frork, and adding to it many raluable additions and improrements amounting to about 100 pages of new matter, included in which will be found the adoption and incorporation in the text of the "newo nomenctature of the Royal College of Physicians of London;" to which are added the Definitions and the Foreign equivalents for thair English names.

The subjects of Matignant Cholera, of Paralyss, of Epidemic Cerebro-Spinal Meninjitis, and of Intestinat Obstruction have been entirely ro-written; and several other subjects in connection with the treatment of disease, of the greatest importance, are con. sidered for the first time in this edition.

The Press have referred to former editions of this work as "an admirable compilin tion." "The most comprehensire of any in the practice of medieine." "It embodies the most advanced knowledge of the time." "The most valuable elass-book for students get published." "It may be looked upon as tho standard text-book in the English language." “The present work contains information that will not be found in any other Manual of Medicine," \&c., \&e.

The author in this edition has endearored to keep up this high reputation, and to make it in every respect a Representative Book of Nedical Science and the Practice of the day, as understood and followed by the best men of the Profession.

Large additions hare also been made by the American Editor, Dr. Meridith Clyuer, equal to over 500 pages of the London edition, and with particular referenee to the wants of the American Practitioner, included in which are new articles upon the following subjects: Spinal Symptoms of Typhoid Fever, Typho-Malarial Fever, Chronic Camp Dysentery, Cholera Morbus, Cholera Infantum, Hereditary Syphilis, Gonorrhceal Rheumatism, The Delirium of Inanition, Chronic Alcoholism, Syphilitic Disease of the Liver, Epileptiform Neuralgia, Capillary Bronchitis, Plastic Bronchitis, Dilatation of the Bronchia, Fibroid Degeneration of the Lung, Chronic Pyæmia, \&c. \&c. 


\section{Trousseau's Clinical Lectures.}

VOI. III. NOW READY.

Lectures on Clinical Medicine, delivered at the Hotel-dieu, Paris. By A. Trousseau, Professor of Clinical Medicine in the Faculty of Medi. cine, Paris, \&c., \&c.

Trousseau's Lectures on Clinical Medicine, so favorably receired, as well by the profession of the United States as abroad, are published in this country in connection with the New Sydenham Society, nnder whose auspices the translation of Vols. II. and III. have been made. Either of these volumes can be furnished separately, and in order to still further extend the circulation of so valuable a work, the Publishers have now reduced the price to Five Dollars per volume.

Contents of Votume I.-Translated and Edited by P. Victor Bazire, M. D., \&c.Lecture 1. On Tenesection in Cerebral 11 morrlage and Apoplexy. 2. On Apoplectiform Cerebral Congestion, and its Relations to Epilepsy and Eclampsia. 3. On Epilepsy. 4. On Epileptiform Neuralgia. 5. On Glosso-laryngeal Paralysis. 6. Progressive Locomotor Ataxy. 7. Un Aphasia. 8. Progressive Muscular Atrophy. 9. Facial l'aralysis, or Bell's l'aralysis. 10. Cross-paralysis, or Alternate Hemiplegia. 11. Infantile Convulsions. 12. Eelampsia of Pregnant and Parturient Tomen. 13. On Tetany. 14. On Chorea. 15. Senile Trembling and I'aralysis Agitans. 16. Cerebral Fever. 17. On Neuralgia. 18. Cerebral Rhenmatism. 19. Exophthalmic Goître, or Graves' Disease. 20. Angina Pectoris. 21. Asthma. 22. Hooping Cough. 23. On Hydrophobia.

Contexts of Volume II.-Translated from the Edition of 1868 (being the last revised and enlarged cdition), by John Rose Cormack, M. D., Edin., F.R.S.E. \&.c. - Leacture 1. Small-pox. 2. Variolous Inoculation. 3. Cow-pox. 4. Chicken-pox. 5. Scarlatina. 6. Measles, and in particular its unfavorable Symptoms and Complications. 7. Rubeola. 8. Erythema Nodosum. 9. Erythema Papulatum. 10. Erysipelas, and in particular Erysipelas of the Face. 11. Humps. 12. Urticaria. 13. Zona, or Ilerpes Zoster. 14. Sudoral Exanthemata. 15. Dothinenteria, or Typhoid Fever. 16. Typhus. 17. Membranous Sore Throat, and in particular llerpes ot the Pharynx. 18. Gangrenous Sore Throat. 19. Inflammatory Sore Throat. 20. Diphtheria. 21. Thrush.

Costexts of Tolune IH. - Translated from the Edition of 1868, by John Rose Cormack, M.D., Edin., F.R.S E., \&c.-Lecture 22. Spe\&ific Element in Disease. 23. Contagion. 24. Ozæna. 25. Stridulous Laryngitis, or False Croup. 26. Edema of the Larynx. 27. Aphonia: Canterization of the Larynx. 28. Dilatation of the Bronchi and Bronchorrhoa. 29. Hemoptysis. 30. Pulmonary Phthisis. 31. Gangrene of the Lung. 32. Pleurisy: l'aracentesis of the Chest. 33. Trammatic Effusion of Blood into the Pleura: Paracentesis of the Chest. 84. Hydatids of the Lung. 35. Pulmonary Abscesses and Peripneumonic Tomice. 36. Treatment of Pueumonia. 37. Paracentesis of the Pericardium. 38. Organic Affections of the Heart. 52. Alcoholism. 62. Spermatorrhwa. 63. Nacturnal lncontinence of Urine. 64. Glucosuria: Saccharine Diabetes. 65. Polydipsia. 67. Vertigo a Stomacho Lreso.

3 Volumes Octavo, handsomely bound in Cloth, Price $\$ 500$ each.

\section{OPINIONS OF THE PRESS.}

"Trousseau furnishes us with an example of the best kind of Clinical teaching. It is a book that deserves to be popularized. The translation is perfect." - Yedicul Times and Gazetle.

"The great reputation of Prof. Trousseau as a practitioner and teacher of Medicine in all its branches, renders the present appearance of his Clinical Leatures particularly "relcome." - Medical Pross and Circular.

"The publication of Trousseau's Lectures will furnish us with one of the very best practical treatises on discase as seen at the bedsile." - British and Foreign HedicoChirurgical Review.

"A clever translation of Prof. Trousseau's admirable and exhaustire work, the best back of reference upon the Practice of Medicine." - Indian Medical Gazette.

- The Lectures of Trousseau, in attractivencss of manner and richness of thoroughly practical matter, worthily takes a place beside the classical lectures of Watson and Graveg." - British Medical Journal.

"Trousseau is essentially the French Graves, and his lectures should sooner than this have been translated into English." - Lancet. 


\section{Meigs and Pepper on Children. \\ FOURTH EDITION, ENLARGED AND IMPROVED.}

The publishers have selected the following notice, from a late number of the London Lancet, of the New Edition of this work, as indicating, nerhaps, more fully than any other of the numerous favorable eritieisms that bare appeared of it elsewhere, its great value to the Practitioner and Student of Mledicine.

"It is not necessary to say much, in the way of eriticism, of a work so well known as Meigs on Diseases of Clilliren,' especially when it his reached a fourth edition. Our duty is welluigh restricted to the point of ascertaining how far, under an old color, it preserves the freshness and the vilue of a new book - how fal it incorporates what is new with what is old withont unseemly marks of mere joining, There is some advantage in starting entirely atresh, in being merely elinical, or in being very short, and limiting one's self to the expression of one's own views and experience. But such is not the rature of this book, and the advantages of it are different. It is a work of more than 900 good American pages, and is more eneyclopredial than elinical. But it is clinical, and withul most effectually brought up to the light, pathological and therapentical, of the present day.

"The book is like so many other good American dical books which we have lately Lad occasion 10 notice; it marvellously combines a résumé of all the best European literature and practice with evidence throughout of gond personal judgment, knowledge, and experience. It is gratifying to see how our English author's are quoted, and especially how the labor's of Ilillier, who died so prematurely, are recognized. But the book abounds in exposition of Ameriean experience and observation in all that relates to the diseases of children. Not the least interesting additions to the volume are several extensive tables, exhibiting the mortality in I'hilatelphia of some of the most common and fatal diseases in comnection with the rariations of the temperature, aud prepared with great care from the recolds of the Board of Health.

"The thoroughty fresh nature of the book is especially seen in the care with which certain articles have been written. Such are those on Rickets and Tuberculosis, Intantile Atrophic Paralysis, and Progressire Paralysis. No book now on diseases of ehildren is complete which does not treat specially of constitutional or diathetic diseases, such as rickets and tuberculosis, syphilis, \&ic.

"Among other articles of great interest am value we would mention those on Disenses of the Cacum and Appendix Vermiformis, on Indigestion in Children, on Diarrhosa, on Entero-Calitis, on Intussusception, on Chronic IIydrocephalus, and on Croup and the value of Tracheotomy.

"The difỉculties of eliting a new edition of a medieal book of some standing are not more felt in the region of pathology and the classitication of disease than in that of therapeutics. In this work this difficulty has been fairly faced by the authors. They have to confess to having changed their practice very materially in the treatment of acute diseases, to having given up mercury in most intlammatory diseases, and almost given up bloolletting. We recommend the views of these authors as to the injurious eflects of calomel and antimony to careful consideration. 'They do not entirely abjure the use of bloolletting in cerrain cases of pneumonia and meningitis. Indeed, we think they will find reason in future editions to talk a little less freely than they do about bleeding and cupping very young children in certain circumstances of pneunonin, and in certain cases of simple meningitis. With a few exceptions of this kind, the therapentics are sound aud commeudible, great importance being given to proper feeding and the general manarement of infancy and childhood. It is due to authors of so much fairness and experience to publish widely their opinion of the injurious and depressing effects of antimony in the inflammations of chizdren. To infants under two years of age they thiuk it best to give no antimony even in pneumonia. They do tot use tartar emetic at all in the cases of children, but small doses, such as the twelfth of a grain. of the precipitated sulphuret of antimony, every two, three, or four hours, watehing its effects, and withdrawing it quickly if symptoms of prostration appear, perhaps without any vomiting.

"We are glad to add this work to our library. There are few diseases of children which it. does not treat of tully and wisely in the light of the latest physiological, pathological, and therapeutical science." - London Lancet, July 23, 1870.

l'rice, handsomely bound in Cloth.

" "

LINDSAY \& BLAKISTON, PUBLISHERS, 


\title{
Cazeaux's Great Work on Obstetrics.
}

\section{THE MOST COMPLETE TEXT-BOOK NOW PUBLISHED.}

\author{
GREATLY ENLARGED AND IMPROVED.
}

\section{CONTAINING 175 ILLUSTRATIONS.}

A Theoretical and Practical Treatise on Midwifery, including the Diseases of Pregnancy and Parturiticn, by P. Cazeaux, Member of the Imperint Academy of Medicine; Adjunct Professor in the Faculty of Mledicine of Paris, etc., etc. Revised and Annotated by S. TArnier, Adjunct I'rofessor in the Faculty of Medicine of Paris; Former Clinical Chief of the Lying-in-Hospital, elc., elc. Fifth American from the Seventh French Edi. tion. Translated by WM. R. BulLock, M.D. In one volume Royal Octavo, of over 1100 pages, with numerous Lithographic and other Illustrations on Wood.

Price, bound in Cloth, bevelled boards, " " Leather, . . . . . . 7.50

M. Cazcaux's Great Work on Obstetrics has become classical in its character, and almost an Encyclopædia in its fulness. Written expressly for the use of students of medicine, and those of midwifery especially, its tenchings are plain and explicit, presenting a condensed summary of the leading principles established by the masters of the obstetric art, and such clear, practical directions for the management of the pregnant, parturient, and puerperal states, as have been sanctioned by the most authoritative practitioners, and confirmed by the author's own experience. Collecting his materials from the writings of the entire body of antecedent writers, carefully testing their correctness and value by his own daily experience, and rejecting all such as were falsified by the numerous cases brought under his own immediate observation, he has formed out of them a body of doctrine, and a system of practical rules, which he illustrates and enforees in the clearest and most simple manner possible.

\section{OPINIONS OF THE PRESS.}

"It is nnquestionably a work of the highest excellence, rich in information, and perhaps fuller in details than any text-book with which we are acquainted. The author bas not merely treated of every question which relates to the business of parturition, but he has done so with judgment and ability." British and Foreign Medico-Chirurgical Review.

"The translation of Dr. Bullock is remarkably well done. We can recommend this work to those espeeially interested in the subjects treated, and can especially recommend the American edition." Medical Times and Gazette.

"The edition before us is one of unquestionable excellence. Every portion of it has undergone a thorough revision, and no little modification; while copious and important additions have been made to nearly every part of it. It is well and beautifully illustrated by numcrous wood and lithographio engravings, and, in typographical exeeution, will bear a favorable comparison with other works of the same class."-American Medical Journal.

"In the multitudinous collection of works devoted to the propagation of human beings, and to the details of parturition, none, in our estimation, bears any comparison to the work of Cazeaux, iu its entire perfectness; and if we were called upon to rely alone on one work on accouebments, our choice would fall upon the book before us without any kind of hesitation." - West. Jour. of Med. and Surgery.

"We do not hesitate to say, that it is now the most complete and best treatise on the subject in the English language."- Buffalo Medical Journal.

"We know of no work on this all-important branch of our profession that we can recommend to the itudent or practitioner as a safe guide before this."-Chicago Medical Journal.

"Among the many valuable treatises on the science and art of obstetrics, the work of Cazeaux stands pre-eminent."-St. Louis Med. and Surg. Journal.

"M. Cazeaux's book is the most complete we have ever seen upon the snhject. It is well translated. sad reflects great credit upon $\mathrm{D}-$. Bullock's intelligence and industry." $-\boldsymbol{N}$. A. Medirs-Chirurg. Revieu 


\section{LINDSAY \& BLAKISTON'S}

MEDICAL PUBLICATIONS.

"The Representative Book of Medical Science."-London Lancet.

\section{Aitken's Science and Practice of Medicine.}

SECOND AMERICAN from the FIFTH LONDON EDITION.

In 2 Volumes, containing 2000 Royal Octavo Pages, a COLORED MAP, a LITHOGRAPIIIC PLATE, and ONE HUNDRED AND TIIIRT ILLUSTRATIONS ON WOOD.

Price, bound in Cloth, bevelled boards . . . . . \$12.00

" " . . . . . . . . 14 Leather

Dr. Aitken's work is now the most comprehensive Text-Book on the Practice of Medicine in the English Language; tho present edition has been enlarged and carefully revised by the author, as well as by the American editor, Meninitu CLYMFR, M.D., the latter having made additions of original matter equal to nearly 500 pages of tine Eusclish Edition, with special reference to the wants of the Americau Practitioner.

\section{Acton on the Functions and Disorders of the Reproductive Organs.}

Second American from the Fourth London Edition. Carefully revised by the author, with additions. Just Ready, octavo, . . . \$3.00

"We thlnk Mr. Acton hse done good service to society by grappling manfully with sexusl vice, and we trust that others, whose position as men of science and teachers enable then to speak with anthority, will egsist in comlrating and arresting the evils which it entails. We are of the opinion that the spirit which pervales it is one that does credit equally to the head and to the heart of the author." - The British and Foreign Medica-Chirurgical Revieno.

\section{Anstic on Stimulants and Narcotics.}

Their Arutual Relations, with Special Researches on the Action of Alcohol, Ether, and Chloroform on the Vital Organism. By Francis E. Anstie, MI.D., Assistant Physician to Westminster Hospital, Lecturer on Mrateria Medica and Therapeutics, etc., etc. Octavo, . . . . \$33.00

\section{Althans' Medical Electricity. A New and Very JIuch Enlarged Edition.}

A Theoretical and Practical Treatise, and its Use in the Treatment of Paralysis, Neuralgia, and other Diseases. By Julius Althava, M. D., Nember of the Royal College of Physicians, \&e. Second Edition, revised, enlarged, and for the most part rewritten. In One Volume Octavo, with a Lithographic Plate and sixty-sico Illustrations on Wood Price, 


\section{WORKS BY DR. LIONEL S. BEALE, F.R.S.,}

Fellow of the Royal College of Plysicians, Physician to King's College Hospital.

Seventh Thousand. Four Hundred Illustrations, some Colored.

HOW TO WORK WITH THE MICROSCOPE.

This work is a complete manual of microscopical manipulation, and contain a ful description of many new processes of investigation, with directions for examining objects under the highest powers, and for taking photographs of microscopic objec s. Octavo. Cloth. Price, \$7.50.

\section{ON KIDNEY DISEASES, URINARY DEPOSITS, AND CALCULOUS}

DISORDERS: - The Tliril Edition.

Including the Symptoms, Diugnosis, and Treatment of Urinary Diseases. With full Directions for the Chemical and Nicroscopical Analysis of the Urine in Health and Disease. 70 Plates, 415 figures, copiel from Nature. Octavo. P'rice, $\$ 10.00$.

\section{THE USE OF THE MICROSCOPE IN PRACTICAL MEDICINE.}

For Students aud Practitioner's, with tull directions for examining the rarious secretions, fic.. in the Microscope. 4th Edition. 500 lllustrations. Octaro. Price, $\$ \mathbf{7 . 0 0}$

MEDICAL PROGRESS. IN MEMORIAM ROBERT BENTLEY'TODD. l'rice, 50 cts.

\section{LIFE, MATTER, AND MIND; OR PROTOPLASM.}

With Original Observations on Hinute Structure, and numerous New Colored Drawings. Sccond Édition, very much enluryed. Eight Plutes. Pricc, \$3.00.

DISEASE GERMS: THEIR SUPPOSED NATURE. With Colored Plates.

An Original Investigation with the Aid of the llighest Powers yet made.

This work contains a critical examination of Dr. Tyndall's statements concerning 'Uust and Disease.' 12mo. Cloth. Price, \$1.75.

DISEASE GERMS: THEIR REAL NATURE. Ticenty-four Plates.

An Original Investigation. Twenty-four Plates, sixteen of which are Colored.

Including the minute pathology of contagious diseases. Price, $\$ 1.00$.

$$
\text { Nearly ready. Four Colorcd Plates. }
$$

PHYSICAL THEORIES OF LIFE: THEIR INFLUENCE UPON RELIGIOUS THOUGHT.

With Observations on the IIypotheses recently adrocated by Tyndall and Huxley.

\section{ON DISEASES OF THE LIVER, AND THEIR TREATMENT.}

A Second Edition, much enlarget, of the Author's Work on the Anatomy of the Liver. Numerous Plates. Preparing.

THE ARCHIVES OF MEDICINE. Part XVII, now ready. Price, \$1.50.

Contexts.-IIedicine under the Liomans-Action of Eyelids in Expiration; F.

C. Donders, Utrecht-Lacunæ and Canaliculi-Nerves in Mescnteric Glands; Dr. Toppcr, St. Petersburg-German Criticism and British Medical Science-Homology of Coracoacromial Ligament; J. B. Perrin-Sulpho-carbolates, by Dr. SansomStructure of the Liver; Editor-Sputum in Phthisis.

DISEASE : ITS NATURE AND TREATMENT. Preparing.

New Rescarches on Inflammation and Fever, and on the Nature of Contagion. With Observations on the Cattle Plague and on Cholera. Numerous Colored Plates. Being the Third Course of Lectures delivered at Oxford by direction of the liadeliffe Trustees.

All these Worlis contain the results of the Author's original investigations. They are Illustrated with upwards of 2000 Engravings, eopierl from the actual oljects, all of which huve been drawn on wood by the Author himself, or under his immediute superintendenee.

LINDSAY \& BLAKISTON, Publishers, Phiadelphia. 


\section{Beasley's Book of Prescriptions.}

\section{A NEW, REVISED, AND ENLARGED EDITION.}

Containing 3000 Prescriptions, collected from the Practice of the most Eminent Physicians and Surgeons - English, French, and American; comprising also a Compendious History of the Materia Medica, Lists of the Doses of all Officinal and Established Preparations, and an Index of Diseases and their Remedies. By Henny Beasley. Second American from the last London Edition. Octavo. . . • . $\$ 4.00$

"The editor, carefully selecting from the mass of materials at his disposal, has compiled a volume, in which both physician and druggist, prescriber and compounder. may find, under the heal of each remody, the natnner in which that renuely may be nost effectively adninistered, or combined with other medicinee in the treatment of various disease日. Tho alphabetical urrangenent adopted renders this easy; and the value of the volume is still further enhanced by the short acconnt giren of each medicine, and the liste of doses of ita several preparations. It is really a most useful aud important fublication, and, from the great aid which it la capalle of affording in prescribing, should be in the possession of every medical practitioner. Amongst ather atvantiges is, that, by giving the prescriptions of some of the most able and successful practitioners of the day, it uffords au insight into the methorls of trentment pursued by them, and of the remedies which they chiefly employed in the treatment of different diseuses." - Lancet.

\section{Beasley's Druggists' General Receipt Book.}

\section{SIXTH AMERICAN EDITION, REVISED AND IMPROVED.}

Comprising a copious Veterinary Formulary, numerous Receipts of Patent and Proprietary IIcdicincs, Druggists' Nostrums, etc.; Perfumery and Cosmetics, Beverages, Dictetic Articles and Condiments, Trade Chemicals, Scientific Processes, and an Appendix of Useful Tables, by Henry Beasley, Author of the Book of Prcseriptions, etc., etc. Sixth American from the Last London Edition. Octavo. $3.50

"This is one of the class of books that is indispensahle to every Druggist and Pharmaceutist as a book of reference for such information as is wanted, not contuined in works used in the regular line of his business, and we can recunmend it as one of the best of the kind." - American Druggists' Circular.

\section{Barth \& Roger's Manual of Auscultation and Percussion.}

A new American Translation from the Sixth French Edition. 16mo. \$1.25.

"This is nne of the most nseful and practical manuals of its sort that has ever yet appeared, and we crunot too strongly recommend it to every student of medicine. It is sufficiently comprehensive without being lengthy, and the principles, which are emiuently sound, can easily be mastered and understood." - Dedical Record.

\section{Bouchardat's Annual Abstract}

Of Therapeutics, Materia Menica, Pharmacy, and Toxicology, For 1867, with an Original Mlemoir of Gout, Gravel, Urinary Cal. culi, \&c. By A. Bouchardat, Professor of Hygiene to the Facully of Medicine, Paris, \&c. Translated and Edited by M. J. De RosseT, M.D., Adjunct to the Professor of Chemistry in the University of Mlaryland. In one Volume. Price, in cloth, . . \$1.50. 
Andrews' Hand-Book of the Practice of Medicine. In Preparation.

\section{Bull on the Maternal Management of Chil- dren in Health and Disease.}

A New and Improved Edition. By Thomas Bull, M. D., Ilember of the Royal College of Physicians. 12mo. . . . . . \$1.25

Reports on the Progress of Medicine, Surgery, and the Allied Sciences. Containing:

1. Report on Pirsiologr, by Henty Power, F.R.C.S., M.B., Lond.

2. Report on Practical Medicive, by Francis Edmund Anstie, M.D., etc.

3. Repolrt on Surgery, by T. Holmes, Esq., M.A., F.R.C.S., etc.

4. Report on Ophthalmic Medicine and Surgery, by Thomas Windsor, M.D.

5. Report on Midwfery and the Diseases of Women axd Children, by R. Barnes, M.D., F.R.C.P.

6. Report on Medical Jurispredexce, by C. Hilton Fagge, M.D., F.R.C.P.

7. Refort on Materia Medica and General Therapeutics, by C. Hilton Fagge, M.D., F.R.C.P.

8. Report on Public Health, by C. Hilton Fagge, M.D., etc.

In One Tolume, handsomely bound in cloth, Price,

"This volnme, publisbed under the anspices of the New Sydenham Society, now makes its welcome appear. ence, at a low price, in the United States. It is the most carefully prepared retrospect with which we are scquainted. Each department is in charge of a gentleman of reputation, and everything is done to sum. marize, in a very reatiale way, all the more important advances of medicine over the globe. It is hand. comely bound and elegantly printed."-N. Y. Medical Record.

Birch on Constipation. From the Third London Edition. CONSTIPATED BOIVELS; the various Causes and the Different Means of Cure. By S. B. Brirci, M. D., Member of the Royal College of Physicians of London, \&c. The Third Edition. One volume, 16 mo.

Price,

\section{Braithwaite's Epitome of the Retrospect of Practical Medicine and Surgery.}

Two large Octavo Volumes of 900 pages each, . . . \$10.06

\section{Braithwaite's Retrospect of Practical Medi- cine and Surgery.}

"The cream of medical literature."

Published half-yearly, in January and July, at $\$ 2.50$ per annum, if paid in alvance; or $\$ 1.50$ for single parts. 


\section{British and Foreign Medico-Chirurgical Re- view, and Quarterly Journal of Practical Medicine and Surgery.}

Published in London on the 1st of January, April, July, and October, at 6 slillings per number, or 24 shillings per annum, and furnished in this country at $\$ 10.00$ per annum; being much less than the present cost of importation of English books. Containing Analytical and Critical Reviews, a Bibliographical Record, Original Communications, and a Chron. icle of Dledical Science, chiefly Foreign and Contemporary.

This is considered the leading Medieal Review in the English language. It is everywhere looked upon as high authority. It presents in its pages a large amount of valuable and interesting matter, and will post the physician who reads it, fully up to the present day in medical literature.

\section{Chambers's Lectures on the Renewal of Life.}

\section{A New American from the Fourth London Edition.}

Lectures chiefy clinical, illustrative of a Restorative System of Mledicine. By Thos. K. Chinuers, M. D., Physician to St. MIary's Hospital. Author of "The Indigestions," \&c., \&c. Octaro, . . \$5.00

"The medical profession in this country are under obligntions to the American publishers for this reprint of Dr. Chambers' Leetures - a work whose time is forever, cverywhere in its place, admirable in tone, full of raluable instructions and practical teachings, and written in elear, compact, and often epigrammatic English. We can offer but a brief notice of this iutrinsically good book, which is certain of finding a wide circle of readers, and we should hope a place in overy medical library."-Neı York Wedical Juurnal.

"This work is of the highest merit, written in a clear, nasterly style, and devoid of technicalities. It is simply what it professes to be, Lectures Clinieal, delivered from cases olserved at the betside; therefore more valuable as enunciating the views and experiences of a practicul mind aided by retnal observation. They are of deep interest, and replete with facts having a practical bearing, aud will well repay perusal. . . . . We can recommenil Dr. Chambers' book freely and witb confidence, as the work of a great mind practical in its bearing, and simple to the nuderstanding of all." - Canada Aedical Journal.

\section{Chew on Medical Education.}

A Course of Lectures on the Proper Method of Studying Medicine. By Samuel Chew, MI.D., Professor of the Practice and Principles of Medicine and of Clinical Medicine in the University of Maryland. $12 \mathrm{mo}$.

\footnotetext{
* The topics discusserl in this volnme are of books - of time to be devoted to study — and the manner - of the order of medical studies - of the taking of notes - of clinical instruction - dissections - auscultation meuical schools, \&c

"Dr. Chew was an eminevt member of the medical profession, and a well-known teacher of medicine. Ilo was, thererore, well fitted for the judicious performance of this task, upon which he secms to bave entered with interest and pleasure. It is a well-timed book, and will serve as a most excellent mannal for thestuden as well a a refreshing and suggentive one to the practitioner." - Luncet and Observer. 


\section{Cazeaux's Great Work on Obstetrics.}

\section{The Fifth American Edition. 175 Illustrations.}

A Theoretical and Practical Treatise on Midwifery. Including the Diseases of Pregnancy and Parturition, and the attention required by the Child from its Birth to the Period of Weaning. BY P. CAzEAUx, Member of the Imperial Academy of Medicine, Adjunct Professor in the Faculty of Hedicine of Paris, \&c., \&c. Revised and annotated by S. Taknin, Aljunct Professor to the Faculty of Medicine, Paris, ic., \&e. Translated by W. R. Bullock, M. D. With new Lithographic and other Illustrations on Wood. One volume Royal Octavo, of over 1100 pages.

Price, bound in Cloth, Bevelled Boards, . . . . . . $\$ 6.50$ " ". Leather, . . . . 7.50

"Written expressly for the nse of students of medicine, and those of midwifery especially, its teachings are phuin and explicit, prenenting a condensal smmary of the leading principles establinhed by the mastergof tre obstetric art, and auch c'ear. practicat directions for the mangement of the pregnant, pat turient, and pus, neral states, as have been sanctined ly the most anthoritative practitioners, aud confirmed by the mithor s own experience. Collectiug his materials from the writings of the entire boly of antecedeut writers, carefully testing their correctness and whe l,y his own daily experjence, nnd rejecting all snch as were filajfed by the uumerous cases bronght under his own immerliate olservation, he lats formed ont of them a body of doctrine, and a system of practical rules, which he illustrates and eutorces in the clearest and most simple munuer possible." - Examaner.

\section{Canniff's Manual of the Principles of Surgery.}

Based on Pathology, for Students, by Wr. CAxsiff, Licentiate of the Medical Board of Lpper Canada; M.D. of the University of New York; M.R.C.S. of England; formerly House Surgeon to the Seamen's Hospital, New Fork; late Professor of General Pathology and the Principles and Practice of Surgery, Cnirersity Victoria College. C. W. Octaro.

"This mammal is evidently the production of a man who is well informed on his sulject, and who moreover bas hat cxperience as it teacher arel as a practitioner. Jle has profitel ly the study of the hest anthors on the principies of enrgery, tested prictically tleir doctrines, abl has gresented his own riews, well arrangtd and cleariy expressed, for the advantige of others." - A merican Joumal of Med. Science.

\section{Cleaveland's Pronouncing Medical Lexicon.}

\section{A NEW AND IMPROVED EDITION (THE EILEVENTH).}

Containing the Correct Promunciation and Definition of most of the Terms used by Speaters and Writers of Wedicine and the Collateral Sciences. By C. H. Cleavelaxd, M.D., Nember of the American Medical Association, etc., etc. A small Pocket Volume. . . . . . \$ \$1.25

This little work is both hrief and comprehensive; it is not only a Lexicon of all the words in common use in Iledicine. but it is also a Pronouncing Dictionary, a feature of great value to lledical Students. To the Dispenser it will prove an excellent aid, and also to the Pharmaceutical Student. This edition contains a List of the Abbrevis. tions used in Prescriptions, together with their meaning; and also of Poisons and their Antidotes: two valuable additions. It has received strong commendation both from the Medical Preas and from the profession. 


\section{Cohen on Inhalation.}

Its Therapentics and Practice. A Treatise on the Inhalation of Gases, Vapors, Nebulized Fluids, and Powders; including a Description of the Apparatus employed, and a Record of Numerous Experiments Physiological and Pathological; with Cases and Illustrations. By I. Solis Collen, M.D. 12mo. Price, . . . . . $\$ 250$

"We recognize in this bonk the work of a persevering Physician who has fa; thfully studied his subject, and alled to its literature much that is useful from his owu experience. It treats respectively of the inhalations of nebulized fluids; of medicated airs, gases, and zapors, and of powders. Dr. Cohen has given us briefly and clearly whatever is valuable in relation to the insuflation of powders in respiratory affections, with the experimental proofs and pathologieal evidence of their penetration into the bronchial tubes and lung tissues." - American Journal of Medical Science, July, 1868.

\section{Prof. Carson's University of Pennsylvania.}

A History of the Mledical Department of the University of Pennsylvania, from its Foundation in 1765: With sketches of the Lires of Deceased Professors. By Josepil Canson, M.D., Professor of Materia Hedica and Pharmacy in the University of Pennsylvania; Hember of the American Philosophical Society, etc. In one volume octavo. Cloth. Price, .

$\$ 200$

"The history of the University of Pennsylvania has a national as well as a loca, interest, from the early date of its origination, and the connection with it of men of illustrious public reputation, such as Drs. Franklin, Rush, Physick, Gibson, Dewees, Chapman, Wood, \&c., \&c.

"For fidelity and carefulness of statement and maintenance of the dignity of the Institution, as well as for skill in literary execution, the task of extending and continuing this record cuuld bave been confided to no better hands than those of Professor Carson.

"For the labor and love which he has spent in preparing this most interesting and valuable work, Prof. Carson has earned the gratitude of the alumni of the University, and of all others interested in medical education in this country." - American Joumal of Medical Science.

\section{Dixon on the Eye. $\}$ A New Edition, thoroughly Revised, and a great portion Re-written.}

A Guide to the Practical Study of Diseases of the Eye, with an Outline of their Medical and Operative Treatment, with Test Types and Illustrations. By Jaurs Dixon, F.R.C.S., Surgeon to the Royal London Ophthalmic Hospital, \&c., \&c. In one volume. Price, . \$2 50

"Mr. Dixon's book is essentially a practical one, written by an observant author, who brings to his special subject a sound knowledge of general Medicine and Surgery." - Dublin Quarterly.

"Our object is not to review, but to recommend this work to students, with the confdent assurance that they will rarely be disappointed in their appeals to it as a reliabie guide to the practical study of the Diseases of the Eye." - American Medical Journai.

"We have taken great pleasure in a sareful perusal of this book, which, both in styre and matter, is unsurpassed in any language. It embraces quite a wide range of topics, and furnishes a very valuable practical guide in the medical and surgical treatucat of diseases of the eye." - Buffalo Medieal Journal. 


\section{Durkee on Gonorrhœa and Syphilis.}

\section{The Fifth Edition, Revised and Enlarged, with Portraits and Colored Illustrations.}

A Treatise on. Gonorrhoca and Syphilis. By Sruas Durkee, M.D., Fel low of the Massachusetts Medical Society, \&.c., \&.c. A New and Revised Edition, with Eight Colored Illustrations. Octavo. . . \$ \$5.00

This work of Dr. DurkeE's has received the unqualified approval of the Nedical Press and the Profession both in this country and in Europe. The author has deroted himself especially to the treatment of this class of discases, and his 25 or 30 years experience in doing so is here recorded. No one reading his work can fail in receiving very valuable information from it.

"It is the work of a praetical man, the subject is treated in a plain, shrewd manner. The book is a good one, and the therapeutics aro laid down with discrimination." - London Medical Times and Gazelte.

"Dr. Durkee's production is one of those, the perusal of which impresses the reader in favor of the author. The general tone, the thorough honesty every where eviuced, the f hilanthropis spirit observable in many pasBages, and the energetic advocacy of professional ructimde, slieak highly of the moral excellence of the writer; nor is the resder bess attracted by the skill with which the book is arranged, the manner in which the facts are cited, the clever way in which the author's experience is lironeht in, and the lucidity of the reawoning. the frequent anil extremely fair allusinus to the labors of others, and the care with which the the rapeutics of venereal complaints are treated." - Laneet.

\section{Fuller on Rheumatism, Rheumatic Gout, and}

\section{Sciatica. a New EDITION PREPARING.}

Their Pathology, Symptoms, and Tratment. By Hexry Wildias Fuller, M.D., Fellow of the Royal College of Plysicians, London; Physician to St. George's Hospital, cte. From the last London Edition. Octavo.

\section{Graves' Clinical Lectures on the Practice of} Medigille. By Robert Janes Graves, M.D., F.R.S., Professor of the Institutes of Medicine in the School of Physic in Ireland. Edited by J. Moone Meligav, M.D. From the Second Revised and Enlarged Edition. Complete in One Tolume. Octaro. Price, . . $\$ 6.00$

Goff's Combined Day-Book, Ledger, and Daity Register of Patients, combining not only the Accuracy and Essential Puints of a regular Day-Book and Ledger System, without any of the labor and responsibitity, but is also a Daily Register of Patients, \&c., \&c. A large Quarto Volume, strongly bound in half-russia. Price, \$12.00 The alvantages of this book are - The account of a whole family for an entire year can be kept in a very small space. (See Mitchell's account.) No transfer of accounts from one book to another, or from one part of the book to another. No protracted search fin an account when wanted. Shows the exact state of an account at any moment. 


\section{Gross' American Medical Biography of the Nineteenth Century.}

Edited by Samuen D. Gross, M.D., Professor of Surgery in the Jefferson Medical College, Philadelphia, \&:e., \&·c. With a Portrait of Bexuamin Rush, M.D. Octavo. • • • • • • • • $\$ 3.50$

\section{Greenhow on Bronchitis, especially as Connected with}

Gout, Emphysema, and Diseases of the Heart. By E. Headuan Green How, M.D., Fellow of the Royal College of Physicians, \&c., \&c.

Price, \$2.00

"In vivid pictures of the sort of chses which a practitioner encounters in his daily walka, and in examples cf the way in which a student onght to turn them over in his mint and make them tools for self-improvewent, we have rarely seen a volnine richer." - Brit. and For. Bedico-Chirurg. Review.

\section{Garratt's ( Alfred C.) Guide for Using Medical Batteries.}

Showing the most approived Apparatus, Methods, and Rules for the Medical Employment of Electricity in the Treatment of Nerrous Diseases, \&.c., \&.c. With numcrous Illustrations. One Volume, octavo. \$2.00

"The large work on the same subject, and by the same autlior, is pretty well known to the Profesaion, but It is bulky and cumbrous, and by no means so jractically usefnl. The present comparatively brief volume contains every thing of importance in regart to the varions apparatuses useful to the Medical Electrician. and the various modes of uplication tor therapentic purposes." - Lancet and Observer.

\section{Hewitt on the Diseases of Women.}

\section{SECOND EDITION, REWRITTEN AND ENLARGED.}

The Diagnosis and Treatment of Diseases of Women, including the Diamosis of Pregnancy. Funnded on a Course of Lectures delivered at st. Mary's Hospital Hedical School. By Graily Hewite, M. D. Lond., M. R. C. I'., I'hysician to the British Lying-in Hospitad; Lecturer on Miduifiry and Diseases of Women and Children at St. Mary's Hospital Medical Sithonl; Honorary Secretary to the Obstetrical So. ciety of London, ide. With a new Series of Illustrations.

P'rice, in cloth, $\$ 5.00$; in leather, $\$ 6.00$.

\section{Hillier's Clinical Treatise on the Diseases of}

Children. By Thomas IILlier, M.D., Physician to the Hospital for Sick Children, and to University College Hospital, \&c., \&c. Octavo.

Price, $\$ 3.00$

"Our space is exhausted, but we have said enough to indicate and illustrate the excellence of Dr. Llillier"s rolume. It is uminently the kind of book needed by all medical men who wish to cultivate clivical sor racy. and sound practice." - London Lancet. 


\section{Headland on the Action of Medicines in the System.}

By F. W. Headuand, M.D., Fellow of the lioyal College of Physicians. \&.c., \&.c. Sixth American from the Fourth London Edition. Reviscd and enlarged. One Volume, ortavo. . . . . . \$3.nn

Dr. Headland's work has been out of print in this country nearly two years, arrniting the revirions of the althor, which now appear in this edition. It gives the only scientific and satisfactory view of the action of medicine; and this not in the way of idle speculation, but by demonstration and experiments, and inferences almost as indisputable as demonstrations. It is truly a great scientific work in a small compass, snd deserves to be the handbook of every lorer of the Profession. It has received the most unqualified approbation of the Medical Press, both in this country and in Europe, and is pronounced by them to bo the most original and practically useful work that has bcen published for many years.

\section{Hille's Pocket Mristomist.}

Being a Complete Description of the Anatomy of the Human Body; for tho Use of Students. By M. W. HiLLEs, formerly Lecturer on Anatomy and Physiology at the Westminster Hospital School of Dredicine.

Price, in cloth, . . . . . . . . $\$ 1.00$

" in Pocket-book form, . . . . . . . . 1.25

\section{Heath on the Injuries and Diseases of the Jaws.}

The Jacksonian Prize Essay of the Royal College of Surgrons of England, 1967. By Christopher II Eati, F. R. C. S., Assistant Surgeon to Univeraty College Hospital, and Téacher of Operative Surgery in University College. Containing over 150 Illustrations. Octavo. Price, $\$ 6.00$ Hodge on Foticide, or Criminal Abortion

By Huan L. IIodar, M. D., Emerilus Professor in the University of Penn:yluania. A Small Pocket Volume. Price in paper covers, 30

flexible cloth, 50

This little book is intended to place in the hands of professional men and others the means of answering atisfactorily and intelligently any inquiries that may be made of them in connection with this imprtient subject.

\section{Holmes' Surgical Diseases of Infancy and}

Childhood. By J. Housies, M.A., Surgeon to the Hospital for Si:k Children, \&c. Second Edition. Revised and Enlarged. Octavo. Price, $\$ 9.00$

\section{IHufeland's Art of Prolonging Life. Edited br}

Erasmus Witison, M.D., F R.S. Author of "A System of Human Anatomy," "Diseases of the Skin," \&ce, \&c. 12mo. Cloth." \$1.25 


\section{Mackenzie on the Laryngoscope, Diseases of the Throat, \&c. Second Edition.}

The Use of the Laryngoscope in Diseases of the Throat. With additions, and an Essay on Hoarseness, Loss of Voice, and Stridulous Breathing in relation to Nervo-Muscular affections of the Larynx, by MonELJ Mackenzie, M.D., Physician to the Hospital for Diseases of the Throat, \&c., \&c. Second Edition, with additions, and a Chapter on the Nasal Passages, by J. Sorrs Conen, M.D., Author of "Inhalation, Its" Thera. peutics and Practice," \&c. Illustrated by two lithographic plates, and 51 engravings on wood. Octavo. Price, . . . . $\$ 3.00$

"Thile laryngoscopy was in its infaney, ond hefore it had begun to engage fo any extent the attention of the Profession. it was stmlied with the gieatest care amt enthusism by thit anthor of this treatixe. A peraonal friend of Czermak's, who has detie wore thin any uther contiuental physician to introduce the latryugoscope into practice, be bas profited by the ofpurtunities which he thus possixsed of beconing aejuainterl with the anatomy and mortid anatomy of the lerynx. But he has done much mere thitn this. As will be seen by a perusal of this trentise, he his mobified the instruments at parsent in use for the eximination of tho larynx, and has inventell others for theripentical purpinet. Those who are anxious to stuly the diseases of Whe baynx and the mode of using the laryntoscope, 'annot do better than parehase the treatise before as, as it is by fir tho best which has been published, aud is thoior ahy to be relied upon."-Glasgow Medical Journal.

\section{Morris on the Pathology and Therapeutics of Scarlet Fever.}

By Casper Morris, M.D., Fellow of the College of Physicians of Philadelphia, \&c., \&.c. A New Enlarged Fistion. Octavo. . \$1.50

\section{Meigs and Pepper's Practical Treatise on the Discases of Children.}

Fourth Edition, thoroughly Revised and greatly Enlarged.

By J. Forsyth Meigs, M. D., Fellow of the College of Physicians of Philadelphia, \&c., fe., and WILIsam Pepper, M.D., Physician to the Philadelphia Hospital, de., de., forming a Royal Octavo Volume of over 900 pages. Price, bound in Cloth, . . . $\$ \$ 6.00$

Leather,

Dr. Meigs' work las been out of print for some years. The rapid sale of the three previous enlition, and the creat demand for a new edition, is sufficient evidence of its great popularity: while the very large practice of many yeat's' siaudiso of the anthor in the speciality of "Disetses of Chilulen," insparts to it a value unquallei, probably, by any otlier work on the sume subject now before the Profession. This present edition has beril alnost entirely rewritten and rearranged, and no effort or labor has been spared by either Drs. Meigr

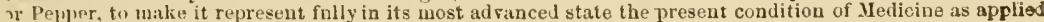
to Children's Diseases.

\section{Murply's Review of Chemistry for Students.}

Adapted to this Courses as Tanght in the Principal Medical Schools in the United Sixles. By Jonx G. Murpmr, M.D. In One Volume. \$1.25.

" whis is an exceedingly well-arranged and converlent Manual. It gives the most important facis and principles of Chemistry in a clear and very concise manner, so as to subserve most admirably the alject for which It was designed" - North Western Afedical and Surgical Journal. 


\section{Maxson's Practice of Medicine.}

a New Text-Book on the Practice of Mcclicine. By Edwin R. Maxson, M.D., formerly Lecturer on the Institutes and Practice of Mledicine in the Geneva Medical College. In One Volume. Royal 8ro. . \$4.00

\footnotetext{
"Judging from his work, he must te a correct observer, of plain, atrong common senso, baving the proress and perfection of the healing art, and the amelioration of sufferiug, enrnestly at hoart, freo from pre fulice, and open to conviction. The fact of employing, aml thereupon reconmending valuable remedis ageots, as yet, for various reasons, under the lan, and misnulerntood liy uany fibysicians, is an honor to aim, and gives a certain achiitiona! value to his book." - Americun Medical Monlhly.
}

\section{Mendenhall's Medical Student's Vade Mecum.}

A Compendium of Anatomy, Physiology, Chemistry, The Practice of Medicine, Surgery, Obstetrics, Diseases of the Skin, Materia Medica, Pharmacy, I'oisons, \&c., \&c. By George MI exdeximal, MI.D., Professor of Obstetrics in the Mcdical College of Ohio, Member of the American Medical Association, \&c., \&c. The Eiglth Edition, Revised and Enlarged; with 224 Illustrations. . . . . . . $\$ 2.50$

"This volnme puts the stndent in possession of a condensed medical library. Its accuracy is a strong recommendition, while the portibility of a rolume containing the wlwle circle of medical science is a matter that will have weiglit with thoso for whose servict tho luok was originally designed. The work is offered, tow, extremely cheap, aud will he found a valuablo assistant even to a woll-lnformed practitioner of any branch of isedicine." - Boston Malical and Sargical Journal.

\section{Pagret's Lectures on Surgical Pathology.}

Delivered at the Royal College of Surgeons of England, by Janes Paget, F.R.S., Surgeon to Bartholomew and Christ's Ilospital, \&.c., \&.c. The Third American from the Second London Edition, Edited and Revised by Wrillian Tumer, MI.B., Lond. Senior Demonstrator of Anatomy in the University of Edinburgh, \&c., d'c. In One Volume, Royal Octavo; with Numerous Illustrations.

Price, in bevelled cloth,

" in leather, . . . . . . . . . . 7.00

\section{Pennsylvania Hospital Reports. Edited by a Com-} mittee of the Hospital Staff, J. M. DaCosta, MI.D., and William Hunt, M.D. Tols. 1 and 2 , for 1868 and 1869 , each rolume containing upacards of Twenty Original Articles, by former and present llembers of the Staff, now eminent in the Profession, with Lithographic and other Illustrations. Price per volume,

$\$ 4.00$

At last, however, the work bas been commenced, the Philadelphia Plysicians beirg the first to occupy this field of usefulness, having issued the first rolume of the Reports of the above hospital last year, and the second volume on January 1st, 1869 . The first Reports were so favorably received on both sides of the Atlantic, that it is hardlv necessary to speak for this volume the universal welcome of which it is deserving. We cannot close our remarks without stating that the papers are all valnable contributions to the literature of medicine, reflecting great credit upon their authors, and the work is one of which the Pennsylvania Hospital may well be proucl. It will do much toward derating the profession of this country in the estimation of their foreign brethren." 


\section{Pereira's Physician's Prescription Book.}

Containing Lists of Terms, Phrases, Contractions, and Abbreviations, usen in Prescriptions, with Explanatory Notes, the Grammatical Constructions of Prcseriptions, Rules for the Pronunciation of Pharmaccutical Terms, A Prosodiacal Vocabulary of the Names of Drugs, etc., and a series of Abbreviated Prescriptions illustrating the use of the preceding terns, etc.; to which is added a Kcy, containing the Preseriptions in an unablreviated Form, with a Litcral Translation, intended for the use of Medical and Phamaceutical Students. By Joxathan Pereira, M.D., F.R.S., etc. From the Fourteenth Londoin Edition.

Price, in cloth, . . . . . . . . \$1.25 " in leather, with Tucks and Pocket, . . . 1.50

This little work has passed through fourteen editions in London and several in this country. The present edition of which this is a reprint has been carefully revised and many additions made to it. Its great value is proven both by its large sale and the many farorable notices of it in the Medical Press.

\section{Physicians Visiting List. Published annually.}

\section{SIZES AN], PRICE.}

For 25 Patients weekly. Tucks, pockets, and pencil, . . . . . . $\$ 100$

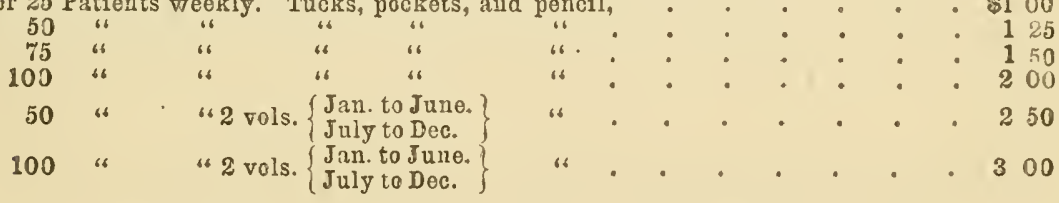

\section{Also, AN INTERLEAVED EDITION,}

for the use of Country Plysicions and others who enmpound their own Preseriptions. or furnish Medicines to their patients. The additional pages can also be used for Speciul II moranda, recording important cases, Se., Sc.

For 25 Patients weekjy, interleaved, tucks, pockets, etc., . . . . . \$1 50

50 " " " " " . . . 175

50 "s "2 vols. $\left\{\begin{array}{l}\text { Jan. to June. } \\ \text { July to Doc. }\end{array}\right.$ " " . . . . 300

\section{Prince's Orthopedic Surgery.}

Ortnopedics: A Systcmatic Work upon the Prevention and Cure of Deformities. By David Prince, M.D. With Numerons Illustrations. Octavo.

"This is a good book, upon an important practical suljoct; carefully written, abundintly illustrated, uud vell printed. It gors over the whole ground of deformities of ail degrees - from cleft-palate and club-foot, to cpinal curvatures and ununited fractures. It appears, moreover, to be an original book, so far as one chiefly of cumpilation can be so. Such a book was wanted, and it deserves euccess." - Ned. \& Surg. Reperter.

\section{Prince's Plastic Surgery.}

4 New Classification and a Brief Exposition of Plastic Surgery. By David Prince, M. D. In One Volume Qctavo. With Numerous Illus. trations. Price, . . . . . . . . . $\$ 1.50$ 


\title{
Radcliffe's Lectures on Epilepsy, Pain, Pa- ralysis,
}

And certain other Disorders of the Nervous System, by Charies Bland Radcliffe, MI.D., Fellow of the Royal College of Physicians of London, Physician to the Testminster Hospital, etc., etc. With Illustrations. 12mo. . . . . . . . . . . . $\$ 2.00$

\begin{abstract}
"The reputation which Dr. Radcliffe possesses as a very able anthority on nervons affections, will commend h1s work to every medical practitioner. Disorders of the nervons system are very inperfectly comprehender, uncli concerning them being involved in mystery; and while Dr. Radcliffe has taken alvantage of the ampls loom to theorize, which his subject afforded, he has not failed to bring forwaril strong and formidable facts to prove the deductions lie attempts to draw. We recommend it to the notice of our readers as a work that will) throw much light upon the Pliysiology and Pathology of the Nervous System." - Cunada Medical Journal.
\end{abstract}

\section{Robertson's Manual on Extracting Teeth.}

Founded on the Anatomy of the Parts involved in the Opcration; the Kinds and Proper Construction of the Instruments to be used; the Accidents liable to occur from the Opcration, and the Proper Remetlics to retrieve such Accidents. By Amrailay Robritsox, D.D.S., M.D., Author of "Priže Essay on Extracting Tceth," \&e. In One Volume, with Illustrations. Second Edition. Revised aud Improved. • . $\$ 1.50$

"The anthor is well kunw as a contributor to the literature of the Profession; and, as a clear, terse, forsil, le writur, he has dewoted consilernble care to tho subject, and treated it with his usual alility. The work is valuahle, mot unly to the dental student and practitioner, but also to the nedical stulent and surgeon: and especially so to the milit:iry surgeon, who, in times like the present, is called upos so frenuently to perforn the operation of extracting teeth." - Dental Cusmos.

\section{Ranking's Half-yearly Abstract of the Medi- cal Sciences.}

Price, per annum, if paid in advance, $\$ 2.50$. IIalf-yearly volumes, $\$ 1.50$. 'The first thirty-two volumes, bound in sixteen volumes, leather, can be furnished each at $\$ 2.00$. Half-yearly volumes, in paper eovers, from 1 to 34 , each at $\$ 1.00$.

\section{Renouard's History of Medicine.}

History of Medicine from its origin to the Nineteenth Century. With an Appendix containing a Philosophical and Historical Review of Medi. cine to the present time. By P. V. ReNOUARD, M.D. Translated from the French by Convelius G. Coneays, M. D., Professor of the Institutes of Hedicine in the Mledical College of Ohio, ete. In One Volume Octavo. Price, . . . . . . . $\$ 4.00$

"Yrom the pages of Dr. Benonard, a rery accurate acquaintance may be obtiined with the history of medisino-its relation to civilization, its progress compared with other sciences and arts, its nost distinguished caltivators with the several theories and systems proposed by them, and its relationship to the reigning phib. wesphicil dogmas of the several periods. Ilis historical uarration is clear aul concise, tracing the progresa of medicine through its three ages or epochs - that of foundation or origin, that of tradition. aud that of reuoratiou."-American Journal of Medical Science.

"The best history of medicine extant, and one that will find a place in the library of every physician who sims at an acquaintance with the past history of his profession. There are many items in it we should like w offer for the iustruction and amusement of our readers." - American Journal of Pharmacy. 


\section{Ryan's Philosophy of Marriage.}

In its Social, Moral, and Physical Relations, with an Account of the Diseases of thie Genito-Urinary Organs. The Physiology of Generation in the Animal and Vegetable Kingdoms, \&c., \&e. By Michael Ryan, M.D., Nember of the Royal College of Physicians and Surgeons in London, \&c. $12 \mathrm{mo}$. .

«Dr. Ryan if above reproach or suspicion; and with a singular degree of candor and independence, explaing in s true and philosophical manner, every lranch of the subject which be considers essential to be ander stood by all intelligent persons." - Boston Medical and Surgical Journal.

\section{Reese's Analysis of Physiology.}

Being a Condensed View of the most important Facts and Doctrines, designed especially for the Use of Students. By JonN J. ReEse, M.D., Professor of Medical Jurisprudence, including Toxicology, in the University of Pennsylvania, \&c., \&c. Second Edition, Enlarged. $12 \mathrm{mo} . \$ 1.50$

\section{Reese's American Medical Formulary.} $12 \mathrm{mo}$

\section{Reese's Syllabus of Medical Chemistry.}

\section{Stillé's Epidemic Meningitis;}

Or, Cerebro-Spinal Meningitis. By Alfred Strlité, M.D., Professor of the Theory and Practice of Medicine in the University of Pennsylvania. \&.e. \& e. In One Volume Octavo. . . . . . \$2.00

"This monograph is a timely publication, comprehensive in its scope, and presenting within amall compass a fair digest of our existing knowledge of the disease, particularly acceptable at the present tine. It is just such a one as is needed, and may be taken as a model for similar works." - Am. Journal Med. Sciences.

\section{Sydenham Society's Publications. New Series, 1859}

to 1870 inclusive, 12 years, 50 vols. Subscriptions received, and back years furnished at $\$ 10.00$ per year. Full prospectus, with the Reports of the Society, and Lists of Books published, furnished free upon application.

\section{Stillé's Elements of General Pathology.}

A Practical Treatise on the Causes, Forms, Symptoms, and Results of Dus. ease. By Alfred Stillé, M.D., Professor of the Theory and Practice. of Dedicine in the University of Pennsylvania, \&ie.

(In Preparation.). 


\section{Sansom on Chloroform.}

Its Action and Administration, by Arthur Ernest SAnsom, M.I3., Physician to King's College Hospital, etc., etc. 12mo. . . . $\$ 2.00$

"The work of Dr. Sansom may be characterized as most excellent. Written not alone from a theoretical print of view, hut showing very considerable experimental study, and an intimate clinical acquaintance with the administration of these remedies, - passing concisely over the whole ground, giving tha latest information apon every point, - it is just the work for the student and practitioner. The author may rest ussured that, although in his preface he oljects to the 'hackneyed expression of endeavoring to supply a want,' this is just what he has done - supplied and well supplied a want, for no such book existed before in our language." American Medical Joumal.

\section{Scanzoni's Practical Treatise on the Diseases of the Sexual Organs of Women.}

Translated from the French of Drs. H. Dor and A. Socis, and annotated with the approval of the authors. By A. K. Garderer, A.MI., M.D., Professor of Clinical Midwifery, \&c., \&c., in the New York Medical College. Wilh Numerous Illustrations. Octavo. . . . \$5.00

In the etiology, pathology, and therapeutics of female diseases, with all the improvements which have been realized during the last twenty years, this volume is exceedingly rich; while in its arrangement it is so methodical that it must constitute one of the best text-books for students, and one of the most reliable aids to the busy practitioner.

\section{Stokes on the Diseases of the Heart and the Aorta.}

By IVilliam Stokes, Regius Professor of Phasic in the University of Dublin; Author of the Treatment and Diagnosis of the Diseases of the Chest, \&c., \&c. Second American Edition. Octavo. - . \$3.00

\section{Spratt's Obstetrical Tables.}

Comprising Graphic Mlustrations, with Lesrriptions and Practical Remarks exhibiting on Dissected Plates many important subjects in Mídwifery. By G. SPRATt, Surgeon Accoucheur. First American from the Fourth and Greally Improved London Edition, carefully Revised, with Additional Notes and CoLoned Plates. One Volume Quarto. Price, . . . . . . . . . . \$3.00

\section{Skoda on Auscultation and Percussion.}

By Josepi SKoda. Translated from the Fourth German Edition, by W. O. Markinam, M.D., Assistant Physician to St. Mary's Hospilal. 12 mo. 


\section{Tanner's Practice of Medicine.}

\section{FIFTH AMERICAN EDITION.}

The Practice of Medicine, iy Thonas Hawkes Tanner, M.D., Fellow of the Royal College of Physicians, Author of A Practical Treatise on the Diseases of Infancy and Childhood, etc., etc. Fifth American from the Sixth London Edition. Greatly Enlarged and Improved.

Price, bound in cloth, . . . . . . . \$6.00 " " in leather $\quad . \quad . \quad . \quad . \quad .700$

Dr. Tanner's work on the Practice of Medicine is so well known in this country, and has had such an extensive and rapid sale, that it seems almost unnecessary to say anything iu reference to it; the present edition, however, contains such substantial additions and altcrations as almost to constitute it a new work, and from being a comparatively small volume it now forms a haudsome octavo of nearly 1000 pages; all that was useful and practical in the smaller volume has been retained and much new matter added, written in the same condensed and easy style.

"The leading feature of this book lo its essentially practical character. Dr. Tanner has produced a mors complote System of Medicine than any with which we are acquaibted. It is the result of long experience and hard practice, and it is therefore valuable as a guide, utd trustworthy as an exemplar." - iondon Lancet.

\section{Tanner's Practical Treatise on the Diseases of Infancy and Childhood.}

\section{Octavo.}

This book differs from other works of the kind, in embracing a wider range of subjects than is usually contained in treatises on children's diseases; besides the ordinary complaints of those subjects, it includes many affections which, though common to adults and children, yet offer some modification in form, or in the indications for treatment, when occurring in the latter. Thus, we hare an account of diseases of the eye, ear, and skin, of small-pox, scrofula, tuberculosis, syphilis, bronchocele, and cretinism, diseases of the kidneys and genital organs, and some of the accidents common to childhood. The style of the work is condensed, and the book might with truth be called a manual, rather than a treatise, but there is nothing superficial about it; - every.thing really important is given, while the discussion of disputed subjects, and, in fact, of everything which is not of practical importance in the study and treatment of children's diseases, is omitted.

\section{Tanner's Index of Diseases and their Treat- ment.}

With upwards of 500 Formula for Medicines, Baths, Dineral Waters, Climates for Invulids, \&c., \&.c. Octavo. . . . . . \$3.00

"Dr. Tanner has been peculiarly bappy in apprecinting and supptying the wants of the Profession. IIis Index of Diseases gives the derivation of words after the manner of a good Medical Dictionary; an outline of every disease, including many surgical discases, with their symptoms and mode of treatment; an admiruble collectiou of Formula, asd an account of the clinates of the various parts of the world suitalle for invalids. It also contains at the beginning of the work a tabular syuopsis of subjects, which does double duty at once, a Nosology and an index. It will be found a most valuable companion to the judicioue practitioner." -- Lancet.

\section{Tanner's Memoranda of Poisons.}




\section{Troussean's Lectures on Clinical Medicine.}

Delivered at the Hotel Dieu, Paris, by A. Trousseau, Professor of Clini. arl Medicine in the Faculty of Medieine, Paris. Translated and edited, with Notes and Appendices, by P. Victone Bazire, MI.D., Assistc ut Pliysician to the National Hospital for the Paralyzed and Epileptic, \&e. Volume One. Cloth, . . . . . . . . . 500 Volume Two, . . . . . . . . . 500 Folume Three, now Ready, . . . . . . . . 500

"This book fnrnishes us with an example of the best kind of clinical teachiug, and we are mnch indebted to the translator for siaplying the Profession with these almirable l.ectures. It is a book which deserves te be popularized. We scarcely know of auy work hetter fitted for jresentation to a young num when entering apon the practical work of his life. The delineation of the recorded cases is graplaic, and their narration deroid of that prohixity which, desirable as it is fir puryoses of extendeel nulysis, is highly undesirable when the object is to joint to a practical lesson." - Lomion Medical Tomes and Guzette.

\section{Tyler Smith's Obstetrics.}

A Course of Leetures. By Willan Trlere Surti, M.D., Physician, Acvoncheur, and Lecturer on Midwifery, and the Diseases of Females, in S't. Mary's Hospital, Medieal School, \&e., \&e. With Nimerous Illustrations. Edited by A. K. Gariver, M.D., Fellow of the New York Academy of Medicine, \&c., \&e. Octavo. . . . . . . . $\$ 5.00$

l'oynbee on Diseases of the Ear. Their Nature, Diagnosis, and Treatment. A new London Edition, with a Supplement. By Jasies Hinton, Aural Surgeon to Guy's Hospitul, de. With Illustrations. Octavo. . . . . Price, $\$ 5.00$

\section{Thompson's Clinical Lectures on Pulmonary Consumption. \\ Octavo. $\$ 2.00$.}

\section{Tyson's Cell Doctrine:}

Its History and Present State, with a Copious Bibliography of the Sub. ject, for the use of Students of Medicine and Dentistry. By Janes Trson, M. D., Lecturer on Microscopy in the University of Pennsylvamia, \&.c., \&.c. In One Tolume, with a Colored Plate, and numerous Illustrations on Wood. Price, . • . . . $\$ 2.00$

\section{Tilt's Elements of Health, and Principles of Female Hygiene.}

By F. J. Trlt, M.D., Senior Physician to the Lying-in Charity, Author of Works on the Diseases of DIenstruation, Uterine Therapeutics, \&e., \&.c. $12 \mathrm{mo}$. . . . . . . . . . . $\$ 150$

"Dr. Tilt divides life into the septennial epochs so Jong adopted by philosophers and medical nen, liscussing, under the different ages, the physical and moral relations, diseases, \&c., peculiar to ench. The chapter devoted to the age from fourteen to twenty-one years contain much valuable advice respecting the menstrual function during that period. Tables showing the value of life at each of the different periods of life, are wpended in their proper places; and the work also contains other statistics of value and interest. The wholo work has been prepared with great care, and contaius a large amount of valuable information, which professional mer. may coneult with profit." $-N$. $F$. Medical Times. 


\section{Taylor's Theory and Practice of the Move- ment-Cure.}

Or, the Treatment of Lateral Curvature of the Spine, Paralysis, Indigestion, Constipation, Consumption, Angular Curvatures, and other Defornities, Diseases Incident to Women, Derangements of the Nervous System, and other Chronic Affections, by the Swedish System of Localized MIovements. By Chari.es TAYLor, M.D. With Illustrations. 12mo. - $\$ 1.50$

The work of Dr. Taylor is a systematic treatise, containing the principles on which this treatment is based, and full and explicit directions in their application to individual diseases. The author diseusses the nutritive processes, muscular contraction, and the physiology of general exercise, the subjects of the first three chapters, in a most satisfactory manner. The work is purely of a scientific character, and commends itself as such to the attention of all physicians.

\section{Virchow's Cellular Pathology.}

As based upon Pliysiological and Pathological History. Translated from the Second Edition of the Original. By Frank Chance, B.A., M.A., Cantab Licentiate of the Royal College of Physicians, \&·c., \& $\&$. With Notes and Numerous Emendations, principally from MISS. Notes of the Author, and Illustrated by 144 Engravings. Octavo. . . $\$ \$ 5.00$

Prof. Virehow and his writings are well known wherever the science of medicine is studied. This work has been selected by the Medical Bureau of the United States for general distribution in the hospitals and medical stations of the army; recording, as it does, the researches in this branch of science down to the present time.

The importance of the subject, the new ideas advanced, and the established reputation of the author, induced the publication of this book, and has made it a standard work throughout Europe and in this country.

\section{Virchow on Morbid Tumors.}

\section{IN PREPARATION.}

\section{Walker on Intermarriage.}

Or, the Mode in which, and the Causes why, Beauty, Health, and Intellect result from certain Unions, and Deformity, Disease, and Insanity from others. With Illustrations. By Alexander Walker, Author of "Woman," "Beauty," \&c., \& c. 12 mo.

* The author is evidently a careful observer, and a proper thinker, and has presented ns with a vast amount of information, derived woth from man and the inferior animals. He has aimed to be useful, by pointing out now bodily deformities and mental infirmities may be forestalled; and how marriages among blood relationa tend to the degeneracy of the offspring. He also shows how, by carefully assorted marriages, the means of inıproving general organization and beanty of countenance, as well as mental and physical vigor, are, in a great degree, under the control of man. Although not strictly a medical work, we cannot refrain from com. mending it to the pe usal of the Prefsesion, as it contain much that is valuable in a hygienic point of view." Ledical Examiner 


\section{Wythes' Physician's Pocket, Dose, and Symp- tom Book.}

Containing the Doses and Uses of all the Principal Articles of the Drateria Medica, and Original Preparations; A Table of Weights and Mea. sures, Rules to Proportion the Doses of Medicines, Common Abbren viations used in Writing Prescriptions, Table of Poisons and Antidotes, Classification of the MIateria MIcdica, Dietetic Preparations, Table of Symptomatology, Outlines of General Pathology and Therapeutics, \&c. By Josepn H. Wrtmes, A.M., M.D., \&c. The Eighth Revised Edition.

Price, in cloth, . . . . . . . . . $\$ 1.00$ " leather, tucks, with pockets, . . . . . 1.25

This little manual has been reccived with much favor, and a large number of copies sold. It was compiled for the assistance of students, and to furnish a vade mecum for the general practitioner, which would save the trouble of reference to larger and more elaborate works. The present edition has undergone a careful revision. The therapeutical arrangement of the Materia Medica has been added to it, together with sucis other improvements as it was thought might prove of value to the work.

\section{Waring's Manual of Practical Therapeutics.}

Considered chiefly with reference to Articles of the Materia Medica. By Edward Join Waring, F.R.C.S., F.L.S., \&c., \&c. From the Second London Edition. Royal Octavo.

Price, in cloth, $\cdot+. \quad \$ 6.00$ " in lcather, . . . . . . . . 7.00

There are many features in Dr. Waring's Therapeutics which render it especially valuable to the Practitioner and Student of Medicine, much important sand reliable in. formation being found in it not contained in similar works; it also differs from them in its completeness, the convenience of its arrangement, and the greater prominence given to the medicina? application of the various articles of the Materia Medica in the treatment of morbid conditions of the Human Body, \&c., \&c. It is divided into two parts, the alphabetical arrangement being adopted throughout the volume. For the further convenience of the reade: there is also added an INDEx of DISEAsEs, with a list of the medicines applicable as remedies, and a full InDEx of the medicines and preparations noticed in the work.

\section{"Our admiration, not only for the immense industry of the suthor, but also of the great prastical value of the volute, lacrenses with every reading or consultation of it. We wish a copy could be put in the liands w every student or practitioner in the country. In our estimation it is the best book of the kind ever mritten." - N. Y. Meuical Journal. \\ IVeber's Clinical Hand-Book of Auscultation and Percussion.}

An Exposition from First Principles of the Method of Tnvestigating Diseases of the Re:spiratory and Circulating Organs. Tianslated by Joun Cockse, M. D. With Illustrations. Price, . . \$1.0 


\section{Walton's Operative Ophthalmic Surgery.}

By Harnes Walpon, F.R.C.S., Surgeon to the Central London Ophthab. mic Hospital, \&c. With 169 Illustrations. Edited by S. Littel L, M.D., Surgeon to the Wills Hospital for the Diseases of the Eye, \&c. Octavo.

"It is eminently a practical work, evincing in its author great research, a thorough knowledge of his subfect, and an accurate and most observing mind." - Dublin Quarterly Journal.

\section{Watson's Practice abridged.}

A Synopsis of the Lectures on the Principles and Practice of Physic. Delivered at King's College, London, by Thomas Watson, M.D., Fellow of the Royal College of Physicians, \&c., \&.c. From the last London Edition. With a concise but Complete Account of the Properties, Uses, Preparations, Doses, \& 8. of all the Medicines mentioned in these Lectures, and other Valuable Additions, by J. J. MEYLor, A.M., M.D., \&c., \&c. A neat Focket Volume bound in cloth flexible. . . . $\$ 2.00$

\section{Wells' 'Treatise on the Diseases of the Eye,}

illustrated by Ophthalmoscopic Plates and Numerous Engravings on Food. By J. Selebere WV Elds, Ophthalmic Surgeon to King's College Huspital, \&e. Second london Edition, cloth, $\$ 6.50$; leather, $\$ 7.50$.

This is the anthor's own edition, printerl in London under his supervision, and issued in this country by special arrangement with him.

\section{Wright on Headaches.}

Their Causes and their Cure. By Henry G. Whight, M.D., Member of the Royal College of Physiciuns, \& c. \& c. From the Fourth London Edition. $12 \mathrm{mo}$. Cloth.

"Few affections are more unmanageable and more troublesome than those of which this essay treats; and we doubt not that any suggestions by which we can relieve them will be gladly received by physicians. The author's plan is simple and practical. He treats of headaches in childhood and youth, in adult life and old age, giving in each their varietses and symptoms, and their causes and treatment. It is a most satisfactory monograph, as the mere tact that this is a reprint of the fourth edition, testifies.

"The great pains which the author takes to clear up the differential diagnosis of the different varieties, and establish a satisiactory basis for rational treatment, are everywhere visible. While such a valuable fund of information is offered to the practitioner at the cost of a single visit, he should not let his patient suffer for want of it." Medial and Surgical Reporter.

Wells on Long, Short, and Weak Sight, and their Treatment by the Scientific Use of Spectacles. Third Eitition Re. vised, with Additions and Numerous Illustrations. By J. SoenBera WELLS. Octavo. 


\section{LINDSAY \& BLAKISTON,}

No. 25 South Sixth Street, above Chestnut,

PHILADELPHIA.

\section{Marris's Principles and Practice of Dentistry.}

THE TENTII REVISED EDITION.

In great part lie-written, Re-arranged, and with many

New and Important Illustrations.

\section{INCLUDING}

1. Dental Anatony and Plysiology.

2. Dental Pathology and Therapentics.
3. Dental Surgery.

4. Dental Mechanics.

By Chapix A. Harris, M.D., D.D.S., \&c. The tenth edition, revised and edited by P. H. Austex, M.D., Professor of Dental Science and Mechanism in the Baltimore College of Dental Surgery, with nearly 400 Illustrations, ineluding many new ones made especially for this edition.

One volume, Royal Octavo, bound in Cloth, . . . . $\$ 6.50$

" " " Leather, . . 7.50

The subscribers, in presenting to the Dental Profession Time Texti Revised Edition of the late Prof. Harris's Text-Book of Dentistry, desire to call their attention to the thorough revision, and morlification in its arrangement which it has been found neccssary to make, in order to bring it fully up to the present advanced state of the science, and to preserve for it that reputation which it has for so many years sustained, as being the first and leading book on the subject in the English language.

So great have been the adrances in Dental Physiology, Pathology, Surgery, and Mechanism, that a revision in all its parts, more complete and thorough than has been made to any previous edition - amounting, in some cases, to a re-writing of the articles or subjects - had become imperative; and in order to have this done in the most efficient manner, the publishers were, fortunately, able to secure the valuable services of Prof. Austen, of the Baltimore Dental College, who, alded to his experience of twenty years as a teacher, was, for a long time, the associate and friend of Dr. Harris. He was thus peculiarly fitted for this task. In order, however, to render the work the more perfect and acceptable to the Profession, Dr. Austen associated with him Prof. Gorgas and Thomas S. Latimer, M.D., of the same school, both gentlemen of acknowledged proficiency in their respective departments, and further obtained valuable original articles from Prof. Kingsley, of the New York Dental College, whose reputation is well known, and other gentlemen of the Profession of equal repute in their respective specialties. The publishers, therefore, offer this edition to the Profession with the utmost confilence that it will be found most complete in every respect as a text-book for the student and a guide and companion for the experienced practitioner. 


\section{Harris's Dictionary.}

THE THIRD REVISED ED."TION.

A Dictionary of Medical Terminology, Dental Surgery, and 'The Colllateral Scrences. By Chapin A. Harris, M.D., D.D.S., Profcssor of the Principles of Dental Surgery in the Baltimore College, Nember of the American Medical Association, \&.c. \&c. The Third Edition, carefully revised and enlarged, by FERdinand J. S. Gorgas, M.D., D.D.S., Professor of Dental Surgery in the Baltimore College, \&.c., \&.e. In one volume, Royal Octavo, bound in Cloth, . $\$ \$ 6.50$

$$
\text { " " " } " 7.50
$$

For a long period after the death of Dr. Harris his Dictionary remained out of print, but a constantly increasing demand for it from the Profession and from students urged the publishers to the preparation of a new edition. The many advances in dental science made during this interim rendered the incorporation of many new terms and formulix absolutely necessary to the student of Dentistry, as well as to the dental practitioner. The present edition has been thoroughly revised by Prof. Gorgas, Dr. Harris's successor iu the Baltimore Dental College; and in a very satisfictory manner, nearly three thousand new words have been incorporated into it, besides additions and corrections being made to many others. The doses of the more prominent medicinal as "nts have also been added, and in every way the book has been greatly improved, and its value enhauced as a work of reference. To those studying or practising Dentistry it must prove invaluable.

\section{Heath on the Injuries and Diseases of the Jaws.}

The Jacksonian Prize Essay of the Royal College of Surgeons of England, 1867. By Cirristopher Heath, F.R.C.S., Assistant Surgeon to University College Hospital, and Teacher of Operative Surgery in University College. Containing over 150 Illustrations. Octavo, . . \$6.00

This work is of the highest practical value. It treats of dislocations, fractures, deformities, and other disorders of the jaws, with the means for relief, illustrated by drawings of displacements, apparatus, norbid growths, portraits of patients, etc., to the number of 154. It embodies American as well as European experience, and contains quite a large appendix of cases in detail. The practical character of its contents, with good paper, print, and engravings, strongly commend this book to the profession. Every surgeon and dentist should have a copy. - Dental Cosmos.

It is impossible to over-estimate the value and importance of this comprehensive work. - British Journal of Dental Science.

The concise descriptions and excellent arrangement make this monograph valuable for reference. - British Medical Journal.

\section{Coles on Deformities of the Mouth,}

Congenital and Acquired, with their Mechanical Treatment. BY JAMES OAKley Coles, D.D.S., Nember of the Odontological Society, \&c., \& c. Sccond Edition, Revised and Enlarged, with 8 Colored Engravings and 51 Illustrations on Wood.

The second edition of this work shows that the anthor has continued to derote himself with zeal to the investigation and treatment of a very interesting class of cases. Mr. Coles has especially stuclied the congenital cleft palate, and has, with the mirror, detected, in several cases, growths in the naso-pharyngeal tonsil. Very beautiful colored drawings are given in illustration of the subject of cleft palate. Mr. Coles gives the preference to mechanical treatment, in both congenital and pathological perforations of the palate, and his experience as to the good results obtained is certainly most encouraging. We recommend the work to the study of both surgeons and dentists. - - London Lancet. 


\section{Taft's Practical Treatise on Operative Den-}

\section{tistry. a New EDITION, THOROUGHLY REVISED.}

By Jonathan Taft, D.D.S., Professor of Operative Dentistry in the Ohio College of Dental Surgery, \&.c. The Second Edition, thoroughly Revised, with additions, and fully brought up to the present state of the Science. Contuining over 100 Illustrutions. Octavo. Leather, . .\$4.50

The first edition of Dr. Taft's work imparted a new impulse to the practice of Oper. ative Dentistry. In the early days of the profession, excellence, as an operator, was attainable only by long years of industrious practice. Now, many of the best operators are found among those comparatively young; and perhaps no single agency has done more to produce this result than this volume of l'rofessor Taft in systematically and clearly setting forth the attained truths of our science in such a way as to be acces sible to all.

The second edition contains nearly fifty additional pages of matter, and the whole work is carefully revised, with a labor but little, if any, short of the preparation of an entirely new volume. - Dental Register

The first edition of this "Practical Treatise on Operative Dentistry," by Prof. Taft, published in 1859 , has beenme very familiar to the dental practitioner and student, and its value universally acknowlerlged. It is with pleasure, therefore. that we note the issue of a revised edition, which the author's intustry has made still more worthy of professional attention. - American Journal of Dental Seience.

Professor Taft has done good service to the profession in thus emborlying, in a separate volume, a comprehensive view of Operatire Dentistry. This gentlemnu's position as a teacher must have renlered him familia. with the most recent views which are entertained in Americi on this matter. while his extensive experience and well-earned reputation in practice must bave rendered him a competent judge of their merits. We willingly comment Prof. 'Taft's able and useful work to the notice of the profession. London Lental lieview.

\section{Richardson's Practical Treatise on Mechani cal Dentistry. sacoxd editrox, much extarged.}

By Josepit Ricmardsox, D.D.S., Professor of Mechunical Dentistry in the Ohio College of Dental Surgery, \& c. With over 150 beautifully executed Illustrations. Octavo. Leather . . . . . \$4.50

When the first edition of this work malle its appearance, we gave a favorable notice of it. It is only necessary now to add that the second contains all the improvements in this department of Dentistry of the past ten years. Many additions have been made and of her parts have been entirely re-rritten. - Dental Cosmos.

Dr. Richardson's work is justly regarded as indispensable to the dentist. Rejecting all that is useless, and conreying his ideas without the verbiage in which too many encumber their thoughts, he has given to us a concise statement of what is to be done by the mechanical dentist, and how to do it.-Dental Laboratory.

Prof. Richarison is thoroughly and minutely acquainted with every topic he attempts to discuss, both in theory and praetice. He exhibits a kuowledge of the hidden nooks and corners of the dental laboratory quite refreshing, in view of the superficial treatment the subject has usually received. II selects one great and important branch of dental science, and exhansts it, placing it in such plain and practical form so that every student sees and understauds its merits at a glance. $-\Lambda$. I. Dental Journal.

This work loes infinite credit to its author. Its comprehensive style has in no way interfered with most elaborate details where this is neeessary; and the numerous and beautifully executer wood-cuts with which it is illustrated rerifler this volume as attractive as its instruetions are easily understood. - Edinburgh Med. Journal.

After a careful perusal, we have no hesitation in commending the book to our readers as a gool practical work, the illustratious of which greatly surpass, so far as we are aware, any other American work on the subject. - London Lancet.

The scope of the whole work is thoroughly carried out, and to any one desiring a theoretical knowledge of Dental Mechanics, Dr. Richardson's book will be found a most efficient guide. - British and Foreign Medico-Chirurg. Review. 


\section{Robertson's Manual on Extracting Teeth.}

\section{A NEW REVISED EDITION.}

Founded on the anatomy of the parts involved in the opcration, the kinds and proper construction of the instruments to be used, the accidents likely to occur from the operation, and the proper remedics to retrieve such accidents. By A. Robertson, M.D., D.D.S., \&c. Second Edition. \$1.50.

The author is well known as a contributor to the literature of the profession, and as a clear, terse, and practical writer. The subject is one to which he has devoted consilerable attention, and is treated with his usual eare and ability. The work is valualle, not only to the dental student and practitioner, but also to the medical student and surgeon. - Dental Cosmos.

\section{Bond's Practical Treatise on Dental Med- icine. FOUR'TH EDITION IN PREPARATION.}

\section{'T'omes' System of Dental Surgery.}

With 208 Illustrations

\section{Fox on the Human Teeth.}

Their Natural History, Structure, and Treatment of the Diseases to which they are Subject. With 250 Illustrations. . . . . \$4.00

\section{Sansom on Chloroform.}

Its Action and Administration, by Arthur Ernest Sansom, M.B.,

Physician to King's College Hospital, \&c., \&c. 12mo . . \$2.00

The work of Dr. Sansom may be charncterized as most excellent. Written not alone from a theoretical point of view, but showing very considerable experimental study, and an intimate clinical acquaintance with the administration of these remedies; passing concisely over the whole ground, giving the latest information upon every point, it is just the work for the student and practitioner. The anthor may rest assured that, although, in his preface, he objects to the "hackneyed expression of endeavoring to supply a want," this is just what he has done - supplied, and well supplied, a want: for no such book existed before in our language. - American Medical Journal.

\section{Anstie on Stimulants and Nareotics.}

Their Mutual Relations, with Special Resenrches on the Action of Alcohol, Ether, and Chloroform on the Fital Orgunism. By Francis E. Anstie, M.D., Assistant Physician to Westminster Hospital, Lecturer on Materia Medica and Therapeutics, \&c. \&c. Octavo. . . . . $\$ 3.00$

\section{Handy's 'Text-Book of Anatomy,}

And Guide to Dissections. For the use of Students of Medicine and Dentul Surgery. With 312 Illustrutions. Octavo. . . . \$t.0()

\section{IN PREPARATION.}

TFE PATE纴OGY OF TEE TEETE.

By Prof. WEed, of the University of Tiemna. Translated by Francis H. Brown, M.D., and Edited by Thos. B. Hıтснсоск, M.D., Professor of Dental Pathology and Therapentics in the Dental School of Harvard. University, Cambridge. IVith numerous Illustrations. 


\section{Cooley's Toilet and Cosmetic Arts.}

The Toilet and Cosmetic Arts, in Ancient and Modern Times. With a Review of the Different Theories of Beauty and copious allied Information, Social, Hygienic, and Medical, including Instructions and Cautions respecting the Selection and Use of Perfumes, Cosmetics, and other Toilet Articles; and a Comprehensive Collection of Formula, and Directions for their Preparation. By ArNold J. Cooley, Author of " Cyclopcedia of Receipts: Processes, Data, and Collateral Information, \&.., in the Arts and Manufactures." With INDEx to about 5000 Matters of Interest, Use or Caution. Demi-Octavo. . . . . . . . $\$ 3.00$

\section{Ott on the Manufacture of Soaps and Candles.}

Including the Most Recent Discoveries, embracing all kinds of Ordinary Hard, Soft, and Toilet Soaps, especially those made by the Cold Process; and the Modes of Detecting Frauds in the Manufacturing and the Making of Tullow and Composite Candles. By ADOLPH OTT, Practical and Analytical Chemist. 12mo. With Illustrations. (Just ready.) $\$ 2.50$

The author, in preparing this volume, has been careful to give a clear and concise pocount of the art of soap and candle making, as now practised, so as to make the work as practical in its character as possible. Appropriate illustrations have been adued, and critical explanations of the rarious manipulations and mechanical arrangements, by which they are effected. Much new matter has also been incorporated in the book, never before published.

\section{Piesse's Whole Art of Perfumery.}

A NEW REVISED AND ENLARGED EDITION.

And the Methods of Obtaining the Odors of Plants; with Instructions for the Manufacture of Perfumes for the Handkerchief, Scented Powders, Odorous Vinegars, Dentifrices, Ponatums, Cosmetics, Perfumed Soaps, \&.c.; to which is added an Appendix, on Preparing Artificial Fruit Essences, \&c. By G. W. Septinus Pirsse, Analytical Chemist. A new American from the Third London Edition. 12mo. With Numerous Illustrations. . . . . . . . . . $\$ 3.00$

Dr. PIEsse's volume covers the entire ground of the subjost upon which it treats. It is full of Useful and Curious Information, including also many Valuable Formulæ; an I will be found of equal importance and interest to the practical man as to the general reader.

\section{Overman's Practical Mineralogy, Assaying and Mining.}

With a Description of the Useful Minerals, and Instructions for Assaying, according to the simplest Methods. By Frederick Ovenusan, Mining Engineer, \&c. $12 \mathrm{mo}$.

The object of this volume is to place before the public the characteristics and use? of minerals, in a popular style, avoiding, as far as possible, the use of scientific and technical terms. The subject is divided into three parts: - Mineralog 5 , or a Description of the Appearance of Ninerals, with the localities in which they may or have bec'n found; Assaying, or an Investigation of the value of Mlinerals, by means which are within the reach of every one; and Practical Mining in its simplest form.

\section{Piggott on Copper Mining and Copper Ore.}

Containing a full Description of some of the Principal Copper Mines of the United States, the Art of Mining, the Mode of Preparing the Ore for Market, \&ic., \&r. By A. Snowden Piggotт, ML.D., Practical Chemist. $12 \mathrm{mo}$. 


\section{Morfit's Chemical and Pharmaceutical Man- ipulations.}

A Manual of the Chemical and Chemico-Mechanical Operations of the Laboratory. By CAMPBeld Morfit, Professor of Analytic and Applieal Chemistry in the University of Maryland, assisted by Clarence Morfin, Assistant Melter and Refiner in the United States Assay Office. The Second Edition, Revised and Greatly Enlarged, with over 500 Illustrutions. Octavo.

$\$ 5.00$

"The arrangement of the whole is snch, that every student will be able to go through the work witlout a guilie to lead him, provided the necessary apparatus are at his command. But even without them, a careful stuity of the book will give the attentive student a very nsetil insight in all the nianipulations of the pharmacentical chemist, and thousands, no doubt, who are prevented f:om attending the schools of ruarmacy asd abemigtrs, will gladly avail thenselves of the ouly means left them for self-improvement. The amuunt of perseverance and industry displayed in the getting up of this work is truly astonishing, the clearneas of exuression in every seutence, and the accurateness of the 500 illustrations, are sbove prajse. "Morfit's Mianipulalions' rauges iu utility immediately after the United States Dispensatory." - Chemical Gazet'e.

\section{Branston's Hand-Book of Practical Receipts.}

A Manual. for the Chemist, Druggist, Medical Practitioner, \&c., \&c. Comprising the Officinal Medicines, their Uses, and Modes of Preparation, and Formulæ for Trade Preparations, Mineral Waters, Pouders, Beverages, Dietetic Articles, Perfumery, \&c.; with a Glsssary of Medical and Chemical Terms, and a Copious Index. By Thoms F. Branston. From the Seconal Revised and Enlarged Edition. $12 \mathrm{mo}$.

\section{Campbell's Manual of Scientific and Practical Agriculture.}

A Systematic Arrangement of all Scientific Knowledge bearing in any manner on the great work of Farming. For the use of Schools and Farmers. By Prow. J. L. Campbell, of Washington College, Va. 12mo. With Illustrations.

$\$ 1.50$

This volume has been prepared to supply those already engaged in the eulture of the soil with a guide, the study or perusal of which will enable them to improve upnn the oll systern, or rather want of system, whick has worn out so much of our best land, and has rendered the pursuit, in so many instances, unprofitable; and also to meet the uemanls of teachers for a text-book of the right kind, which will give the student sucb information as will tit him for the intelligent pursuit of agriculture as a business.

\section{Darlington's Flora Cestrica;}

Or, Herborizing Companion. Containing all the Plants of the Middle States, their Linncean Arrangement, a Glossary of Botanical Terms, a complete Index, etc. By IVilliam Darlington, M.D. The Third Edition, enlarged. $12 \mathrm{mo}$. . . . . . . . \$2.25

\section{Miller on Alcohol, and Lizars on Tobacco.}

Alcohol: Its Place and Power. By James Miller, F.R.S.E., Professor of Surgery in the University of Edinburgh; President of the MedicoChirurgical Society; Author of MrLler's Principles and Practice of Surgery, etc., etc. The Use and Abuse of Tobacco. By John Lizars, late Professor of Surgery to the Royal College of Surgeons, etc., etc. The Tu'o Essays in One Volume. $12 \mathrm{mo}$.

$\$ 1.00$

The first of these treatises was prepared by Prof. Miller at the request of the Scotsish Teuperance League, who were anxious to have a work of high autho-ity, presenting the medical view of the Temperanee question. It has passed through a great number of editions in Scotland, and has had a large sale in this country. The second was prepared by Prof. Lizars to show the pernicious consequences of excessive or babilual smoking. If purchased in quantities, either together or separately, by Tem. perance or other societies, theg will be furnished at a reduced price 


\section{NEIV STDENHAI SOCIETY'S PUBLICAPIONS.}

\section{LINDSAY \& BLAKISTON, Philadelphia,}

Are now prepared to receive suhseriptions for the publications of THE NEw SrdenHax Societr for the new year, at Ten Dollars, payable in eurreney, and invariably in advance, and to furnish any of the previous years at the same rate and on the me terms.

The Practienl Character and Permanent Value of these publieations, and the very low price at which they are furnished, commend them to the favorable attention of the Medical Profession in the United States.

\section{WORIS ALREADY PUBLISHED.}

1859. (First Iear.)

Tot. 1. Did.Ay on Infantile Syphilis.

2. Fooch on Diseises of Women.

3. MEMolrs on lijhtleria.

4. VAX DER liozk on tho Spinal Cord, \&c.

5. Jonogkaphs (Kusmmal \& Tenuer, Græfe, Witguer, sc.)

1560. (Second Iear.)

Voz. 6. Dr. Brigh on Abdominal Tumors.

7. Frerichs on Diseases of the liver. Vol.'T.

8. A Y FaRBOOK for 1859.

9. Arras of Portraits of Skin Diseases. (1st Fasciculus.)

\section{1. (Third Year.)}

VoL. 10. A YEARBOok for 1560.

11. Moxorraphs (Cyermak, Dnsch, Radicke, \&c.)

12. Casper's Forensic Merlicine, Vol. I.

14. AThas of Purtraits ot Slin Diseases. (2nd Fisciculus.)

1862. (Furth Fear.)

Tol. 13. Frericus on Distises of the Liver. Vol. II.

15. A YEARBOOK for 1961 .

16. Casper's Forensic Medicine. Vol. I1.

17. Atras of Portraits of Skin Disenses. (3d Fasciculus.)

\section{3. (Fifth Fear.)}

Vol. 18. KrAMER on Disenses of the Ear.

19. A YEARDOOK for 1862 .

20. NeUDAUER and Yoget on the Urine.

\section{S64. (Sixth Jear.)}

Vou. 21. Casper's Forensic Iledicine. Tol. III.

2.) Dondirs on the Accommutition aud Refraction of the Eye.

23. A Yearbook for 1863.

24. ATLAS of Portraits of Skin Diseases. (4th Fasciculus.)
1S65. (Seventh Tear.)

Tol. 25. A YEAEBOOK tor 1864

26. Cisper's Forensic II dicine. Tol. IV.

27. Aruas of Portraits of Skin Diseases. (5th Fasciculus.)

\section{6. (Eighth Fear.)}

VoL. 2S. BERxU1z \& froupil on the Diseases of Women. 29. Athas of loriraits of Skin Diseases. (6th Fusciculns.)

30. IIEBRA on Diseases of the Skin. Vol. T.

31. Bensutz \& Guepil on Diseases of Wumen. Vol. 11.

1867. (Finth Fear.)

Voz. 32. A Bievial Retrospect of Medicine and Sur-

gery.
33. Griesixger on Jlentil Pathology and Therapeutics.

34. Atlas of Portraits of Skin Disenses. (7th Fiasciculus.

35. Troussedu's Clinical Medicine. Vol. I. 1s68. (Tenth Jear.)

Voz. 36. The Collected Works or Dr. Addison.

37. IIEBRA on SlituD istatses. Vul. 11.

38. IAverreatx's Trutise on Sypluilis, Vol. I.

39. Athas of Portraits of Skin Diseases; ( 8 th Fasciculus.)

40. A Catalooue of the Portraits issued in the Society's Atlas of Shin Diseases. (Part T.) 1569. (Eleventh Year.)

Fol. 41. Trousseau's Clinical Medicine. Translated and edıted by Dr. Ruse Cormack. Vol. 1I.

42. Biennial Retrospfet of Medicine and SCRQERY, for $186 \overline{-}-8$. Elited liy Dr. Anstie, Dr. Barnes, Mr. Ilolmes, Mr. Power, Mr. Cirter, and Dr. Underwod.

43. Lancereacx on Syphilis. Trumslated by Dr. Whitley. Vol. II., completing the Hirrk.

44. A Ninth Fasciculus of the Atras of PoBTRAITS OF SEIN DJSEASES

\section{WORKS FOR 1870. - 12th YEAR.}

Trousseau's Clinical Medicine. Vol, III.

Stricker's Manual of Histology. Vol, I.

Niemeyer on Phthisis.

A Tenth Fasciculus of the Atlas of Skin Diseases.

Subscribers at a distance can have their Volumes mailed to them, postage paid, as they appear, by - emitting $\$ 150$ in addition to the subscription price for the year.

Non-Subscribers can obtain the books published during any one year by s'ubscribing and paying for that year $\$ 10.00$, but no volumes or books can be had otherwise or separately excet the following:

The Year 15ooks for $1859,{ }^{\prime} 60,{ }^{2} 61$, and $' 62$, for . . . . . . . . . . . \$10 50

Portxaits of Skin Diseases. Fasciculi 1 to 9, for . . . . . . . . . . 4230

A Descriptivn Catalogue of the Society's Atlas of Portraits of Diseases of the Skin, and their last BEPORT, will be furnished gratis npon application, 


\section{AMERICAN \& BRITISH PERIODICALS SUPPLIED BY}

\section{IINDSAY \& BIAKISTON, \\ P H I L A D E L P H I A.}

Subscriptions Payable in Advance.

NAMES.

WHERE PUBLISHED, PRIVE

\section{IEARLY.}

Per Arman

The Pennsylvania IIospital Reports,

The Physician's Visiting List, for 1871, prices reduced.

Clinical Society's Transactions,

St. Andrew's Medical Graduates' Aszociation Reports,

St. Thomes' Hospital Reports,

The Liverposl " "

St. George's " " "

St. Bartholomew "

Guy's

Obstetrical Society's Transactions,

Pathological "

Medico-Chimergical Society's Transactions,

The New Sydenham Society's Publications. 3 to 4 volumes published annually,

Vols. 1 \& 2, each, See Catalogue.

London,

London, :

"

16

66

66

66

66

6

66

$\$ 400$

HALF-YEARLI.

Braithwaite's Retrospect of Medicine and Surgery, . Ranking's Half-Yearly Abstract "

\section{QUARTERLY.}

American Journal of the Merlical Sciences, .

British and Foreign Medico-Chirurgical Review, .

The Dublin Quarterly Journal of Medicine. .

American Journal of Syphilography and Dermatology,

Journal of Anatomy and Plysiology. \$2.00 per Number,

Microsioprcal Journal,

The American Joumal of Obstetrics,

Journal of Psychological Medicine,

The New Orleans Journal of Medicine,

The Ophthalmic Hospital Reports,

Reprint, - . 260

1000

250

Pbiladelphia, . . 500

London, . . . 1000

Dublin, : . 10 U:

New York, $\quad: 300$

London, : : 800

New York, : $\quad 400$

500

New Orleans, . . 500

BI-MONTHLY.

The American Journal of Pharmacy,

MONTHLY.

Journal of the Gynæcological Society,

The London Lancet,

The Pharmacentical Journal,

The Medical and Surgical Journal,

The Practitioner. Edited by F. E. Anstie, M. D., .

The Americun Chemist,

The Medical Archives,

The Richmond and Lourisville Medical Journal,

The Chicago Medical Journal,

New York Medical Journal, .

London, .

Philadelphia,

300

Boston - . 500

Reprint, . . 500

London, : : 600

Edinburgh, : 800

Reprint, . . 400

New York, $\quad 500$

St. Louis, • • 300

Louisville, . $\quad 500$

3 ल0

400

\section{SEMI-MONTHLY.}

The Medical Times,

The Mcdical Record,

The London Lancet,

" Medical Times and Gazette, .

"British Medical Journal,

"Medical and Surgical Reporter,

"* Medical and Surgical Journal,

\section{WEEKLY.}

Philadelphia,

New York,

London, - . . 1200

- " . . 1200

Philadelphio - 1200

400 


\section{Marshall's Physiological Diagrams.}

-

\section{I.IFE-SIZE, AND BEAUTIFULLY COLORED.}

On account of their large size and the great distinctness of the figures on them, there has been a growing demand in this country for these Maps for the Lecture Roou and for lecturing from in MEDicAl as well as oTHER ScHools. In orcer to supply this demand on more favorable terms, we have recently completed an arrangement with the publishers in London, by which we can sell them to the trade and others at a reduced price and on better terms than heretofore.

The series, illustrating the whole Human Bolly, are life-size, each map printed on a single sheet of paper, made specially for the purpose, 7 feet long and 3 feet 9 inches broad, colored in fac-simile of the Original Trawings. There are nine diagrams, as follows:

No. 1. The Skeleton and Liganents. $\quad$ No. 5. The Lymphaties or Absorbents.

No. 2. The Mluseles and Joints, with Ani- No. 6. The Digestive Organs. mal Jlechanics.

No. 3. The Viscera in Position. - The No. 8. The Organs of Sense and Toice. Structure of the Lungs.

No. 4. The lleart and principal Bloodressels.

Prepared under the direction of Jomn Marshall, F.R.S., F.R.C.S., Professor of Surgery, University College, and Surgeon to University College Hospitul.

Price of the Set, Nine Maps, in Sheets, $\$ 50.00$ " " " " " handsomely Mounted on

Canvas, with Rollers, and Varnished, . . . . . $\$ 80.00$

Though designed more especially for purposes of general education, supplying an acknowledged necessity of moderu teaching, these diagrams will be found not inapplicable to the requirements of professed Medical Schools, affording. as they do, a correct preliminary view of the various systems and organs in the human body. For PUBLIC School Pulposks, for Lectures at Literary, Scikstric, and other Institutes, they will be found invaluable; and also to stulents of Artistic Anatomy, imparting, as they do, when suspended on the walls of the Lecture-hall, School-room, or Studio, a familiar acruaintance with the whole human system.

\section{An Explanatory Key' to the Physiological} Diagrams. By John Marishald, F.R.S., F.R.C.S., \&c. Octavo. Paper covers. . . . . . . . 50 cts.

\section{Description of the Human Body.}

Its Structure and Functions. Illustrated by Physiological Diugrams, Designed for the Use of Teachers in Schools and Young Men destined for the Medical Profession, and for popular Instruction generally. New Eilition. By Johr Marshall, F.R.s., F.R.C.S., Profes:or of Surgery, University College, and Surgeon to the University College Hospital.

The work contains 260 quarto payes of Text, bound in cloth, and 193 Colured Illustrutions, arranged in Nine Folio Diugrams, carejully colored and reduced firom Prof. Harshall's large work. 22 vols. Cloth. . \$10.00

\section{Beeton's Book of Household Management.}

With Sanitary, Medical, and Legal Nemorandums; also, a History of the Properties and Uses of all things connected with Home Life and Comforts. With 72 eolored and 600 other Illustrations. By Mrs. J. BeEton. A New Edition, 1100 pages. Demy-octavo, half-roan. - $\$ 3.25$ 


\section{PRICES REDUCED OF}

\section{LINDSAY \& BLAKISTON'S I'HYSICIAN'S VISITING LIST.}

\section{IN PREPARATION FOR 1872.}

"The simplest of all the visitisg uists published, it must continue to holl, what it now has, the preference over all other forms of this indispensable cotnpanion for the l'hsician."- New lork Hed. Journal.

\section{CONTENTS.}

1. Tuble of Sigus, cr Guide for Registering Visits, En- 7. Memoranda pages for every montly in the year. gingements, \&c.

2. An Almanist:

3. Marshall II:all's Rearly Metbor in Asphyria.

4. Joisonsa and their Alitidisteg

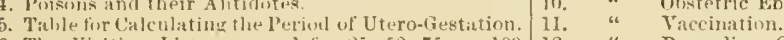

6. The Visiting List arranged for $25,50,75$, or 100 12. " Recording Obstetric Cases, Denths, and Patients.

\section{SIZES A ND PRICE.}

For 25 Patients weokly. Tucks, pockets, and pencil, . . . . . \$1 00

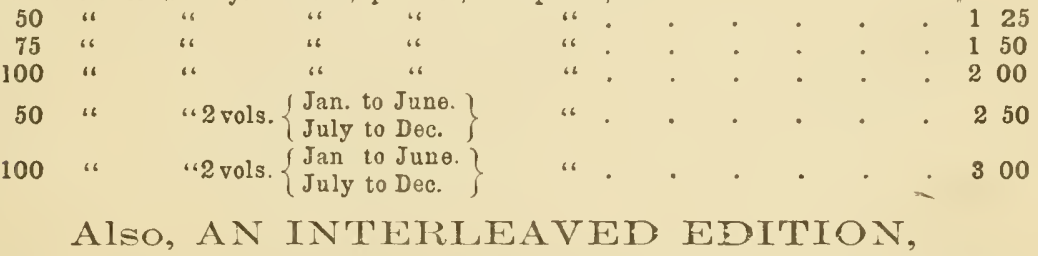

for the use of Country Physicians and others who compound their own Prescriptions, or furnish Medicines to their patients. The arlditional pages can also be used for Special Mrmoranda, recording important cases, \&c., \&c.

For 25 Patients weekly, interleaved, tucks, pockets, etc., . . . . \$1 50

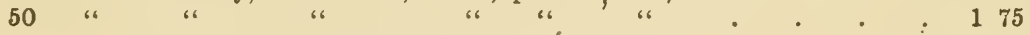

50 " " 2 vols $\left\{\begin{array}{l}\text { Jan. to June. } \\ \text { July to Dec. }\end{array}\right\}$ " " * . 300

This $V$ Isiting List has now been published for Twenty Years, and has met with such uniform and hearty approval from the Profession, that the demand for it has steadily increased from year to year.

The Publishers, in order to still further extend its circulation and usefulness, and to keep up the reputation which it has so long retained, of being

\section{THE CHEAPEST AND BEST,}

as well as the OLDEsT VIsitring List published, have now made a very considerable reduction in the price.

It can be procured from the principal booksellers in any of the large cities of the United States and Canada, or copies will be forwarded by mail, free of postage, by the Publishers, upon receipt by the of $0^{t}$ he retail price as annexed.

In ordering the work from other booksellers, order

\section{Iindsay d Blakiston's Physician's Visiting List.}

And in all cases, whether ordering from the Publishers or otherwise, pecify the size, style, \&c., wanted. 

postrye, mon receipt of the retril prite.

ArTrEn's Science and Practice of Medicine, from the fifth Loudon edition, with Arerican additions equal to 500 pages of the Lond. edit. 2 vols. 8 vo. $\$ 12.00$ Althans's Medical Electricity. New Edition

Acton $n$ the Reproductive Organs Anstie on Stimulants and Narcotics

Byford on Diseases of Women

3.00

5.00

Bildle's Materia Jledica. Third edition. 4.00

Branton's Practical Receipts . . . 1.50

Beale's How to Work the Microscope . T.50

Microscope in Practical Medicine $\mathbf{7} .00$

Beale on the Kidneys, Urine, \&c. 400 Ill. 1000

Beasley's Book of Prescriptions . . 4.00

Drugrist's Receipt Book . $\quad 3.50$

Barth \& Roger"s Auscultation . . 1.25

Birch on Constipation. Tlird edition 1.00

Bouchardat's Annual of Therapeuties, \&c.1.50

Bull's Maternal Management of Chililren J.25

Braithwaite's Epitome, 2 vols.

lieale's l'rotoplasnı, 2d edition, plates, $\mathbf{3 . 0 0}$

On Disease Germs, do.

1.75

Hodge on Foeticirle. Paper 30 cts. : cloth, 50 Cbambers' Lectures. Renewal of Lite 5.00 Chew on Medical Education . . 1.00 Coben's Therapeutics of Inlalation . 2.50

Cazbaux's Obstetrical Text-Book, the

Fifth American edition, very much enlarged. 175 Illustrutions

Sleaveland's Pronouncing Medical Lexicon. Eleventh edition

Carson's History Medical Department University of Pennsylvania

Goff's Physician's Day-Book, Ledger, \&c.12.00 Dixon on Diseases of the Eye, new ed. 2.50 Kirkes I Inual of Physiology, 7 th Lon.ed. 5.00 l.egg's Guicle to the lixamination of Urine, 75 Durkee on Gonorrhoe and Syphilis, Fifth edition, revised and improved

Fuller on Rheumatism. A new edition.

Garratt on Medical Batterics

Grares' Clinical Merlicine. New ed.

Greenhow on Chronic Bronchitis

Gross' Anıerican Medical Biography

Ileadland on the Action of Medicine

Ileath's Diseases and Injuries of the Jaws

Ilewitt on the Diseases of Women.

Hilles' Pocket Anatonist

Holmes' Surgical Diccases of Children Ilufeland's Art of Prolonging Life.

Hillier's Diseases of Children

Mackenzie on the Laryngoscope, Rhinoscopy, and Diseases of the Throat

Morris on Searlet Fever

Míns' \& PePper's Treatise on Diseases of Children. Fourth edition, rewritten and very much enlarged

Marson's Practice of Medicine

Mentenball's Medical Student's Vade Meeum. Eighth erlition

rennsylvania Hospital Reports. Vols. 1 and 2, each

2.00

6.00

2.00

3.50

3.00

6.00

5.00

1.00

9.00

1.25

3.00

3.00

1.50

Paget's Leetures on Surgical Pathology 6.00

Pereira's Physician's Prescription Book 1.25

Pbysician's Visiting List. Various sizes end priecs. See Catalogue.
Prince's Orthopedic Surgery

Prinee's Plastic Surgery. Illustrated 1.50

Renouard's History of Medicine . $\quad 4.00$

Radelitte on Epilepsy, Pain, Paralysis, \&c. 2.00

Lawson's Complete Text-Book of Dis-

eases and Injuries of the Eye

2. 50

Ruppaner on Laryugoseopy, \&c. . . ₹00

Ryan's Philosophy of Marriage . 1.00

Reese's Aualysis of Physiology . . . 1.50

Reese's American Mellical Formulary 1.50

Sydenham Society's Biemial lietrospect 2.0C

Stille's Epilemic Meniugitis . . 2.00

Sansom on C'bloroform, its Action, Modes

of Administration, \&c., \&c. . . . 2,00

Stokes on Diseases of the Heart . 300

Spratt's Obstetric Tables. 4to, eol'd Pl. 8.00

Skoda on Auscultation and P'ercussion I 5,0

Sydenham Society's Pub. Per year, 10.00 'Tyson's Cell Doctrine. Illustrated. , 2.00 Tanner's l'ractice of Medicine, 5th ed. 6.00 Tanner on Diseases of Children

Tanner's Index of Diseases . . . 8.00

Tanner's .II'molanda of Poisons . . .50

Tronssenu's Élinisal Medicine. Vols.

1, 2 , and 3 , each . . . . . 5.00

Thompson on Pulnonary Consumption 1.25

Tilt's Elements of Female Hygiene . $\mathbf{1 . 5 0}$

Taylor's Novement Cure . . . 1.50

Virchow's Cellular Pathology . . 5.00

Soelberg Wells on the Eye. 2d London

Edition, with Illustrations . . . 6.50

Walker on Intermarriage . . . . 1.50

Wythe's P'ocket Dose and Symptom Book. Fighth edition. . . 1.00

Waring's I'ractical Therapcutics : 6.00

Walton's Operative Ophthalmic Surgery 4.00

Watson s I'racticc, Abridged . . 2.00

Wright on llend-Aches . . . . 1.25

Wells on Long, Short, and Weak Sight. Third edition

Weber's Clinical Hand-Book of Auscultation and Pereussion

Ilaris' Dictionary of Medical Terminology and Dental Surgery

Harris' Principles and Practice of Dental Surgery. Tenth edition $\quad 6.00$

Bond's Dental Nledicine . . . . $\mathbf{8 . 0 0}$

Rohertson on Extracting Teeth . . . 1.50

Taft's Operative Dentistry . . . 4.50

Fox on the IImman Teeth . . . 4.00

Richardson's Mechanical Dentistry . 4.50

Handy's Text-Book of Anatomy . . 4.00

Coles on Deformities of the Mouth. $2 d$

Edition. Colored Illustrations $\quad 2.50$

Tomes' System of Dental Surgery . 4.50 SCIFNTIFIC.

Cooley's Toilet and Cosmetic Arts . . 3.00

Ott on the Manufacture of Soap and Candles

Piésse on Perfumery. A new edition 3.00

Overman's Mineralogy, Assaying, and Mining

Piggott on Copper Mining . • • $\quad 1.50$

Morfit's Chemical Manipulations . $\quad$. 5.00

Darlington's Flora Cestrica . . . . 2.25

Miller \& Lizars on Alcohol and Tobacco 1.00 


$3=0$ 
WQRKS BY DR. LIONEL S. BEALE, F.R.S., Eellow of the Royal College of Physicians, Physician to King's College Ilospitat.

Seventh Thousand. Four Hundred Illustrations, some Colored.

\section{HOW TO WORK WITH THE MICROSCOPE.}

This work is a complete manual of microscopical manipulation, and contain 3 a ful description of many new processes of investigation, with lirections for examining objects under the highest porrers, and for taking photographs of microscopic ohjec:s Octavo. Cloth. Price, \$7.50.

\section{ON KIDNEY DISEASES, URINARY DEPOSITS, AND CALCULOUS}

DISORDERS: - The Third Edition.

Including the Symptoms. Diagnosis, and Treatment of Urinary Diseases. With full Directions for the Chemical and Microscopical Analysis of the Urine in Health and Disease. 70 Plates, 115 fignres, copied from Niture. Octaro. Price, $\$ 10.00$.

THE USE OF THE MICROSCOPE IN PRACTICAL MEDICINE.

For Students and Practitioners, with full directions for examining the various secre-

tions, \&o., in the Microscope. 4th Edition. 500 Illustrations. Octavo. Price, \$7.00.

\section{MEDICAL PROGRESS. IN MEMORIAM ROBERT BENTLEY TODD.}

$$
\text { Price, } 50 \text { cts. }
$$

LIFE, MATTER, AND MIND; OR PROTOPLASM.

With Original Observations on Minute Structure, und numerous New Colored Drawings. A Nrw L'dition, very much enlarged. Eight Plates. Price, \$

DISEASE GERMS : THEIR SUPPOSED NATURE. With Colored Plates. An Original lnrestigntion with the did of the llighest Powers yet made.

This work contrins a critical examination of Dr. Tyndall's statements con. cerning 'Dust amel Discase.' 12mo. Cloth. P'rice, \$1.75.

DISEASE GERMS: THEIR REAL NATURE. Twenty-four Plates.

An Original Invistigntion. Twenty-four Plates, sixteen of which are Colored.

Including the minute pathology of contagious diseases. Price, St.00.

Now ready. Four Colored Plates.

\section{PHYSICAL THEORIES OF LIFE: THEIR INFLUENCE UPON RE. LIGIOUS THOUGHT. P'rice $\$ 2,50$.}

With Obscrvations on the llypotheses recently advoeated by Tyndall and Huxley.

ON DISEASES OF THE LIVER, AND THEIR TREATMENT.

A Sccond Edition, nuch enlarged, of the Author's Work on the Anatomy of the Liver. Numerous Plates. I'reparing.

THE ARCHIVES OF MEDICINE. Part XVII. now ready. Price, $\$ 1.50$. Contents.-Medicine nnder the Romans-Action of Eyelicts in Expiration; F. C. Donders, Utrecht-Lacuno and Canaliculi-Nerres in Mesenteric Glands; Dr. Popper, St. Peterșburg-German Criticism and British Medical Science-Homology of Coracoacromial Ligament; J. B. Perrin-Sulpho-carbolates, by Dr. SansomStrueture of the Liver; Editor-Sputum in Plithisis.

DISEASE : ITS NATURE AND TREATMENT. Preparing.

New Researches on Inflimmation and Fever, and on the Nature of Contagion. With Observations on the Cattle Plague and on Cholera. Numerous Colored Plates. Being the Third Course of Lectries delivered at Oxford by direction of the Radcliffe Trustees.

All these Wurks contain the results of the Author's oriyinal inecstigations. They are Illustruted with upwards of 2000 Engravings, copierl from the actual objects, rill of rahich have been drazou on wood by the Author himself, or under his immediate superintendence.

LINDSAY \& BLAKISTON; Publishers, Phiadelphia. 